Copyright

by

Laura Jean Loiacono

2010 
The Dissertation Committee for Laura Jean Loiacono

certifies that this is the approved version of the following dissertation:

\section{Measurement of the Muon Neutrino Inclusive Charged Current Cross Section on Iron Using the MINOS Detector}

Committee:

Sacha Kopp, Supervisor

Karol Lang

Christina Markert

Jack Ritchie

Christopher Sneden 


\title{
Measurement of the Muon Neutrino Inclusive Charged Current Cross Section on Iron Using the MINOS Detector
}

by

Laura Jean Loiacono, BS

\author{
Dissertation \\ Presented to the Faculty of the Graduate School of \\ The University of Texas at Austin \\ in Partial Fulfillment \\ of the Requirements \\ for the Degree of
}

Doctor of Philosophy

The University of Texas at Austin

May 2010 
To my family. 


\section{Acknowledgments}

This thesis would not have been possible without the help and support of many people. I would like to thank my committee for their patience and understanding regarding the lateness of this thesis and for their useful comments. I would like to thank all of those who have contributed to the large amount of work that has made this thesis possible and those who have shared their knowledge and advise including Jesse Chvojka, Jason Koskinen, Rustem Ospanov, Zarko Pavlovich, Jasmine Ratchford, and Bob Zwaska among many others.

I especially want to thank my adviser, Prof. Sacha Kopp for his enormous support, advise, time and patience, especially during these last months. I really cannot thank him enough.

And finally, I thank my family. Thank you for your great support and and unlimited amount of understanding. No words can express my gratitude to you. Thank you from the bottom of my heart. 
The University of Texas at Austin

May 2010 


\section{Measurement of the Muon Neutrino Inclusive Charged Current Cross Section on Iron Using the MINOS Detector}

Publication No.

Laura Jean Loiacono, Ph.D.

The University of Texas at Austin, 2010

Supervisor: Sacha Kopp

The Neutrinos at the Main Injector (NuMI) facility at Fermi National Accel-

erator Laboratory (FNAL) produces an intense muon neutrino beam used by the Main Injector Neutrino Oscillation Search (MINOS), a neutrino oscillation experiment, and the Main INjector ExpeRiment $\nu$-A, (MINER $\nu \mathrm{A})$, a neutrino interaction experiment.

Absolute neutrino cross sections are determined via $\sigma_{\nu}=N_{\nu} / \phi_{\nu}$, where the numerator is the measured number of neutrino interactions in the MINOS 
Detector and the denominator is the flux of incident neutrinos. Many past neutrino experiments have measured relative cross sections due to a lack of precise measurements of the incident neutrino flux, normalizing to better established reaction processes, such as quasielastic neutrino-nucleon scattering. But recent measurements of neutrino interactions on nuclear targets have brought to light questions about our understanding of nuclear effects in neutrino interactions.

In this thesis the $\nu_{\mu}$ inclusive charged current cross section on iron is measured using the MINOS Detector. The MINOS detector consists of alternating planes of steel and scintillator. The MINOS detector is optimized to measure muons produced in charged current $\nu_{\mu}$ interactions. Along with muons, these interactions produce hadronic showers. The neutrino energy is measured from the total energy the particles deposit in the detector.

The incident neutrino flux is measured using the muons produced alongside the neutrinos in meson decay. Three ionization chamber monitors located in the downstream portion of the NuMI beamline are used to measure the muon flux and thereby infer the neutrino flux by relation to the underlying pion and kaon meson flux. This thesis describes the muon flux instrumentation in the NuMI beam, its operation over the two year duration of this measurement, and the techniques used to derive the neutrino flux. 


\section{Contents}

Acknowledgments $\quad$ v

Abstract vii

1 Neutrino Interactions with Matter 1

1.1 Neutrino Interactions . . . . . . . . . . . . . . . . 3

1.1.1 Low Energy . . . . . . . . . . . . . 5

1.1.2 Intermediate Energies . . . . . . . . . . 6

1.1.3 High Energies . . . . . . . . . . . . . . . . 17

1.2 Past Cross Section Experiments . . . . . . . . . . . . . 19

1.3 Goal of this Thesis . . . . . . . . . . . . . 26

2 The MINOS Experiment 28

2.1 NuMI Beamline . . . . . . . . . . . . . . . . . 29

2.1.1 The NuMI Target . . . . . . . . . . . . . 33

2.1.2 The NuMI Horns . . . . . . . . . . . . . . . 35

2.1.3 Decay Volume and Hadron Absorber . . . . . . . . . 41

2.1.4 Variable Energy Beam . . . . . . . . . . . . 43

2.1.5 Beamline Instrumentation . . . . . . . . . . . 48 
2.2 The MINOS Detector . . . . . . . . . . . . . 56

2.2 .1 Calibration . . . . . . . . . . . . . 58

2.2.2 Event Reconstruction . . . . . . . . . . . . . . . . . . . 60

2.2 .3 Event Selection . . . . . . . . . . . . . . . . . . 67

2.3 Summary . . . . . . . . . . . . . . . . . . 70

3 Muon Flux Measurement System $\quad 74$

3.1 Muon Flux System . . . . . . . . . . . . . . . . . 75

3.1 .1 Ionization Scale . . . . . . . . . . . . . . . . 79

3.1.2 Gas Supply System and Purity . . . . . . . . . . . 81

3.1.3 Ambient Pressure and Temperature . . . . . . . . . 83

3.1.4 Rate-Dependent Effects _. . . . . . . . . . . . 84

3.2 Measurements of Backgrounds . . . . . . . . . . . . 88

$3.2 .1 \quad$ No-Target Spills . . . . . . . . . . . . . . . . . . . 89

3.2.2 Beam-Missing-Target Spills _ . . . . . . . . . . . 90

3.2 .3 Summary . . . . . . . . . . . . . . . . . . . 93

3.3 System Performance . . . . . . . . . . . . . . . 96

3.3.1 Long-Term Performance . . . . . . . . . . . . . 100

3.4 Horn Current Scan Data Sets . . . . . . . . . . . . . . 110

3.4 .1 Systematic Effects . . . . . . . . . . . . 116

$3.4 .2 \quad$ Summary . . . . . . . . . . . . . . . . 120

4 Neutrino and Muon Flux Calculation and Uncertainty 126

4.1 Monte Carlo Simulation of the Beamline . . . . . . . . . 127

4.2 Meson Production in Nuclear Targets . . . . . . . . . . . . 133

4.3 Meson Decay to Neutrinos and Muons . . . . . . . . . . . . . 142

4.4 Muons in the Muon Monitors . . . . . . . . . . . . 155 
4.4.1 Simulation of Hadron Absorber and Muon Filter . . . . 155

4.4 .2 Ionization by Muons . . . . . . . . . . . . . 157

4.4.3 Cross Check of Absorber Backgrounds . . . . . . . . 163

4.5 Summary ....................... 166

5 Neutrino Flux Measurement 172

5.1 Introduction . . . . . . . . . . . . . . . . 172

5.2 Muon Alcove Acceptances . . . . . . . . . . . . . . . . . . . . 175

5.3 Horn Current Scans . . . . . . . . . . . . . . . . . . 176

5.4 Empirical Fit to Particle Production . . . . . . . . . . . . 185

5.5 Constraints on the Fit . . . . . . . . . . . . . . 187

5.6 The $\nu_{\mu}$ Flux Measurement . . . . . . . . . . . . . . . . . . . . 189

5.7 Summary . . . . . . . . . . . . . . . . 195

$6 \quad$ Neutrino Flux Systematics $\quad 197$

6.1 Systematic Errors . . . . . . . . . . . . . . . . 197

6.1.1 Systematics from Backgrounds . . . . . . . . . . 198

6.1.2 Systematic Uncertainty from the $K^{+} / \pi^{+}$Ratio . . . . 201

6.1.3 Systematic Uncertainty from the $\pi^{+} / \pi^{-}$Ratio . . . . . 203

6.1.4 Charge Collection ............... 205

6.2 Normalization . . . . . . . . . . . . . . . . 210

$\begin{array}{lll}7 & \text { Cross Section Measurement } & 219\end{array}$

7.1 Introduction . . . . . . . . . . . . . . . . . . 219

7.2 The Method . . . . . . . . . . . . . . . . 221

7.3 Systematics . . . . . . . . . . . . . . . . . . . . . . 229

7.3.1 Detector and Event Selection . . . . . . . . . . 229 
7.3.2 The Neutrino Flux . . . . . . . . . . . . . . . . . 231

7.4 Measurement of $\sigma\left(\nu_{\mu} F e \rightarrow \mu^{-} X\right) \ldots \ldots \ldots \ldots \ldots$

7.5 Cross Checks . . . . . . . . . . . . . . 236

8 Conclusions and Outlook 240

8.1 Summary . . . . . . . . . . . . . . . . . 240

8.2 Future Hardware Improvements . . . . . . . . . . . . . . . 244

8.3 Future Analysis Improvements . . . . . . . . . . . . . . . 245

8.4 Future MINER $\nu$ A Experiment . . . . . . . . . . . . . . 248

A Pressure and Temperature Corrections for the Hadron and Muon Monitors 251

A.1 Introduction . . . . . . . . . . . . . . . . 251

A.2 In-situ Corrections . . . . . . . . . . . . . . 252

A.2.1 Pressure Corrections . . . . . . . . . . . . . 255

A.2.2 Temperature Corrections . . . . . . . . . . . . . 255

A.3 Summary . . . . . . . . . . . . . . . . . . . 261

B Alignment of the Proton Beam at the Target 264

B.1 The Hadron Monitor . . . . . . . . . . . . . . . . . 265

B.2 The Muon Monitors . . . . . . . . . . . . . . . . . 271

B.3 Beam Position Corrections for the Muon Monitor Signals . . . 273

C Flux Measurement Systematics 282

$\begin{array}{ll}\text { D Cross Section Measurement Systematics } & 289\end{array}$

$\begin{array}{ll}\text { Bibliography } & 303\end{array}$ 
Vita

312

xiii 


\section{Chapter 1}

\section{Neutrino Interactions with Matter}

The discover of the large energy releases in $\alpha, \beta$ and $\gamma$ decay in radioactivity ushered in the discovery of new forces, the strong and weak nuclear forces, expanding our understanding of nature being governed by just the gravitational and electromagnetic forces. The process of beta decay, furthermore, ultimately enlarged our picture of the particles that make up the universe, introducing the concept of the neutrino. In beta decay, a neutron decays to a proton and an electron and a neutrino are emitted

$$
n \rightarrow p+e^{-}+\bar{\nu}_{e}
$$

In early experiments, all that was seen is the emission of the electron. If, indeed, only the electron were emitted and the law of conservation of energy held then the energy of the electrons emitted from beta decays should be a fixed value. Measurements of the electron energy spectrum showed that the 
it was in fact not discrete but continuous. For the next 15 years, the law of conservation of energy was in question, until 1930 when Wolfgang Pauli proposed [1], in a letter to his colleagues attending a physicists' workshop, that a neutral, spin 1/2 particle was emitted along with the electron, which he named "neutron". Pauli's particle necessarily had a mass on the same order as the electron. The neutron is discovered a couple years later and Pauli's "neutron" is renamed "neutrino" by Enrico Fermi. Using the quantum mechanics developed by Dirac, Fermi builds the theory of of beta decay, also known as the theory of the weak interaction.

The introduction of Pauli's neutrino also ushered in a new understanding of the weak interaction itself - namely that it is very weak. A particle as light as an electron should readily be detected if it interacts strongly with other matter. Its not detection signals the feebleness of the weak interaction. In fact, in 1934, H. Bethe and R. Peierls [2] showed that the neutrino interaction cross section, $\sigma$, probability of interaction, was less than $10^{-44} \mathrm{~cm}^{2}$ for a $6 \mathrm{MeV}$ neutrino, which corresponded to a penetration of $10^{16} \mathrm{~km}$ in matter. They concluded that, even though Fermi's model showed an increase in cross section with energy, "it seems highly improbable that, even for cosmic ray energies, $\sigma$ becomes large enough to allow the process to be observed" [2]. Direct detection of the neutrino occurs only in 1956 when Fredrick Reines and Clyde Cowan observed the reaction

$$
\bar{\nu}_{e}+p \rightarrow n+e^{+}
$$

by placing a detector of liquid cadmium chloride and scintillator next to a nuclear reactor which produces a $\bar{\nu}_{e}$ flux on the order of $10^{13} \nu / \mathrm{s} / \mathrm{cm}^{2}$. Their 
measured cross section was $11 \pm 2.6 \times 10^{-44} \mathrm{~cm}^{2}$. [3]. Such may be compared to strong interaction cross sections, $\sim 10^{-26} \mathrm{~cm}^{2}$, or electromagnetic cross sections, $\sim 10^{-30} \mathrm{~cm}^{2}$. Even today, the neutrino continues to be useful as a probe to measure the very feeble weak interaction.

\subsection{Neutrino Interactions}

In his theory of beta decay, Fermi used quantum mechanical perturbation theory to represent the interaction between the initial state before decay and the final state after the decay. He found the transition rate is given by

$$
W=\frac{2 \pi}{\hbar}\left|M_{i f}\right|^{2} \frac{d n}{d E_{f}}
$$

where $M_{i f}$ is the matrix element for the transition from an initial state, $|i\rangle$, to a final state, $|f\rangle$, and $d n / d E_{f}$ is the energy density of available final states. The matrix element is

$$
M_{i f} \equiv\langle f|V(r)| i\rangle=\int \psi_{f}^{*} V(r) \psi_{i} d^{3} \mathbf{r}
$$

Thus, it can be interpreted as the overlap integral of the initial- and final-state wave functions in the presence of the perturbing potential. In 3-dimensional Euclidean space the density of states can be written as

$$
\frac{d n}{d E_{f}}=\frac{p^{2} d \Omega}{(2 \pi \hbar)^{3}} \frac{d p}{d E_{f}} g_{f}
$$

where $E_{f}$ is the final state energy, which is the total energy in the center of momentum frame, $p$ is the final state momentum also in the center of 
momentum frame, $d \Omega$ is the solid angle containing all of the final-state particles and $g_{f}$ is the multiplicity of the final state spins.

The cross section is the transition rate, $W$, per incident particle flux per target particle, $\phi$. For a general reaction $a+b \rightarrow c+d$,

$$
W=n_{a} \sigma v_{i}
$$

is the number of interactions per target particle, where $n_{a}$ is the density of incident particles and $v_{i}$ is the relative velocity of $a$ and $b$. Using Equations 1.31.6 the cross section can be written as

$$
\frac{d \sigma}{d \Omega}=\frac{1}{(2 \pi)^{2} \hbar^{4}}\left|M_{i f}\right|^{2} \frac{p^{2} g_{f}}{v_{i} v_{f}}
$$

where conservation of energy gives $d p / d E_{f}=E_{c} E_{d} / E_{f} p=1 / v_{f}$, where $v_{f}$ is the relative velocity of $c$ and $d$ in the final state.

Fermi postulated that the matrix element should describe the strength of the weak interaction and it should be equal to a constant, $G_{f}$. This holds true for beta decay where the available energy is on the order of $\mathrm{MeV}$. In fact, the near-constant value of the matrix element $M_{i f} \sim G_{f}$ holds for neutrino energies up to $\sim 100 \mathrm{GeV}$, since the actual force carrier for the weak interaction, the $W^{ \pm}$or $Z^{0}$, have masses in the $80-90 \mathrm{GeV}$ range. Thus, Fermi's approximation of $M_{i f} \sim G_{f}$ is reasonable for the neutrino energies covered in this thesis. 


\subsubsection{Low Energy}

For beta decay we know that the interaction rate, $W$, is just $1 / \tau_{n}$, where $\tau_{n}$ is the lifetime of the neutron in its rest frame. The density of states is,

$$
\frac{d n}{d E_{f}}=\frac{1}{4 \pi^{4}} \frac{1}{\hbar^{6} c^{3}} \int_{0}^{p_{f}} p_{e}^{2}\left(E_{f}-E_{e}\right)^{2} d p_{e}
$$

Then the rate is

$$
W=\frac{1}{\tau_{n}}=\frac{\left|M_{i f}\right|^{2} m_{e}^{5} c^{10}}{2 \pi^{3} \hbar(\hbar c)^{6}}\left\{\frac{\int_{0}^{p_{f}} p_{e}^{2}\left(E_{f}-E_{e}\right)^{2} d p_{e}}{m_{e}^{5} c^{7}}\right\}
$$

giving a value for the matrix element according to:

$$
\left|M_{i f}\right|^{2}=\frac{2 \pi^{3} \hbar c(\hbar c)^{6}}{c \tau_{n}} \frac{1}{\int_{0}^{p_{f}} p_{e}^{2}\left(E_{f}-E_{e}\right)^{2} d p_{e}}
$$

This expression for the weak matrix element, along with a measurement of the neutron lifetime, may be used to compute the cross section for neutrino interactions with matter. The inverse beta decay is

$$
\bar{\nu}_{e}+p \rightarrow e^{+}+n
$$

In the case of a neutrino beam incident on a stationary target, the density of states is

$$
\frac{d n}{d E_{f}}=\frac{1}{(2 \pi)^{3}} p^{2} \frac{d p}{d E_{f}}
$$

where $p$ is the momentum of the outgoing positron in the center of momentum 
frame. Assuming, ultra-relativistic positrons, i.e. $v_{f}=c$, the cross section is

$$
\sigma=\frac{1}{\pi} \frac{1}{\hbar c^{2}}\left|M_{i f}\right|^{2} p_{f}^{2} \approx \frac{1}{4 \pi} \frac{1}{\hbar c^{2}}\left|M_{i f}\right|^{2}\left(m_{p} E_{\nu}\right)^{2}
$$

where $p_{f}$ is the momentum of the center of momentum frame. In the lab frame

$p_{f} \approx \frac{1}{2} m_{p} E_{\nu}$ where $E_{\nu}$ is the energy of the neutrino beam incident on a proton target. The interaction strength of these four particles should be the same in the forward and reverse directions so the matrix element for inverse beta decay is the same as in beta decay. So substituting Equation 1.10, and $\tau_{n}=$ 886 sec gives

$$
\sigma \simeq 12 \times 10^{-44} \mathrm{~cm}^{2} \quad \text { for } E_{\nu}=2 \mathrm{MeV}
$$

which is very close to the measurement of Reines and Cowan. The approximately linear dependence of $\sigma_{\nu}$ on the neutrino energy is demonstrated in a wide range of experiments as shown in Figure 1.1. For $\sim \mathrm{MeV}$ electrons and neutrinos, the deBroglie wavelength is $\sim 10^{-11} \mathrm{~cm}$, which is much larger than the size of the nucleus, $\mathrm{R} \sim 10^{-13} \mathrm{~cm}$. Thus, for inverse beta decay using $\sim \mathrm{MeV}$ electrons and neutrinos the nucleus looks like a point particle.

\subsubsection{Intermediate Energies}

In general, the structure of the interacting particles must be taken into account. In this case,

$$
\frac{d \sigma}{d \Omega}=\left(\frac{d \sigma}{d \Omega}\right)_{\text {point }} F\left(q^{2}\right)
$$

where

$$
F\left(q^{2}\right)=\int \rho(\mathbf{R}) e^{i \mathbf{q} \cdot \mathbf{R}} d^{3} \mathbf{R}
$$




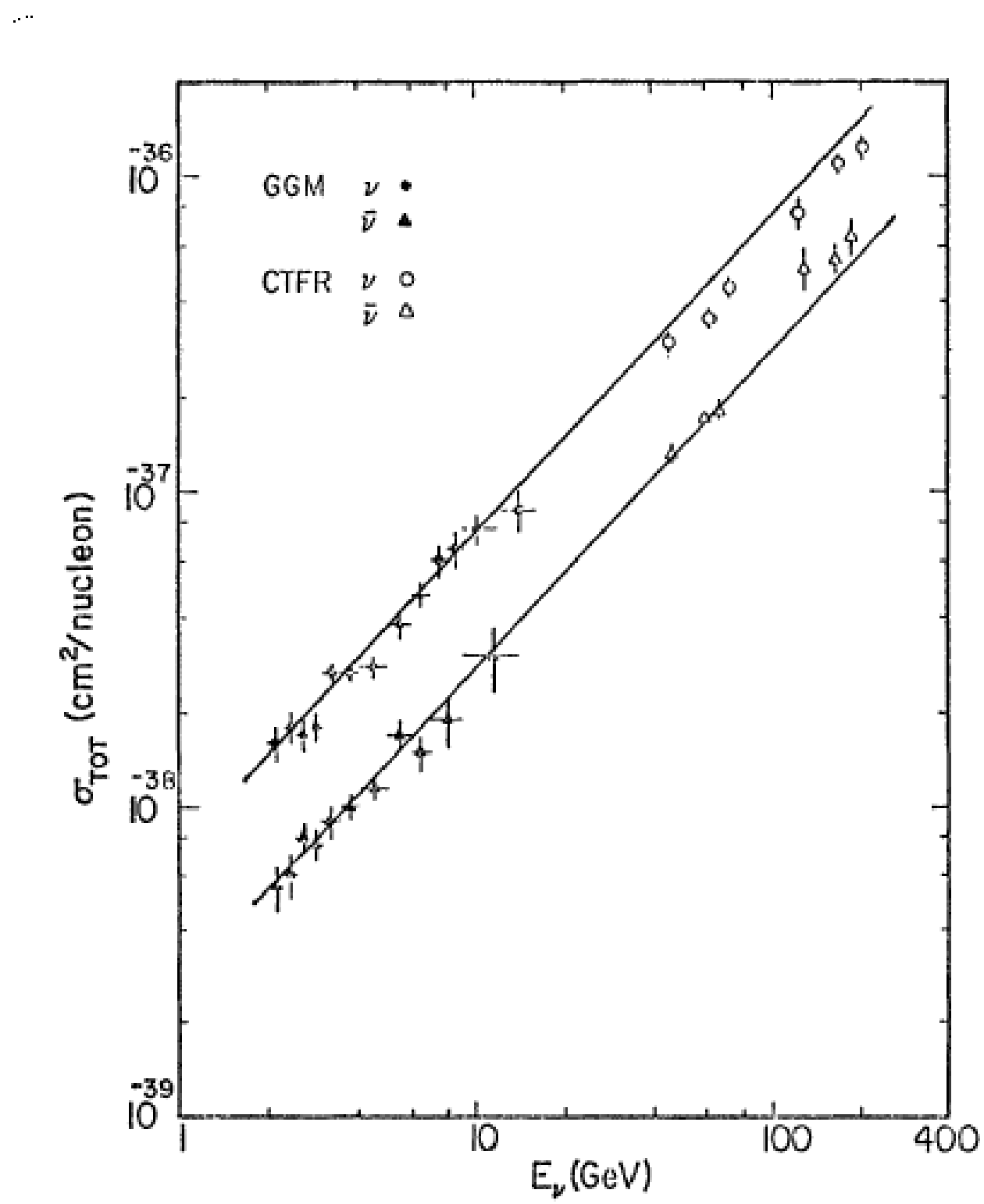

Figure 1.1: The measured charged current neutrino and antineutrino total cross sections (points) from the Gargamelle and CITFR experiments. The curves are best fits to the Gargamelle data extrapolated to higher neutrino energies. This demonstrates the linear rise in neutrino cross section with energy.(taken from Ref [4]) 
is the form factor and $\rho(\mathbf{R})$ is the density distribution of the scattering target. The form factor, Equation 1.16, is the Fourier transform of the density distribution in the target. The form factor provides information about the structure of the target particle. A measurement of the scattering cross section provides a measurement of the internal structure of the target.

Electrons and neutrinos with energies of $0.1-1 \mathrm{GeV}$ have wavelengths of order $\lambda \approx h c / p c \approx 1 \mathrm{fm}$ which is comparable to the size of the nucleus. Thus at these energies they are able to probe the structure of protons and neutrons with in the nucleus. For, electron scattering, the nucleon structure is defined by two form factors, $G_{E}\left(q^{2}\right)$, the electric form factor, and $G_{M}\left(q^{2}\right)$ the magnetic form factor. The form factors for the proton and neutron obey the scaling

$$
\left.G_{E}^{p}\left(q^{2}\right)=\frac{G_{M}^{p}\left(q^{2}\right)}{\left|\mu_{p}\right|}=\frac{G_{M}^{n}\left(q^{2}\right)}{\left|\mu_{n}\right|}=G_{(} q^{2}\right) \quad \text { and } \quad G_{E}^{n}\left(q^{2}\right)=0
$$

where $\mu_{p}$ and $\mu_{n}$ are the magnetic moments of the proton and neutron, respectively, and

$$
G\left(q^{2}\right)=\left(1+\frac{q^{2}}{M_{V}^{2}}\right)^{-2}
$$

Figure 1.2 shows measurements of the proton form factors from electron scattering. The dipole data fit well to the dipole form of Equation 1.18 where $M_{V}^{2}=0.71 \mathrm{GeV}^{2}$. Using equation, Equation 1.16, this dipole form factor can be interpreted as the Fourier transform of an exponential charge-magnetic moment distribution of the proton density given by $\rho(R)=\rho_{0} e^{-M_{V} R}$.

While $G_{E}\left(q^{2}\right)$ and $G_{M}\left(q^{2}\right)$ are the result of vector, parity conserving, currents in the interaction, neutrino interactions being weak interactions do not conserve parity. Thus, the description of neutrino interactions also in- 

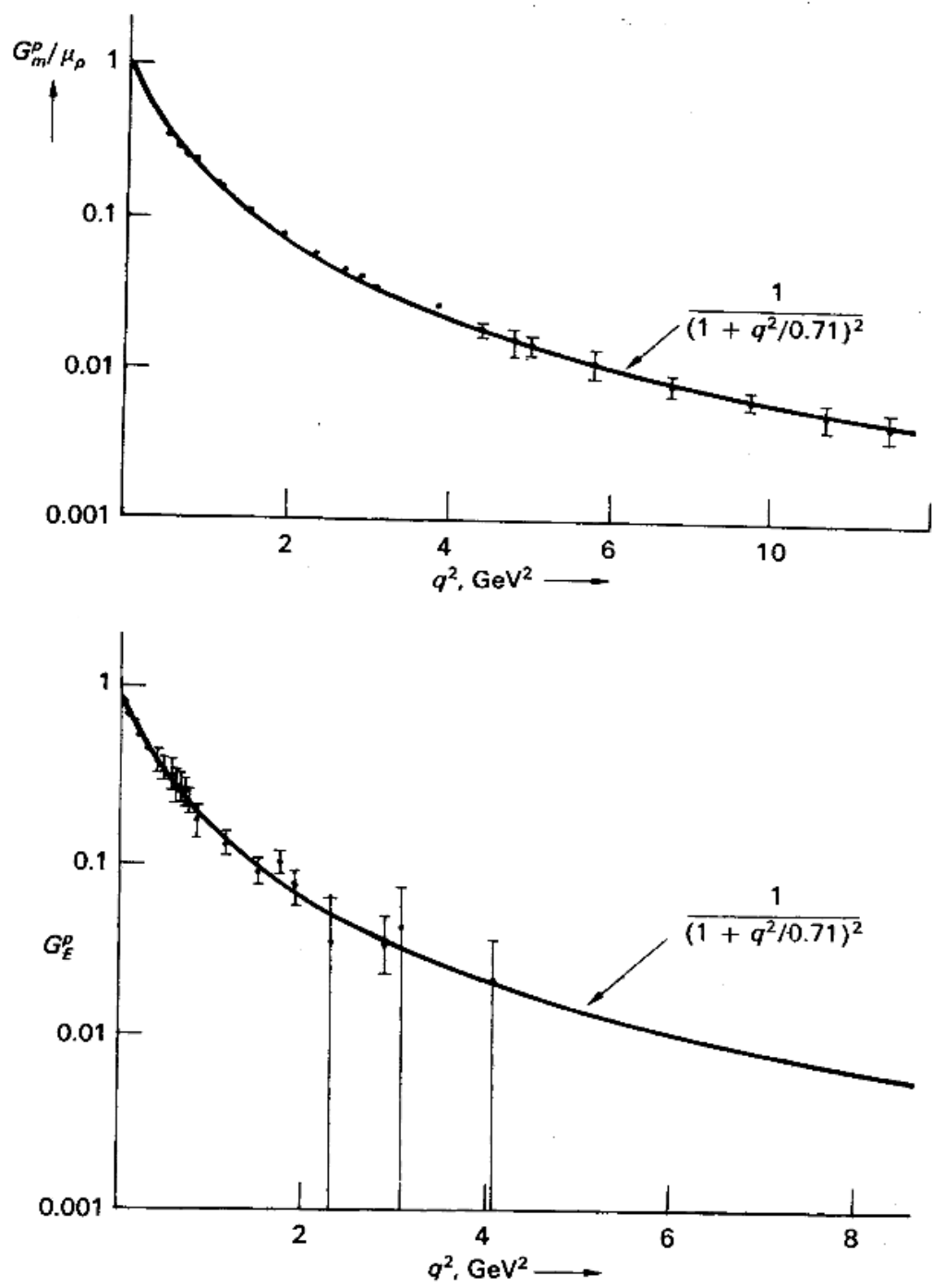

Figure 1.2: The measured (points) proton electric and magnetic form factors from electron scattering. The fit (curve) shows the dipole dependence of the form factors. (taken from Ref [5]) 
volves axial vector currents. The combination of the vector and axial vector currents allows for neutrinos of the proper helicity, left-handed neutrinos and right-handed antineutrinos. As a result, neutrino interactions in this intermediate energy region, also called quasielastic interactions, are described by the vector form factors in Equation 1.17 and also an axial vector form factor approximated as

$$
F\left(q^{2}\right)=F_{A}(0)\left(1+\frac{q^{2}}{M_{A}^{2}}\right)^{-2}
$$

where $F_{A}(0)=1.267$ is the axial coupling derived from beta decay experiments and $M_{A}$ is called the axial mass. The full mathematical description of the neutrino cross section in this formalism can be found in Ref [6].

In neutrino scattering experiments, the vector form factor measurements from electron scattering are utilized such that the only free parameter is $M_{A}$. Figure 1.3 shows the quasielastic cross section as a function of $q^{2}$ from the MiniBoone Experiment. They have fit their data to the form of Equation 1.19 to determine the value of the axial mass $M_{A}$. Figures 1.4 and 1.5 show charged current quasielastic cross sections from numerous neutrino scattering experiments on a variety of targets. The cross sections are determined from measurements of the axial mass parameter. The data largely agree with the dipole form factor description of the target nuclei structure.

An added complication to the formulation of the cross section for neutrino scattering in this region is the production of resonances which occurs for neutrino energies $\sim 0.5-10 \mathrm{GeV}$. Resonances occur when the bombarding neutrino has enough energy to excite the nucleon into one of the known excited states, or resonances. The resonance decays producing one or more pions in the final state. This is shown in Figure 1.6 for electron scattering on protons. 


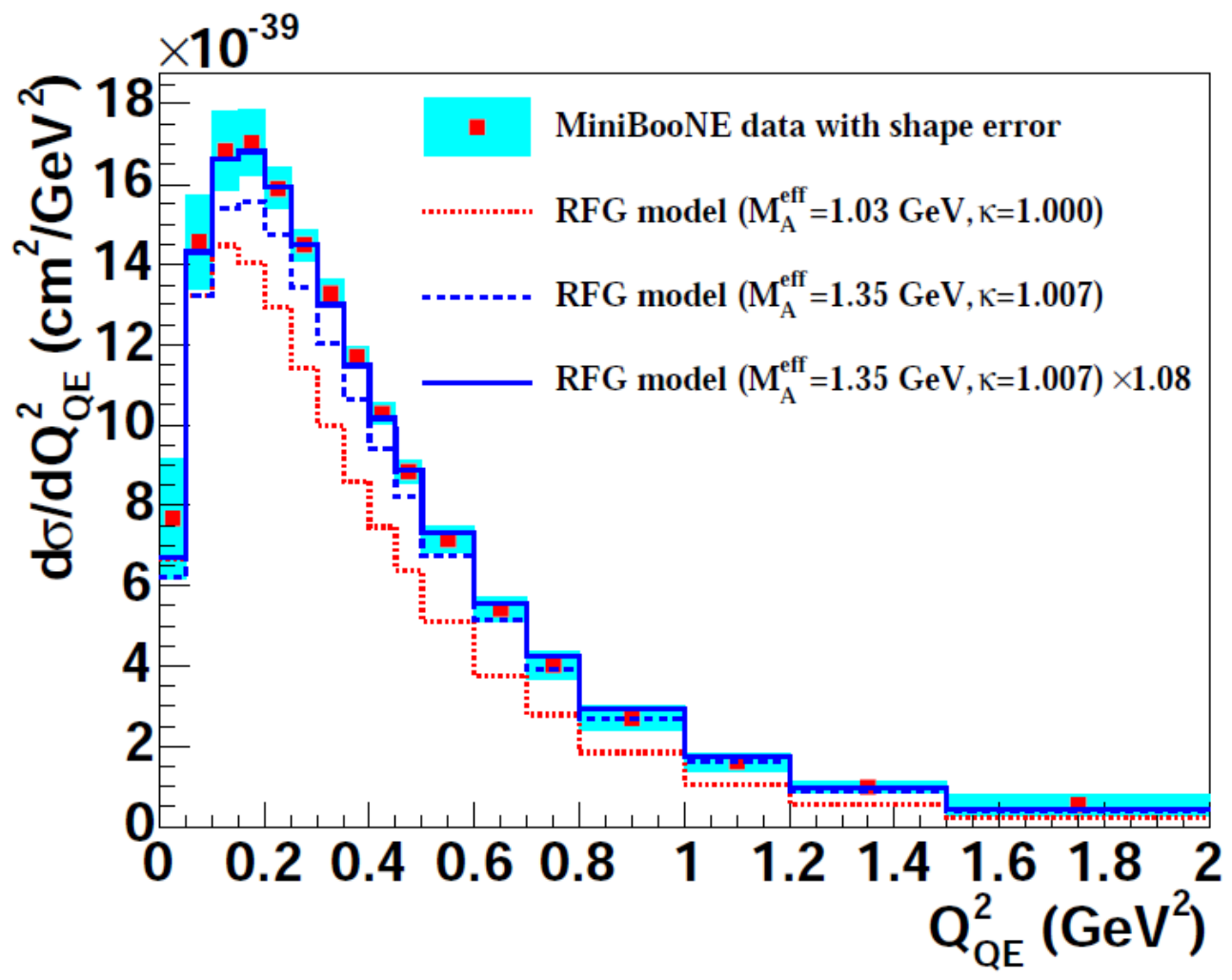

Figure 1.3: The neutrino quasielastic cross section as a function of $q^{2}$ measured by the Miniboone experiment. Shown are fits to the theoretical Relativistic Fermi Gas Model for neutrino scattering with various values of the axial mass $M_{A}$ parameter. They found that when including an extra parameter, $\kappa$, which they describe as an empirical Pauli-Blocking parameter, they achieved the best fit to their data. Refer to Ref [7]. 


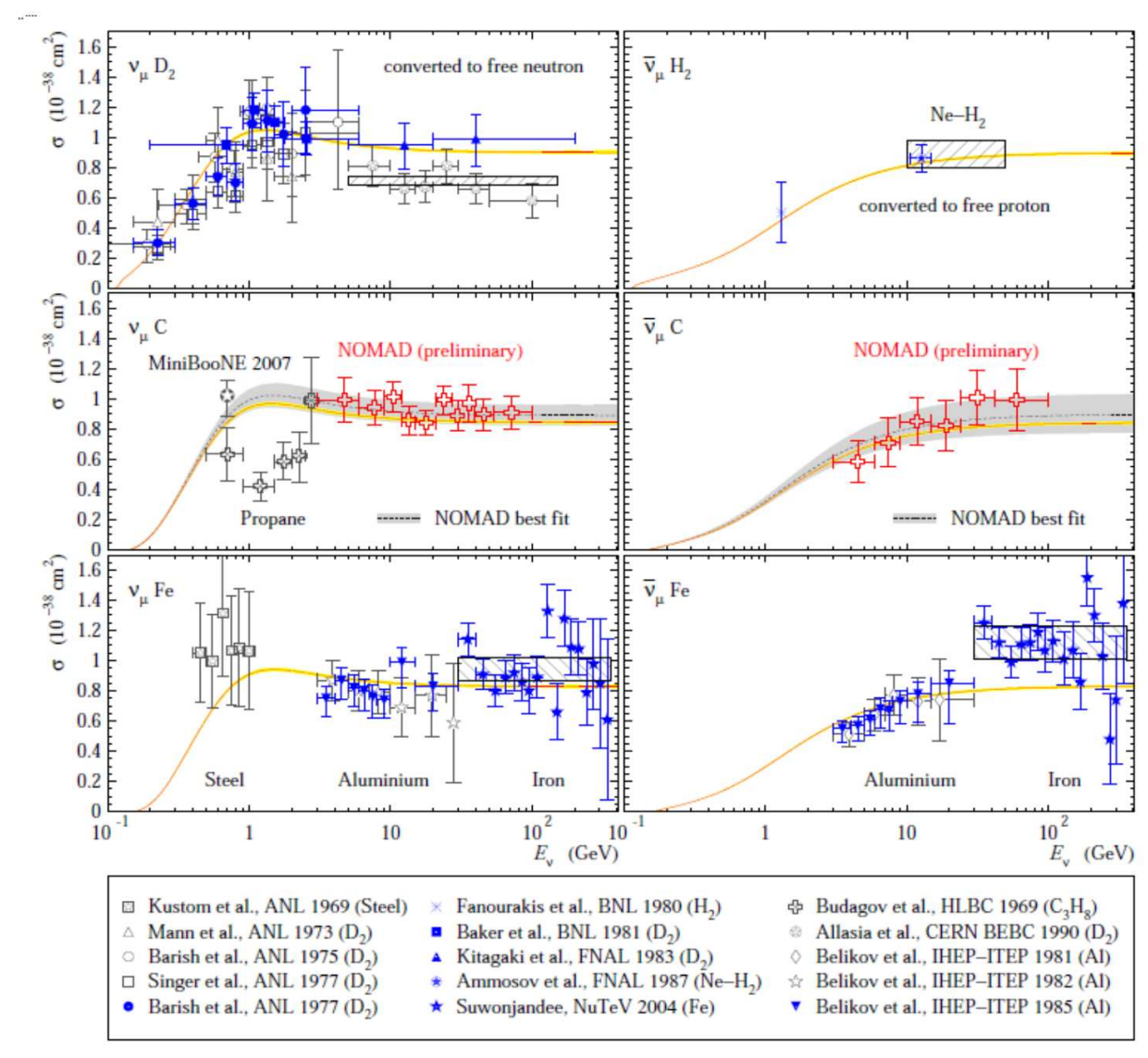

Figure 1.4: Muon neutrino, $\nu_{\mu}$ and antineutrino, $\bar{\nu}_{\mu}$, charged-current quasielastic cross section measurements from numerous neutrino scattering experiments which used various target materials as a function of the neutrino energy. The curves are theoretical predictions from the model utilized in Ref [8]. The model assumes an axial mass of $M_{A}=0.999 \pm 0.011 \mathrm{GeV}$. (taken from Ref [8]). 


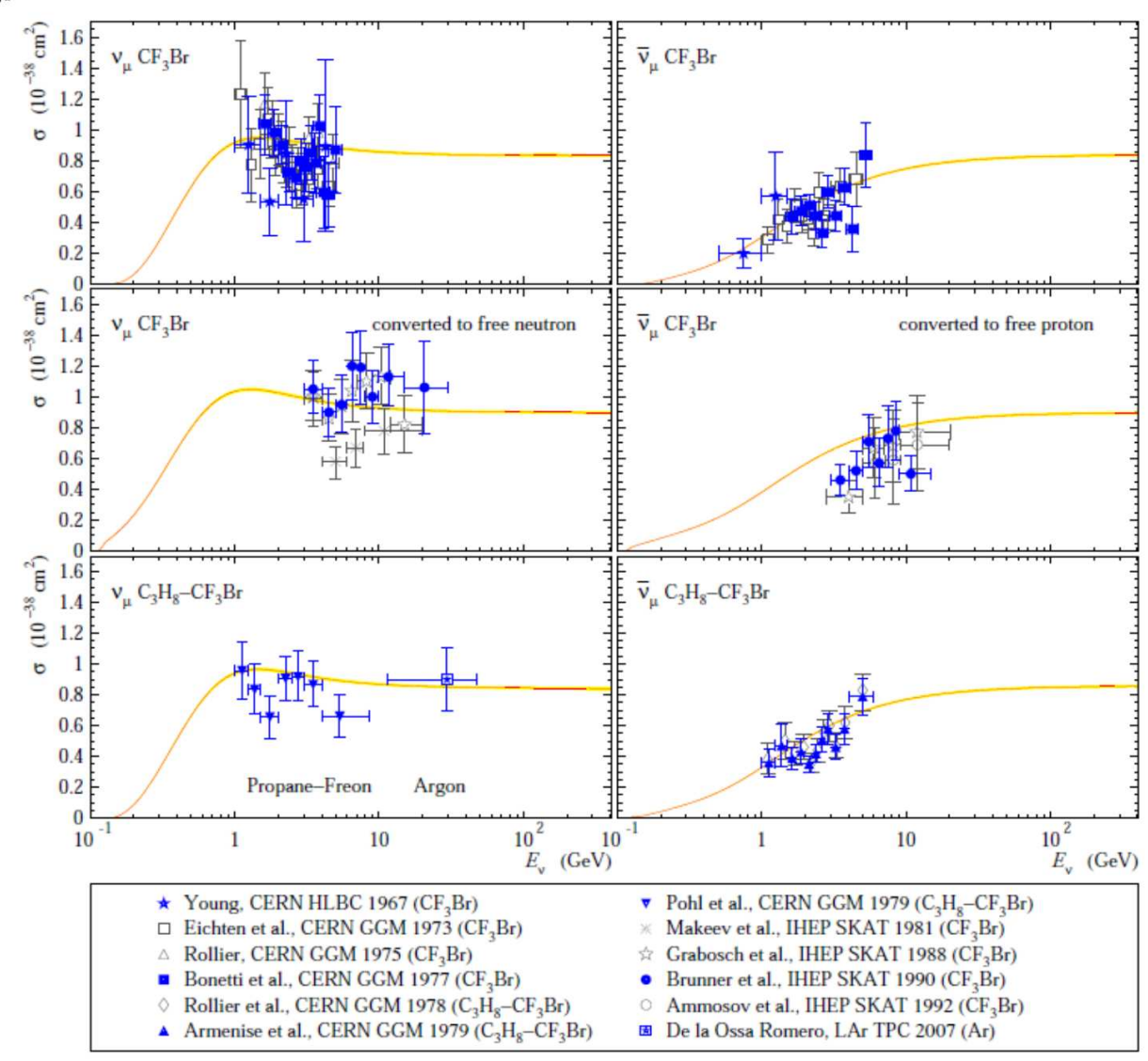

Figure 1.5: Muon neutrino, $\nu_{\mu}$ and antineutrino, $\bar{\nu}_{\mu}$, charged-current quasielastic cross section measurements from numerous neutrino scattering experiments which used various target materials as a function of the neutrino energy. The curves are theoretical predictions from the model utilized in Ref [8]. The model assumes an axial mass of $M_{A}=0.999 \pm 0.011 \mathrm{GeV}$. (taken from Ref [8]). 


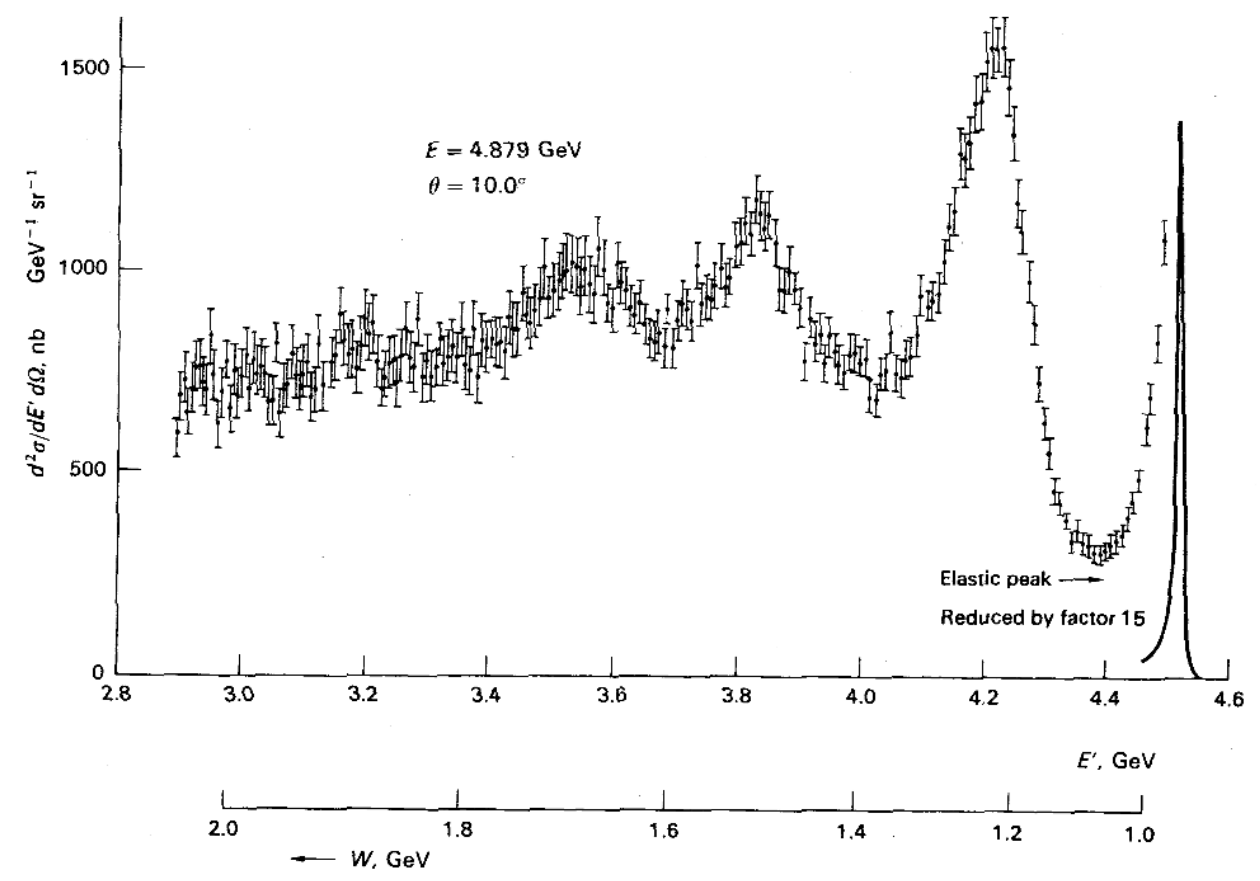

Figure 1.6: The differential cross section for incident electrons of $E=$ $4.879 \mathrm{GeV}$ on protons as a function of $E^{\prime}$, the energy of the scattered electron, and $W$, the mass of the produced hadron state. The peaks at $W=1232,1450$, and $1688 \mathrm{MeV}$ correspond to nucleon resonances. (taken from Ref [5].) 


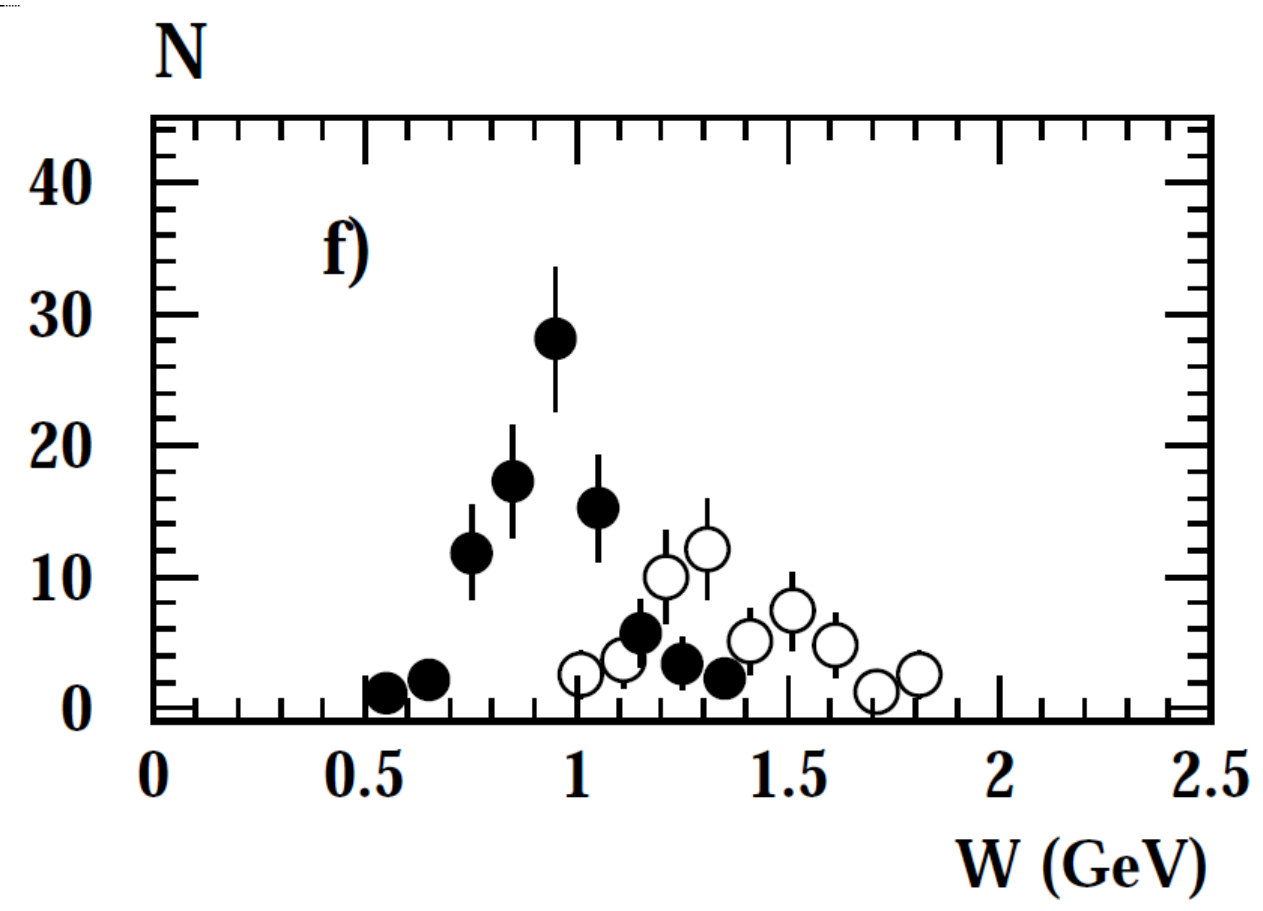

Figure 1.7: The mass, $W$, distribution of the hadronic system for $\nu_{\mu} N \rightarrow$ $\mu^{-} N^{\prime}(\pi)$. The open circles correspond to one pion and the full circles correspond to zero pions. (taken from Ref. [9]) 


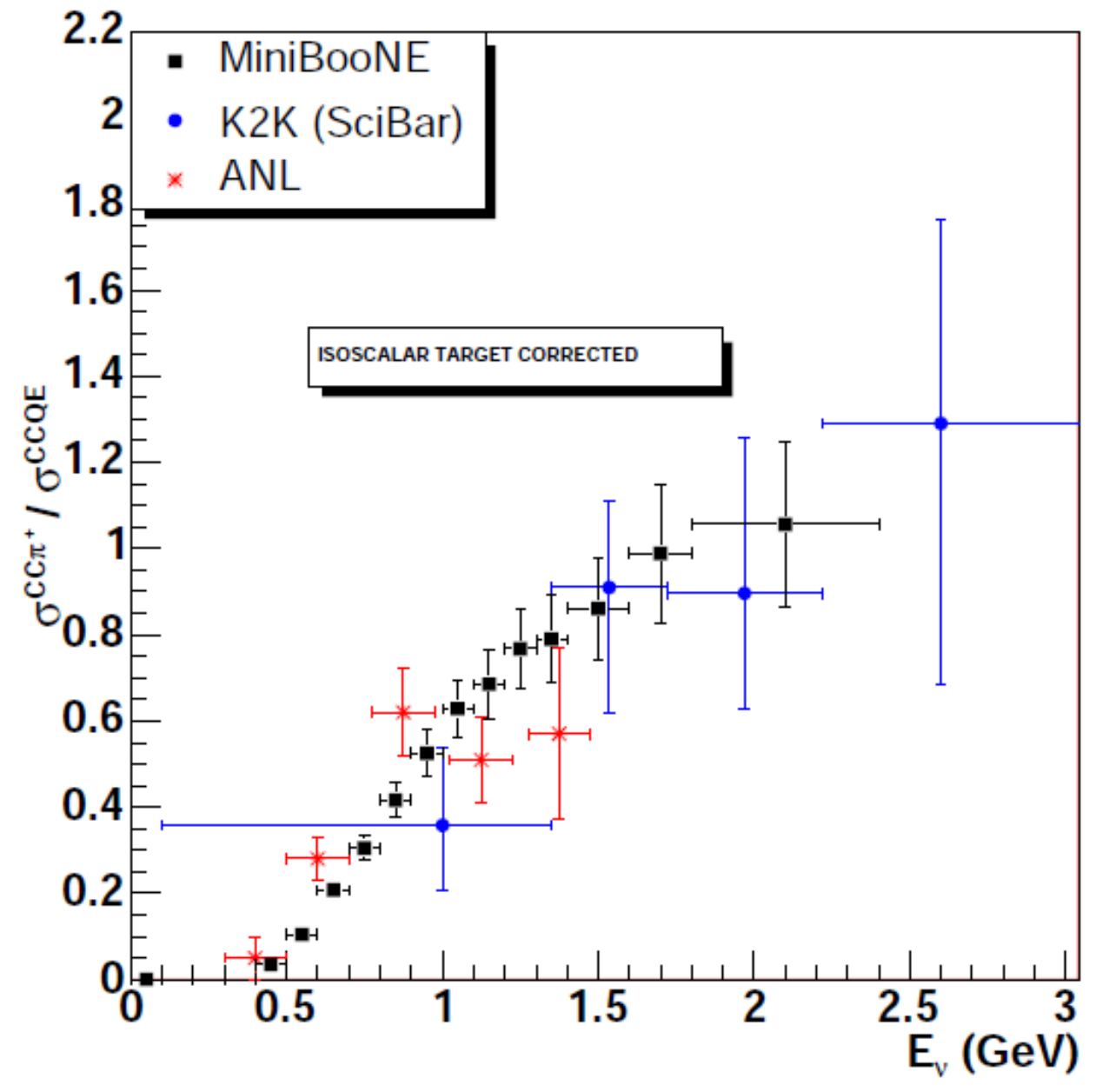

Figure 1.8: Measurements by the Miniboone collaboration and others, furthermore, show that resonance production increases, as expected, as the neutrino energy is raised above $\sim 0.5 \mathrm{GeV}$. (taken from Ref. [10]) 
Plotted in the figure, is the mass, $W$, of hadronic states created in the electron proton interaction. The strong peak at $W=940 \mathrm{MeV}$ corresponds to elastic scattering, ep $\rightarrow e p$. The peaks at different masses, $W$, indicate nucleon resonances produced by the electron scattering correspond to nucleon resonances, such as the at $\Delta(1232)$ etc.

Similar observations are made in neutrino-nucleon scattering. Data from the SKAT [9] neutrino experiment, for example, at $E_{\nu} \sim 3 \mathrm{GeV}$, show a similar behavior in $\nu_{\mu} N \rightarrow \mu^{-} N^{\prime}(\pi)$ interactions. As shown in Figure 1.7, if no final state pion is produced, the reaction is a quasi-elastic $\nu_{\mu} n \rightarrow \mu^{-} p$ and the final mass, $W$, of the hadronic state is peaked at $940 \mathrm{MeV}$. If a final state pion is observed the mass distribution, $W$, has additional peaks such as the $\Delta(1232)$. As shown in Figure 1.8 Measurements by the Miniboone collaboration and others, furthermore, show that resonance production increases, as expected, as the neutrino energy is raised above $\sim 0.5 \mathrm{GeV}$. However, the pion(s) may not be detected due to absorption of the pion by the struck nucleus or absorption in the passive detector material. [7]

\subsubsection{High Energies}

Above $\sim 10 \mathrm{GeV}$, the cross section is dominated by deep inelastic scattering, where the electrons and neutrinos scatter off partons in the nucleons. The nucleus breaks up as a result of the collisions and a shower of hadrons are produced. This type of scattering is parameterized by parton structure functions which describe the structure of bound partons. Figure 1.1 shows that, even as the neutrino energy goes from the few $\mathrm{GeV}$ to the $100 \mathrm{GeV}$ range, the cross section continues to increase approximately linearly with energy as predicted 


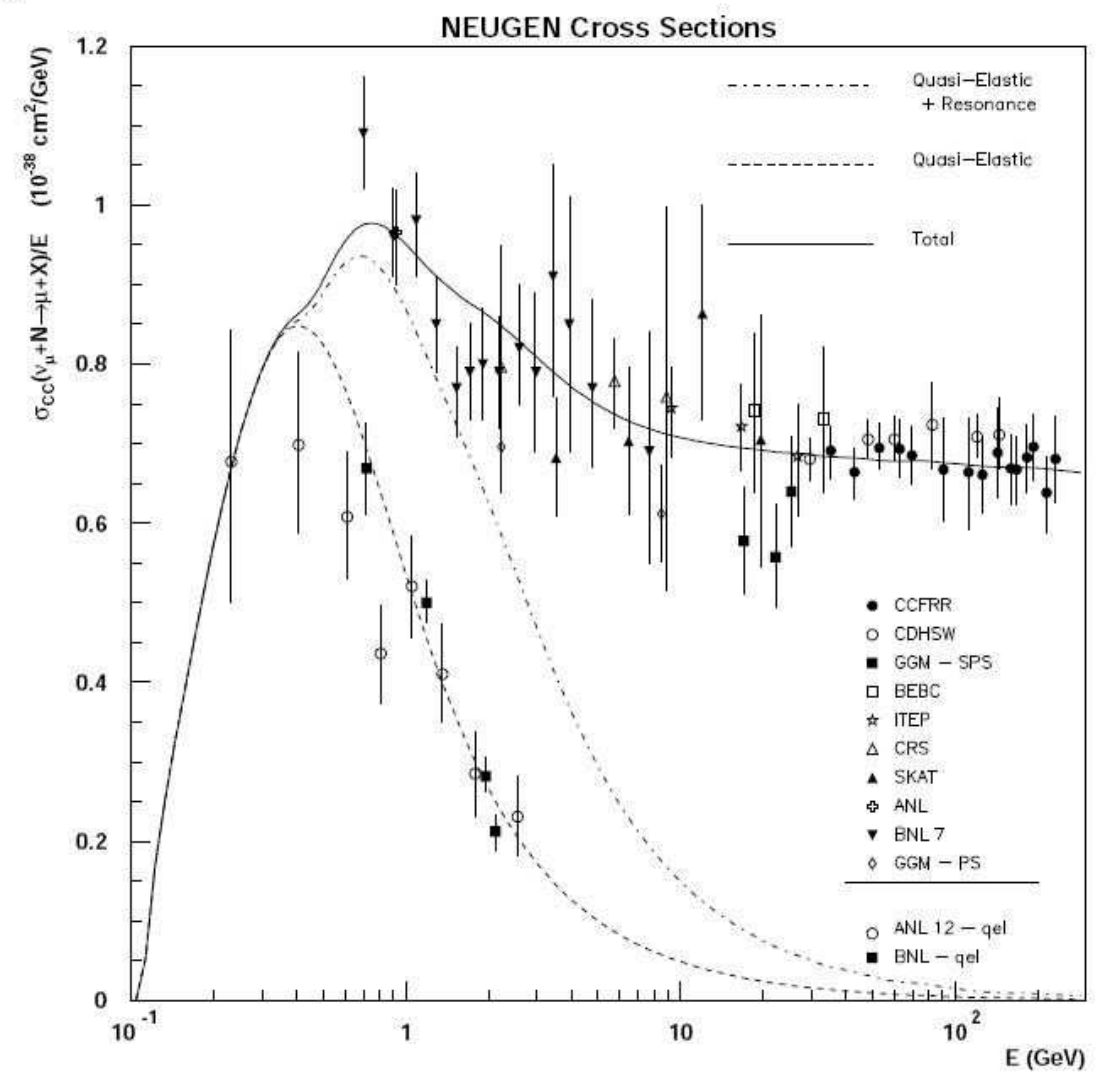

Figure 1.9: The differential muon neutrino charged current cross sections as a function of neutrino energy. The points show the measured cross-sections from several experiments. The curves are theoretical predictions from NEUGEN; shown are the quasielastic and quasielastic+resonance contributions to the total cross section as well as the total cross section. 
by Equation 1.13. Figure 1.9 shows the charged current muon neutrino cross section divided by the neutrino energy over the range of intermediate and high neutrino energies. Shown are data from several experiments and predictions from the NEUGEN cross section model [11]. For muon neutrinos, the cross section overcomes the kinematic barrier of producing a muon at $E_{\nu} \sim 0.1 \mathrm{GeV}$, then passes through the quasi-elastic, resonance and deep inelastic regimes.

For neutrino energies above $\sim 25 \mathrm{GeV}$ the uncertainty on the data is significantly less than the error on data taken at neutrino energies above $25 \mathrm{GeV}$. This is because the experimental data below $25 \mathrm{GeV}$ were taken with Narrow Band Beams(NBB) as compared to the use of Wide Band Beams(WBB) below this energy.

\subsection{Past Cross Section Experiments}

Particle Accelerators are used to produce well controlled beams of neutrinos for use in neutrino experiments. The interaction of a beam of protons with a fixed target produces pion and kaon mesons which subsequently decay giving neutrinos. The mesons diverge from the production target at a typical angle of $\sim 2 / \gamma$, where $\gamma$ is the relativistic boost of the meson. High energy neutrinos come from the decay of high energy mesons and, likewise, low energy neutrinos come from the decay of low energy mesons. Thus, the beam of high energy mesons off of the production target is more collimated compared to that of low energy neutrino beams. This allows the meson beam to be passed through momentum selecting magnets prior to decay. The neutrino beams produced in this way have a narrow energy spectrum. The collimation of the beam and the narrow momentum range permits monitoring devices to be placed in the 
meson beam providing a measurement of the flux incident to the experiment.

The production of mesons off of the particle production target was and remains the largest uncertainty in neutrino scattering experiments. The ability to actually measure the secondary meson beam allowed the NBB experiments to precisely measure the incident neutrino flux providing for precise neutrino cross section measurements. The large transverse size of the secondary meson beam in wide band beams (WBBs), makes in situ measurements of the flux much more difficult than for NBB, but nonetheless beneficial. In the next section, measurements of the neutrino flux from wide band neutrino beams are discussed.

In the decays $\pi \rightarrow \mu \nu_{\mu}$ and $K \rightarrow \mu \nu_{\mu}$, which predominantly contribute to the production of neutrinos for accelerator based neutrino beams, muons are produced alongside the neutrinos. Thus, the muon flux may be used to both monitor and measure the neutrino flux. Numerous past experiments, going nearly as far back as the first accelerator neutrino beam experiment in the early 1960's, have used muon monitoring systems to monitor their tertiary beams and to infer or constrain the fluxes to the experiment $[12,13,14,15,16,17]$. One such beam that used muon monitors is the 1967 CERN PS neutrino beam [13], shown in Figure 1.10. Muons from $\pi / K \rightarrow \mu \nu$ were ranged out by $\sim 20 \mathrm{~m}$ of shielding upstream of the neutrino detector. Ionization chambers were placed in transverse channels within the shielding.

A Monte Carlo of the beamline used a Sanford-Wang [18] parameterization for the production of $\pi^{+}$and $K^{+}$off of the target in order to predict the flux delivered to the neutrino experiments. When comparing the yields of muons in these muon detectors with the Monte Carlo prediction the experimenters found that the Sanford-Wang model had to be increased by $\sim 50 \%$ in 


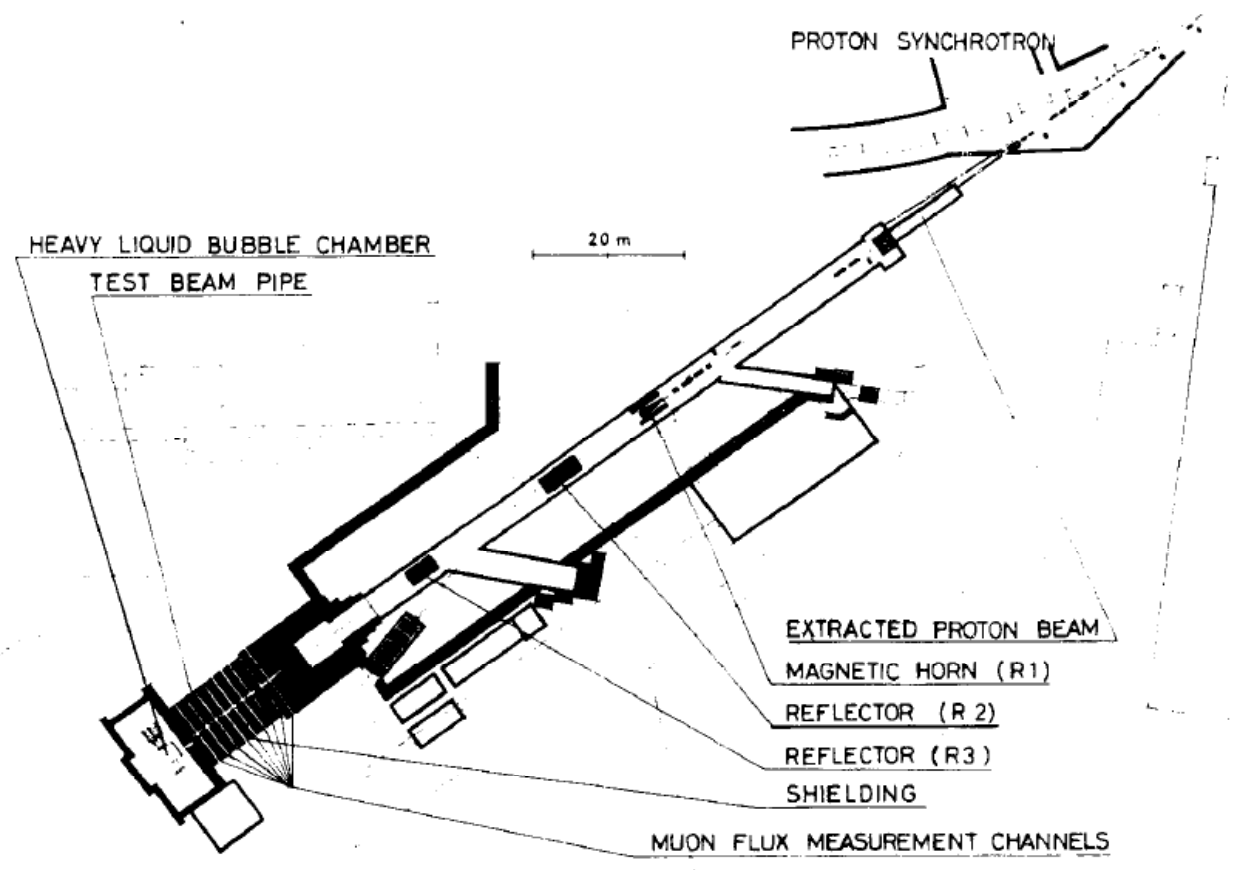

Figure 1.10: Diagram of the 1967 CERN PS neutrino facility. Ionization chambers were placed within the muon flux measurement channels located within the downstream muon filter(shielding) and used to determine the neutrino flux. Figure caption from Ref [13] reads: "CD - co-ordinate detectors; T target; FS - focusing system; DT - decay tunnel; 1-16 - muon filter slits; IC ionization chambers." 


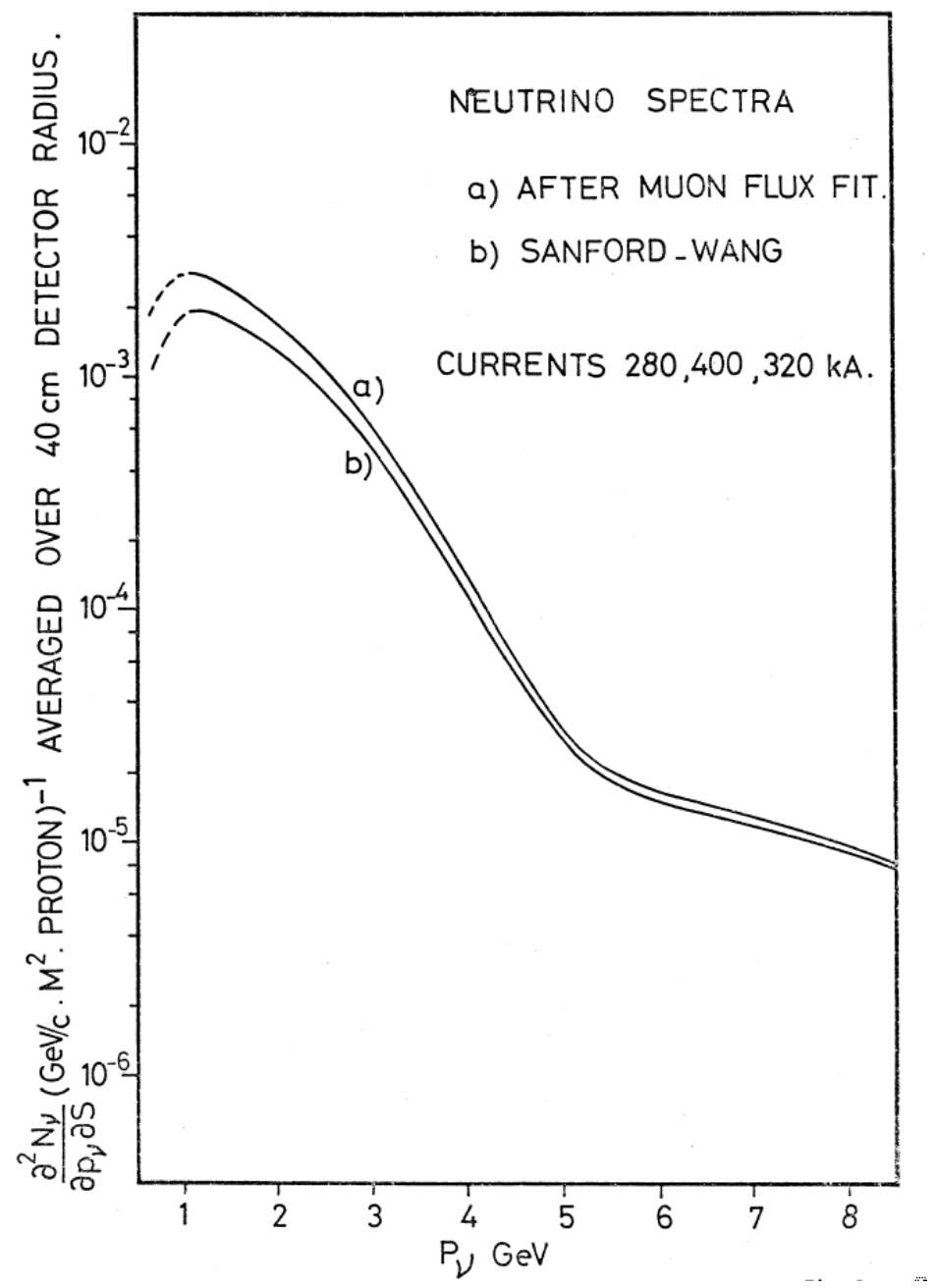

Figure 1.11: The 1967 CERN predicted and measured neutrino flux. The curves are fitted to data using a Sanford-Wang parameterization of pion production. The neutrino flux calculated with the original Sanford-Wang parameterization (b), and after a fit to the muon monitor flux data (a) [14]. 
order to correctly describe the muon flux yields.

Figure [?] shows the neutrino flux inferred from the pion and kaon spectra before and after the fit to the muon data, showing that their prediction is about $50 \%$ different from the in-situ measurement of the neutrino flux using their muon monitors. The stated uncertainty is $10-25 \%$ depending on neutrino energy. Shortly after this analysis $K^{+}$and $\pi^{+}$production data from a boron carbode target with $\sim 20 \mathrm{GeV} / c$ protons, particularly relevant to this neutrino beam, became available and were found to agree with the measured muon and neutrino fluxes within $10 \%$.

In the 1970's, the IHEP-Serpukhov neutrino beam show in Figure 1.12 also used ion chambers to measure the muon flux in the downstream muon filter [16]. Similar to the CERN 1967 beam, the IHEP beam line had transverse slits or channels within the downstream iron shielding in which ionization chamber were placed. Figure 1.13 shows the measured lateral muon distribution(profile) within slits 1,5 and 10 corresponding to depths of $3,6.8$ and $18.5 \mathrm{~m}$ of Fe within the muon filter. It is noted in Ref [16] that the profiles exhibit asymmetries resulting from imperfect focusing of parent pions and kaons off of the target.

It these beamlines the decay volume was short, $\sim 50 \mathrm{~m}$ in the CERN beam, so the radial width of the muon beam at the monitors was small enough that nearly the entire muon profile was measured by the muon monitors. Furthermore, there were numerous longitudinal channels within the muon filter allowing for good sampling of the differential muon flux, $d \phi_{\mu} / d p_{\mu}$.

The Neutrinos at the Main Injector (NuMI) facility at Fermi National Accelerator Laboratory (FNAL, Fermilab) is an accelerator based neutrino source supplying a beam of neutrinos to the several neutrino experiments. The NuMI beamline has a system of 3 muon monitors located with in its 


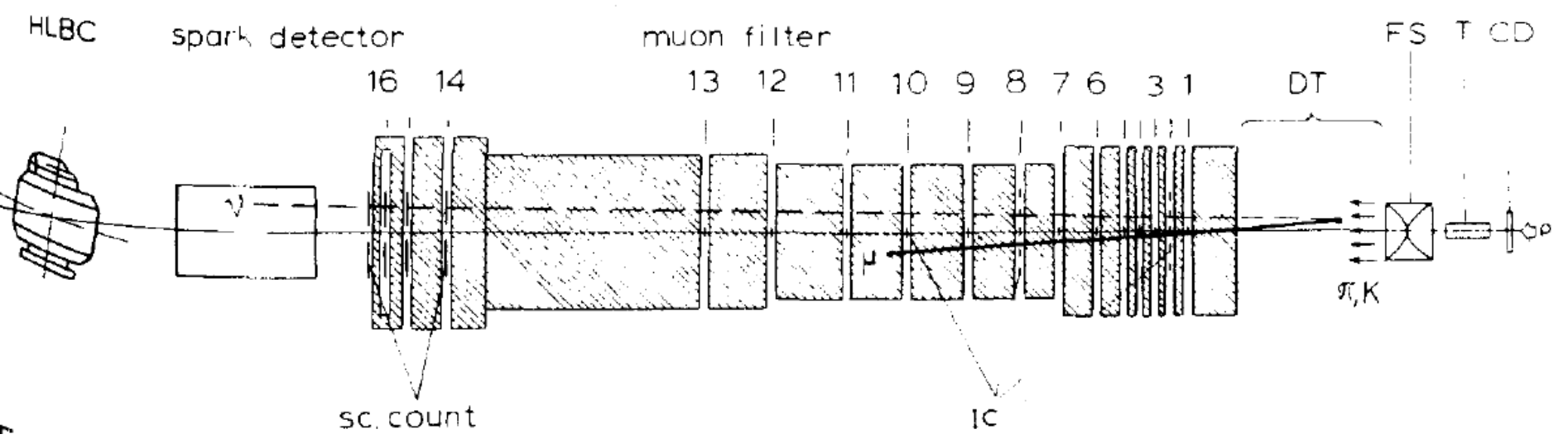

Figure 1.12: Diagram of the 1970's IHEP-Serpukhov neutrino facility. Ionization chambers were placed within the downstream muon fiter channels and used to measure the muon flux [16]. 


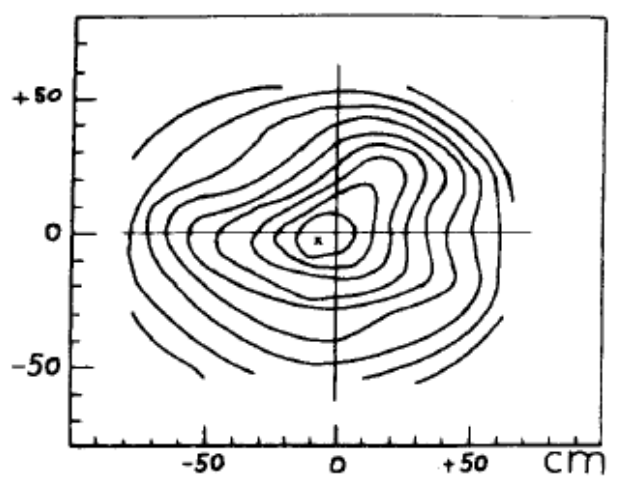

1.

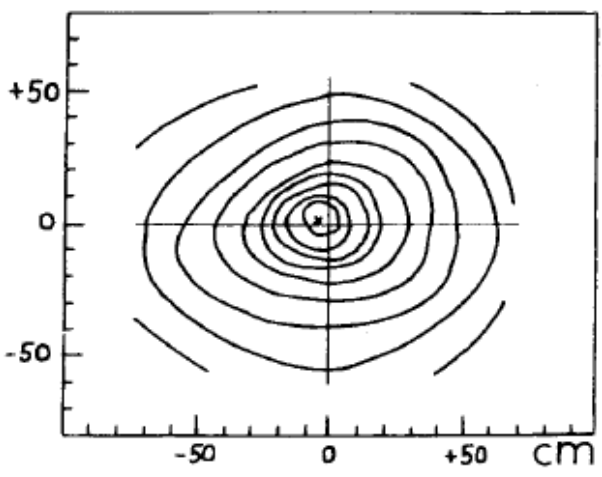

2.

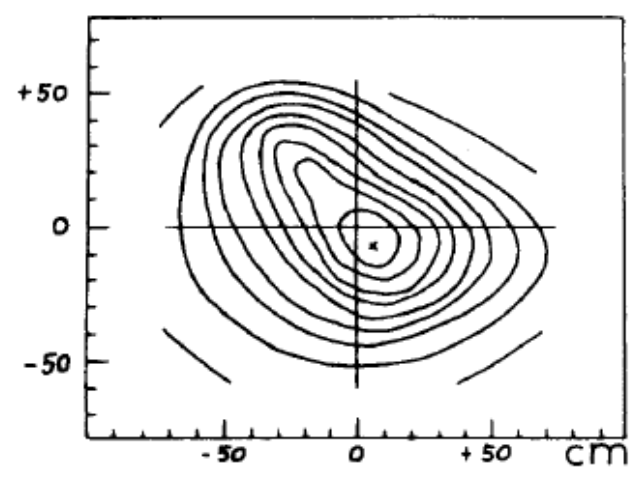

3.

Figure 1.13: Lateral muon distribution within slits $1(3 \mathrm{~m}$ of Fe)(top), $5(6.8 \mathrm{~m}$ of $\mathrm{Fe}$ )(middle) and $10(18.5 \mathrm{~m}$ of $\mathrm{Fe}$ (bottom) in the muon filter of the IHEP neutrino beamline. The lines are contours of equal muon flux in increments of $0.1 \times$ the maximum muon density in each profile. 
muon filter which are used to monitor and also measure the flux. Each muon monitor consists of a $9 \times 9$ array of ionization chambers. In this thesis, we will use the NuMI muon monitors to determine the neutrino flux using much the same procedure as done at the CERN PS and IHEP experiments.

\subsection{Goal of this Thesis}

The NuMI beamline is a wide band beam producing a peaked neutrino flux in the range from 3 to $6 \mathrm{GeV}$. As mentioned in the previous section, neutrino cross sections in this energy range suffer large uncertainty from knowledge of the incident neutrino flux, providing an opportunity for us to improve the experimental knowledge in this region. The large transverse distribution of the pion and kaon secondaries from the particle production target and the wide range of energies makes measurements of the neutrino flux from wide band beams challenging. Typically, experiments rely on measurements of the

pion and kaon spectra made by dedicated particle production experiments to predict their neutrino flux, resulting in significant uncertainties in the flux and hence in the measured cross sections. $[19,20]$ Thus, it is ideal to measure the neutrino flux from the beamline data itself.

The goal of this thesis is to present a measurement of the muon neutrino $\left(\nu_{\mu}\right)$ charged current inclusive cross section on iron using the neutrino beam produced by NuMI. We also present a measurement of the NuMI $\nu_{\mu}$ flux obtained from a measurement of the muon flux using the 3 muon monitors in the NuMI beamline. We then utilize this flux to measure the $\left(\nu_{\mu}\right)$ charged current cross section using the MINOS detector located downstream in the NuMI beamline. Chapter 2 describes the NuMI beamline and MINOS detec- 
tor. Chapter 3 describes the NuMI Muon Monitors and data sets collected for use in measuring the muon flux from the NuMI beamline. Chapter 4 discusses the calculation of the neutrino and muon fluxes. It also discusses studies of the flux uncertainty due to beamline conditions and components. Chapter 5 describes the measurement of the neutrino flux derived from a measurement of the muon flux and Chapter 6 describes the systematic uncertainty on this flux measurement. Chapter 7 describes the measurement of the $\left(\nu_{\mu}\right)$ charged current inclusive cross section. The last chapter, Chapter 8, provides and summary of this thesis and discusses future potential improvements. 


\section{Chapter 2}

\section{The MINOS Experiment}

The Main Injector Neutrino Oscillation Search (MINOS) is a two detector neutrino oscillation experiment. The Neutrinos at the Main Injector (NuMI) facility at Fermi National Accelerator Laboratory (FNAL, Fermilab) is an accelerator based neutrino source supplying a beam of neutrinos to the MINOS experiment. The neutrinos are produced in the decays of mesons created in the interaction of a beam of protons with a fixed target.

The MINOS oscillation experiment uses two functionally identical neutrino detectors. The MINOS Near Detector is on the Fermilab site $100 \mathrm{~m}$ underground and $1 \mathrm{~km}$ from the neutrino source. The MINOS Far Detector is located $735 \mathrm{~km}$ away in the Soudan Underground Laboratory in Soudan, Minnesota. The MINOS Far Detector measures the neutrino spectrum after oscillations have occurred while the close proximity of the MINOS Near Detector to the neutrino source ensures that it measures the neutrino spectrum before any oscillations occur. The close proximity of the Near Detector to the neutrino source also means that a large, sample of neutrino interactions can be acquired. This makes the Near Detector useful for measuring neutrino 
interaction cross sections.

In this chapter a detailed overview of the NuMI neutrino beamline is provided. Additionally, the MINOS Near Detector is described and details of neutrino interactions in the detector are discussed. The MINOS Far Detector is not used for this analysis.

\subsection{NuMI Beamline}

Figure 2.1 shows a schematic view of the NuMI beamline. The production of the neutrino beam begins with the extraction of $120 \mathrm{GeV}$ protons from the Fermilab Main Injector in pulses $10 \mu$ s long and consisting of anywhere between $1 \times 10^{11}$ and $4.5 \times 10^{13}$ protons. The pulses, commonly referred to as spills, are typically extracted every 2.2 seconds. The protons are transferred to the NuMI target hall where they collide with a fixed carbon graphite target. The target is discussed in detail in Section 2.1.1.

Pions and kaons are produced in the interaction of the proton beam with a fixed target. The decay of these mesons produce the beam of neutrinos used in the experiment. The branching ratios for those mesons which contribute significantly to the neutrino beam are :

$$
\begin{aligned}
& \pi^{ \pm} \rightarrow \mu+\nu_{\mu} \quad \mathrm{BR} \approx 99.99 \%, \tau \approx 26 \mathrm{~ns} \\
& K^{ \pm} \rightarrow \mu+\nu_{\mu} \quad \mathrm{BR} \approx 63.4 \%, \tau \approx 12 \mathrm{~ns} \\
& K_{L}^{0} \rightarrow \pi+\mu+\nu_{\mu} \quad \mathrm{BR} \approx 27.2 \%, \tau \approx 52 \mathrm{~ns}
\end{aligned}
$$

The beam is predominantly composed of muon neutrinos, (92.9\%), with a small component of anti-muon, 5.8\%, and electron and anti-electron (1.3\%), 

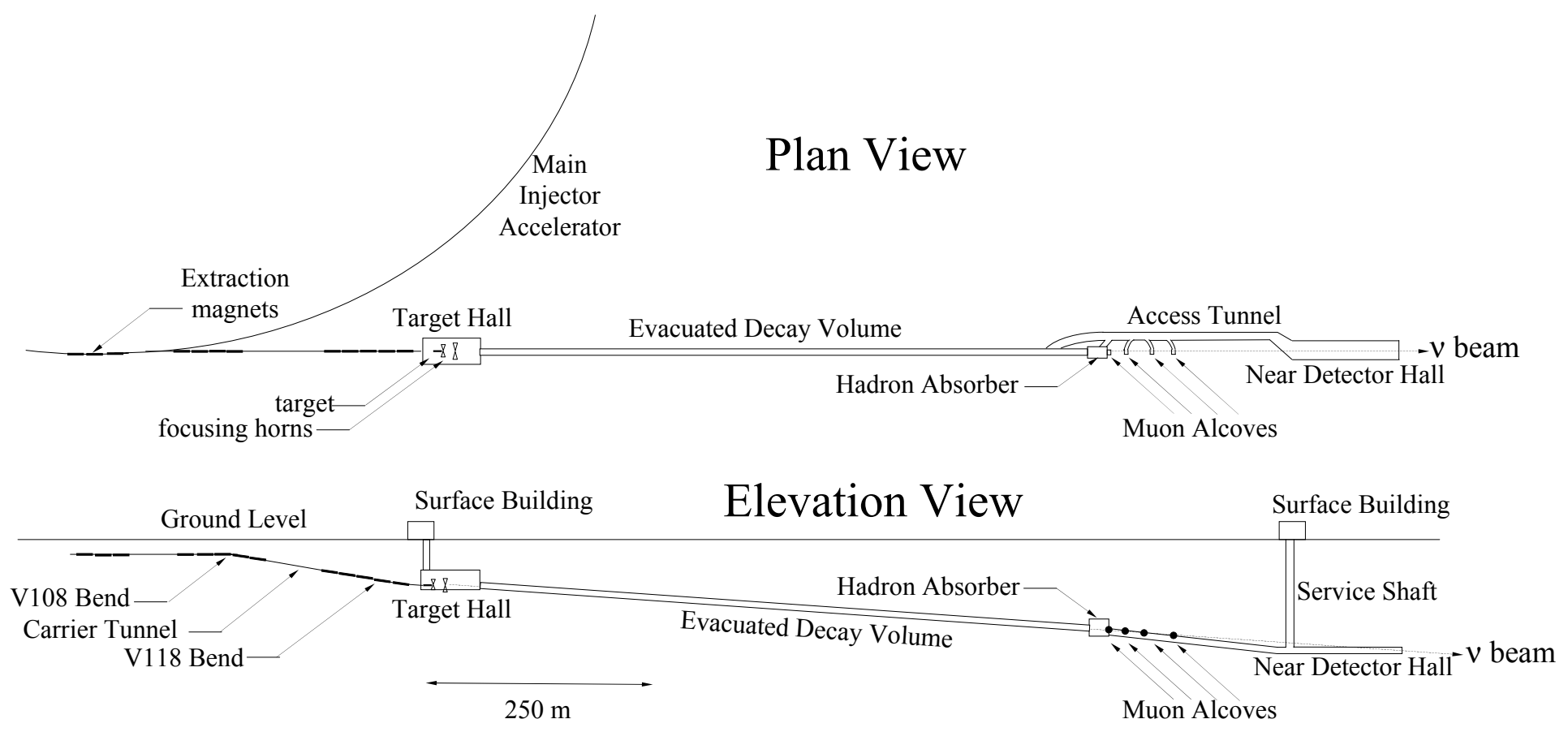

Figure 2.1: Plan and elevation views of the NuMI neutrino beamline. A beam of protons from the Fermilab Main Injector is collided with a fixed target producing pion and kaon mesons. The mesons are focused into the decay volume where they decay into neutrinos producing the neutrino beam. 
neutrinos [21]. Muons are produced along with the muon neutrinos. In later chapters it will be shown that these muons can be used to measure the number of neutrinos delivered to the MINOS experiment also referred to as the neutrino flux, $\phi_{\nu}$. Directly measuring the neutrino flux is a significant experimental challenge and is essential for the goal of this thesis which is the measurement of neutrino cross sections which is discussed in Chapter 7.

Immediately following the target are two magnetic focusing horns. Particles escape the target with some angular divergence with respect to the center of the beamline. The horns act as lenses, removing this divergence, focusing particles parallel to the beamline towards the MINOS detectors. Like a lens system, the horns have a focal length. The focal length is proportional to the momentum of the charged particle. Thus, those particles with a momentum closest to the focal length are most perfectly focused parallel to the beamline. This selects the peak energy of the neutrino beam. This is discussed further in Section 2.1.2. Furthermore, depending on the polarity, the horns focus positively charged particles and defocus negatively charged particles, producing a predominantly muon neutrino beam, or focus negatively charged particles and defocus positively charged particles, producing a predominantly anti-muon neutrino beam. For the first 4 years of operation the horns were operated to produce a muon neutrino beam. All data used in the analysis discussed herein were collected within the first 2 years of operation.

The target and horns are contained within a $45 \mathrm{~m}$ long shielded enclosure. Immediately following this enclosure is a $2 \mathrm{~m}$ diameter $675 \mathrm{~m}$ long decay volume. For the first 3 years of operation the decay volume was evacuated. In November 2007 the decay volume was filled with helium near atmospheric pressure. All data used in the analysis discussed herein were collected while 
the decay volume was evacuated. The purpose of the decay volume is to allow the focused mesons to decay in free space producing neutrinos. All kaons and most pions $(95 \%$ at $5 \mathrm{GeV} / c)$ decay before the end of the decay volume. Those that do not decay are stopped at the end of the decay volume by the hadron absorber.

The hadron absorber is block of concrete steel and aluminum immediately adjacent to the end of the decay volume. It removes all remaining hadrons from the neutrino beam. These predominantly include protons that did not interact in the target and undecayed pions. The hadron absorber is discussed in more detail in Section 2.1.3. Contained within the hadron absorber is the hadron monitor which is an array of ionization chambers used to monitor the beam at the end of the decay volume.

After the beam passes through the hadron absorber it is composed of neutrinos and muons produced alongside the neutrinos in meson decay. The muons are removed from the beam by $\sim 200 \mathrm{~m}$ of unexcavated earth following the hadron absorber. The earth is largely composed of dolomite rock. The energy of the proton beam from the Main Injector is $120 \mathrm{GeV}$. Thus, the maximum energy of any muon is also $120 \mathrm{GeV}$. At this energy, about $200 \mathrm{~m}$ of rock is required for a muon to loose all energy by electromagnetic interactions with the rock. Thus, after the $200 \mathrm{~m}$ of rock the beam is only composed of neutrinos. In the upstream portion of the rock, before all of the muons are removed from the beam are three muon monitors. The first monitor is located $7 \mathrm{~m}$ after(downstream) of the hadron absorber and is separated from the hadron absorber by air. The other two monitors a located within small excavated alcoves downstream of the first monitor. The second monitor is separated from the first by $12 \mathrm{~m}$ of rock and the third is located after $18 \mathrm{~m}$ 
more rock. At the end of the $200 \mathrm{~m}$ of rock is the MINOS Near Detector Hall in which sits the MINOS Near Detector. The MINOS Near Detector is discussed in Section 2.2.

\subsubsection{The NuMI Target}

The NuMI meson production target is shown in Figure 2.2. The beam impinges upon the target from the left. The target consists of 47 carbon graphite segments $6.4 \mathrm{~mm}$ wide, $18 \mathrm{~mm}$ high and $20 \mathrm{~mm}$-long the beam direction and spaced $0.3 \mathrm{~mm}$ apart. The total length of the target is $95.38 \mathrm{~cm}$. There is a 48th target segment located $\sim 15.7 \mathrm{~cm}$ upstream of and rotated at $90^{\circ}$ to the main target segments. It is used for aligning the target with respect to the proton beam.

The target length corresponds to 2 interaction lengths. This increases the fraction of the incident proton beam that will interact in the target and produce mesons which decay producing neutrinos. However, even at this length

approximately $13.5 \%$ of the proton beam will remain unreacted, escape the target and travel downstream to the hadron absorber. The length of the target also increases the number of reinteractions of particles produced in the primary interaction of the proton beam within the target. This has implications for the neutrino flux and is discussed in Chapter 4.

The segmentation of the target reduces thermodynamic stress and allows particles produced in interactions of the incident proton beam with the graphite to escape the target, reducing the number of particles produced from reinteractions in the target material.

At $6.4 \mathrm{~mm}$, the width of the target also provides particles produced in 


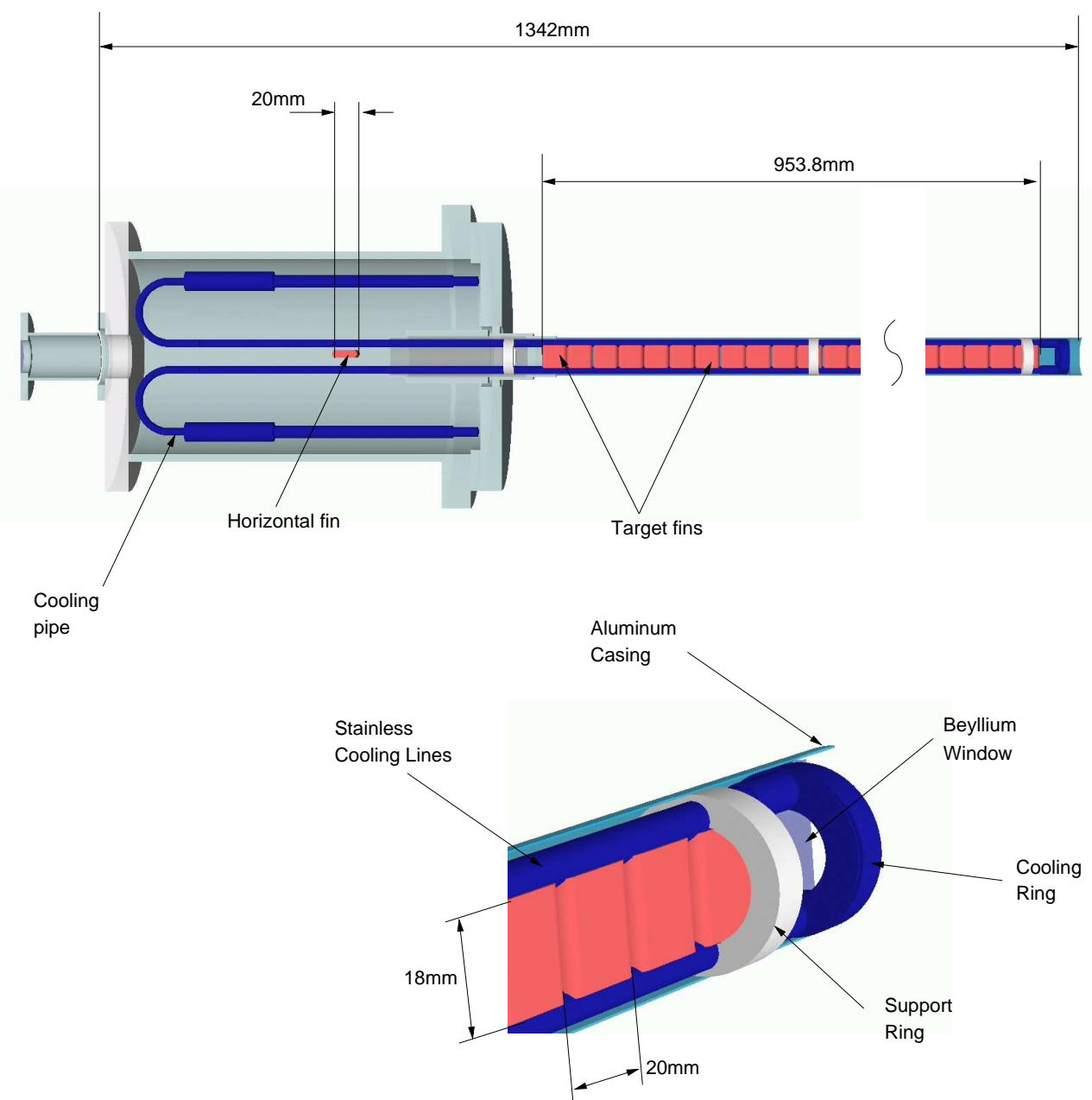

Figure 2.2: The NuMI hadron production target. Interactions of a beam of $120 \mathrm{GeV}$ protons with the graphite target produces mesons which decay producing the neutrino beam. [22] 


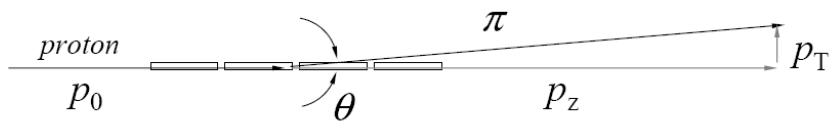

Figure 2.3: Schematic of a particle diverging out of the target with some transverse, $p_{T}$, and longitudinal, $p_{z}$, momentum. [23]

primary proton-graphite interactions a chance to escape the target without undergoing reinteractions. However, the incident proton beam has an RMS width of $\sim 2.2 \mathrm{~mm}$ making precise alignment of the proton beam and target essential for maintaining target integrity and the production of a consistent neutrino flux. The alignment of the target is discussed in Appendix B.

Located $\sim 2.0 \mathrm{~m}$ upstream of the target is a $1.5 \mathrm{~m}$ long collimating baffle. The baffle is a carbon graphite cylindrical tube with outer diameter $30 \mathrm{~mm}$ and inner diameter $11 \mathrm{~mm}$. The baffle protects the target cooling lines and horns from mis-steered proton beam that could damage these components. Figure 3.10 shows a proton beam's-eye-view of the target-baffle system.

The target and baffle are mounted in a carrier which can move longitudinally with respect to the beamline center. This special ability of the NuMI beam line is important for the physics analysis discussed in this thesis and is discussed in Section 2.1.4.

\subsubsection{The NuMI Horns}

Particles diverge from the target with some transverse, $p_{T}$, and longitudinal, $p_{z}$, momentum as shown in Figure 2.3.

To increase the neutrino flux at downstream neutrino detectors it is desirable to remove this divergence and direct parent pion and kaons towards downstream detectors. NuMI uses horn focusing to accomplish this.

Figure 2.5 shows a diagram of a parabolic horn. The NuMI particle fo- 
cusing system consist of two parabolic horns positioned as shown in Figure 2.9. They consist of a $2.54 \mathrm{~cm}$ thick outer conductor and a $0.2-0.5 \mathrm{~cm}$ thick inner conductor. Both conductors are made of aluminum and the inner conductor has a parabolic shape following $z=a r^{2}$, where $z$ is the longitudinal coordinate parallel to the axis of symmetry, $r$ is the radial coordinate, and $a$ is a constant parabolic parameter. The first horn has an outer and inner diameter of $35 \mathrm{~cm}$ and $1.8 \mathrm{~cm}$ respectively, while the second horn is larger with an outer and inner diameter of $79 \mathrm{~cm}$ and $7.8 \mathrm{~cm}$, so that it can intercept those particles not fully focused by the first horn.

Electrical current flows down the inner conductor and returns along the outer conductor. This generates a toroidal magnetic field between the conductors $B(r)=\mu_{0} I / 2 \pi r$, where $r$ is the radial distance from the symmetry axis. Ideally, there is no magnetic field outside of the region between the conductors. Thus, particles emitted with very small divergence may pass through the field free region inside of the inner conductors of the horns(the "neck" of the horn) receiving no focusing.

The magnetic field of the NuMI horns is measured using a hall probe before placing the horns in the beamline. Figure 2.4 shows the measured magnetic field inside $(\sim 13.5-150 \mathrm{~mm})$ the first NuMI horn. The magnetic field shows the expected $1 / \mathrm{r}$ behavior and the expected, $B(r)=\mu_{0} I / 2 \pi r$, magnitude between the conductors. The field drops to zero outside of the outer conductor at $\mathrm{r}>150 \mathrm{~mm}$. The field inside of the inner conductor $(\mathrm{r}<13.5 \mathrm{~mm})$ was measured separately and was found to be negligibly small [24].

Particles traversing the region between the conductors feel a force, $q \mathbf{v} \times \mathbf{B}$, which directs them towards or away from the center of the beamline depending on the sign of their charge, $\mathrm{q}$. 


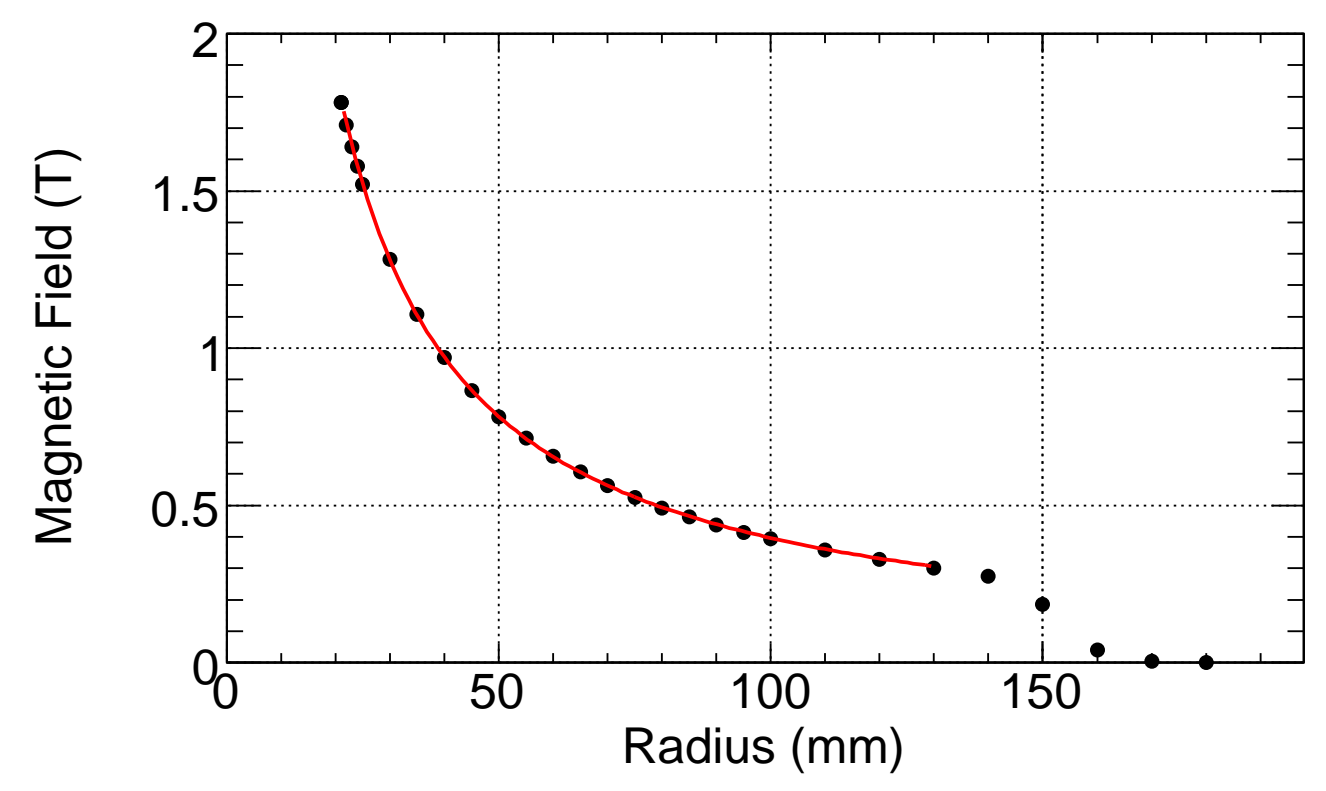

Figure 2.4: The measured (dots) magnetic field inside, $\sim 13.5-150 \mathrm{~mm}$, the first NuMI horn shows the expected $1 / \mathrm{r}$ dependence (curve) and the expected, $B(r)=\mu_{0} I / 2 \pi r$, magnitude. The field drops to zero outside of the outer conductor at $\mathrm{r}>150 \mathrm{~mm}$.

Figure 2.5 shows a schematic of a particle entering a focusing horn through the parabolic inner conductor. Since the force on the particle is perpendicular to its motion, the resulting change in the particle's momentum is described by the change in direction as

$$
|\Delta \theta|=\left|\theta_{\text {out }}-\theta_{\text {in }}\right|=\frac{\left|\Delta p_{T}\right|}{p}
$$

using the fact that $p_{T} \ll p$ and so $\sin \theta \simeq \theta$. The magnitude of the particle's momentum change is

$$
\left|\Delta p_{T}\right|=\int B(r) d \ell \approx \frac{\mu_{0} I}{2 \pi r} \cdot a r^{2} .
$$




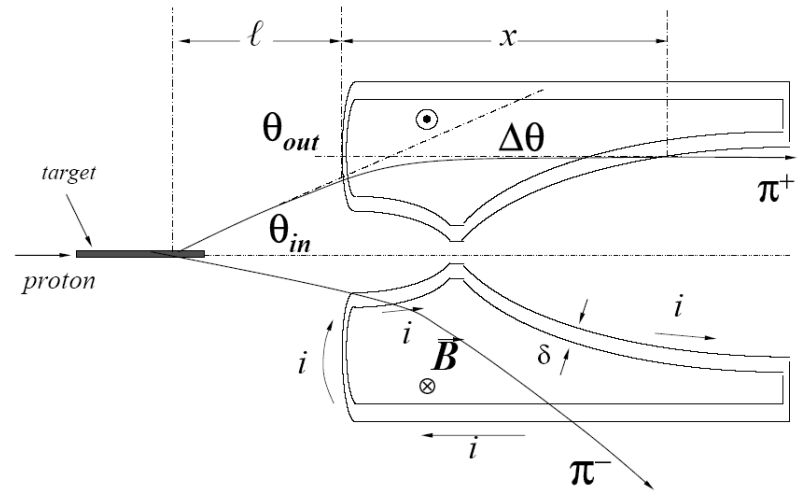

Figure 2.5: Schematic view of particles going through a focusing horn with parabolic shape of inner conductor. Due to the shape of the conductor, the particles entering at bigger radius will see greater $\int B d \ell$ resulting in a greater change in transverse momentum. Horz and vert scales not same. Taken from Ref [23].

Perfect focusing occurs when $\theta_{\text {out }}=0$ so that $\left|\Delta p_{T}\right|=p_{T}$. In the approximation that the source is far from the horn, the focal length, $f$, of the horn is $[25]$

$$
f \approx \frac{r}{p_{T}} p=\frac{2 \pi}{\mu_{0} I a} p
$$

This shows that the focusing power of the horn depends on the particle momentum and the current in the horn. Figure 2.6, shows a Monte Carlo simulation of the muon neutrino flux at the MINOS Near Detector when the current in the horns is varied while maintaining a fixed distance between the target and horns. The peak energy of the neutrino beam changes with the current in the horns. Thus, the current in the horns selects the particle momentum that is most perfectly focused parallel to the beamline. Particles that pass through the necks of the horns are high energy and thus contribute to the high energy neutrino flux outside of the focusing peak.

It is also possible to change the peak energy of the neutrino beam while 


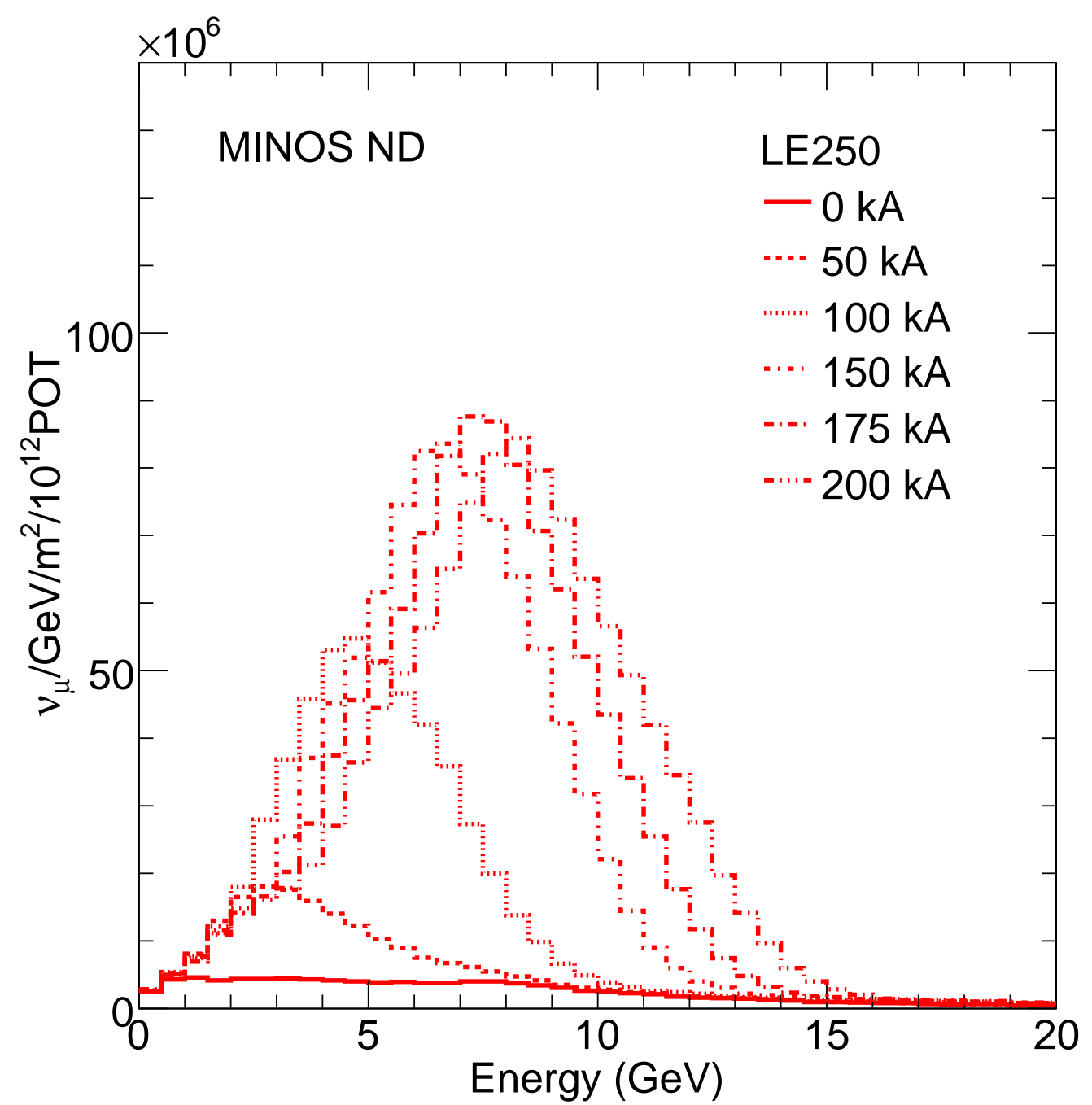

Figure 2.6: The Monte Carlo simulated muon neutrino flux at the MINOS Near Detector when the current in the horns is varied while maintaining a fixed distance between the target and horns. The peak energy of the neutrino beam changes with the current in the horns. The current in the horns selects the particle momentum that is most perfectly focused parallel to the beamline. 
keeping the horn current fixed but varying the the distance between the target and horns. This is discussed in Section 2.1.4.

Figure 2.6 also shows that the horns greatly increase the flux of neutrinos downstream along the beamline. The flux of neutrinos with energies less than $\sim 20 \mathrm{GeV}$ overwhelmingly comes from the decay of pions. The typical angle of divergence of pions off of the target is [23]

$$
\theta_{\pi}=\frac{p_{T}}{p_{\pi}} \approx \frac{\left\langle p_{T}\right\rangle}{E_{\pi}} \approx \frac{280 \mathrm{MeV}}{\gamma m_{\pi}}=\frac{2}{\gamma}
$$

where $\gamma$ is the relativistic boost factor and $280 \mathrm{MeV}$ is the Fermi momentum of partons in a nucleon. This angle is larger than the typical divergence of neutrinos produced in pion decay, $\theta_{\nu} \sim 1 / \gamma$ (Chapter 4$)$. The flux of neutrinos at an angle, $\theta$, with respect to the parent pion direction is given by

$$
\phi_{\nu} \approx \frac{1}{4 \pi}\left(\frac{2 \gamma}{1+\gamma^{2} \theta^{2}}\right)^{2}
$$

We are interested in the neutrino flux with respect to the center of the beam line so in the case of no focusing $\theta=3 / \gamma$ and for the case of perfect focusing $\theta=\gamma$. Substituting into Equation 4.12 gives [23]

$$
\frac{\phi_{\text {focus }}}{\phi_{\text {no focus }}}=\frac{\left(1+\gamma^{2} \theta_{\text {no focus }}^{2}\right)^{2}}{\left(1+\gamma^{2} \theta_{\text {focus }}^{2}\right)^{2}}=\frac{\left(1+\gamma^{2}(3 / \gamma)^{2}\right)^{2}}{\left(1+\gamma^{2}(1 / \gamma)^{2}\right)^{2}}=\frac{100}{4}=25 .
$$

So the flux of neutrinos to downstream neutrino experiments is increased by a factor of 25 by removing the divergence of particles coming from the target. 


\subsubsection{Decay Volume and Hadron Absorber}

Approximately $30 \mathrm{~m}$ after the second horn, the evacuated decay volume begins. ${ }^{1}$ The purpose of the decay volume is to allow pions and kaons to decay in free space unimpeded by interactions with matter. It is true that the majority of pions and kaons that contribute to the neutrino flux at the MINOS Near Detector decay unimpeded in the decay volume. However, as the horns only provide perfect focusing for single particle momentum, off-momentum particles receive partial focusing and still have some angular divergence. This angular divergence may be large enough that the particle does collide with the walls of the decay pipe. Particles produced in interactions with the walls do produce neutrinos which contribute to the neutrino flux at the MINOS Near Detector. This is a source of uncertainty in physics analyses.

The hadron absorber is located at the end of the $675 \mathrm{~m}$ long decay volume. Its purpose is to remove hadrons remaining in the beam at the end of the decay volume in a controlled manner. A breakaway view of the hadron absorber produced from the GEANT4 [22, 26, 27] beam Monte Carlo is shown in Figure 2.7. It has an innermost core composed of aluminum and steel surrounded by steel shielding blocks. The aluminum and steel core is further surrounded by concrete blocks. The metal core serves to simultaneously create and contain hadronic showers. The interactions do create a gas of neutrons and the outer concrete shielding serves to attenuate neutrons.

Further discussion and details of the hadron absorber geometry can be found in $\operatorname{Ref}[29,28]$.

After passing through the hadron absorber the beam contains only neu-

\footnotetext{
${ }^{1}$ For the first 3 years of beamline operation the decay volume was evacuated. In November 2007 the decay volume was filled with helium near atmospheric pressure.
} 


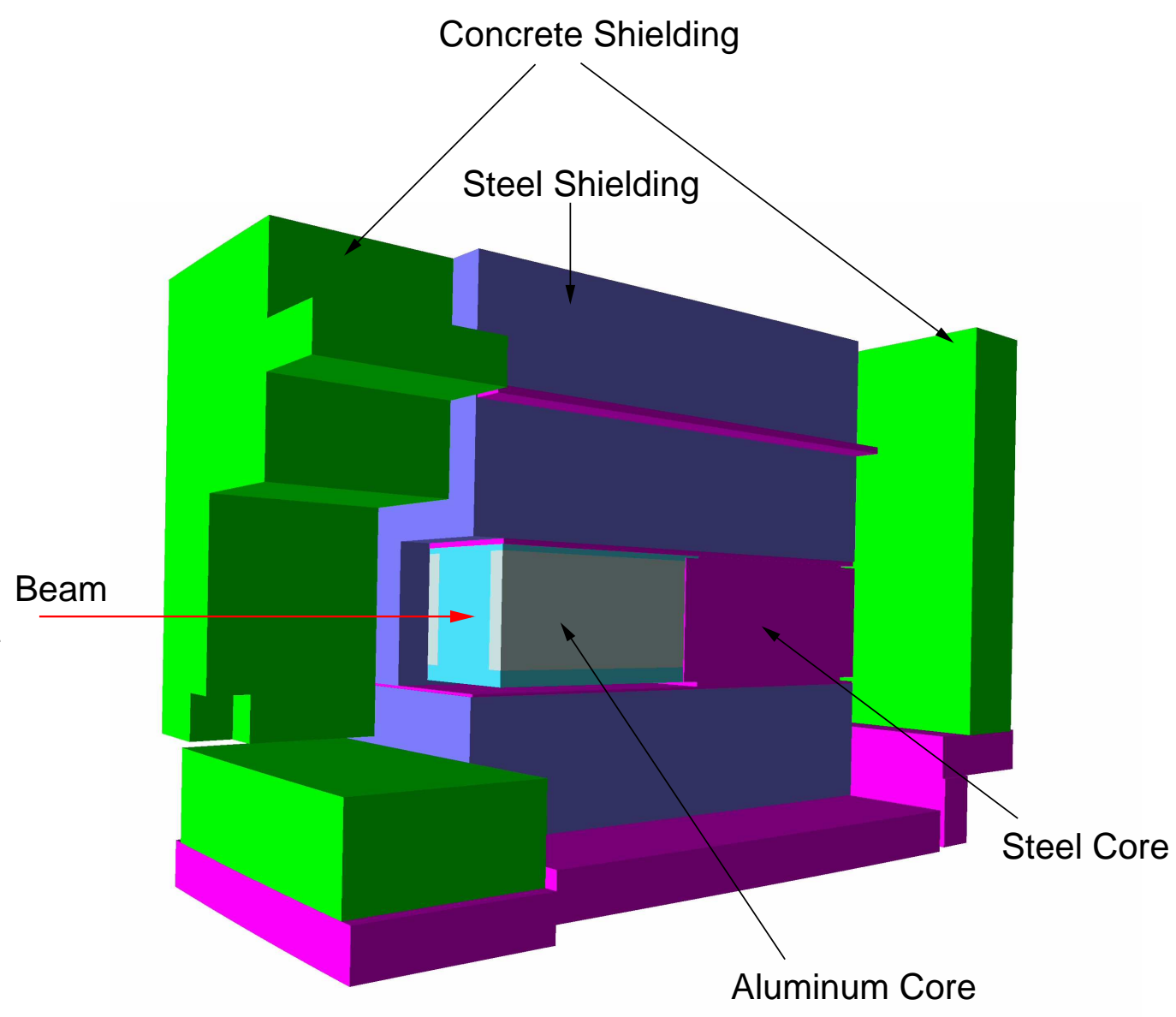

Figure 2.7: A breakaway view of the hadron absorber created from the GEANT4 implementation of the hadron absorber geometry. located at the end of decay pipe. The Aluminum core is $2.4 \mathrm{~m}$ long and the following steel core is $2.3 \mathrm{~m}$ long. [22, 28] 
trinos and muons produced from the decay of mesons in the decay volume. By the time the beam reaches the downstream neutrino detectors, it must only contain neutrinos. The region downstream of the hadron absorber but before the MINOS Near Detector hall is designed to remove muons from the neutrino beam and is thus referred to as the muon filter.

Figure 2.8 shows a side view of the muon filter. It consists of about $200 \mathrm{~m}$ of unexcavated earth with the exception of four excavated muon alcoves. The three upstream most alcoves contain a muon monitor that is used to monitor the muon beam and measure the muon flux. The muon monitors are discussed in detail in Chapter 3 and in Ref [30].

The earth is a composition of Dolomite rock and Maquoketa Shale. Details on the rock composition can be found in Ref [29]. For the maximum energy muon of $120 \mathrm{GeV}$, about $200 \mathrm{~m}$ of rock is required for a muon to loose all energy by electromagnetic interactions with the rock. Hence, the $200 \mathrm{~m}$ of rock between the hadron absorber and the MINOS Near Detector Hall is sufficient to ensure that the MINOS detector is not overwhelmed by beam muons, but rather can record neutrino interactions. The MINOS Near Detector is discussed in Section 2.2.

\subsubsection{Variable Energy Beam}

According to Equation 2.4, the magnetic horns have a variety of focal lengths for particles with different momenta. The average momentum of focused particles is fixed by the longitudinal placement of the target with respect to the horns. In the NuMI beamline, the average momentum of focus particles can be varied by changing the separation between the target and horns. This locates 


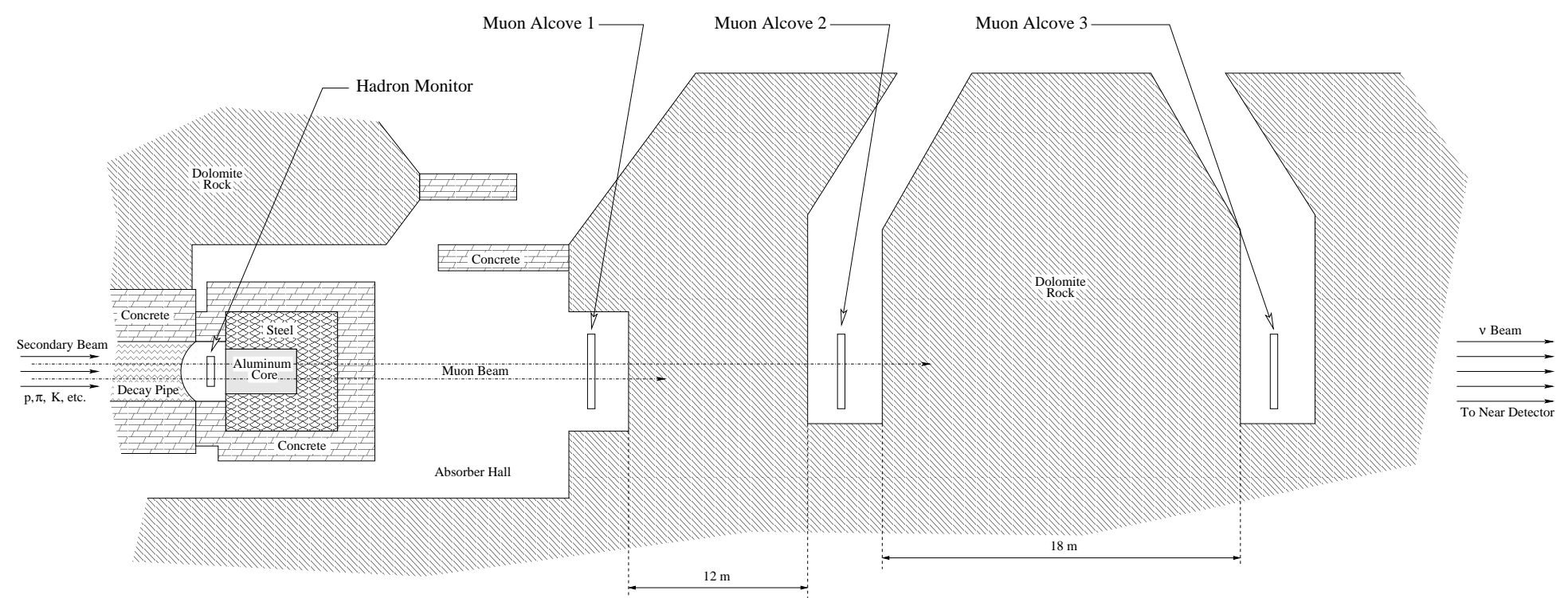

Figure 2.8: Bird's-eye view of the downstream hadron absorber and muon region of the NuMI beamline. At the end of the decay volume the beam consists of unreacted protons, secondary hadrons, muons and neutrinos. The hadron monitor, located at the upstream face of the hadron absorber measures the hadron content of the beam. Hadrons are absorbed by interactions within the hadron absorber so that the beam contains only muons and neutrinos as it exists the downstream end of the absorber. Three muon monitors located within excavated alcoves downstream of the hadron absorber measure the muon flux at each location. Muons range out in the rock so that the beam contains only neutrinos by the time it reaches the MINOS Near Detector. [31] 
a different portion of the target at the focal length of the horns, effectively changing the focal length of the horns. As the typical divergent angle of pions out of the target is $2 / \gamma$, higher momentum pions diverge less than do lower momentum pions. Thus, increasing the relative difference between the target and horns results in higher momentum pions receiving full focusing. A higher momentum pion beam directly results in a higher energy neutrino beam.

The target and baffle are mounted in a carrier on rails. The system may travel a maximum distance of $2.5 \mathrm{~m}$ along the rails. Shown are four of the five target-horn configurations in which the NuMI beam has operated to produce different energy neutrino beams. The fifth(not shown) is LE150. In the nominal configuration, LE or LE000, the target is inserted into the first horn with only $35 \mathrm{~cm}$ outside of the horn. The LE000 configuration produces the lowest possible neutrino energy beam. Higher energy beams are produced my moving the target away from the horns. In principal the target may be positioned at any distance with respect to the horns up to $2.5 \mathrm{~m}$.

Figure 2.10 shows the Monte Carlo simulated neutrino flux at the MINOS Near Detector in the LE010, LE100, LE150 and LE250 beam configurations. The peak energy of the neutrino flux increases as the target is moved further from the horns.

The ability of the NuMI beamline to produce a variety of different energy neutrino beams is essential in understanding the neutrino flux. This is discussed further in Chapters 3-5. 


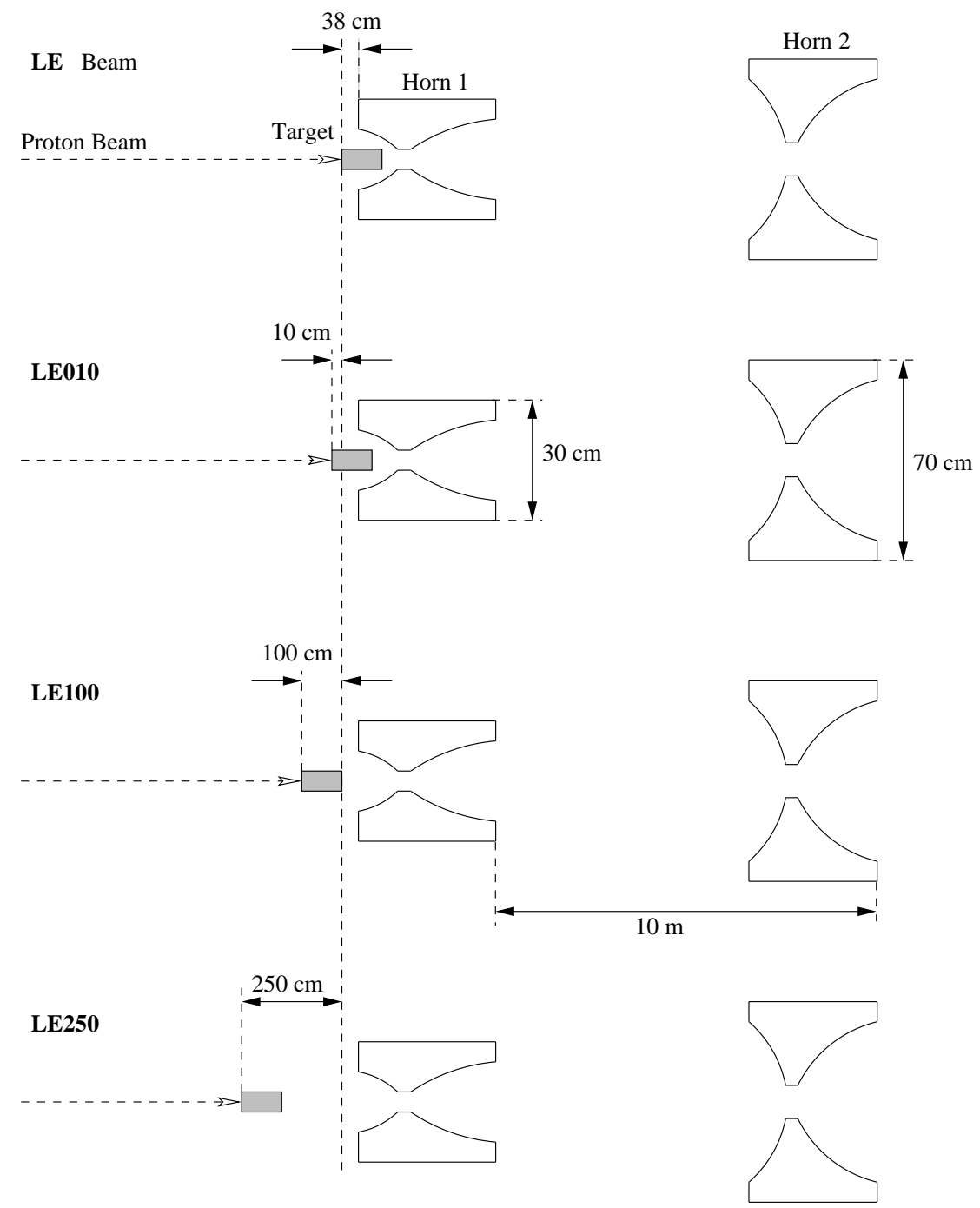

Figure 2.9: Shown are four of the five target-horn configurations in which the NuMI beam has operated to produce different energy neutrino beams. The fifth is LE150. In the nominal configuration, LE or LE000, the target is inserted into the first horn with only $35 \mathrm{~cm}$ outside of the horn. The LE000 configuration produces the lowest possible neutrino energy beam. Higher energy beams are produced my moving the target away from the horns. In principal, the target may be positioned at any distance with respect to the horns up to $2.5 \mathrm{~m}$. 


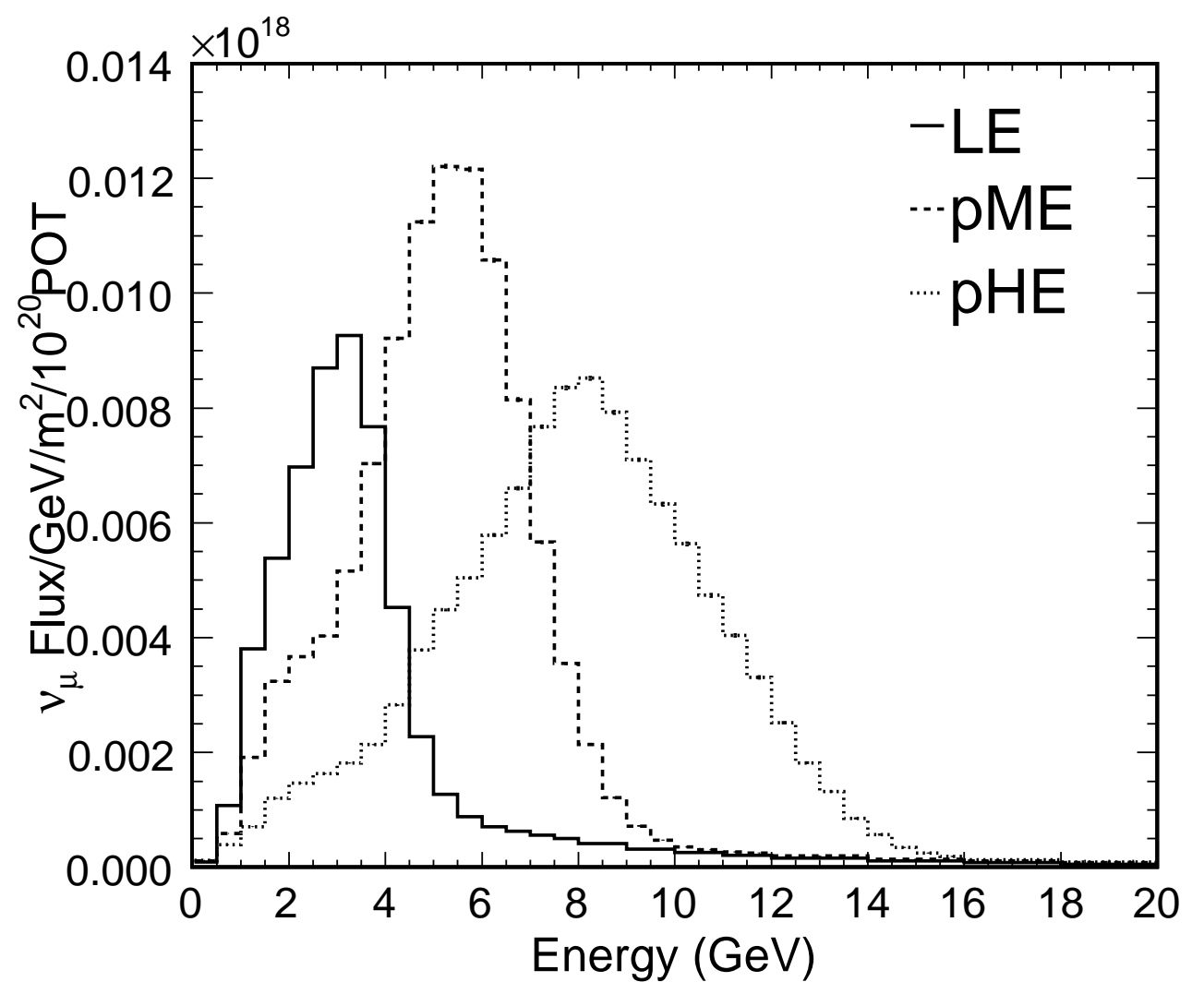

Figure 2.10: Monte Carlo simulation of the neutrino flux at the MINOS Near Detector in the LE010, LE100, LE150 and LE250 beam configurations. The peak energy of the neutrino flux increases as the target is moved further from the horns. 


\subsubsection{Beamline Instrumentation}

The cross section measurement presented in this thesis we will rely on a neutrino flux from the NuMI beam which is derived partially from calculations and partially from direct measurements.

The NuMI beam has extensive instrumentation systems for the primary proton beam, the secondary hadron beam off of the target, and the tertiary muon beam that accompanies the neutrino beam. Use of these instrumentation systems allows precise constraints to be placed on many aspects of the flux. A brief summary of each system is provided here.

From the point of view of physics analyses, it is important to understand the quality of the primary proton beam on a spill-by-spill basis. Various devices monitor the proton beam along the transfer line from the Main Injector to the NuMI target.

The neutrino flux directly depends on the number of protons impacting the target. Three quantities of the proton beam are of particular importance: the intensity, the position at the target and the lateral distribution at the target. The proton beam intensity is measured by two Toroid Intensity Monitor Integrators. One is located at the upstream end of the transfer line near the Main Injector extraction point and the other is located immediately upstream

of the target. These devices provide a $1 \%$ measurement of the number of protons delivered to the target for every spill $[22,32]$.

The position of the proton beam is measured by 24 capacitative "Beam Position Monitors" (BPMs) [33] at various points along the transfer line. The resolution on the measured position of the beam at the target is $<70 \mu \mathrm{m}$ [22].

Secondary Emission Monitors (SEMs) measure the beam position and 
profile [34]. Each monitor consists of two planes of $5 \mu \mathrm{m}$ thick segmented titanium foils. The planes are oriented transverse to the beam and perpendicular to each other such that the strips of one are oriented vertically while the strips of the other are oriented horizontally. Figure 2.11 shows the horizontal profile of the proton beam as measured by the SEM located just upstream of the target. The location of the target and upstream collimating baffle is superimposed. The center of the beam, corresponding to the peak of the profile, is aligned with the center of the target. Over the course of the run, the proton beam was observed to be centered on the target with less than $70 \mu \mathrm{m}$ spill to spill variation. Additionally, less than $0.2 \%$ of the proton beam envelope was observed to fall off of the side of the target.

The secondary beam, defined by pions and kaons produced in proton beam interactions with the target, is monitored by an array of ionization chambers located at the downstream end of the decay volume known as the hadron monitor. The hadron monitor is a $7 \times 7$ array of ionization chambers spanning a total of $76 \times 76 \mathrm{~cm}^{2}$ transverse to the beam. It is located immediately after the end of the decay volume at the very front of the hadron absorber, as shown in Figure 2.8. At this location, the flux of particles through hadron monitor is dominated by hadrons such as undecayed pions and unreacted primary protons.

Figure 2.12 shows the lateral distribution of particles at the hadron monitor from a typical beam spill where the proton beam impinges upon the center of the target. This shows that the RMS distribution of hadrons is typically $\sim 30 \mathrm{~cm}$. Figure 2.12 also shows the lateral distribution of particles measured by the hadron monitor when the proton beam impinges on the center of a gap between the target and baffle and the distribution when the proton beam im- 


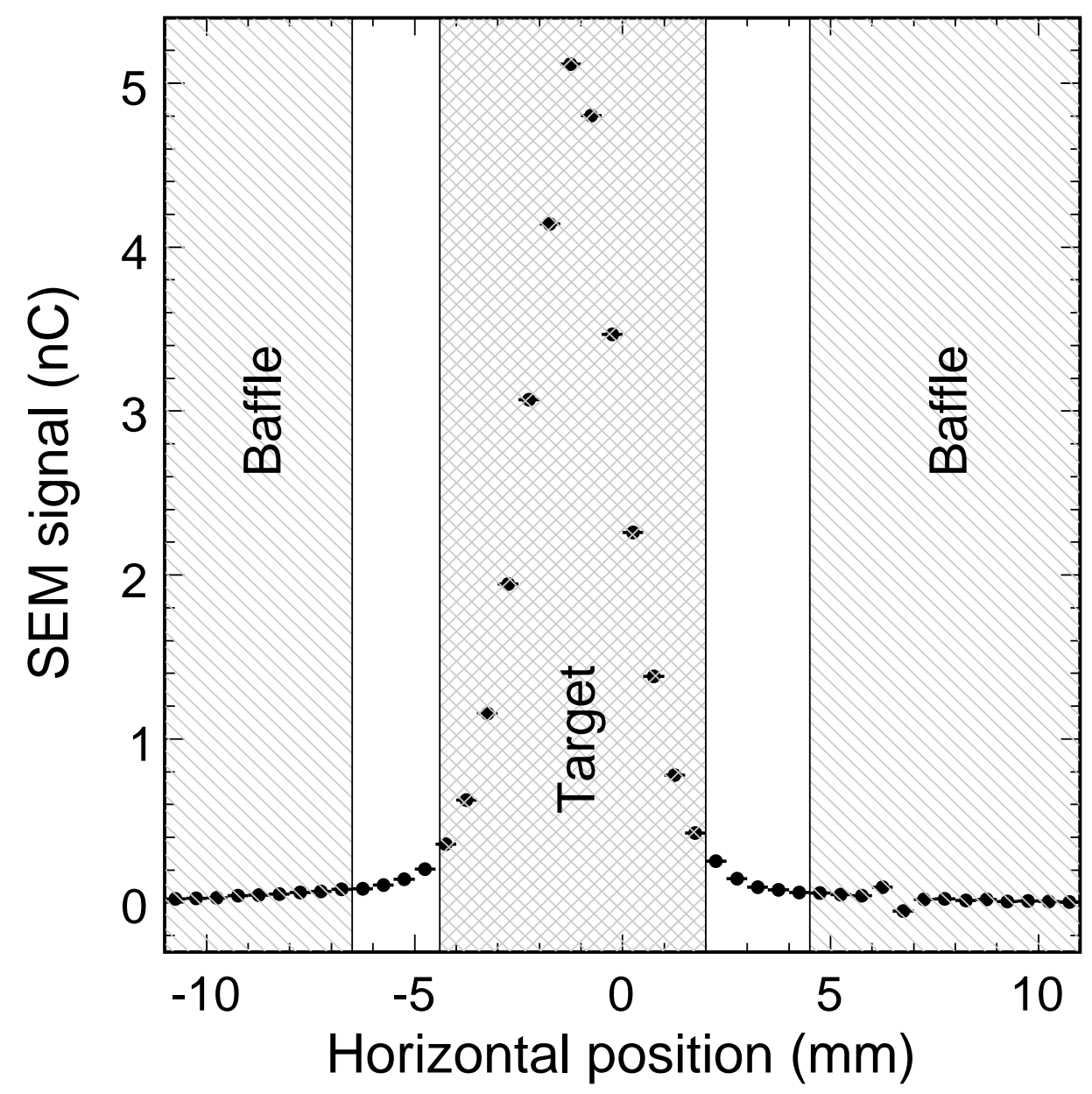

Figure 2.11: Horizontal profile of the proton beam measured by SEM closest to the target. The locations of the target and baffle as seen by the proton beam are superimposed. The spill is centered at $\mathrm{x}=1.1 \mathrm{~mm}$ with width $\sigma_{x}=1.2 \mathrm{~mm}$. $[22]$. 

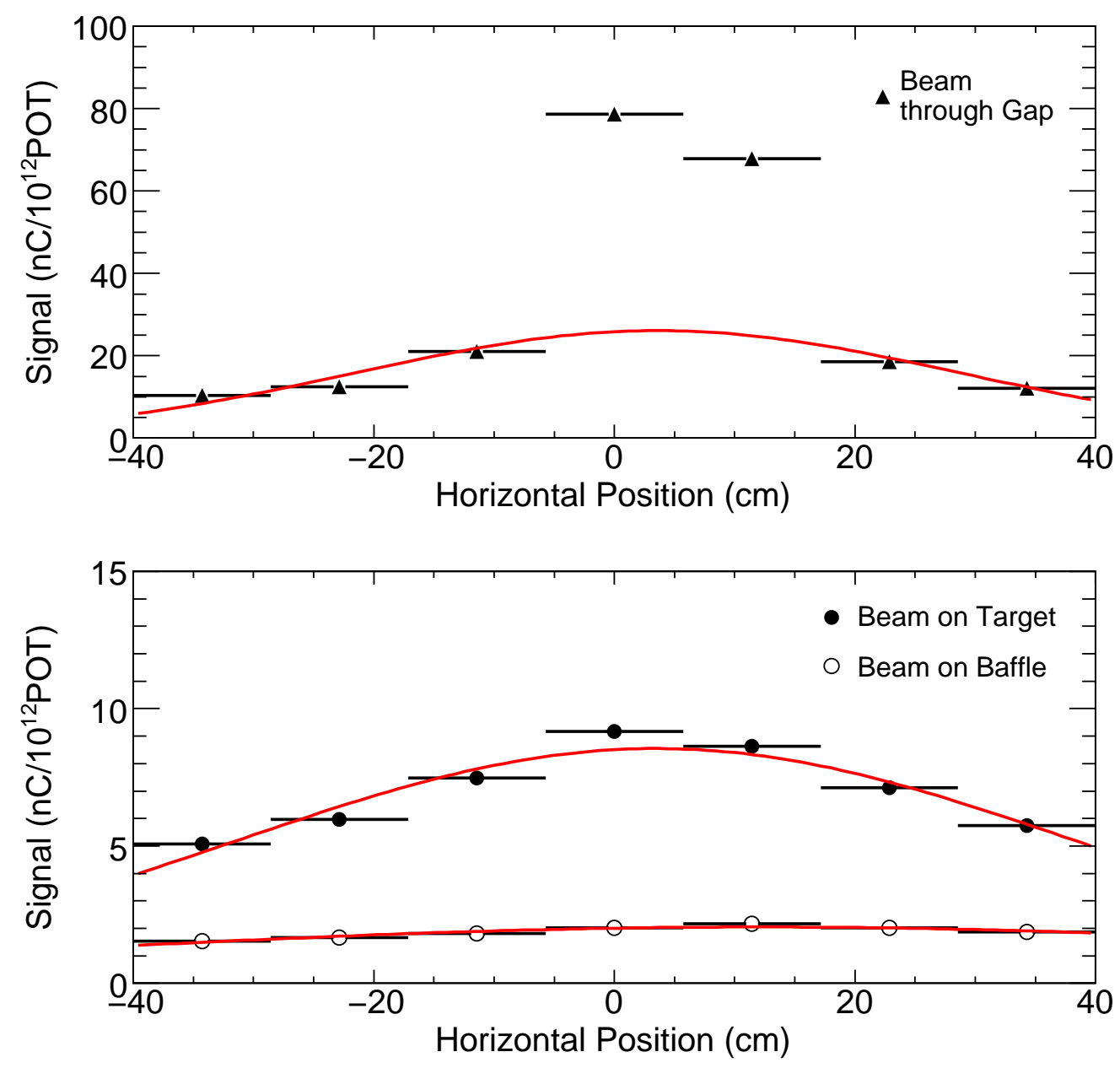

Figure 2.12: The horizontal distribution of particles at the hadron monitor when the proton beam impinges on the center of a gap between the target and baffle(top), when the proton beam impinges upon the center of the target(bottom), and when the proton beam impinges on the baffle(bottom). The curve is a fit to a Gaussian distribution. 
pinges upon the baffle. The proton beam passes largely uninteracted through the target-baffle gap and is transported directly to the hadron absorber. This results in a much larger beam intensity at the hadron monitor compared to when protons are attenuated in the target and a highly compact and peaked lateral distribution resulting from the lack of scattering that would occur from interactions within the $\sim 1 \mathrm{~m}$ target. On the other hand, the beam profile measured by the hadron monitor when the proton beam impinges upon the baffle shows reduced hadron intensity and a wider hadron distribution resulting from increased proton beam attenuation and scattering within the longer $2.5 \mathrm{~m}$ baffle.

The tertiary muon beam produced along with neutrinos in meson decay is measured by a system known as the muon monitors. Three muon monitors are located within excavated alcoves downstream of the hadron absorber as shown in Figure 2.8. Since they are located downstream of the hadron absorber, the flux of particles through the muon monitors is dominated by muons produced along with the neutrinos in the decay of pions and kaons in the decay volume. Like the hadron monitor each muon monitor is a 9 by 9 array of ionization chambers. Each muon monitor is separated from the decay volume by successively more shielding. The shielding imposes a momentum threshold for muons to reach each monitor of 4,11 , and $21 \mathrm{GeV} / c$, for alcoves 1,2 and 3 , respectively. This is to say that muons produced in the decay volume with momenta less than $4 \mathrm{GeV} / c$ will range out in the hadron absorber and never intercept muon monitor 1 , etc. Thus the muon monitors measure an integral of the muon flux above each of these thresholds.

A key role of the muon and hadron beam instrumentation is the verification of the neutrino beam's performance. As described in Ref $[22,31,35]$ and 
in Appendix B, precise manipulation of the proton beam can, when combined with measurements of particle fluxes in these downstream detectors, be utilized to align the target and magnetic horns. The results of these past studies are summarized in Chapter 4.

As a result, many neutrino flux uncertainties are directly constrained by data to be quite small [22]. Thus, attention may be paid to the measurement of particle fluxes off of the target. The knowledge of pion and kaon fluxes off of the target are the dominant uncertainty in the neutrino flux. Chapters 5 and 6 are concerned with using the muon monitors to directly measure these fluxes.

Consistency of the neutrino flux is important for experiments utilizing the neutrino beam. As muons are produced alongside neutrinos in meson decay, the muon flux is a good monitor of the neutrino flux. Figure 2.13 shows the muon flux measured in muon monitors 1 and 2 normalized by the number of protons on the target(ppp) for each spill over a 1 week period. During this time the proton beam quantities are very stable; the proton beam position at the target varies by less than $0.2 \mathrm{~mm}$ and the proton beam width varies by less than $0.1 \mathrm{~mm}$. Also, the current in the focusing horns varies by less than $0.4 \mathrm{kA}$. So it is not surprising that the muon flux is also very stable. The signal from both muon monitor 1 and muon monitor 2 varies by less than $\sim 1 \%$. Figure ?? shows the flux in the muon monitors during a 1 week period when proton beam conditions were not as stable leading to slight variation in the muon flux.

It is important that the neutrino beam is centered on downstream neutrino detectors. The angular size of the MINOS Near detector at a distance of $1 \mathrm{~km}$ from the target is $4 \mathrm{mrad}$. The solid angle of the MINOS Far Detector is 

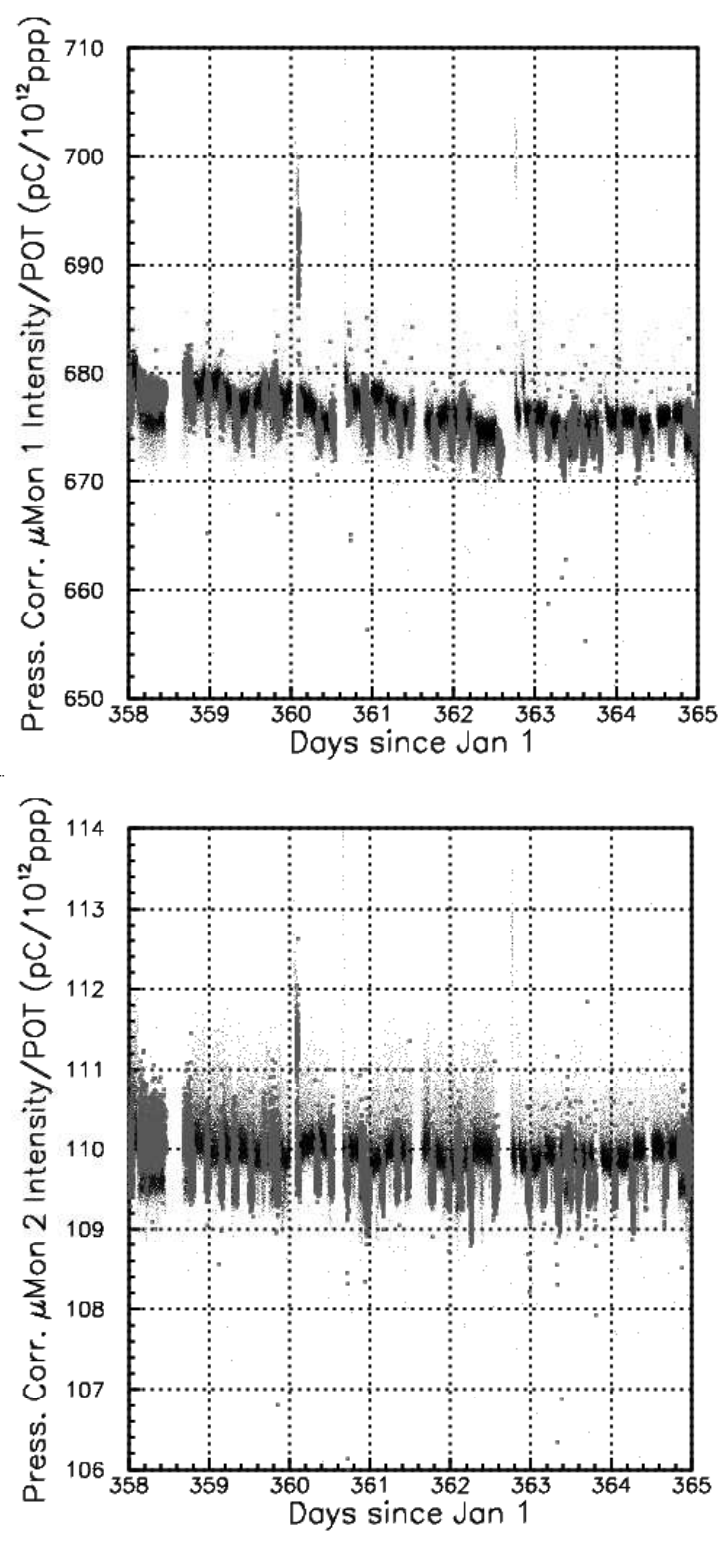

Figure 2.13: The muon flux measured in muon monitors 1(top) and 2(bottom) normalized by the number of protons on the target(POT,ppp) for each spill over a 1 week period. The muon flux is stable to $\sim 1 \%$ during this time due to stable proton beam conditions. The horizontal axis is "Days since January 12005 " thus the full range corresponds to Dec. 25, 2005(day 358) to Jan. 1, 2006(day 365). The vertical axis is the signal from muon monitors 1 and 2 normalized to the number of protons impacting the target. 


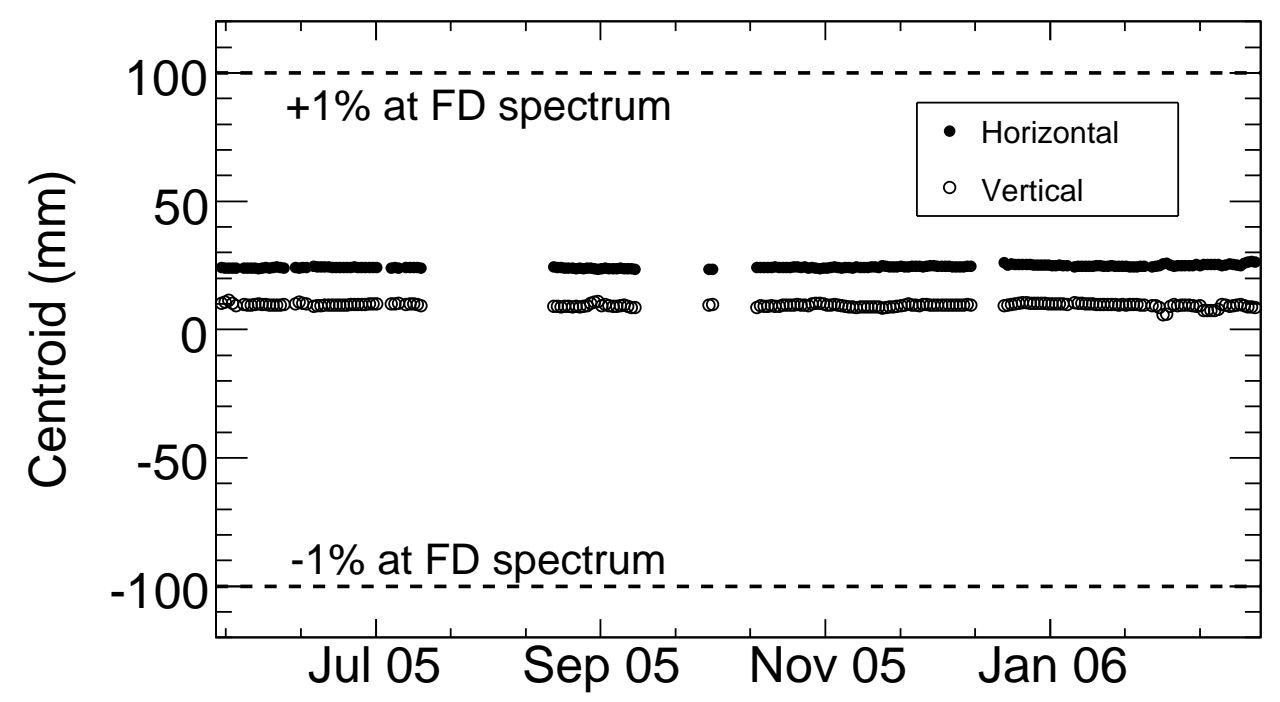

Figure 2.14: Horizontal and vertical muon beam centroid measured by first muon monitor station. The direction of the muon beam is the same as the direction of the neutrino beam. The observed variations in the centroid correspond to deviations of less than $10 \mu \mathrm{rad}$ in the beam angle. 
$\sim 735$ times smaller since it is 735 times farther from the target. Thus, small angles of the proton beam with respect to the beamline center can result to large offsets of the neutrino beam at downstream detectors. Given the large, $725 \mathrm{~m}$, distance between the muon monitors and the target, the direction of the muon beam and thus the neutrino beam can be determined to a few microradians. Figure 2.14 shows the centroid of the muon beam in the horizontal and vertical directions as measured in the first muon monitor over $\sim 1$ year. Deviations in the direction of the muon beam are less than $\sim 10 \mu \mathrm{rad}$.

\subsection{The MINOS Detector}

The MINOS Detector is located on the Fermilab site $100 \mathrm{~m}$ underground and $1 \mathrm{~km}$ from the NuMI target. It is a sampling calorimeter composed of alternating planes of $1 \mathrm{~cm}$ thick scintillator arranged in planes of strips and $2.45 \mathrm{~cm}$ thick steel planes. It is designed to measure the momenta of muons produced in charged current muon neutrino interactions, $\nu_{m u}+\mathrm{Fe} \rightarrow \mu^{-}+X$. The detector is magnetized allowing for the separate identification of $\mu^{+}$and $\mu-$ from $\bar{\nu}_{\mu}$ and $\nu_{\mu}$ charged current interactions, respectively. This also allows for a measurement of muon momentum by the curvature of the muon track. The energy of the hadronic state, $X$, is measured calorimetrically.

Figure 2.15 shows a diagram of the 980 ton MINOS Detector. It is a "squashed" octagon $\sim 3.8 \mathrm{~m}$ in diameter and $16.8 \mathrm{~m}$ long. The steel-scintillator planes are spaced $5.95 \mathrm{~cm}$ center-to-center for a total of 282 planes. The planes alternate in $+45^{\circ}$, called $\mathrm{U}$, and $-45^{\circ}$, called $\mathrm{V}$, orientation with respect to the horizontal direction. The upstream 120 planes comprises the calorimeter portion of the detector. All planes are instrumented to measure hadronic 


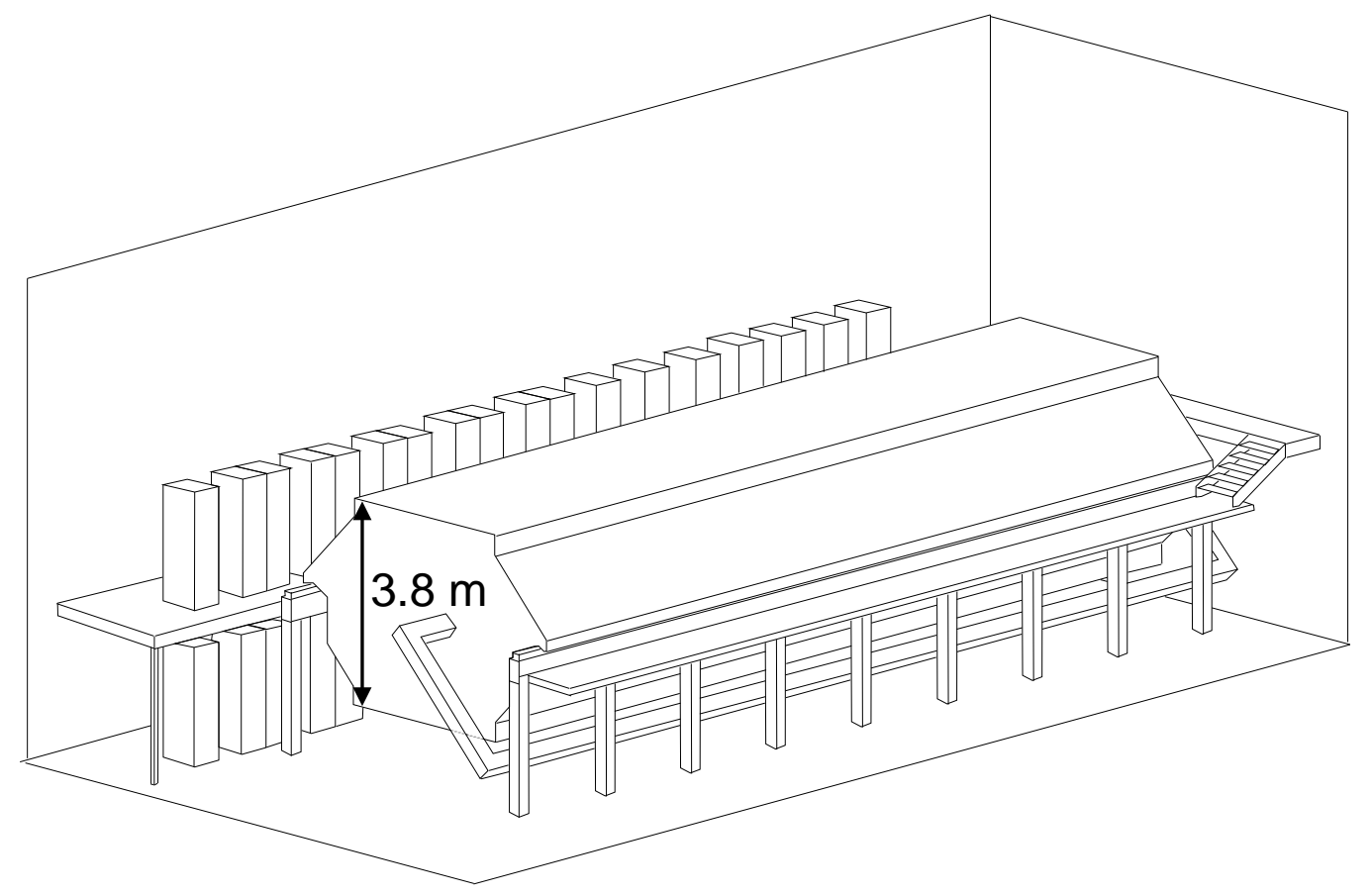

Figure 2.15: The MINOS Near Detector. The Near Detector is $16.6 \mathrm{~m}$ long and consists of alternating planes of steel and scintillator. [21] 
showers produced in neutrino interactions. The downstream 162 planes form the spectrometer section. Every 5th plane is instrumented to track muons produced in muon neutrino interactions. The magnetic coil is offset from the center of the detector $\sim 0.56 \mathrm{~m}$. The neutrino beam is centered on the left side of the detector between the left edge and the coil.

Figure 2.16 shows a diagram of a MINOS scintillator strip and demonstrates the scintillation process. Each polystyrene scintillation strip is $4.1 \mathrm{~cm}$, $1.0 \mathrm{~cm}$ thick and up to $6 \mathrm{~m}$ long(in the Near Detector) and coated with a titanium-dioxide $\left(\mathrm{TiO}_{2}\right)$ doped polystyrene reflective layer to maximize reflection and protect the strip. A wavelength shifting(WLS) fiber is inserted into a $2.3 \mathrm{~mm}$ deep grove cut into the wide face of the strip. Charged particles ionize the scintillator producing scintillation light at a characteristic $420 \mathrm{~nm}$. The WLS fibers absorb radiation peaked at $420 \mathrm{~nm}$ and re-emit light at $470 \mathrm{~nm}$. The light travels along the length of the fiber to the end where it impinges upon a photo-multiplier tube(PMT). Each strip is connected to one PMT

pixel. Only one end of each strip is readout, the other end is coated with a highly reflective material. Further details of the MINOS detector construction can be found in [36].

\subsubsection{Calibration}

The momentum from curvature is determined by a fitting algorithm which can compare the curvature and the range of stopping muons as a cross check of each measurement. The muon momentum from curvature is measured to 4\%. [37] 


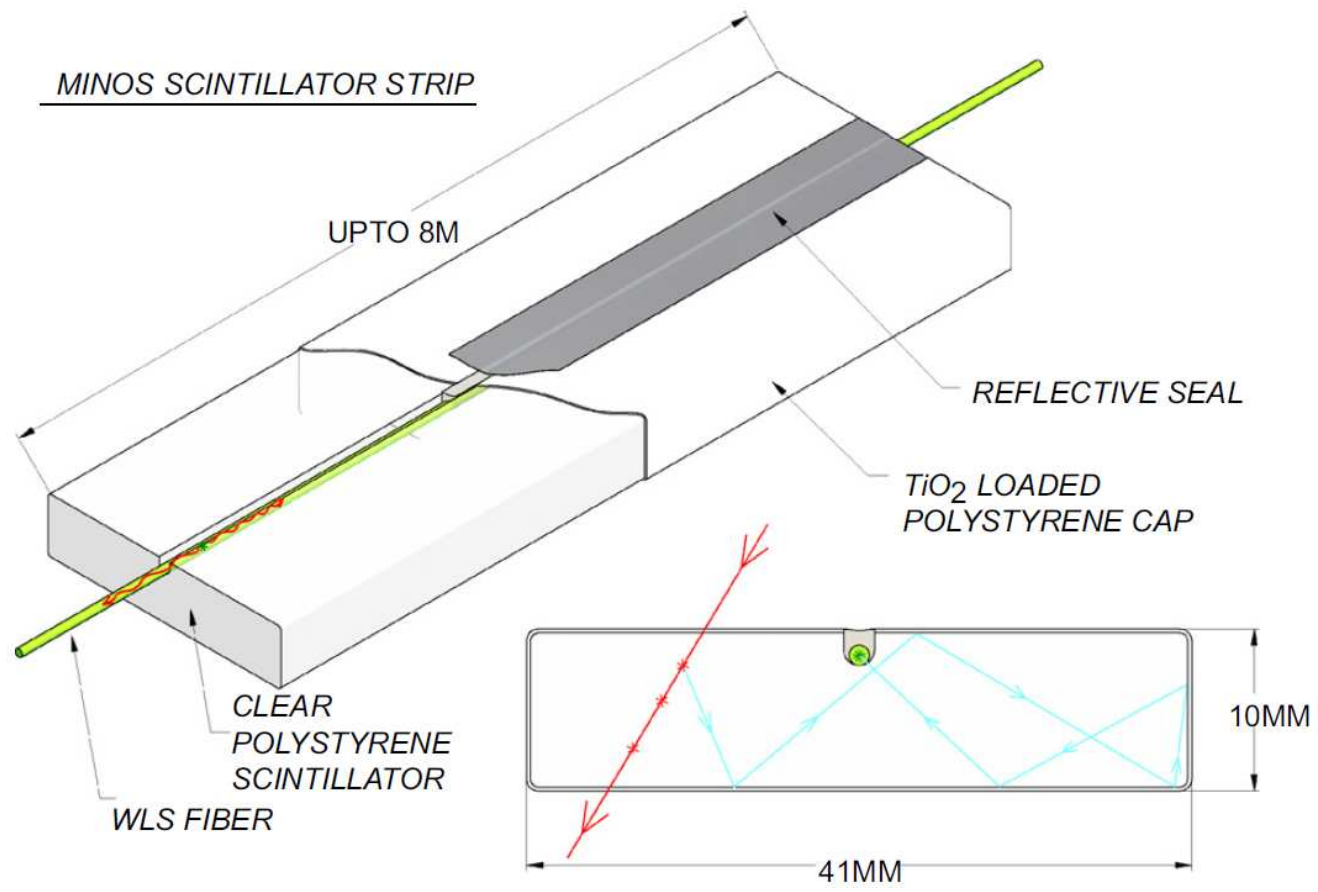

Figure 2.16: Diagram of a MINOS scintillator strip showing the outer $\mathrm{TiO}_{2}$ coating and WLS fiber. Scintillation light reflects within the scintillator until it is absorbed by the WLS fiber. Light is re-emitted by the fiber and travels down it's length to the collection system). [36] 


\subsubsection{Event Reconstruction}

Neutrino interactions or events with the MINOS detector are either charged current(CC) $\nu_{\mu}+\mathrm{Fe} \rightarrow \mu^{-}+X$ or neutral current (NC) $\nu+\mathrm{Fe} \rightarrow \nu+X$, the difference being the production of a charged lepton in the final state of a charged current interaction. The lepton allows for identification of the incident neutrino flavor. Thus, in NC interactions, the neutrino flavor cannot be determined.

During a proton beam spill, $\sim 10$ neutrino events occur in the detector due to the large neutrino flux. Figure 2.17 shows neutrino events in the detector during one proton beam spill. Spacial and timing information are used to distinguish individual events during reconstruction. [38] Figure 2.18 shows the timing distribution of hits from the neutrino events shown in Figure 2.17.

The scintillator strips measure the charge deposited by the particles produced in the interaction. The energy deposition of a charged particle in a scintillator strip is called a hit. The collection of hits is reconstructed to form the neutrino event. The reconstruction code pieces together successive hits forming muon tracks and particle showers. The reconstruction identifies muon tracks and hadronic showers from the same neutrino event by determining the origin of the track or shower, referred to as the event vertex. Figure 2.19 shows the distribution of neutrino event vertices in data and Monte Carlo.

Muon tracks from interaction of beam neutrinos in the detector should propagate longitudinally away from the beam direction. Figure 2.20 shows the distribution of the direction of the muon track from selected $\nu_{\mu} \mathrm{CC}$ events with respect to the incident neutrino beam. Neutrino events selected(refer to Section 2.2.3) for analysis predominantly produces muons parallel to the 


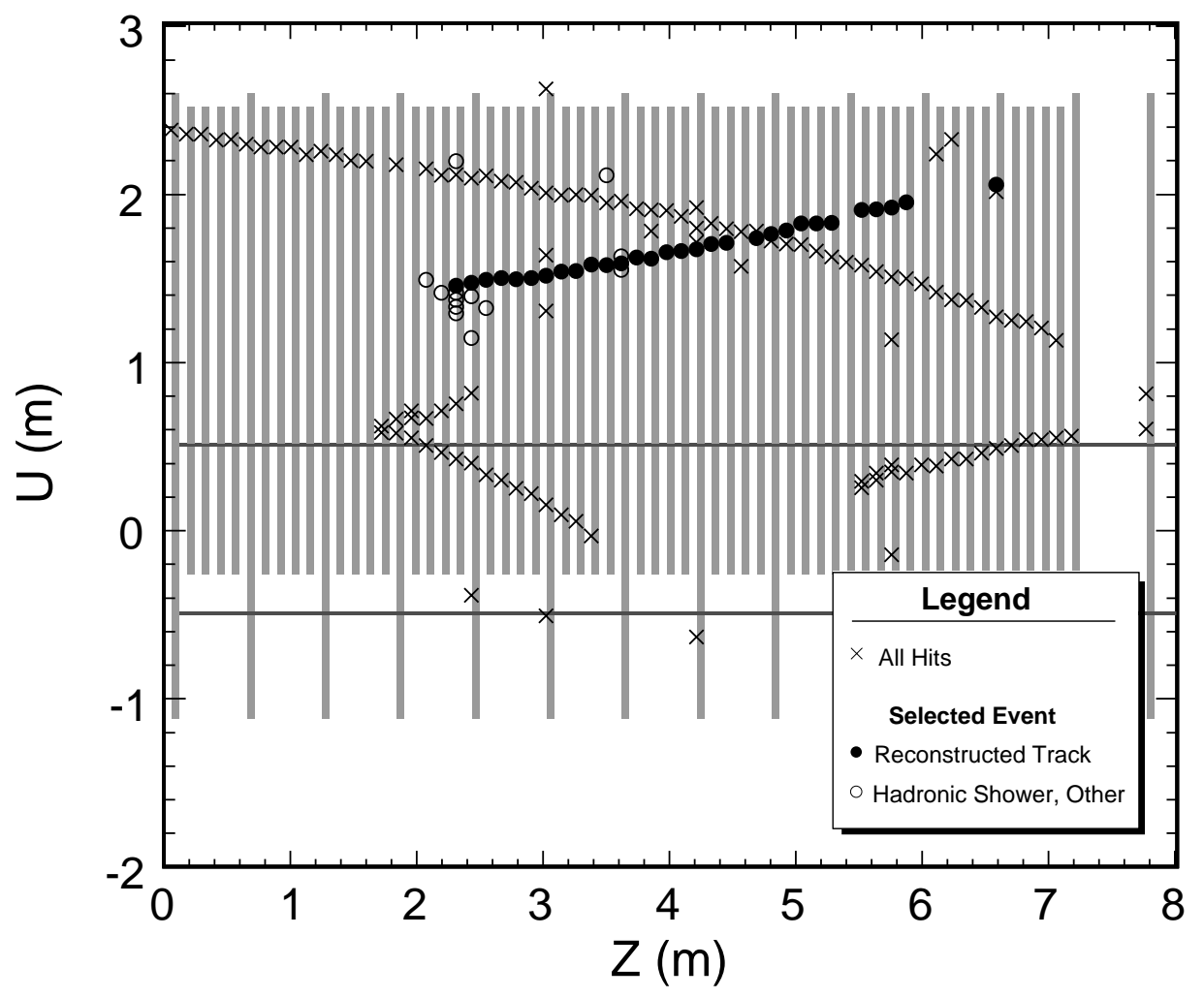

Figure 2.17: Event display in the MINOS detector for one proton beam spill. Four neutrino interactions are visible. Typically the number of events/spill is larger but a spill with fewer events is chosen for clarity. The timing distribution is shown in Figure 2.18. [21] 


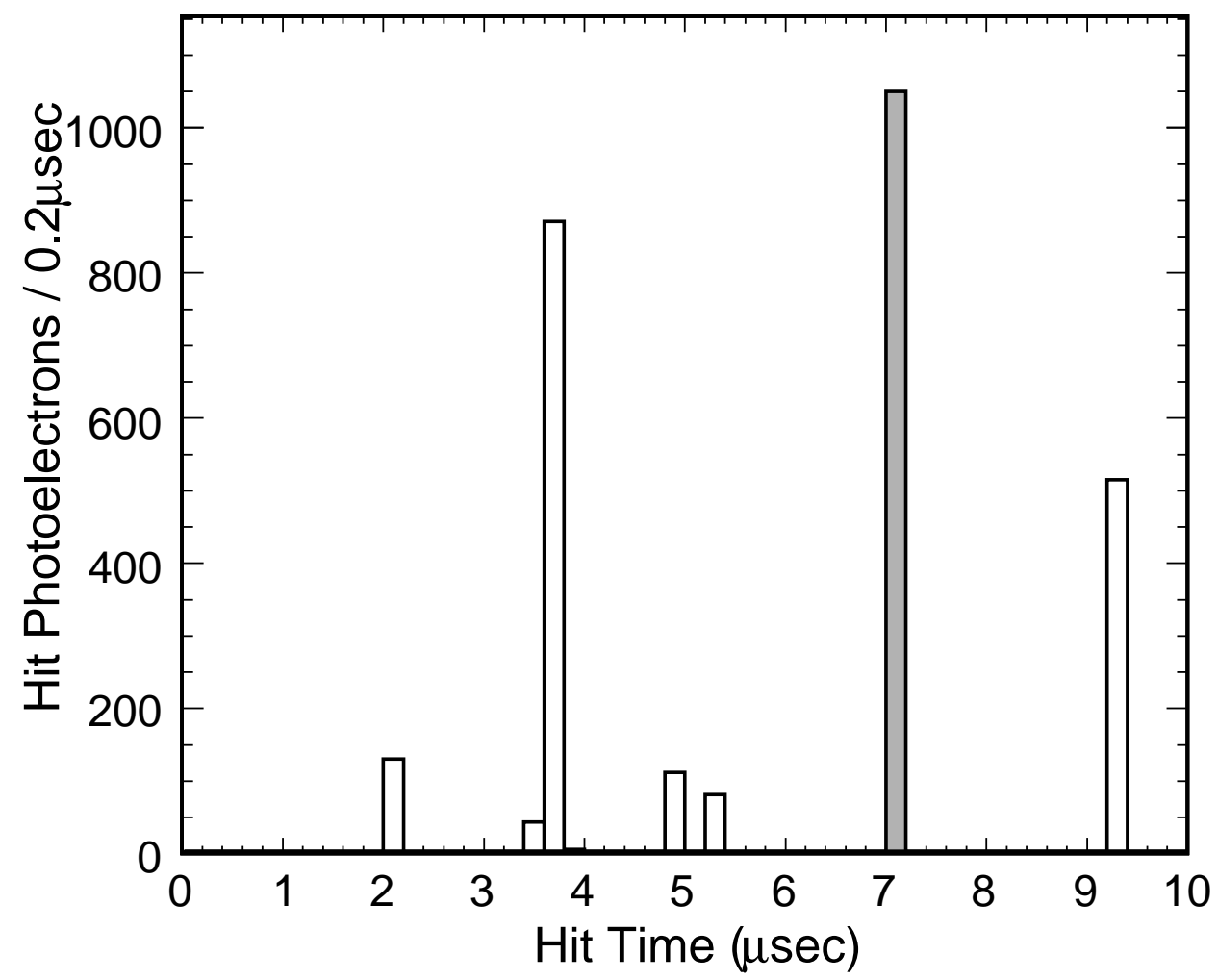

Figure 2.18: The timing distribution of detector hits from the neutrino events shown in Figure 2.17. [21] 

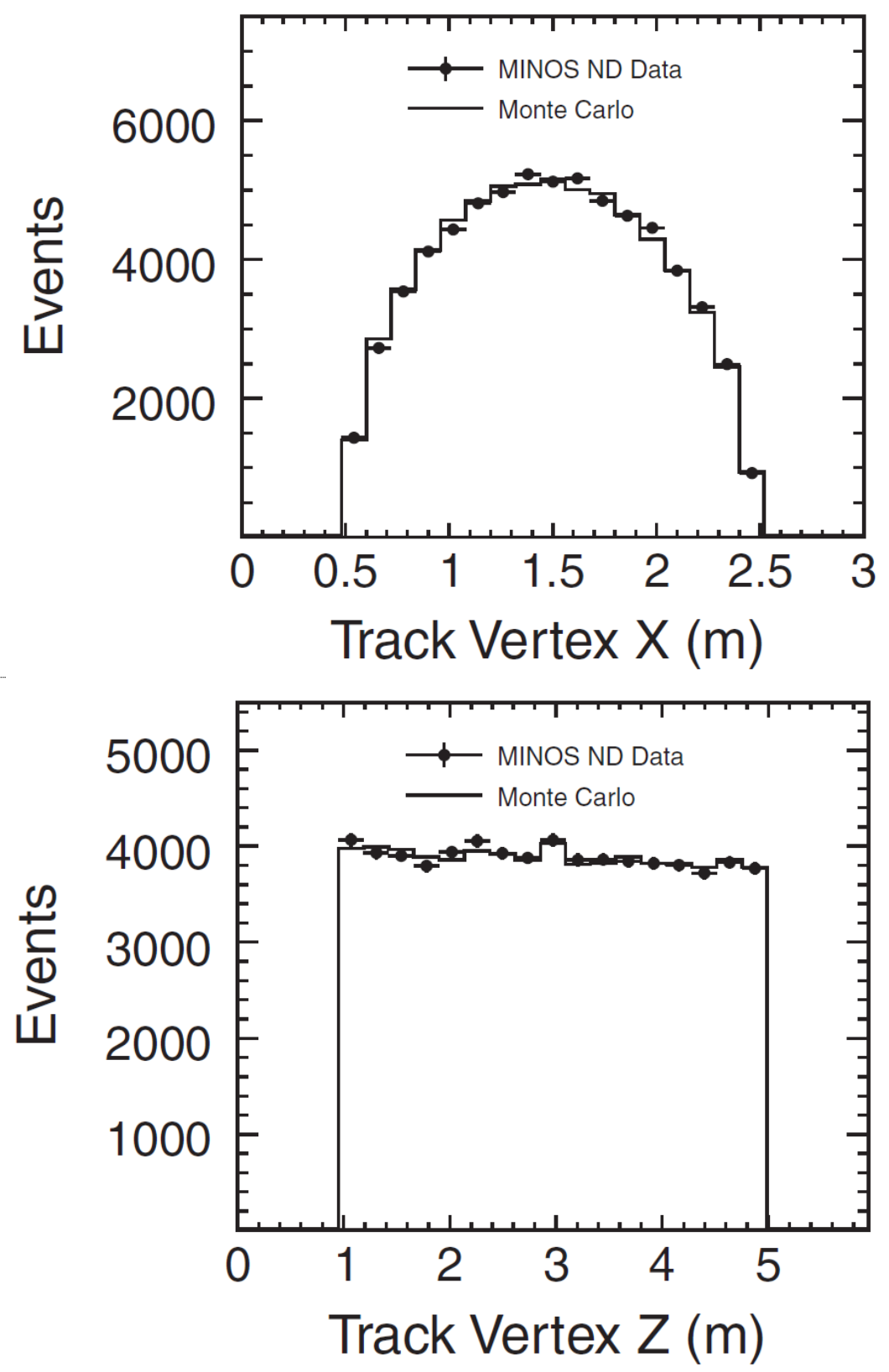

Figure 2.19: The reconstructed $\mathrm{x}$, horizontal, and $\mathrm{z}$, longitudinal to the detector, neutrino interaction vertices, defined by the muon track, for selected(refer to Section 2.2.3) $\nu_{\mu}$ charged current events in MINOS Detector. The Data(points) are in good agreement with Monte Carlo(histogram). [21] 


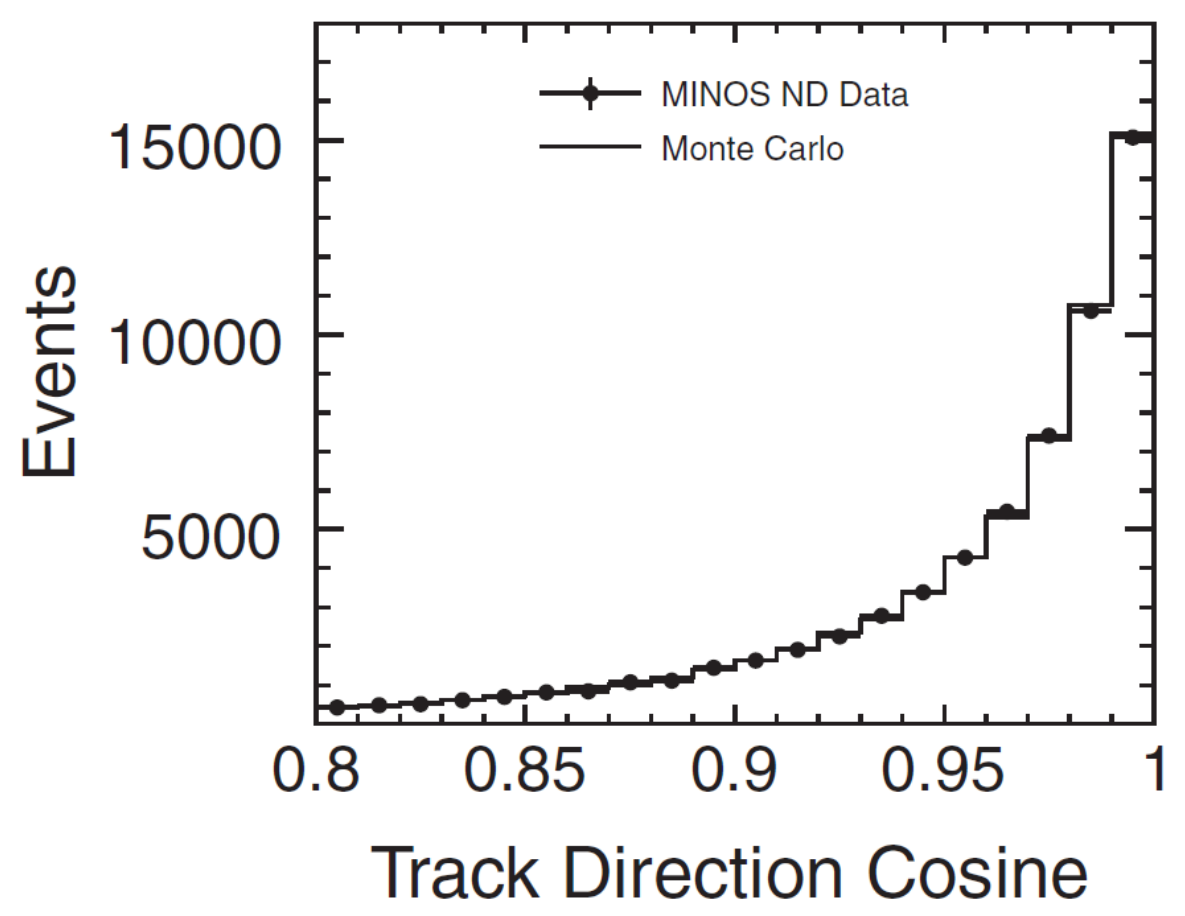

Figure 2.20: The direction of the muon track from selected(refer to Section 2.2.3) $\nu_{\mu} \mathrm{CC}$ events with respect to the incident neutrino beam. The Data(points) are in good agreement with Monte Carlo(histogram). [21] 
incident neutrino beam.

Figure 2.21 shows three types of neutrino events that occur in the MINOS detector. The charged current $\nu_{\mu}$ event is characterized by the long well defined muon track penetrating the vertex shower activity as seen in the upper(U-view) and middle(V-view) left plots of the figure. The charged current $\nu_{\mathrm{e}}$ and neutral current events are characterized by an electromagnetic and a hadron shower respectively. The electromagnetic shower tends to have a compact transverse profile while the hadronic shower is transversely diffuse.

If the energy of the muon from a $\mathrm{CC} \nu_{\mu}$ event is low, it may range out within the detector before escaping the hadronic shower near the vertex. The reconstruction may fail to reconstruct the track in that event. Alternatively, "fake" particle tracks may be reconstructed from clusters of hits. Occasionally tracks may also be produced from protons or pions within an hadronic shower. Thus, a CC event may look like a NC event and a true NC may appear to have a muon track. A particle identification technique was developed which uses event topology and physics characteristics to identify muon tracks in neutrino events and thus separate charged current muon neutrino events from NC events. This is discussed in Section 2.2.3.

The neutrino energy is reconstructed from the visible energy in the detector as

$$
\mathrm{E}_{\nu}=\mathrm{E}_{\mu}+\mathrm{E}_{h a d}
$$

where $\mathrm{E}_{\mu}$ is the visible muon energy and $\mathrm{E}_{\text {had }}$ is the visible hadronic energy in a neutrino event. However, due to uncertainties in final state interactions and hadronization, the process of the formation of hadrons out of quarks and gluons, the visible energy in the detector is not the true neutrino energy. The 


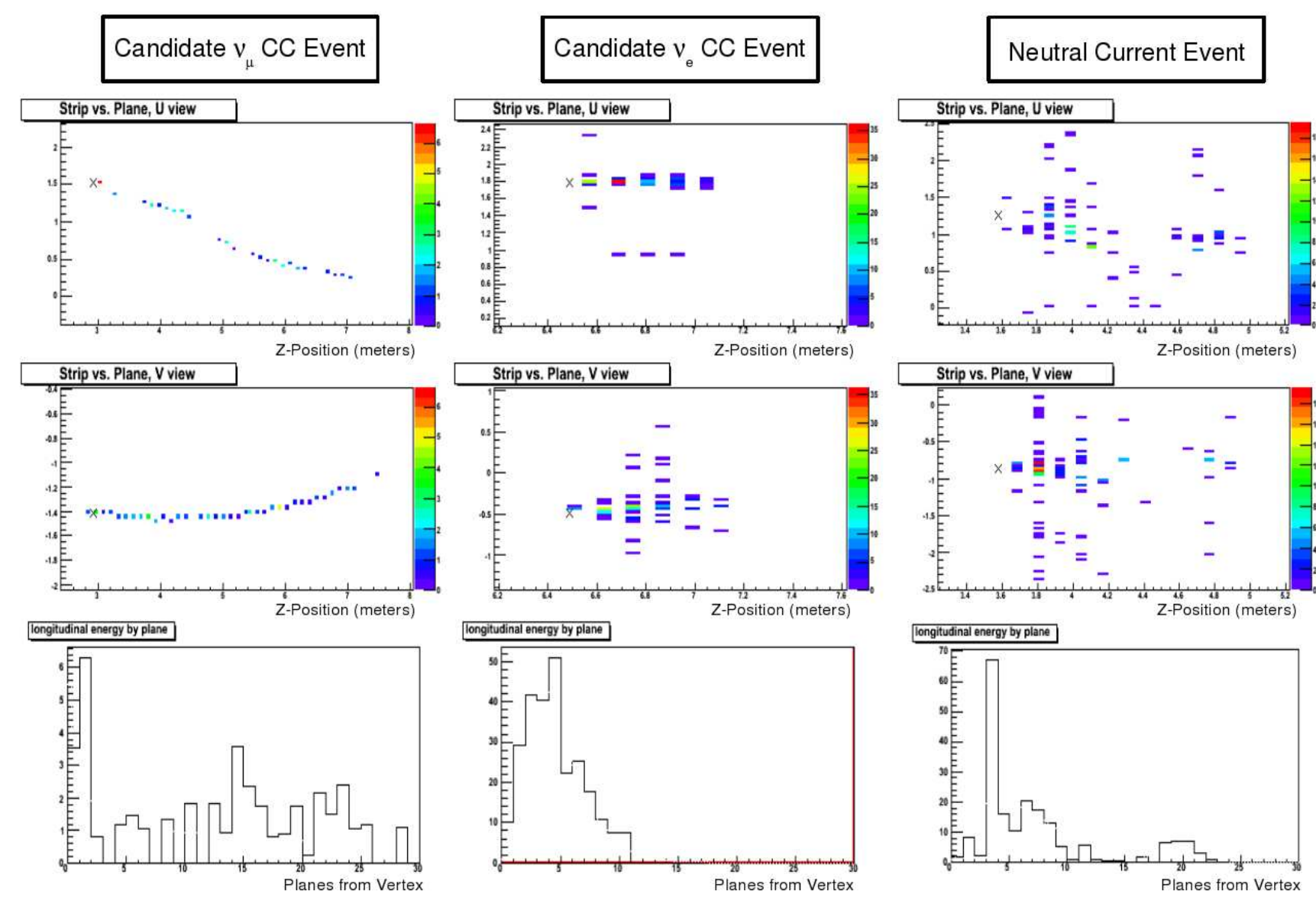

Figure 2.21: A charged current $\nu_{\mu}$ (left column), a charged current $\nu_{\mathrm{e}}$ (middle column), and a neutral current event candidate in the MINOS Detector. The first row is shows the U-view orientation and the second row shows the $\mathrm{V}$-view orientation of the detector scintillator planes. The last row shows the total pulse height of all strips vs the longitudinal position in the detector. The $\mathrm{CC} \nu_{\mu}$ event produces a muon which appears as a long track in the detector. The $\mathrm{CC} \nu_{\mathrm{e}}$ and $\mathrm{NC}$ events produce electromagnetic and hadronic showers, respectively, which differ in the width and length of their longitudinal profiles. 
smearing in energy from the true incident $\nu_{\mu}$ energy to the value measured in the detector must be accounted for in the cross section analysis and is discussed in Chapter 7.

The MINOS Detector Monte Carlo simulates neutrino interactions using the NEUGEN event generator [11]. The NEUGEN model uses current knowledge of neutrino cross sections and incorporates models for final state interactions, such as pion charge exchange, absorption and rescattering in the nucleus and hadronization. For neutrinos of the same energy the total energy deposited in the detector may vary from interaction to interaction and the energy deposited in the detector may not be the true energy of the incident neutrino. The uncertainty on the hadronic shower energy measured in the detector is determined by varying the model assumptions in NEUGEN and comparing the results. Figure 2.22 shows the total uncertainty on the hadronic energy as the quadrature sum of the uncertainty on the hadronic shower energy due to these nuclear effects(energy dependent), the $5.6 \%$ single particle energy scale(energy independent), and the $2.3 \%$ detector calibration uncertainty.

\subsubsection{Event Selection}

Events are pre-selected by requiring that the even vertex is reconstructed within a $3.2 \mathrm{~m}$ long, $0.8 \mathrm{~cm}$ radius cylindrical volume starting $0.8 \mathrm{~cm}$ from the upstream face of the detector and centered around the beam axis, the fiducial volume. Hadronic showers from events originating outside of the fiducial volume may not be fully contained within the detector leading to poor event reconstruction. Events are also required to have a reconstructed track 


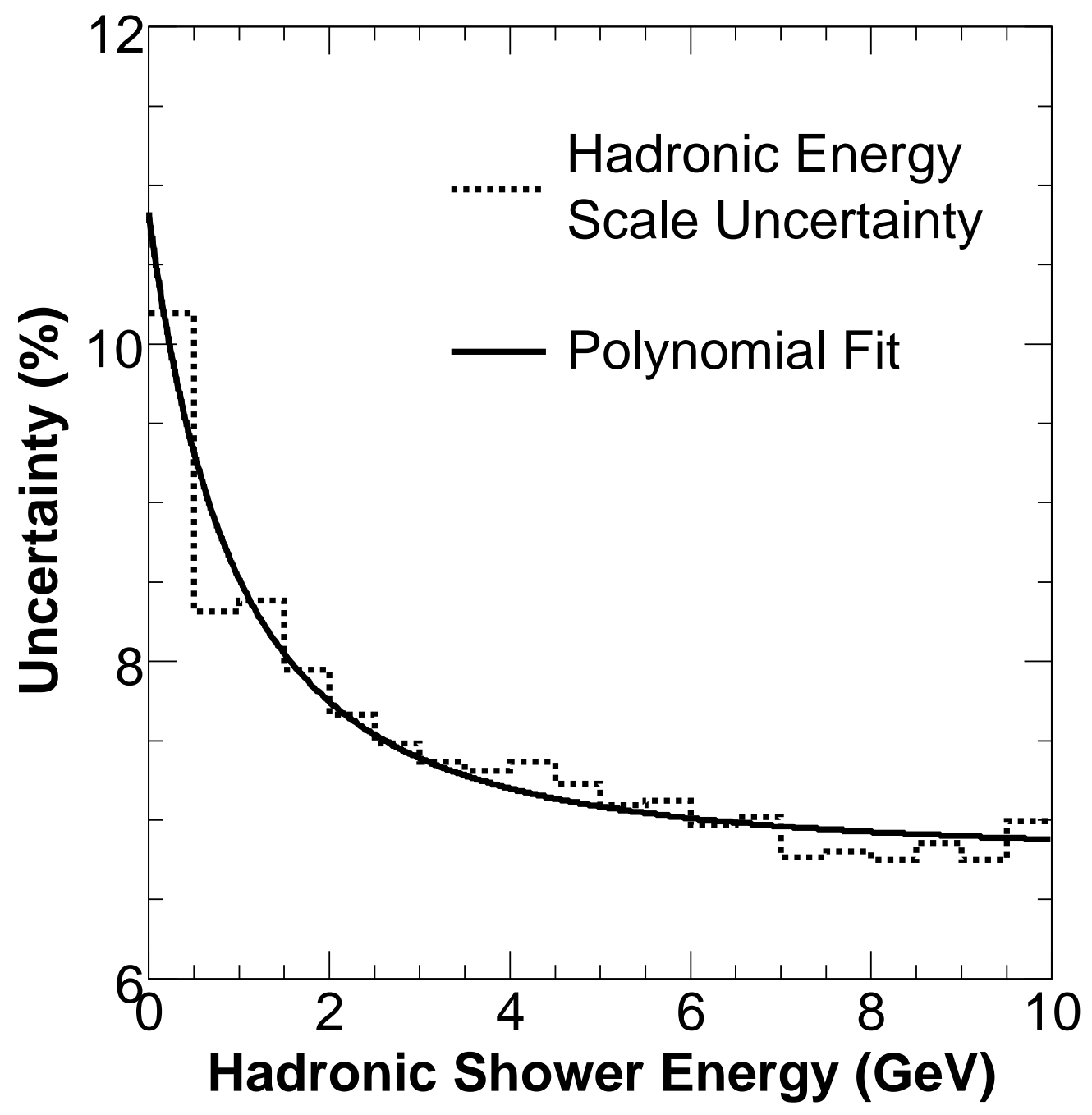

Figure 2.22: The uncertainty in the hadronic energy scale in the MINOS detector. The histogram is the quadrature sum of the uncertainty in the simulation of hadron production in neutrino-iron interactions [39], an energy independent $5.6 \%$ uncertainty in detector response [40, 41, 42]. and a $2.3 \%$ uncertainty in detector calibration [21, 43]. The curve is a polynomial fit. 
that spans 5 or more detector planes in each U-view and V-view.

For the analysis of this thesis we are interested in using charged current $\nu_{\mu}$ events to measure the charged current $\nu_{\mu}$ cross section and use the MINOS $\nu_{\mu} \mathrm{CC}$ event particle identification algorithm $[44,37]$ to identify $\nu_{\mu} \mathrm{CC}$ events for use in the analysis. Four variables which differ for muon and non-muon tracks are used to classify events as $\mathrm{CC} \nu_{\mu}$ or background.

Compared to hadrons, muons have a lower $d E / d x$ which means that muons tend to have longer tracks than do hadrons. This makes the length of the track a good discriminating variable. Also, few $\mathrm{GeV}$ muons tend to loose energy mostly by ionization which means that the average energy deposited in each strip varies little. Hadrons may undergo nuclear processes which lead to large variations in the energy deposited along the track. Furthermore, the muons deposit energy in a narrow window close to their path whereas hadrons tend deposit energy in a broader window transverse to their path. Thus, the four variables, the length of the track, the light yield of the track, the ratio of the sum of the lowest light yield hits and the sum of the highest light yield hits composing the track, and the fraction of hits in a plane that belongs to the track exhibit characteristics that distinguish muon and non-muon tracks.

The variable are inputs in a k-nearest neighbor $(\mathrm{kNN})$ algorithm which classifies events as $\nu_{\mu} \mathrm{CC}$ or background based on these variables. A Monte Carlo sample of events is used to populate the 4 dimensional space defined by these four variables. In Monte Carlo it is known whether the a given event is $\nu_{\mu} \mathrm{CC}$ or a background event. Each data event is compared to the nearest Monte Carlo events in the 4-dimensional variable space. The nearest events are defined by the Euclidean distance, where the coordinates are the variables, between the data event and the surrounding population of Monte Carlo events. 
A discriminating variable, the "kNN value" is defined as the fraction of $\nu_{\mu} \mathrm{CC}$ events among the $\mathrm{k}$-nearest neighbors around the data event. A $\mathrm{kNN}$ value is assigned to each data event.

Figure 2.23 shows the distribution of the $\mathrm{kNN}$ value for the pre-selected events in the MINOS detector. Also, shown are the expected curves for the $\mathrm{kNN}$ variable for $\mathrm{CC}$ and $\mathrm{NC}$ event in the MINOS Monte Carlo. Clear separation between events with a muon and non-muon track is achieved by this method. Those events having a $\mathrm{kNN}$ value greater than 0.3 are classified as $\nu_{\mu}$ $\mathrm{CC}$ events otherwise they are classified as background events. A small sample of true background events are selected as signal, $\nu_{\mu} \mathrm{CC}$ events. Figure 2.24 shows the efficiency and purity of the selected events as a function of the measured neutrino energy. The efficiency is the fraction of true $\nu_{\mu} \mathrm{CC}$ events that are selected and drops as the neutrino energy decreases because the muons are lower energy and travel shorter distances in the detector. The purity is the fraction of the total selected sample which are true $\nu_{\mu} \mathrm{CC}$ events. It also drops as the neutrino energy decreases because hadronic tracks may mimic short muon tracks. Further details of the event selection algorithm can be found in Refs. [44, 37].

\subsection{Summary}

This chapter describes the experimental apparatus to measure the total charged current cross section of muon neutrinos on iron. The neutrino source is the Neutrinos at the Main Injector, NuMI, beamline. The neutrino detector is the 980 ton MINOS detector located $1 \mathrm{~km}$ from the source target. The apparatus has several essential features for the cross section measurement. First, 


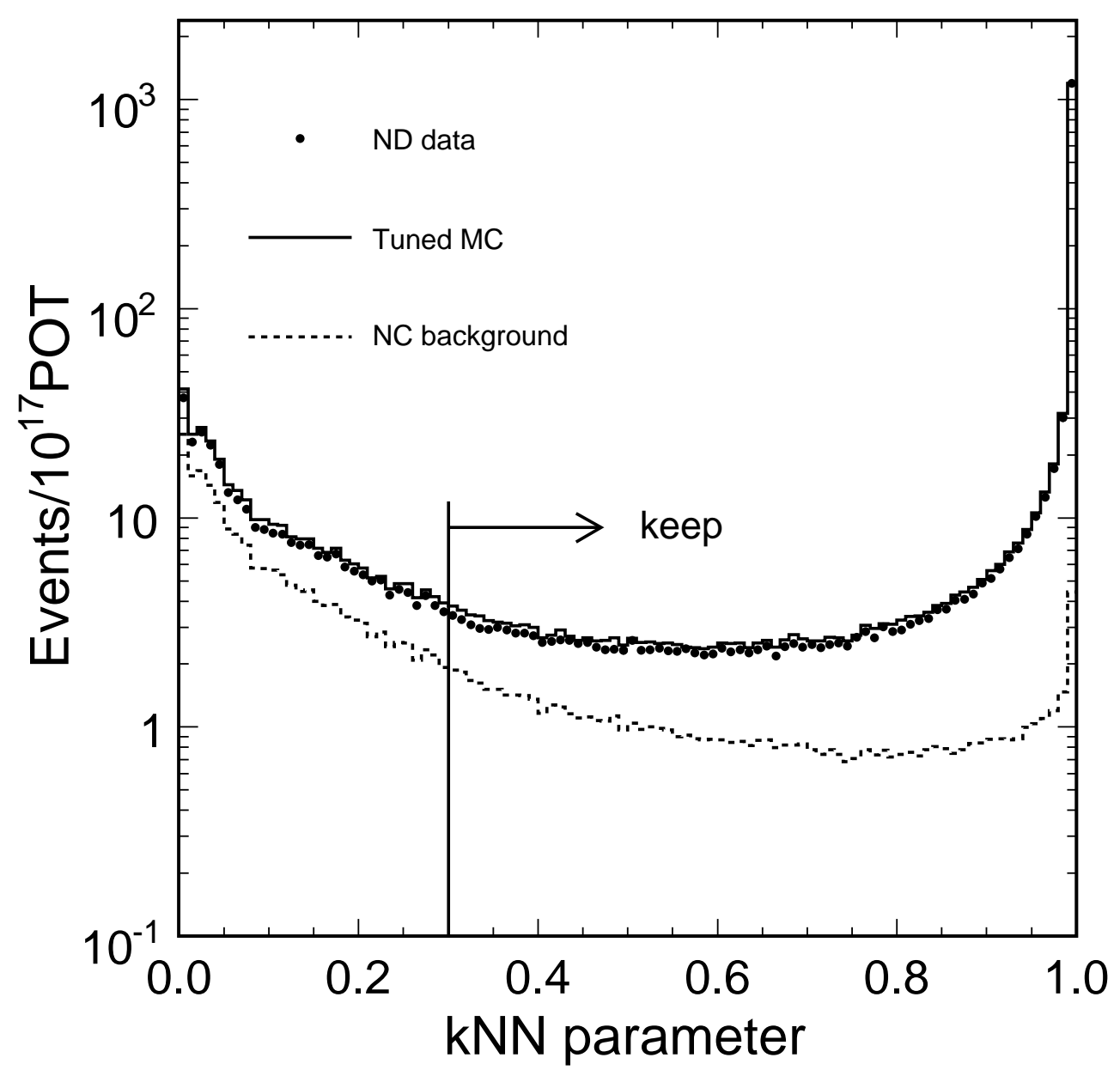

Figure 2.23: The distribution of $\mathrm{kNN}$ value for neutrino events in the MINOS detector. Those events having a $\mathrm{kNN}$ value greater than 0.3 are classified as $\nu_{\mu} \mathrm{CC}$ events otherwise they are classified as background events. Courtesy R. Ospanov. 


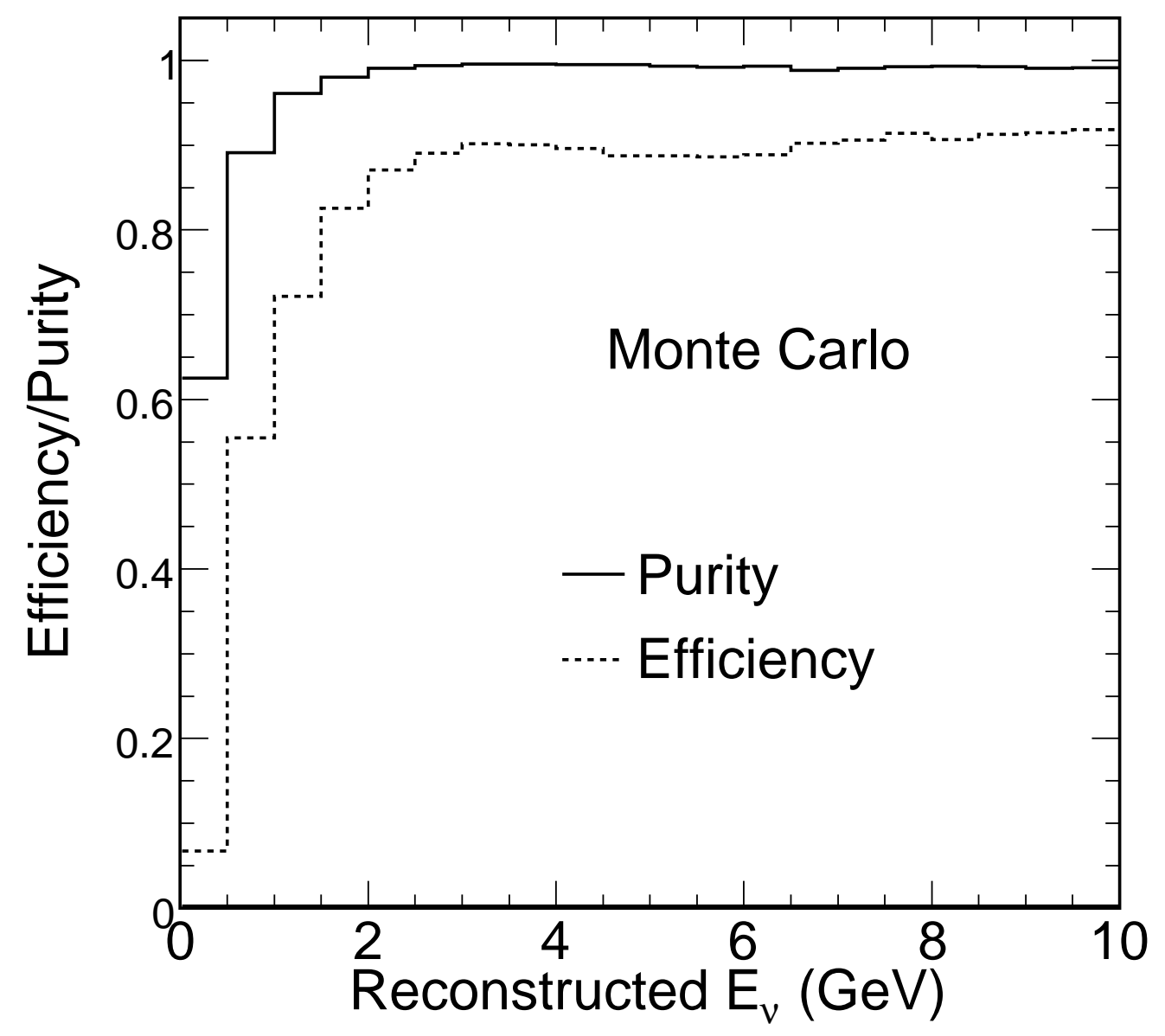

Figure 2.24: The efficiency and purity of the selected $\nu_{\mu}$ charged current data sample as a function of the measured (reconstructed) neutrino energy. The efficiency is the fraction of true $\nu_{\mu} \mathrm{CC}$ events that are selected and drops as the neutrino energy decreases because the muons are lower energy and travel shorter distances in the detector. The purity is the fraction of true $\nu_{\mu} \mathrm{CC}$ in the total selected sample. It also drops as the neutrino energy decreases because hadronic tracks may mimic short muon tracks. Courtesy R. Ospanov. 
the NuMI beamline provides an intense beam that spans $1-16 \mathrm{GeV}$ peaking at $\sim 4 \mathrm{GeV}$ which enables neutrino cross section measurements to be made at low energies. Second, the NuMI beamline is flexible, and enables changes in the flux spectrum which can be used to cross-check the results. Third, the beamline has extensive instrumentation which, when combined with the flexible design, permits measurement of the neutrino flux. Fourth, the neutrino detector separates $\nu_{\mu}$ and $\bar{\nu}_{\mu}$ interactions, permitting study of $\nu_{\mu}$ cross sections free from contamination from $\bar{\nu}_{\mu}$ in the beam. These features enable the analysis presented in this thesis. 


\section{Chapter 3}

\section{Muon Flux Measurement System}

The NuMI beamline produces neutrinos by allowing pion and kaons to decay in flight along the evacuated tunnel. $\pi \rightarrow \mu \nu_{\mu}$ and $K \rightarrow \mu \nu_{\mu}$ decays also produce muons, thus a measurement of the muon flux may be used to infer the neutrino flux. This chapter begins with a brief discussion of the muon detector system hardware. Section 3.2 discusses the backgrounds which contribute to the signal in the muon detectors. Section 3.3 describes the operational performance of the muon monitors over the time period relevant for the cross section analysis. The last section describes specialized data sets taken with the muon flux system which will be used in Chapters 5 and 6 to derive the neutrino flux measurement. 


\subsection{Muon Flux System}

The muon flux system consists of 3 muon monitors located downstream of the hadron absorber as shown in Figure 2.8. Figure 3.1 shows a schematic of a single muon monitor. There are nine $10.2 \times 10.2 \mathrm{~cm}^{2}$ ionization chambers aligned from top to bottom inside each of 9 tubes forming a $9 \times 9$ array. The chambers are spaced $25.4 \mathrm{~cm}$, center-to-center, so that a muon monitor spans a total of $2.2 \times 2.2 \mathrm{~m}^{2}$ transverse to the beam. A tube is rectangular with dimensions $15.24 \mathrm{~cm}$ wide(horiztonal) and $228.6 \mathrm{~cm}$ high on the front and back faces the thickness of the plates forming the tube is $0.3175 \mathrm{~cm}$. The ionization chamber plates are ceramic, $\mathrm{Al}_{2} \mathrm{O}_{3}$, and are $10.16 \mathrm{~cm}^{2}$ and $0.1 \mathrm{~cm}$ thick. Thus a particle passing perpendicularly to the monitor at the center of a tube would pass through $0.635 \mathrm{~cm}$ of aluminum and $0.2 \mathrm{~cm}$ of ceramic. The $3 \mathrm{~mm}$ region between the chamber plates is filled with helium gas at NTP.

As shown in Figure 3.2, a single ionization chamber consists of two parallel plate electrodes separated $3 \mathrm{~mm}$. The chambers are filled with helium gas. A bias voltage is applied across the electrodes which causes the ions liberated by the traversing muon beam to drift towards the cathode. The resulting current is measured and digitized by the readout electronics.

Figure 3.3 shows "plateau" curves for the central chamber(pixel) of the first muon monitor. Plateau curves are obtained at several proton beam intensities an beam configurations to check rate dependent effects [31]. The fact that these curves show a flat region between 50-100 V indicates full collection of charge ionized in the chamber without significant loss due to recombination, irrespective of space charge build-up in the ion chamber. Ideal operation of the ion chamber is achieved on this plateau. The muon monitors are operated 


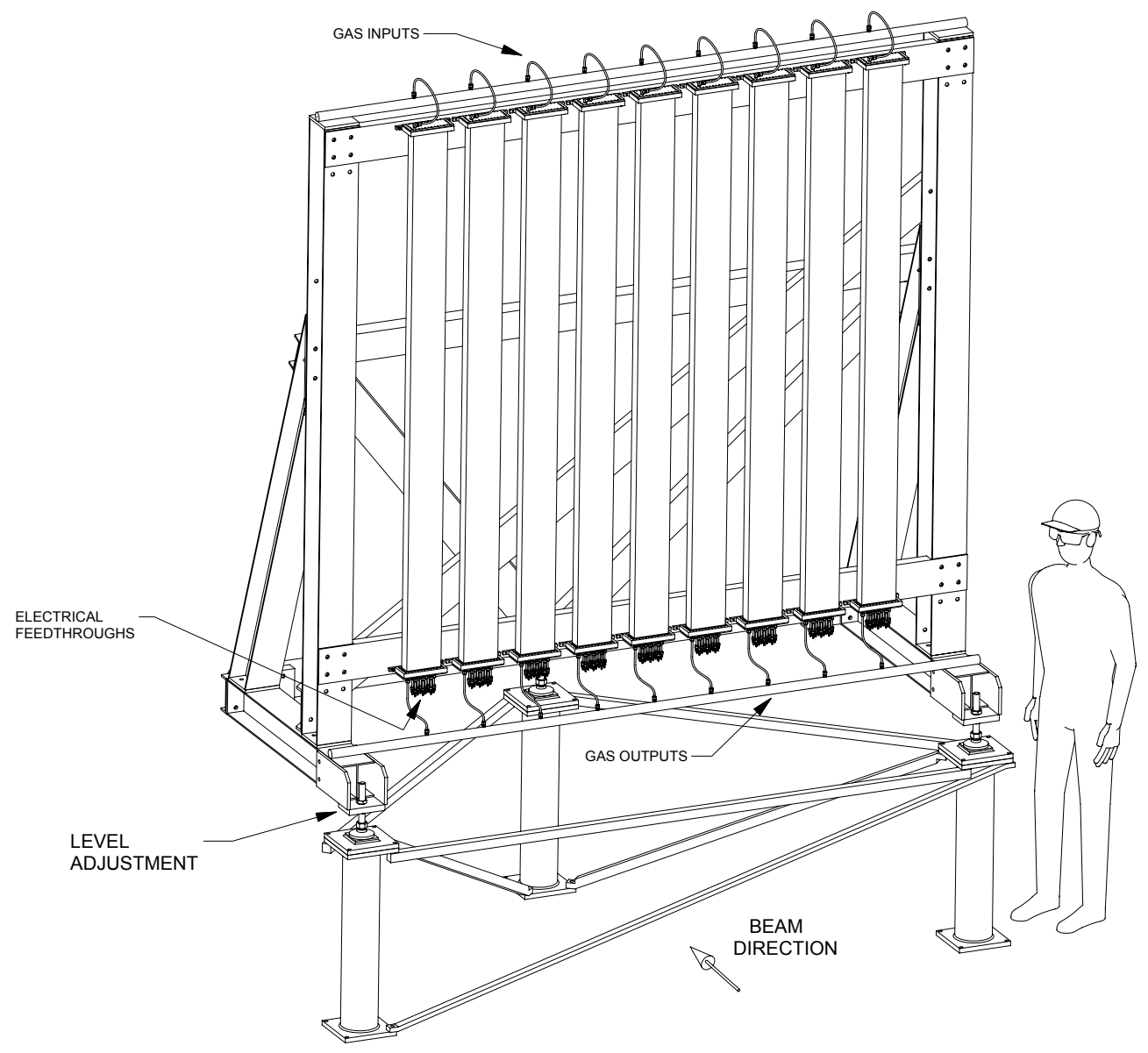

Figure 3.1: One of the three Muon Monitor arrays. There are nine ionization chambers in each of nine tubes. 


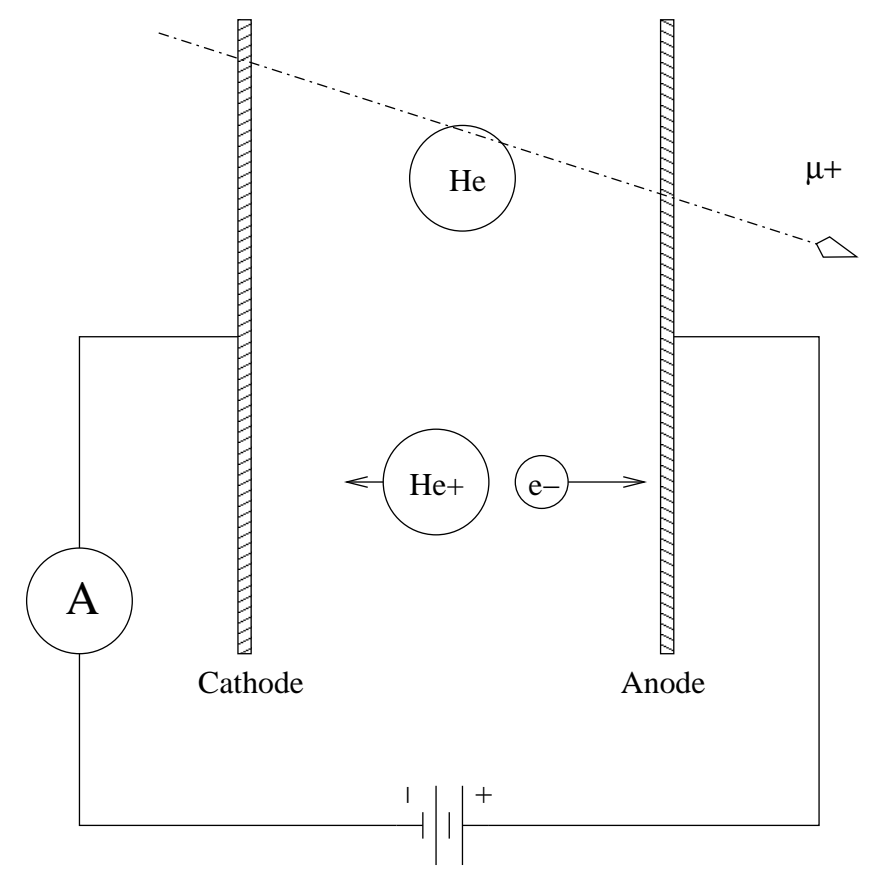

Figure 3.2: Schematic of a parallel plate ion chamber. The chamber is filled with helium gas. A bias voltage is applied across the plate electrodes of the chamber producing an electric field across the gap. Charged particles ionize the helium producing an ion-pair. The ion and electron are accelerated towards their respective electrodes. The resulting current is measured and digitized by the readout electronics. 


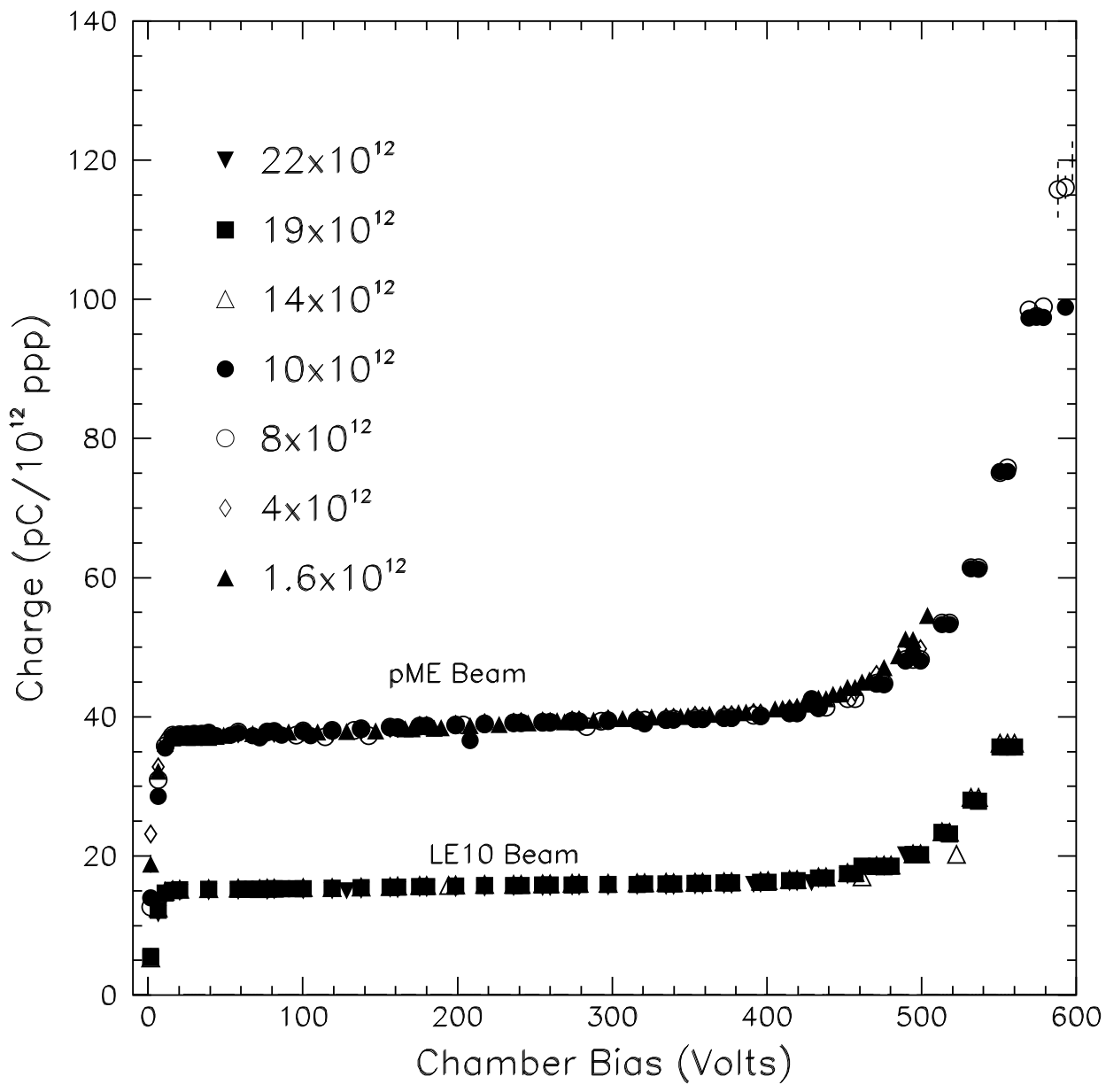

Figure 3.3: Plateau curves of the central chamber(pixel) of muon monitor 1. Plateau curves are obtained at several proton beam intensities and beam configurations to check rate dependent effects. Ideal operation of the ion chamber is achieved for bias voltages ranging from $\sim 100$ to $\sim 400 \mathrm{~V}$. The muon monitors are operated at $300 \mathrm{~V}$. [31] 
at $300 \mathrm{~V}$.

\subsubsection{Ionization Scale}

In order to know the number of muons passing through each muon monitor, the ionization scale, that is the charge collected by the monitors per intercepting muon, of the monitors must be known. Theoretical and experimental values from past experiments of the ionization of helium gas by charge particles are summarized in Ref [31]. Two beam tests using prototype muon monitor ionization chambers were performed at FNAL [45] and BNL [46] from which the ionization scale may be extrapolated.

The beam test performed at FNAL placed two prototype $8 \times 8 \mathrm{~cm}^{2}$ parallel plate ionization chambers (IC), with $1 \mathrm{~mm}$ and $2 \mathrm{~mm}$ electrode spacings, in the $8 \mathrm{GeV}$ proton Booster beam. The chambers were filled with He gas from a commercial bottle of labeled $99.998 \%$ purity.

The upper plot of Figure 3.4 shows the normalized IC signal as a function of the proton beam intensity for three different applied bias potentials. Without charge amplification, recombination or screening effects [31] such curves are flat with intensity. Except at the lowest intensities, the curves demonstrate non-linearity due to these effects except at the lowest intensities. The least affected curve, $250 \mathrm{~V}$, extrapolates to $3.2 \mathrm{pC} / 10^{7}$ particles as the intensity goes to zero. Since the muon monitor chambers have an anode-cathode spacing of $3 \mathrm{~mm}$, the charge per particle scale factor for the muon monitors is $9.6 \mathrm{pC} / 10^{7}$ particles.

The beam test performed at BNL utilized the Accelerator Test Facility (ATF) which provides $42 \mathrm{MeV}$ electron bunches with pulse charges from $1 \mathrm{pC}$ to 

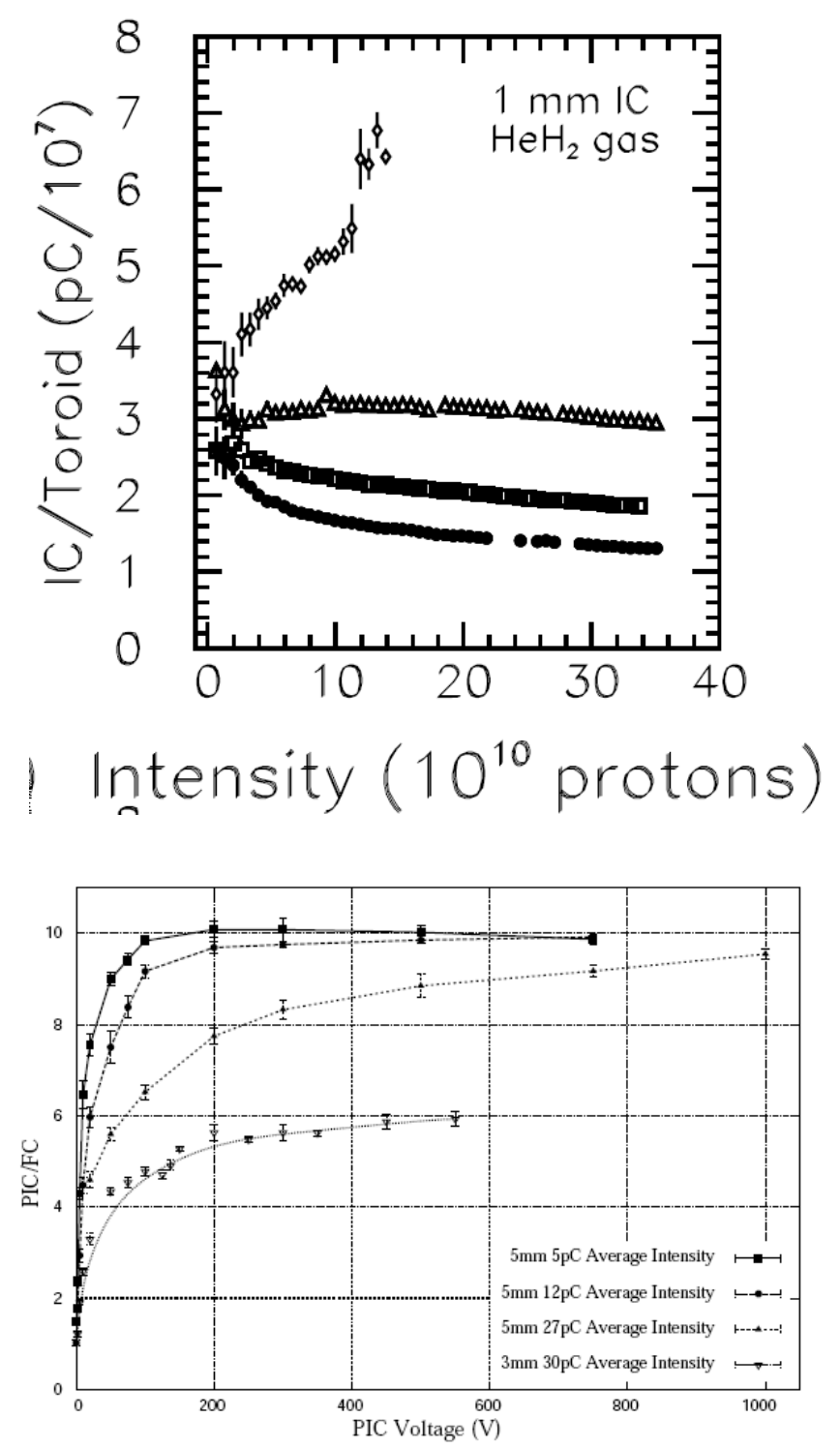

Figure 3.4: Top: The normalized ion chamber (IC) signal as a function of the proton beam intensity for applied bias potential of $100 \mathrm{~V}(\bullet), 150 \mathrm{~V}(\square), 200 \mathrm{~V}$ $(\triangle)$, and $250 \mathrm{~V}(\diamond)$ measured by the beam test conducted at FNAL. Bottom: The normalized PIC signal as a function of the applied bias voltage across the chamber for several beam intensities as measured by the beam test conducted at BNL. The beam intensity is measured in $\mathrm{pC} / \mathrm{spill}$. The vertical axis is the ratio of the ion chamber to beam charge. 
$1 \mathrm{nC}$. The prototype ionization chamber (PIC) was of similar construction and size to the FNAL prototype chambers. The chamber was filled with $99.999 \%$ pure He gas. The chamber was tested with $3 \mathrm{~mm}$ and $5 \mathrm{~mm}$ anode-cathode spacings.

The lower plot of Figure 3.4 shows the normalized signal as a function of the applied bias voltage across the chamber for several beam pulse charges. At the lowest voltages the ion chamber signal suffers from insufficient bias potential to collect all of the ionized charge. Under ideal conditions the curves plateau over the mid-range voltages indicating that the charge collected is directly proportional to the charge ionized. The $5 \mathrm{~mm}$ chamber plateaus above $200 \mathrm{~V}$ for beam charges of $5-12 \mathrm{pC}$. For the $5 \mathrm{~mm}$ chamber the charge per particle is $15.6 \mathrm{pC} / 10^{7}$ particles. Extrapolating to the $3 \mathrm{~mm}$ electrode spacing of the NuMI muon monitors gives $9.4 \mathrm{pC} / 10^{7}$ particles.

\subsubsection{Gas Supply System and Purity}

The NuMI muon monitors are supplied helium from 8 commercial gas cylinders of helium rated at $99.998 \%$ purity. A single gas line runs from the bottle manifold, located above ground, $\sim 300 \mathrm{~m}$ to an equipment rack, located below ground, where it is fanned out to each of the 3 muon monitors and the hadron monitor. Helium is continuously flowed through the monitors requiring gas bottle exchange every $\sim$ month to 8 days depending on the flow rate. The flow

rate of helium through the monitors has been changed several times over the 5 years of operation to purge impurities from the system.

A typical impurity found in commercial helium bottles is oxygen and typical levels are p.p.m.(parts per million). As oxygen has a lower ionization 


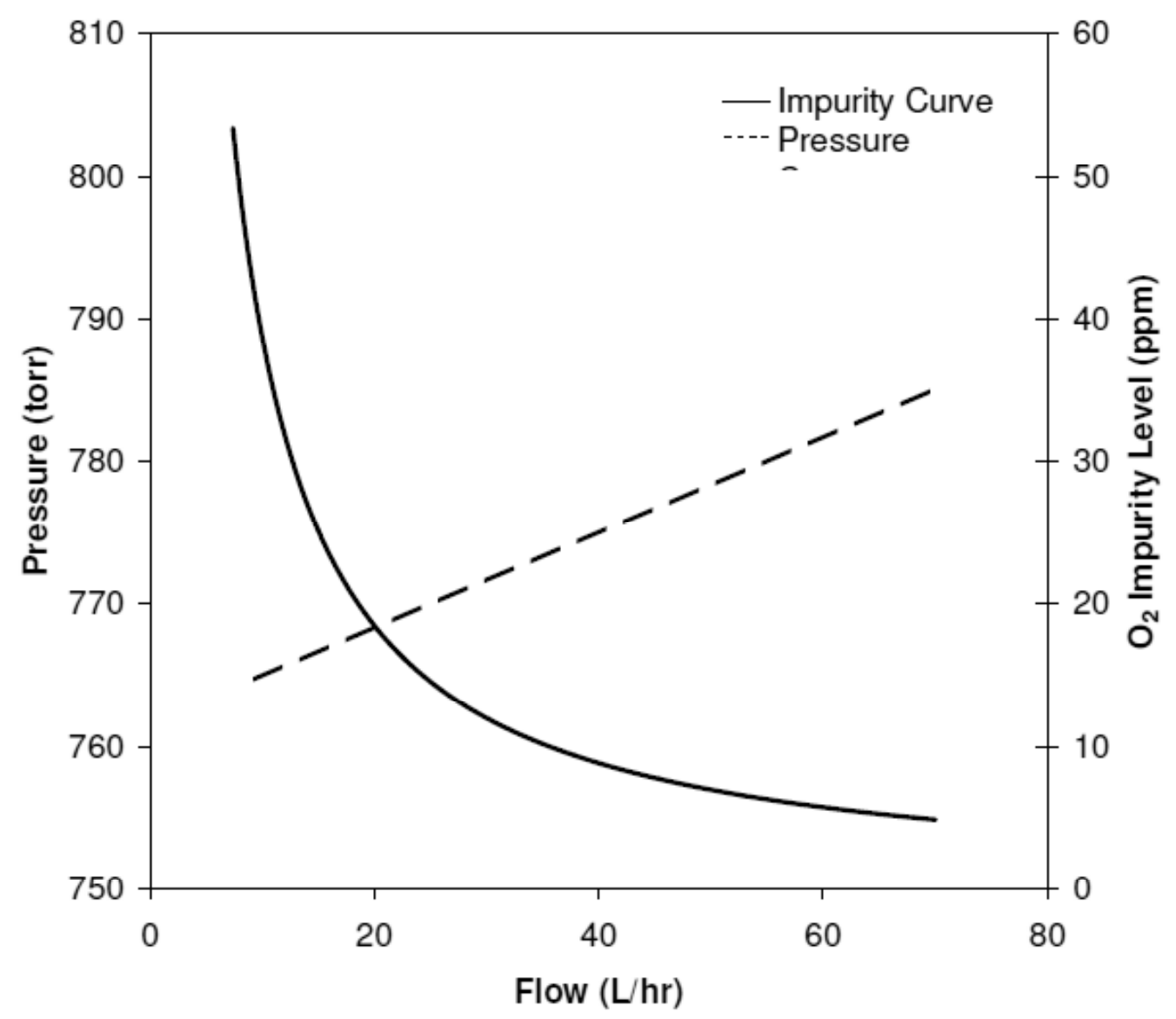

Figure 3.5: Measurements of the impurity level and corresponding gas pressure in the exhaust of muon monitor 1 were taken at flow rates of 10, 20, 30, 42, and $60 \ell / \mathrm{hr}$. The curves are fits to the data 
potential than helium, oxygen contaminants increase the ionization scale of the monitors resulting in an increased ionization signal. Figure 3.5 shows measurements of impurity level as well as the pressure of the gas versus flow rate. The results show a decrease in the impurity level with increased flow rate. Unfortunately, effect of impurity level on the signal of the monitors has not been systematically measured. But, it is suspected that changes in impurity

levels on the order of several p.p.m. have a significant $(\sim 5-10 \%)$ effect on the ionization scale.

On numerous occasions large increases in the ionization signal from the muon monitors have been seen, particularly at the times of gas bottle exchanges. As a result, ionization calibration chambers with internal $\mathrm{Am}^{241} \alpha$ sources were installed in the exhaust lines of each muon monitor in 2007. Pressure transducers were installed in the gas line alongside the chambers so the variations in the ionization signal due to pressure may be calibrated out. The calibration chambers are located outside of the alcoves and are not subject to ionization of particles produced by the beam. Thus, they are sensitive to the gas quality within the muon monitors and may be used to calibrate out effects of gas purity in the muon monitor signals. Furthermore, bubblers were installed at the exhausts to reduce back diffusion of air into the monitors.

\subsubsection{Ambient Pressure and Temperature}

Ambient pressure and temperature in the muon alcoves affect the ionization signal from the muon monitors because they increase or decrease the density of the gas within the monitors. Barometric pressure variations of $\sim 20$ torr can occur over a period of hours due to high and low pressure weather systems. 
Figure 3.6 shows the total signal from muon monitors 1 and 2 as a function of He gas pressure over 2 days during which the barometric pressure varied significantly.

The absolute temperature at a given muon alcove varies little over time since the temperature underground is not significantly affected by temperature changes above ground. However, there is a considerable difference in the temperature between alcoves 1, 2 and 3. The temperature at the hadron monitor is affected by the penetrating hadron beam and therefore can vary significantly over several weeks. Figure 3.7 shows the total signal from the hadron monitor as a function of ambient temperature over $\sim 3$ weeks.

Pressure and temperature effects can be readily corrected for and these corrections are described in detail in Appendix A.

\subsubsection{Rate-Dependent Effects}

Ion chambers are subject to non-linear behavior due to space-charge build up between the electrodes. An increase in ion density within the ion chamber can screen the potential seen by surrounding electrons and ions as the rate of ionization increases. The upper plot of Figure 3.4 demonstrates is effect. Without screening effects the ionization signal should be directly proportional to the incident particle intensity and the curves shown in this figure should be flat with intensity. As the intensity of incident particles is increased, the increased number of ions becomes more and more effective at screening ion-pairs from the applied potential so that a "dead zone" forms nearest the cathode where the electric field is zero. As the intensity increases the downward sloping curves suffer from space-charge induced recombination and the upward sloped 

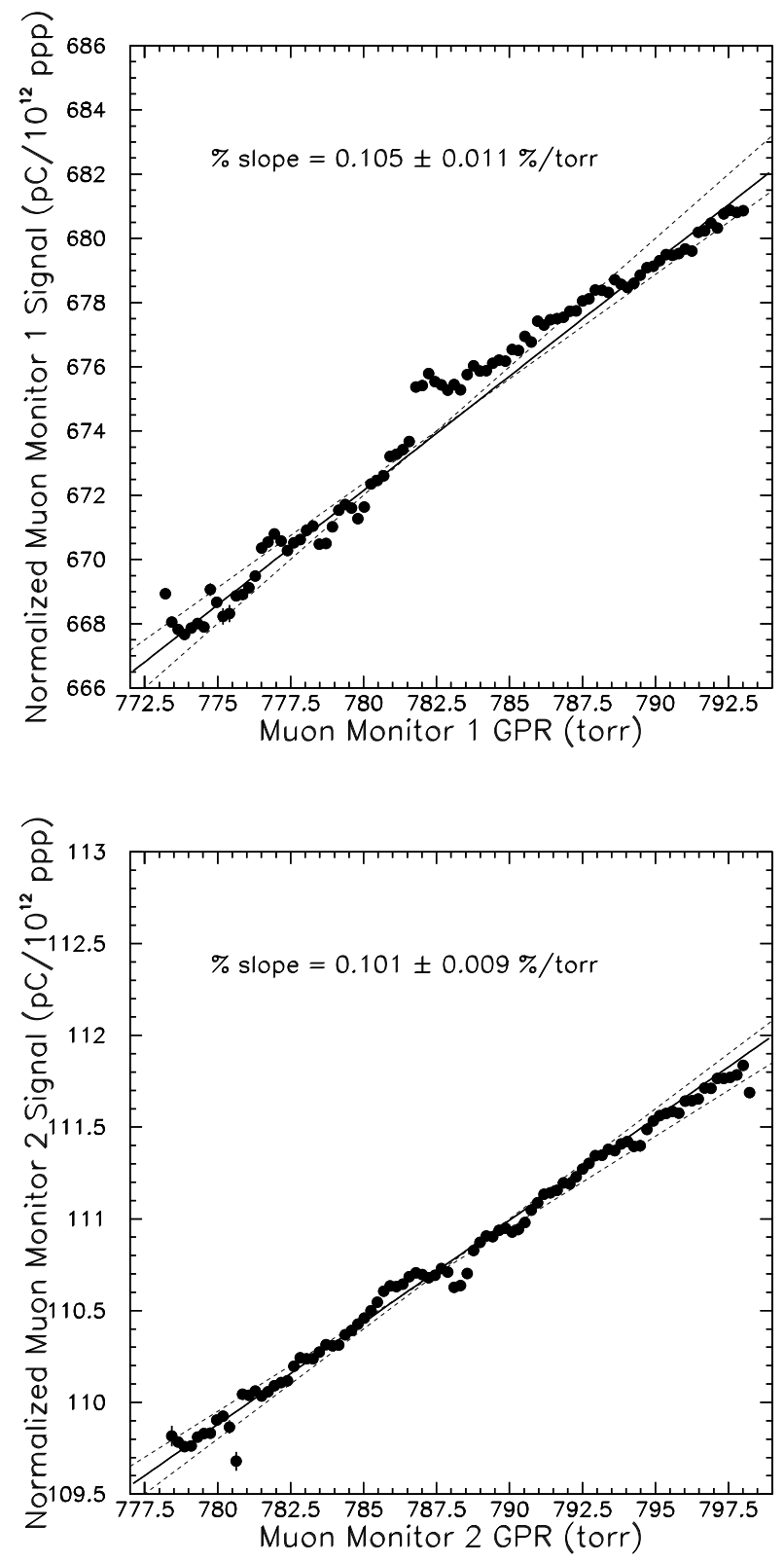

Figure 3.6: Total signal from muon monitors 1(top) and 2(bottom) as a function of He gas pressure over 2 days during which the barometric pressure varied significantly. A correction for the muon monitor signals with gas pressure is developed in Appendix A. 


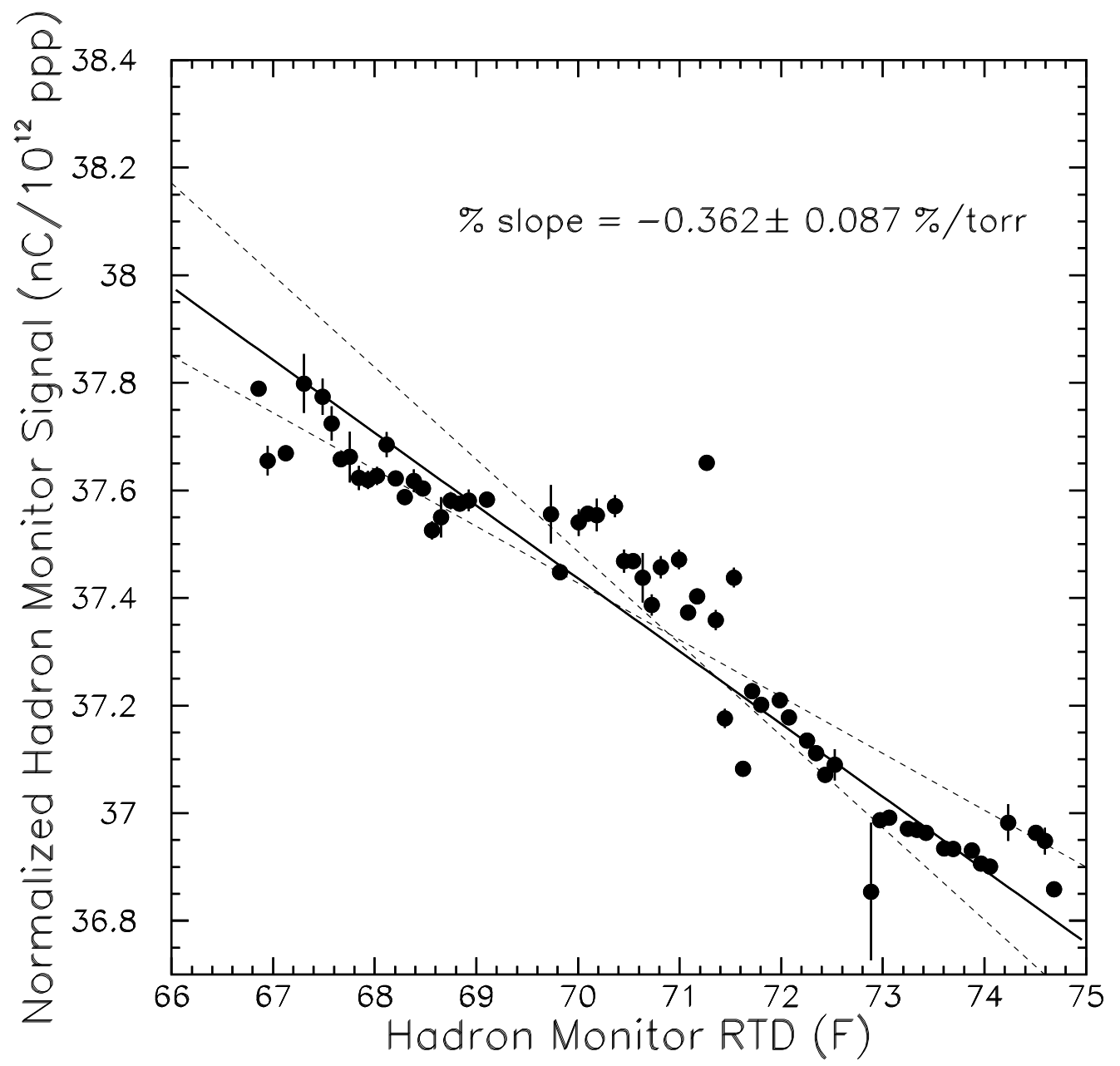

Figure 3.7: Total signal from the hadron monitor as a function of ambient temperature over $\sim 3$ weeks. A correction for the signal with temperature is developed in Appendix A. 

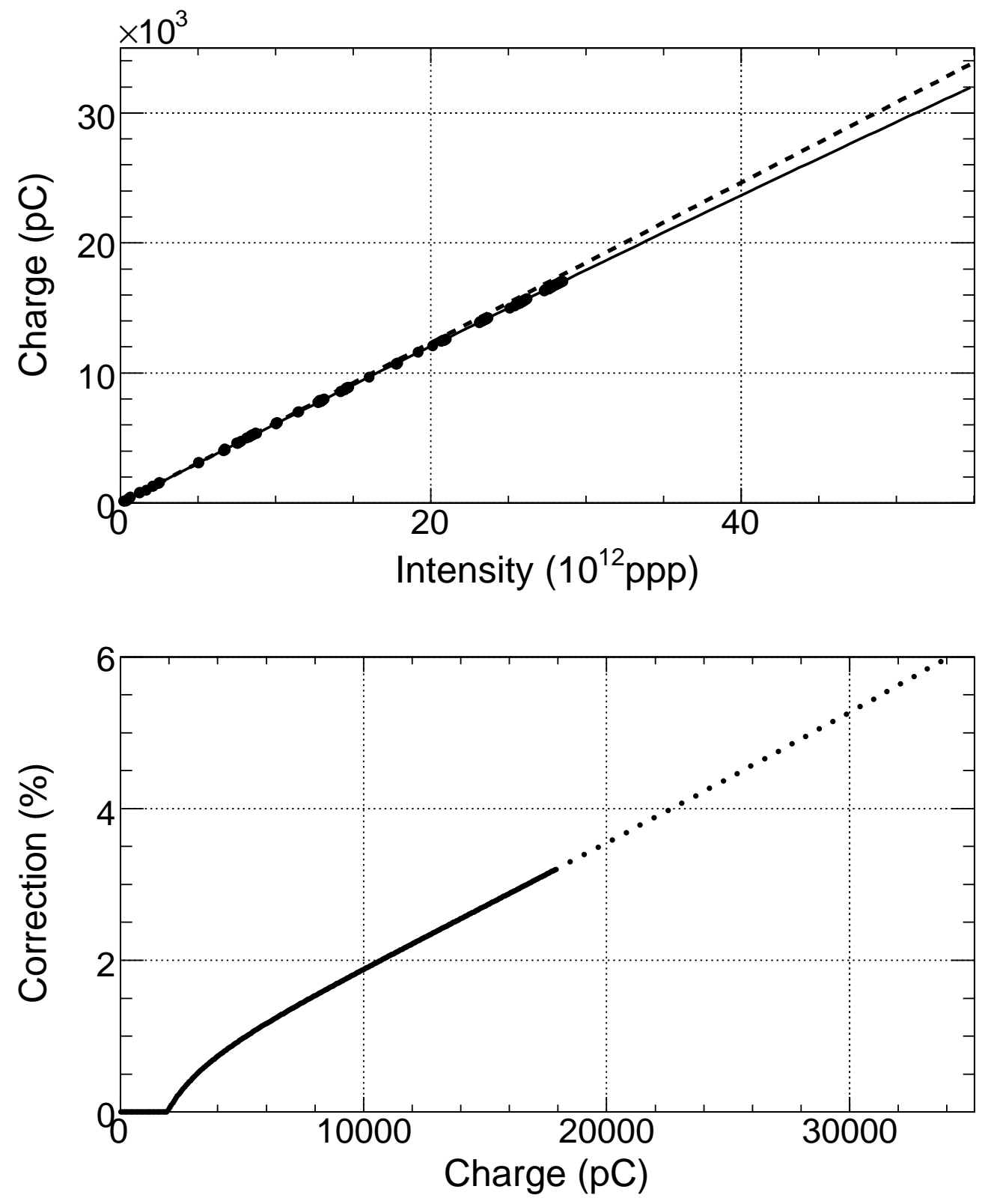

Figure 3.8: Top: Charge collected in Muon Monitor 1 as a function of protons on target. The solid curve is a quadratic fit to the data. The dashed curve is a linear fit below $5 \times 10^{12}$ ppp. Bottom: The percent difference between the quadratic and linear fits to the data. 
curve suffers from space-charge induced multiplication [31].

The muon monitors were designed to have relatively small non-linear rate dependent effects [30], but the small non-linearity does require a few percent correction. The upper plot in Figure 3.8 shows the charge collected in Muon Monitor 1 vs. the proton beam intensity(ppp) while other beam conditions are kept constant. In this case the response of the chamber is expected to be linear and any non-linearity provides a measure of the ratedependent effect in the chambers. The solid curve is a quadratic fit to the data which reasonably describes the data. The dashed curve is a linear fit to the data below $5 \times 10^{12}$ ppp where space-charge effects are expected to be smaller. The lower plot in Figure 3.8 shows the percent difference between the curves as a function of collected charge. This difference is taken to be the correction for rate-dependent effects. The error on the correction is taken to be the greater of half the correction or 0.01. Similar curves are developed for muon monitors 2 and 3.

\subsection{Measurements of Backgrounds}

The dominant contribution to the muon monitor ionization signal comes from muons produced by meson decay in the decay volume. However, there are other particles that pass through the monitors, ionize the gas and contribute to the ionization signal. These particles constitute a background to muon flux measurements in the muon monitors. Thus, it is necessary to determine the amount of the muon monitor signal which comes only from muons produced in meson decay in the decay volume. Particles that impact the absorber interact within the absorber producing background particles to muons from meson- 
decay-in-flight. Such backgrounds arise in part because $\approx e^{-2}=13.5 \%$ of the primary proton beam passes through the target unreacted and is transported to the beam absorber, where it can create pions and kaons which decay to muons. These muons from the beam absorber are produced in close proximity to the muon alcoves. Additionally, Alcove 1 sees a background from the absorber due to $10-100 \mathrm{MeV}$ neutrons not fully contained in the absorber which can elastically scatter in the chamber gas and produce heavily-ionizing Helium recoil nuclei, as was studied in Ref. [47]. Monte Carlo simulations indicate that, depending on the beam configuration, $92-88 \%$ of the backgrounds produced in the absorber originate from the unreacted proton beam.

The background signal in the muon monitors from interactions of unreacted protons with the absorber is determined by two independent in situ studies. The first study involves the use of a set of special runs in which the proton beam is transported directly to the absorber (no target in place). The second takes advantage of the fact there exist gaps on either side of the target in between the baffle. During scans of the proton beam across the target (Appendix B), the proton beam is positioned over these gaps and is largely transported directly to the absorber.

\subsubsection{No-Target Spills}

Figure 3.9 shows distributions in the muon alcoves, averaged over several pulses, for two beam configurations. The left column of plots shows the muon beam profiles which arose during a special run in which the target was removed from the beam and the proton beam was transported directly to the beam absorber. The right column of plots shows the muon beam profiles which were 
observed when the horn was set to zero current. ${ }^{1}$ These two data sets were acquired approximately 1 hour apart. As anticipated, the close proximity of the absorber to the alcoves results in a dump-muon signal that is comparable (per proton) as the muon flux produced by decays-in-flight when the proton beam hits the target. ${ }^{2}$

To estimated the expected backgrounds in the monitors, the no-target data are scaled by $13.5 \%$ to the level anticipated during normal running. The results are given in Table 3.1 .

While this measurement of the dump-related backgrounds has the merit of being quite direct, in practice the uncertainties from this technique range from $9 \%$ in Alcove 1 to $75 \%$ in Alcove 3 , due to the extremely low $\left(8 \times 10^{11} \mathrm{ppp}\right)$ spill intensities in use during this particular beam study.

\subsubsection{Beam-Missing-Target Spills}

At several occasions studies have been performed in which the proton beam is scanned horizontally across the target for alignment purposes (Appendix B and $[31,35])$. The diagram of Figure 3.10 shows the geometry of the target and baffle system. The region in between each pair of solid vertical lines on the plot of muon monitor signal vs. proton beam position is the gap in between the target and the baffle. The dashed vertical lines indicate the center of each gap.

The proton beam is in fact small enough to partially be transmitted

\footnotetext{
${ }^{1}$ The no-target data was collected in place in order to perform alignment scans across the horn cross hairs [31].

${ }^{2}$ Interestingly, the no-target profiles are strongly peaked at the centers of the muon arrays and do not show the peaks in Alcove 1 due to the cracks in the absorber as is seen in the no-horn, beam on target, profiles.
} 

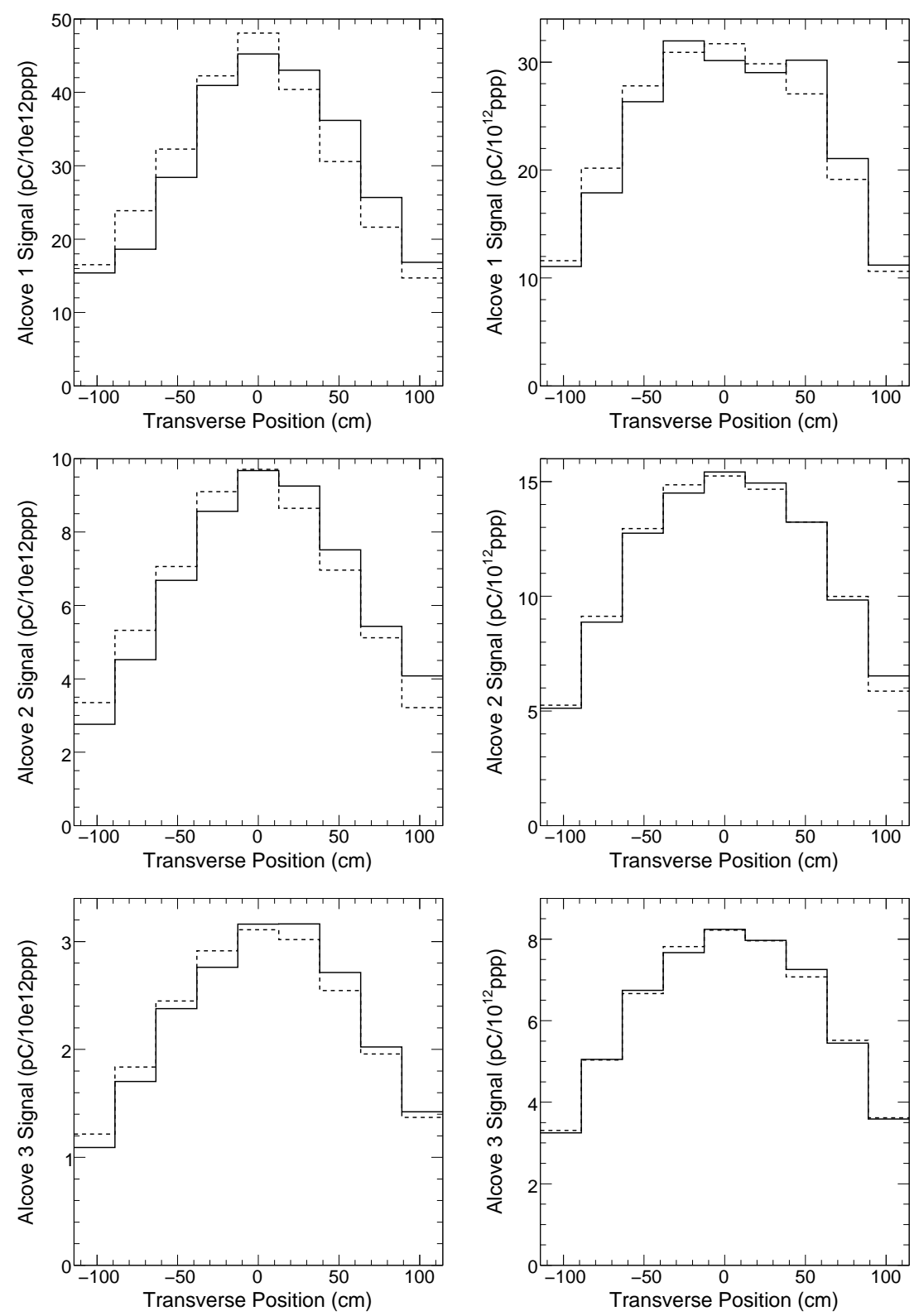

Figure 3.9: Signal in the muon alcoves in the horizontal (solid lines) and vertical (dashed lines) projections. Left column is taken from pulses in which the proton beam was transported directly to the beam absorber (no target in place), while plots in the right column have a target in place, but the horns were not pulsing. Rows 1, 2, 3 are Muon Monitors 1, 2, 3 . 

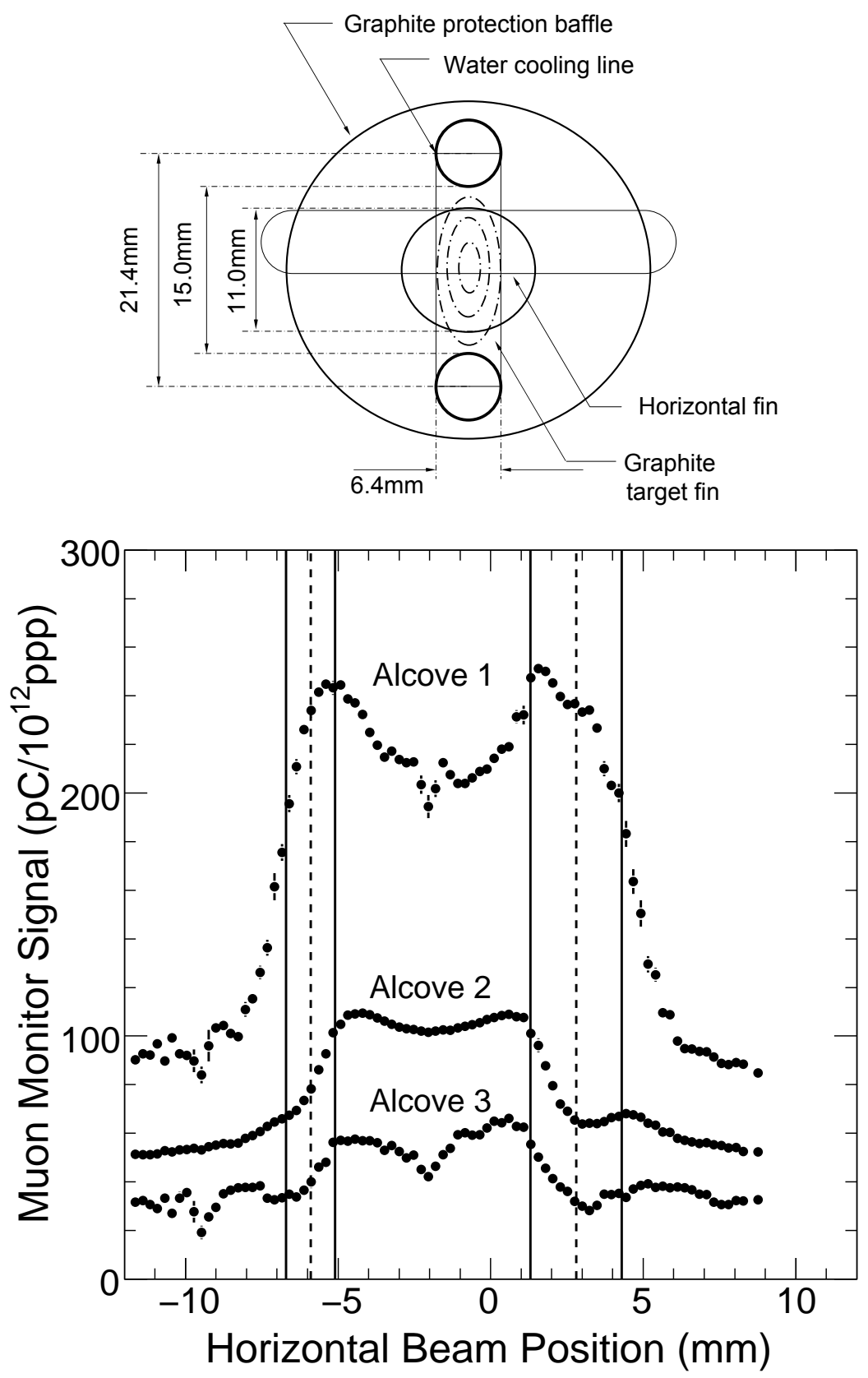

Figure 3.10: Top: Proton beam's-eye-view of the target-baffle system. Data, showing scans of the proton beam across the target and baffle in the horizontal direction for the target at the $z=0 \mathrm{~cm}$ position, without the horns being pulsed. The clear aperture between the target and baffle can permit approximately $93 \%$ of the proton beam to be transported directly to the absorber if it is steered at $x=2.8 \mathrm{~mm}$. 
without interruption through the gap between the target and the inner aperture of the baffle. During the period of March-May, 2005, during which the the scan shown in Figure 3.10 was performed, the $6.4 \mathrm{~mm}$ wide target was horizontally displaced within the $11 \mathrm{~mm}$ circular aperture of the baffle so that a $3.0 \mathrm{~mm}$ gap was created to the right of the target. This $3.0 \mathrm{~mm}$ gap should be compared to the $\sigma_{x} \simeq 0.82 \mathrm{~mm}$ horizontal beam widths typical during the beam scans at that time, suggesting that approximately $93 \%$ of the proton beam could be steered between the baffle and target $\left( \pm 1.5 \sigma_{x}\right)$. When the beam is at $x=2.8 \mathrm{~mm}$, approximately $93 \%$ of the beam should be transported directly to the absorber, with $3.4 \%$ striking the target and $3.4 \%$ striking the baffle.

To determine the signal in the muon monitors which arises from protons hitting the absorber, we take the signal level when the beam is centered at $x=2.8 \mathrm{~mm}$, subtract off $3.4 \%$ of the signal level for $x=1.3 \mathrm{~mm}$ and $6 \%$ of the signal level for $x=4.3 \mathrm{~mm}$. This "sideband-subtracted" quantity is then scaled up by $1 / 0.93$ so that the signal per proton is correctly normalized (the toroids measure the total beam/spill). These results are summarized in Table 3.1. These beam scans were conducted at relatively high intensity (a couple $\left.10^{12} \mathrm{ppp}\right)$. The uncertainties in Table 3.1 are estimated by variation of the level which should be subtracted off the $x=2.8 \mathrm{~mm}$ value, taken to be $5 \%$, as well as an effective $2 \%$ uncertainty in the $93 \%$ transmitted beam value.

\subsubsection{Summary}

Backgrounds in the muon monitors arise from particles produced in the beam dump by the remnant high energy proton beam. These particles include neu- 
trons (mostly in muon monitor 1), as well as muons produced in hadronic showers, and vary in level from muon monitor 1 to 3 .

The expected $30 \mathrm{pC} / 10^{12} \mathrm{ppp}$ dump muon background is $15 \%$ of the Alcove 1 horn-off signal, for example. The dump-related backgrounds amount to just $8 \%$ of the Alcove 3 horn-off signal. As expected, the background level is higher in the first alcove. 


\begin{tabular}{c|c|c|c}
$\begin{array}{c}\text { Muon } \\
\text { Alcove }\end{array}$ & $\begin{array}{c}\text { Signal Before } \\
\text { Gas Corrections } \\
\left(\mathrm{pC} / 10^{12} \mathrm{ppp}\right)\end{array}$ & $\begin{array}{c}\text { Signal After } \\
\text { Gas Corrections } \\
\left(\mathrm{pC} / 10^{12} \mathrm{ppp}\right)\end{array}$ & $\begin{array}{c}\text { Extrapolated to } \\
13.5 \% \text { Unreacted } \\
\text { Proton Beam }\end{array}$ \\
\hline \hline & \multicolumn{2}{|c|}{ Data from No-Target Spills (Section 3.2.1) } \\
\hline \hline 1 & $270 \pm 22$ & $251 \pm 21$ & $34 \pm 3$ \\
2 & $59 \pm 5$ & $54 \pm 5$ & $7.3 \pm 1$ \\
3 & $20 \pm 15$ & $12 \pm 9$ & $1.6 \pm 1.2$ \\
\hline \hline & \multicolumn{3}{|c}{ Data from Target Scans (Section 3.2.2) } \\
\hline \hline 1 & $236 \pm 13$ & $223 \pm 16$ & $30 \pm 2$ \\
2 & $63 \pm 4$ & $58 \pm 4$ & $7.8 \pm 0.5$ \\
3 & $29 \pm 2$ & $20 \pm 3$ & $2.7 \pm 0.4$ \\
\hline
\end{tabular}

Table 3.1: Backgrounds in the Muon Monitors as determined in spills with no target in the beam line (Section 3.2.1), and in spills in which the beam is intentionally steered to the right of the target (Section 3.2.2). In both cases, all or a significant fraction of the primary proton beam is transported directly to the NuMI beam absorber, permitting a measurement to be made of the fluxes of particles in the monitors produced by backgrounds in the absorber. 


\subsection{System Performance}

The muon monitors can detect small $(<1 \%)$ changes in flux. Such changes arise from varying proton beam conditions such as varying beam width, focusing effects such that arise from small changes in horn current, and small misalignments of the proton beam with respect to beamline components.

Figure 3.11 shows the proton beam size and horn current for each spill over a 1 week period in December, 2005. Over this time the horizontal and vertical proton beam size at the target varies by as much as $30 \%$ and horn current varies by $0.2 \%$. Figure 3.12 shows the measured muon flux in muon monitors 1 and 2 over the same time period. A $1 \%$ change in the muon flux is measured by muon monitor 2 at the same time that the proton beam size at the target changes by $30 \%$. This is consistent with more protons impacting the edges of the target, reducing reinteractions within the target, and producing more high energy mesons which decay to high energy muons and neutrinos (see additional discussion in Appendix B). Further confirmation comes from the hadron monitor, also shown in Figure 3.12. The signal increases in the hadron monitor as the same time the proton beam size increases, consistent with a larger number of protons escaping out of the sides of the target be-

fore interacting or passing through the target-baffle gap completely missing the target altogether. The opposite effect, i.e. a decrease in the low energy muon flux, may be expected to be observed in muon monitor 1, except that the simultaneous increase in the horn current seems to cancel out the effect. However, an increase of $\sim 0.6 \%$ is observed in the muon flux in muon monitor $1>$ day 343 corresponding to $\mathrm{a} \sim 0.2 \%$ increase in the current through the horns. 

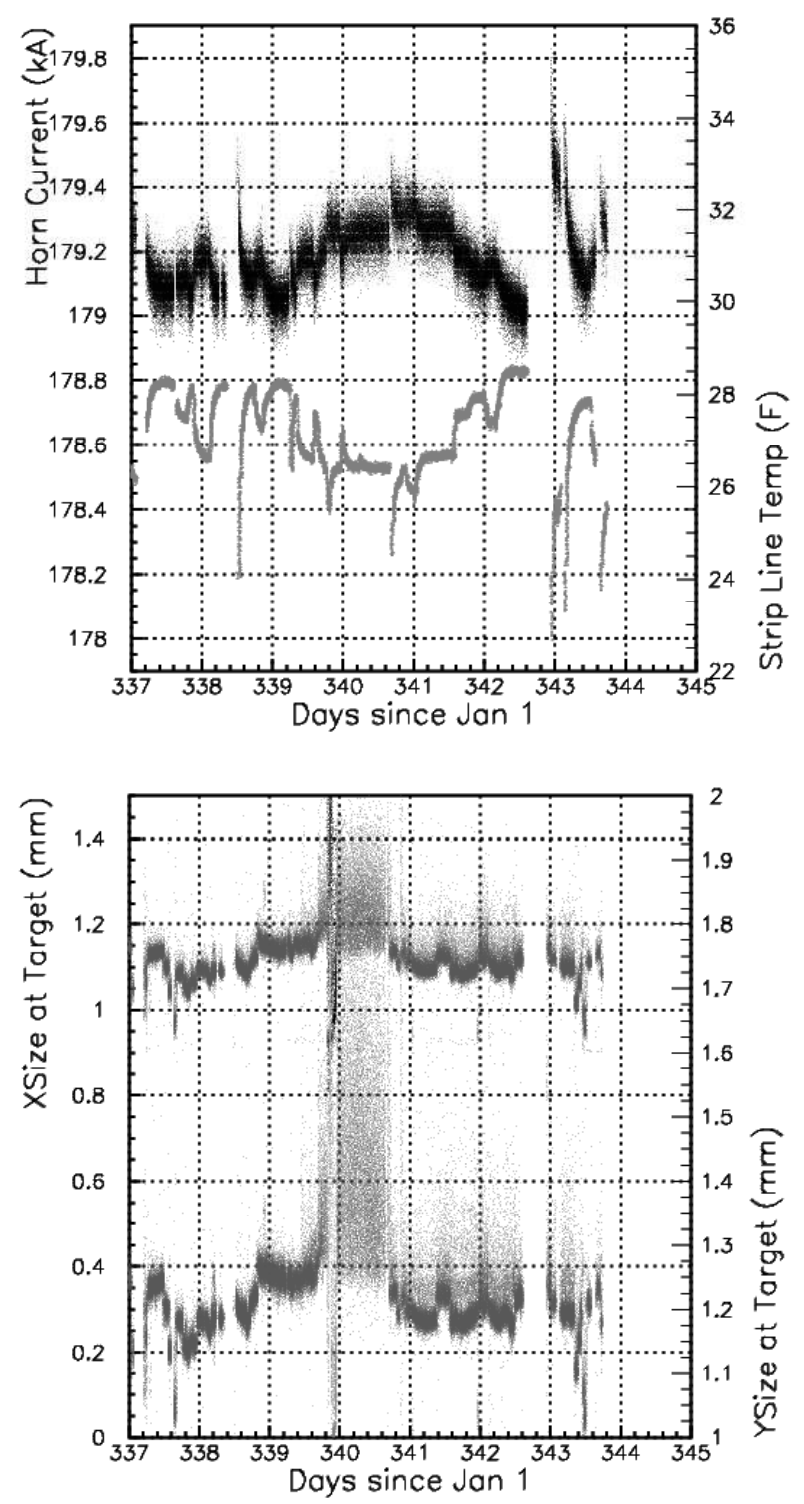

Figure 3.11: Proton beam size and horn current and horn temperature for each spill over a 1 week period. (The strip line carries current to the horns.) Variations in the quantities produce measurable changes in the muon and hadron flux measured in the muon and hadron monitors (see Figure 3.12). (The horizontal axis is "Days since January 12005 " thus the full range corresponds to Dec. 04, 2005, day 337, to Dec 12, 2005, day 345.) 

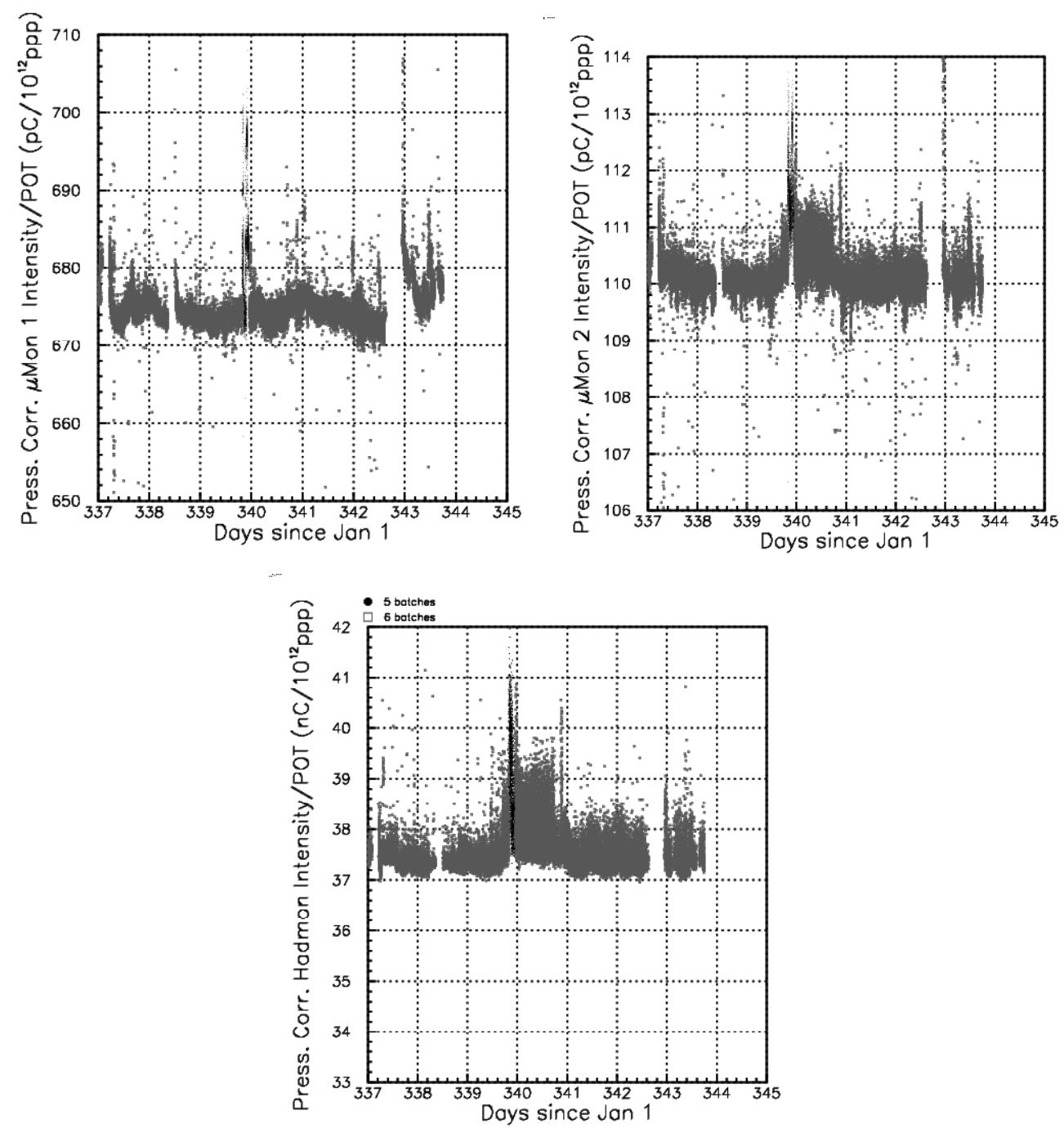

Figure 3.12: The muon flux measured in muon monitors 1(top right) and 2(top left) normalized by the number of protons on the target(ppp) for each spill over a 1 week period. The variations in the monitor signals corresponds to variations in the proton beam size and horn current as show in Figure 3.11. (The horizontal axis is "Days since January 12005 " thus the full range corresponds to Dec. 04, 2005, day 337, to Dec 12, 2005, day 345. The vertical axis is the pressure corrected(see Section 3.1.3) signal from the monitors normalized to the number of protons impacting the target.) 


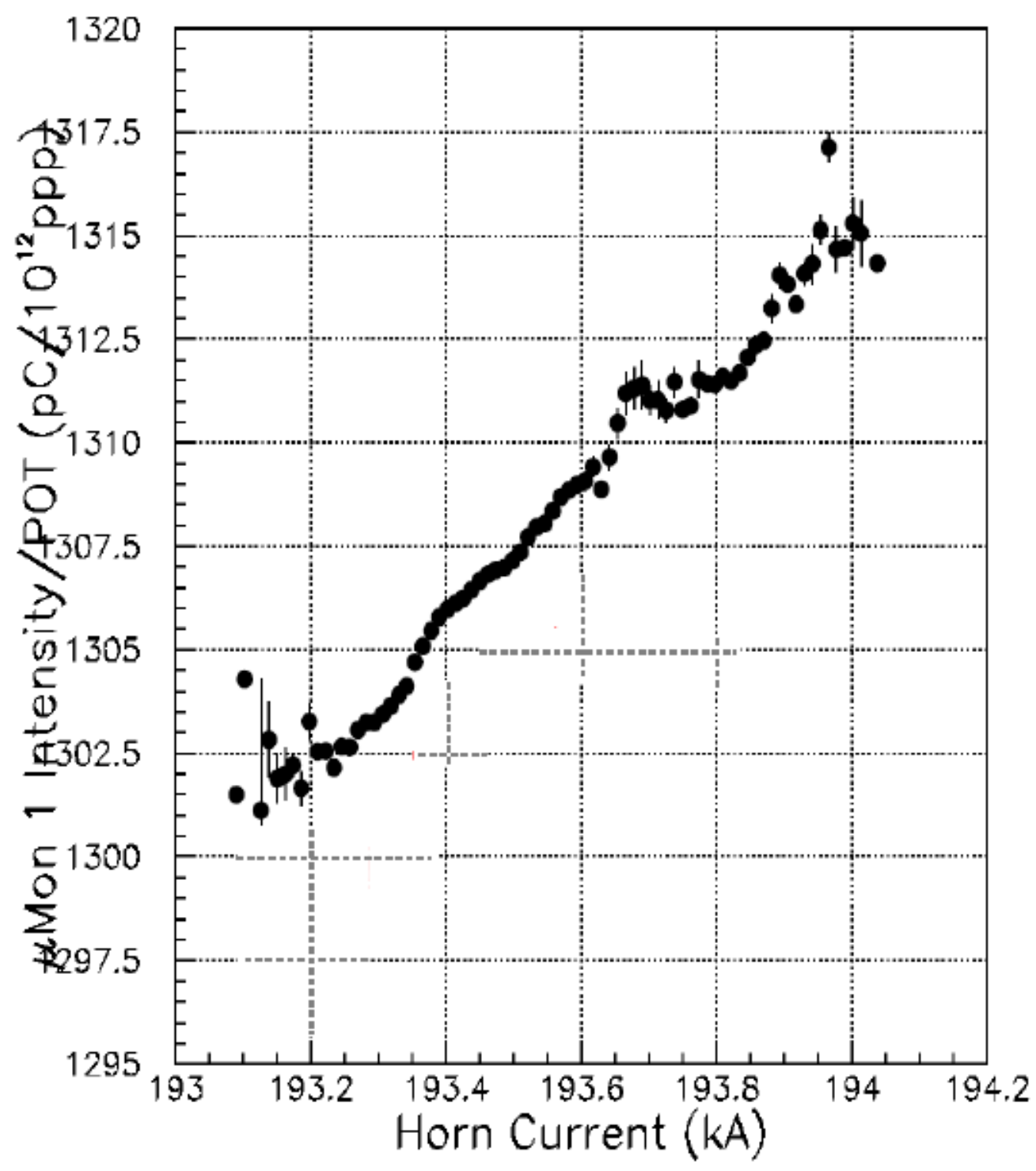

Figure 3.13: Muon Monitor 1 signal versus the current in the horns in the LE150/200 kA beam configuration over several days. The current in the horns is decreasing over time due to heating in the target hall. 
Figure 3.13 shows a strong correlation between the signal in Muon Monitor 1 and the horn current in the LE150/200 kA beam configuration over a several day period. The current in the horns is decreasing over time due to heating in the target hall.

\subsubsection{Long-Term Performance}

This section documents the stability of the beam flux using the muon monitors between January 2005 and August 2006. Table 3.2 lists the target positions during the time this time. For the purpose of this thesis, this time period is divided into 3 smaller time periods listed in Table 3.3.

The muon monitors measure the muon beam flux by intercepting the muons produced in hadronic decays in the upstream decay volume. Muons ionize helium gas, which continuously flows through the chambers, producing an electronic signal which is proportional to the flux of intercepting particles.

Variations in the density of the helium gas caused by changes in the ambient pressure and temperature result in changes in the amount of charge ionized per incident particle. Corrections to the ionization signal for the gas pressure and temperature are developed in Appendix A. Impurities in the helium will also result in a change in the ionization signal per incident particle.

\section{Run I Part I}

Figures 3.14 and 3.15 show the beam conditions and muon monitor signals during Run I Part I. During this time period the beam is being commissioned. Beam conditions such as the position of the beam at the target vary greatly. There are numerous target scans, during which the beam is scanned from -10 


\begin{tabular}{c|c|c}
$\begin{array}{c}\text { Beam } \\
\text { Configuration }\end{array}$ & $\begin{array}{c}\text { Start Date } \\
(\text { UTC) }\end{array}$ & $\begin{array}{c}\text { End Date } \\
\text { (UTC) }\end{array}$ \\
\hline \hline LE100 & Jan. 20, 2005 & Feb. 27, 2005 \\
LE250 & Feb. 27, 2005 & Mar. 04, 2005 \\
LE100 & Mar. 04, 2005 & Mar. 07, 2005 \\
LE000 & Mar. 08, 2005 & Mar. 23, 2005 \\
LE100 & Apr. 30, 2005 & May. 12, 2005 \\
LE250 & May. 12, 2005 & May. 20, 2005 \\
LE010 & May. 20, 2005 & Feb. 25, 2006 \\
LE150 & Jun. 01, 2006 & Jun. 11, 2006 \\
LE250 & Jun. 11, 2006 & Aug. 13, 2006 \\
\hline \hline
\end{tabular}

Table 3.2: Beam configurations between January 2005 and August 2006.

\begin{tabular}{c|c|c}
$\begin{array}{c}\text { Time } \\
\text { Period }\end{array}$ & $\begin{array}{c}\text { Start Date } \\
\text { (UTC) }\end{array}$ & $\begin{array}{c}\text { End Date } \\
\text { (UTC) }\end{array}$ \\
\hline \hline Run I Part I & Jan. 20, 2005 & May. 20, 2005 \\
Run I Part II & May. 20, 2005 & Feb. 25, 2006 \\
Run II Part I & Jun. 01, 2006 & Aug. 13, 2006 \\
\hline \hline
\end{tabular}

Table 3.3: Time periods discussed in this section. 

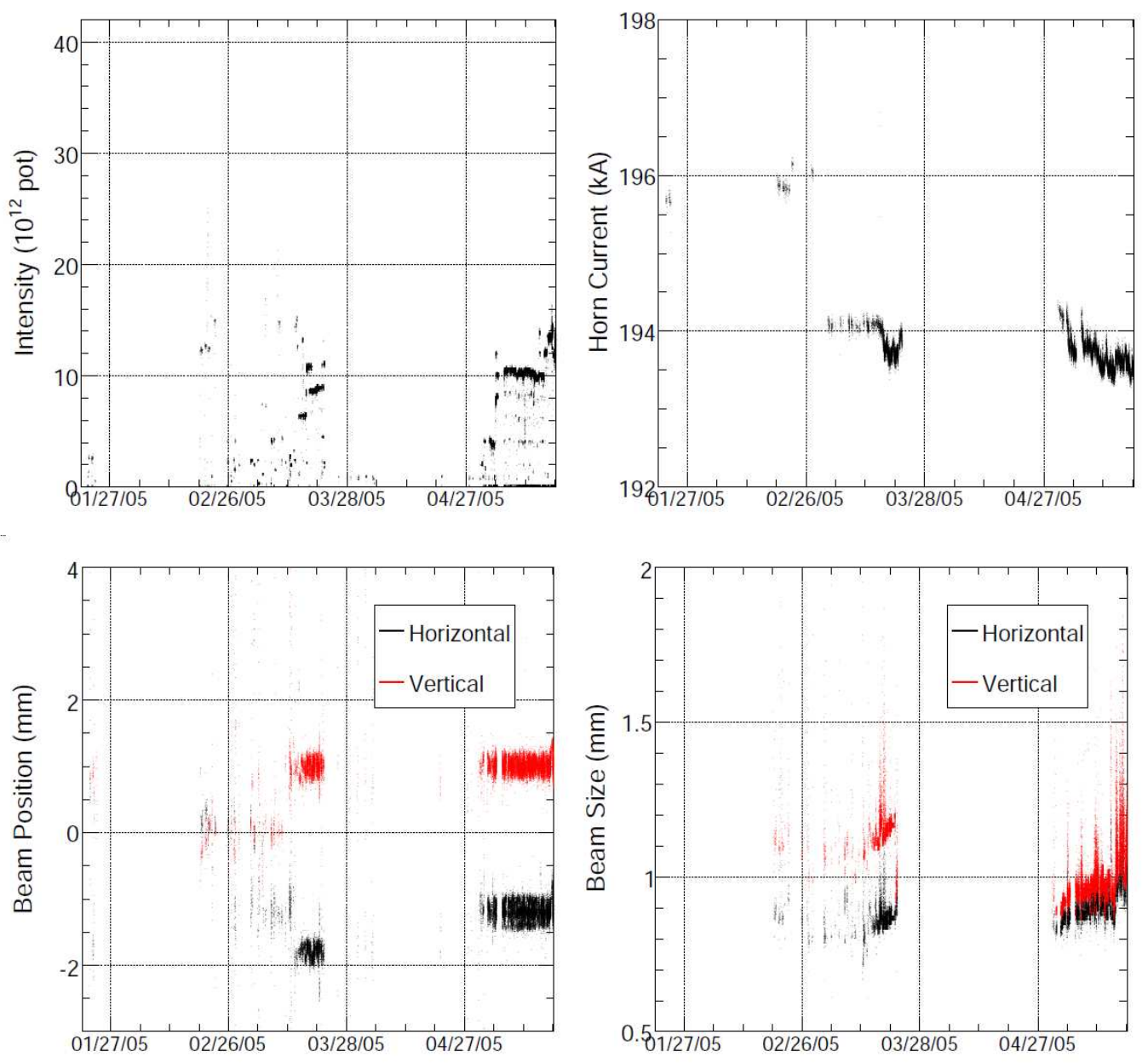

Figure 3.14: Beam conditions during Run I Part I. Top Left: The number of protons per spill impacting the target. Top Right: The current in the focusing horns. Bottom Left: The position of the proton beam at the target. Bottom Right: The RMS transverse size of the proton beam at the target. 

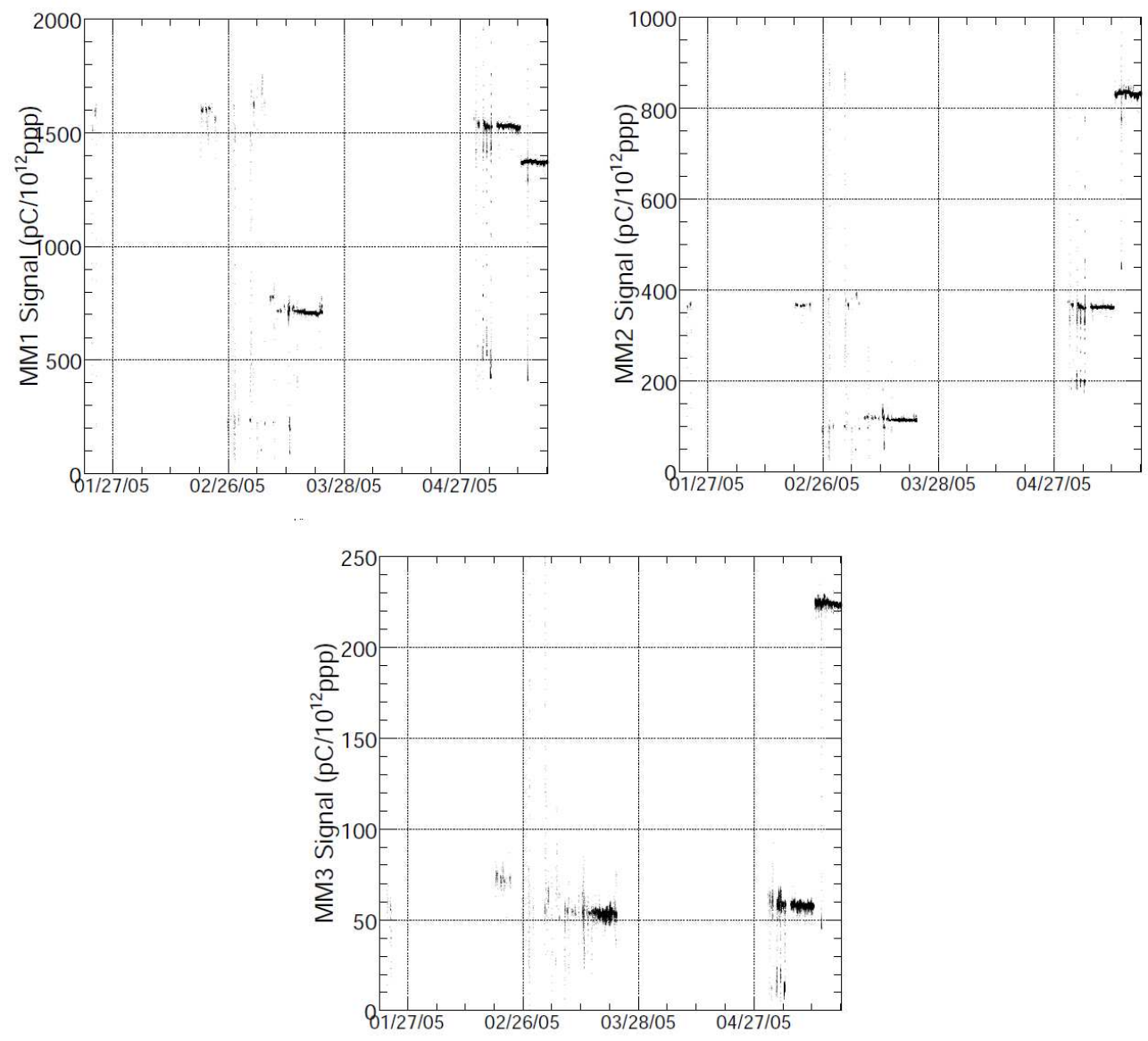

Figure 3.15: Muon Monitor 1(top), 2(middle) and 3(bottom) signals during Run I Part I. Only spills with proton beam intensity greater than $1 \times 10^{12} \mathrm{ppp}$ are included. During this time period commissioning of the NuMI beam was occurring. Various special tests of the proton beam and the muon monitors were being conducted and the beam was operated in several different target positions, LE000, LE100, LE250. 
to $10 \mathrm{~mm}$ horizontally and vertically across the face of the target, performed during this time period. Also, Autotune, which is used to steer the beam onto the center of the target, is not being used for most of this time period. There are also several instances in which the current in the horns is 0kA. And the target position changes several times as listed in Table 3.2. The muon monitor signals vary greatly over this time consistent with the changing beam conditions.

\section{Run I Part II}

Figures 3.16 and 3.17 show the beam conditions and muon monitor signals during Run I Part II. During this time period the target is in the LE010 position and the beam conditions are more stable than during the previous time period. The muon monitor gas flow rate is nominally $10 \ell / \mathrm{hr}$.

On July 8, 2005, it was discovered that a valve in the gas line leading to muon monitor 3 was left open and was closed. This means that the impurity level of the gas in muon monitor 3 is higher prior to July 8 than the impurity level of the gas in muon monitor 1 and 2. This is evidenced by higher signal in alcove 3 prior to July 8.

Around July 15, the helium gas supply is exchanged and the signals in the monitors begin to drift. Between July 15 and Aug 9, it appears that inferior gas causes changes in the monitor signals. On Aug 9 a new supply of helium is placed in the gas system and the signals begin to drop. To expedite the purging of the system, on Aug 12 the gas flow is increased from $10 \ell / \mathrm{hr}$ to $40 \ell /$ hr causing the signal to drop rapidly. On Aug. 15, the gas flow is returned to $10 \ell / \mathrm{hr}$ and the signals stabilize at their pre-Jul. 15 levels in alcove 1 and 2. A similar event occurs on September 22. 

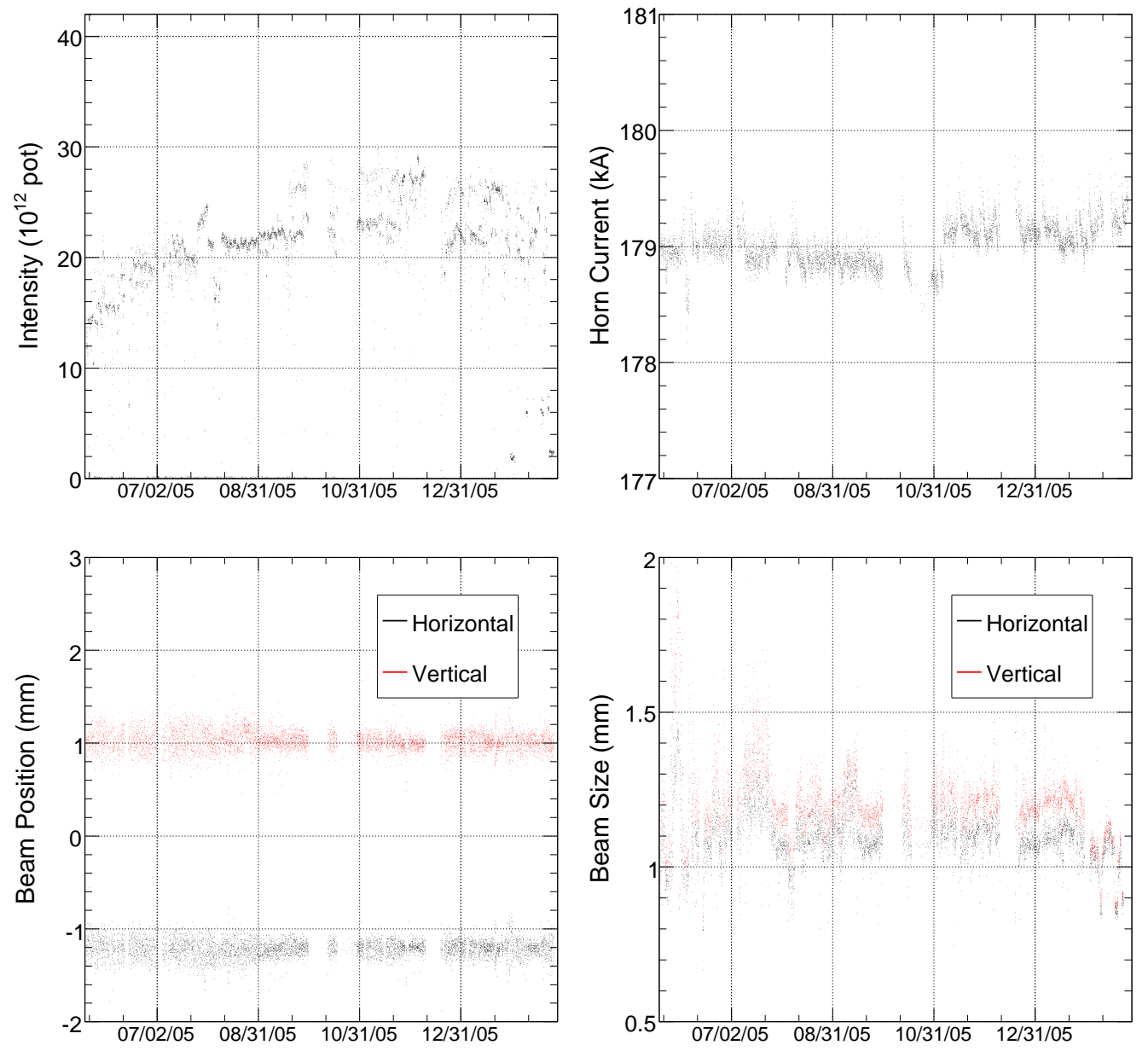

Figure 3.16: Beam conditions during Run I Part II. Top Left: The number of protons per spill impacting the target. Top Right: The current in the focusing horns. Bottom Left: The position of the proton beam at the target. Bottom Right: The RMS transverse size of the proton beam at the target. 

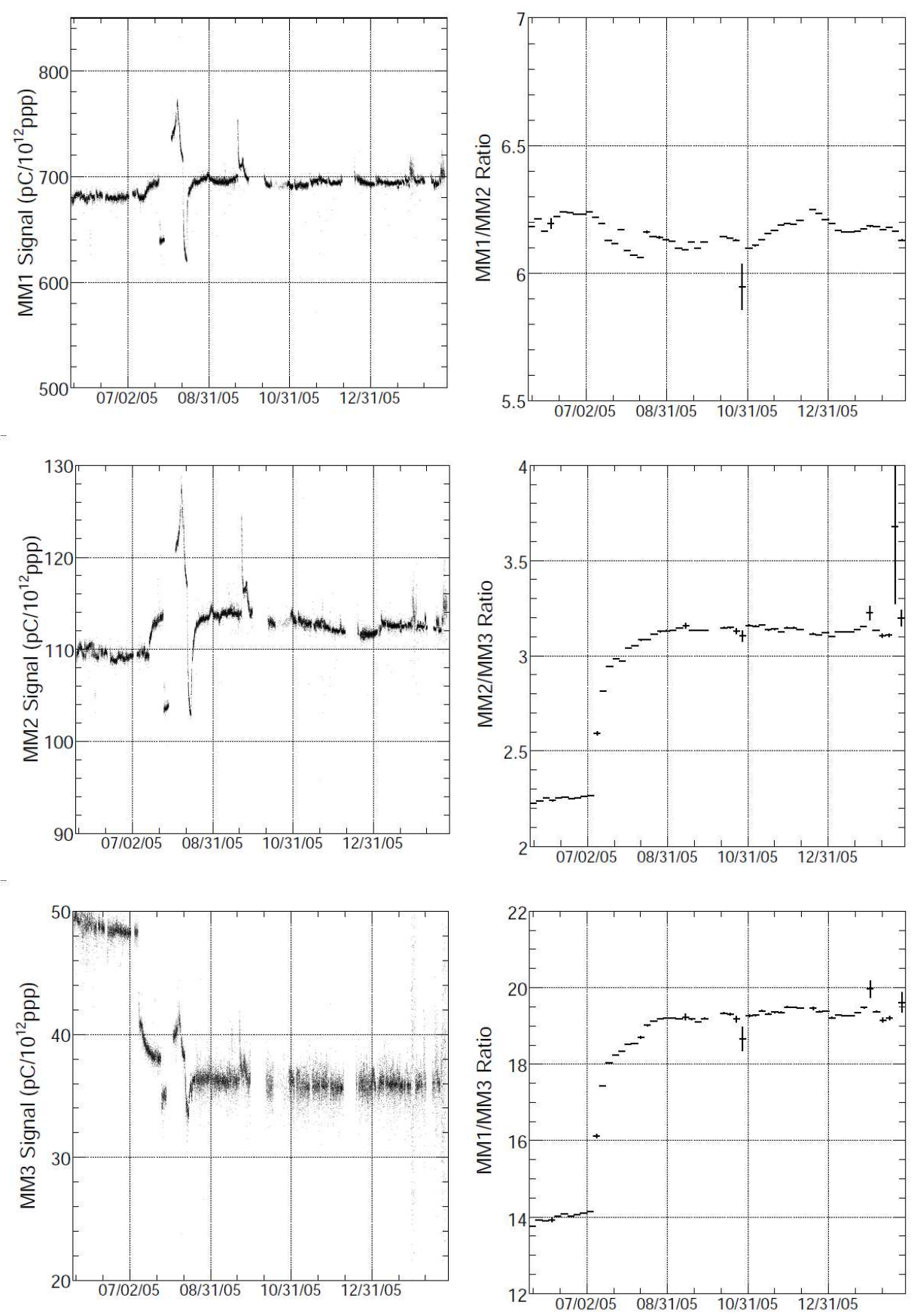

Figure 3.17: Muon Monitor 1(top left), 2(middle left), and 3(bottom left) signals during Run I Part II and the ratios of the signals in muon monitor 1 to 2 (top right), muon monitor 2 to 3 (middle right) and muon monitor 1 to 3 (bottom right). 
The signal ratio of alcove 1 to alcove 2 is consistent throughout this time, indicating similar impurity levels in the two monitors. The ratio of alcove 1 to alcove 2 during Run I Part II is stable to $3 \%$. The ratio of alcove 1 to alcove 3 prior to July 8 is stable to $3 \%$. After about Sep. 1 , it is stable to $2 \%$. The ratio of alcove 2 to alcove 3 prior to July 8 is stable to $2 \%$ and after Sep. 1 , it is stable to $1 \%$.

\section{Run II Part I}

Figures 3.18 and 3.19 show the beam conditions and muon monitor signals during Run II Part I. The muon monitor gas flow rate is nominally $20 \ell / \mathrm{hr}$. From Jun. 01 to Jun. 11 the target is in LE150 position. From Jun. 11 to Aug. 13 the target is in LE250 position.

On Jun. 5, the gas supply begins to run out causing an increase in the monitor signals until Jun. 10 when a new helium gas supply is placed in the system and the signals begin to drop.

On Aug. 2, the helium gas supply is exchanged and the signals start to increase. On Aug 4 the gas flow was increased from $20 \ell /$ hr to $30 \ell / \mathrm{hr}$ causing the signals to drop. On Aug. 7 the gas flow was decreased to $20 \ell / \mathrm{hr}$ and shortly thereafter increased to $30 \ell / \mathrm{hr}$ causing the signals to increase then decrease. On Aug 11 the gas was changed and the signals decrease towards their new equilibrium values at $30 \ell / \mathrm{hr}$.

As during Run I Part II, the ratios of the alcoves are unaffected by the gas quality changes because the impurity levels in the three monitors are similar causing the absolute signals to change proportionally.

During the LE150 running, the ratio of alcove 1 to alcove 2, alcove 1 to alcove 3 and alcove 2 to alcove 3 is stable to 1, 4, and $4 \%$ respectively. 

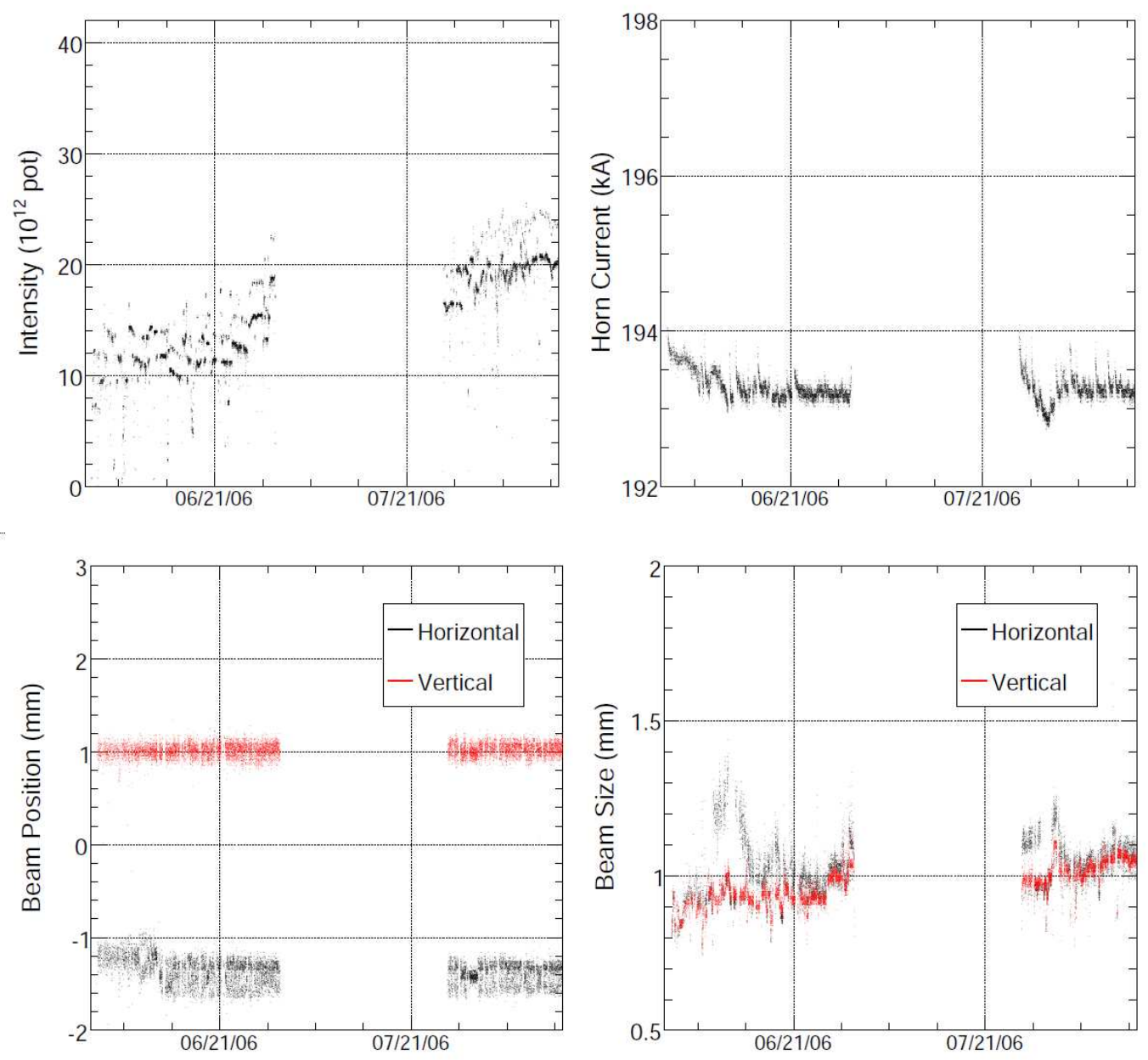

Figure 3.18: Beam conditions during Run II Part I. Top Left: The number of protons per spill impacting the target. Top Right: The current in the focusing horns. Bottom Left: The position of the proton beam at the target. Bottom Right: The RMS transverse size of the proton beam at the target. 

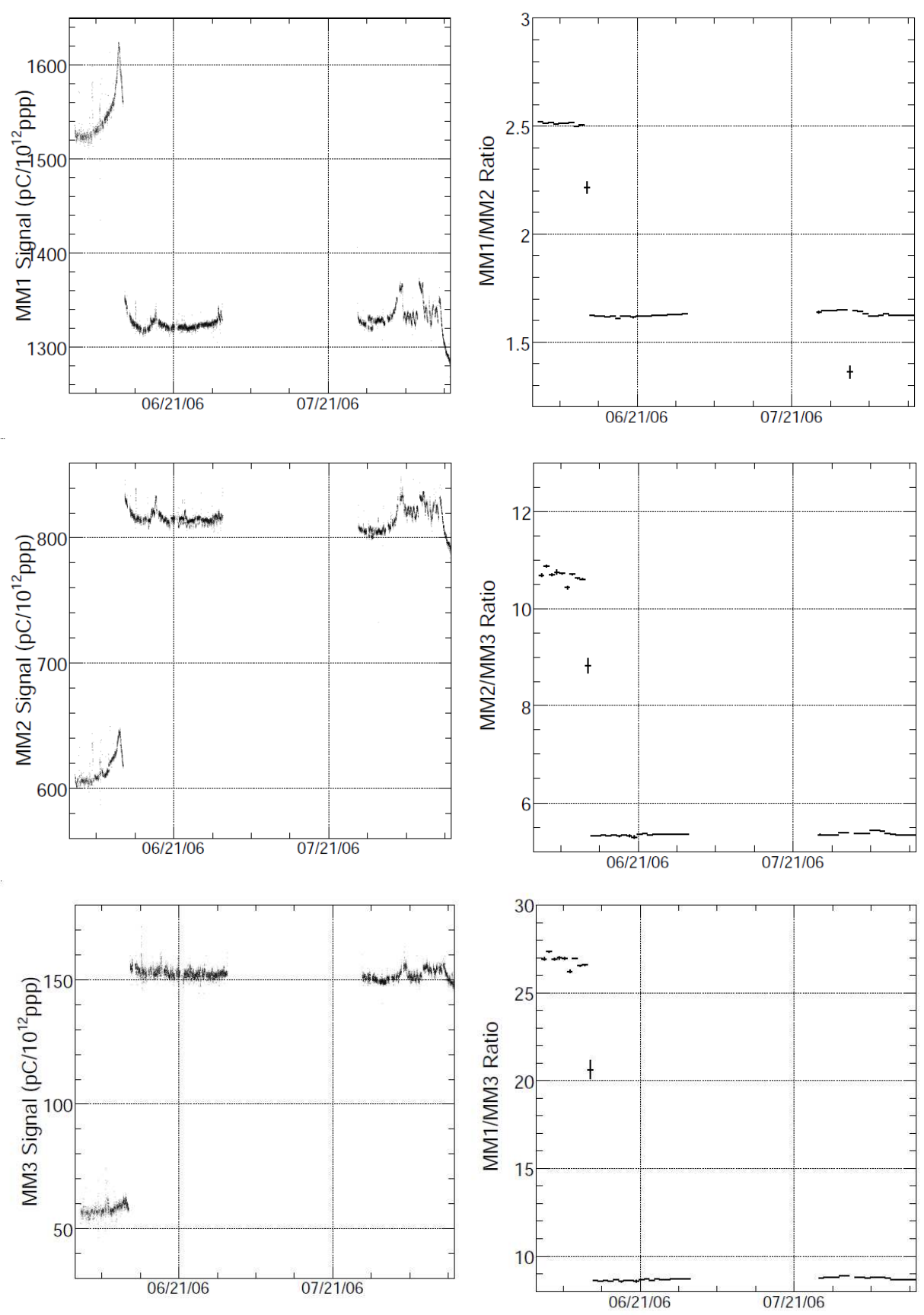

Figure 3.19: Muon Monitor 1(top left), 2(middle left), and 3(bottom left) signals during Run II Part I and the ratios of the signals in muon monitor 1 to 2 (top right), muon monitor 2 to 3 (middle right) and muon monitor 1 to 3 (bottom right). 
During the LE250 running. The ratio of alcove 1 to alcove 2, alcove 1 to alcove 3 and alcove 2 to alcove 3 is stable to 2 , 4 , and $3 \%$ respectively.

\section{Summary}

The muon monitor data has been thoroughly characterized over the course of Run I and the Run II LE150 and LE250 beam running. Although, variations in gas quality caused variations in the absolute signal in the monitors, with the exception of muon monitor 3 prior to July 2005, the relative calibration between the monitors is not affected. A correction is made to the data taken in muon monitor 3 to account for the inferior gas quality. Also, during these time periods the muon monitors show that there are no large fluctuations in the relative muon flux measured; the relative muon flux is stable in each beam configuration.

\subsection{Horn Current Scan Data Sets}

The muon flux is directly related to the neutrino flux, as the muons are produced alongside neutrinos in pion and kaon decay. Chapters 4-6 will describe the relationship between the muon and the neutrino flux and how measurements in the muon monitors can be translated into a neutrino flux measurement.

The NuMI beamline can produce a variety of neutrino fluxes with peak energies in the range from $\sim 1$ to $\sim 16 \mathrm{GeV}$ by varying the current in the focusing horns and by varying the longitudinal distance between the target and the horns. In doing so, a different momentum of pion and kaon is most fully focused parallel to the beamline. Likewise, the muon beam changes when 
the target position or horn current are altered: muons from the decay of the most optimally focused parent particles diverge the least with respect to the beamline center, and thus form the peak of the muon flux at the downstream muon monitors.

During the first 2 years of operation, the NuMI beam operated in several different target configurations, LE000, LE010, LE100, LE150 and LE250 (see Table 3.2). During each beam configuration, the current in the horns was scanned from 0kA to 200kA providing a large data set of measurements of the muon fluxes in each target configuration in the muon monitors. Each scan of the horn current from $0 \mathrm{kA}$ to $200 \mathrm{kA}$ at a single LEXXX is called a "horn current scan". Each horn current scan consists of between 1 and 10's of proton beam spills at each horn current, which varies from 8 to $\sim 50$ different horn currents, for a total of 100-600 proton beam spills per horn current scan. The dates that each horn current scan was performed are listed in Table 3.4.

The remainder of this chapter describes the muon monitor horn current scan data sets and the corrections to the data necessitated by varying ambient and beam conditions. Although the statistical error on the flux measurement at each LEXXX/YYYkA data point is negligible, the systematic effects such as those described in the previous section of this chapter must be corrected and accounted for. Differences in ambient and beam conditions during the scans are summarized in Table 3.4.

Figures 3.20 through 3.22 show the raw data from the six scans of Table 3.4 in each of the three alcoves before any corrections, with the exception that electronics "pedestals", have been applied. Each point is an average of the signal from all spills taken at the same horn current, which ranges from 1 to 10 's of proton beam spills. A couple of differences between the scans are 


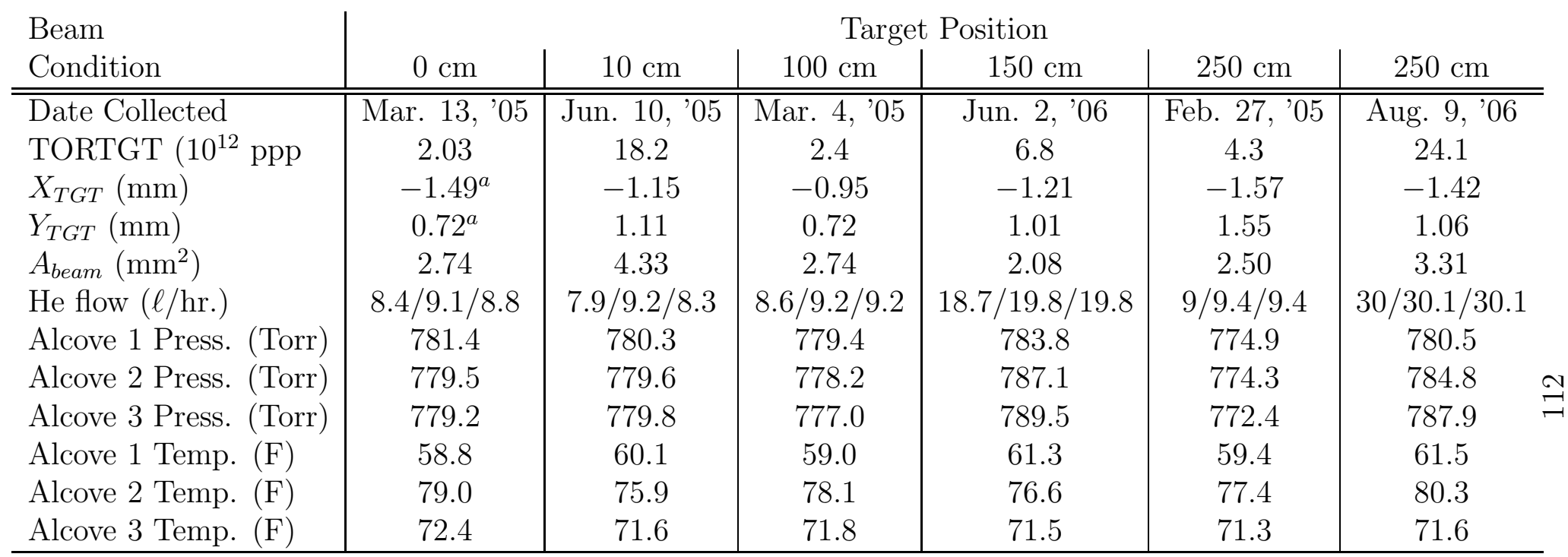

${ }^{a}$ Determined with the profile monitors(SEMs).

Table 3.4: Beam configurations and data sets accumulated for this analysis. For each data set is given the average intensity of the proton beam per spill, the average position of the proton beam (horizontal and vertical), average proton beam spot size, He gas flows and pressures and temperatures in the muon monitors (in Alcoves 1, 2, and 3). 


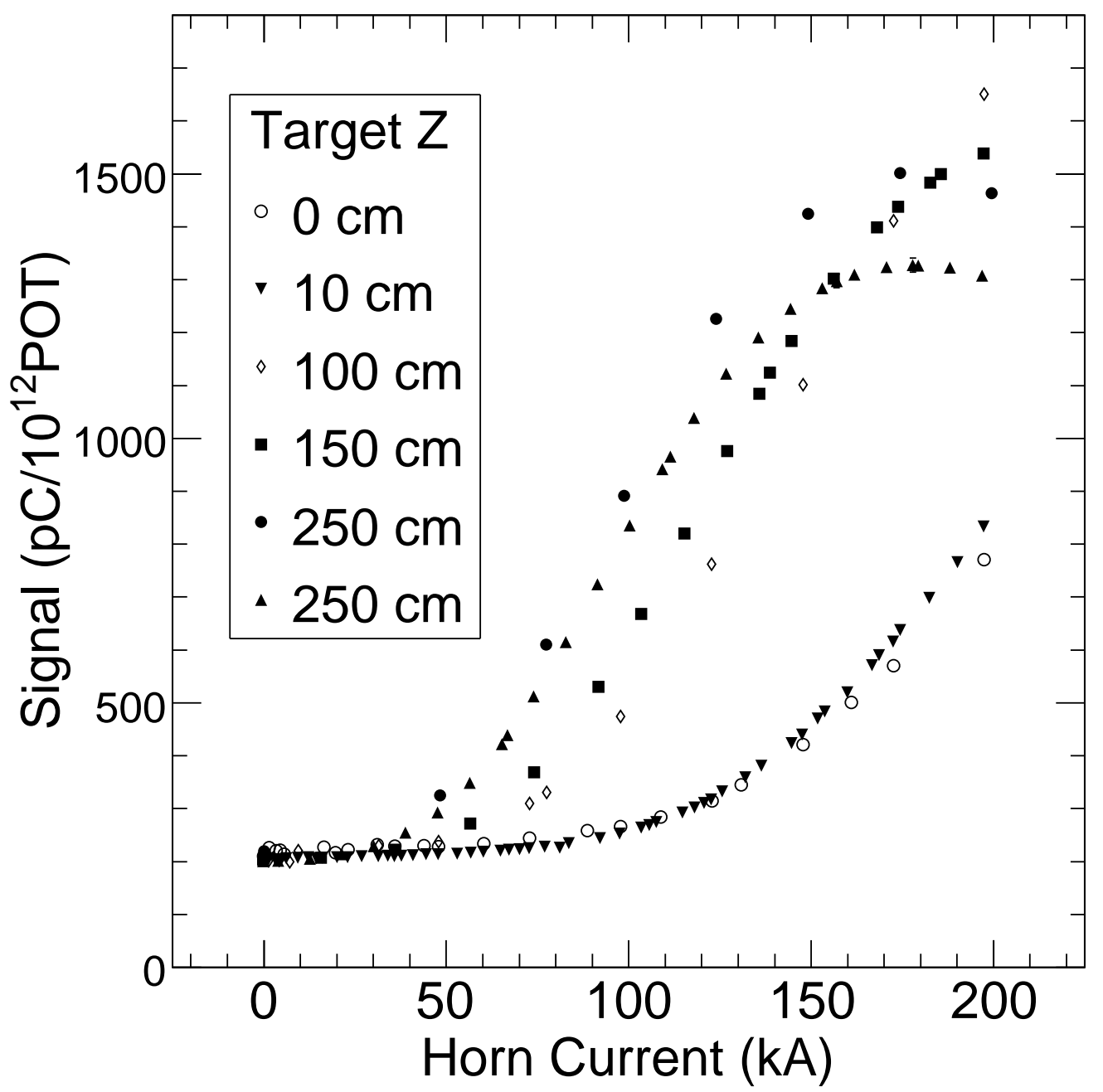

Figure 3.20: The raw signal from Muon Monitor 1 plotted as a function of the current in the horn and several target positions Each scan was performed under different beam and chamber conditions. 


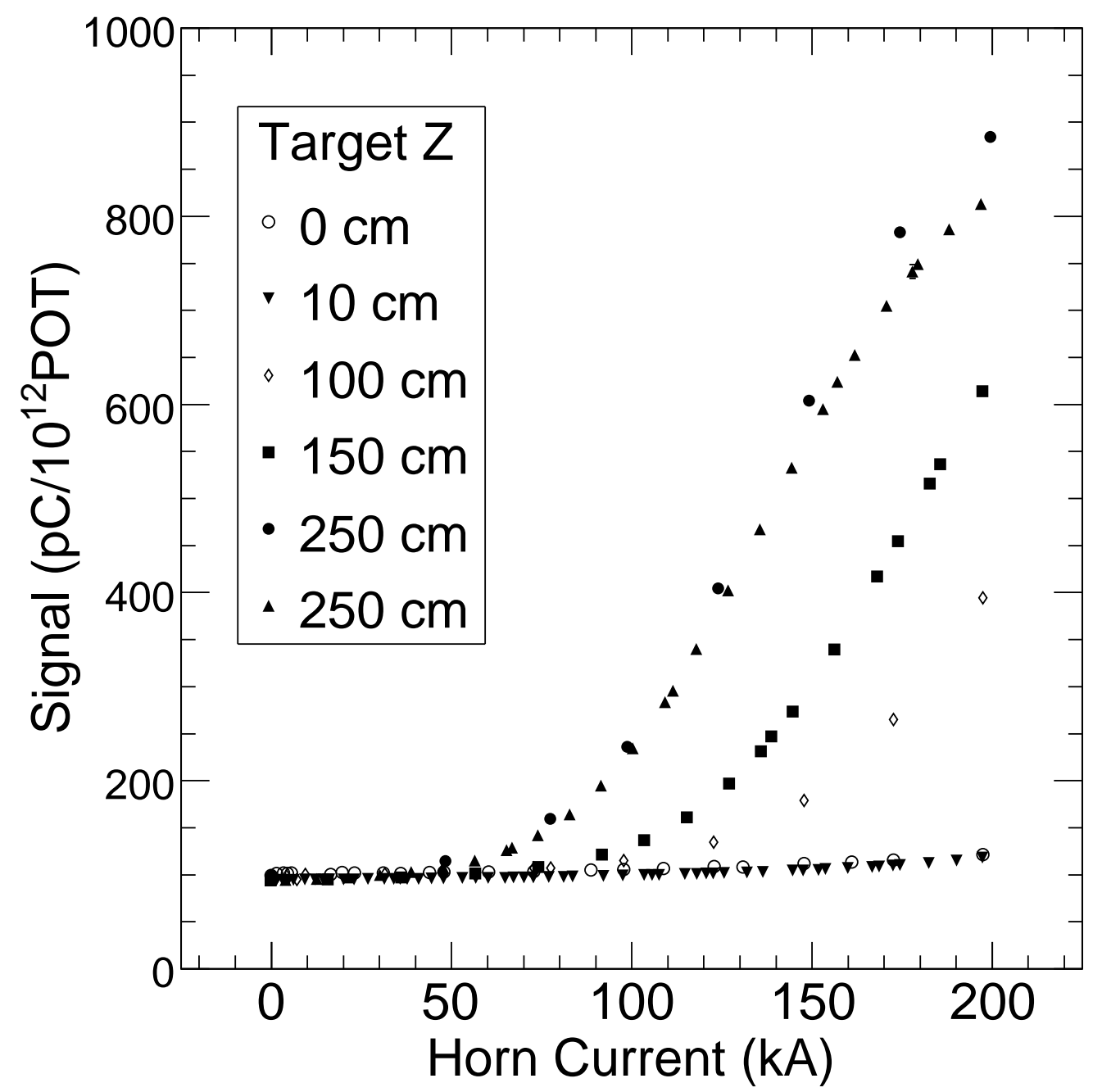

Figure 3.21: The raw signal from Muon Monitor 2 plotted as a function of the current in the horn and several target positions Each scan was performed under different beam and chamber conditions. 


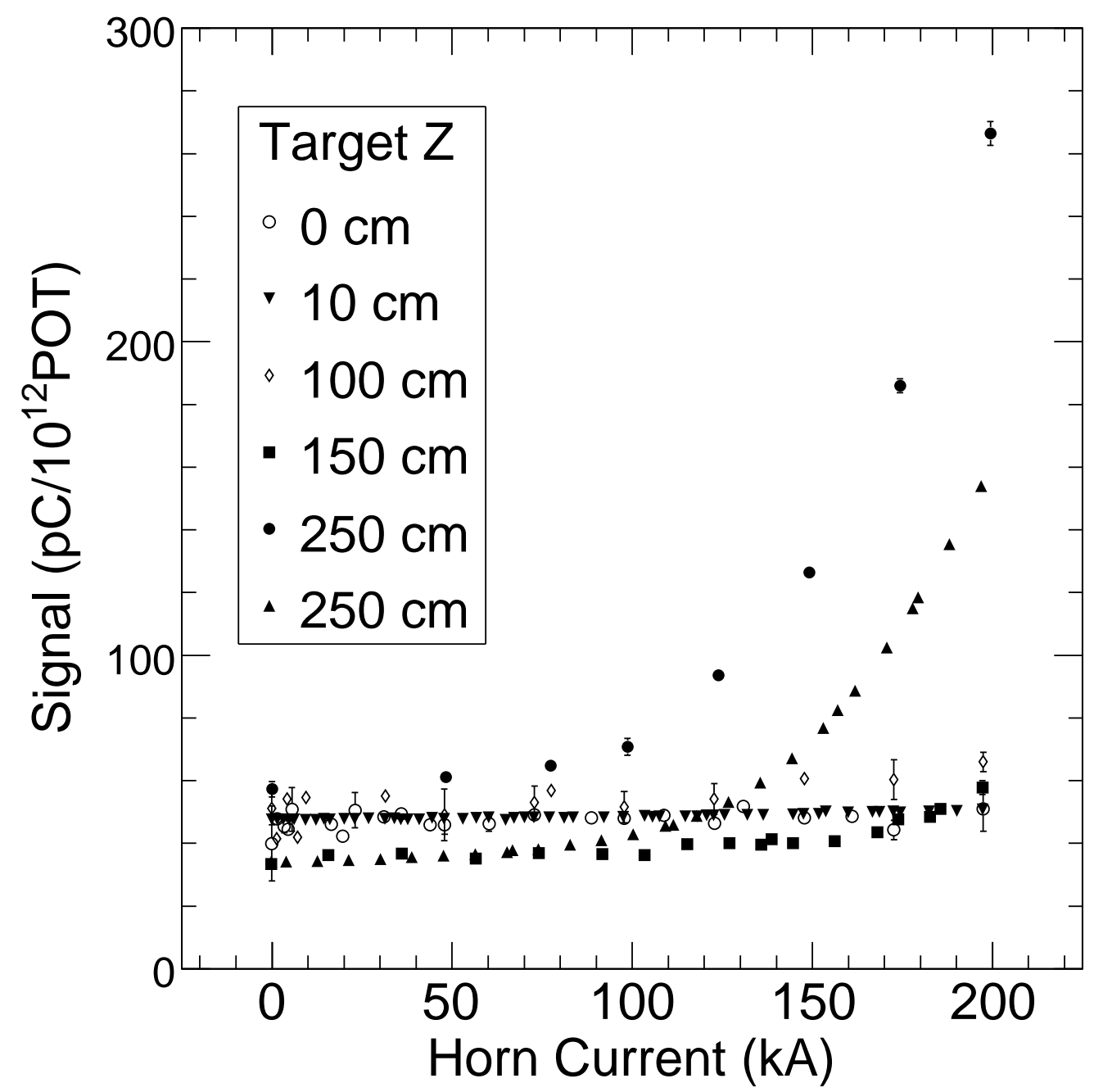

Figure 3.22: The raw signal from Muon Monitor 3 plotted as a function of the current in the horn and several target positions Each scan was performed under different beam and chamber conditions. 
readily evident from these plots. First, as can be seen in Figures 3.20 and 3.21, the two LE250 scans do not agree, reflecting different conditions under which each scan was performed. Second, as can be most easily seen in Figure 3.22, the scans performed at different target positions do not agree at $0 \mathrm{kA}$. The, at most, $2.5 \mathrm{~m}$ difference in target position between the scans is nearly negligible compared to the $\sim 725 \mathrm{~m}$ distance between the target and the muon monitors. Thus, without horn focusing there should be no measurable difference in muon flux at the muon monitors. Corrections to the scans are evaluated below.

\subsubsection{Systematic Effects}

In this section, we apply corrections to the data of Figures 3.20 through 3.22. We will correct for ambient effects such as barometric pressure, temperature and flow rates, as well as variations in beam running conditions which would be expected to cause variation in rates. These include proton beam spot size and position at the target, as well as intensity per spill. These are discussed in turn.

Ambient pressure and temperature conditions vary across these data sets as summarized in Table 3.4. As discussed in Section 3.1.3 and in Ap-

pendix A, a correction was developed to account for such variations. The dominant correction is for the difference in the temperature between alcoves 1, 2 and 3. Because the temperature at each monitor varies little over time, a temporal correction was not necessary. Since hadron monitor is essentially identical to the muon monitors in construction and function, the temperature correction obtained for the hadron monitor (see Appendix A) is applied to the muon monitor data. 
As indicated in Table 3.4, the horn current scans were performed at different instantaneous beam intensities (protons-per-pulse), with the earlier data sets acquired at significantly lower intensity. Also, the flux of particles through the muon monitors depends strongly on horn current. As discussed in Section 3.1.4, a correction must be applied to the muon monitor signals in order to compare data taken at different rates of ionization resulting from varying proton beam intensity and horn current. The correction shown in Figure 3.8 is used to correct the horn current scan data sets. Figure 3.23 shows the percent correction applied to each data point in the horn scan data sets.

The average position of the proton beam at the target during the horn scans varies from $\left\langle X_{T G T}\right\rangle=-0.84 \mathrm{~mm}$ to $-1.57 \mathrm{~mm}$ in the horizontal direction and $\left\langle Y_{T G T}\right\rangle=0.2 \mathrm{~mm}$ to $1.6 \mathrm{~mm}$ vertically. The nominal center of the target is at $X=-1.2 \mathrm{~mm}$ and $Y=+1 \mathrm{~mm}$. As discussed in Appendix B, as the proton beam is steered closer to the edge of the target, fast pions exiting the same side of the target have a greater probability of exiting without reinteracting in the target material. An off-center proton beam can therefore increase the flux of high-energy neutrinos and muons. Because a target alignment scan using the proton beam was performed each time the target was moved into a different longitudinal position, there exist horizontal and vertical target scans performed in each beam configuration, LE000, LE010, LE100, LE150 and LE250. As discussed in Appendix B, these data were used as the basis for a correction based on proton beam position. Table 3.5 lists the resulting corrections applied to each horn scan data set in each of the muon monitors.

As indicated in Table 3.4, the gas flow has been increased on a couple of occasions which affect the ionization scale of the monitors. Further, the 


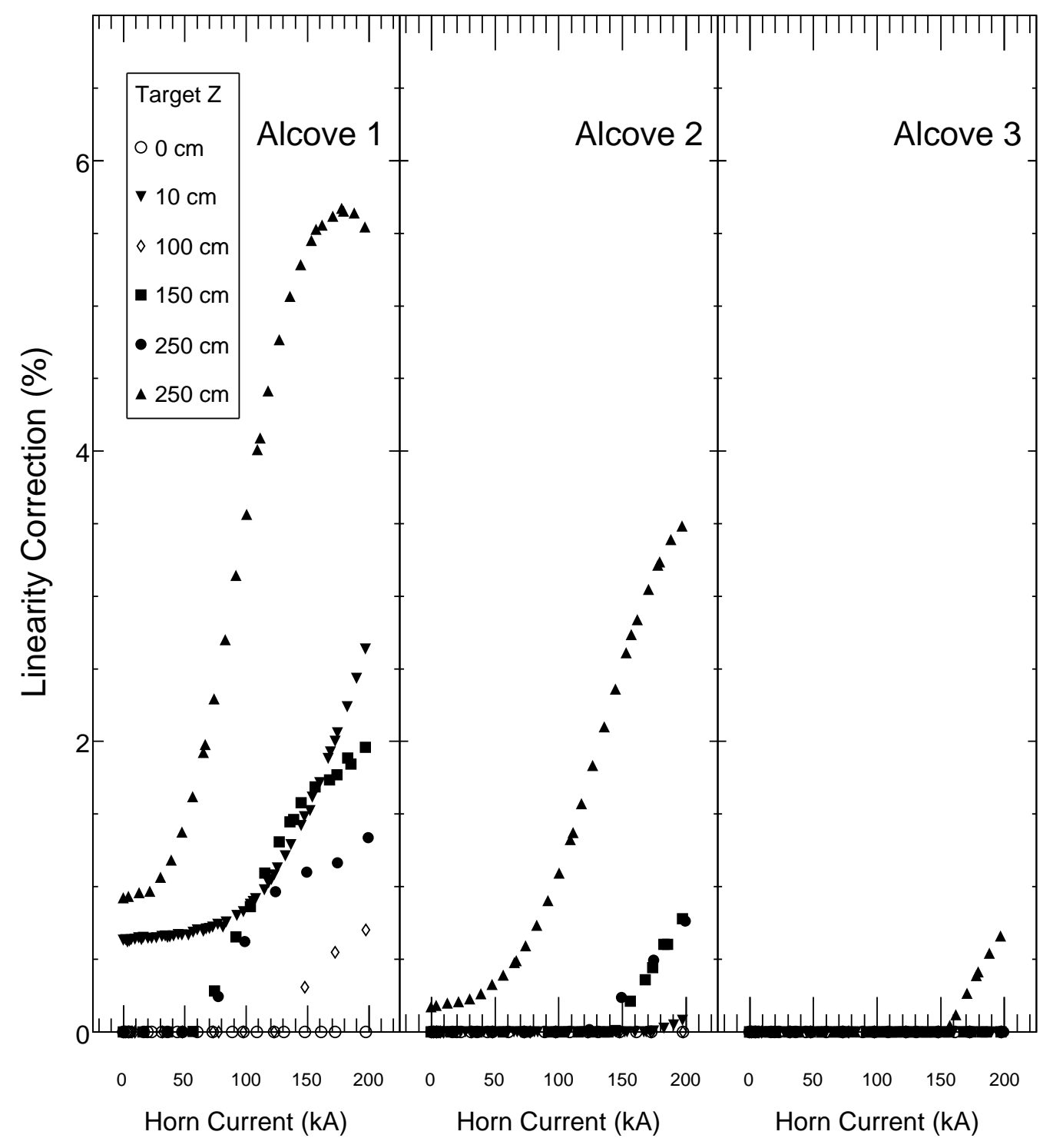

Figure 3.23: Magnitude of the non-linearity correction applied to the horn scan data. The plot legend refers to the horn scan data sets in Table 3.4. The corrections come from comparing the ionization rate at each horn scan data point to the curve in Figure 3.8. 


\begin{tabular}{|c|c|c|c|c|}
\hline \multicolumn{2}{|c|}{ Data Set } & \multicolumn{3}{|c|}{ Systematic Effect:Beam Position } \\
\hline Alcove & $\begin{array}{c}\text { Target } \\
\text { Position }\end{array}$ & $\begin{array}{c}\text { Correction to } \\
X_{T G T}=-1.2 \mathrm{~mm} \\
(\%)\end{array}$ & $\begin{array}{c}\text { Correction to } \\
Y_{T G T}=+1.0 \mathrm{~mm} \\
(\%)\end{array}$ & $\begin{array}{c}\text { Total } \\
\text { Correction } \\
(\%) \\
\end{array}$ \\
\hline $\begin{array}{l}\text { Muon } \\
\text { Alcove } \\
\quad 1\end{array}$ & $\begin{array}{c}0 \mathrm{~cm} \\
10 \mathrm{~cm} \\
100 \mathrm{~cm} \\
150 \mathrm{~cm} \\
250 \mathrm{~cm}^{a} \\
250 \mathrm{~cm}^{b}\end{array}$ & $\begin{array}{c}0.2 \pm 0.5 \\
-0.1 \pm 0.5 \\
0.0 \pm 0.5 \\
0.0 \pm 0.5 \\
0.0 \pm 0.5 \\
0.6 \pm 0.5\end{array}$ & $\begin{array}{c}0.1 \pm 0.5 \\
0.0 \pm 0.5 \\
-0.4 \pm 0.5 \\
-0.1 \pm 0.5 \\
0.7 \pm 0.5 \\
0.0 \pm 0.5\end{array}$ & $\begin{array}{c}0.3 \pm 0.7 \\
-0.1 \pm 0.7 \\
-0.4 \pm 0.7 \\
-0.1 \pm 0.7 \\
0.7 \pm 0.7 \\
0.6 \pm 0.7\end{array}$ \\
\hline $\begin{array}{l}\text { Muon } \\
\text { Alcove } \\
\quad 2\end{array}$ & $\begin{array}{c}0 \mathrm{~cm} \\
10 \mathrm{~cm} \\
100 \mathrm{~cm} \\
150 \mathrm{~cm} \\
250 \mathrm{~cm}^{a} \\
250 \mathrm{~cm}^{b} \\
\end{array}$ & $\begin{array}{c}0.0 \pm 0.5 \\
0.0 \pm 0.5 \\
-0.3 \pm 0.5 \\
0.0 \pm 0.5 \\
0.8 \pm 0.5 \\
0.5 \pm 0.5 \\
\end{array}$ & $\begin{array}{c}0.0 \pm 0.5 \\
0.0 \pm 0.5 \\
-0.3 \pm 0.5 \\
-0.1 \pm 0.5 \\
0.8 \pm 0.5 \\
-0.1 \pm 0.5\end{array}$ & $\begin{array}{c}0.0 \pm 0.7 \\
0.0 \pm 0.7 \\
-0.6 \pm 0.7 \\
-0.1 \pm 0.7 \\
1.6 \pm 0.7 \\
0.4 \pm 0.7\end{array}$ \\
\hline $\begin{array}{l}\text { Muon } \\
\text { Alcove } \\
\quad 3\end{array}$ & $\begin{array}{c}0 \mathrm{~cm} \\
10 \mathrm{~cm} \\
100 \mathrm{~cm} \\
150 \mathrm{~cm} \\
250 \mathrm{~cm}^{a} \\
250 \mathrm{~cm}^{b}\end{array}$ & $\begin{array}{c}2.0 \pm 0.5 \\
1.2 \pm 0.5 \\
0.1 \pm 0.5 \\
0.1 \pm 0.5 \\
-1.8 \pm 0.5 \\
2.3 \pm 0.5 \\
\end{array}$ & $\begin{array}{c}0.1 \pm 0.5 \\
0.0 \pm 0.5 \\
0.8 \pm 0.5 \\
0.0 \pm 2.0 \\
1.0 \pm 0.5 \\
-0.1 \pm 0.5\end{array}$ & $\begin{array}{c}2.1 \pm 0.7 \\
1.2 \pm 0.7 \\
0.9 \pm 0.7 \\
0.1 \pm 2.1 \\
-0.8 \pm 0.7 \\
2.2 \pm 0.7 \\
\end{array}$ \\
\hline
\end{tabular}

${ }^{a}$ February, 2005 Data set ${ }^{b}$ August, 2006, Data set

Table 3.5: Corrections and uncertainties in the horn current scan data due to the horizontal, and vertical beam positions. Given for each of the muon monitors and in each of the target configurations. The derivation of these corrections can be found in Appendix B. 
third alcove had an undetected leak in the gas system prior June, 2005, so that its purity level at that time was worse than the other two alcoves. Most recent data, taken at $20 \ell / \mathrm{hr}$. and $30 \ell / \mathrm{hr}$. flow to the alcoves, appears to be fairly stable in signal level, indicating that the impurity problem has been reduced. To correct some of the earlier horn current scan data, we note that the data taken with the horns off should provide a "standard candle." Thus allowing older data collected at higher impurity level to be scaled down to the signal level seen in the most recent data sets at the lower impurity levels. All other corrections ( beam conditions, pressure, temperature) have been applied. These values form the basis for the gas quality correction factors, shown in the last column of Table 3.6. This correction is $\sim 4 \%$ in the first two alcoves, but is $\sim 30 \%$ in Alcove 3 due to the now-fixed leak. Table 3.6 lists the signal levels at zero horn current after the previously discussed corrections have been applied in each of the monitors during each of the scans, which are used to develop the correction factors in Table 3.6.

\subsubsection{Summary}

The corrections for pressure, temperature, non-linearity, proton beam position and gas quality discussed in this chapter and listed in Tables 3.1, 3.5 and 3.6 are applied to the horn scans shown in Figures 3.20 through 3.22. The fullycorrected horn scans, are shown in Figures 3.24 through 3.26. The uncertainty on the data points is the sum in quadrature of the errors associated with the corrections applied to the data and the RMS scatter in the signals from the individual beam spills comprising each point. Close inspection of Figures 3.24 through 3.26 shows very good agreement between the two LE250 horn scans 


\begin{tabular}{c|c|c||c}
\multicolumn{2}{c|}{ Data Set } & $I_{\text {horn }}=0 \mathrm{kA}$ Signals & \\
\hline \multirow{3}{*}{ Alcove } & $\begin{array}{c}\text { Target } \\
\text { Position }\end{array}$ & $\begin{array}{c}\text { Signal After } \\
\text { Corrections } \\
\left(\mathrm{pC} / 10^{12} \mathrm{ppp}\right)\end{array}$ & $\begin{array}{c}\text { Gas Quality } \\
\text { Correction } \\
\text { Factor }\end{array}$ \\
\hline \hline & $0 \mathrm{~cm}$ & $209.0 \pm 10.7$ & $0.927 \pm 0.048$ \\
Muon & $10 \mathrm{~cm}$ & $198.0 \pm 0.9$ & $0.979 \pm 0.005$ \\
Alcove & $100 \mathrm{~cm}$ & $200.6 \pm 8.5$ & $0.966 \pm 0.041$ \\
1 & $150 \mathrm{~cm}$ & $193.8 \pm 0.6$ & 1.0 \\
& $250 \mathrm{~cm}^{a}$ & $211.2 \pm 3.2$ & $0.918 \pm 0.014$ \\
& $250 \mathrm{~cm}^{b}$ & $194.7 \pm 0.1$ & $0.995 \pm 0.003$ \\
\hline \multirow{3}{*}{ Muon } & $0 \mathrm{~cm}$ & $102.3 \pm 2.6$ & $0.918 \pm 0.023$ \\
Alcove & $100 \mathrm{~cm}$ & $95.1 \pm 0.3$ & $0.987 \pm 0.004$ \\
2 & $150 \mathrm{~cm}$ & $98.4 \pm 1.5$ & $0.954 \pm 0.015$ \\
& $250 \mathrm{~cm}$ & $93.9 \pm 0.3$ & 1.0 \\
& $250 \mathrm{~cm}^{b}$ & $95.6 \pm 0.1$ & $0.982 \pm 0.003$ \\
\hline \multirow{3}{*}{ Muon } & $0 \mathrm{~cm}^{a}$ & $45.1 \pm 13.7$ & $0.729 \pm 0.223$ \\
Alcove & $100 \mathrm{~cm}^{3}$ & $47.7 \pm 0.2$ & $0.688 \pm 0.019$ \\
3 & $150 \mathrm{~cm}^{a}$ & $51.3 \pm 5.2$ & $0.641 \pm 0.067$ \\
& $250 \mathrm{~cm}^{a}$ & $52.9 \pm 0.9$ & 1.0 \\
& $250 \mathrm{~cm}^{b}$ & $34.3 \pm 0.1$ & $0.958 \pm 0.026$ \\
\hline
\end{tabular}

${ }^{a}$ February, 2005 Data set ${ }^{b}$ August, 2006, Data set

Table 3.6: The horn-off signals in the alcoves (in units of $\mathrm{pC} / 10^{12} \mathrm{ppp}$ ), after corrections for barometric pressure, ambient temperature, rate-dependent effects, and beam position are applied. Data for each monitor within columns should be compared. Changes in the gas quality are evident. Based on these changes, a correction factor is derived for the Muon Alcove data which is the ratio of the horn off signal in a particular data set to the $150 \mathrm{~cm}$ data set. It is shown Section 3.3.1 that the ratios between alcoves are accurate. 


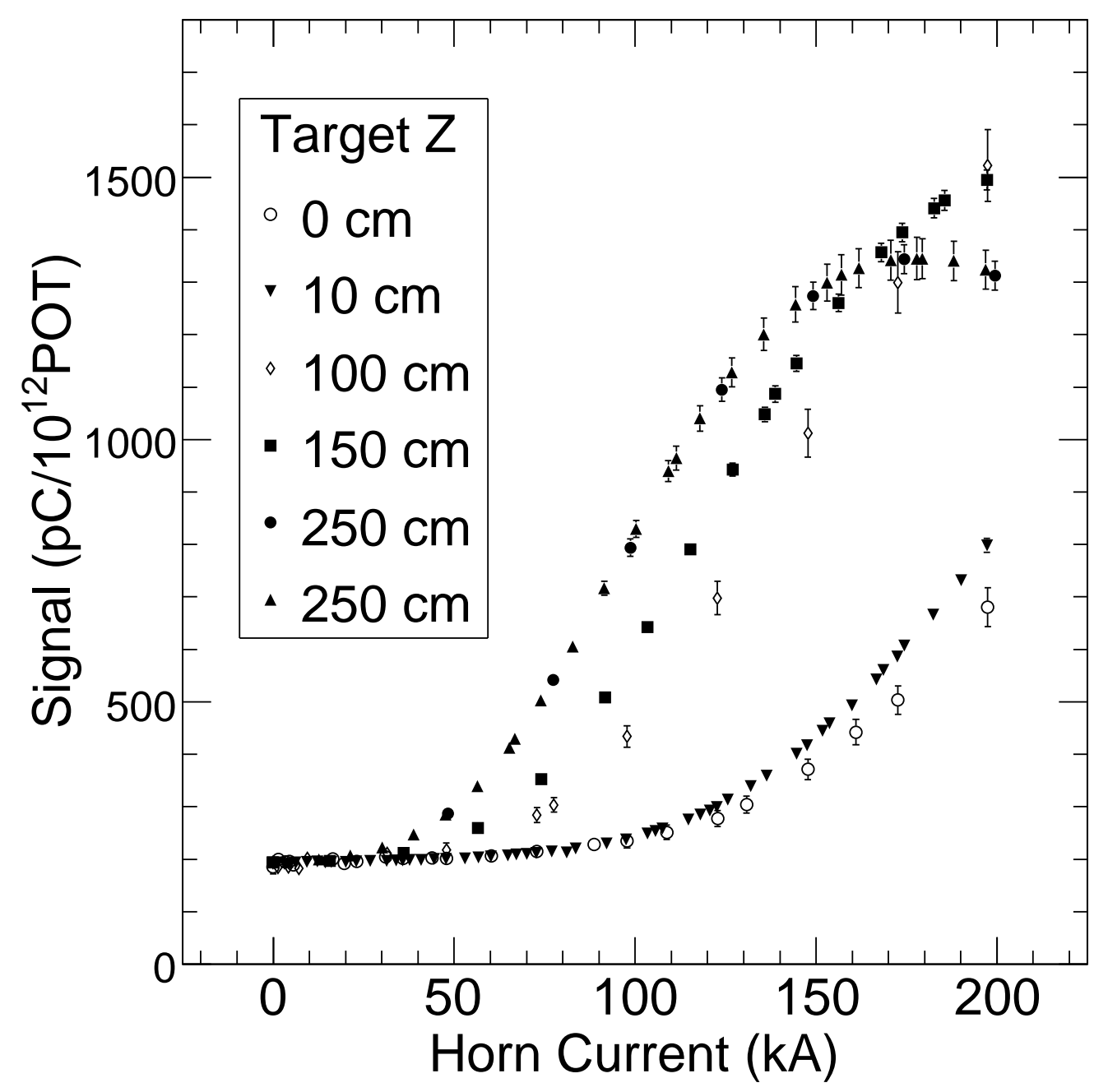

Figure 3.24: Corrected signal from Muon Monitor 1 plotted as a function of the current in the horn during several study periods. Each scan was performed under different beam and chamber conditions. The data are the same as in Figure 3.20, but the corrections for ambient pressure, temperature, linearity, beam position, and gas quality discussed herein are applied to the data. 


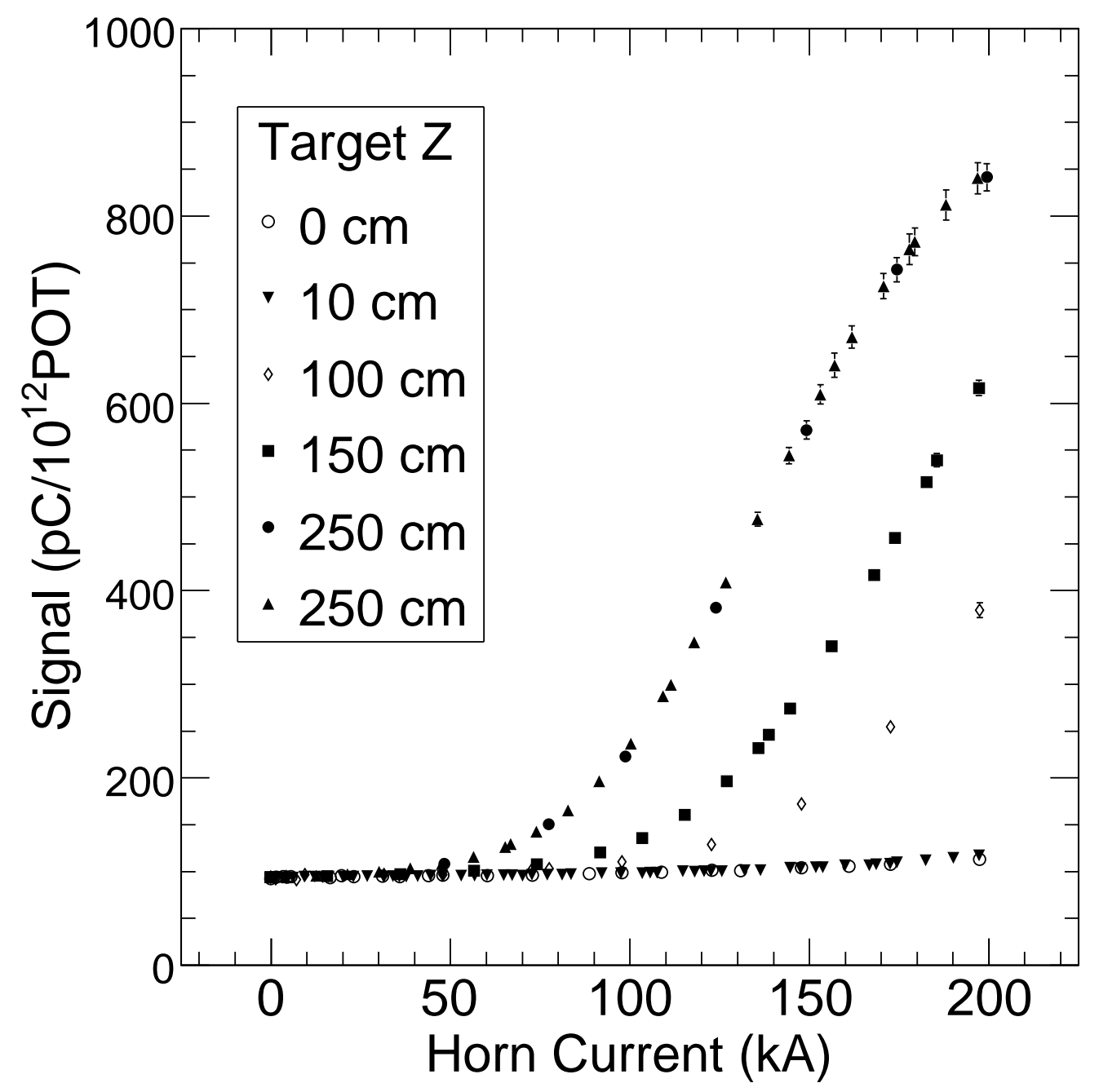

Figure 3.25: Corrected signal from Muon Monitor 2 plotted as a function of the current in the horn during several study periods. Each scan was performed under different beam and chamber conditions. The data are the same as in Figure 3.21, but the corrections for ambient pressure, temperature, linearity, beam position, and gas quality discussed herein are applied to the data. 


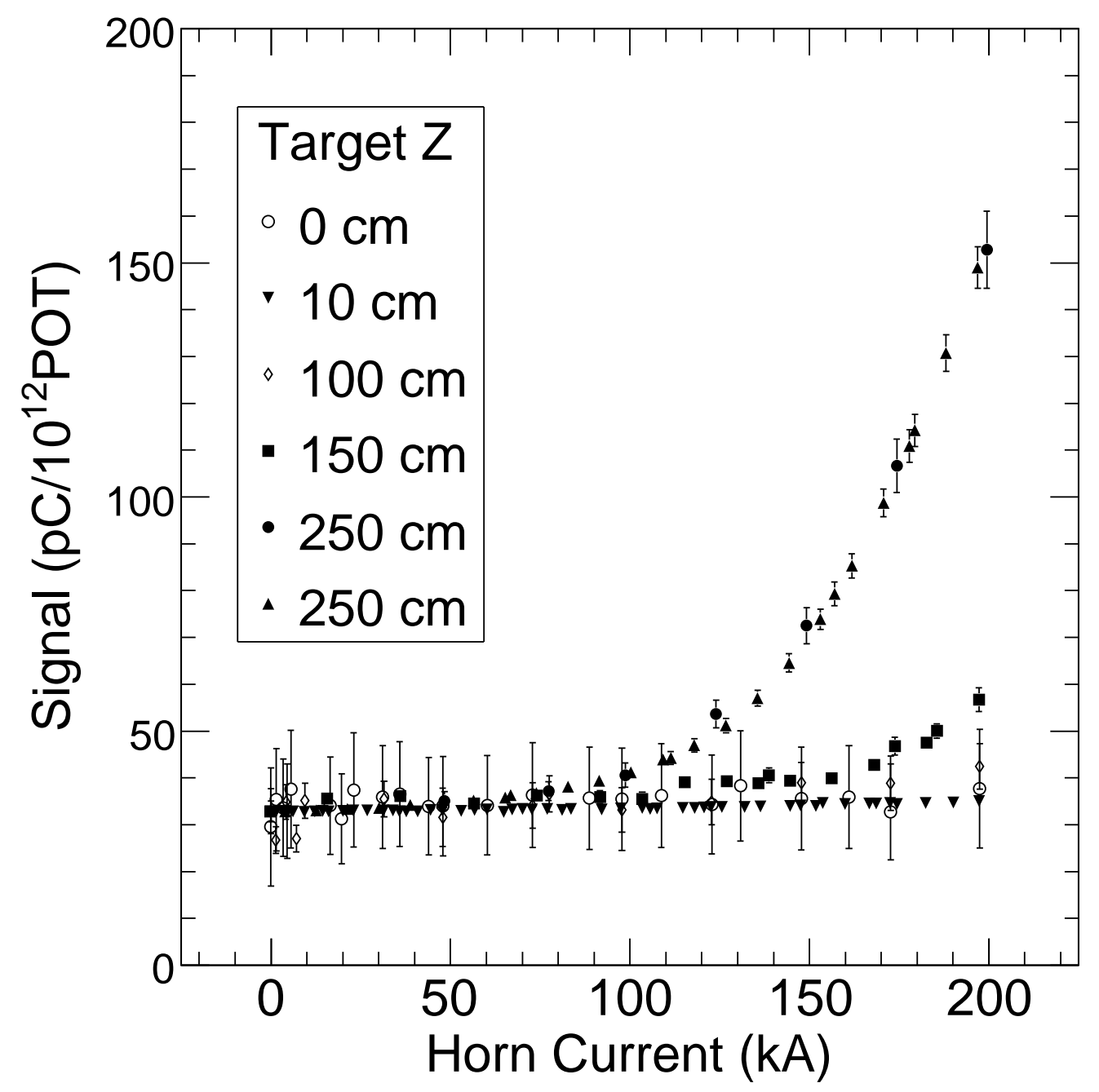

Figure 3.26: Corrected signal from Muon Monitor 3 plotted as a function of the current in the horn during several study periods. Each scan was performed under different beam and chamber conditions. The data are the same as in Figure 3.22, but the corrections for ambient pressure, temperature, linearity, beam position, and gas quality discussed herein are applied to the data. 
is achieved after corrections are applied. Because the two LE250 data sets were taken under very different ambient and beam conditions, this agreement supports the accuracy of the correction procedures.

These horn scan data can be compared to Monte Carlo calculations of the muon flux at the muon monitors. This comparison will be made in the following chapters. 


\section{Chapter 4}

\section{Neutrino and Muon Flux}

\section{Calculation and Uncertainty}

The present chapter describes a calculation of the neutrino flux produced by the NuMI beamline. Analytic relationships, external experimental data and theoretical models of physical processes are used as inputs to the calculation. As it will be seen, it is not essential that each of these inputs are known very precisely. Instead, it will be possible to constrain directly the calculation using data from the NuMI muon beam flux. Because the same calculation yields both the muon and neutrino flux in $\pi \rightarrow \mu \nu_{\mu}$ and $K \rightarrow \mu \nu_{\mu}$ decays. The experimental constraint on the muon flux will, in Chapters 5 and 6 , be utilized to obtain a neutrino flux. In this chapter, we limit our discussion to the details of the muon and neutrino flux calculation.

Calculating the neutrino and muon flux from the NuMI beam involves several steps. First, the spectrum of particles produced in proton-target collisions must be known. Each particle must then be tracked through the focusing 
horns, and decay volume. Care must be taken to account for secondary interactions of mesons with beamline components and scattering of particles within beamline material. The decay of pions and kaons must be performed and the muons from the decays must be tracked through the hadron absorber and muon region. The calculation involves integration over all of these steps. This is accomplished via a Monte Carlo simulation algorithm which tracks particles from production to decay through the NuMI beamline.

This chapter describes the Monte Carlo production and tracking of parent mesons along the beamline is described as well as the decay of those mesons which leads to muons in the muon monitors and neutrinos at the MINOS Near Detector. The results of Monte Carlo studies of the uncertainties in the neutrino flux at the MINOS Detector from uncertainties in particle production and tracking are also described.

\subsection{Monte Carlo Simulation of the Beamline}

In order to predict the fluxes from the NuMI beamline several different Monte Carlo (MC) simulation codes have been developed. Each implements a more detailed and complex geometric representation of the NuMI beamline and each use different physics models to simulate interaction and tracking of particles along the beamline.

For the analysis described in this thesis, the simulation of pion and kaon parents off of the NuMI target is performed using Fluk05 particle production models [48]. The subsequent tracking through the beamline into the decay volume is done with a Geant3 [49] based Monte Carlo called GNUMI. While the tracking of muons starting at the upstream face of the hadron absorber 
through the muon filter is done with G4NUMI, a Geant4 [26] based Monte Carlo.

Geant3(4) provides Fortran $(\mathrm{C}++)$ based computer software tools for describing the geometry of the experimental setup as well as for tracking particles through the geometry. The level of detail and thus presumably accuracy with which beamline components are simulated can be potentially significant. For example, interactions of particles within material along the beamline can cause particles to scatter out of the angular acceptance of downstream detectors. A detailed geometry of the NuMI target is implemented in Fluka. The NuMI horns, beamline shielding and decay volume are represented in Geant3. A detailed representation of the hadron absorber and muon filter is implemented in Geant4. The Monte Carlo tracks particles one at a time through all beamline components. The tracking of particles is a discrete process performed in small enough steps that it is approximately continuous. The simulation determines the distance that each particle takes by calculating the mean-free-path for each physics process a particle may undergo based the current geometric and material attributes of the environment. The physics process with the smallest mean free path is chosen to occur. If after the physics process occurs, the particle still exists, e.g. it did not decay, become absorbed, etc., it is transported the distance of the mean free path and the process of determining the new mean-free-path, etc., is repeated.

The Monte Carlo is an idealized representation of the beamline geometry and functioning. In the Monte Carlo the target system, horns and proton beam are collinear and the proton beam always impinges directly on the center of the target and has a fixed transverse size. During normal operation of the NuMI beam the position of the proton beam on the target and the 
size of the proton beam at the target are typically very stable as discussed in Section 2.1.5 and but small variations do occur as shown in Section 3.3. The procedure for aligning the target-baffle system, as discussed in Appendix B, is precise to within $\pm 0.5 \mathrm{~mm}$. Also discussed in this appendix and in Section 3.4.1 is that protons interacting nearest the edge of the target produce higher energy mesons leading to higher energy neutrino and muon fluxes and protons interacting in the baffle also have a similar effect. Also in the Monte Carlo, the current in the horns is fixed while in reality the measured current sent through the horns has a $1 \%$ uncertainty [22] and also undergoes temporal variations as described in Section 3.3. Another focusing uncertainty is that the distribution of current within the horn conductors is not precisely known since it is expected that the skin depth of current penetration into the conductors is on the same order as the thickness of the conductors [22].

The uncertainty on the flux resulting from these effects has been systematically studied by varying each of beamline properties in Monte Carlo [22]. Figures 4.1 through 4.3 show the resulting uncertainty on the neutrino spectrum in the MINOS Detector (ND), coming from each of the systematic effects listed in the legend. This is also the uncertainty on the neutrino flux at the detector since the parameters listed in the figure effect only the flux and not neutrino interactions in the detector. The "Total error" is the sum in quadrature of the individual effects listed.

The uncertainty from knowledge of meson production off of the target is not included in this particular study. In the next section, this uncertainty is discussed. The uncertainty from meson production exceeds all of the other beamline uncertainties shown in this section by a factor of $\sim 3-4$. 


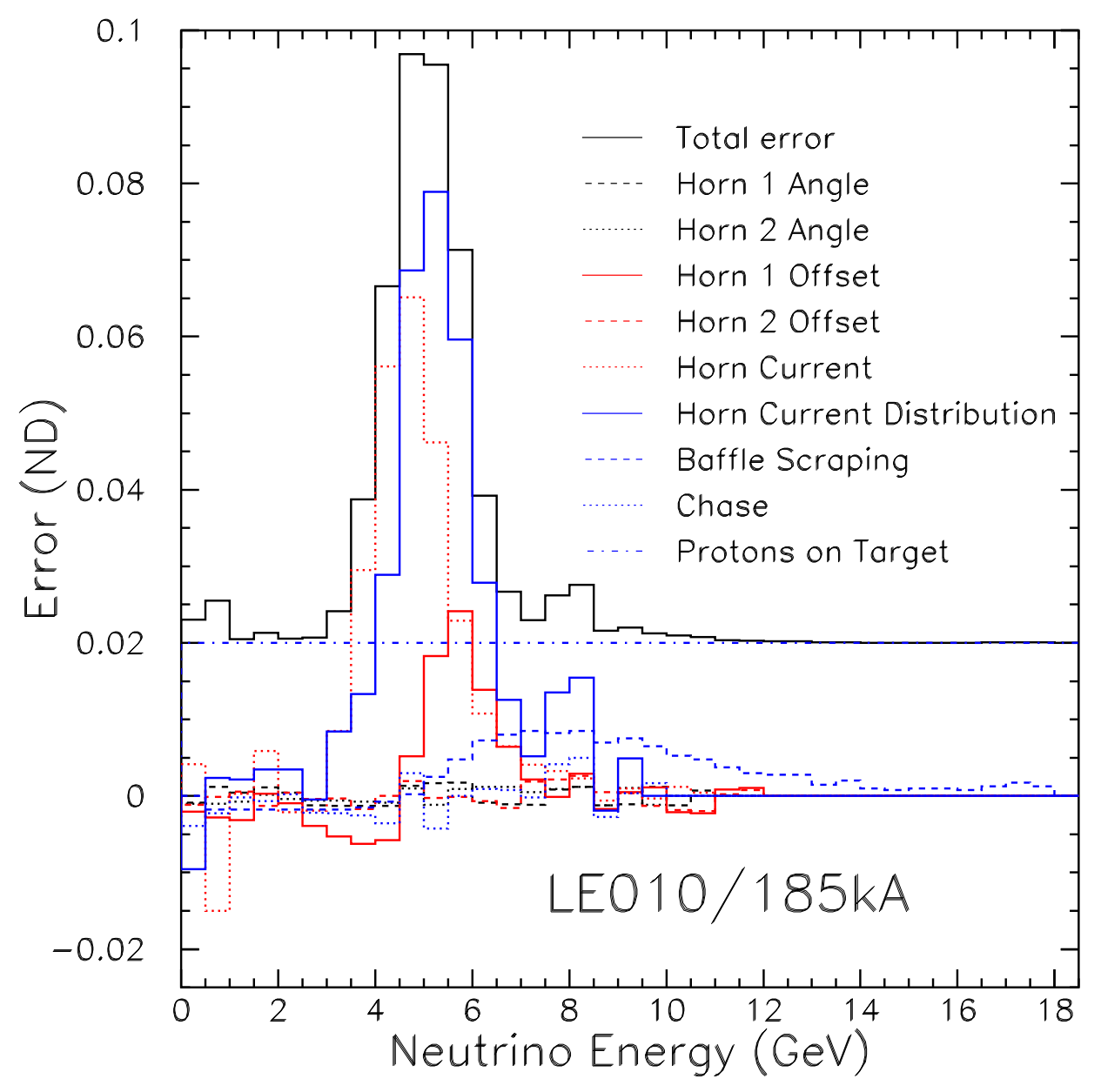

Figure 4.1: The error on the neutrino energy spectrum and neutrino flux at the MINOS Detector(ND) due to the beamline uncertainties listed for the LE010/185kA beam. The "Total error" is the sum in quadrature of the systematic effect listed. Note that uncertainty from hadron production is not included. [22] 


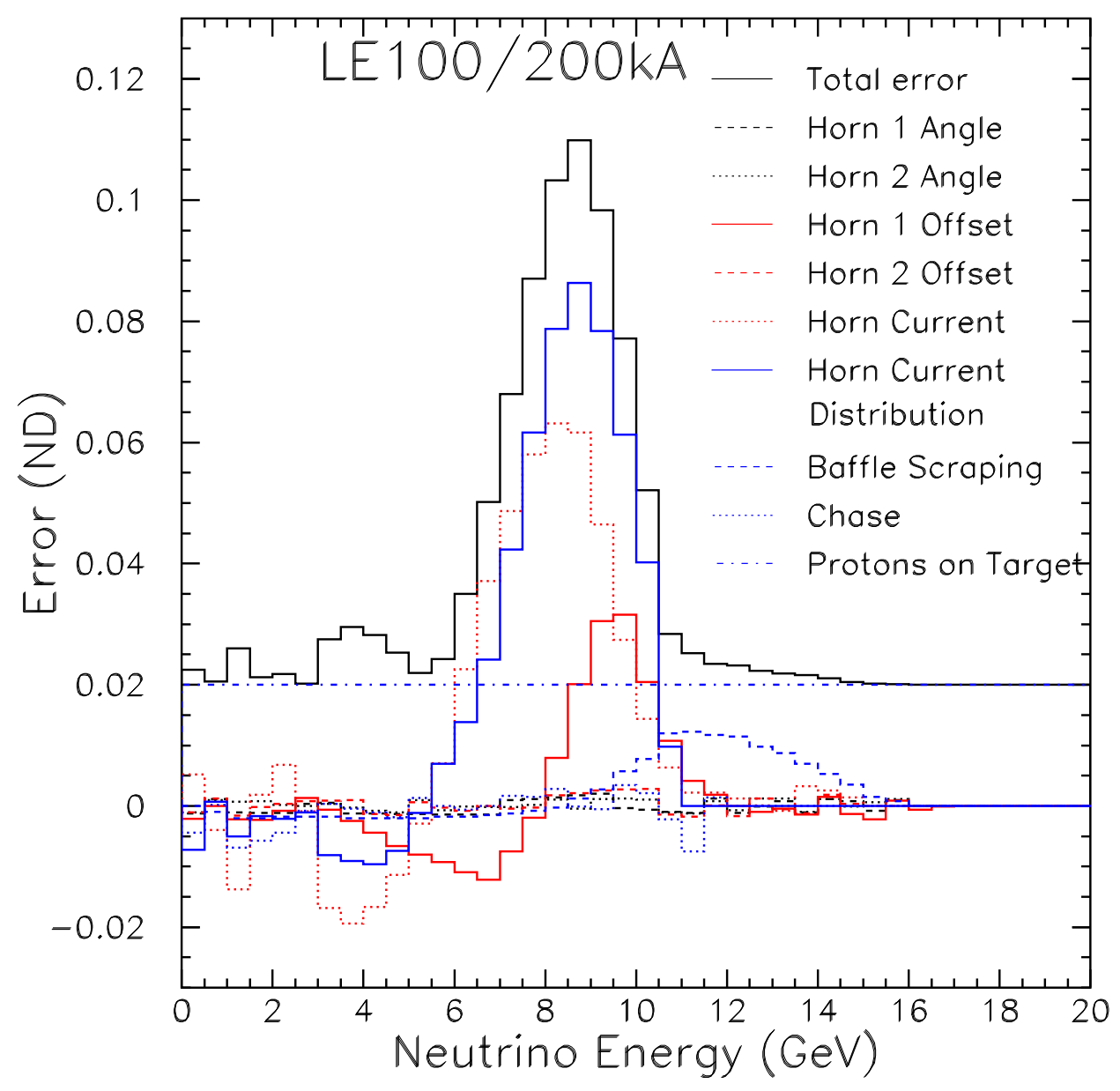

Figure 4.2: The error on the neutrino energy spectrum and neutrino flux at the MINOS Detector(ND) due to the beamline uncertainties listed for the LE100/200kA beam. The "Total error" is the sum in quadrature of the systematic effect listed. Note that uncertainty from hadron production is not included. [22] 


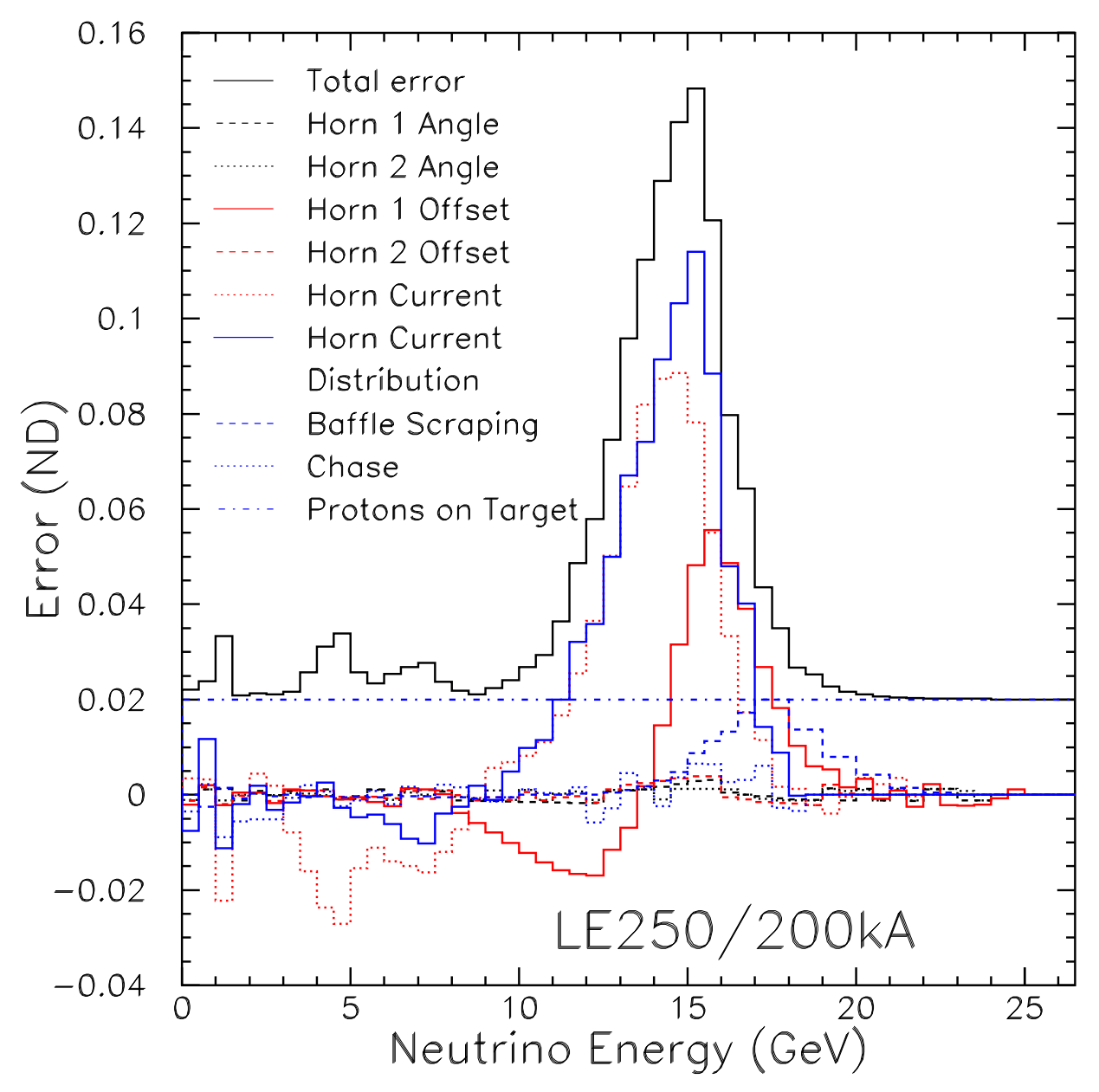

Figure 4.3: The error on the neutrino energy spectrum and neutrino flux at the MINOS Detector(ND) due to the beamline uncertainties listed for the LE250/200kA beam. The "Total error" is the sum in quadrature of the systematic effect listed. Note that uncertainty from hadron production is not included. [22] 


\subsection{Meson Production in Nuclear Targets}

The neutrino and muon fluxes come from the decay of pion and kaon mesons produced in $\mathrm{p}+\mathrm{A}$ collisions within the NuMI target. Predicting the neutrino and muon fluxes requires knowledge of the underlying production of mesons off of the target. Numerous experiments have been performed which specifically studied hadron production in nuclear targets $[50,51,52,53,54]$. For a variety of reasons, none are directly applicable to the NuMI beam. ${ }^{1}$ In some cases, experiments only measure the production of mesons at discrete points in $\left(p_{T}, p_{z}\right)$ space and do not cover the full production spectra of pions and kaons relevant for production from the NuMI target. Figure 4.4 shows the transverse $p_{T}$ vs. longitudinal $p_{z}$ momentum of $\pi^{+}$that contribute neutrinos in the MINOS Detector for the LE010/180kA beam configuration. The overlaid points, which represent hadron production measurements made by the experiments listed in the figure, sparsely cover the phase space of the NuMI beam.

Furthermore, none of these experiments were performed at $p_{\text {beam }}=$ $120 \mathrm{GeV} / c$ on a graphite targe, requiring scaling in beam energy and target nucleus. Further discussion of these effects can be found in Ref [22]. Significantly, the experiments also used thin targets which were less than $1 / 5$ th of an interaction length long compared to the NuMI target at 2 interaction lengths. This is important because reinteractions of particles reduce the energy of mesons that escape the target thus reducing the energy of the neutrino and muon flux. A Monte Carlo study using Fluka [48] particle production models predicts that $\sim 30 \%$ of $\pi^{+}$with momentum above $5 \mathrm{GeV}$ come from

\footnotetext{
${ }^{1}$ The only experiment that is directly relevant for NuMI is the MIPP experiment [55] which made hadron production measurements from a NuMI target. However, these measurements were not available at the time of this analysis and thus, were not used.
} 


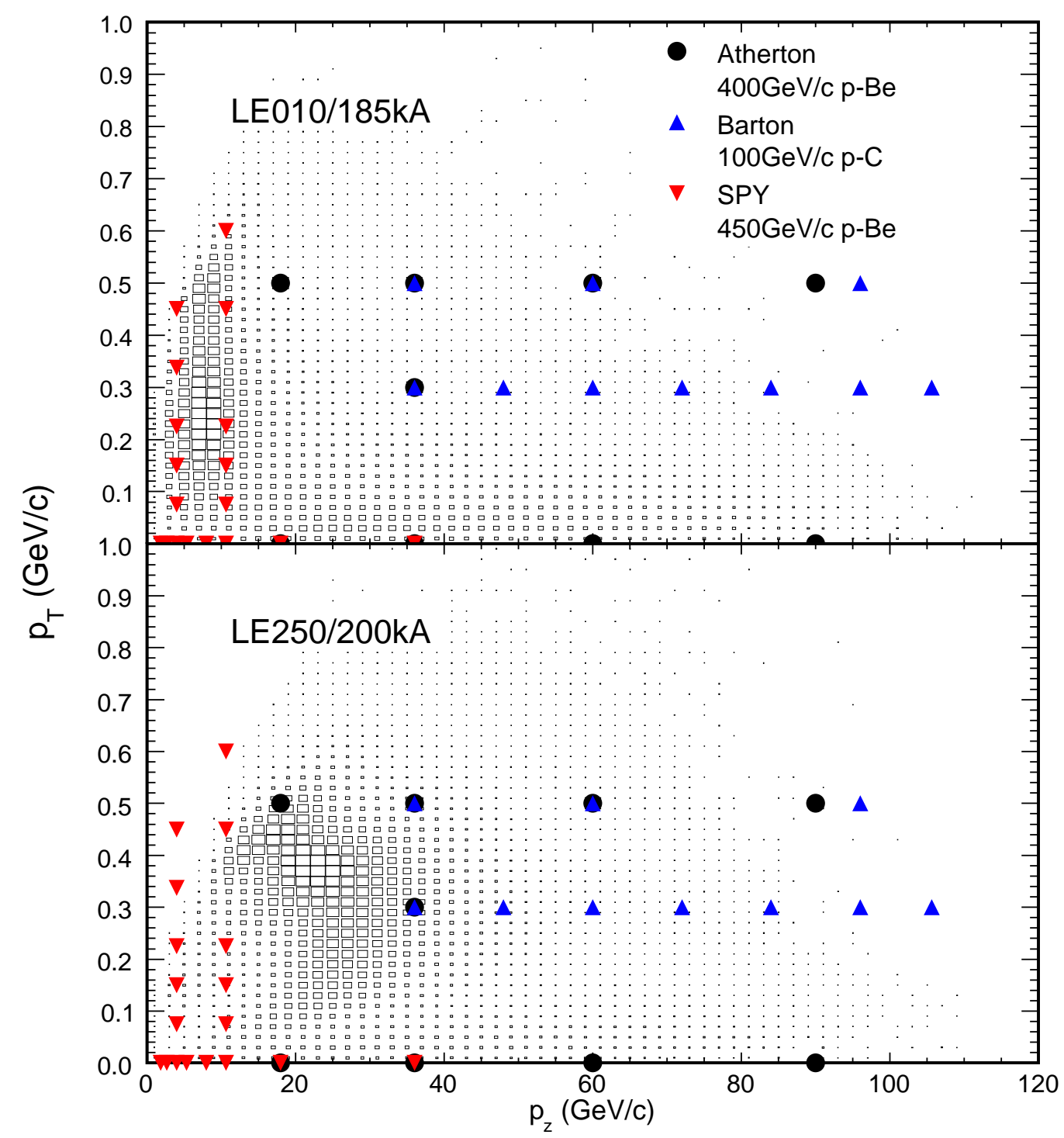

Figure 4.4: The transverse $p_{T}$ vs. longitudinal $p_{z}$ momentum of $\pi^{+}$that contribute neutrinos in the MINOS Detector for the LE010/185kA beam configuration. Overlaid are points for which particle production measurements have been made by the listed experiments [50], [51] and [52]. [22] 
reinteractions within the NuMI target [22].

The data from hadron production experiments are used to develop empirical models of hadron production used in Monte Carlo simulations [56, 48, $57,49,26]$. These models simulate the interaction cascade of particles, thus accounting for reinteractions within long targets. However, simulations of interactions within the NuMI target using several of these models shows that they vary significantly in their predictions of meson production as shown in Figure 4.5 .

Alternatively, the data from hadron production experiments are parameterized analytically in terms of the phase space variables $\left(p_{T}\right.$ and $x_{F}[58,59]$. Note that $x_{F}=2 p_{z}^{*} / \sqrt{s}$ is the Feynman scaling variable [60], where $p_{z}^{*}$ and $\sqrt{s}$ are the longitudinal momentum of the produced particle and the total energy, both in the center of momentum frame. It allows particle production to be quantitatively described in the most simple way possible and allows data collected with different initial proton beam momenta to be compared. Under two conditions, the produced particle is ultrarelativisic, $E \gg m$, and it's divergence is very small, $p_{z} \gg p_{T}$, the Feynman scaling variable can be expressed as $x_{F} \simeq p_{z} / p_{0}$, where $p_{0}$ is the momentum of the incident particle [61, 62]. Both of these conditions hold for pions and kaons contributing to the NuMI neutrino and muon beam, thus when referring to meson production off of the NuMI hadron production target $x_{F}$ and $p_{z}$ will be used interchangeably with the implicit relation $x_{F}=p_{z} / 120 \mathrm{GeV}$ for the NuMI beam.

The MINOS Monte Carlo simulation of particle interactions in the NuMI target implements Fluka05 [48] particle production models. Figures 4.6 and 4.7 show the yield, $d^{2} N / d p_{T} d x_{F}$, of $\pi^{+}, \pi^{-}, K^{+}$and $K^{-}$out of the NuMI target as a function of their transverse momentum, $p_{T}$, for specific values of 


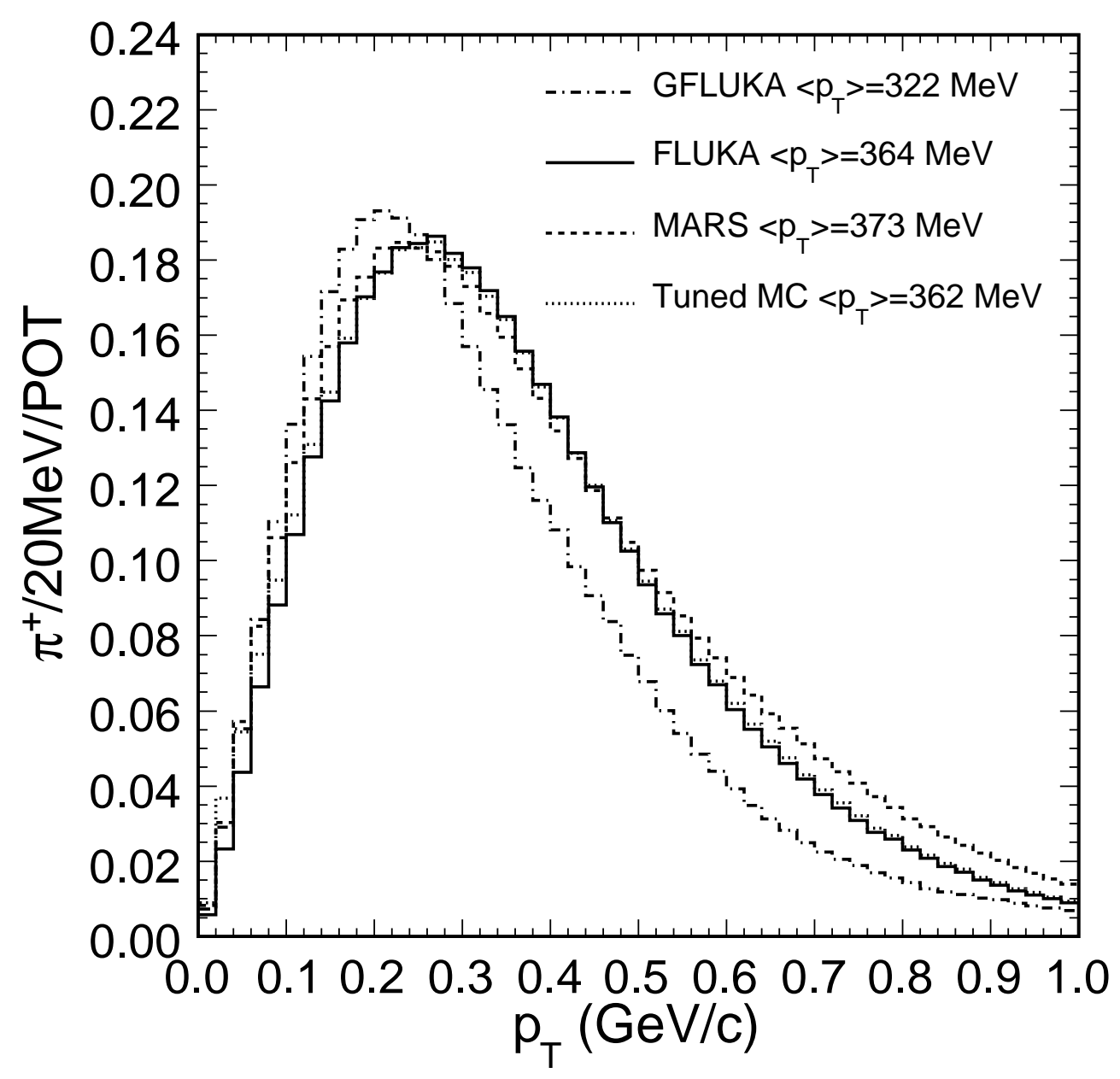

Figure 4.5: The yield of $\pi^{+}$above $p_{z}>0.5 \mathrm{GeV} / c$ from the NuMI target as a function of $p_{T}$ as predicted by various hadron cascade models $[56,48,57]$. The Tuned MC corresponds to a MINOS fit using the MINOS Near Detector neutrino data [22]. 


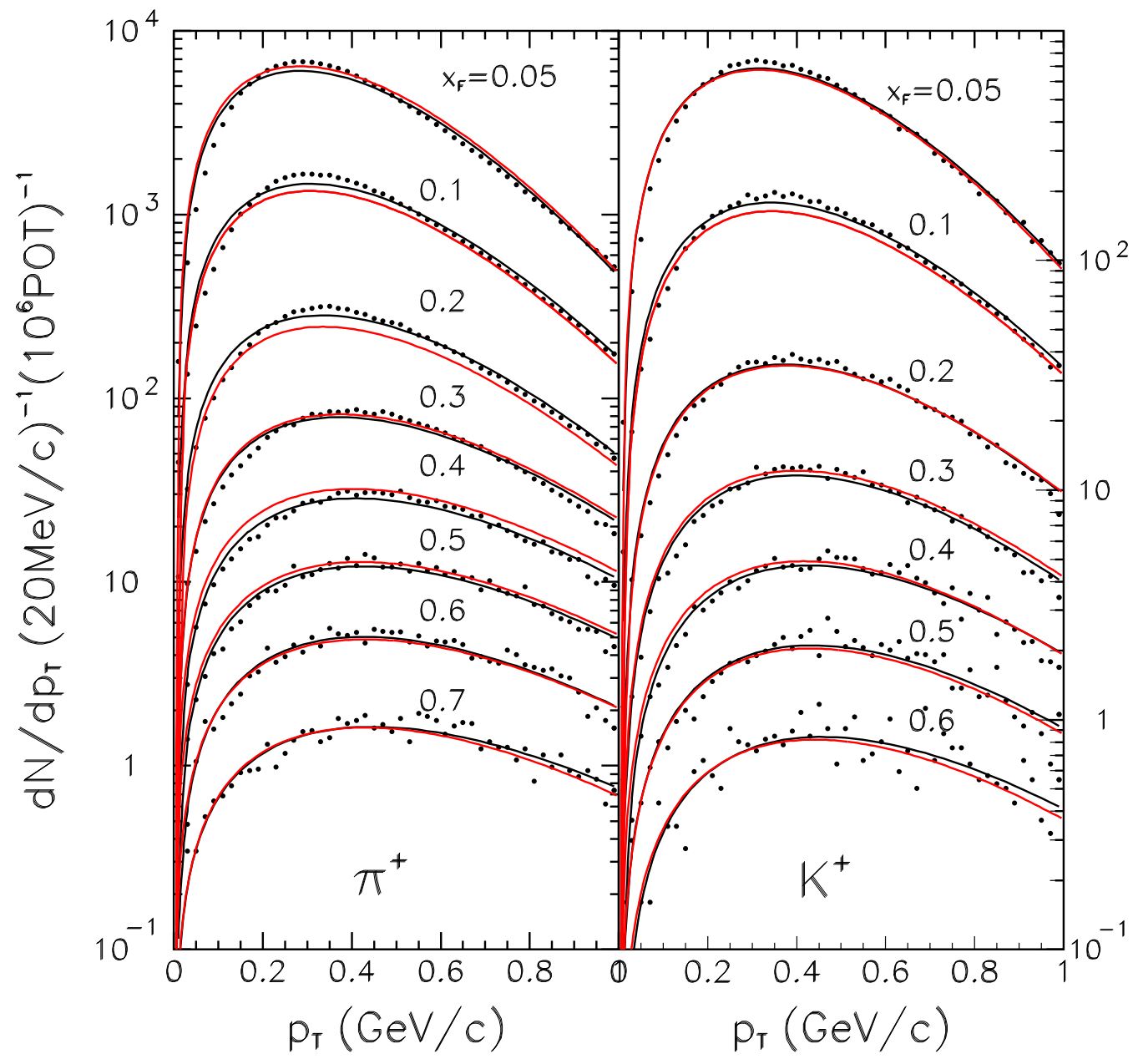

Figure 4.6: The yield of $\pi^{+}$and $K^{+}$from the NuMI target as a function of $p_{T}$ in several $x_{F}$ slices using FLUKA hadron production model (black dots). Black solid line is a fit to a particular $x_{F}$ slice using Equation 4.1 and red line is the overall parameterization using Equation 4.2 [22]. 


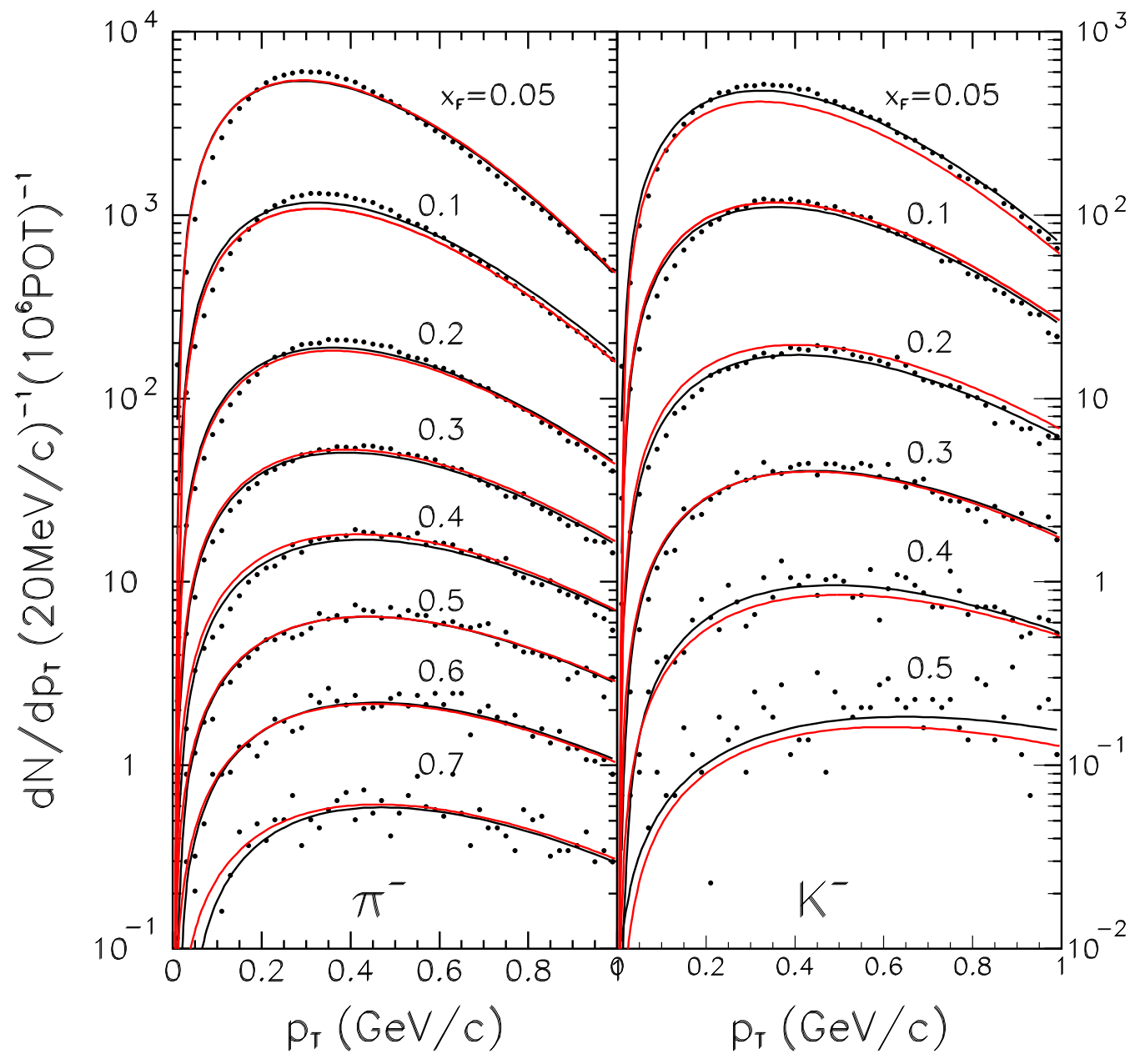

Figure 4.7: The yield of $\pi^{-}$and $K^{-}$from the NuMI target as a function of $p_{T}$ in several $x_{F}$ slices using FLUKA hadron production model (black dots). Black solid line is a fit to a particular $x_{F}$ slice using Equation 4.1 and red line is the overall parameterization using Equation 4.2 [22]. 
$x_{F}$. These Fluka05 yields can also be parameterized in terms of $p_{T}$ and $x_{F}$ similarly to Ref. [58] as [22]:

$$
\frac{d^{2} N}{d x_{F} d p_{T}}=\left[A\left(x_{F}\right)+B\left(x_{F}\right) p_{T}\right] \times \exp \left(-C\left(x_{F}\right) p_{T}^{3 / 2}\right)
$$

This parameterization corresponds to the black curves in Figures 4.6 and Figures 4.7, where each $x_{F}$ curve has a fitted set of $\{A, B, C\}$ constants. A description of how these constants vary as a function of $x_{F}$ is given by [22]:

$$
\begin{aligned}
& A\left(x_{F}\right)=a_{1} \times\left(1-x_{F}\right)^{a_{2}} \times\left(1+a_{3} x_{F}\right) \times x_{F}^{-a_{4}} \\
& B\left(x_{F}\right)=b_{1} \times\left(1-x_{F}\right)^{b_{2}} \times\left(1+b_{3} x_{F}\right) \times x_{F}^{-b_{4}} \\
& C\left(x_{F}\right)= \begin{cases}c_{1} / x_{F}^{c_{2}}+c_{3} & \text { if } x_{F}<0.22 \\
c_{1} / e^{\left(x_{F}-c_{3}\right) c_{2}}+c_{4} x_{F}+c_{5} & \text { otherwise }\end{cases}
\end{aligned}
$$

where the a's, b's and c's are found by plotting the constants A, B, and C as a function of $x_{F}$ and fitting to the resulting curve. $\pi^{+}, \pi^{-}, K^{+}$and $K^{-}$ are fit separately. This procedure is outlined in detail in Ref [22]. Later, in Section 5.4, these functions, $A\left(x_{F}\right), B\left(x_{F}\right)$, and $C\left(x_{F}\right)$ will be modified to fit muon flux Monte Carlo to muon flux data.

Figures 4.8 shows the ratio of the yield of $K^{+}$mesons to $\pi^{+}$mesons predicted by Fluka05. Also shown is the $K^{+} / \pi^{+}$ratio from other particle cascade models. It is seen that these models consistently predict this ratio within $\sim 10 \%$. As a result, while all of the models may vary significantly in individual $\pi$ and $K$ yields, the ratio $K^{+} / \pi^{+}$seems to be consistent. We will use this ratio as a constrain when use the muon monitors to infer the neutrino flux. 


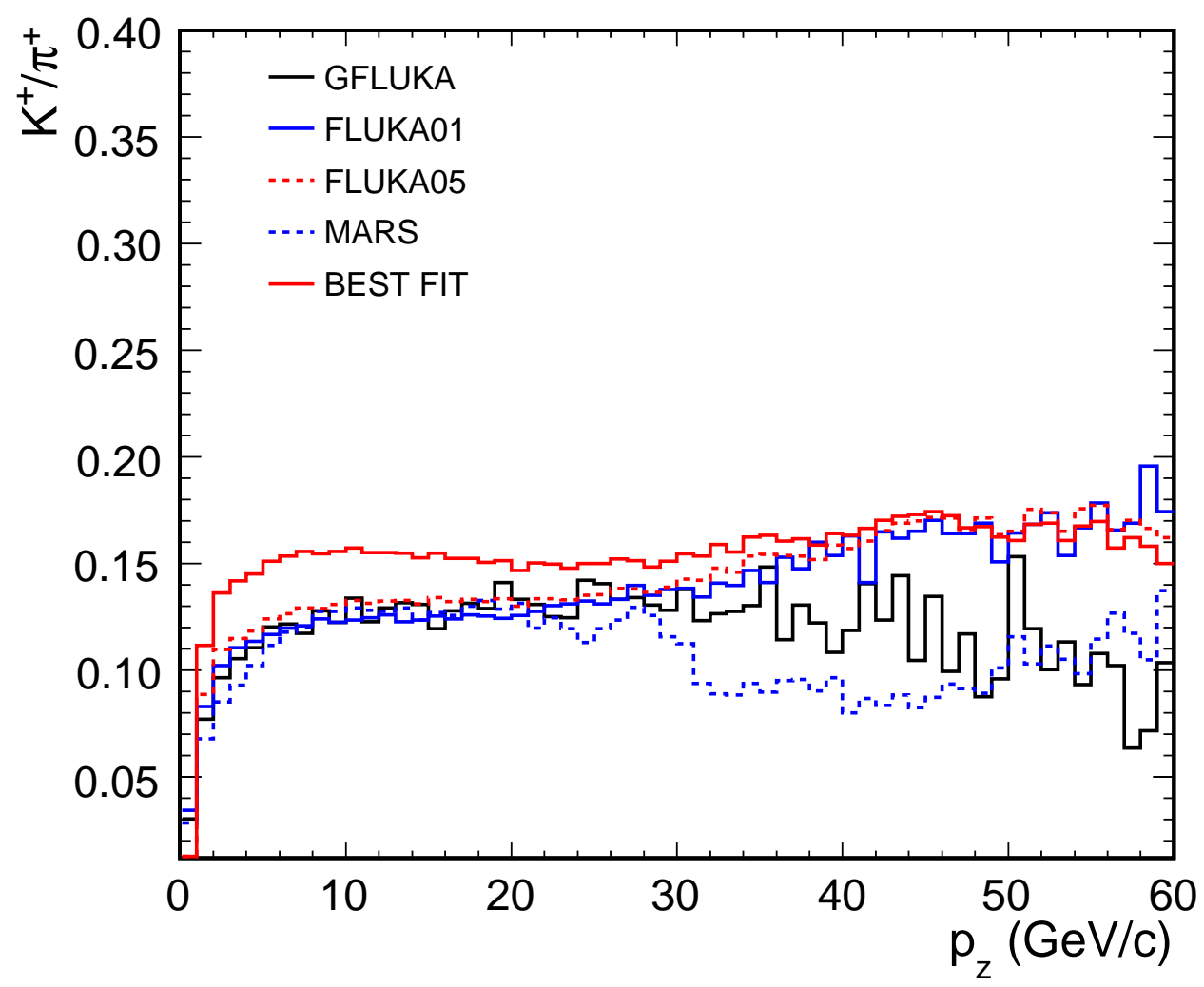

Figure 4.8: The Fluka05 $K^{+} / \pi^{+}$ratio and the $K^{+} / \pi^{+}$from several particle production models, including that obtained from the MINOS Monte-Carlo tuning procedure (labeled "Best Fit"). [22] 


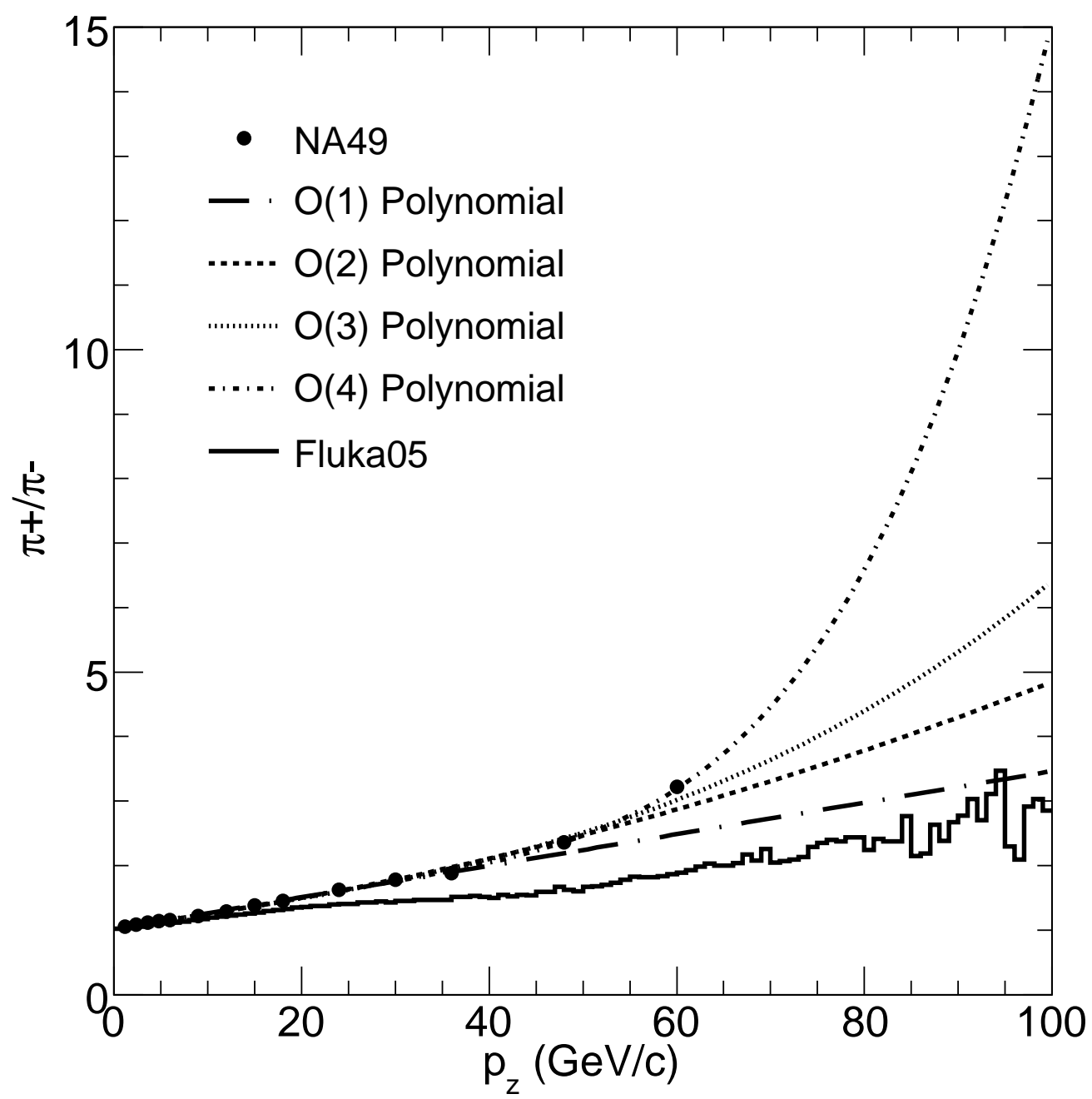

Figure 4.9: The $\pi^{+} / \pi^{-}$ratio. The points are NA49 data. The curves are polynomial fits to the NA49 data. The histogram is the Fluka05 ratio. 
Figure 4.9 shows the ratio of the yield of $\pi^{+}$mesons to $\pi^{-}$mesons predicted by Fluka05. Also shown are data from proton-carbon interactions from the NA49 particle production experiment. The ratios differ significantly. The NA49 experiment did use a $\sim 1 \mathrm{~cm}$ length target, corresponding to $1.5 \%$ of an interaction length. However, a Monte Carlo study of Fluka05 particle yields in a $2 \mathrm{~cm}$ graphite target showed that the predicted $K^{+} / \pi^{-}$ratio was within $5 \%$ of the predicted ratio using the $1 \mathrm{~m}$ long NuMI target.

Figure 4.9 also demonstrates how particle production data sets do not cover the full $\left(p_{T}, p_{z}\right)$ production spectra of pions and kaons relevant for production from the NuMI beam. The NA49 data exist only up to $x_{F}=0.5$, corresponding to $p_{z}=60 \mathrm{GeV}$ for the $120 \mathrm{GeV}$ proton beam energy for NuMI. Thus, in order to utilize this data they must be extrapolated to higher $p_{z}$. The curves are polynomial fits to the NA49 data which will be used when fitting for the flux in Chapters 5 and 6.

\subsection{Meson Decay to Neutrinos and Muons}

The decays that predominantly produce muon flavor neutrinos are $\pi^{ \pm} \rightarrow \mu+\nu_{\mu}$ and $K^{ \pm} \rightarrow \mu+\nu_{\mu}$.

Figure 4.10 shows a schematic of either decay in the lab and center of momentum frames. The four-momenta $(E, \vec{P})$ of the parent meson, $\left(E_{\mu}+\right.$ $\left.p_{\nu}, \vec{p}_{\mu}+\vec{p}_{\nu}\right)$ of the daughter muon and neutrino in the lab frame, and $\left(E_{\mu}+\right.$ $\left.p_{\nu}, 0\right)$ of the daughters in the center of momentum frame are all defined in the figure.

Conservation of momentum requries that the two daughters be emitted back-to-back with a fixed momentum in the center of momentum frame. 


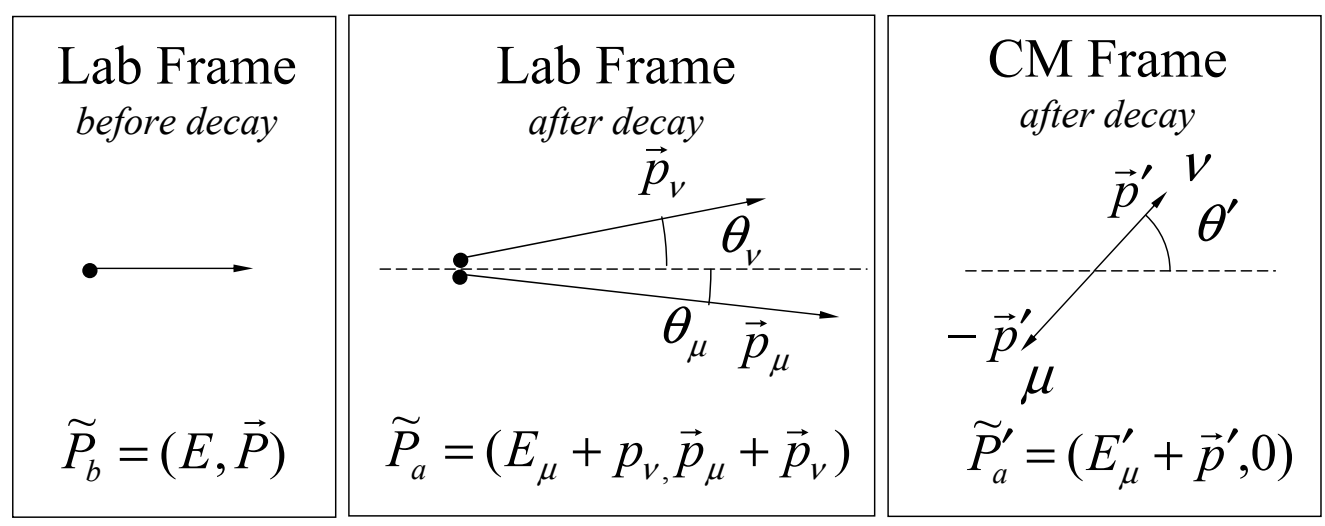

Figure 4.10: Diagram of a 2-body meson decay in the center of momentum and lab frames. The four-momenta $(E, \mathbf{P})$ of the parent meson, $\left(E_{\mu}+p_{\nu}, \mathbf{p}_{\mu}+\mathbf{p}_{\nu}\right)$ of the daughter muon and neutrino in the lab frame, and $\left(E_{\mu}+p_{\nu}, 0\right)$ of the daughters in the center of momentum frame are all defined in the diagram. [23]

Conservation of energy gives the momentum of either as:

$$
p^{\prime}=\frac{m_{m}}{2}\left(1-\frac{m_{\mu}^{2}}{m_{m}^{2}}\right)
$$

where $m_{m}$ is the mass of the $\pi$ or $K$ parent and $m_{\mu}$ is the muon mass. For $\pi(K)$ decay, this gives a momentum of $p^{\prime}=29.8 \mathrm{MeV}(235.6 \mathrm{MeV})$ for both the muon and the neutrino. For neutrinos, $E^{\prime}=p^{\prime}$ and for the muons, $E_{\mu}^{\prime}=\sqrt{\left(p^{\prime}\right)^{2}+m_{\mu}^{2}}=109 \mathrm{MeV}(258 \mathrm{MeV})$ in pion (kaon) decays.

The daughter momenta in the lab are given by the Lorentz transformation:

$$
\begin{gathered}
E=\gamma\left(E^{\prime}+\beta p_{z}^{\prime}\right) \\
p_{z}=\gamma\left(p_{z}^{\prime}+\beta E^{\prime}\right) \\
p_{T}=p_{T}^{\prime}
\end{gathered}
$$


where $\gamma=E_{m} / m_{m}$ is the boost factor, $\beta=\left(1-1 / \gamma^{2}\right)^{1 / 2}$ is the velocity of the center of momentum frame with respect to the lab frame, and $E_{m}$ and $m_{m}$ are the energy of the parent meson in the lab and its mass, respectively.

A relation between the angles $\theta^{\prime}$ and $\theta$ is found by substituting the relations $p_{T}=p \sin \theta, p_{z}=p \cos \theta, p_{T}^{\prime}=p^{\prime} \sin \theta^{\prime}$ and $p_{z}^{\prime}=p^{\prime} \cos \theta^{\prime}$ into the ratio of Equations 4.5 and 4.6 , giving

$$
\gamma \tan \theta=\frac{\sin \theta^{\prime}}{\cos \theta^{\prime}+\left(\beta / \beta^{\prime}\right)}
$$

where $\beta^{\prime}=p^{\prime} / E^{\prime}$ is the daughter velocity in the CM frame. For the neutrino $\beta^{\prime}=1$ allowing this equation to be solved for $\theta^{\prime}$ giving

$$
\cos \theta^{\prime} \approx \frac{1-\gamma^{2} \tan ^{2} \theta_{\nu}}{1+\gamma^{2} \tan ^{2} \theta_{\nu}}
$$

where it is assumed that the parent, $\pi$ or $K$, is very relativistic so the approximation $\beta \approx 1$ can be made. For the case of the NuMI beam which produces pions and kaons with energies of $\mathrm{GeV}$, this is a good approximation for for all relevant pions and for kaons with $\mathrm{E}_{K}>\sim 4 \mathrm{GeV}$.

The neutrino energy in the lab can then be found from Equations 4.4, 4.3 and 4.8 giving

$$
E_{\nu} \approx \frac{2 \gamma p^{\prime}}{1+\gamma^{2} \tan ^{2} \theta_{\nu}}=\frac{\left(1-\frac{m_{\mu}^{2}}{M^{2}}\right) E}{1+\gamma^{2} \tan ^{2} \theta_{\nu}}
$$

In general the angular distribution of daughters is

$$
\frac{d P}{d \Omega}=\frac{d P}{d \Omega^{\prime}} \frac{d \Omega^{\prime}}{d \Omega}=\frac{d P}{d \Omega^{\prime}} \frac{d \theta^{\prime}}{d \theta} \frac{\sin \theta^{\prime}}{\sin \theta}
$$


Since pions and kaons are spin zero, the angular distribution of daughters in the center of momentum frame is isotropic

$$
\frac{d P}{d \Omega^{\prime}}=\frac{1}{4 \pi}
$$

Using this and Equation 4.8 gives the neutrino distribution

$$
\frac{d P}{d \Omega_{\nu}} \approx \frac{1}{4 \pi} \frac{4 \gamma^{2}\left(1+\tan ^{2} \theta_{\nu}\right)^{3 / 2}}{\left(1+\gamma^{2} \tan ^{2} \theta_{\nu}\right)^{2}}
$$

Equations 4.9 and 4.12 can then be used to decay the pions and kaons in the decay volume producing neutrinos that intercept the MINOS detector. A randomized decay in Monte Carlo simulation can produce a neutrino at an angle outside of the solid angle of the MINOS detector. This makes it inefficient to randomly decay the mesons in the Monte Carlo. Instead the mesons are forced to decay such that the neutrino intercepts the MINOS detector. A probability is assigned to the decay using Equation 4.12 and the neutrino energy is then given by Equation 4.9 .

Because $\beta^{\prime}$ for muons is 0.27 or 0.91 for $\pi$ and $K$ decays respectively, Equation 4.8 does not provide a straightforward analytic solution in terms of $\theta_{\mu}$. Also, because muons will be affected by multiple scattering in the rock and the muon monitors are pixelated, it is most efficient to determine the probability that a decay will produce a muon that intercepts the end of the decay volume. As such the probability that a decay of will lead to a muon at the end of the decay volume is performed by numerical integration as

$$
\int \frac{d P}{d \Omega^{\prime}} d \Omega^{\prime} \approx \sum_{i=1}^{\mathrm{N}} \frac{d P}{d \Omega^{\prime}} \Delta \Omega_{i}^{\prime}=1
$$




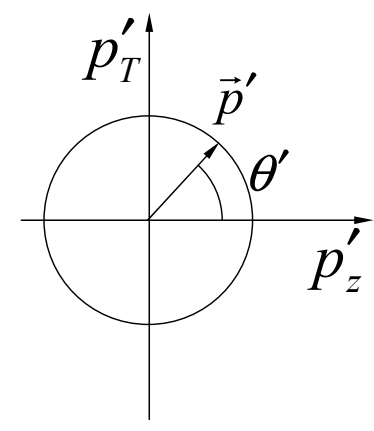

CM Frame

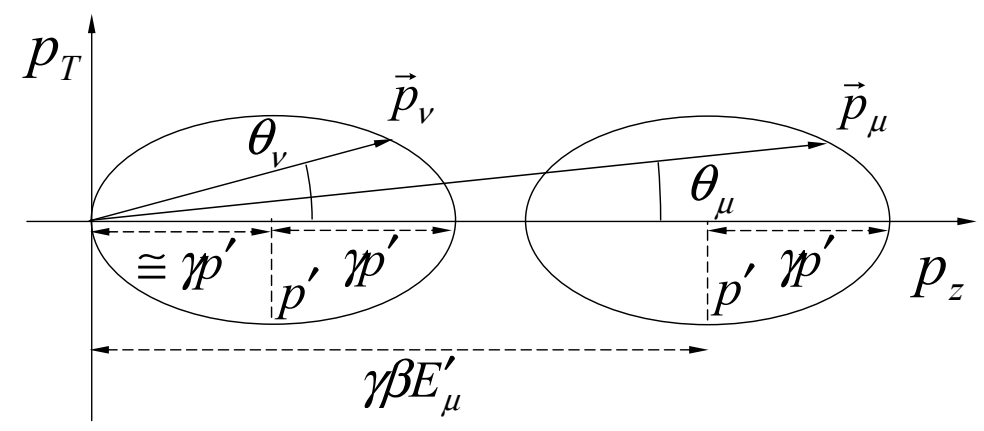

Laboratory Frame

Figure 4.11: The Lorentz transformation of a neutrino and muon momentum vector from the center of momentum frame to the lab frame. In the center of momentum frame the momentum may lie anywhere on a circle whereas in the lab it lies on an ellipse (Equation 4.15). For $\beta \approx 1$ parents as sketched above, the neutrino ellipse intercepts the origin and the muon mass causes the the muon ellipse to be more forward boosted. This diagram represents the decay $\pi \rightarrow \mu \nu$ and so the muon energy in the lab always larger than the neutrino energy. However, for $K \rightarrow \mu \nu$ decays the neutrino and muon ellipses overlap. [23]

Each meson is decayed $\mathrm{N}$ times, where $\mathrm{N}$ can be chosen to accurately sample the distribution but also limit computing resources, producing a muon with a probability weight to intercept the end of the decay volume. Further details of the implementation can be found in [22].

It is possible, however, to develop a picture of what to expect by examining the vector transformation of the momenta from the center of momentum frame into the lab frame. The basic vector relation

$$
p^{2}=p_{T}^{\prime 2}+p_{z}^{\prime 2}
$$

says that the momentum vectors in the center of momentum lie on a circle as shown in Figure 4.11. The corresponding relation in the lab is found by 
substitution of Equations 4.5 and 4.6, giving:

$$
\frac{\left(p_{z}-\beta \gamma E^{\prime}\right)^{2}}{\gamma^{2} p^{\prime 2}}+\frac{p_{T}^{2}}{p^{\prime 2}}=1
$$

which is the equation of an ellipse in the lab centered at $p_{z}=\gamma \beta E^{\prime}$ with semimajor and semi-minor axes of $\gamma p^{\prime}$ and $p^{\prime}$, respectively. Pions with momenta $\gtrsim 1 \mathrm{GeV}$ and kaons $\gtrsim 4 \mathrm{GeV}$ are very relativistic so the approximation $\beta \approx 1$ can be made. For neutrinos $E^{\prime}=p^{\prime}$, so the ellipse approximately intercepts the origin as in Figure 4.11. However, the large muon mass shifts the ellipse to the right.

The maximum and minimum momentum occur when the neutrino or muon is emitted at $\theta^{\prime}=0$ and $180^{\circ}$. Figure 4.11 shows that, for the neutrino, this corresponds to 0 to $2 \gamma p^{\prime}$ which is $0.43 \mathrm{E}_{\pi}$ for pion parents and $0.96 \mathrm{E}_{K}$ for kaon parents.

The energy distribution of neutrinos $\left(\beta_{\nu}=1\right)$ in the lab is a constant as can be shown by the chain rule,

$$
\frac{d P}{d E_{\nu}}=\frac{d P}{d \Omega_{\nu}} \frac{d \Omega_{\nu}}{d E_{\nu}}
$$

and the constant is found to be

$$
\frac{d P}{d E_{\nu}}=\frac{1}{\left(1-\frac{m_{\mu}^{2}}{m_{m}^{2}}\right) E_{m}}
$$

where

$$
0<\mathrm{E}_{\nu}<0.43 \mathrm{E}_{\pi} \quad \text { for } \pi \rightarrow \mu \nu
$$

$$
0<\mathrm{E}_{\nu}<0.96 \mathrm{E}_{K} \quad \text { for } K \rightarrow \mu \nu
$$

This implies that the muon energy distribution must also be constant and 
equal to the neutrino distribution, and by energy conservation in the lab

$$
\frac{d P}{d E_{\mu}}=\frac{1}{\left(1-\frac{m_{\mu}^{2}}{m_{m}^{2}}\right) E_{m}}
$$

where

$$
\begin{array}{ll}
0.57<\mathrm{E}_{\mu}<\mathrm{E}_{\pi} & \text { for } \pi \rightarrow \mu \nu \\
0.04<\mathrm{E}_{\mu}<\mathrm{E}_{K} & \text { for } K \rightarrow \mu \nu
\end{array}
$$

This is demonstrated in Figure 4.12. This is an important fact because it says that, although a muon from a $4 \mathrm{GeV}$ pion which produces a forward going neutrino that intercepts the MINOS detector will not have enough energy to penetrate the shielding and intercept the muon monitors, there are just as many decays in which a $4 \mathrm{GeV}$ pion produces a forward going muon that can intercept the monitors.

Furthermore, from Figure 4.11 it is evident that there exists a maximum angle that the neutrino and muon may diverge from the parent direction. This occurs when the momentum vector in the lab is tangent to the lab ellipse. From the figure it can be seen that this maximum angle for the neutrino is $\pi / 2$. The equation defining this angle is

$$
\gamma^{2} \tan ^{2} \theta \max =\frac{\left(\beta^{\prime}\right)^{2}}{\beta^{2}-\left(\beta^{\prime}\right)^{2}}
$$

which gives for the muon, again taking $\beta=1$,

$$
\theta_{\max }=\left\{\begin{aligned}
0.28 / \gamma & \text { for the } \mu \text { from } \pi \rightarrow \mu \nu \\
2.2 / \gamma & \text { for the } \mu \text { from } \mathrm{K} \rightarrow \mu \nu
\end{aligned}\right.
$$

This means that the muon is more forward boosted than the neutrino 

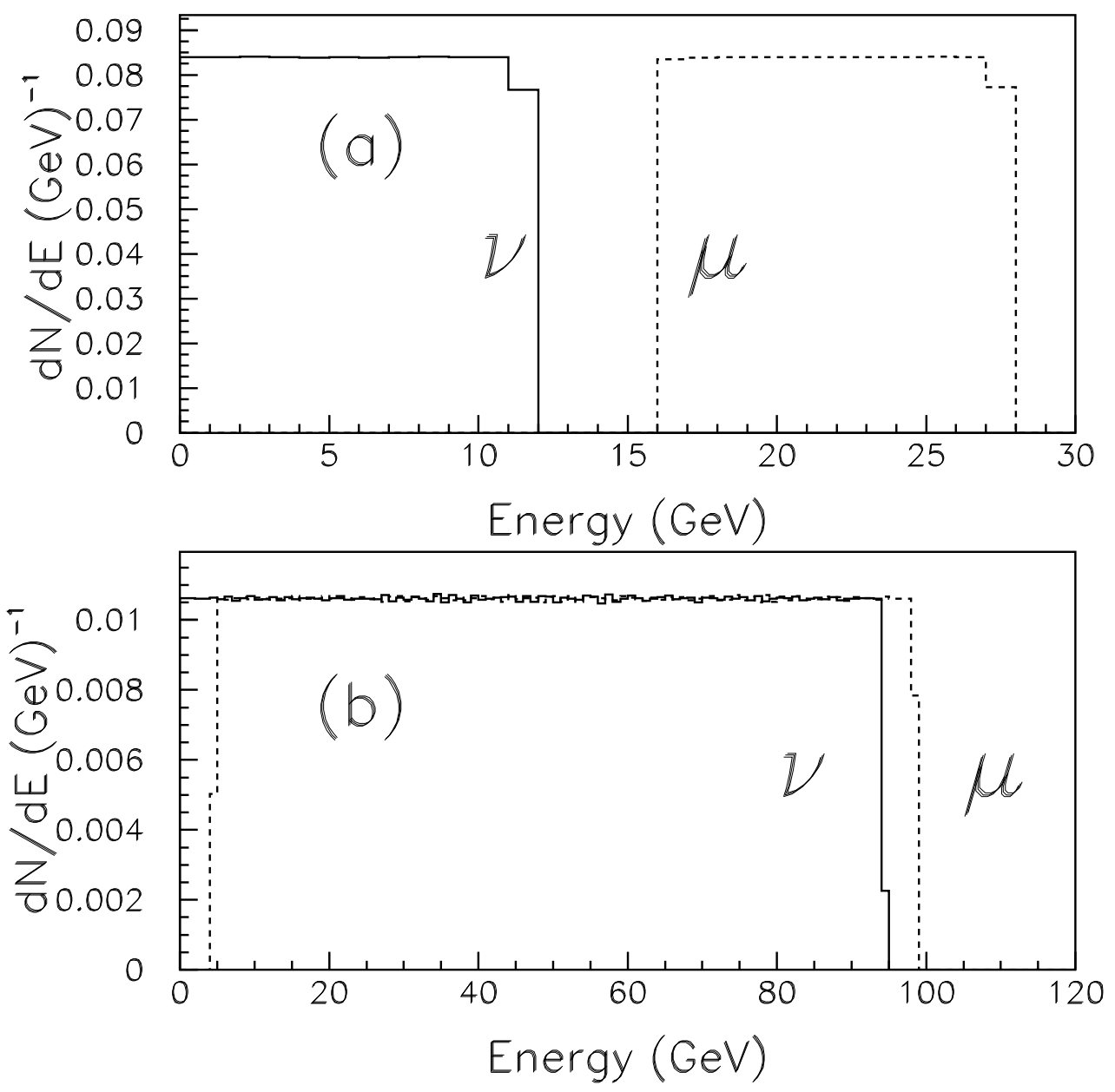

Figure 4.12: The energy distribution of the muon(dashed line) and neutrino(solid line) in meson decays with $\gamma=200$. (a) $\pi \rightarrow \mu \nu$ (b) $K \rightarrow \mu \nu$. [22] 

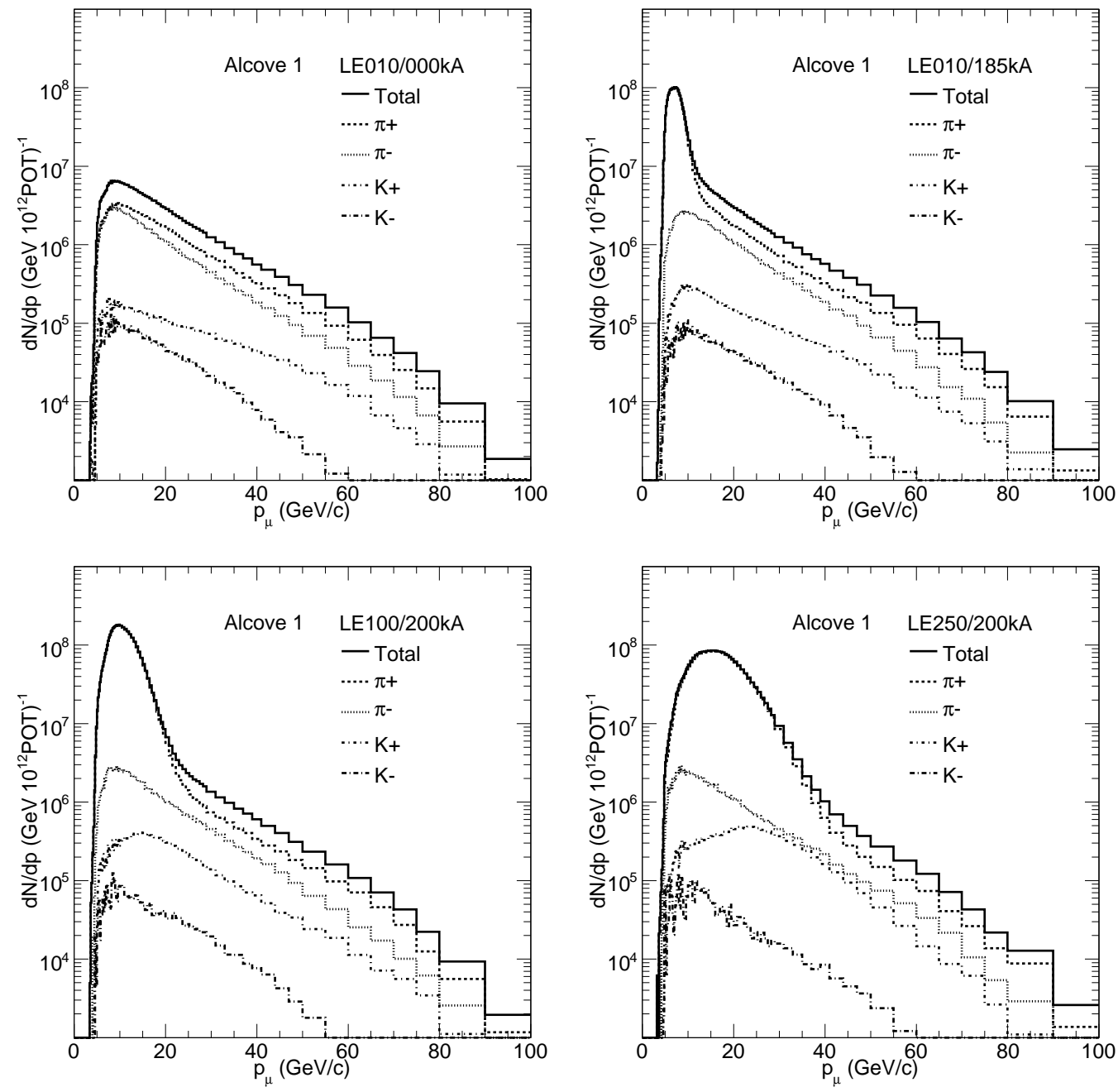

Figure 4.13: Distribution of momenta of $\mu^{ \pm}$arriving in Alcove 1 in the horn off (top left), LE10/185kA (top right), LE100/200kA (bottom left) and LE250/200kA (bottom right) beam configurations. The spectra by parent type are also shown. 

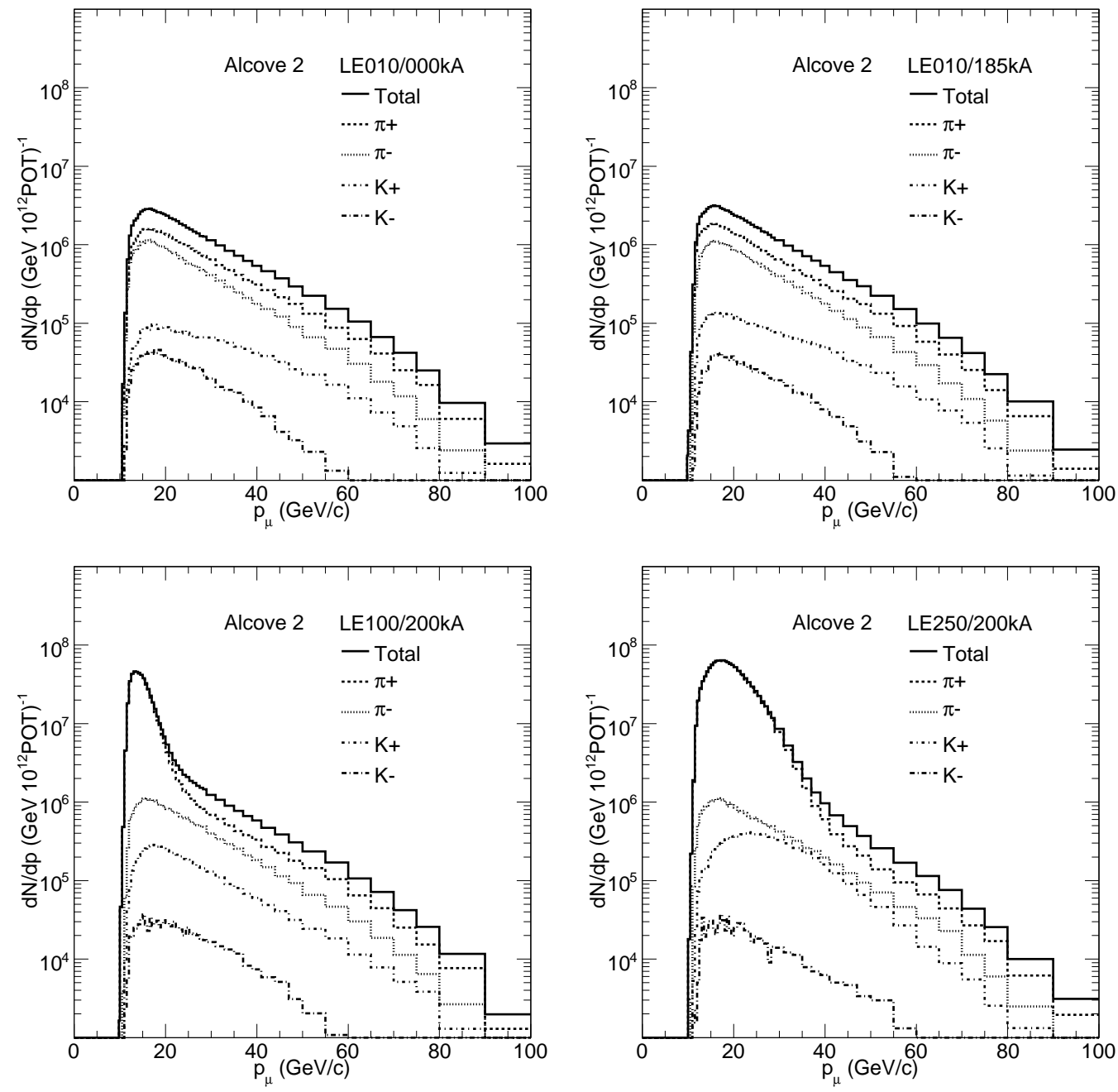

Figure 4.14: Distribution of momenta of $\mu^{ \pm}$arriving in Alcove 2 in the horn off (top left), LE10/185kA (top right), LE100/200kA (bottom left) and LE250/200kA (bottom right) beam configurations. The spectra by parent type are also shown. 

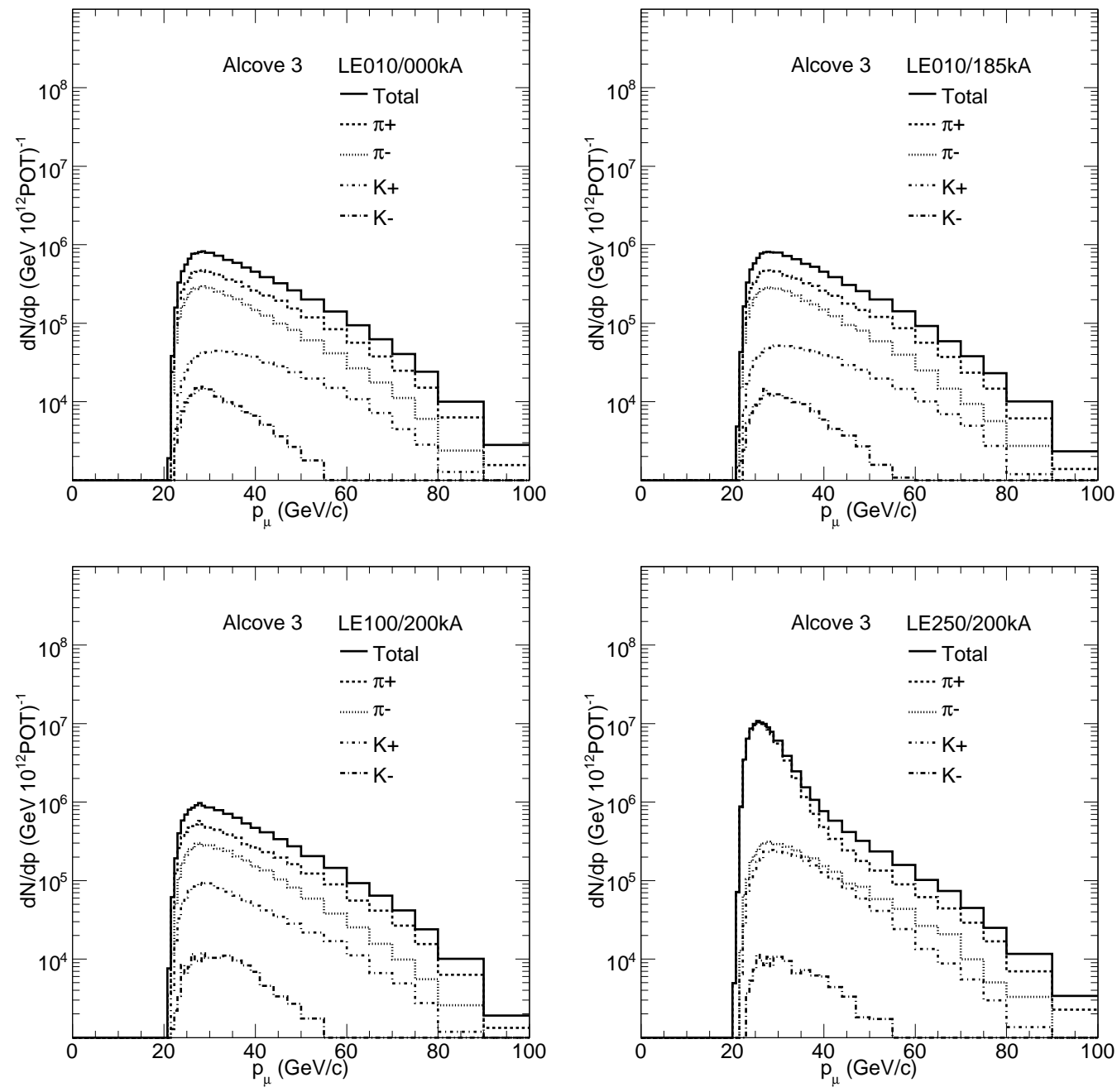

Figure 4.15: Distribution of momenta of $\mu^{ \pm}$arriving in Alcove 3 in the horn off (top left), LE10/185kA (top right), LE100/200kA (bottom left) and LE250/200kA (bottom right) beam configurations. The spectra by parent type are also shown. 
and muons from kaons are emitted at much larger angles than muons from pions. Given that, in the worst case scenario, the solid angle of the monitors from the upstream end of the decay pipe is $\sim 1.5 \mathrm{mrad}$ compared to the $10 \mathrm{mrad}$ and $270 \mathrm{mrad}$ maximum divergence of muons from pions and kaons, respectively, the muon monitors have good acceptance to muons from pions, but less acceptance to muons from kaons. This is confirmed in Figures 4.134.15 which show the distribution of momenta of $\mu^{ \pm}$arriving in Alcove 1 in the horn off (top left), LE10/185kA (top right), LE100/200kA (bottom left) and LE250/200kA (bottom right) beam configurations from pions and kaons parents.

Figure 4.16 shows the momentum distribution of muons in the horn off, LE10/185kA, LE100/200kA and LE250/200kA beam configurations confirming that the rock imposes energy thresholds for muons to reach each muon monitor. The uppermost curve in each figure shows the momentum distribution of all muons produced in meson decays in the NuMI decay pipe(volume). The curve labeled "EODP" (end of the decay pipe) shows the distribution of these muons emitted at sufficiently small angles to reach the end of the decay pipe. The curves labeled by Alcoves 1-3 represent the subset of the EODP muons that penetrate the rock and shielding to reach the monitors. These last three curves employ a Monte Carlo simulation of muons traversing the shielding and rock which is described in the next section. 

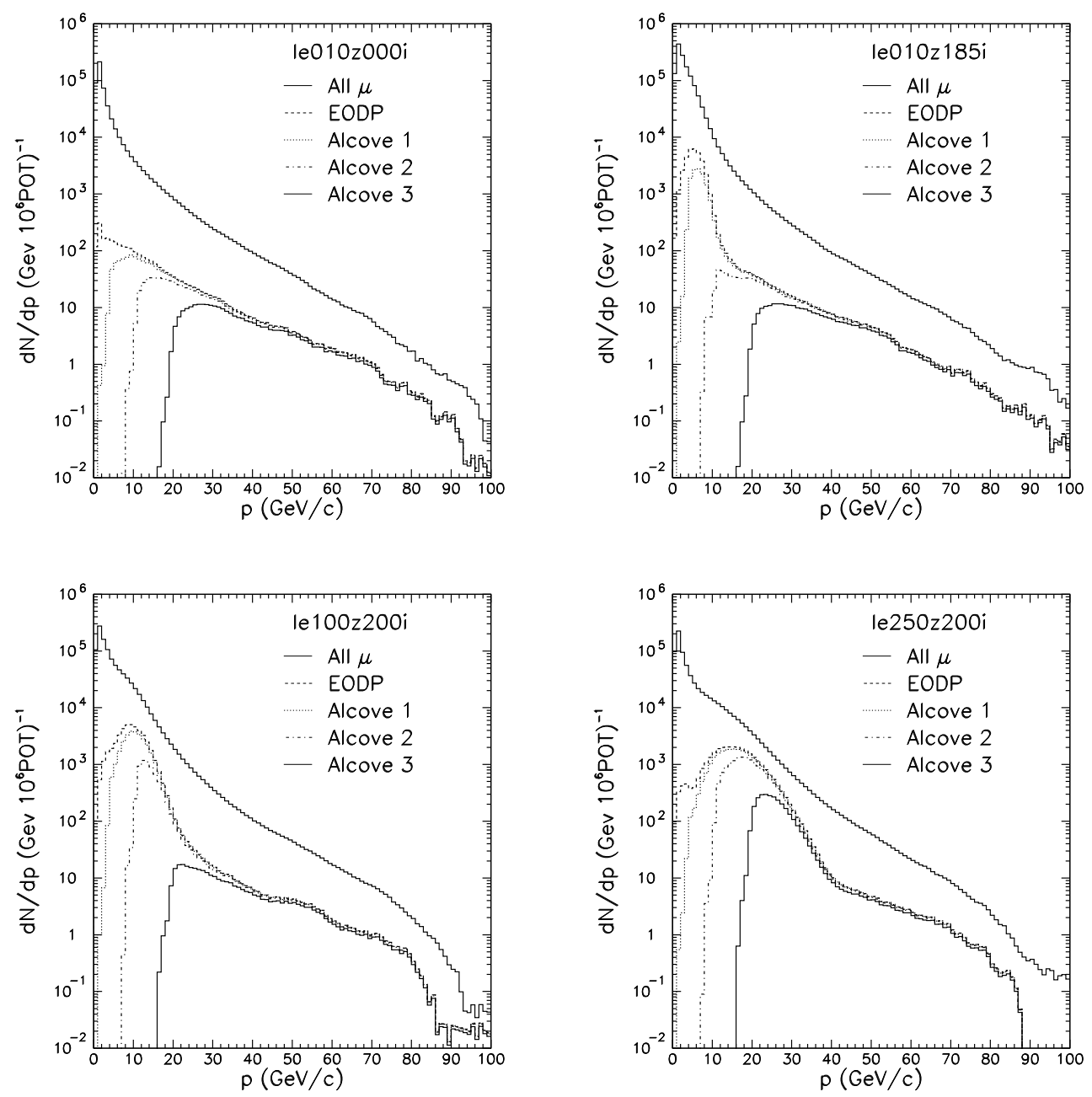

Figure 4.16: Distribution of momenta of muons $\left(\mu^{+}\right.$and $\left.\mu^{-}\right)$in the horn off (top left), LE10/185kA (top right), LE100/200kA (bottom left) and LE250/200kA (bottom right) beam configurations. Shown are the distribution of muons created at any angle and anywhere in the decay pipe, the distribution of those muons arriving at the end of the decay pipe (upstream of the hadron absorber, and in each of the 3 alcoves. 


\subsection{Muons in the Muon Monitors}

\subsubsection{Simulation of Hadron Absorber and Muon Filter}

A diagram of the downstream region of the NuMI beamline which includes the hadron absorber and muon monitor alcoves is shown in Figure 2.8. As mentioned previously, the muon monitors sit in the muon alcoves downstream of the hadron absorber and measure the flux of muons produced along with the neutrinos in meson decay. In order to predict the muon flux reaching the muon monitors an accurate geometrical description of the NuMI hadron absorber and the muon filter is implemented in the Geant4 based Monte Carlo.

The probability for a given muon to penetrate the absorber and subsequent rock to intercept the muon monitors is highly dependent upon the type of material as well as the amount of material traversed by the muon. The development of the $\mathrm{MC}$ involves understanding the precise locations and material composition of the components of the hadron absorber and muon alcoves. This work was performed in Ref [29] but is summarized here.

A diagram of the hadron absorber geometry in the Monte Carlo is shown in Figure 2.7. The hadron absorber is a $18 \times 18.5 \times 28 \mathrm{ft}^{32}$ box composed of aluminum, steel and concrete blocks. There are three layers of blocks extending radially outward from the centerline.

The inner most layer, the inner core, is composed of 8 aluminum and 10 steel blocks with dimensions $0.51 \times 0.51 \times 15.6 \mathrm{ft}^{3}$ centered on the centerline of the beam. The blocks are aluminum and iron alloys which each contain $\sim 2 \%$ by weight impurities of at least 5 other elements. The blocks were chosen such that there is very little variation in the density between the 8 aluminum and,

\footnotetext{
${ }^{2}$ The dimensions are given as horizontal $\times$ vertical $\times$ longitudinal as seen by the beam.
} 
separately, the 10 steel blocks. Numerous water cooling pipes run through longitudinal channels drilled into the inner core blocks.

There is an outer core layer of $0.52 \times 0.52 \times 26 \mathrm{ft}^{3}$ steel blocks surrounding the inner core on all sides. These blocks are made from reclaimed steel and iron and the tolerances in manufacturing were not stringent contributing to uncertainty of $\sim 0.16 \mathrm{~g} / \mathrm{cm}^{3}$ in the density and up to $1.2 \mathrm{~cm}$ variations in surface flatness. These variations allow gaps between adjacent blocks. Furthermore a vertical gap of $1.5 \mathrm{~cm}$ exists between the inner and outer core blocks in which steel slabs of the same thickness were inserted. However, the extent of these slabs is uncertain a may allow for a void where muons may pass unimpeded by matter. The upper right muon monitor beam profile of Figure 3.9 may show indications of the gaps by the slightly higher signals on either side of the center.

The outermost layer is composed of various sized, rebar reinforced, concrete blocks which are modeled as one $3 \times 15 \times 21 \mathrm{ft}^{3}$ rebar reinforced slab placed on all sides and on the downstream end rotated at $90^{\circ}$ of the core. More details of the hadron absorber material and construction and associated uncertainties can be found in Ref. [29].

The composition and dimensions of the rock between the muon alcoves is particularly difficult to understand. From geological surveys, it is known that the rock at the level of the muon alcoves is Dolomite and Maquoketa Shale. A core sample of the rock at several subterranean levels on the Fermilab site was taken very near the location of the of the muon alcoves. From this sample, the density of the rock was found to be $2.78 \pm 0.04 \mathrm{~g} / \mathrm{cm}^{3}$. But the analysis of the samples was performed some time after they were drilled and the water content in these samples may not reflect the actual water content in the rock. 
Furthermore, the longitudinal distance of rock between muon alcoves 1 and 2 is $11.7 \pm 0.027 \mathrm{~m}$ and between 2 and 3 is $18.1 \pm 0.15 \mathrm{~m}$, however there is added uncertainty in these numbers due to the fact that there is a $\sim 10 \mathrm{~cm}$ layer of Shotcrete, concrete sprayed through a hose high velocity, on the upstream and downstream faces of the alcoves. More details can be found in Ref. [29].

This geometry and material composition information used to simulate the hadron absorber and muon filter. As discussed in Section 4.3 pions and kaons are decayed producing muons and the muons are traced to the end of the decay volume. The list of muons at the end of the decay volume is input into the Geant 4 Monte Carlo. The muons are tracked through the hadron absorber and muon filter by Geant4, losing energy and ranging out in the filter.

Figure 4.17 shows the efficiency for muons to reach each muon monitor from the end of the decay pipe as a function of muon momentum at the end of the decay pipe. The Monte Carlo predicts the thresholds for muons to reach alcove 1, 2 and 3 are $4.2,11$, and $21 \mathrm{GeV} / c$, respectively, as shown in Figure 4.17. The active area of a monitor is just under $10 \%$ of the total $2.2 \mathrm{~m} \times 2.2 \mathrm{~m}$ area of the monitor array. Thus the maximum efficiency is near 0.1. The gray bands in Figure 4.17 show how the efficiency changes if when increasing and decreasing the amount of rock between the muon alcoves, the density of the rock and the density of the absorber outer core blocks by the uncertainty in these values as discussed above.

\subsubsection{Ionization by Muons}

As muons are transported through the rock and air surrounding the muon monitors they undergo physics processes as determined by Geant4. One of 


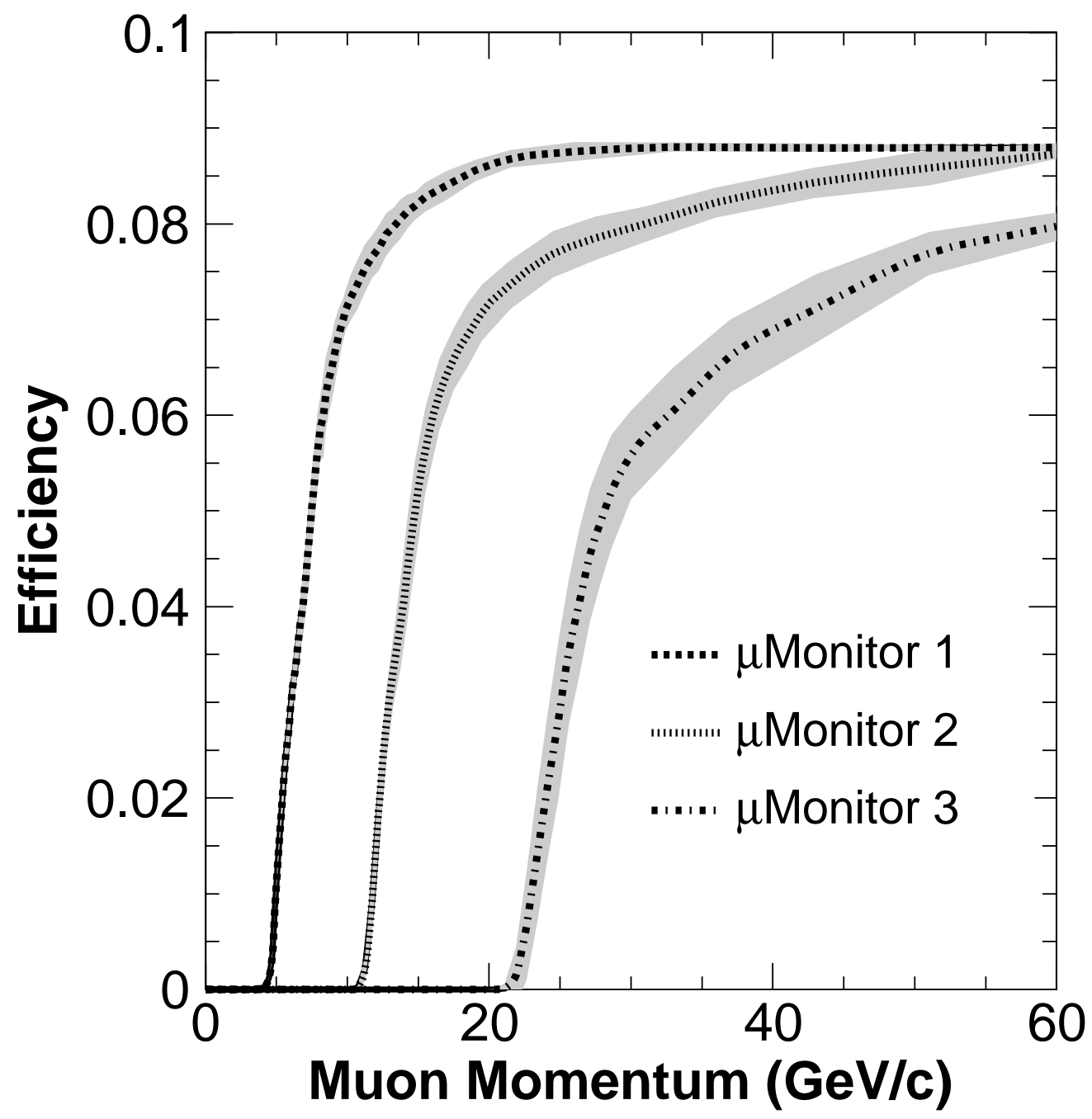

Figure 4.17: The efficiency for muons starting at the downstream end of the decay volume just before the hadron absorber to reach Muon Monitors 1, 2, and 3 as a function of the muon momentum at the end of the decay pipe. It can be seen that the threshold for for muons to reach alcove 1, 2 and 3 are 4.2, 11 , and $21 \mathrm{GeV} / c$, respectively. Because the active area of a monitor is just under $10 \%$ of the total $2.2 \mathrm{~m} \times 2.2 \mathrm{~m}$ area of the monitor array, the maximum efficiency is $\sim 0.1$. 
those physics processes is energy loss through the production of $\delta$-ray electrons $^{3}$. Muon Monitor 1 is directly downstream of the absorber with a $7 \mathrm{~m}$ air gap between the absorber and the monitor. Muon Monitors 2 and 3 are directly downstream of several meters of rock with a $1.3 \mathrm{~m}$ air gap separating the upstream rock and each monitor. $\delta$-rays produced in the rock and the surrounding air can penetrate the monitor and ionize the helium gas within. Furthermore, interactions of the muon and $\delta$-rays with the monitor itself can produce $\delta$-rays which ionize the helium gas within the chambers contributing to the ionization signal measured by the muon monitors.

The simulation of $\delta$-ray production and subsequent ionization was done using a Geant4 biased Monte Carlo with simplified geometry. This Monte Carlo simulates the 9 aluminum tubes of a muon monitor, as shown in Figure 3.1, as well as the parallel plates of each of the 81 individual ion chambers.

The simulation tracks muons with energies ranging from near 0 to $120 \mathrm{GeV}$ through 0.5 meters of Dolomite rock $^{4}$. Downstream of the rock is $7 \mathrm{~m}$ or $1.3 \mathrm{~m}$ of air for alcove 1 and alcoves 2 and 3 respectively after which the monitor is located. $\delta$-rays that are produced in the material and air surrounding the monitors are classified as "external" $\delta$-rays. Those that are produced in the material of the monitor itself are classified as "internal" $\delta$-rays. Those external $\delta$-rays that intercept the muon monitor tubes and ionization chamber plates can also produce $\delta$-rays. If a $\delta$-ray passes through a chamber gap it can ionize the helium gas and thus contribute to the ionization signal from the muon monitors.

\footnotetext{
${ }^{3} \mathrm{~A} \delta$-ray or knock-on electron is an electron liberated from an atom in the medium by the ionizing muon which carries enough energy to itself intercept the monitor chambers

${ }^{4}$ This is thought to be the the amount of rock necessary to produce $\delta$-rays which can escape the rock and intercept the monitors. All $\delta$-rays produced behind more rock will range out in the rock.
} 

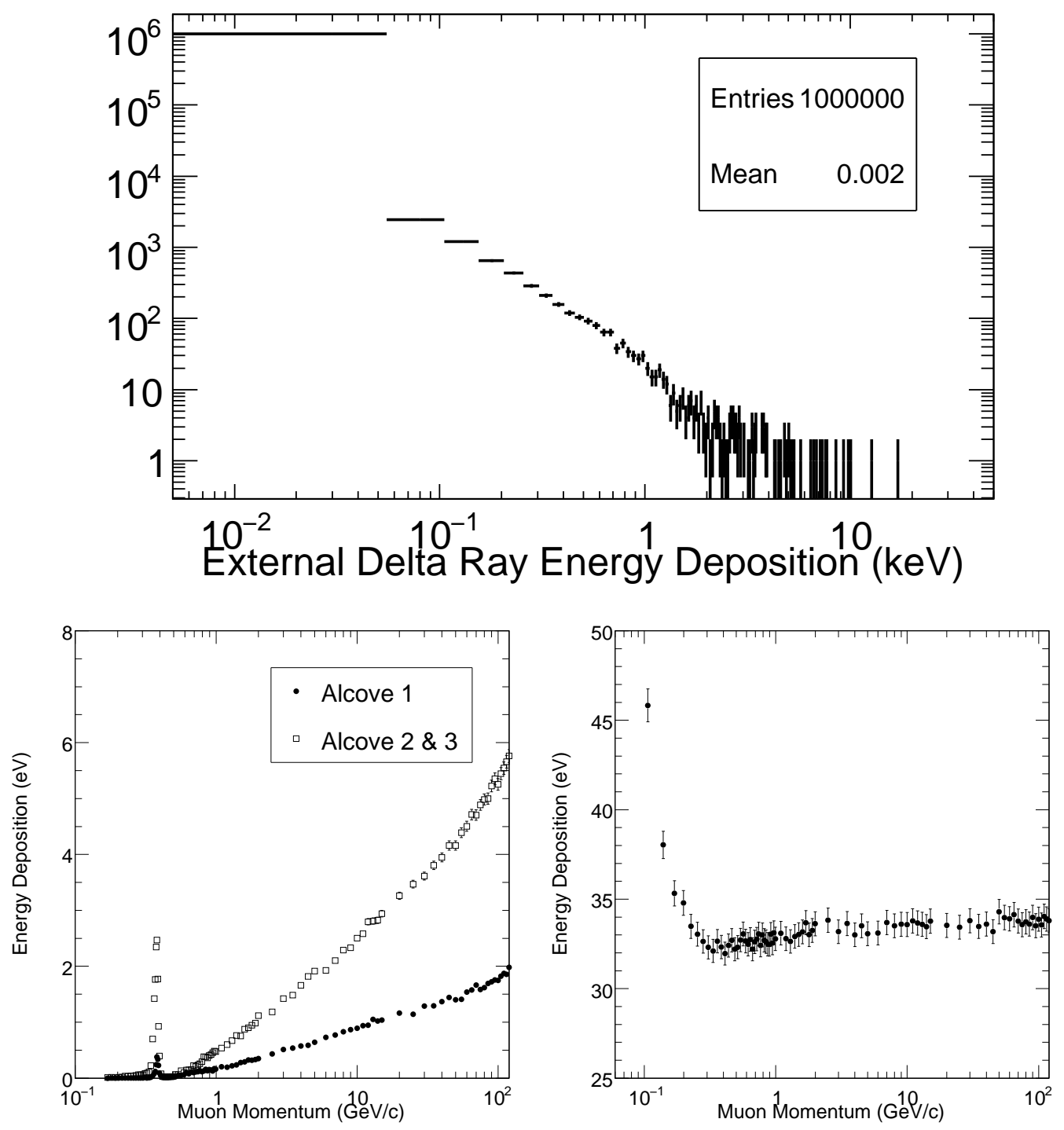

Figure 4.18: Top: Energy deposition from external $\delta$-rays produced from a set of muons which had an initial energy of $5 \mathrm{GeV}$. Bottom Left: The average external $\delta$-ray energy deposition per muon as a function of initial muon momentum. Bottom Right: The average internal $\delta$-ray energy deposition per muon as a function of muon momentum at the monitor. 


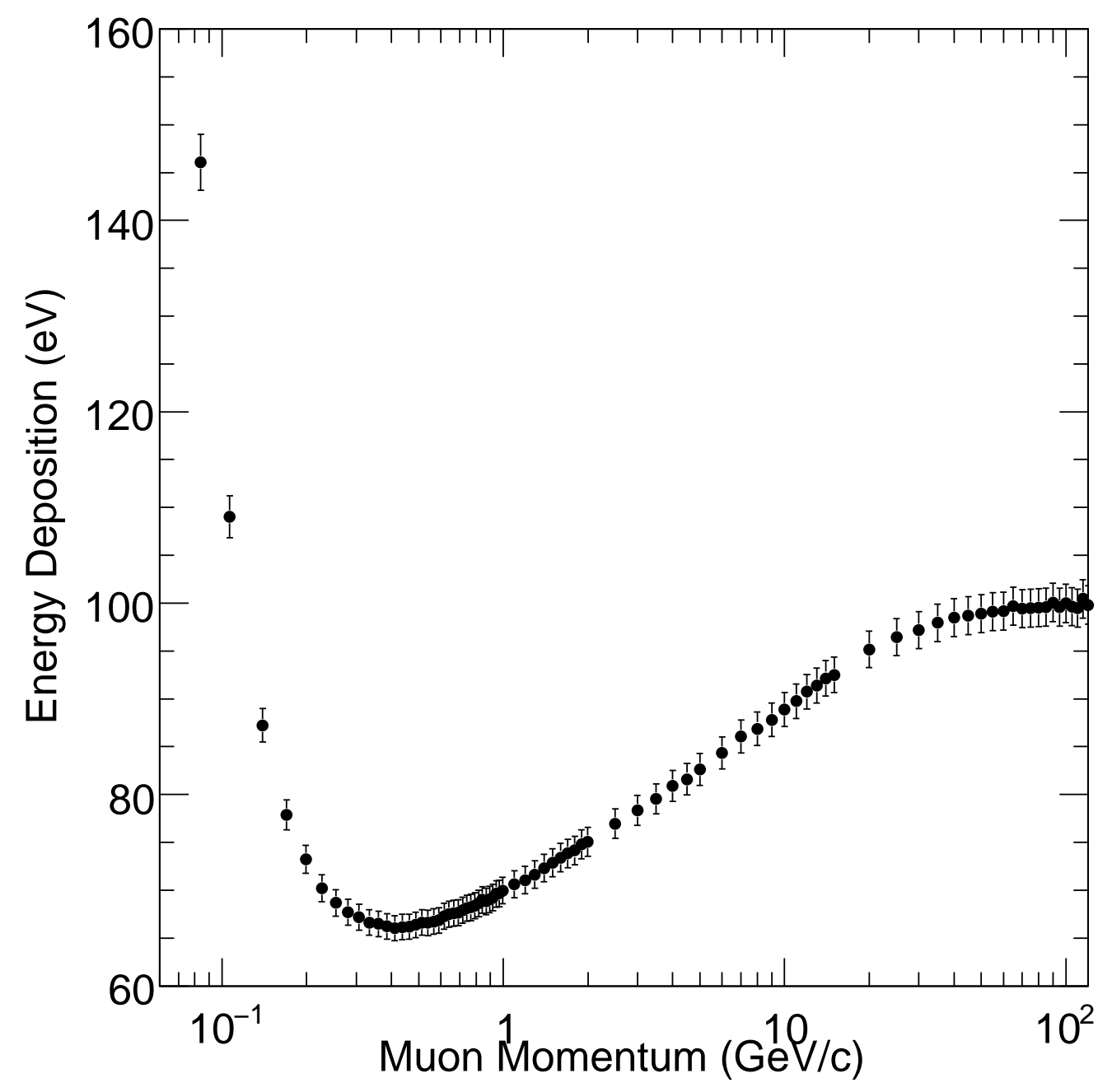

Figure 4.19: A Monte-Carlo simulation of the average energy deposition per muon in the helium in the muon monitor chambers as a function of the muon momentum. The energy deposition goes like Bethe-Block. 
The top plot of Figure 4.18 shows the energy deposited by external $\delta$ rays in the helium gas of all chambers for a sample of muons with initial energy of $5 \mathrm{GeV}$. The spread of energy depositions is large, ranging from 0 to $10 \mathrm{keV}$. There is a large number of muons that do not produce external $\delta$-rays, hence, the large number of entries at $0 \mathrm{keV}$. Also contributing to the entries near 0 $\mathrm{keV}$ are $\delta$-rays which intercept a chamber but do not deposit energy in the helium. For each initial muon energy, the average of this plot is computed and the average external energy deposition per muon is plotted as a function of the initial muon momentum as shown in the lower left plot of Figure 4.18. Because of the different amounts of air in front of Alcove 1 as compared to Alcoves 2 and 3, two separate curves are computed. The result of the larger $7 \mathrm{~m}$ air gap in front of Alcove 1 results in significantly less $\delta$-rays reaching Muon Monitor 1 as compared to Muon Monitors 2 and 3 due to $\delta$-rays ranging out in the air. A similar plot is made for the internal $\delta$-rays. The lower right plot of Figure 4.18 shows the average internal $\delta$-ray energy deposition per muon as a function of the muon momentum at the monitor. Since the monitors are constructed of identical materials only one curve is needed to describe the energy deposition in all three monitors from internal $\delta$-rays.

The above results for energy deposition by $\delta$-rays may be compared to the energy deposition of muons in the helium gas is also studied. Figure 4.19 shows the average energy deposition per muon as a function of muon momentum. As expected the average energy deposition of muons in the helium gas goes like Bethe-Block. When summed over all of the muons that reach each alcove at the various target positions and horn currents the Monte Carlo predicts that the delta rays can contribute as much as $\sim 30 \%$ of the muon monitor signal. 


\subsubsection{Cross Check of Absorber Backgrounds}

In Section 3.2, we discussed measurements of backgrounds in the muon monitors arising from hadrons impinging upon the hadron absorber creating muons and neutrons which can intercept the muon monitors. These are largely unreacted protons but also other hadrons which include undecayed pions and kaons which survive the $675 \mathrm{~m}$ length of the decay pipe and hadrons generated in tertiary particle interactions along the beam line also impact the absorber. For $4 \mathrm{GeV}$ pions(kaons), $5 \%(0 \%)$ are expected to survive the length of the decay pipe. However, for higher energy mesons, as are dominant in the higher energy beam configurations, a larger percentage will survive.

Because of the large energy and multiplicity of the $e^{-2}=13.5 \%$ of protons which traverse the target and reach the hadron absorber, and because of the close proximity of the dump to the muon monitors, it may be imagined that protons arriving at the absorber are the dominant source of backgrounds in the muon monitors arising from the absorber. A Monte Carlo study was performed to verify this assumption.

Using the simulation of the NuMI beamline, which uses Fluka05 models for meson production in the NuMI target, the total energy deposited in the hadron absorber by beam hadrons was simulated. Figure 4.20 shows the total kinetic energy of particles impacting the hadron absorber as a function of the current in the horns for the LE010, LE100 and LE250 beams. The solid curve is the contribution from protons that escape the target without interacting. Since uninteracted protons largely pass through the necks of the horns they are unaffected by the horn current. This contribution is dependent only upon the interaction length of the carbon-graphite beam target. Thus, this contri- 


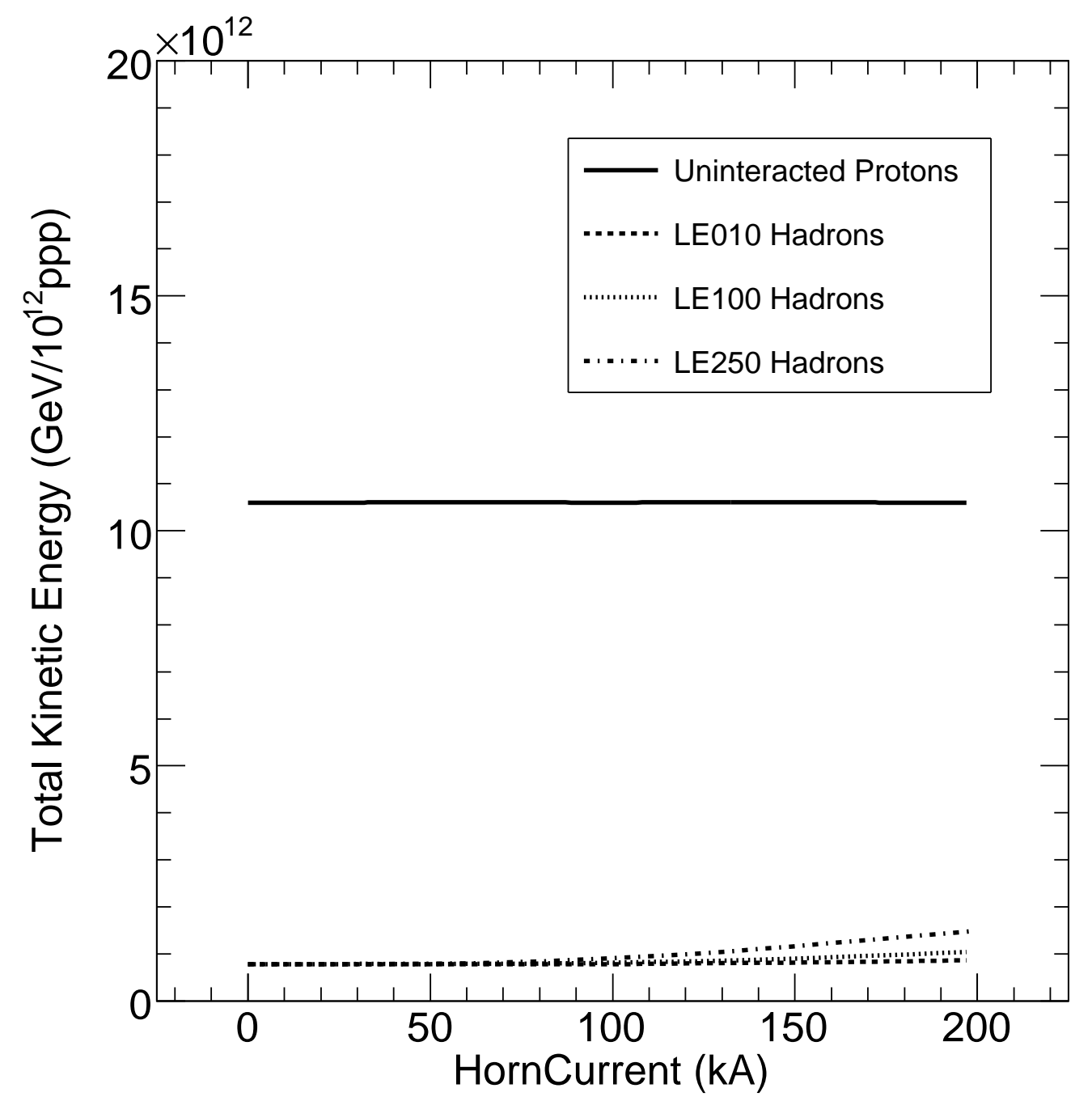

Figure 4.20: A Monte-Carlo simulation of the total kinetic energy of particles impacting the Hadron Absorber as a function of the current in the horns for the LE010, LE100 and LE250 beams. The solid curve is the contribution from protons that escape the target with out interacting. This contribution is the same across all beam configurations. The dotted/dashed lines are the energy contribution from all other hadrons impacting the Hadron Absorber. 
bution is the same across all beam configurations. The energy deposited by uninteracted protons indicates that $9 \%$ of the total primary protons survived to the hadron absorber. This is $4.5 \%$ less than the naive $13.5 \%$ from consideration of only the interaction length of protons in the target as is assumed in Section 3.2. $3 \%$ of the difference is accounted for by the multiple scattering of the uninteracted protons in the target. This results in a transverse angle at the end of the target which is larger than the solid angle to reach the end of the decay pipe. The remaining difference is easily accounted for by interactions of the uninteracted protons in the decay pipe windows.

The dotted/dashed lines are the energy contribution from all other hadrons impacting the Hadron Absorber. Such hadrons include, protons and neutrons from tertiary interactions of particles along the beam line as well as undecayed pions and kaons which survive the $675 \mathrm{~m}$ length of the decay pipe. This contribution is dependent on the beam configurations since these particles or the parents of these particles likely experience horn focusing. Uninteracted protons are the largest contributors to the total energy deposition in the hadron absorber. The energy deposition by other hadrons is at most $12 \%$ of the total energy deposition, which occurs in the LE250/200kA beam configuration.

Because the dump backgrounds are in fact not a large contribution to the muon monitor signal, we continue to use the measured backgrounds listed in Table 3.1. 


\subsection{Summary}

This chapter discussed the prediction of neutrino and muon fluxes and the associated uncertainties. The largest uncertainty in the prediction of the flux is the knowledge of meson production in nuclear targets as discussed in Section 4.2. It was shown that different cascade models predict a different yield $\pi^{+}$from the NuMI target as shown in Figure 4.5. As neutrinos are directly produced from these mesons, it is not surprising that these variations translate to significantly different neutrino spectra predictions in the MINOS Detector as shown in Figure 4.21. MINOS Detector CC $n u_{\mu}$ events are shown in the solid curves. The Monte Carlo spectrum produced using Fluka05 models for meson production are the central value of the bands. The bands are variations in the predicted spectrum from other particle production models [49, 57]. There exists some discrepancy between MINOS detector neutrino event data and the predicted spectrum using Fluka05 particle production models. However, since the number of events depends on neutrino cross sections as well as flux, it is unclear if the discrepency is due to uncertainty in the predicted flux or uncertainty in the cross section model. MINOS uses a procedure to fit for hadron production that uses the MINOS neutrino event data which must utilize a cross section model [22]. We will employ a similar procedure using the muon flux data from the muon monitors to infer the neutrino flux which does not require the use of neutrino cross sections.

The muon monitors have been shown to have good acceptance for the muons produced along side the neutrinos in meson decay. Thus, the muon monitors can be used to measure the muon flux and infer the neutrino flux as is done in Chapter 5. This is accomplished by measuring the muon flux at 
various target positions and horn currents in the muon monitors. Figures 4.224.24 show the horn current data sets discussed in Chapter 3. Superimposed is the muon flux prediction using Fluka05 meson production in the NuMI target. The ionization scale of the muon monitors is taken to be $9.6 \mathrm{pC} / 10^{7} \mu$ 's as discussed in Section 3.1.1 and is used to convert the number of muons reaching each monitor to $\mathrm{pC}$ in the Monte Carlo. The absorber backgrounds discussed in Section 3.2 are included in the Monte Carlo prediction. The error on the Monte Carlo is the variation in the predicted muon flux due to the uncertainties in the hadron absorber and rock geometry and compostion decribed in Section 4.4.1. It is clear that the prediction does not agree with data. Thus, a measurement of the flux will be obtained by fitting the Monte Carlo prediction(curves) to data(points) by varying the parent $p i^{+}$Fluka05 production spectrum using the $\left(p_{T}, p_{z}\right)$ parameterization in Equations 4.1 and 4.2. This is done in Chapter 5. 


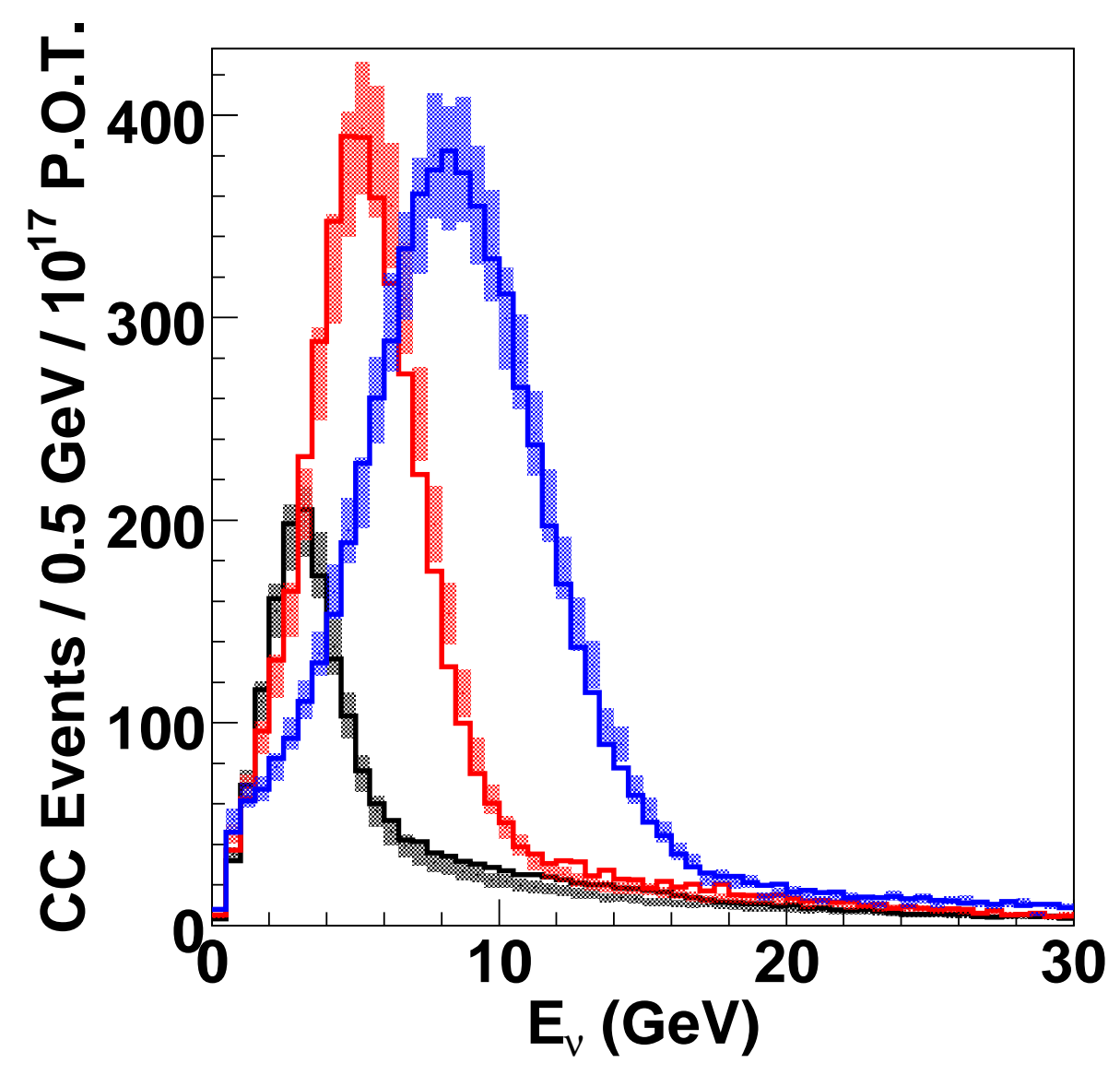

Figure 4.21: MINOS Detector CC $n u_{\mu}$ events are shown in the solid curves for the LE010/185kA, LE100/200kA and LE250/200kA beam configurations. The predicted spectrum produced using Fluka05 models for meson production are the central value of the bands. The bands are variations in the predicted spectrum from other particle production models [49, 57]. [63] 


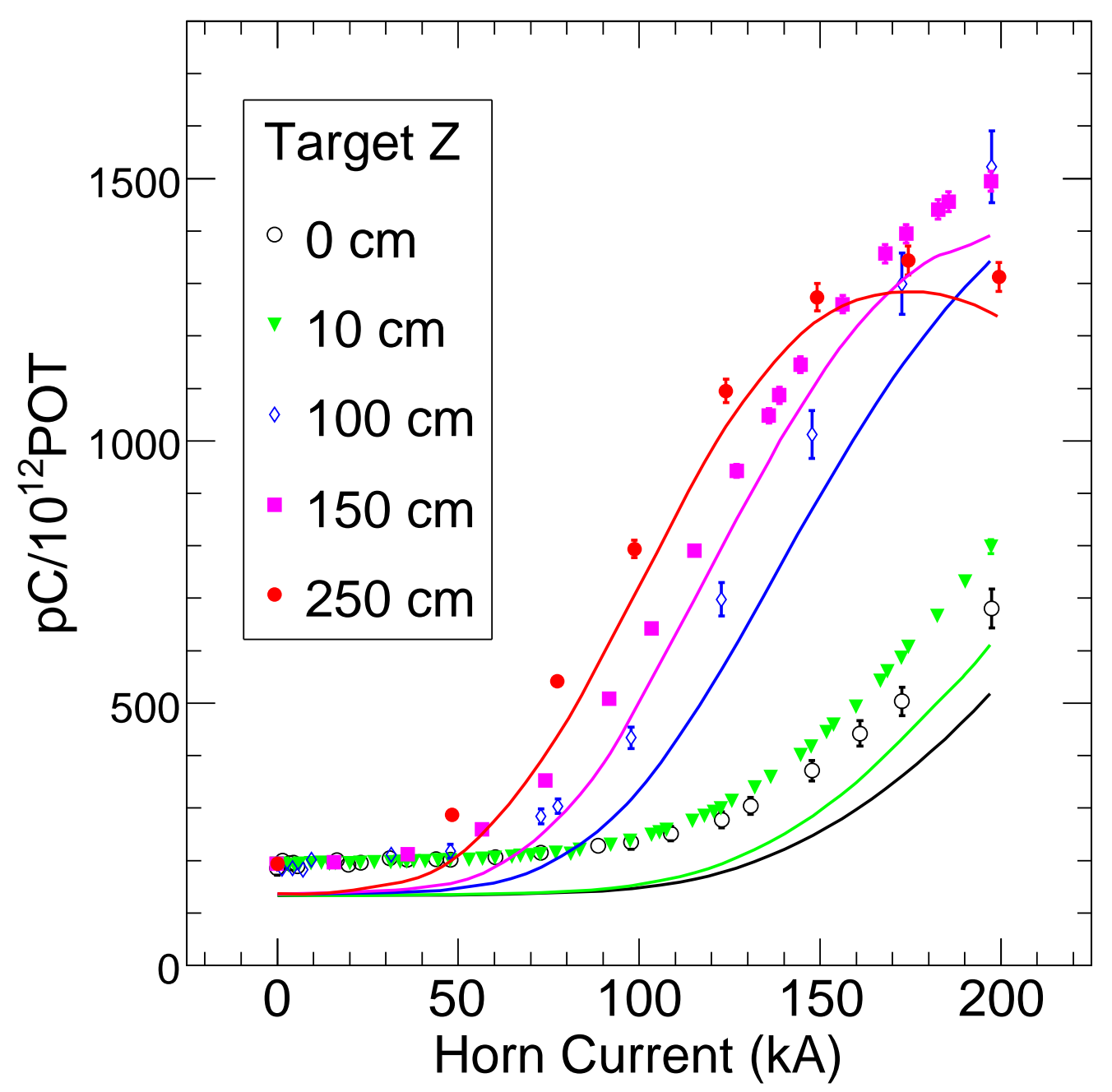

Figure 4.22: The muon flux in Muon Monitor 1 for various target positions and horn currents. The points are the data horn scans shown in Figure 3.24. The curves are the Monte Carlo prediction using Fluka05 models for hadron production in the NuMI target. The ionization scale of the muon monitors is taken to be $9.6 \mathrm{pC} / 10^{7} \mu$ 's as discussed in Section 3.1.1 and is used to convert the number of muons reaching Muon Monitor 1 to $\mathrm{pC}$ in the Monte Carlo. The absorber backgrounds discussed in Section 3.2 are included in the Monte Carlo prediction. The error on the Monte Carlo is the variation in the predicted muon flux due to the uncertainties in the hadron absorber and rock geometry and compostion decribed in Section 4.4.1. 


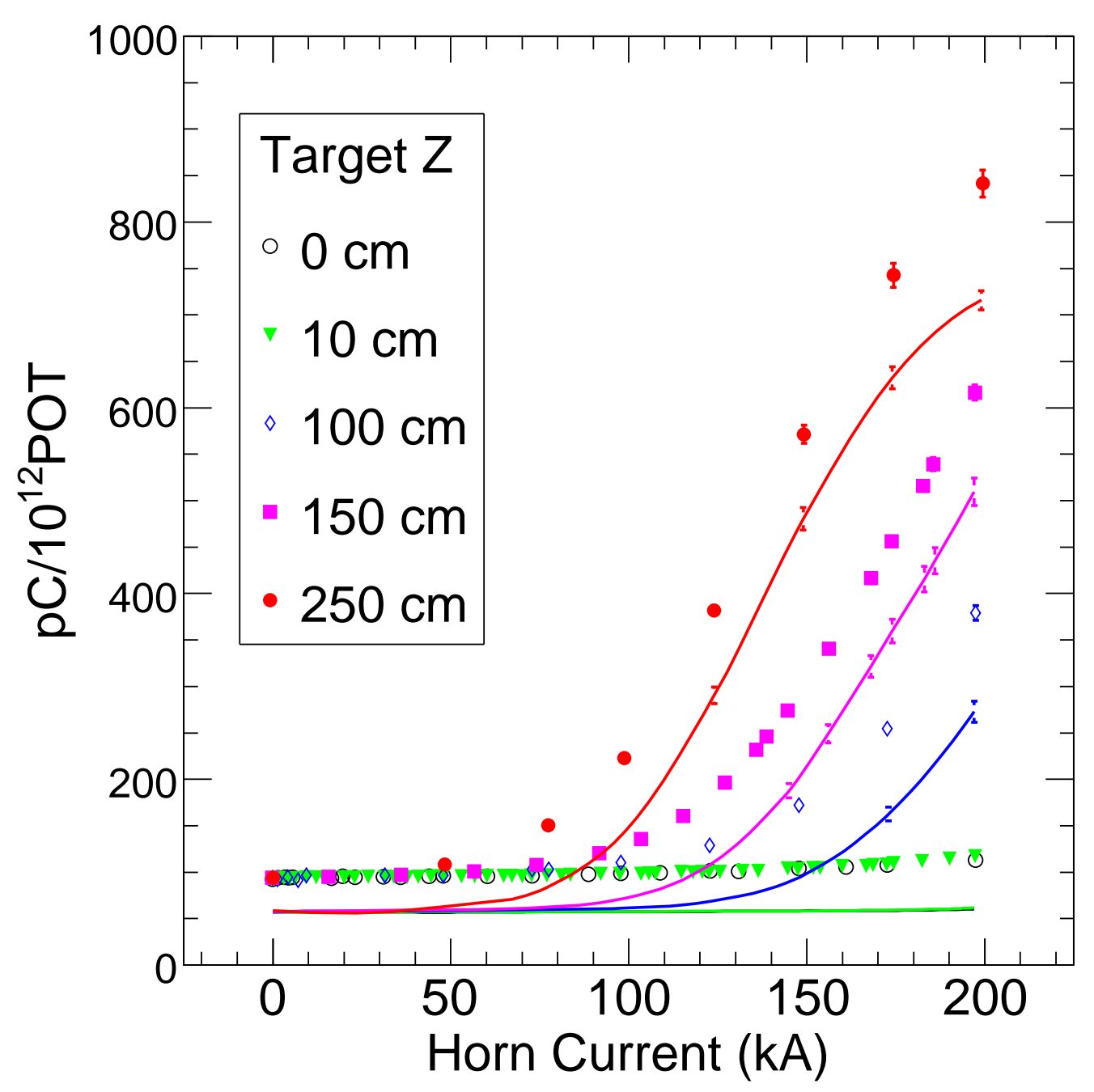

Figure 4.23: The muon flux in Muon Monitor 2 for various target positions and horn currents. The points are the data horn scans shown in Figure 3.25. The curves are the Monte Carlo prediction using Fluka05 models for hadron production in the NuMI target. The ionization scale of the muon monitors is taken to be $9.6 \mathrm{pC} / 10^{7} \mu$ 's as discussed in Section 3.1.1 and is used to convert the number of muons reaching Muon Monitor 2 to $\mathrm{pC}$ in the Monte Carlo. The absorber backgrounds discussed in Section 3.2 are included in the Monte Carlo prediction. The error on the Monte Carlo is the variation in the predicted muon flux due to the uncertainties in the hadron absorber and rock geometry and compostion decribed in Section 4.4.1. 


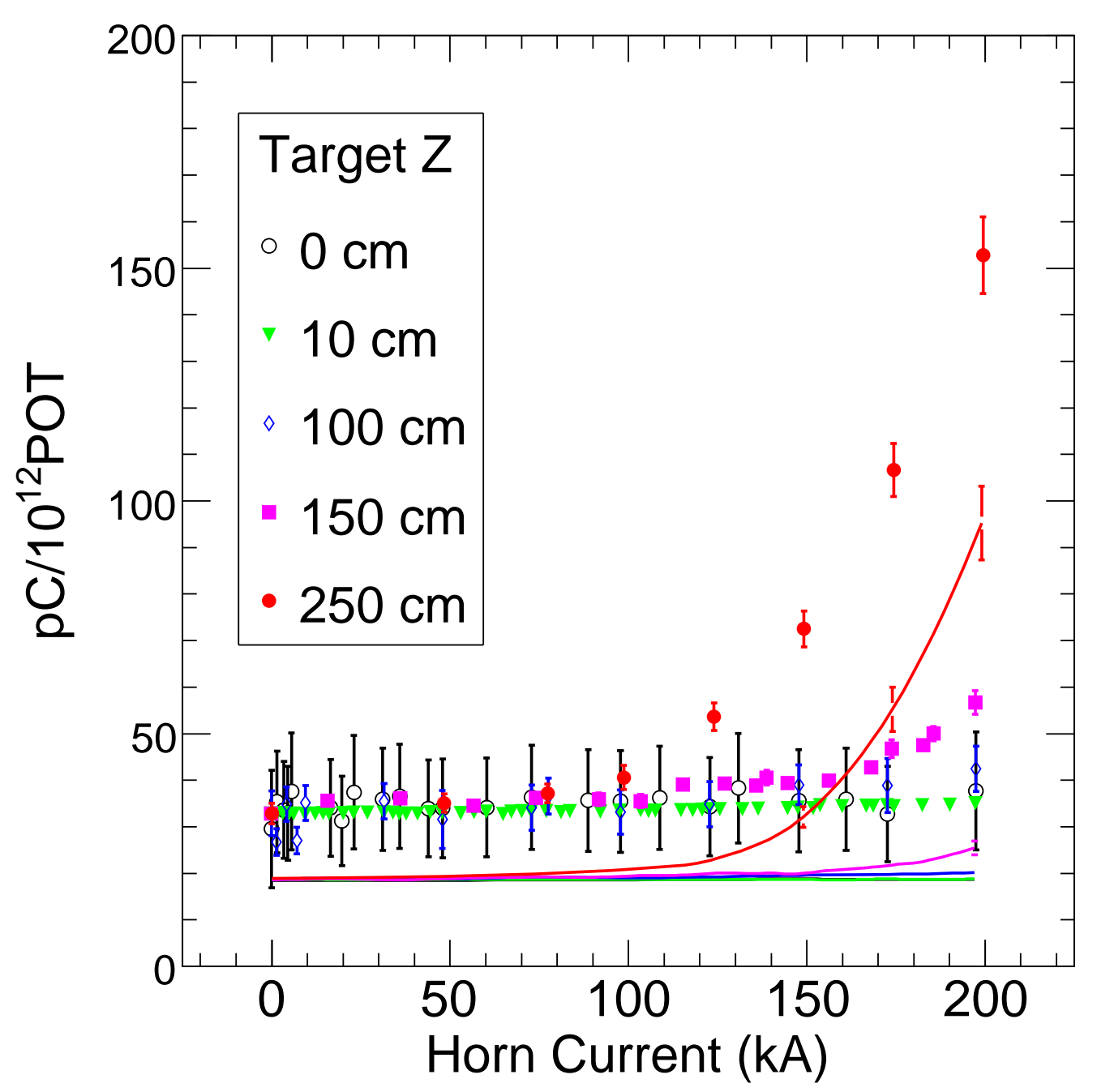

Figure 4.24: The muon flux in Muon Monitor 3 for various target positions and horn currents. The points are the data horn scans shown in Figure 3.26. The curves are the Monte Carlo prediction using Fluka05 models for hadron production in the NuMI target. The ionization scale of the muon monitors is taken to be $9.6 \mathrm{pC} / 10^{7} \mu$ 's as discussed in Section 3.1.1 and is used to convert the number of muons reaching Muon Monitor 3 to pC in the Monte Carlo. The absorber backgrounds discussed in Section 3.2 are included in the Monte Carlo prediction. The error on the Monte Carlo is the variation in the predicted muon flux due to the uncertainties in the hadron absorber and rock geometry and compostion decribed in Section 4.4.1. 


\section{Chapter 5}

\section{Neutrino Flux Measurement}

\section{$5.1 \quad$ Introduction}

In past experiments, muon monitors have been used to infer or constrain the neutrino flux to the experiment $[12,13,14,15,16,17]$. Past experiments have benefited from 9-16 muon alcoves which can be used to perform integral flux measurements, and the large number of alcoves permitted unfolding of the threshold measurements into a differential distribution of muons, and hence neutrinos. Furthermore, these preceding wide-band neutrino lines were rather short (80-140 m long decay pipes) so that the muon monitors were close to the target and could measure the lateral profile of muons; such is beneficial to help constrain the energy spectrum because muons from pions are emitted at smaller angles and large angle muons constrain the neutrinos from kaon parents.

The NuMI beam has only three instrumented alcoves, and the thresholds are higher than these past beam lines, especially for the low-energy beam 
configuration (the effective neutrino energy threshold of Muon Alcove 1 is approximately 1.6 GeV (Chapter 4)). Further, the NuMI decay pipe is quite long, so the radial fall-off of the muon beam becomes rather flat at their location 700-800 $\mathrm{m}$ from the target, especially in the low-energy beam configuration.

Despite these limitations, the muon monitors, when studied in the various configurations of the flexible NuMI beam [64], do have some sensitivity to the neutrino flux. As will be discussed, measurements of muon fluxes in the three alcoves at various target positions and horn currents can help to place constraints on the $\left(x_{F}, p_{T}\right)$ of parent hadrons which lead to neutrinos in the MINOS detector. Thus, the two variables of horn current and target position provide somewhat independent constraints, much like the multiple muon thresholds and muon radial distributions provided in past beam lines.

The present chapter utilizes Monte Carlo tools developed in Chapter 4 to calculate muon fluxes at the alcoves from meson decay-in-flight in the NuMI decay tunnel. First, a weighting scheme was developed [22] to force mesons to decay such that they produce a forward-going muon which exits the end of the decay tunnel. Such a reweighting relies on the similar kinematic formulae utilized in developing weights for neutrinos to arrive at the MINOS Near Detector. Second, an accurate geometrical description of the NuMI beam absorber and the dolomite rock was generated in the Geant-4 based g4numi Monte Carlo $[22,29]$. This geometry was used to obtain the efficiency for muons which exit the decay tunnel to penetrate the shielding and arrive at one of the alcoves. These efficiencies for the alcoves have strong dependence upon the muon momentum, as the upstream shielding defines the effective thresholds for muons to arrive in each alcove as well as the probability for the muons to multiple scatter out of the $2.2 \times 2.2 \mathrm{~m}^{2}$ area of the muon monitors. 
In this chapter, the Monte Carlo calculations of muon rates discussed in Chapter 4 will be compared to data collected during the special runs of the NuMI beam line discussed in Chapter 3. During these studies, 100-500 pulses were acquired at each target location, LE000, LE010, LE100, LE150 and LE250, and over a variety of horn currents.

The muon monitors directly measure the total charge ionized by particles intercepting the helium filled chambers. The particles include not only muons from meson decay-in-flight in the decay tunnel, but also particles from interactions in the absorber and $\delta$-rays. In order to measure the muon flux the the background component of the signal from these latter particles has been studied and included in the Monte Carlo simulated horn scans. The basis for these background estimates is derived in Chapters 3 and 4.

Note that because a conversion factor between charge recorded in the muon monitors and number of incident muons is not known, the present study will be limited to a shape analysis of the beam spectrum, i.e. an absolute rate will not be determined. The original beam tests of the NuMI beam monitor chambers $[45,46]$ were performed at an unknown absolute pressure and temperature, and furthermore had a Helium filling gas whose composition/purity may not be identical to that filling the NuMI chambers. An absolute rate fit to the data is further precluded because a beam muon is often accompanied by $\delta$-rays which cause additional ionization in the chamber gas $[65,66]$ and this effect will be treated as a systematic, not as a correction. 


\subsection{Muon Alcove Acceptances}

It is a somewhat surprising statement that the muon monitors have good acceptance for the muons that make up the low energy neutrino beam. Two effects are commonly cited as limitations of the muon monitors in the lowenergy beam:

- The lower-energy muons in the beam have greater probability of ranging out in the beam absorber or inter-alcove rock than do the muons in the higher energy, LE100/200kA, LE150/200kA or LE250/200kA beams.

- The decays of soft pions, which decay upstream in the NuMI decay volume, can send their muons at off-angles such that they cannot reach the alcoves.

The above two effects are qualitatively correct but quantitatively do not limit the muon monitors' acceptance.

First, a $4 \mathrm{GeV} / c$ pion produces at most a $1.6 \mathrm{GeV}$ neutrino or (at most) a $4 \mathrm{GeV} / c$ muon (though not in the same decay). The $\sim 4 \mathrm{GeV} / c$ threshold for a muon at the end of the decay pipe to reach alcove 1 , therefore, implies that the alcoves can monitor the parent hadrons down to an equivalent $\sim 1.6 \mathrm{GeV}$ neutrino, which is below the peak of the LE010 focusing (see Figure 2.10).

With regard to the angular acceptance of the muon monitors, the muons in pion decay are more forward-boosted than the neutrinos. The muons are all contained within a cone of $\tan \theta_{\max }=\beta^{\prime} /\left(\gamma \sqrt{\beta^{2}-\left(\beta^{\prime}\right)^{2}}\right.$, where $\beta$ and $\gamma$ describe the pion/kaon velocity and boost factor in the lab frame, and $\beta^{\prime}$ is the muon velocity in the rest frame of the parent meson $(=0.28$ for $\pi$ decays and 0.9 for $K$ decays). This works out to about $10 \operatorname{mrad}(4 \operatorname{mrad})$ for $4 \mathrm{GeV} / c$ 
$(10 \mathrm{GeV} / c)$ pion decays. Thus, the $\sim 1.4 \mathrm{mrad}$ solid angle acceptance of the monitors spans an appreciable fraction of the meson decays in the focusing peak, even for the LE010 target configuration. To be sure, the lateral profile of the muons at the alcoves will be quite broad for the decays of low-energy pions, but the rate should be still measurable.

Figures 5.1 through 5.3 attempt to demonstrate the preceding argument and may be compared to Figure 4.16. (Figure 5.1 is for the LE010/185kA beam, Figure 5.2 is for the LE100/200kA beam, and Figure 5.3 is for the LE250/200kA beam). These show the acceptance for the underlying parent pions. Shown in the upper left of each figure are the $\left(p_{T}, p_{z}\right)$ of parent pions, as they emerge from the target, that contribute to the neutrino flux in the MINOS Detector. The upper right plot shows the same information, but for those $\pi^{+}$which contribute a muon which reaches Alcove 1. The distribution looks quite similar to that for the MINOS ND, with the exception of the

threshold $p_{z}=4 \mathrm{GeV} / c$ for Alcove 1 . The $\left(p_{T}, p_{z}\right)$ sensitivities of Alcoves 2 and 3 are also shown, in the bottom left and right plots, which have higher effective parent thresholds of $11 \mathrm{GeV} / c$ and $21 \mathrm{GeV} / c$, respectively. That the Alcove 1 acceptance is so similar to the MINOS detector reinforces the expectation that the muon monitors can observe most of the relevant flux muons which contribute to the flux of neutrinos seen in MINOS.

\subsection{Horn Current Scans}

To be useful as a tool to measure the differential flux with respect to neutrino energy, the muon monitors must overcome the fact that they consist of only three arrays, with three very coarse threshold bins in energy (to be contrasted 

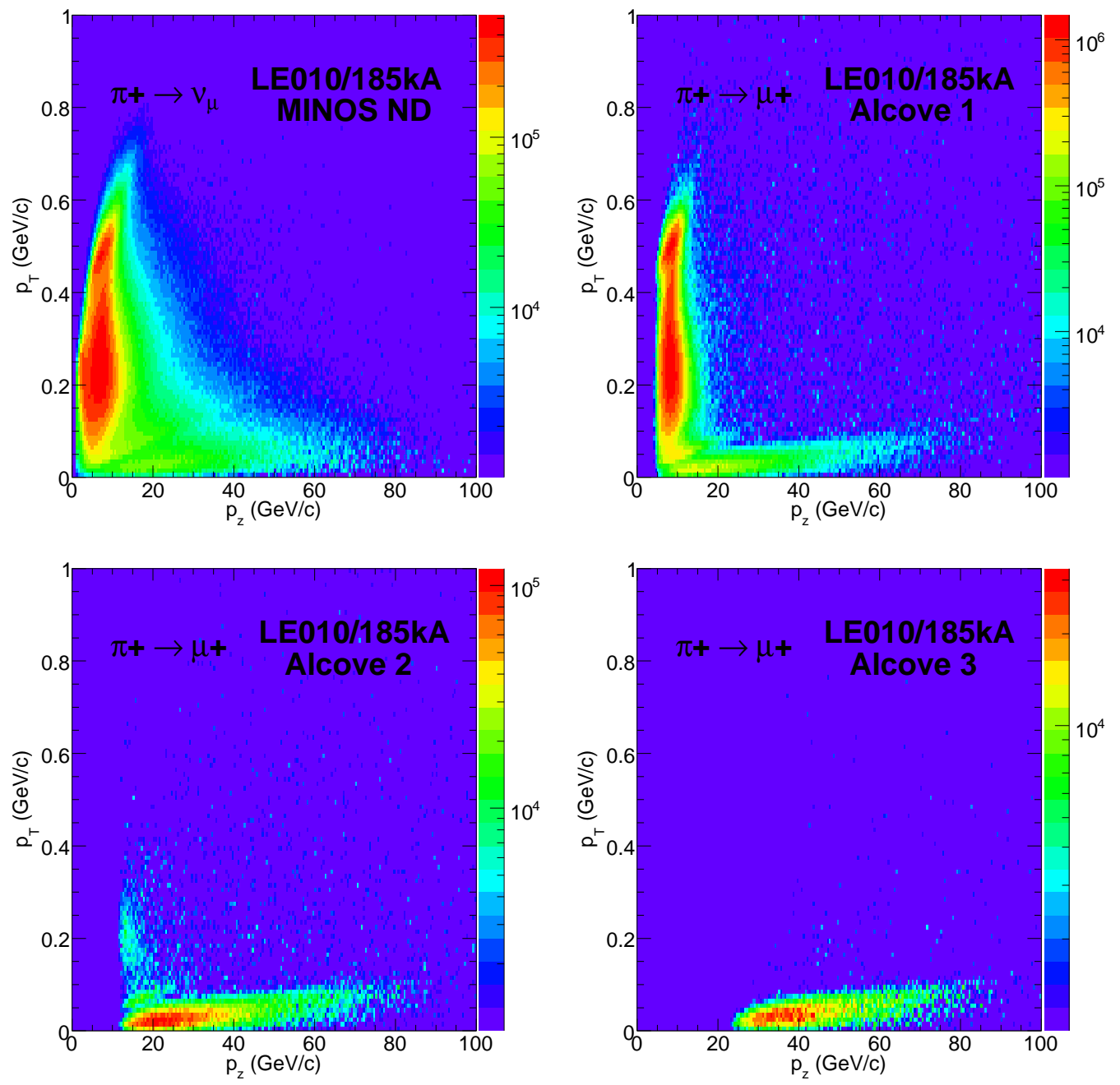

Figure 5.1: Fluka 2005 Monte Carlo prediction of the $p_{T}$ and $p_{z}$ of pions which contribute a CC neutrino in the MINOS Detector (upper left plot), or a muon in Alcoves 1 (upper right), 2 (lower left), or 3 (lower right). Distributions are for the LE010/185kA beam configuration. The distributions for Alcoves 2 and 3 are similar to Alcove 1, but with higher thresholds of $11 \mathrm{GeV} / c$ and $21 \mathrm{GeV} / c$, respectively. 

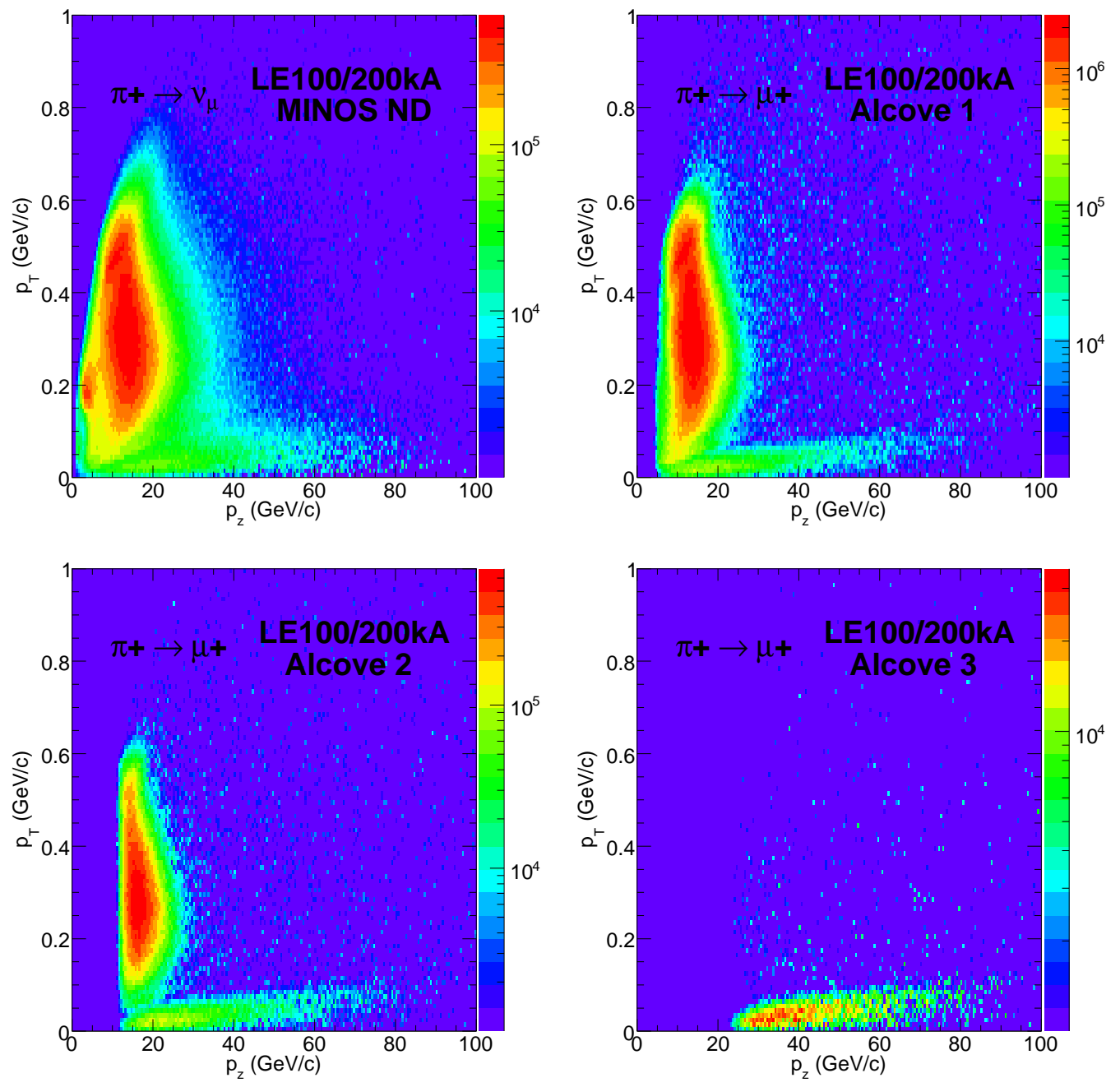

Figure 5.2: Fluka 2005 Monte Carlo prediction of the $p_{T}$ and $p_{z}$ of pions which contribute a CC neutrino in the MINOS Detector (upper left plot), or a muon in Alcoves 1 (upper right), 2 (lower left), or 3 (lower right). Distributions are for the LE100/200kA beam configuration. The distributions for Alcoves 2 and 3 are similar to Alcove 1, but with higher thresholds of $11 \mathrm{GeV} / c$ and $21 \mathrm{GeV} / c$, respectively. 

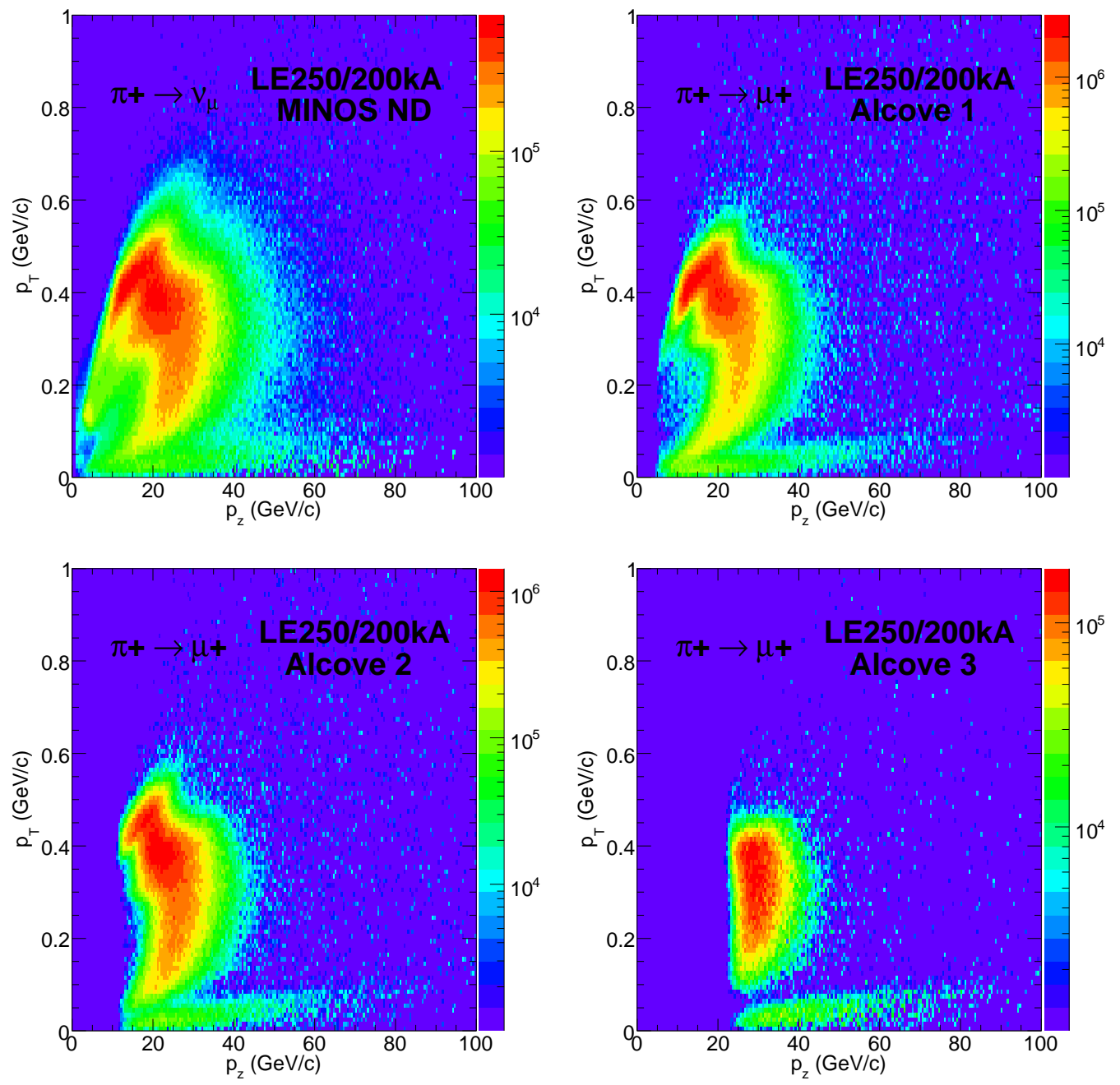

Figure 5.3: Fluka 2005 Monte Carlo prediction of the $p_{T}$ and $p_{z}$ of pions which contribute a CC neutrino in the MINOS Detector (upper left plot), or a muon in Alcoves 1 (upper right), 2 (lower left), or 3 (lower right). Distributions are for the LE250/200kA beam configuration. The distributions for Alcoves 2 and 3 are similar to Alcove 1, but with higher thresholds of $11 \mathrm{GeV} / c$ and $21 \mathrm{GeV} / c$, respectively. 
with the 9-16 alcoves in previous beam lines). This is done using the ability in the NuMI beam to vary both the horn current, $I_{\text {horn }}$, which affects the $\left\langle p_{T}\right\rangle$ kick given to particles by the horns, and also vary the position, $z_{\text {target }}$, of the target, which changes the $\left\langle p_{z}\right\rangle$ of parent mesons focused by the horns. As discussed in Chapter $3, \sim 1$ hour runs varying both of these parameters were taken and the Muon Monitor data recorded. While such measurements could in principle be taken using neutrinos in the MINOS detector as well, the power of the Muon Monitors is that they can record relatively accurate (integral) fluxes in just a few beam spills.

Figures 5.4 through 5.6 demonstrate the ability to sweep in both $p_{T}$ and $p_{z}$ using $\left(I_{\text {horn }}, z_{\text {target }}\right)$. Shown are the $p_{T}$ and $p_{z}$ of $\pi^{+}$that contribute muons to Alcove 1 at a variety of horn currents in the LE010, LE150, and LE250 target configurations. As can be seen in the LE250 beam, variation of the horn current sweeps the $p_{T}$ of focused particles to larger values. In the LE010 configuration, variation of the horn current sweeps in both the $p_{T}$ and $p_{z}$ directions. Alcoves 2 and 3 , though not shown in these figures, add the information of independent flux measures above the thresholds of $p_{z}=11$ and $21 \mathrm{GeV} / c$, respectively. Similar information is shown in Figure 5.7, which shows the spectra of pion $p_{T}$ in several $\left(I_{\text {horn }}, z_{\text {target }}\right)$ configurations. As expected, larger $I_{\text {horn }}$ focuses larger $\left\langle p_{T}\right\rangle$.

To be fair, the muon monitors do no measure directly these underlying $\left(p_{T}, p_{z}\right)$ distributions. All that is seen in the monitors is a signal proportional to the total number of muons, in effect the integral under any one of the surface plots in Figures 5.4 through 5.6. The situation similar to the fitting of hadron production models to the MINOS Near Detector data described in Ref [22]. The one data point provided by a single muon alcove rate is analogous to a 


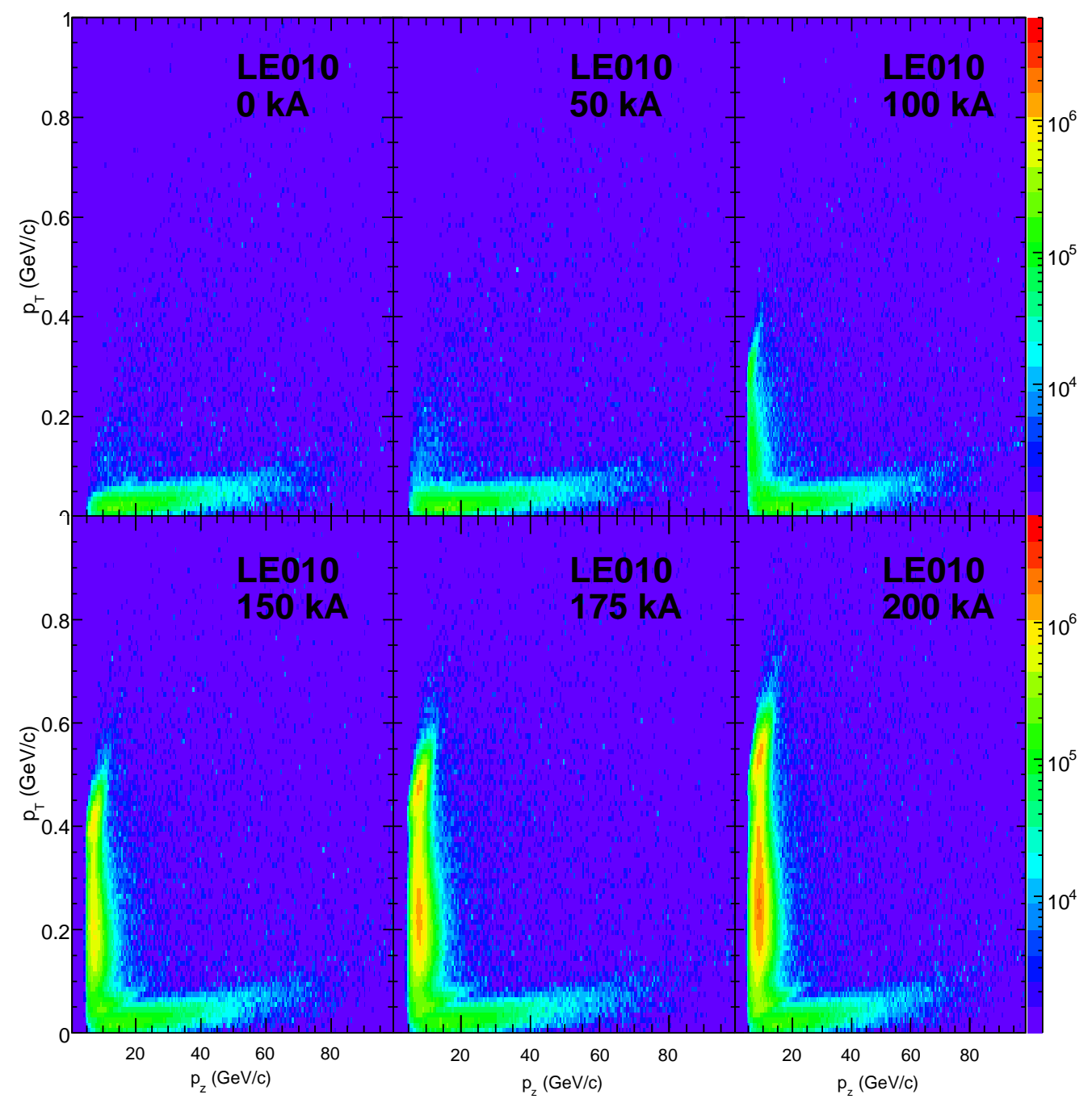

Figure 5.4: Fluka 2005 Monte Carlo prediction of the $p_{T}$ and $p_{z}$ of pions which contribute a muon in Alcove 1. Distributions are for the LE010 beam configuration with $0,50,100,150,175$, and $200 \mathrm{kA}$. The distributions for Alcoves 2 and 3 are similar to Alcove 1, but with higher thresholds, but with higher thresholds of $11 \mathrm{GeV} / c$ and $21 \mathrm{GeV} / c$, respectively. 


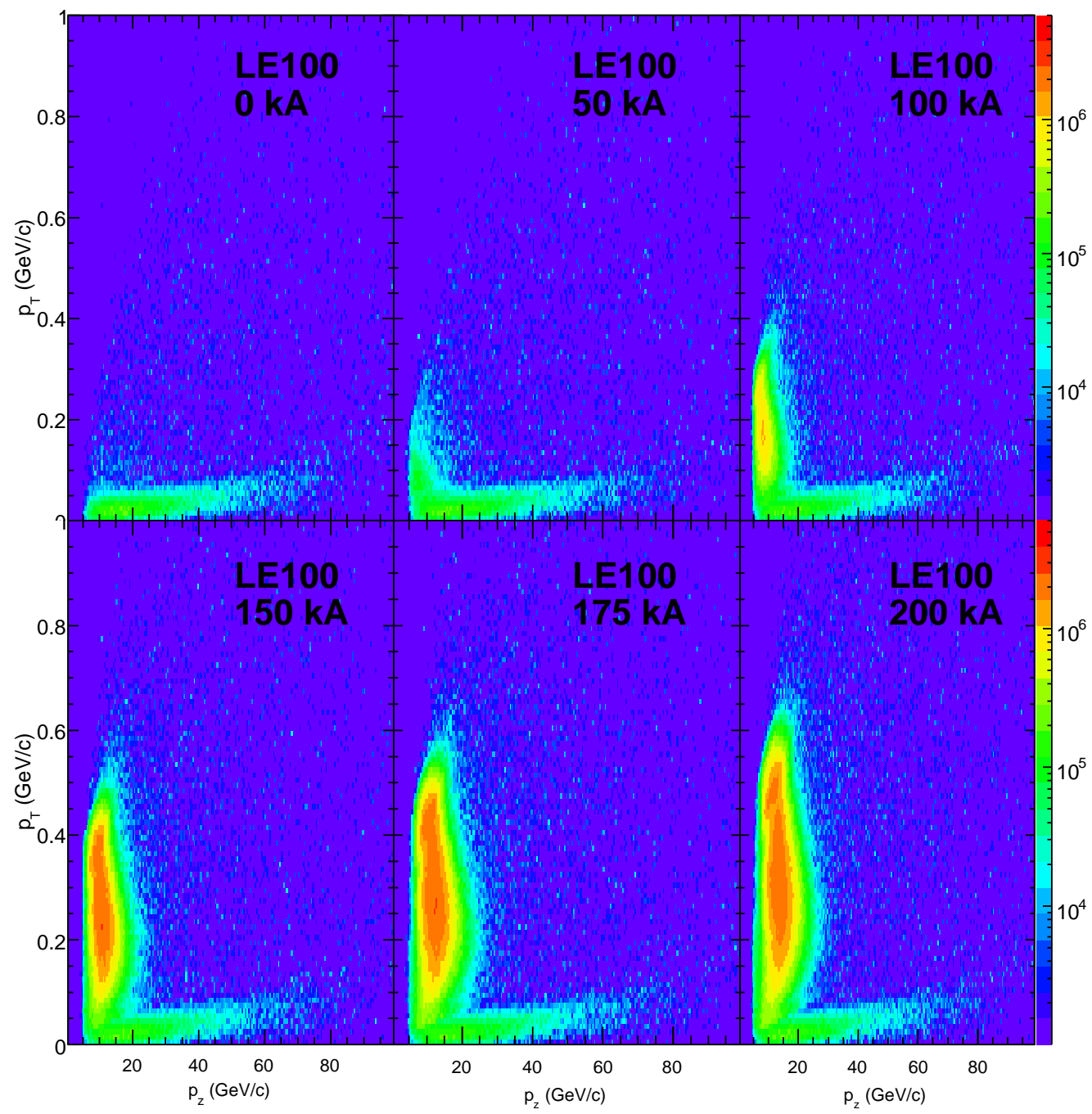

Figure 5.5: Fluka 2005 Monte Carlo prediction of the $p_{T}$ and $p_{z}$ of pions which contribute a muon in Alcove 1. Distributions are for the LE100 beam configuration with $0,50,100,150,175$, and $200 \mathrm{kA}$. The distributions for Alcoves 2 and 3 are similar to Alcove 1, but with higher thresholds, but with higher thresholds of $11 \mathrm{GeV} / c$ and $21 \mathrm{GeV} / c$, respectively. 


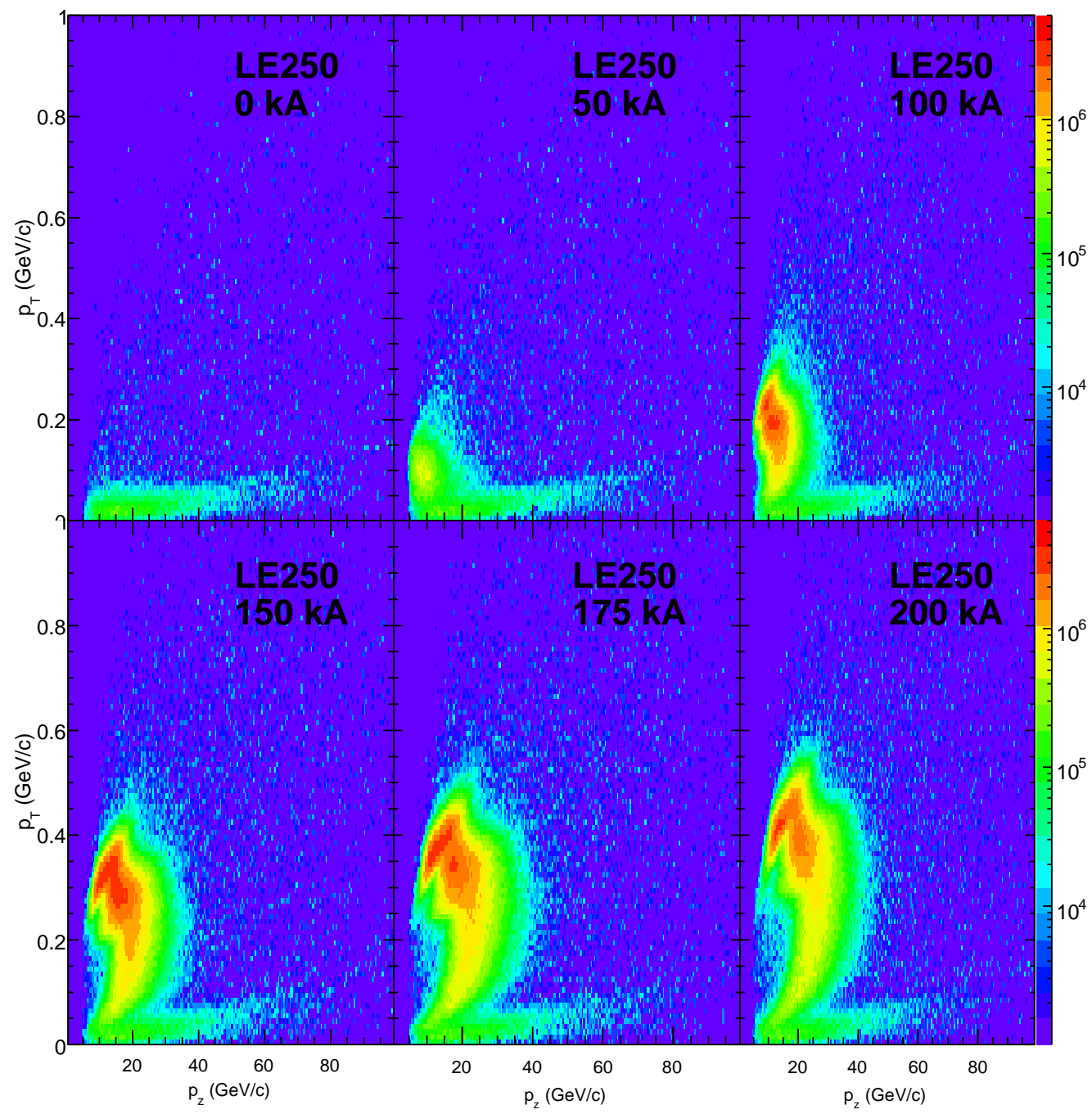

Figure 5.6: Fluka 2005 Monte Carlo prediction of the $p_{T}$ and $p_{z}$ of pions which contribute a muon in Alcove 1. Distributions are for the LE250 beam configuration with $0,50,100,150,175$, and $200 \mathrm{kA}$. The distributions for Alcoves 2 and 3 are similar to Alcove 1, but with higher thresholds, but with higher thresholds of $11 \mathrm{GeV} / c$ and $21 \mathrm{GeV} / c$, respectively. 

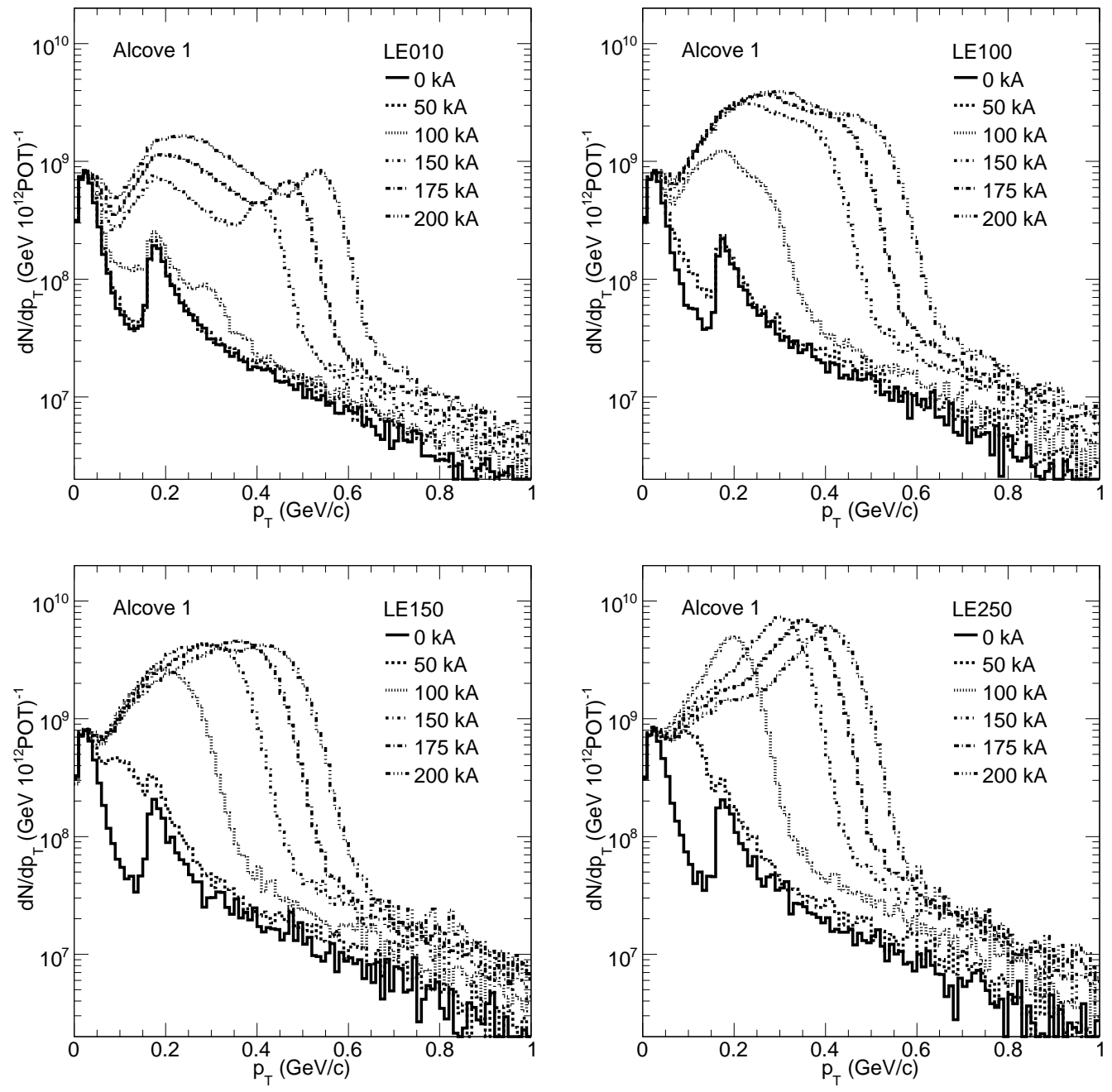

Figure 5.7: Fluka 2005 Monte Carlo prediction of the $p_{T}$ pions which contribute a muon in Alcove 1. Distributions are for the LE010, LE100, LE150 and LE250 beam configurations with $0,50,100,150,175$, and $200 \mathrm{kA}$. Increase of the horn current is seen to increase the $\left\langle p_{T}\right\rangle$ of focused particles. 
bin of neutrino energy in the MINOS Near Detector. The power of the Near Detector data is that a single data set consists of 60 neutrino energy bins, and just a few beam configurations were acquired. The power of the muon monitors is not in the number of energy bins (there are just three for the three alcoves), but in the larger number of beam configurations acquired in the $\left(I_{\text {horn }}, z_{\text {target }}\right)$ data set. Chapter 3 describes the data sets in which $I_{\text {horn }}$ and $z_{\text {target }}$ were varied. Chapter 4 describes the Monte Carlo which will be compared to the data.

\subsection{Empirical Fit to Particle Production}

A measurement of the $\nu_{\mu}$ flux is obtained by fitting the horn current scan Monte Carlo to the horn current scan data by varying the underlying parent particle production off of the NuMI target.

The muon and neutrino flux derives directly from the underlying parent particle flux. The parent particle production from the NuMI hadron production target has been empirically parameterized according to Equations 4.1 and 4.2. The hadron production off of the NuMI target can be tuned from the Fluka05 model by allowing the $A\left(x_{F}\right), B\left(x_{F}\right)$, and $C\left(x_{F}\right)$ functions to warp according to [22]

$$
\begin{aligned}
& A^{\prime}\left(x_{F}\right)=\left(p_{1}+p_{2} x_{F}\right) A\left(x_{F}\right) \\
& B^{\prime}\left(x_{F}\right)=\left(p_{3}+p_{4} x_{F}\right) B\left(x_{F}\right) \\
& C^{\prime}\left(x_{F}\right)=\left(p_{5}+p_{6} x_{F}\right) C\left(x_{F}\right)
\end{aligned}
$$

where the $p_{i}$ 's are the tuning(fitting) parameters. The scale factor, $\mathrm{W}\left(x_{F}, p_{T}\right)$, 
by which the Fluka05 hadron production, at a given $\left(x_{F}, p_{T}\right)$, must be increased or decreased is given by

$$
W\left(x_{F}, p_{T}\right)^{\pi^{+}} \text {or } K^{+}=\frac{\left[A^{\prime}+B^{\prime} p_{T}\right] \times \exp \left(-C^{\prime} p_{T}^{3 / 2}\right)}{\left[A+B p_{T}\right] \times \exp \left(-C p_{T}^{3 / 2}\right)}
$$

for $\pi^{+}$and $K^{+}$and

$$
W\left(x_{F}, p_{T}\right)^{\pi^{-}} \text {or } K^{-}=\left(p_{13}+p_{14} x_{F}\right) \times W\left(x_{F}, p_{T}\right)^{\pi^{+}} \text {or } K^{+}
$$

for $\pi^{-}$and $K^{-}$.

The horn current scan Monte Carlo is tuned to fit the horn current scan data of Chapter 3 using this parameterization. A fit is performed in which the $\chi^{2}$ defined as

$$
\chi^{2}=\sum_{I_{\text {horn }}, z_{\text {target }}} \frac{\phi^{\text {data }}\left(I_{\text {horn }}, z_{\text {target }}\right)-\phi^{M C}\left(I_{\text {horn }}, z_{\text {target }}, p_{T}, p_{z}\right)}{\sigma^{2}{ }_{\text {data }}+\sigma^{2}{ }_{M C}}
$$

is minimized. $\phi^{\text {data }}$ is the measured muon flux at $\left(I_{\text {horn }}, z_{\text {target }}\right) . \phi^{M C}$ is the Monte Carlo predicted muon flux at $\left(I_{\text {horn }}, z_{\text {target }}\right)$. $\sigma_{\text {data }}$ is the error on the data described in Chapter 3 and $\sigma_{M C}$ is the error on the Monte Carlo described in Chapter 4. As the $\left(p_{T}, p_{z}\right)$ of parent mesons is varied in the fit, the Monte Carlo muon flux prediction will vary accordingly. The set of $\left(p_{T}, p_{z}\right)$ parameters that minimizes the $\chi^{2}$ provides the muon flux measurement and the neutrino flux measurement when used to reweight the neutrino Monte Carlo. The LE000, LE010, LE100, LE150 and 2 LE250 horn current scans as well as the horn current scans in each monitor, shown in Figures 4.22-4.24, are fit simultaneously. 


\subsection{Constraints on the Fit}

In the preceding chapters, the technical aspects of correcting the horn current scan data and generating the best Monte Carlo prediction of the horn current scan data sets have been discussed at length. The data and Monte Carlo horn current scans in Figures 4.22-4.24 represent the best data and Monte Carlo estimation of the ionization current produced within the muon monitor chambers by charged particles that intercept the chambers under the following conditions:

- All data corrections discussed in Chapter 3 are applied to the data.

- $9.6 \mathrm{pC} / 10^{7} \mu$ 's is used to convert the $\mathrm{MC}$ from muons to $\mathrm{pC}$, and is applied to all muons independent of muon momentum.

- The measurements of the backgrounds from unreacted protons discussed in Chapter 3 are added to the MC.

- No contribution from $\delta$-ray backgrounds are included.

The data and Monte Carlo horn current scan data sets produced under these conditions will henceforth be referred to as the nominal data and Monte Carlo horn current scan data sets.

In order to tune the default Fluka05 horn current scan Monte Carlo to the horn current scan data some constraints are placed on the parameterization. The muon monitors cannot distinguish between $\mu^{+}$and $\mu^{-}$. The $\pi^{+}$are tuned according to Equations 5.1 and the weights are calculated according to Equation 5.2. However, the corresponding parameters for $\pi^{-} \mathrm{s}$ are not varied 
in the fit. Instead, the $\pi^{-}$weights are given by

$$
W\left(x_{F}, p_{T}\right)^{\pi^{-}}=R_{\pi} \times W\left(x_{F}, p_{T}\right)^{\pi^{+}}
$$

where $R_{\pi}$ is computed such that the NA49 $\pi^{+} / \pi^{-}$is preserved [?]. Since the NA49 data only cover $x_{F}$ up to $0.5\left(p_{z}=60 \mathrm{GeV}\right.$ for NuMI $)$ a reasonable extrapolation to higher $x_{F}$ is obtained by fitting the data to several polynomials as shown in Figure 4.9. The 3rd order polynomial is taken to be the best fit to the data and the $\pi^{+} / \pi^{-}$is fixed to this ratio for the nominal muon monitor fit. The other curves are used to determine the systematic error in the nominal flux measurement from the variation of the $\pi^{+} / \pi^{-}$discussed in Chapter 6 . Also, since muons from kaons are produced farther upstream and at larger angles than muons from pions, the muon monitors have little sensitivity to muons from kaons as discussed in Section 4.3. Therefore, the $K^{+}$and $K^{-}$ weights are given by

$$
W\left(x_{F}, p_{T}\right)^{K^{+}} \text {or } K^{-}=R_{K / \pi}
$$

where $R_{K / \pi}$ is computed such that the Fluka05 $K^{+} / \pi^{+}$ratio, shown in Figure 4.8 , is preserved. Figure 4.8 also shows the $K^{+} / \pi^{+}$from several particle production models, including that obtained from the MINOS fit to the neutrino data [22]. Fluka05 is in good agreement with other models, so it is not unreasonable to use the Fluka05 $K^{+} / \pi^{+}$as a constraint. The systematic error on the flux from this constraint is discussed in Chapter 6 .

In summary, the following constraints are placed on the fitting procedure.

- For the tuning, the $\pi^{+} / \pi^{-}$is fixed to the NA49 data fitted with a third 
order polynomial.

- The $K^{+} / \pi^{+}$is fixed to the Fluka05 $K^{+} / \pi^{+}$.

These constraints will be referred to as the nominal fitting constraints.

\subsection{The $\nu_{\mu}$ Flux Measurement}

The fitting procedure produces a set of weights in $\left(p_{T}, p_{z}\right)$ for $\pi^{+}, \pi^{-}, K^{+}$ and $K^{-}$. The $\pi^{+}$weights produced by the fit are shown in Figure 5.8. These weights are values which scale up or down the predicted yield, $d^{2} N / d p_{T} d x_{F}$, for pions and kaons as predicted by Fluka05 to match the muon data. These weights are used to adjust the neutrino GNUMI Monte Carlo flux. With the adjusted Monte Carlo yields, a final prediction for the neutrino flux is produced. Figure 5.9 shows the $\nu_{\mu}$ flux at the Near Detector for the LE010/185kA, LE100/200kA, LE150/200kA and LE250/200kA configurations. The flux is normalized to MINOS Near Detector data in the region between $E_{\nu}=26$ and $40 \mathrm{GeV}$ using the procedure described in Section 6.2. The gray hatched region represents an extrapolation of the measurement due to the shielding constraints of the muon monitors discussed in Chapter 4. The flux measurement is limited to $E_{\nu}<26 \mathrm{GeV}$, because the region between $E_{\nu}=26$ and $40 \mathrm{GeV}$ is used for normalization. Furthermore, the neutrino flux above $40 \mathrm{GeV}$ predominantly comes from kaons for which the muon monitors have limited sensitivity.

Figures 5.10 through 5.12 show the muon monitor data and Monte Carlo expectation before and after fitting. The solid red curves result from the fit, while the blue curves show the original Fluka expectation. The error 


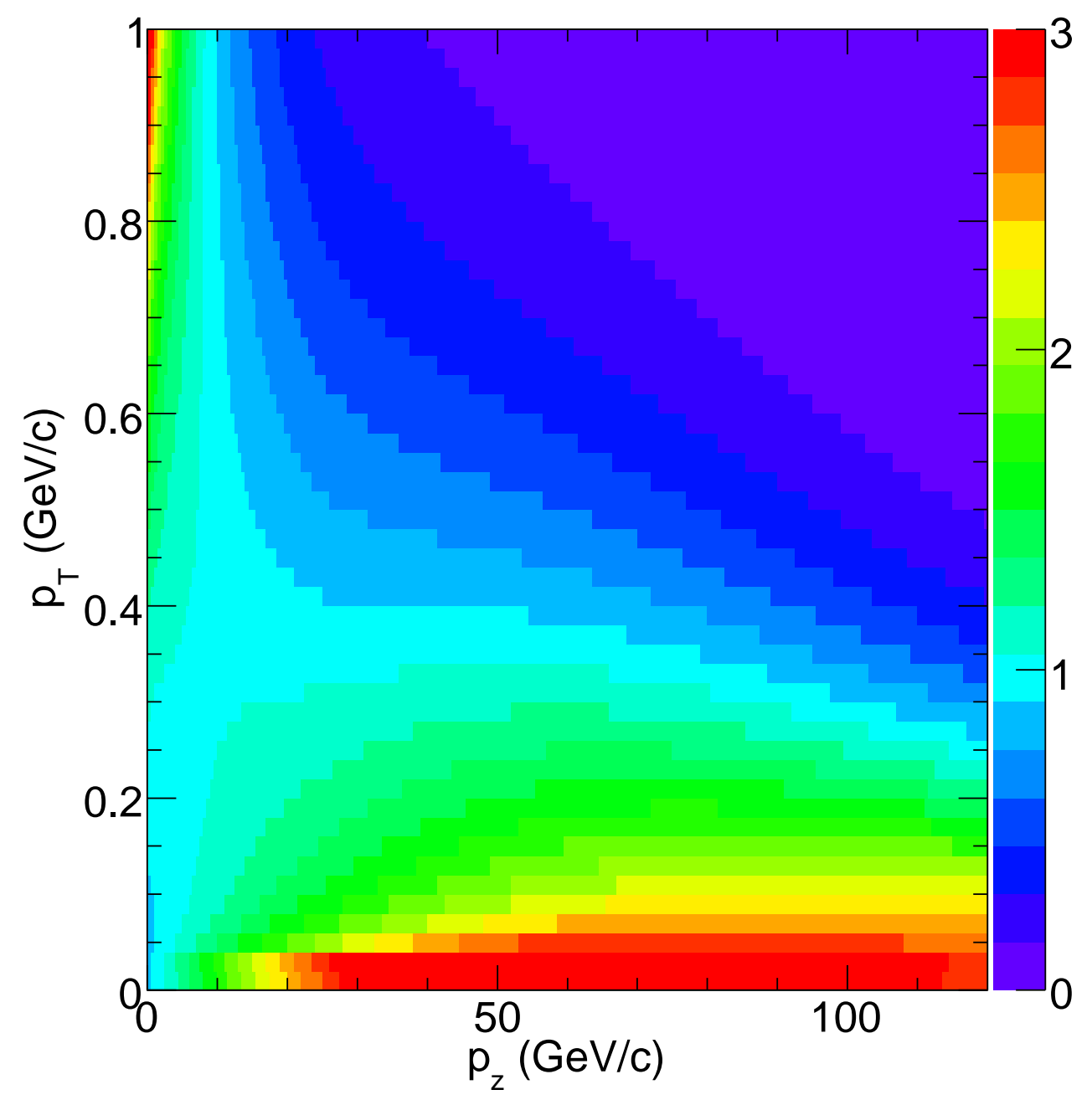

Figure 5.8: The $\pi^{+}$weights in $\left(p_{T}, p_{z}\right)$ from tuning the horn scan data to MC. 


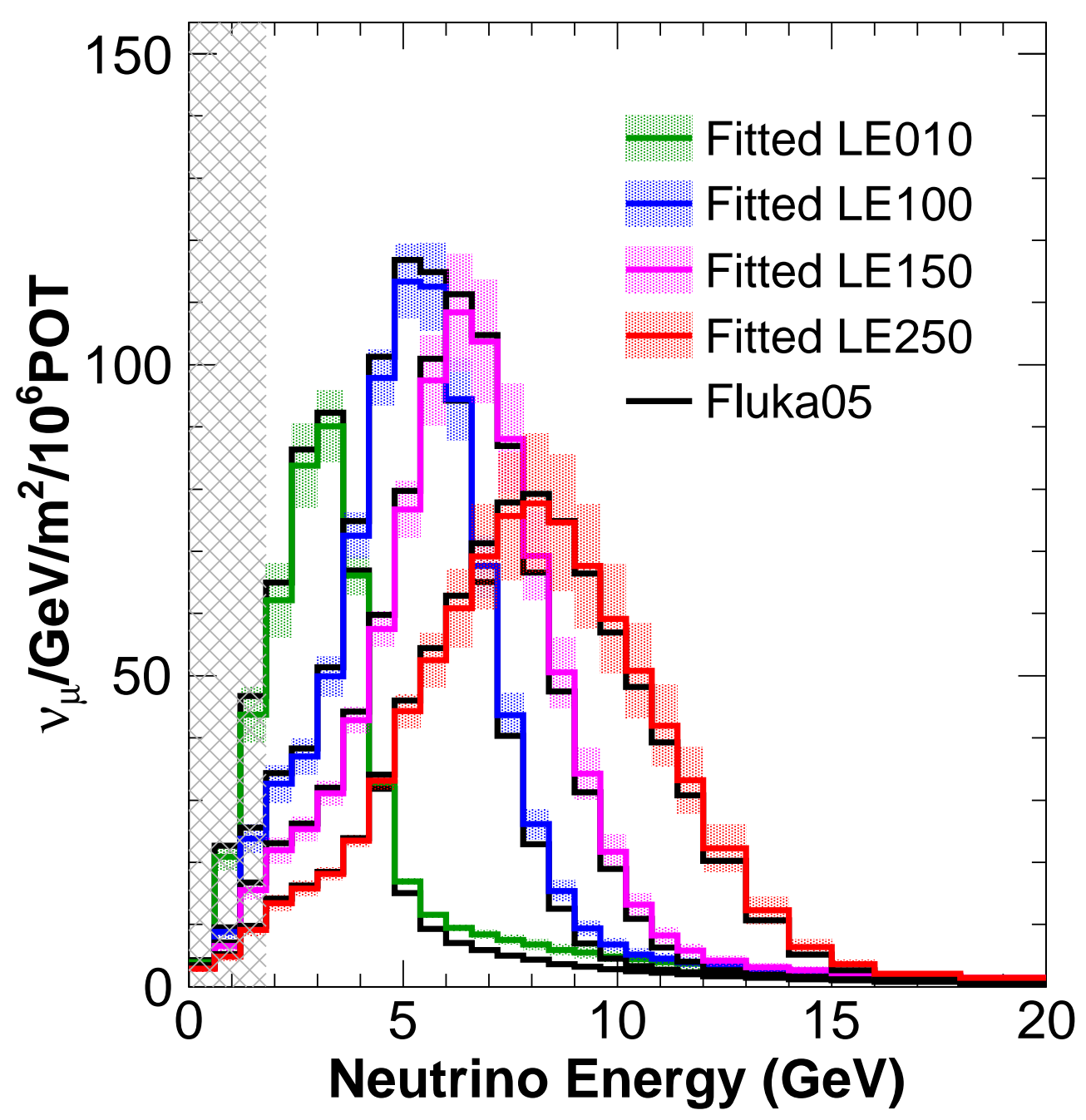

Figure 5.9: The normalized fitted Monte Carlo $\nu_{\mu}$ flux at the MINOS Near Detector for the all beams. The colored curves are the nominal fit reweighted Monte Carlo flux. The error bands are the total errors on the nominal flux after normalization as discussed in Chapter 6. The black curve is the default Near Detector Monte Carlo flux generated with Fluka05 hadron production. The default Monte Carlo is shown only for reference. The gray hatched region is an extrapolation of the fitted hadron production parameters. The region $E_{\nu}>40 \mathrm{GeV}$ are is used for normalization. 


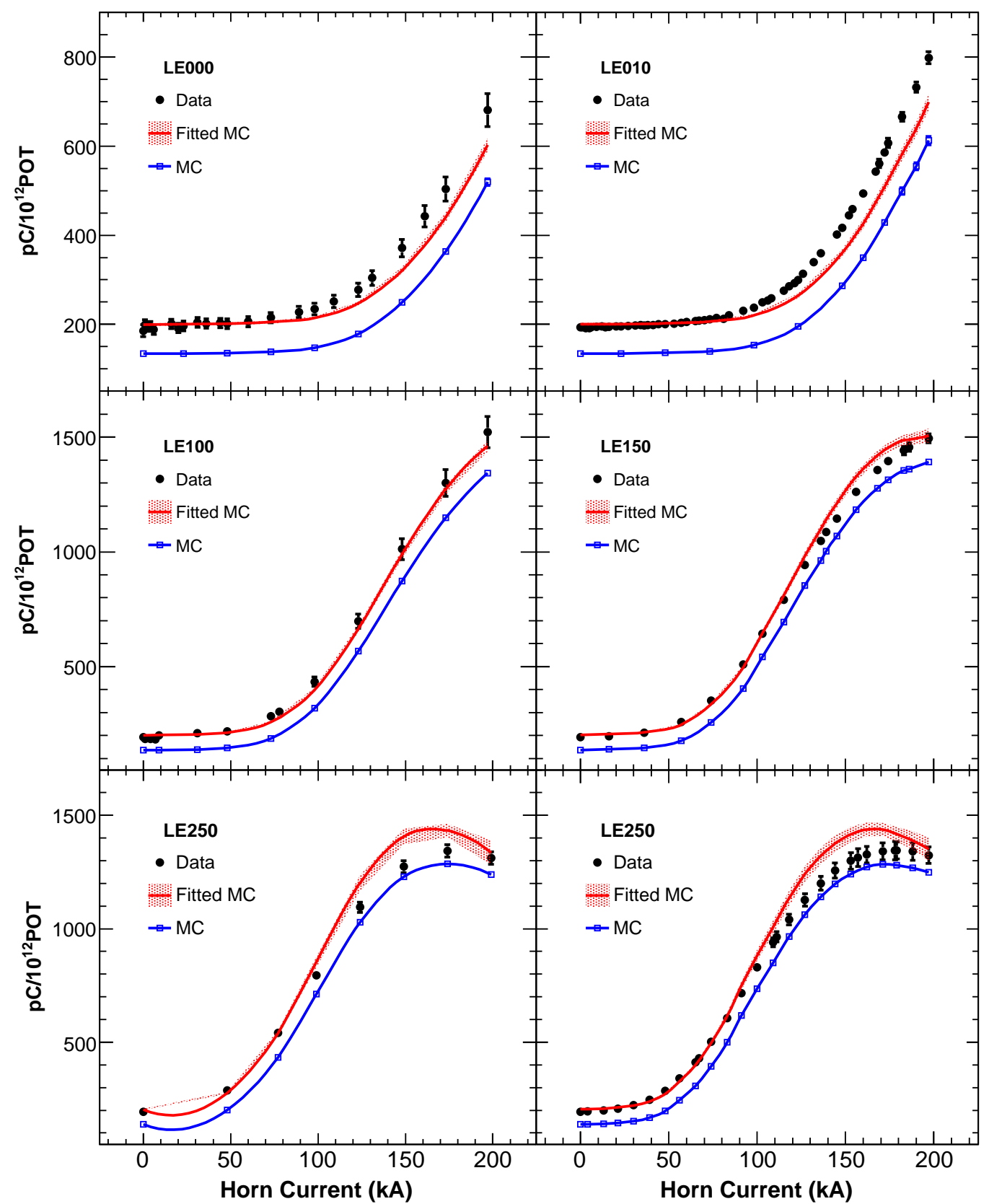

Figure 5.10: The Muon Monitor 1 Monte Carlo horn scans before and after fitting to data. The solid red curves result from the nominal fit. The error band is the sum in quadrature of the difference between the nominal curve and the curves produced by the alternative fits. Better data-Monte Carlo agreement is achieved after fitting. 


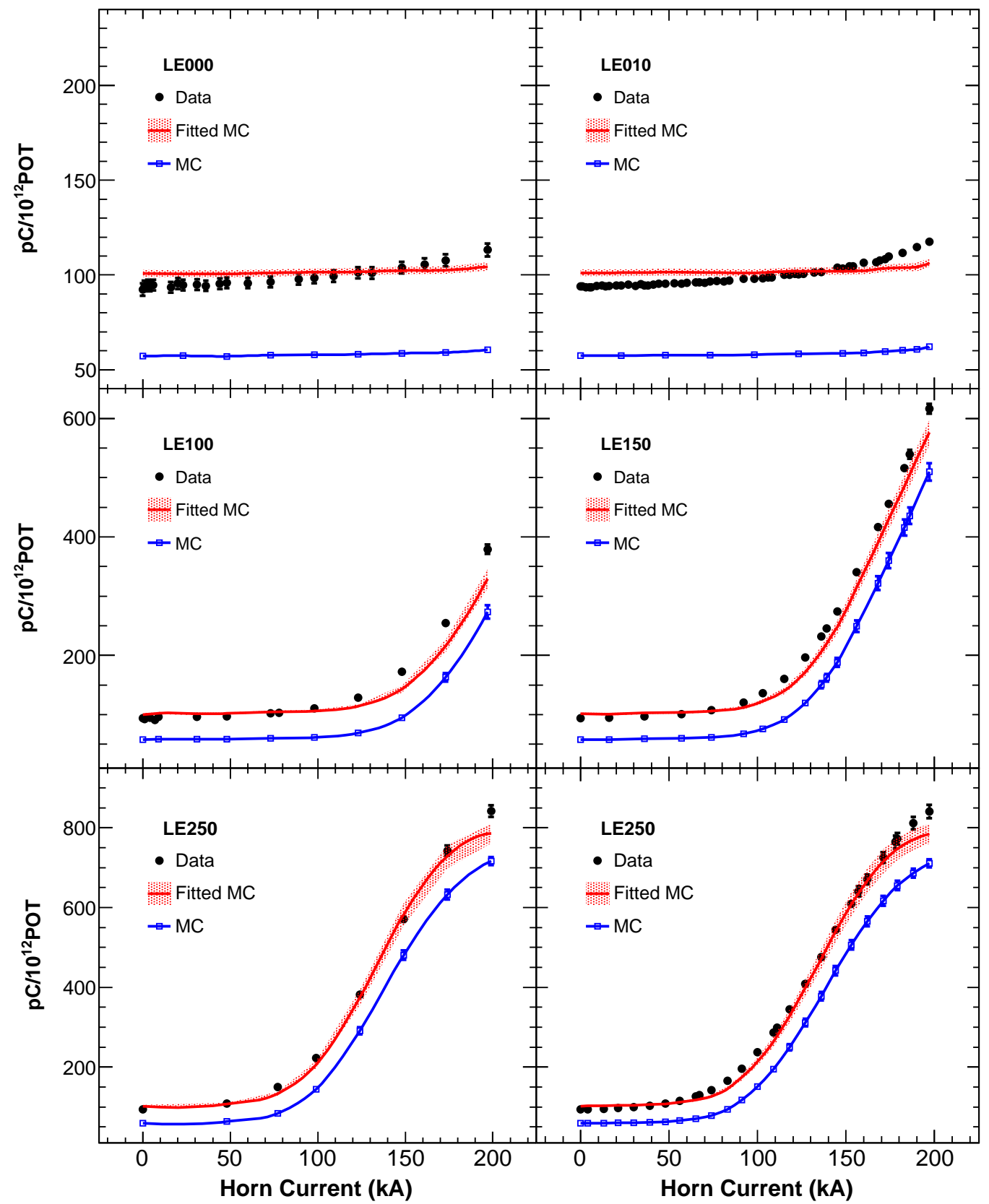

Figure 5.11: The Muon Monitor 2 Monte Carlo horn scans before and after fitting to data. The solid red curves result from the nominal fit. The error band is the sum in quadrature of the difference between the nominal curve and the curves produced by the alternative fits. Better data-Monte Carlo agreement is achieved after fitting. 


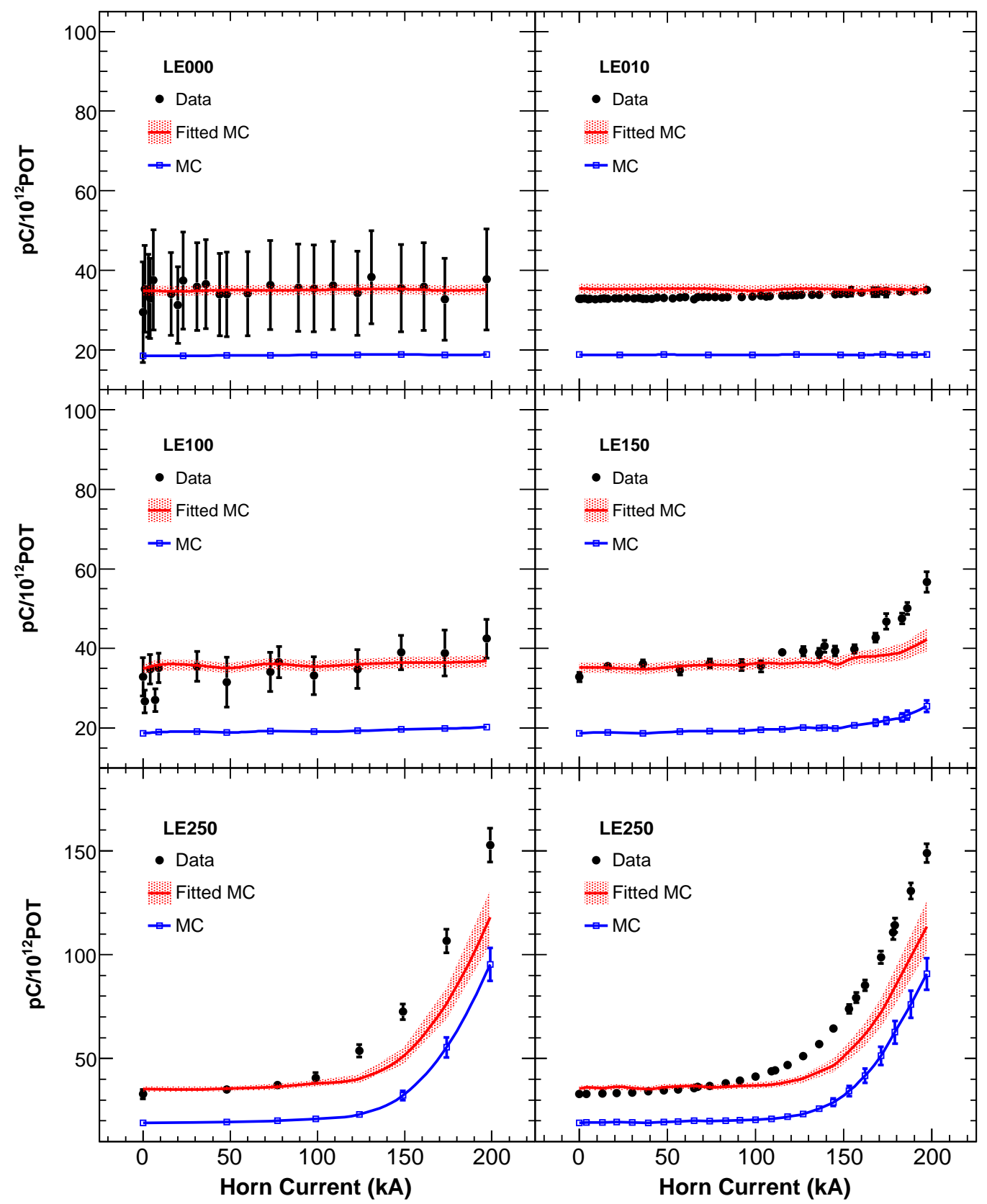

Figure 5.12: The Muon Monitor 3 Monte Carlo horn scans before and after fitting to data. The solid red curves result from the nominal fit. The error band is the sum in quadrature of the difference between the nominal curve and the curves produced by the alternative fits. Better data-Monte Carlo agreement is achieved after fitting. 
band is the sum in quadrature of individual systematic uncertainties discussed in detail in Chapter 6. Better data-Monte Carlo agreement is achieved after fitting but the fit shows tension between the data and Monte Carlo horn scan sets. This is evidenced by the fact that there is agreement in the LE250 Muon Monitor 1 data-fitted Monte Carlo horn scan but still significant disagreement in the LE250 Muon Monitor 3 data-fitted Monte Carlo horn scan. Muon Monitor 1 measures the muons that Muon Monitor 3 measures, so a change in Muon Monitor 3 would result in the same change in Muon Monitor 1. As such, the fit is not perfect, and it may be hoped that residual disagreements between the muon monitor data and the tuned MC are covered by the systematic errors. Further discussion of this can be found in Chapter 8 .

\subsection{Summary}

The muon monitors have been shown to have good acceptance for muons from pions which contribute neutrinos in the MINOS detectors. Measurements of the muon fluxes in the three alcoves at various target positions and horn currents are used to constrain the $\left(x_{F}, p_{T}\right)$ of parent hadrons which lead to neutrinos in the MINOS detectors. A comprehensive Monte Carlo was developed for comparison to data sets taken at various target positions and horn currents. The Monte Carlo has been fit to the muon monitor horn scan data by tuning the underlying $\left(x_{F}, p_{T}\right)$ of parent hadrons. A measurement of the NuMI beam $\nu_{\mu}$ flux as well as a comprehensive study of the associated systematic errors has been made using the muon monitors. A detailed discussion of systematics is presented in Chapter 6. In Chapter 7 the measured $\nu_{\mu}$ flux is used to measure the charged current inclusive $\nu_{\mu}$ cross section on iron using 
the MINOS Detector. 


\section{Chapter 6}

\section{Neutrino Flux Systematics}

\subsection{Systematic Errors}

In Chapter 5, a Monte Carlo model which relates the fluxes observed in the muon monitors to the $\nu_{\mu}$ flux observed at the MINOS Detector is presented. Adjusting the Monte Carlo prediction to match the muon monitor data provides a data-driven estimate of the $\nu_{\mu}$ flux that is unique because it does not use the neutrinos themselves in the estimate. However, the muon monitor data do not, by themselves, possess enough kinematic information to uniquely determine the $\left(x_{F}, p_{T}\right)$ of pions and kaons off of the target. As a result, we necessarily imposed some constraints on the model. These constraints or assumptions are not without error and changes in these corrections, conditions, and constraints result in a slightly different neutrino flux. To quantify the systematic error on the neutrino flux measurement, these conditions are varied and an alternative fit performed for each variation. In each case an alternative set of weights in $\left(p_{T}, p_{z}\right)$ are obtained from the fit producing and alternative 
neutrino flux. Each alternative flux is compared to the flux from the nominal fit and the difference is taken as an error on the nominal flux.

The systematic errors on the neutrino flux measurement are divided into four categories as follows.

1. Backgrounds in the muon detectors

2. $K^{+} / \pi^{+}$ratio off the target

3. $\pi^{+} / \pi^{-}$ratio off the target

4. Charge collection in the muon detectors

The remainder of this section discusses these categories in detail.

\subsubsection{Systematics from Backgrounds}

Backgrounds to muons in the muon monitors are divided into two categories

1. Absorber Backgrounds.

2. $\delta$-rays and Bethe-Block corrections to muon energy deposition.

The background from particles produced in the hadron absorber, discussed in Chapter 3, are directly determined from muon monitor data. In the nominal fit, the backgrounds are fixed to the average value from Table 3.1. To determine the systematic error on the nominal flux from this background, the background in each monitor is varied by $\pm 1 \sigma$, where the uncertainty is also listed in Table 3.1, and reapplied to the MC. Fits are run for both cases $(+1 \sigma$ and $-1 \sigma)$ giving alternative neutrino fluxes. Figure 6.1 shows the percent error on the nominal neutrino flux measurement from varying the absorber 

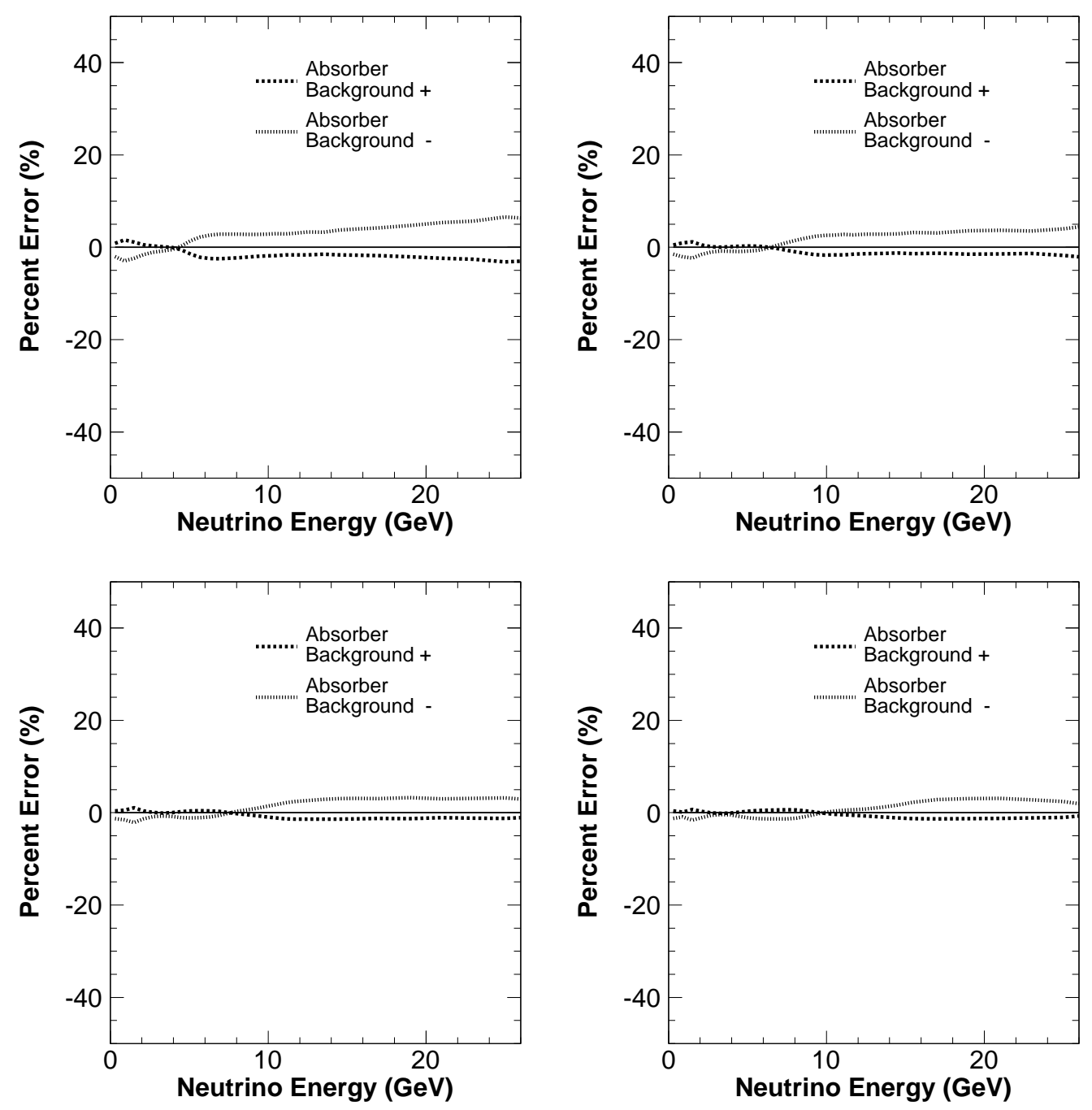

Figure 6.1: The percent error on the neutrino flux measurement before normalization for the LE010/185kA(top left), LE100/200kA(top right), LE150/200kA(bottom left), and LE250/200kA(bottom right) beams from increasing $(+)$ or decreasing $(-)$ the absorber backgrounds in each muon monitor by $1 \sigma$. The + and - curves result from refitting for the flux after either increasing or decreasing the background. 
background in the muon monitors. The effect is relatively small and largely affects the high energy region. This is reflective of the fact that the backgrounds, being an additive quantity, are a larger percentage of the low horn current muon flux region of the horn scans. The low horn current muon flux corresponds to the high energy neutrino flux.

Another component of the uncertainty arises from the simulation of electromagnetic interactions of muons with surrounding media. For example, the energy deposited by a muon in the helium gas of the chambers is dependent upon the energy of the muon according to Bethe-Block [67]. On the other hand the beam tests discussed in Section 3.1.1 used single energy particles, $8 \mathrm{GeV}$ protons in the FNAL test and $42 \mathrm{MeV}$ electrons in the BNL test. The ionization scale obtained from these tests is reflective of the charge deposited by an, for example, $8 \mathrm{GeV}$ proton plus any $\delta$-rays produced by interactions of the protons with the chamber material.

Furthermore, muons passing through the rock and the air in front of the monitors ionize these materials producing external delta rays which intercept the monitor chambers and ionize the helium gas. The bottom left plot in Figure 4.18 shows the energy deposited by these $\delta$-rays in the monitors predicted by Monte Carlo. Like the muon energy deposition, the energy deposited by an external $\delta$-ray depends on the momentum of the $\delta$-ray which in turn depends on the momentum of the muon that created it.

To understand the effect on the fitted neutrino flux, the Monte Carlo generated energy deposition curve discussed in Chapter 4 and shown in Figure 4.19, the internal $\delta$-ray energy deposition curve shown in the bottom right plot of Figure 4.18, and the external $\delta$-ray energy deposition curve, shown in the same figure, are applied to the Monte Carlo horn scans. The beam test 
results are used to convert "energy deposition" to "ionized charge". An $8 \mathrm{GeV}$ proton corresponds in momentum to a muon of $1 \mathrm{GeV} / c$. The total energy deposition at a muon momentum of $1 \mathrm{GeV} / c$ is scaled to be $9.6 \mathrm{pC} / 10^{7} \mu$ 's. Then an alternative set of Monte Carlo horn scans are generated by "looking up" the charge contributed by each muon with a given momentum from each

of these 3 curves. A fit is run giving an alternative neutrino flux. Figure 6.2 shows the resulting percent error on the nominal neutrino flux measurement. The effect is two-fold in that the neutrino flux is scaled up overall but at the same time does exhibit a neutrino energy dependent scaling. This correction is muon momentum dependent which means that it effects the muon flux in the individual beam configurations, target positions and horn currents differently and thus results in an neutrino energy dependent effect on the flux. The effect of adding both the Bethe-Block correction and the delta ray background to the Monte Carlo horn scans is that "too much" muon flux is predicted in the LE150 and LE250 horn current scans in Alcove 1 and 2 compared to horn current scan data. Thus hadron production must decrease in the $\left(p_{T}, p_{z}\right)$ regions affecting these beams resulting in a greatly reduced neutrino flux with respect to the nominal flux. The flux normalization procedure discussed in Section 6.2 reduces the flux error from this effect but this effect remains the largest contribution to the error.

\subsubsection{Systematic Uncertainty from the $K^{+} / \pi^{+}$Ratio}

Figure 4.8 shows the ratio of yields of $K^{+} / \pi^{+}$from a $2 \mathrm{~m}$ long graphite target as predicted by several particle production models. This ratio, or even the yield individually from kaons, is not very well measured in particle production 

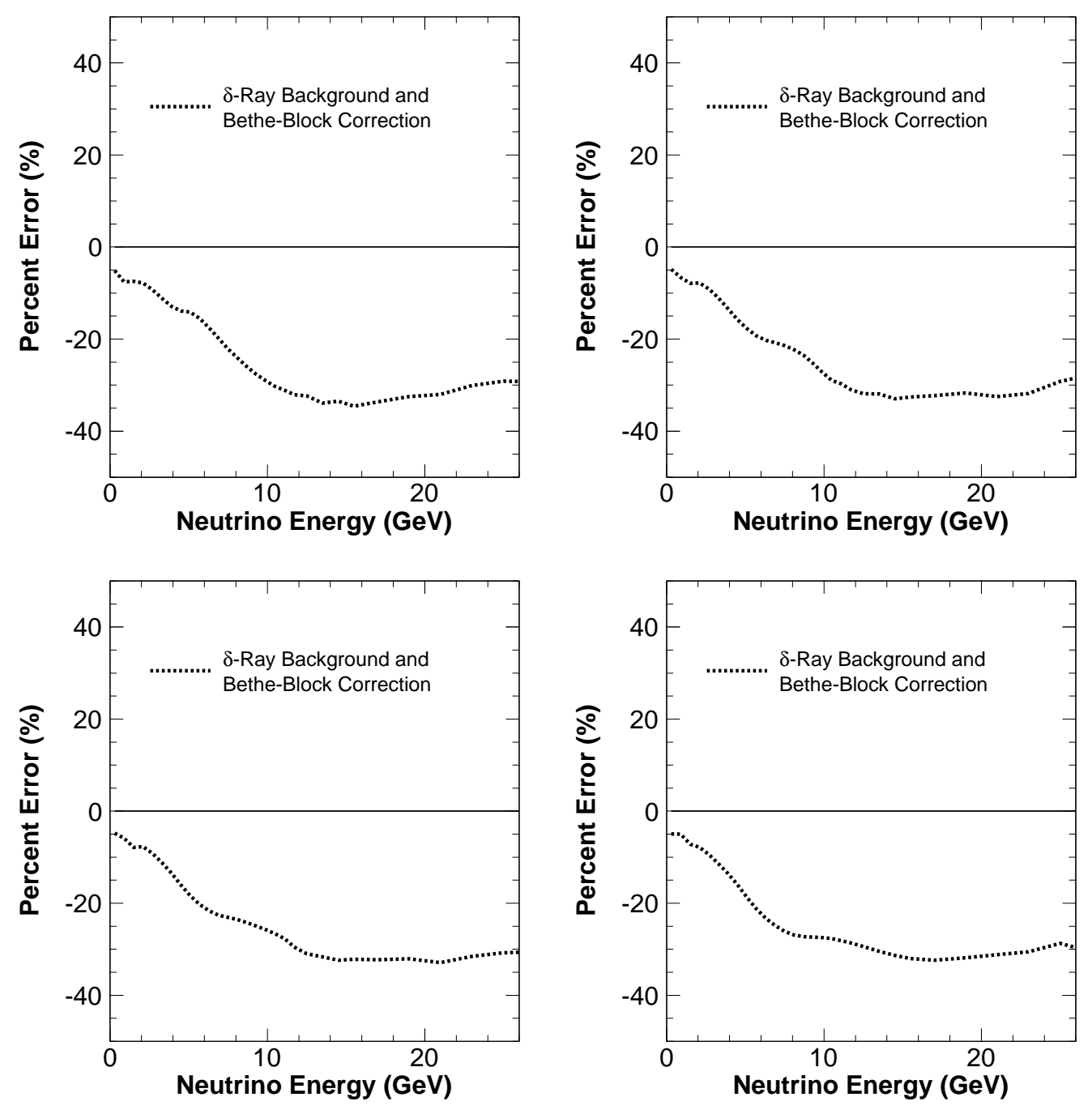

Figure 6.2: The percent error on the neutrino flux measurement before normalization for the LE010/185kA(top left), LE100/200kA(top right), LE150/200kA(bottom left), and LE250/200kA(bottom right) beams from applying Bethe-Block to calculate the momentum dependent energy deposition of charged particles in the muon monitors and from including $\delta$-ray backgrounds in the muon monitors. The curve results from refitting for the cross section after applying these changes. 
experiments $[50,51,52,53]$. The different models are in relatively good agreement with each other, and are in relatively good agreement with a different MINOS analysis [22] which fits for $K / \pi$ yields, within loose constraints, using data in the MINOS Near Detector. Thus, a reasonable error on the Fluka05 $K^{+} / \pi^{+}$is $\pm 10 \%$. Figure 6.3 shows the resulting percent error on the nominal neutrino flux measurement from alternative flux measurements obtained by fitting with the $K^{+} / \pi^{+}$varied by $\pm 10 \%$ of the Fluka05 ratio. The effect of varying the Fluka05 $\mathrm{K}^{+} / \pi^{+}$by $\pm 10 \%$ is about a $\pm 10 \%$ change in the nominal flux at high neutrino energies. While kaons contribute largely low energy muons in the muon monitors, they contribute high energy neutrinos in the MINOS detector. Thus the high energy region of the neutrino flux is effected by changes in the $K^{+} / \pi^{+}$.

\subsubsection{Systematic Uncertainty from the $\pi^{+} / \pi^{-}$Ratio}

The muon monitors see $\mu^{ \pm}$from $\pi^{ \pm}$decays but cannot distinguish the difference in charge. Since, the horns focus $\pi^{+}$and defocus $\pi^{-}$, the muon flux at the muon monitors is dominated by $\mu^{+}$so the muon monitors have good sensitivity to the $\nu_{\mu}$ flux arising from $\pi^{+} \rightarrow \mu^{+} \nu_{\mu}$ decays. Nonetheless, the comparatively small "background" flux of $\mu^{-}$arising from $\pi^{-} \rightarrow \mu^{-} \bar{\nu}_{\mu}$ must be subtracted from the total muon flux in the muon monitors. ${ }^{1}$.

The best constraint on the relative $\pi^{+} / \pi^{-}$yields comes from the NA49 experiment [?], but the NA49 $\pi^{+} / \pi^{-}$only covers half of $x_{F}$ space. A reasonable parameterization of the NA49 $\pi^{+} / \pi^{-}$ratio is a third order polynomial, but other not unreasonable parameterizations exist as shown in Figure 4.9. In

\footnotetext{
${ }^{1}$ The flux of $\mu^{ \pm}$from $K^{ \pm}$decays is smaller by a factor of $10-15$ due to the $K / \pi$ ratio off of the target.
} 

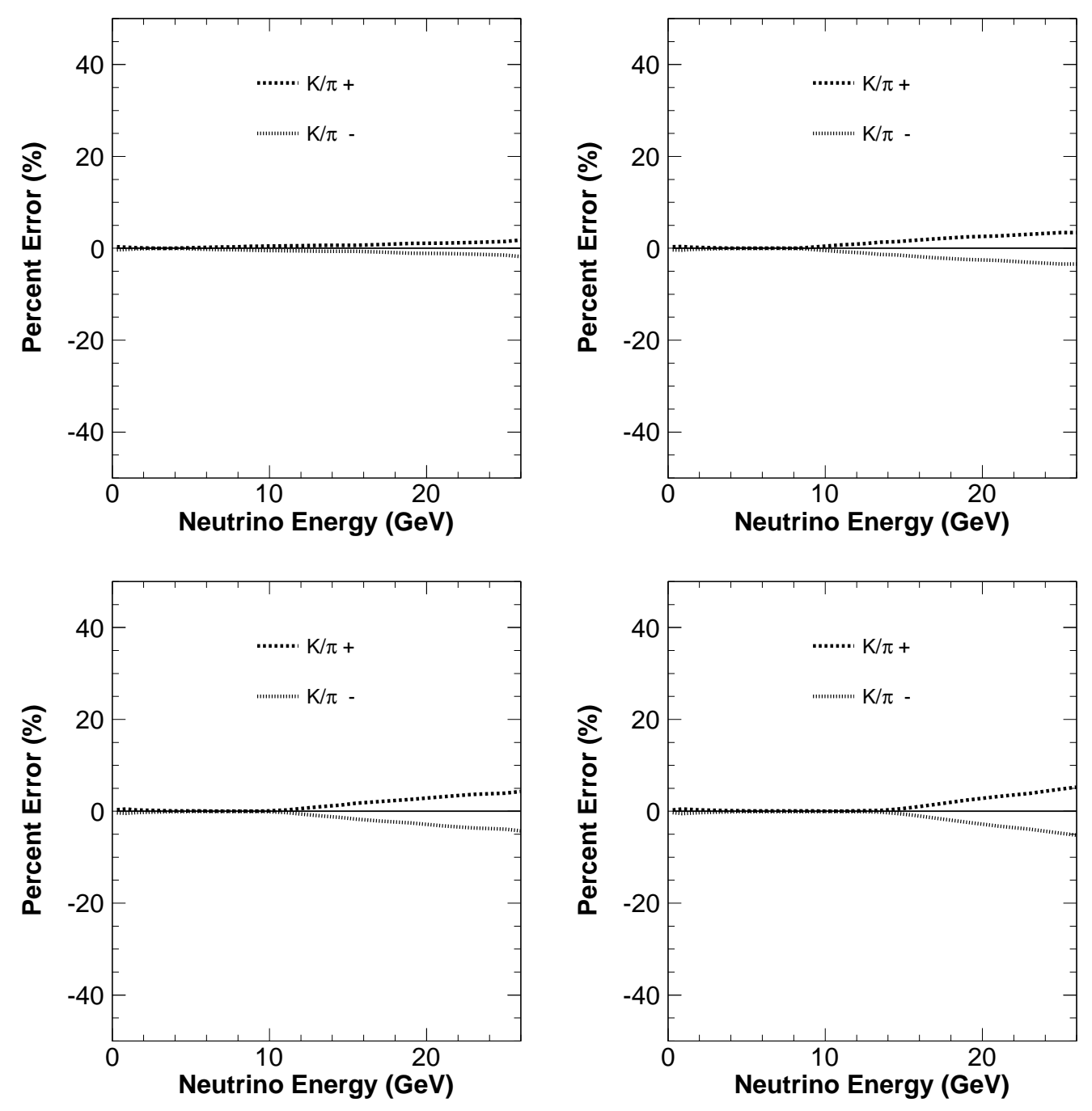

Figure 6.3: The percent error on the neutrino flux measurement for before normalization the LE010/185kA(top left), LE100/200kA(top right), LE150/200kA(bottom left), and LE250/200kA(bottom right) beams from increasing $(+)$ or decreasing $(-)$ the Fluka05 $K^{+} / \pi^{+}$ratio by 10 percent. The + and - curves result from refitting for the flux after either increasing or decreasing the $K^{+} / \pi^{+}$ratio. 
order to quantify the uncertainty in the flux due to different parameterizations of the $\pi^{+} / \pi^{-}$, the $\pi^{+} / \pi^{-}$is fixed to the Fluka05 $\pi^{+} / \pi^{-}$in an alternative fit to see the effect of decreasing the $\pi^{+} / \pi^{-}$on the neutrino flux. Likewise, to to see the effect of increasing the $\pi^{+} / \pi^{-}$on the neutrino flux, an alternative fit is run taking the $\pi^{+} / \pi^{-}$fixed to the NA49 data fit with a fourth order polynomial. Figure 6.4 shows the resulting percent error on the neutrino flux measurement.

The NuMI horns focus positively charged particles and defocus negatively charged particles. Thus, negatively charged pions that contribute muons in the muon monitors and neutrinos in the MINOS Near detector are high energy and pass through the necks of the focusing horns. Changing the $\pi^{+} / \pi^{-}$ ratio affects primarily the high energy tail of the neutrino flux. The focusing peak shifts to higher neutrino energies for higher energy beam configurations. Thus, changing the $\pi^{+} / \pi^{-}$changes the flux for $E_{\nu}$ greater than $5,8,10$, and 15 for the LE010/185kA, LE100/200kA, LE150/200kA and LE250/200kA beams, respectively, as seen in Figure 6.4.

\subsubsection{Charge Collection}

Uncertainties in the conversion of the muon chamber signal into a muon flux come in in two categories: 1 . the ionization scale and 2. non-linearities in the charge collection.

The ionization scale factor obtained from the beam tests mentioned in Section 3.1.1 has an uncertainty of $\pm 10 \%$. In particular the data from the FNAL beam test behaves non-linearly for all bias voltages tested. Thus, the charge per muon scale factor obtained from this data likely has considerable 

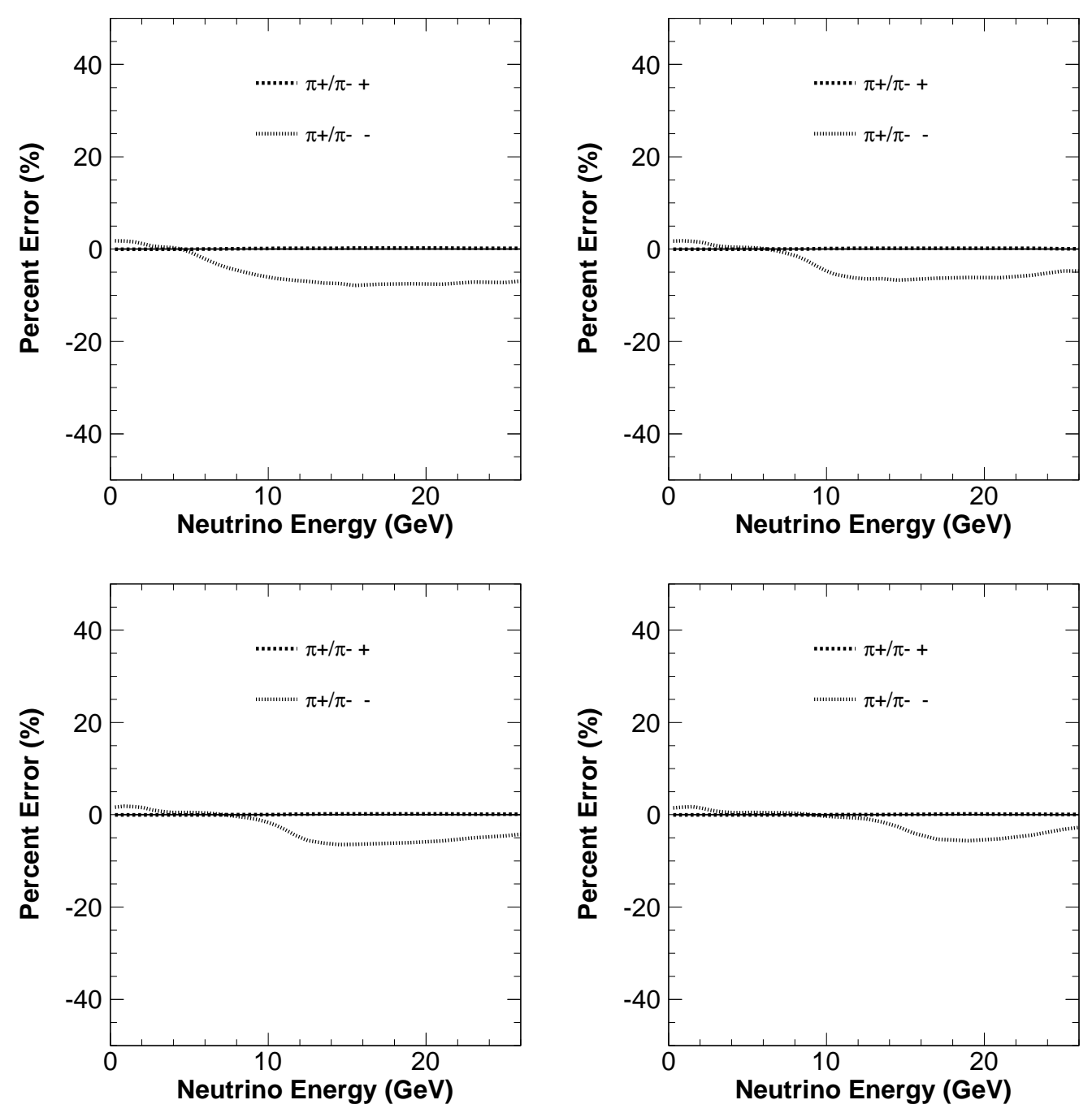

Figure 6.4: The percent error on the neutrino flux measurement before normalization for the LE010/185kA(top left), LE100/200kA(top right), LE150/200kA(bottom left), and LE250/200kA(bottom right) beams from fixing the $\pi^{+} / \pi^{-}$ratio to the NA49 measurement fit with a fourth order polynomial $(+)$, as shown in Figure 4.9, or fixing the $\pi^{+} / \pi^{-}$ratio to the Fluka05 $\pi^{+} / \pi^{-}$ratio (-), also shown in Figure 4.9. The former results in an increase in the $\pi^{+} / \pi^{-}$ratio with respect to nominal(the NA49 measurement fit with a third order polynomial) and the latter results in a decrease in the $\pi^{+} / \pi^{-}$ ratio with respect to nominal. The + and - curves result from refitting for the neutrino flux after either increasing or decreasing the $\pi^{+} / \pi^{-}$ratio. 
error. Also, the charge per muon is dependent upon purity of the helium gas in the chambers. Both the gas used in the beam tests and in the muon monitors is rated $99.998 \%$ pure. However in-situ measurements of gas purity in the chambers are not made and experience has shown that the gas purity in the chambers does fluctuate (Section 3.3). Because of this the horn scan data are scaled to agree at $0 \mathrm{kA}$ as discussed in Section 3.4 and the flux measurement requires normalization as discussed in Section 6.2. The normalization corrects for large variations in the ionization scale. Thus, an error of $\pm 10 \%$ is taken on the scale factor. The ionization scale factor, $9.6 \mathrm{pC} / 10^{7} \mu$ 's, is changed by by $\pm 10 \%$ and reapplied to the Monte Carlo. Figure 6.5 shows the resulting percent error on the nominal neutrino flux measurement from alternative flux measurements obtained by fitting with the ionization scale different by $\pm 10 \%$.

The effect of changing the ionization scale factor is a $\pm 10 \%$ change in the neutrino flux, independent of neutrino energy. This implies that after normalization the error on the neutrino flux measurement from the muon monitor ionization scale will be negligible. This is confirmed in Appendix C.

The linearity correction to the muon monitors also results in an uncertainty in the flux measurement because of the wide range of particle fluxes in the in the muon monitors with changing beam configurations. This nonlinearity correction is determined from muon monitor data as discussed in Section 3.4. The error on this correction is taken to be the greater of half the correction or 0.01. The correction on each data point is shown in Figure 3.23. The linearity correction is varied by $\pm 1 \sigma$ and two alternative fits run. Figure 6.6 shows the resulting percent error on the neutrino flux measurement. The largest effect on the neutrino flux occurs in the high neutrino energy region. This is because the low horn current muon flux, which is most affected 

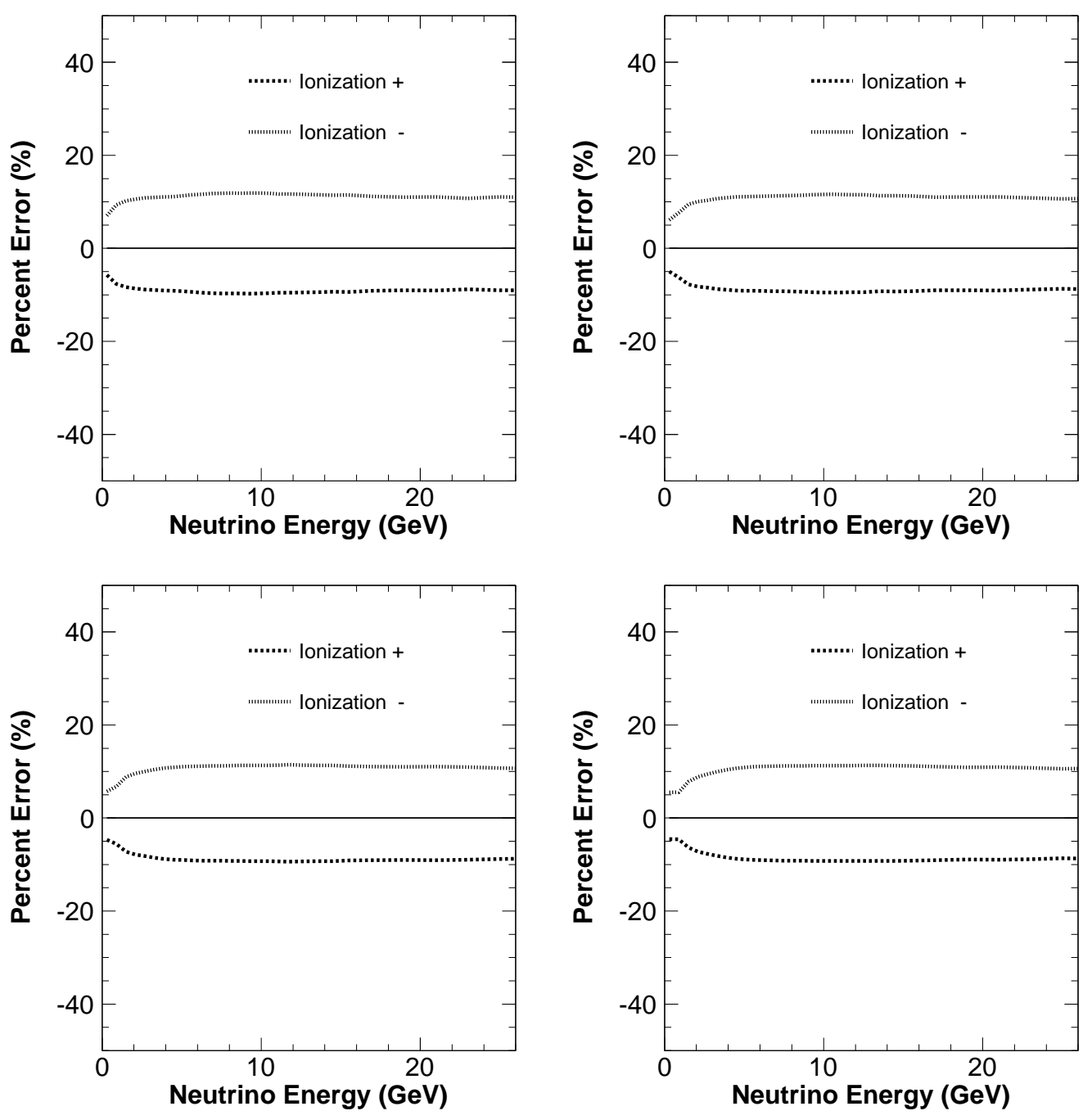

Figure 6.5: The percent error on the neutrino flux measurement before normalization for the LE010/185kA(top left), LE100/200kA(top right), LE150/200kA(bottom left), and LE250/200kA(bottom right) beams from increasing $(+)$ or decreasing (-) the muon monitor ionization scale factor by 10 percent. The + and - curves result from refitting for the flux after either increasing or decreasing the ionization scale factor. 

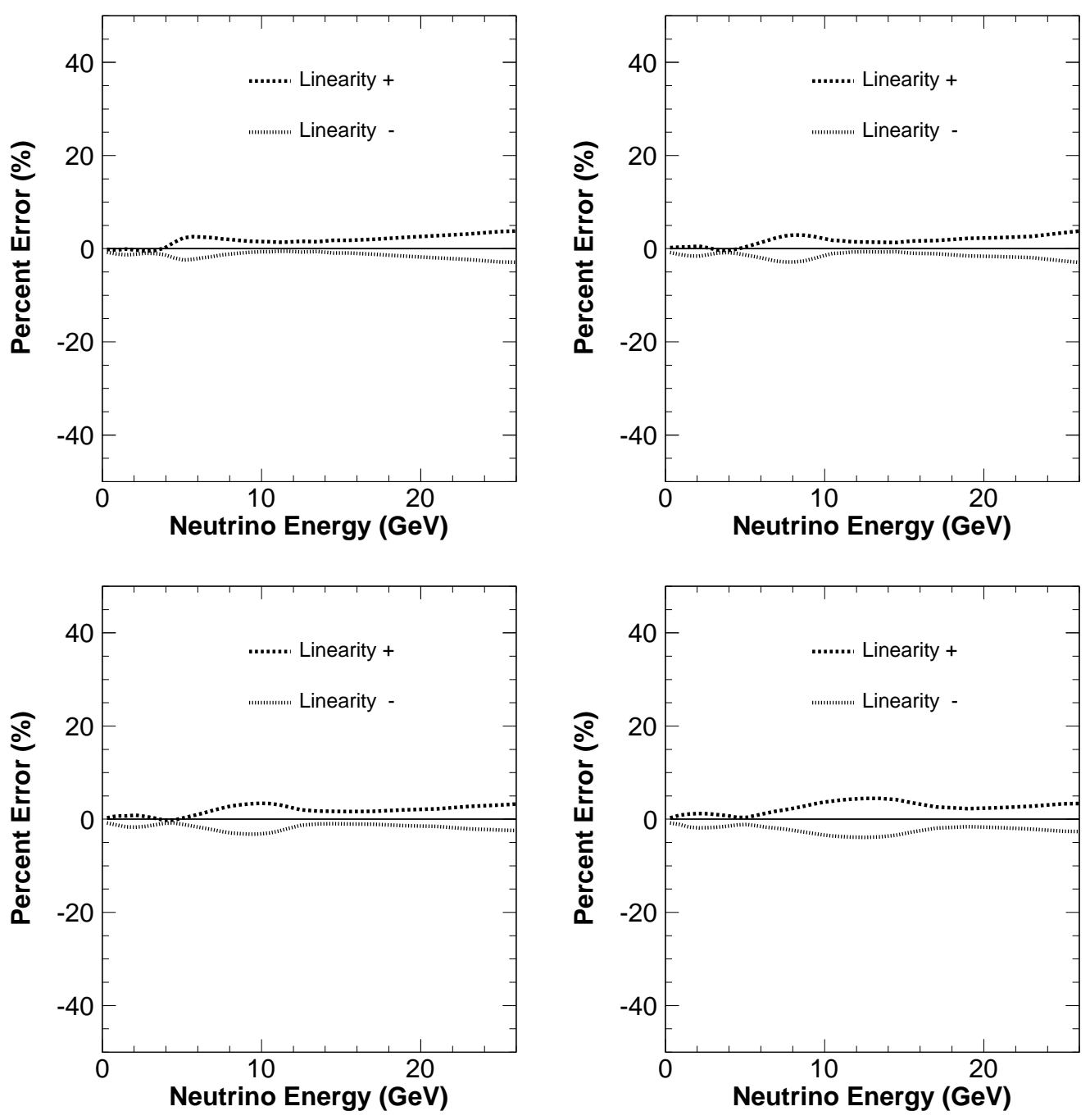

Figure 6.6: The percent error on the neutrino flux measurement before normalization for the LE010/185kA(top left), LE100/200kA(top right), LE150/200kA(bottom left), and LE250/200kA(bottom right) beams from increasing $(+)$ or decreasing $(-)$ the muon monitor the linearity correction by $1 \sigma$. The + and - curves result from refitting for the flux after either increasing or decreasing the the linearity correction. 
by the non-linearity correction, corresponds the the high energy neutrino flux.

\subsection{Normalization}

Beam tests of prototype muon monitor ionization chambers serve well to provide an estimate of the charge ionized per intercepting muon. However, this estimate is not without potentially substantial error. The beam tests of the NuMI beam monitor chambers $[45,46]$ were performed at an unknown absolute pressure and temperature. Although, the gas used in the beam tests and in the muon monitors is rated $99.998 \%$ pure, in-situ measurements of gas purity in the prototype chambers and in the muon monitors were never made. Experience has shown that the gas purity in the chambers does fluctuate as discussed in Section 3.3.1. Thus, an absolute rate fit of the horn scan Monte Carlo to the horn scan data, and therefore an absolute neutrino flux measure-

ment, is not currently possible. The fitted neutrino fluxes are normalized by the following procedure.

The normalization procedure relies on the precise measurements of high energy neutrino cross-sections [68, 69]. Figure 1.9 shows charged current neutrino cross section measurements from several experiments along with theoretical predictions from the NEUGEN neutrino interaction generator. The experimental data above $26 \mathrm{GeV}$ have considerably smaller errors due to the fact that these experiments used narrow band neutrino beams. This facilitated more precise neutrino flux measurements than can be obtained by wide band beam experiments. The MINOS neutrino Monte Carlo uses NEUGEN to model neutrino interactions in the neutrino detectors. As can be seen in Figure 1.9 the NEUGEN charged current inclusive cross-section agrees well 
with experimental data at high neutrino energies. Thus, the MINOS Monte Carlo is used to normalize the fitted flux by scaling the flux reweighted MINOS Monte Carlo to agree with the charged current neutrino event data for neutrino energies between 26 and $40 \mathrm{GeV}$.

The charged current neutrino event data are the black points shown in Figures 7.1 through 7.4. A normalization scale factor for the fitted flux of 0.92 is obtained from the ratio of the charged current neutrino event data, with the flux reweighted event Monte Carlo in the region between $E_{\nu}=26$ and $40 \mathrm{GeV}$. Neutrino cross sections are given by $\sigma_{\nu}=N_{\nu} / \phi_{\nu}$, where $N_{\nu}$ is the number of neutrino events measured in a neutrino detector and $\phi_{\nu}$ is the neutrino flux. By scaling the flux by 0.92 , the cross section obtained by dividing the number of neutrino events in the MINOS Detector with the normalized flux will agree with the NEUGEN cross section and experimental data in the region between $E_{\nu}=26$ and $40 \mathrm{GeV}$.

The normalization constraint, though unsatisfying because it makes this cross section measurement relative to values measured for $E_{\nu}>26 \mathrm{GeV}$, serves to reduce the effect of systematic uncertainties. This occurs because the normalization constraint is always applied last, and in any systematic study in which an alternate Monte Carlo is utilized(eg: one with a different $\pi^{+} / \pi^{-}$ ratio off the target) the normalization constraint serves to reduce some of the variation introduced at high neutrino energies by the alternate Monte Carlo.

Figures 6.7 and 6.8 show the percent error on the flux measurement before and after application of the normalization constant. The 4 dashed curves correspond to the 4 categories of flux systematics listed in Section 6.1. The Backgrounds systematic error is the sum in quadrature of the absorber background systematic error and the $\delta$-ray background systematic error with the 

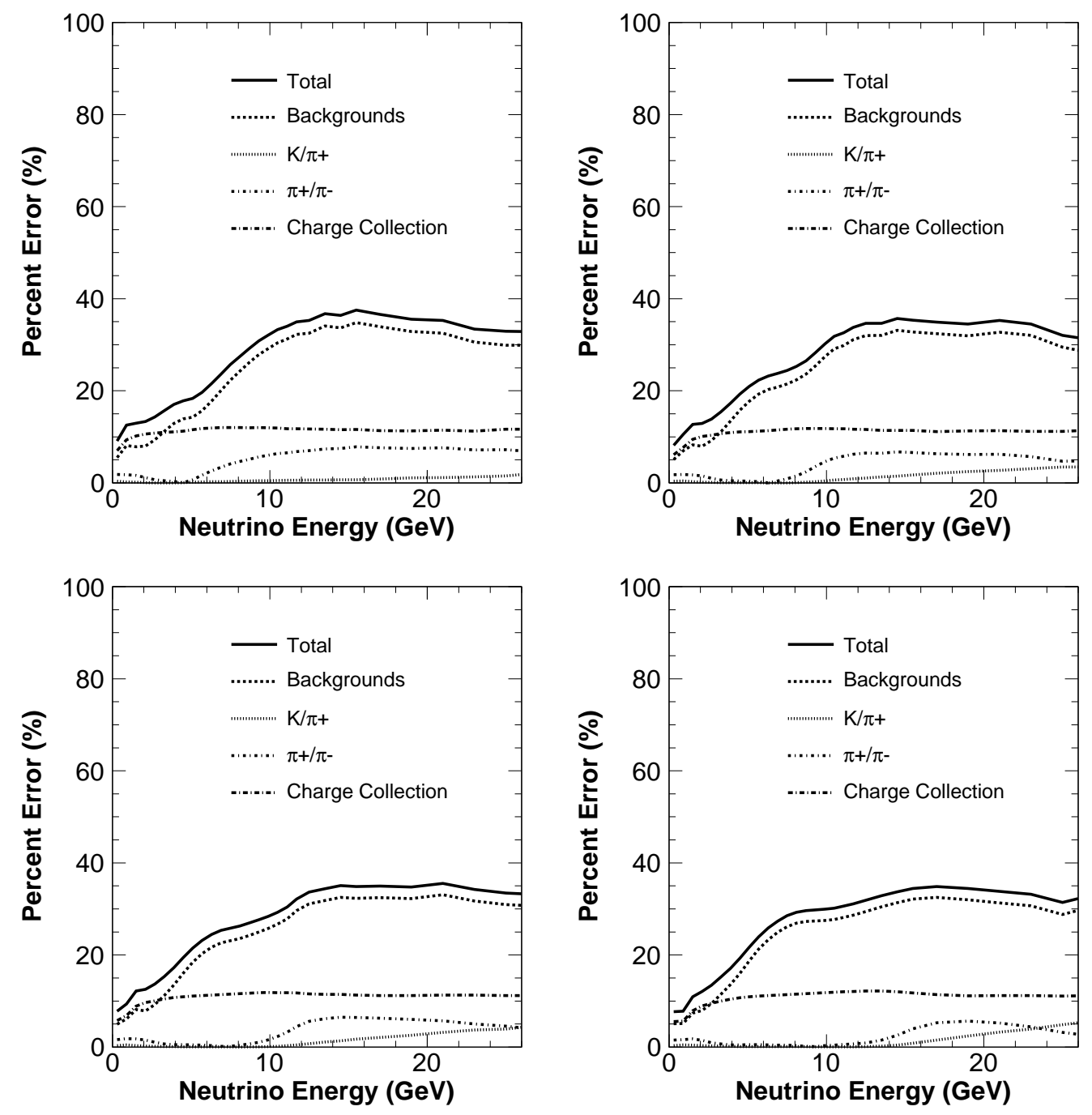

Figure 6.7: The percent error on the neutrino flux measurement before normalization for the LE010/185kA(top left), LE100/200kA(top right), LE150/200kA(bottom left), and LE250/200kA(bottom right) beams. The 4 dashed curves correspond to the 4 categories of flux systematics listed in Section 6.1. The Backgrounds systematic error is the sum in quadrature of the absorber background systematic error and the $\delta$-ray background systematic error with the correction for the Bethe-Block energy loss by charged particles. The Charge Collection systematic error is the sum in quadrature of the ionization energy scale systematic error and the muon monitor linearity systematic error. The Total error is the sum in quadrature of the errors from all of the systematic effects. 

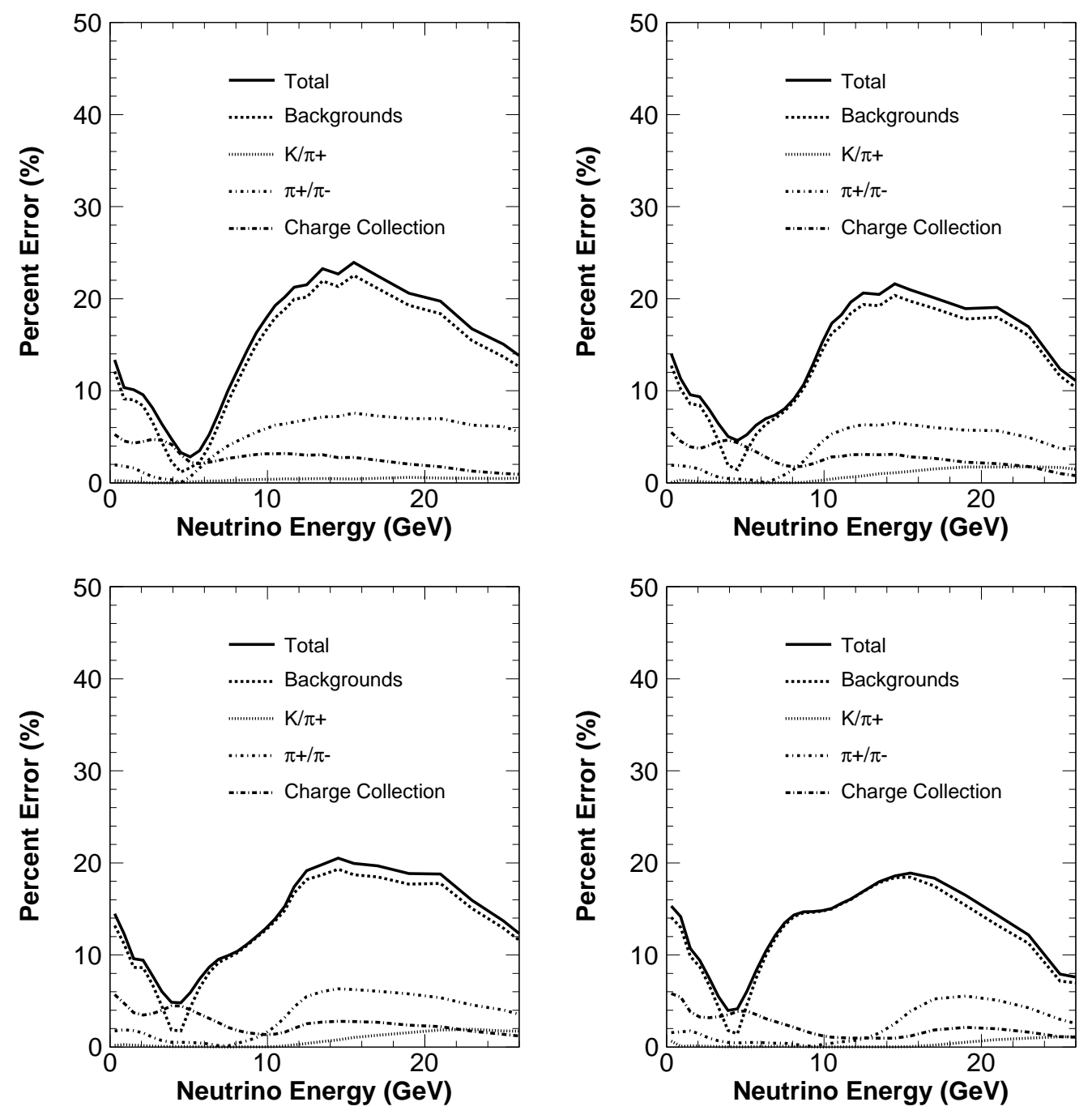

Figure 6.8: The percent error on the neutrino flux measurement after normalization for the LE010/185kA(top left), LE100/200kA(top right), LE150/200kA(bottom left), and LE250/200kA(bottom right) beams. The 4 dashed curves correspond to the 4 categories of flux systematics listed in Section 6.1. The Backgrounds systematic error is the sum in quadrature of the absorber background systematic error and the $\delta$-ray background systematic error with the correction for the Bethe-Block energy loss by charged particles. The Charge Collection systematic error is the sum in quadrature of the ionization energy scale systematic error and the muon monitor linearity systematic error. The Total error is the sum in quadrature of the errors from all of the systematic effects. 
correction for the Bethe-Block energy loss by charged particles. The Charge Collection systematic error is the sum in quadrature of the ionization energy scale systematic error and the muon monitor linearity systematic error. The Total error is the sum in quadrature of the errors from all of the systematic effects. As can be seen in Figure 6.8, the percent error on the nominal flux measurement from the systematics after normalization is reduced.

Appendix $\mathrm{C}$ shows the percent error on the nominal flux measurement contributed by each of the individual systematics after normalization. The error on the nominal flux is significantly reduced by normalization.

Figures 6.9-6.12 provide more detailed views of the flux for each of the 4 beam configurations, also showing the high energy portion of the beam spectra. 

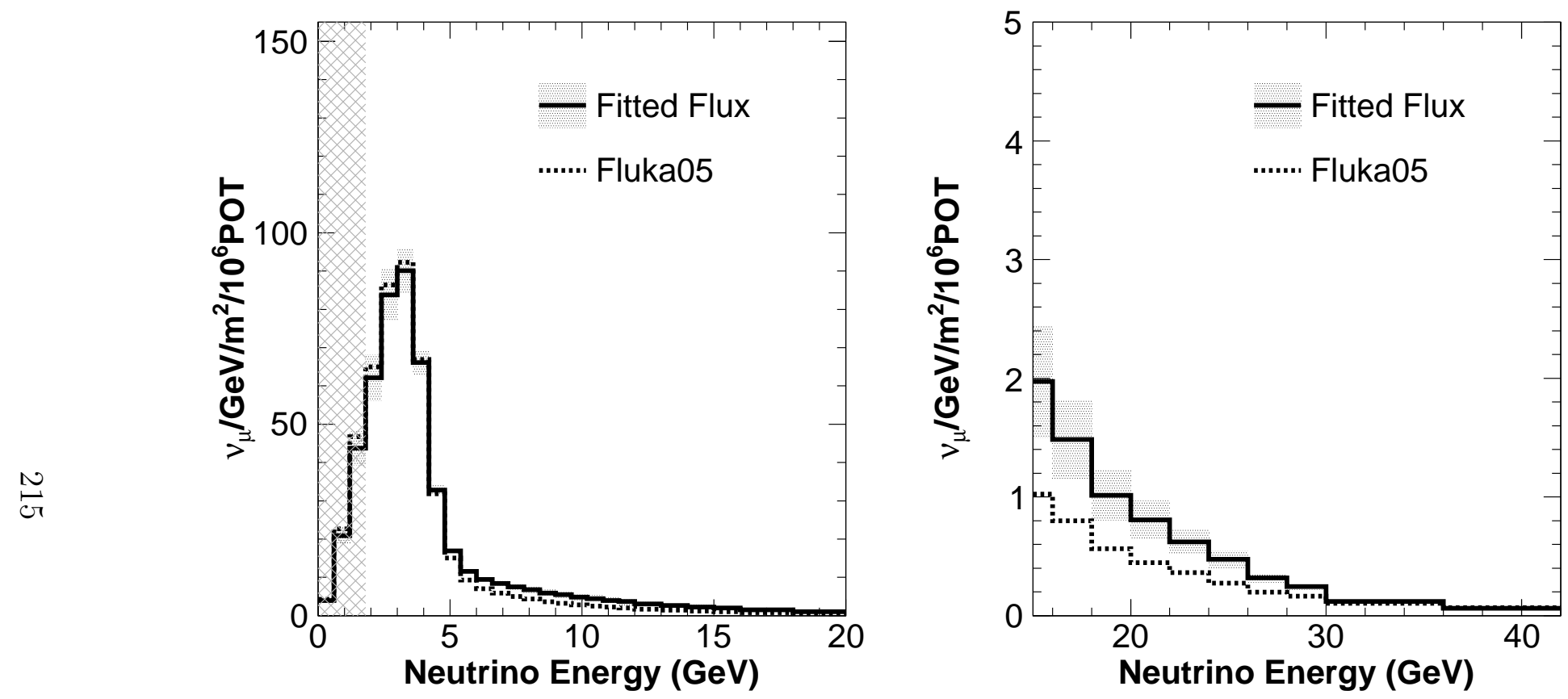

Figure 6.9: The normalized fitted Monte Carlo $\nu_{\mu}$ flux at the MINOS Near Detector for the LE010/185kA beam. The solid black curve is the nominal fit reweighted Monte Carlo flux. The gray error bands are the total errors on the nominal flux after normalization as discussed in Chapter 6. The dashed black curve is the default Near Detector Monte Carlo flux generated with Fluka05 hadron production. The default Monte Carlo is shown only for reference. The gray hatched region is an extrapolation of the fitted hadron production parameters The region $E_{\nu}>40 \mathrm{GeV}$ are is used for normalization. 

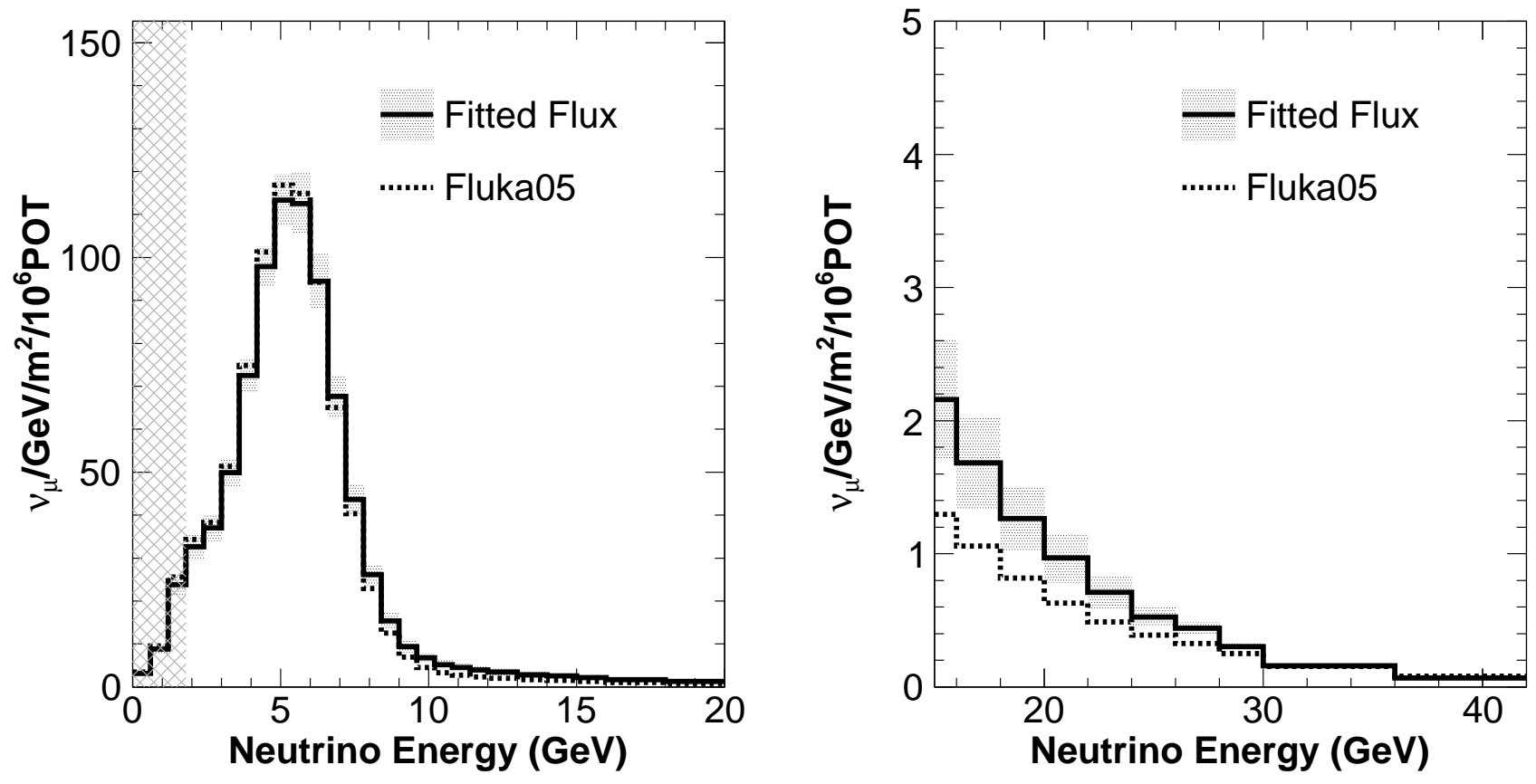

Figure 6.10: The normalized fitted Monte Carlo $\nu_{\mu}$ flux at the MINOS Near Detector for the LE100/200kA beam. The solid black curve is the nominal fit reweighted Monte Carlo flux. The gray error bands are the total errors on the nominal flux after normalization as discussed in Chapter 6 . The dashed black curve is the default Near Detector Monte Carlo flux generated with Fluka05 hadron production. The default Monte Carlo is shown only for reference. The gray hatched region is an extrapolation of the fitted hadron production parameters The region $E_{\nu}>40 \mathrm{GeV}$ are is used for normalization. 

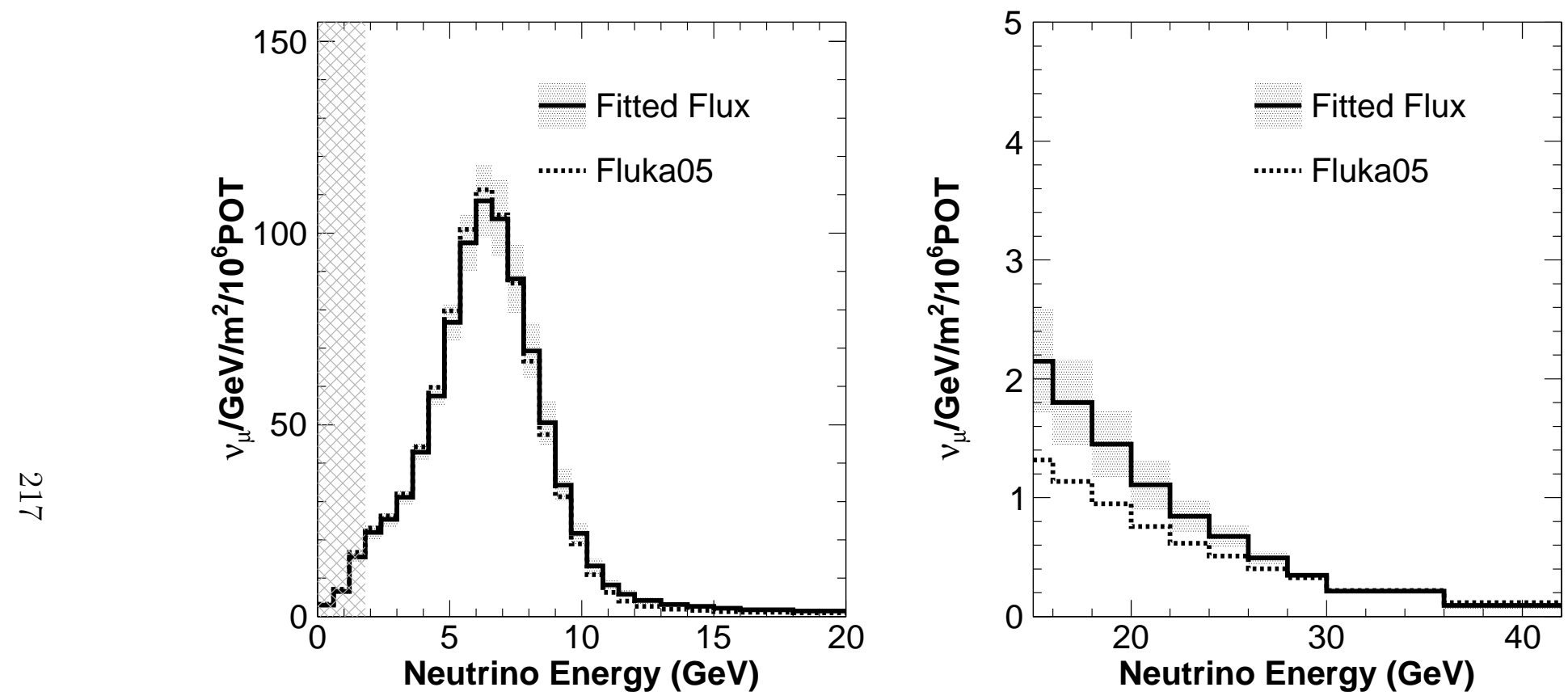

Figure 6.11: The normalized fitted Monte Carlo $\nu_{\mu}$ flux at the MINOS Near Detector for the LE150/200kA beam. The solid black curve is the nominal fit reweighted Monte Carlo flux. The gray error bands are the total errors on the nominal flux after normalization as discussed in Chapter 6. The dashed black curve is the default Near Detector Monte Carlo flux generated with Fluka05 hadron production. The default Monte Carlo is shown only for reference. The gray hatched region is an extrapolation of the fitted hadron production parameters The region $E_{\nu}>40 \mathrm{GeV}$ are is used for normalization. 

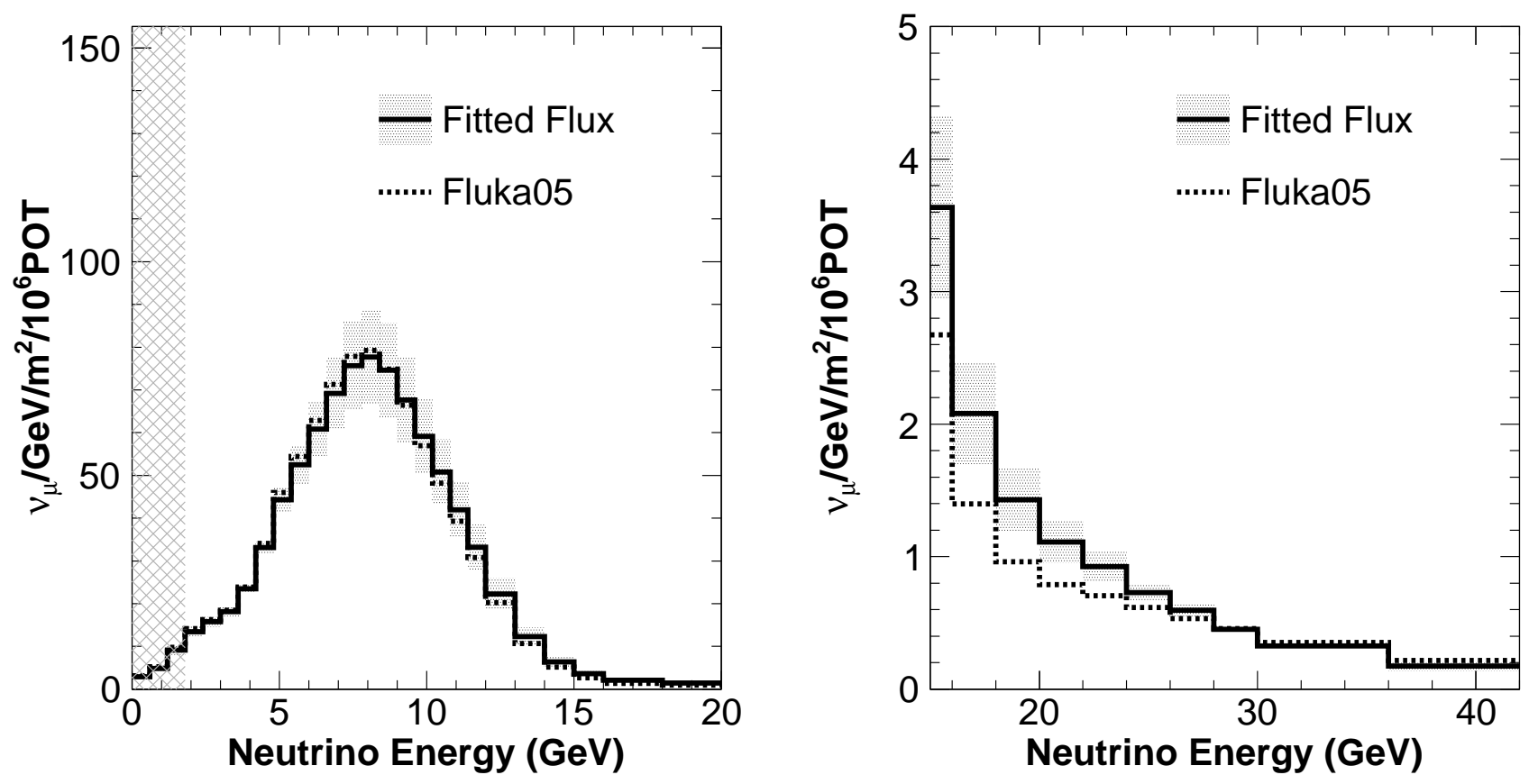

Figure 6.12: The normalized fitted Monte Carlo $\nu_{\mu}$ flux at the MINOS Near Detector for the LE250/200kA beam. The solid black curve is the nominal fit reweighted Monte Carlo flux. The gray error bands are the total errors on the nominal flux after normalization as discussed in Chapter 6 . The dashed black curve is the default Near Detector Monte Carlo flux generated with Fluka05 hadron production. The default Monte Carlo is shown only for reference. The gray hatched region is an extrapolation of the fitted hadron production parameters The region $E_{\nu}>40 \mathrm{GeV}$ are is used for normalization. 


\section{Chapter 7}

\section{Cross Section Measurement}

\subsection{Introduction}

The goal of this thesis is to measure the cross section for charged current interactions of muon neutrinos on iron as a function of the energy of the incident neutrino. By definition the interaction cross section for neutrinos in a neutrino detector is

$$
\sigma_{\nu}\left(E_{\nu}\right)=\frac{N_{\nu}\left(E_{\nu}\right)}{\phi_{\nu}\left(E_{\nu}\right)},
$$

where $N_{\nu}\left(E_{\nu}\right)$ is the number of neutrino interactions in the fiducial volume of the neutrino detector in terms of the true neutrino energy and $\phi_{\nu}\left(E_{\nu}\right)$ is the neutrino flux through the fiducial volume of the neutrino detector in terms of the neutrino energy. The variable $E_{\nu}$ is the actual energy of the neutrino to be contrasted with the energy as measured by the detector.

The neutrino detector measures both the rate and energy of neutrino $\mathrm{CC}$ interactions but with uncertainties in both. Uncertainties in the rate mea- 
surement arise from in efficiencies to identify $\mathrm{CC}$ interactions in the detector and may be corrected for using a Monte Carlo simulation of the detector. Uncertainties in the energy measurement arise from imperfect tracking of the muon and from incomplete measurement of the recoiling hadrons' energy in the calorimeter. Energy mismeasurements are particularly problematic because neutrinos of an energy $E_{\nu}^{\text {true }}$ are recorded in the detector at an incorrect energy $E_{\nu}^{r e c o}$. The relationship between the reconstructed number of events and the true number is

$$
N_{\nu}\left(E_{\nu}^{\text {true }}\right)=\frac{N_{\text {sel }}\left(E_{\nu}^{\text {reco }}\right)-N_{\text {sel }}^{\text {bkg }}\left(E_{\nu}^{\text {reco }}\right)-N_{\text {sel }}^{\text {non-fid }}\left(E_{\nu}^{\text {reco }}\right)}{\epsilon\left(E_{\nu}^{\text {true }}\right)} \times M\left(E_{\nu}^{\text {true }}, E_{\nu}^{\text {reco }}\right)
$$

The neutrino event selection algorithm requires the event to be reconstructed in the fiducial volume. $N_{s e l}^{b k g}\left(E_{\nu}^{r e c o}\right)$ is the number of true background events selected(reconstructed in the fiducial volume) as signal events. $N_{s e l}^{n o n-f i d}\left(E_{\nu}^{r e c o}\right)$ is the number of selected true signal events with true nonfiducial interaction vertex. Thus the numerator is the number of selected true signal events with interaction vertices inside the fiducial volume. Since truth can only be determined in Monte Carlo, both $N_{s e l}^{b k g}\left(E_{\nu}^{r e c o}\right)$ and $N_{s e l}^{\text {non-fid }}\left(E_{\nu}^{r e c o}\right)$ are derived from Monte Carlo. $M\left(E_{\nu}^{\text {true }}, E_{\nu}^{r e c o}\right)$ is a matrix which takes $E_{\nu}^{\text {reco }}$ and converts it to $E_{\nu}^{\text {true }}$. The factor $\epsilon\left(E_{\nu}^{\text {true }}\right)$ is the efficiency of the reconstruction algorithm to identify signal events with true fiducial vertices in the fiducial volume and the efficiency to reconstruct one event for every true event. Since the matrix, $M$, and the efficiency, $\epsilon$, use truth information, they are derived from Monte Carlo.

For this analysis, the Monte Carlo simulation of neutrino interactions in 
the MINOS Detector is used to derive the matrix $M\left(E_{\nu}^{\text {true }}, E_{\nu}^{r e c o}\right)$. We do not attempt to invert this large-dimensional matrix, but rather we will fit directly to the reconstructed rate in the MINOS Near Detector, varying the underlying cross section as a function of neutrino energy to obtain a set of fitted values for $\sigma_{\nu}$ in bins of $E_{\nu}^{\text {true }}$.

\subsection{The Method}

A measurement of the $\mathrm{CC} \nu_{\mu}$ inclusive cross section is made by first selecting CC $\nu_{\mu}$ event sample in both the data sample from the MINOS Near Detector and the Monte Carlo simulation of such a data set, using the selection method discussed in Chapter 2. The Monte Carlo sample is then reweighted using the flux parameters determined by the measurement of the muon flux using the muon monitors as described in Chapter 5. At this point, the reweighted Monte Carlo selection represents the best estimate of the neutrino event spectrum in the MINOS Detector. The cross section model used in the MINOS Monte Carlo is NEUGEN [11].

The flux reweighted Monte Carlo CC $\nu_{\mu}$ event sample and the Near Detector data CC $\nu_{\mu}$ sample have been reconstructed identically and thus contain the energy smearing, event misreconstruction and event misidentification. Thus these data and Monte Carlo samples can be compared without the need to quantify these effects as is necessary for the unfolding method.

Table 7.1 lists the proton exposure of the Data CC $\nu_{\mu}$ event samples for each beam configuration. The statistics in the LE010/185kA beam are greater than 8 times the statistics in the other beam configurations. The cross section is measured by fitting each of the beams, LE010/185kA, LE100/200kA, 
LE150/200kA and LE250/200kA, simultaneously. In order to allow each beam to contribute equally to the cross section measurement, all event samples are scaled to an exposure of $1 \times 10^{18}$ protons.

\begin{tabular}{c|c} 
Beam Configuration & POT/1e18 \\
\hline \hline LE010/185kA (Run I) & 126.01 \\
LE100/200kA (Run I) & 1.02 \\
LE150/200kA (Run II) & 1.87 \\
LE250/200kA (Run II) & 15.46 \\
\hline \hline
\end{tabular}

Table 7.1: The number of protons on target(POT) in each data CC $\nu_{\mu}$ event sample used for the $\nu_{\mu}$ cross section measurement.

Figures 7.1 through 7.4 show the event rate in the MINOS Detector as a function of the energy of the neutrino, as recorded in the detector. Hence, the horizontal axis has implicit in it all the resolution smearing of energy which arises from the imperfect reconstruction of the detector. The vertical axis is event rate in the fiducial volume of the detector (Chapter 2), and implicitly has all of the backgrounds, detector inefficiencies, as well as the beam flux and interaction cross sections. The MINOS data are shown as data points. The Monte Carlo, including the flux measurements from Chapter 4, is shown in blue, where the shaded band represents the flux error from Chapter 5. Clearly, discrepancies exist between the observed event rate and the flux-tuned Monte Carlo calculation. These discrepancies are attributed to in accuracies in the NEUGEN cross section model utilized in the Monte Carlo prediction. Therefore, we use the ratio of the data and the blue Monte Carlo curve as the correction factors to the NEUGEN model to obtain a measurement for the cross section. Because these are in fact 4 beam spectra and because we want to obtain these correction factors as a function of the actual neutrino 


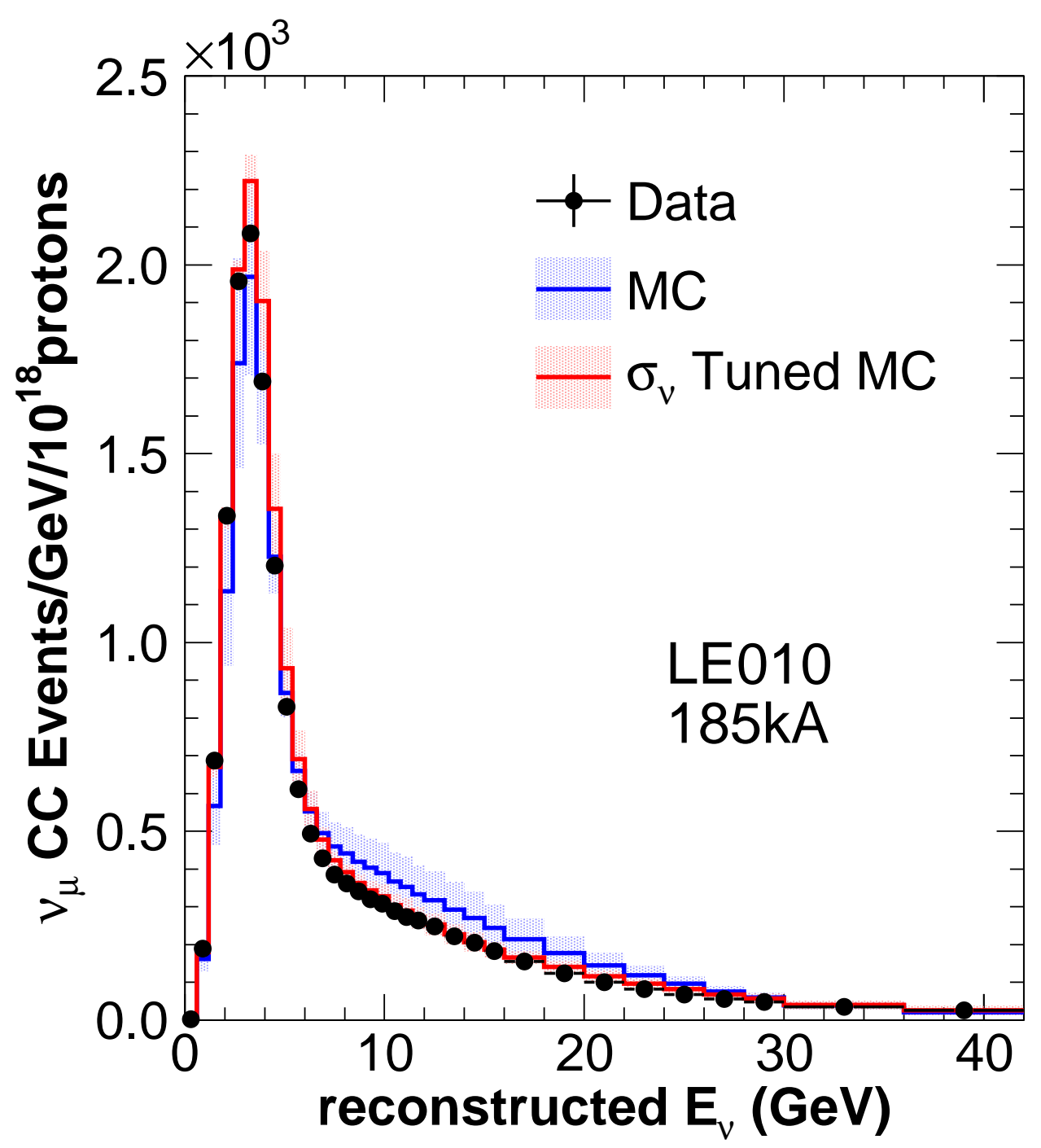

Figure 7.1: MINOS Detector spectrum in the LE010/185kA beam configuration. The black points are MINOS Detector Data selected with the $\nu_{\mu}$ event selection with statistical errors. The blue histogram is the flux reweighted MINOS Detector Monte Carlo. The blue shaded bars represent the sum in quadrature of the differences between the nominal Monte Carlo spectrum and each of the alternative spectra (The alternative fiducial volume spectrum and the alternative selection cut spectrum are not included). The red histogram results from fitting the flux reweighted Monte Carlo spectrum(blue histogram) to the Data spectrum(black points). The red shaded bars represent the sum in quadrature of the difference in each alternative fitted spectrum with the nominal fitted spectrum(red histogram). 


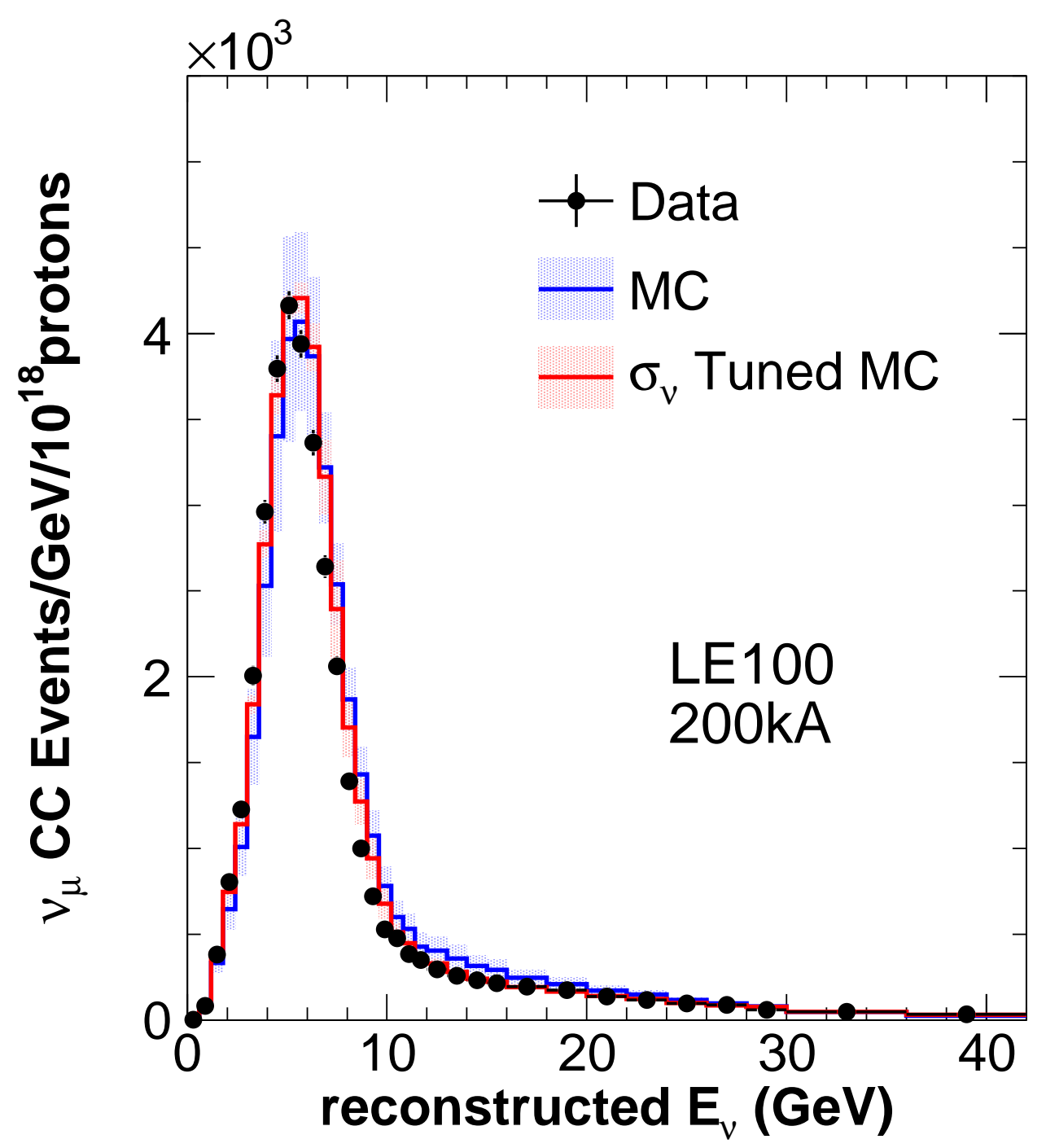

Figure 7.2: MINOS Detector spectrum in the LE100/200kA beam configuration. The black points are MINOS Detector Data selected with the $\nu_{\mu}$ event selection with statistical errors. The blue histogram is the flux reweighted MINOS Detector Monte Carlo. The blue shaded bars represent the sum in quadrature of the differences between the nominal Monte Carlo spectrum and each of the alternative spectra (The alternative fiducial volume spectrum and the alternative selection cut spectrum are not included). The red histogram results from fitting the flux reweighted Monte Carlo spectrum(blue histogram) to the Data spectrum(black points). The red shaded bars represent the sum in quadrature of the difference in each alternative fitted spectrum with the nominal fitted spectrum(red histogram). 


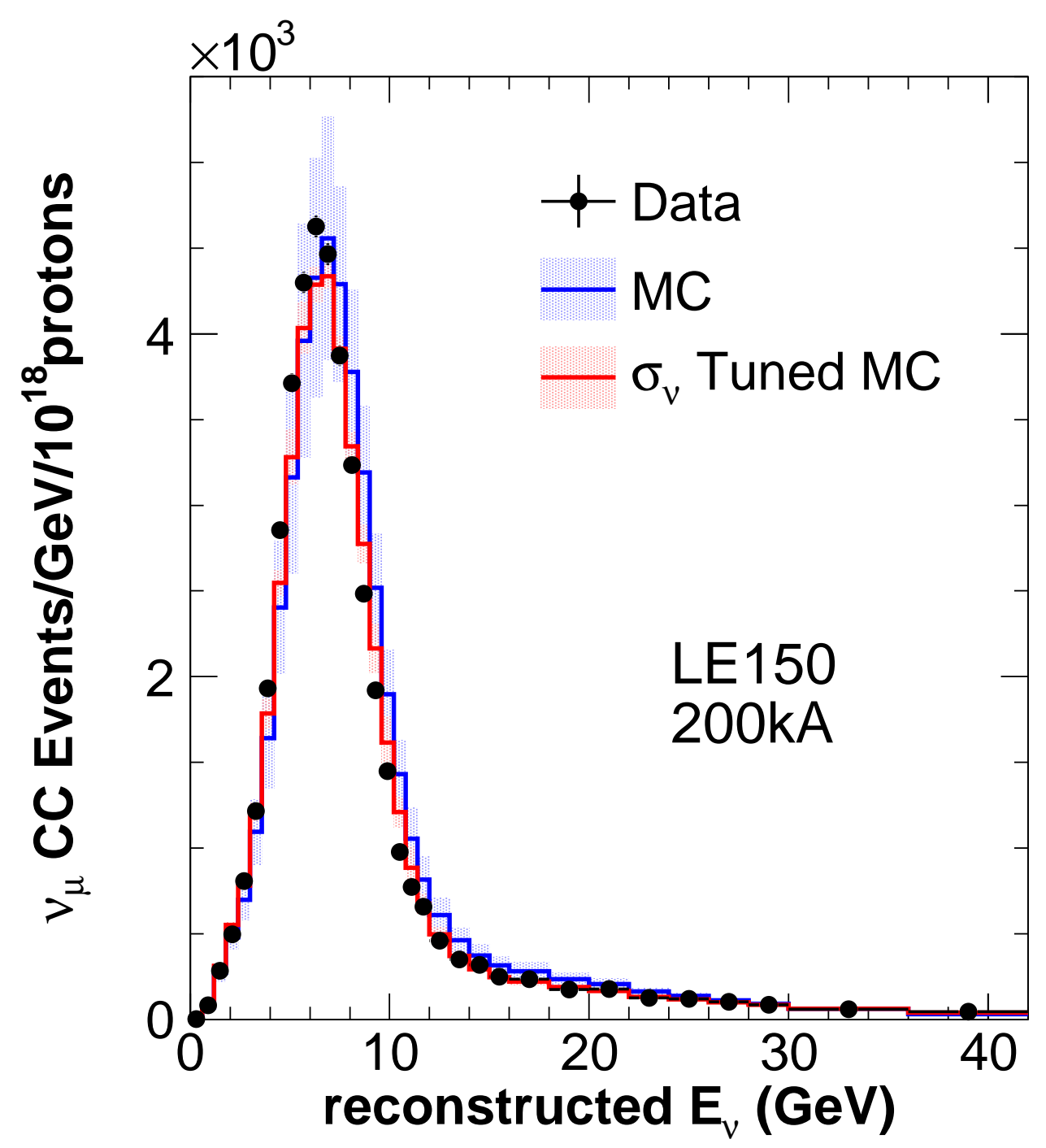

Figure 7.3: MINOS Detector spectrum in the LE150/200kA beam configuration. The black points are MINOS Detector Data selected with the $\nu_{\mu}$ event selection with statistical errors. The blue histogram is the flux reweighted MINOS Detector Monte Carlo. The blue shaded bars represent the sum in quadrature of the differences between the nominal Monte Carlo spectrum and each of the alternative spectra (The alternative fiducial volume spectrum and the alternative selection cut spectrum are not included). The red histogram results from fitting the flux reweighted Monte Carlo spectrum(blue histogram) to the Data spectrum(black points). The red shaded bars represent the sum in quadrature of the difference in each alternative fitted spectrum with the nominal fitted spectrum(red histogram). 


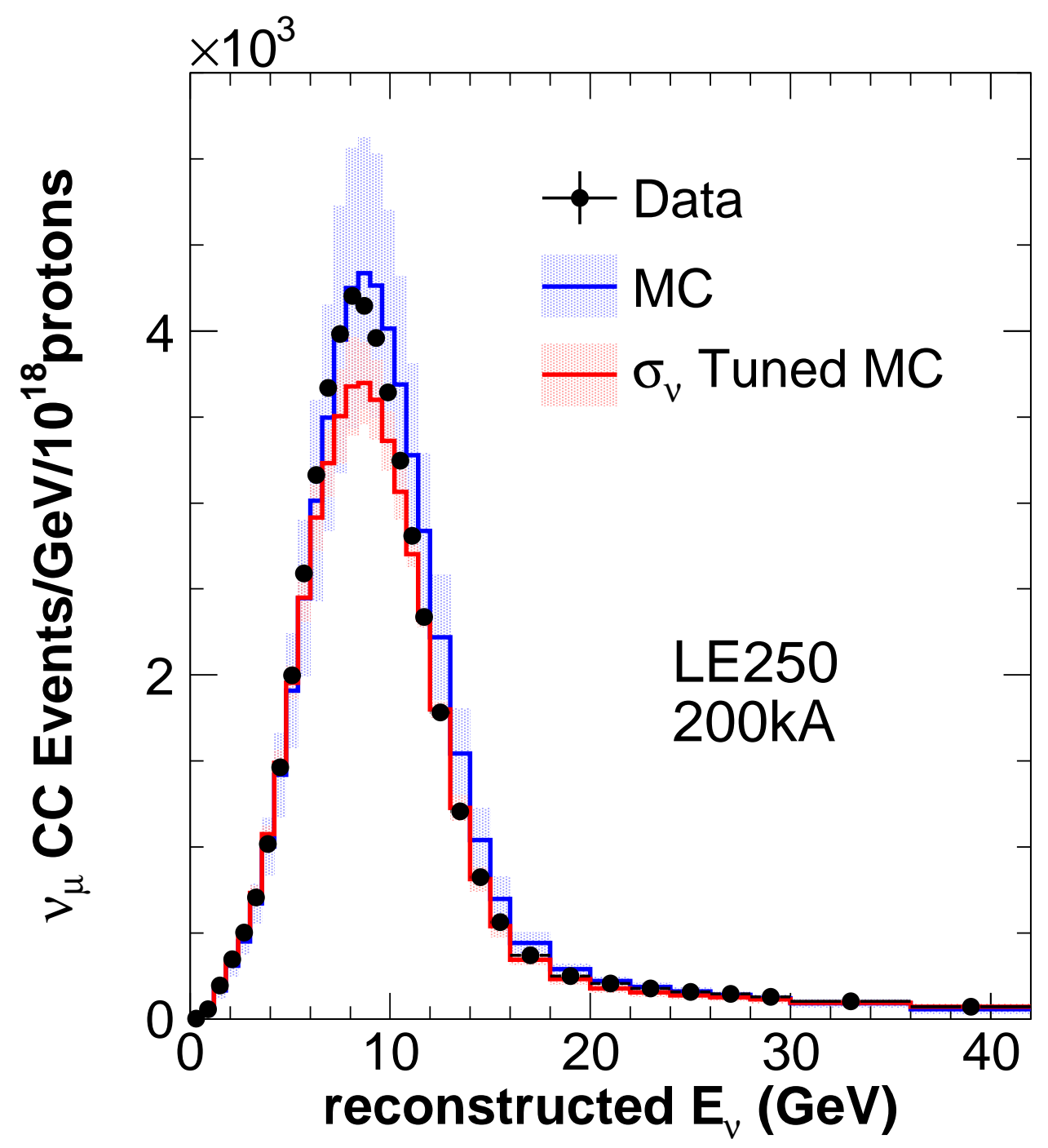

Figure 7.4: MINOS Detector spectrum in the LE250/200kA beam configuration. The black points are MINOS Detector Data selected with the $\nu_{\mu}$ event selection with statistical errors. The blue histogram is the flux reweighted MINOS Detector Monte Carlo. The blue shaded bars represent the sum in quadrature of the differences between the nominal Monte Carlo spectrum and each of the alternative spectra (The alternative fiducial volume spectrum and the alternative selection cut spectrum are not included). The red histogram results from fitting the flux reweighted Monte Carlo spectrum(blue histogram) to the Data spectrum(black points). The red shaded bars represent the sum in quadrature of the difference in each alternative fitted spectrum with the nominal fitted spectrum(red histogram). 
energy $E_{\nu}^{\text {true }}$ and not in terms of reconstructed energy $E_{\nu}^{r e c o}$, a $\chi^{2}$ fit to the data is performed for each bin of $E_{\nu}^{\text {true }}$ which fits the observed spectrum to the $\mathrm{MC}$, determining correction factors to the NEUGEN cross section model for each $E_{\nu}^{\text {true }}$. These correction factors are listed in Table 7.2. The red curves in Figures 7.1 through 7.4 show the result of this tuning procedure for the cross section.

Figure 7.5 shows the measurement of the cross section for $\nu_{\mu} \mathrm{CC}$ scattering on iron as a function of neutrino energy from the above procedure. The horizontal axis is true energy of the incident neutrino. The cross section plotted on the vertical axis is obtained from the fitting procedure applied to Figures 7.1 through 7.4 described above. 


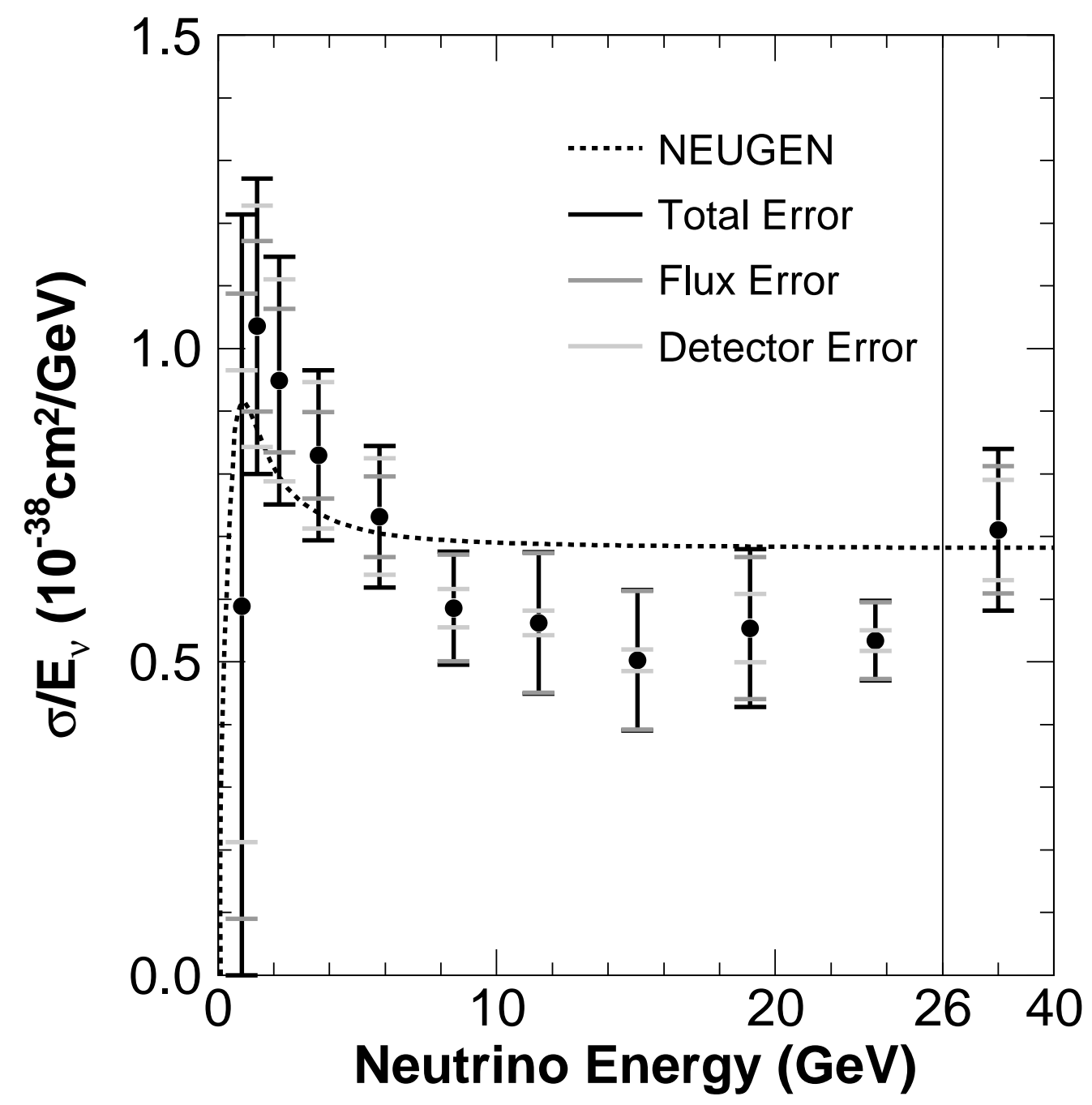

Figure 7.5: The MINOS isoscalar iron charged current $\nu_{\mu}$ inclusive differential cross section measurement. The total error is the quadrature sum of the detector and event selection error and the neutrino flux error. The solid curved is the NEUGEN cross section, which is used to generated the default MINOS Detector event Monte Carlo. Table 7.2 lists the values and errors of the data points. 


\subsection{Systematics}

The systematic errors on the cross section can be divided into two groups. One group is MINOS near detector and charged current $\nu_{\mu}$ event selection systematics. The other group is systematic error sources from the flux measurement.

\subsubsection{Detector and Event Selection}

The systematics from detector and event selection sources derive from uncertainties into four categories.

1. Hadronic Energy Scale: The uncertainty in the hadronic energy scale is described in Chapter 2.

2. Muon Energy Scale: The uncertainty in the muon energy scale is comprised of the uncertainty in the muon energy determined from a measurement of the muon curvature in the detector (4\%) and the uncertainty in the muon energy as determined from the measurement of the muon range in the detector $(2 \%)$ as described in Chapter 2.

3. Fiducial Volume: The Fiducial volume is a $3.267 \mathrm{~m}$ long cylindrical volume with radius of $0.8 \mathrm{~m}$ aligned with the axis parallel to the detector axis. Neutrino events that occur at the edge of the fiducial volume have a greater probability to have the vertex reconstructed outside of the fiducial volume than inside of the fiducial volume due to the larger volume just outside the fiducial volume than just inside. Also, events that occur closer to the edge of the fiducial volume may not be fully contained within the detector leading to poorly reconstructed events. To account for uncertainty in the cross section measurement due to the 
fiducial volume definition, a CC $\nu_{\mu}$ sample is selected with a larger and smaller fiducial volume. The size of the increase or decrease in the fiducial volume is determined by the vertex resolution in the detector. The transverse event vertex resolution is conservatively $\sim 3$ scintillator strips which corresponds to $12 \mathrm{~cm}$ and the longitudinal event vertex resolution is conservatively $\sim 3$ planes which corresponds to $18 \mathrm{~cm}$ [70]. The length of the fiducal volume is increased and decreased by twice the longitudinal resolution and at the same time the radius of the fiducial volume is increased and decreased by the transverse resolution. The change in the cross section measurement obtained with these event samples compared to the nominal event sample is taken as the error on the cross section measurement due to the fiducial volume definition.

4. Event Selection: Selection of charged current $\nu_{\mu}$ events is described in Chapter 2. Sources of error on the cross section measurement from the selection of the charged current $\nu_{\mu}$ sample come from uncertainties in the event classification cut value, the neutral current contamination and the antineutrino contamination [37]. To account for uncertainties from the event classification cut value, the cut value is varied by \pm 0.1 and charged current $\nu_{\mu}$ events are reselected. The uncertainty on the cross section measurement from the misclassification of neutral current and antineutrino events as charged current $\nu_{\mu}$ events is accounted for by increasing and decreasing the number of neutral current and antineutrino events in the charged current $\nu_{\mu}$ sample by $50 \%$ and $100 \%$ respectively.

To quantify these systematic effects on the cross section measurement, each systematic variation just discussed is applied to the nominal flux reweighted 
MINOS Detector neutrino event Monte Carlo or to both the Monte Carlo and Data events, as in the case of the event selection uncertainties, to produce an alternative Monte Carlo and/or Data charged current $\nu_{\mu}$ event spectrum. Each alternative Monte Carlo spectrum is fit to the corresponding Data spectrum using the method described in Section 7.2 giving an alternative cross section measurement The difference between the alternative cross section measurement and the nominal cross section measurement is taken as an error on the nominal cross section measurement. Figure 7.6 shows the percent error on the cross section measurement from the detector and event selection systematics. Appendix D describes these systematic errors in more detail.

\subsubsection{The Neutrino Flux}

The systematics from the neutrino flux measurement derive from uncertainties into four categories.

1. Backgrounds in the muon monitors,

2. the $K^{+} / \pi^{+}$ratio off the target,

3. the $\pi^{+} / \pi^{-}$ratio off the target,

4. the ionization scale of the muon monitors.

Refer to Chapter 6 for a detailed discussion of these systematics.

Each of the alternative flux fits discussed in Chapter 6 produce an alternative set of flux weights which account for systematic errors on the neutrino flux. To determine the systematic error on the cross section measurement from the systematics on the flux measurement, each of set of alternative flux 


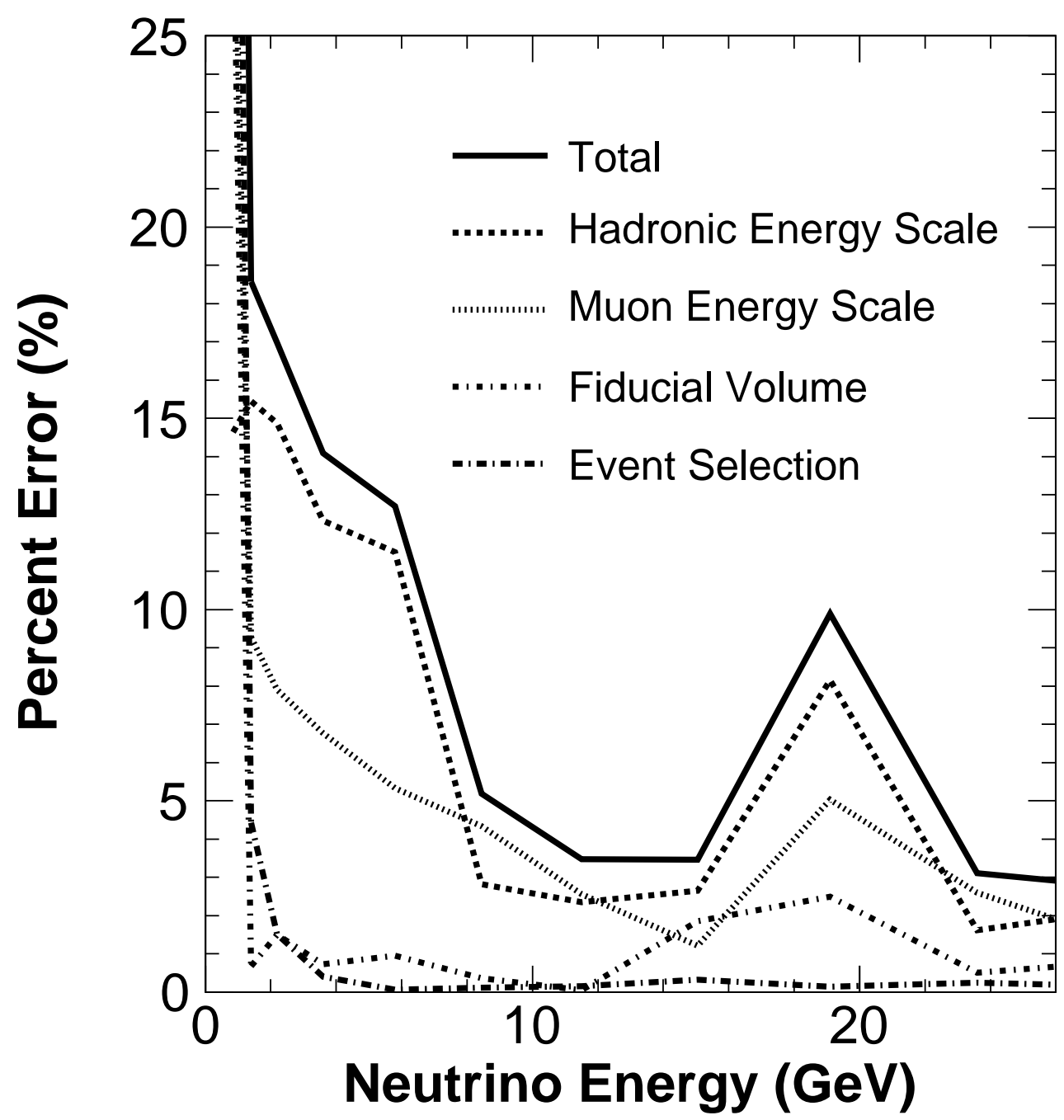

Figure 7.6: The percent error on the cross section measurement from the detector and event selection systematics. The Total error is the sum in quadrature of the 4 systematic categories(dashed curves). The Muon Energy Scale systematic is the sum in quadrature of the muon curvature energy scale and the muon range energy scale systematics. The Event Selection systematic is the sum in quadrature of the the event classification cut value, the neutral current contamination and the antineutrino contamination systematics. 
weights are applied to the default MINOS Detector Monte Carlo. The nominal charged current $\nu_{\mu}$ selection is applied to the Monte Carlo to obtain an alternative charged current $\nu_{\mu}$ Monte Carlo event spectrum. Each alternative Monte Carlo spectrum is fit to the nominal Data spectrum using the method described in Section 7.2 giving an alternative cross section measurement The difference between the alternative cross section measurement and the nominal cross section measurement is taken as an error on the nominal cross section measurement. Figure 7.7 shows the percent error on the cross section measurement from the neutrino flux systematics. Appendix D describes these systematic errors in more detail.

\subsection{Measurement of $\sigma\left(\nu_{\mu} F e \rightarrow \mu^{-} X\right)$}

The charged current $\nu_{\mu}$ inclusive cross section is measured by fitting the nominal flux reweighted MINOS Detector Monte Carlo charged current $\nu_{\mu}$ event sample to the Data charged current $\nu_{\mu}$ event sample using the method described in Section 7.2. The cross section scale factors given by the fit are applied to the NEUGEN isoscalar iron charged current $\nu_{\mu}$ inclusive cross section.

Table 7.2 lists the cross section scale factors an cross section values resulting from the simultaneous 4 beam fit of nominal Monte Carlo to data. Figure 7.5 shows the resulting $\mathrm{CC} \nu_{\mu}$ inclusive differential cross section measurement. The total error is the quadrature sum of the detector and event selection error and the neutrino flux error described in Section 7.3. As discussed in Chapter 6, the flux is normalized such that the flux weighted number of neutrino events in the Near Detector Monte Carlo agrees with experimental 


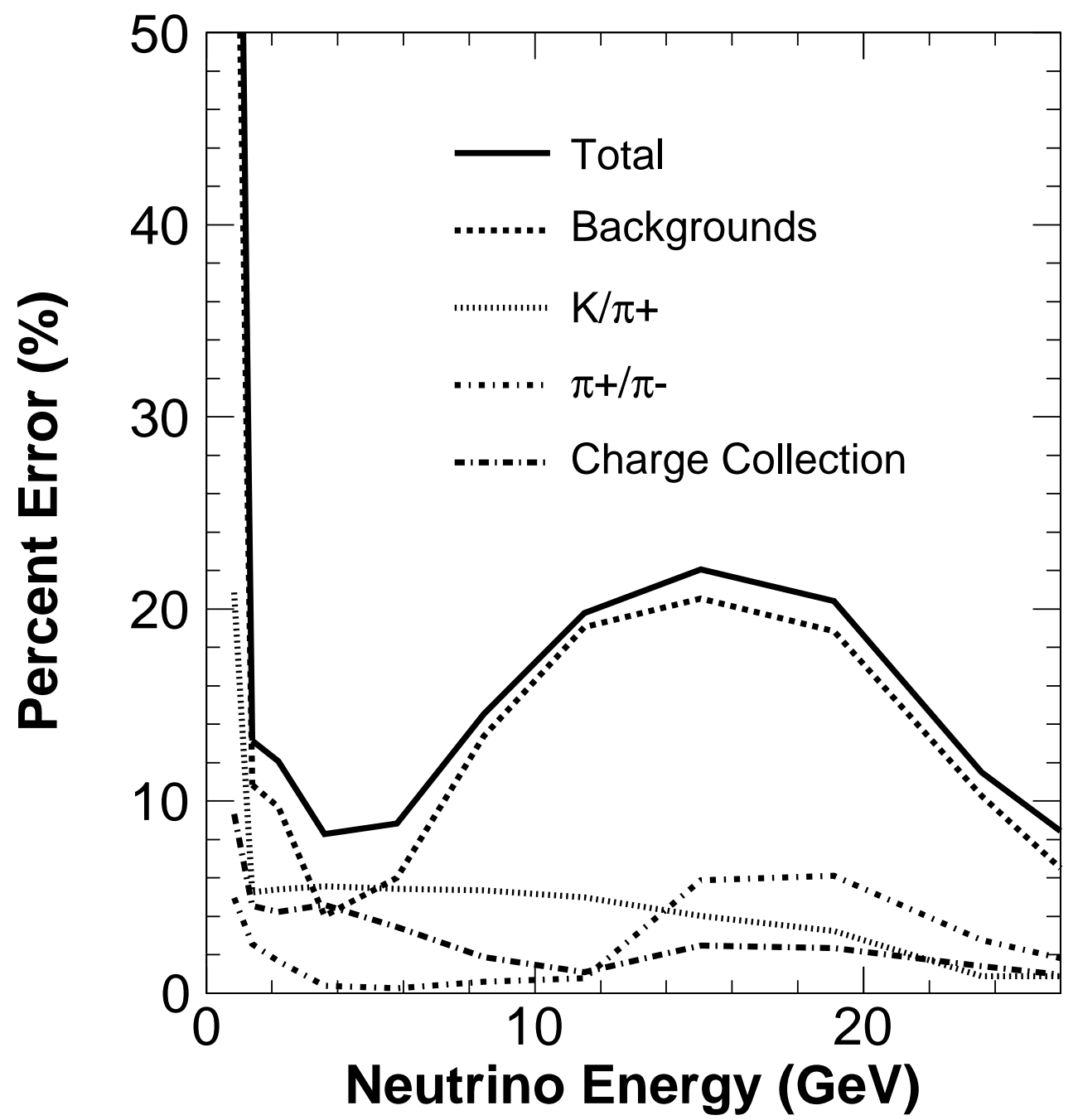

Figure 7.7: The percent error on the cross section measurement from the systematics on the flux measurement. The Total error is the sum in quadrature of the 4 systematic categories(dashed curves) described in detail in Chapter 6 . The Backgrounds systematic is the sum in quadrature of the absorber background systematic and the $\delta$-ray background systematic with the correction for the Bethe-Block energy loss by charged particles. The Charge Collection systematic is the sum in quadrature of the ionization energy scale systematic and the muon monitor linearity correction systematic. 


\begin{tabular}{|c|c|c|c|c|c|}
\hline $\begin{array}{l}\text { Cross Section } \\
\text { Scale Factor } \\
\text { Parameter } \\
\end{array}$ & $\begin{array}{l}E_{\nu}^{\text {true }} \\
\text { Range } \\
(\mathrm{GeV}) \\
\end{array}$ & $\begin{array}{c}\text { Average } \\
E_{\nu}^{\text {true }} \\
(\mathrm{GeV}) \\
\end{array}$ & $\begin{array}{c}\text { Cross Section } \\
\text { Scale Factor } \\
\end{array}$ & $\begin{array}{c}\text { Measured CC } \nu_{\mu} \\
\text { Cross Section } \\
\left(\mathrm{cm}^{-2} \mathrm{GeV}^{-1}\right) \\
\end{array}$ & $\begin{array}{c}\text { Error } \\
(\%) \\
\end{array}$ \\
\hline 0 & $0.7-1$ & $\begin{array}{c}0.85 \\
\end{array}$ & 0.65 & 0.59 & 106 \\
\hline 1 & $1.0-1.8$ & 1.40 & 1.20 & 1.04 & 22.8 \\
\hline 2 & $1.8-2.6$ & 2.20 & 1.20 & 0.95 & 20.8 \\
\hline 3 & $2.6-4.6$ & 3.60 & 1.13 & 0.83 & 16.3 \\
\hline 4 & $4.6-7.0$ & 5.80 & 1.04 & 0.73 & 15.5 \\
\hline 5 & $7.0-9.9$ & 8.45 & 0.85 & 0.59 & 15.4 \\
\hline 6 & $9.9-13.1$ & 11.50 & 0.82 & 0.56 & 20.1 \\
\hline 7 & $13.1-17.0$ & 15.05 & 0.73 & 0.50 & 22.3 \\
\hline 8 & $17.0-21.2$ & 19.10 & 0.81 & 0.55 & 22.7 \\
\hline \multirow[t]{2}{*}{9} & $21.2-26.0$ & 23.60 & 0.78 & 0.53 & 11.9 \\
\hline & $26-40$ & 33 & & 0.71 & 18.2 \\
\hline
\end{tabular}

Table 7.2: The MINOS isoscalar iron charged current $\nu_{\mu}$ inclusive differential cross section measurement. Column 1: The parameter number corresponding to the $E_{\nu}^{\text {true }}$ bin in the fit. Column 2: The upper and lower limits of the $E_{\nu}^{\text {true }}$ bin. Column 3: The fitted cross section scale factor (to be applied to the NEUGEN cross section). Column 4: The measured differential cross section. Column 5: The percent error on the measured differential cross section. 
data in the region between $E_{\nu}=26$ and $40 \mathrm{GeV}$. Thus, the cross section in the region between $E_{\nu}=26$ and $40 \mathrm{GeV}$ is predetermined to agree with the NEUGEN CC $\nu_{\mu}$ cross section. The error in this region represents the uncertainty on the normalization.

\subsection{Cross Checks}

When fitting for the cross section the 4 beams, LE010/185kA, LE100/200kA, LE150/200kA and LE250/200kA, are fit simultaneously. As a consistency check, each beam can be fit independently for the cross section. Figure 7.8 shows the charged current $\nu_{\mu}$ cross section from fits using the LE010/185kA, LE100/200kA, LE150/200kA and LE250/200kA beams independently. The difference in the cross section among the beams is reflective of the tension in the flux measurement discussed previously. The differences are on the order of the uncertainties shown in Figure 7.5.

Also when fitting for the cross section, to ensure that each beam contributes equally to the measured cross section, all of the charged current $\nu_{\mu}$ event samples are scaled to an exposure of $1 \times 10^{18}$ protons. Alternatively, it may be desirable to use the appropriate statistical power of each of the beams to measure the cross section. Table 7.1 lists the proton exposure of the Data $\mathrm{CC} \nu_{\mu}$ event samples for each beam configuration. The statistics in the LE010/185kA beam are greater than 8 times the statistics in the other beam configurations. Figure 7.9 shows the fitted charged current $\nu_{\mu}$ inclusive differential cross section obtained by allowing each beam to contribute the appropriate statistical power in the fit. This is also done for each alternative fit to determine the error on the cross section. 


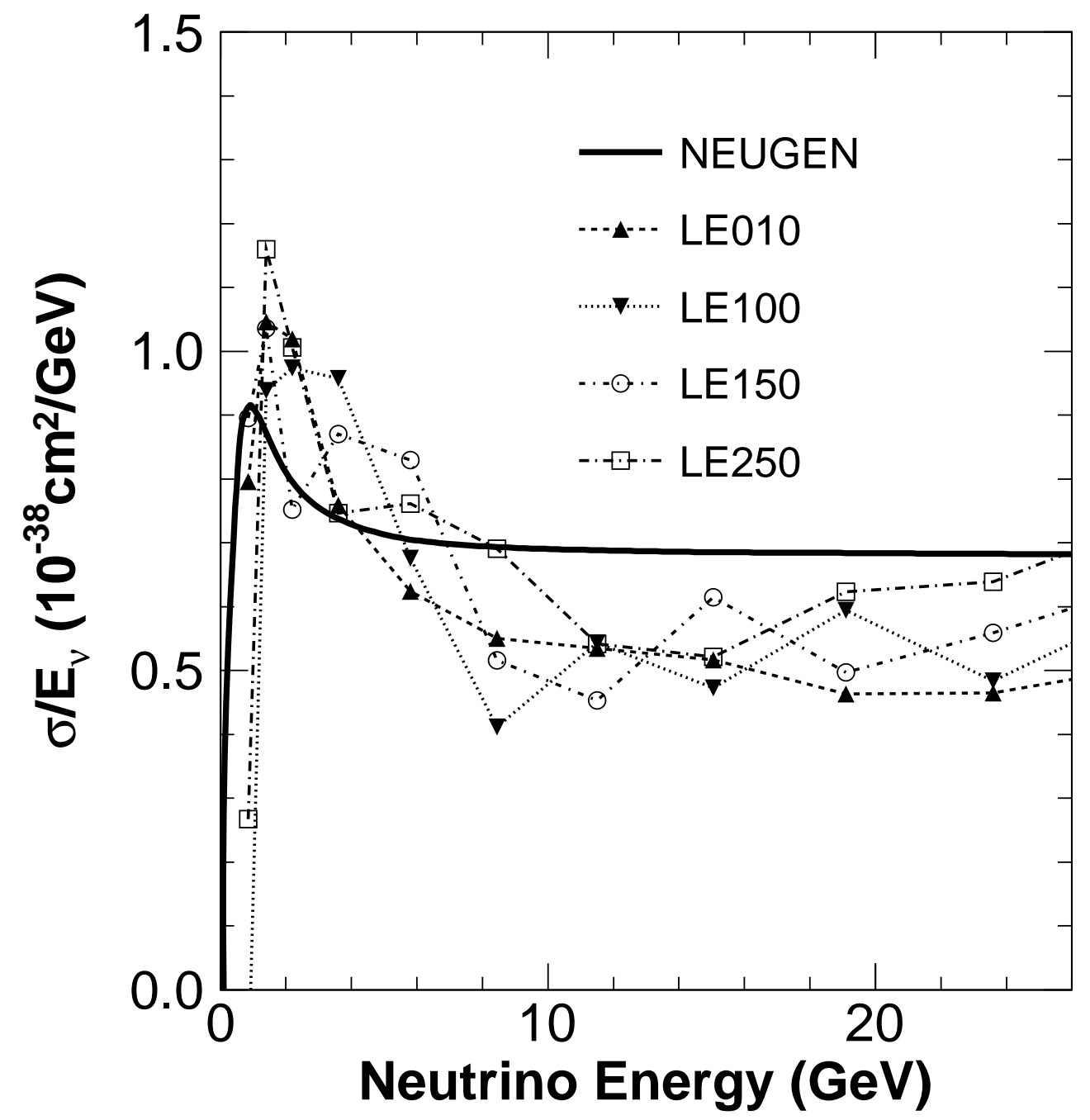

Figure 7.8: The 4 curves correspond to 4 independent fits to the LE010/185kA, LE100/200kA, LE150/200kA, and LE250/200kA data sets in Table 7.1. This set of independent fits differs from the single, simultaneous fit of all 4 data sets to obtain a single cross section result. 


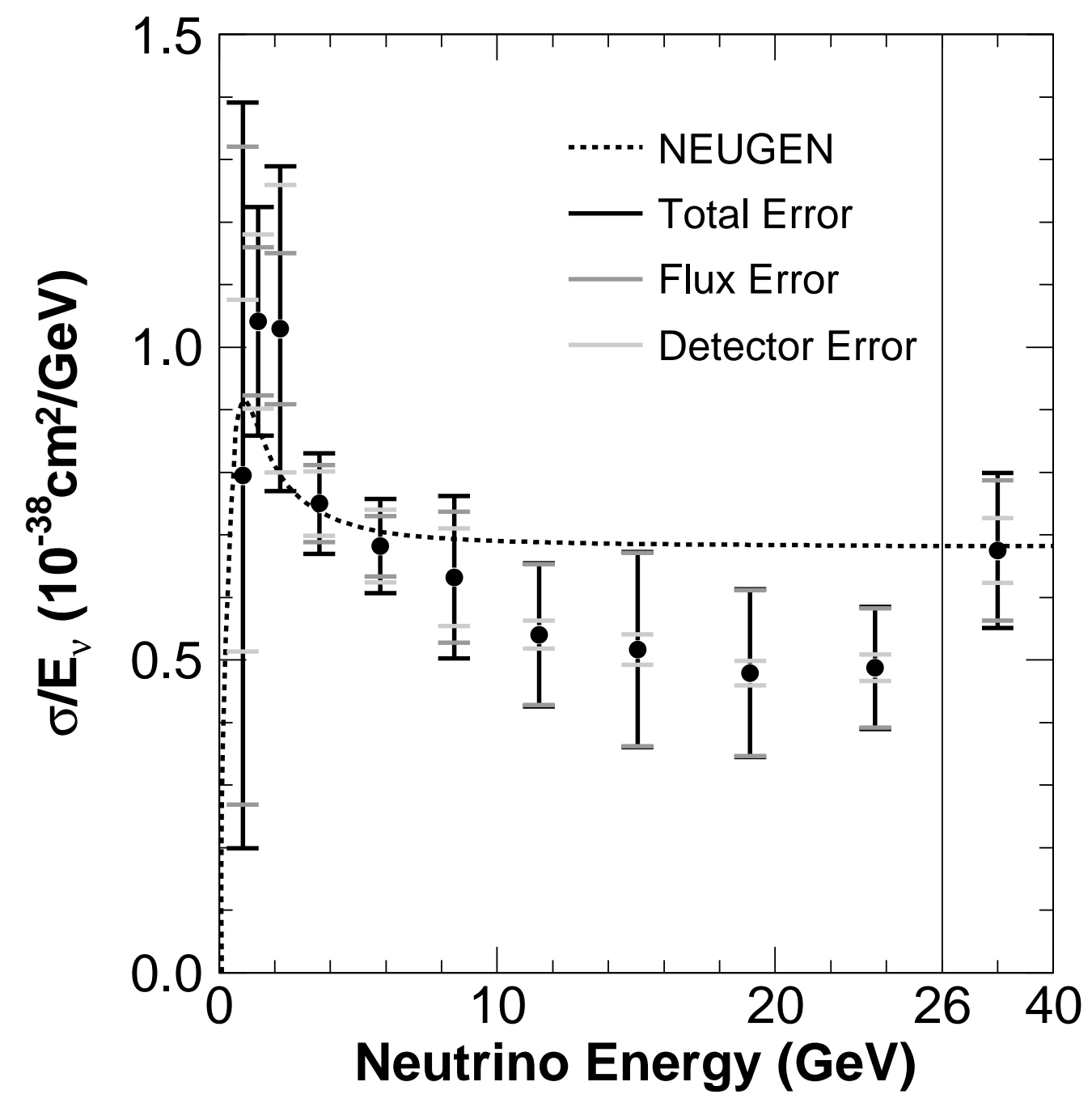

Figure 7.9: The MINOS isoscalar iron charged current $\nu_{\mu}$ inclusive differential cross section resulting from allowing each beam, LE010/185kA, LE100/200kA, LE150/200kA and LE250/200kA, to contribute the appropriate statistical power in the fit. The total error is the quadrature sum of the detector and event selection error and the neutrino flux error. 
By comparing the LE010 cross section curve from Figure 7.8 with Figure 7.9 it is clear that the LE010 beam is dominant in this fit. Also the results of this fit are consistent with the cross section measurement obtained by weighting each of the beams equally. 


\section{Chapter 8}

\section{Conclusions and Outlook}

\subsection{Summary}

In this thesis we have presented a measurement of the $\nu_{\mu}$ charged current cross section on iron using the MINOS detector. As shown in Figure 7.5, the cross section is given in 10 bins of neutrino energy from 1 to $26 \mathrm{GeV}$. The uncertainties on this measurement range from 15-22\% depending on neutrino energy. The data sets utilized corresponds to $1.44 \times 10^{20}$ protons on target acquired by the MINOS experiment in 2005-2006. About $2 \times 10^{6}$ neutrino interactions contribute to this measurement, meaning that it is not statistics limited. In fact, the largest uncertainties arise from our understanding of the MINOS detector and from the neutrino flux.

The result presented here utilizes a technique for measuring the neutrino flux in situ using the muon beam which accompanies the neutrinos in meson decay. This technique has been used by wide band neutrino beam experiments [?]. Past neutrino beams had relatively short decay tunnels and were instru- 
mented with 10-16 muon stations. Our measurement derived a flux using the limited angular acceptance of the NuMI muon stations and overcame the limitation of having just 3 muon stations by utilizing the flexible configurations of the NuMI beam. A set of muon flux data was collected which translates to a neutrino flux, relying only on assumptions about the relative acceptance of the muon and neutrino detectors.

Past neutrino experiments have employed a wide variety of techniques to measure the flux, typically the largest uncertainty in the experiment. At high energies $\left(E_{\nu}>40 \mathrm{GeV}\right)$ narrow band beam techniques can achieve a flux uncertainty as small as $4 \%[?, ?]$. At lower energies neutrinos are most efficiently produced in wide beams, where the flux measurement is more challenging. Some measurements of a total cross section in this region have utilized a combination of external particle production data and muon flux measurements and are shown in Figures 8.1 and 7.5. Other measurements, such as the one shown in Figure 8.2, utilize the neutrino events themselves to extract a flux iteratively and use the resulting flux to measure the cross sections. As can be seen, those measurements using neutrinos event data to extract a flux generally lead to smaller overall uncertainties, albeit at the expense of model assumptions about neutrino interactions. The result presented here makes no such assumptions about how neutrinos interact in the MINOS detector, and compares well to past measurements using a similar technique. 


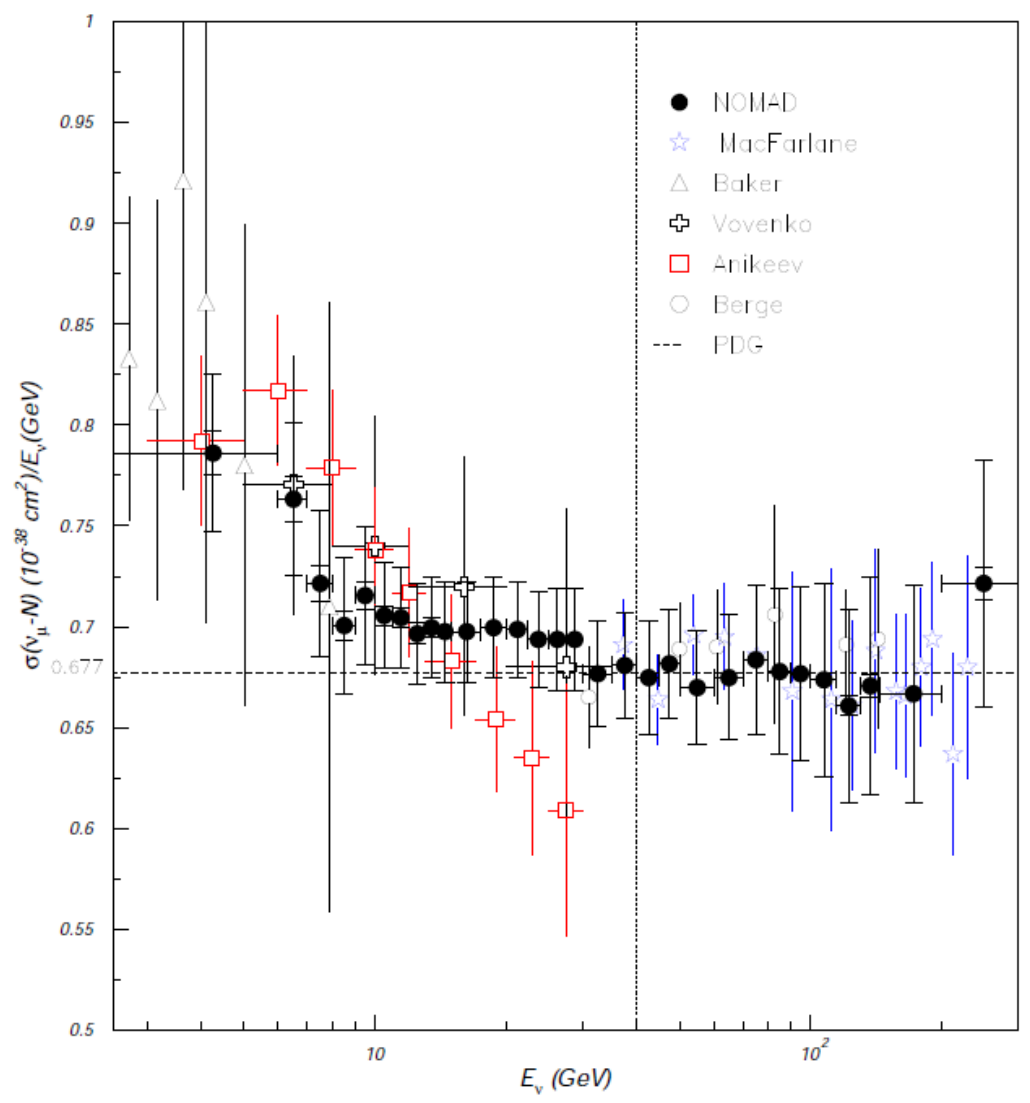

Figure 8.1: The measured $\nu_{\mu}$ charged current inclusive cross section measured by numerous experiments listed in Ref [71]. These experiments have utilized a combination of external particle production data and muon flux measurements to determined their neutrino flux. (taken from Ref [71]) 


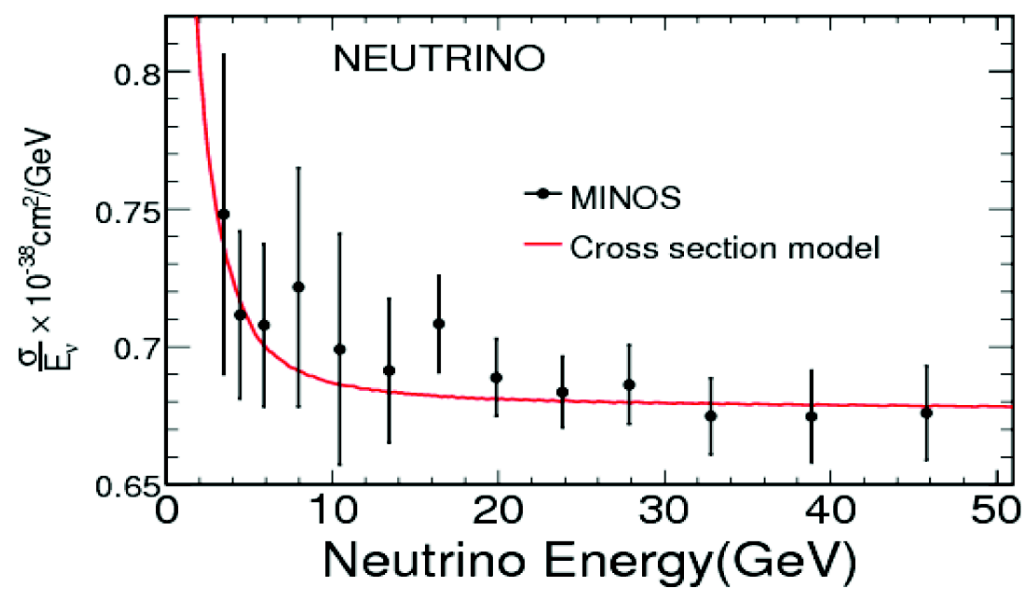

Figure 8.2: The measured $\nu_{\mu}$ charged current inclusive cross section measured using the MINOS detector which utilizes the neutrino event data to extract a neutrino flux. (taken from Ref [72]) 
The analysis in this thesis provides a set of tools which future experiments in the NuMI beam may utilize to obtain absolute neutrino cross section measurements. This analysis may be improved upon in the future. First, the MINOS detector was optimized for measuring 10-20 GeV neutrino interactions [73] and is not fine-grained enough to measure the particles produced in neutrino interactions in the low energy range between 1 and $5 \mathrm{GeV}$. Second, the NuMI muon instrumentation was limited to 3 muon monitors. Also, many lessons about the hardware and analysis tools have been learned through this thesis analysis which future analyses may improve upon. In this chapter we will discuss some of the lessons learned so as to provide guidance to future experiments in the NuMI beam.

\subsection{Future Hardware Improvements}

The large uncertainty in the ionization scale of the muon monitors limited this analysis to a shape only flux and cross section measurement. One reason for the large uncertainty in the ionization scale is the large uncertainty in the purity of the helium gas within the monitors. To ensure stability the gas delivery may be tested and redesigned to understand the mechanisms by which the helium gas may be contaminate and prevent future contamination. The purity of the helium gas in the monitors may also be constantly measured to provide a mechanism to quantitatively evaluate changes in the muon monitor signal due to gas quality and separate these changes from real flux effects. The reference chambers installed in the muon monitor gas system will be a useful tool to further understand and monitor the gas quality within the chambers. The combination of an improved gas delivery system, constant 
purity measurements and reference chamber monitoring would ensure that the ionization scale of the muon monitors may be reliably evaluated in future analyses.

Furthermore, a well controlled beam test similar to those performed at FNAL and BNL would provide further constraints on the absolute ionization scale of the helium within the chambers. Although the FNAL and BNL beam tests provided much useful information, the purity of the helium with in the test chambers was not continuously monitored. As demonstrated by the in situ operation of the muon monitors, the purity of the helium gas may vary over time making constant monitoring a necessity in future testing and operation.

As Muon Monitor 3 is located far downstream, the muon flux at alcove 3 is considerably less than at the other 2 monitors. During low intensity proton beam operations, as may occur while performing horn scans or target scans, the signal to noise ratio is considerable. The single ionization gap pixels of Muon Monitor 3 are planned to be replaced with multiple ionization gap pixels. This will provide for more active ionization volume therefore increasing the ionization signal collected over that of the current single-gap ionization chambers. This will provide for improved measurement sensitivity.

\subsection{Future Analysis Improvements}

As discussed in the previous chapters, tension exists in the measurement of the neutrino flux. The tension in the flux measurement is directly translated to the cross section measurement producing tension among the different neutrino spectra and different cross section results when the individual beam configurations are independently used to measure the flux. Figures 5.10 through 5.12 
show the horn current scan data and the horn current scan Monte Carlo before and after the nominal flux fit. Better overall data-Monte Carlo agreement is achieved after fitting but there remains disagreement for certain monitors, beams, and horn currents. An example of the tension in the fitting is that the fitted Muon Monitor 3 LE250 horn scan remains low compared to the data while the fitted Muon Monitor 1 LE250 horn scan is high. The current fitting parameterization seems unable to achieve simultaneous agreement between these horn scans. It may be the the parameterization doesn't not provide enough flexibility to change regions of the parent $p_{T}, p_{z}$ space without affecting other regions; in other words, the parameterization provide too much correlation between different regions of $p_{T}, p_{z}$ space to fit the muon flux data. Thus, it would be useful to develop other parameterizations that will provide more flexibility.

For this analysis the Monte Carlo used to simulate the muon flux and background flux in the muon monitors was developed from scratch. The previously existing Fluka/Geant3 based Monte Carlo was used to predict the pion and kaon fluxes reaching the NuMI decay volume. The simulation of particle production in the NuMI target utilized Fluka physics models while the subsequent focusing and tracking of the mesons through the beamline implemented Geant3 physics models. The passage of muons through the hadron absorber and muon filter was performed with Geant4 physics models. Particles that contribute muon in the muon monitors are not all directly produced in interactions in the hadron production target. Pions and kaons are produced in tertiary interactions of pions, kaons, protons, and neutrons with material along the beam line, such as the horns, radiation shielding and the widow and walls of the decay pipe. Muons from the decay of pions and kaons produced from 
tertiary interactions are a significant fraction, nearly half, of the muon flux at the lowest horn currents. When used for simulations of particle production in the hadron production target Geant3 is known to significantly over estimate the neutrino flux at the MINOS detectors. Thus, it is logical to suggest that the muon flux at the lowest horn currents may be too large in the Monte Carlo horn current scans. Also, the parameterization of hadron production used to warp the pT,pz of parents contributing muons in the muon monitors was developed using data from controlled experiments of hadron production from a limited number of materials. It is possible that the $p_{T}, p_{z}$ spectrum of particles produced in tertiary interactions is badly described by this parameterization and should not be warped in the same way as particles produced from first generation interactions in the hadron production target. For this analysis, technical limitations of the Monte Carlo did not allow sufficient information about these tertiary particles to be retained for later use. A single Monte Carlo simulation should be developed which utilizes Fluka physics models and contains information about tertiary particle production.

For this analysis the nominal fit does not include backgrounds from $\delta$ rays, instead $\delta$-rays are included as a systematic error. The reason for this is 2-fold. Up until just recently, no in situ measurements of the $\delta$-ray backgrounds in the muon monitors existed. The Monte-Carlo predicts that delta rays may contribute to up to $30 \%$ of the signal in the muon monitors. The piece-meal way in which the $\delta$-rays were included in the full Monte Carlo simulation may contribute large uncertainty in the predicted contribution of $\delta$-rays to the muon monitor signals. Recently a beam test was performed to study $\delta$-ray backgrounds in the muon monitors. In late 2009, this test was performed and in situ measurements of $\delta$-ray backgrounds were made. Analysis of this 
data is crucial for future analyses to precisely understand the effect of $\delta$-ray backgrounds, which may be significant, in the muon monitors and on the flux and cross section measurements.

As a first analysis, the integral muon flux from all of the 81 ionization chambers was analyzed. The muon beam RMS size as the muon monitors is on average $70 \mathrm{~cm}$. The RMS beam size does slightly vary by $\sim 10 \mathrm{~cm}$ at different monitors for different target positions and horn currents. If the

pT,pz distribution of the muon parents has some radial dependence at the muon monitors which do vary significantly, then incorporating the radial muon distribution into the flux fitting would provide greater sensitivity to the parent hadrons. In the worst case, if there is little pT,pz dependence or variation in the radial distribution, the radial muon distribution could still be used as a constraint to prevent the $\mathrm{pT}, \mathrm{pz}$ parametrization from being unphysically warped.

\subsection{Future MINER $\nu$ A Experiment}

The newest experiment to utilize the high intensity NuMI neutrino beam is the MINER $\nu \mathrm{A}$ experiment. The MINER $\nu \mathrm{A}$ experiment is a neutrino scattering experiment specifically optimized to measure neutrino cross sections. The MINER $\nu \mathrm{A}$ detector is a fine grained scintillator detector and is located immediately in front of the MINOS detector $1 \mathrm{~km}$ from the NuMI particle production target. Some neutrino events in the MINER $\nu$ A detector are shown in Figure 8.3.

In order to precisely measure absolute neutrino cross sections in the NuMI wide band neutrino beam, MINER $\nu \mathrm{A}$ will require precise knowledge 

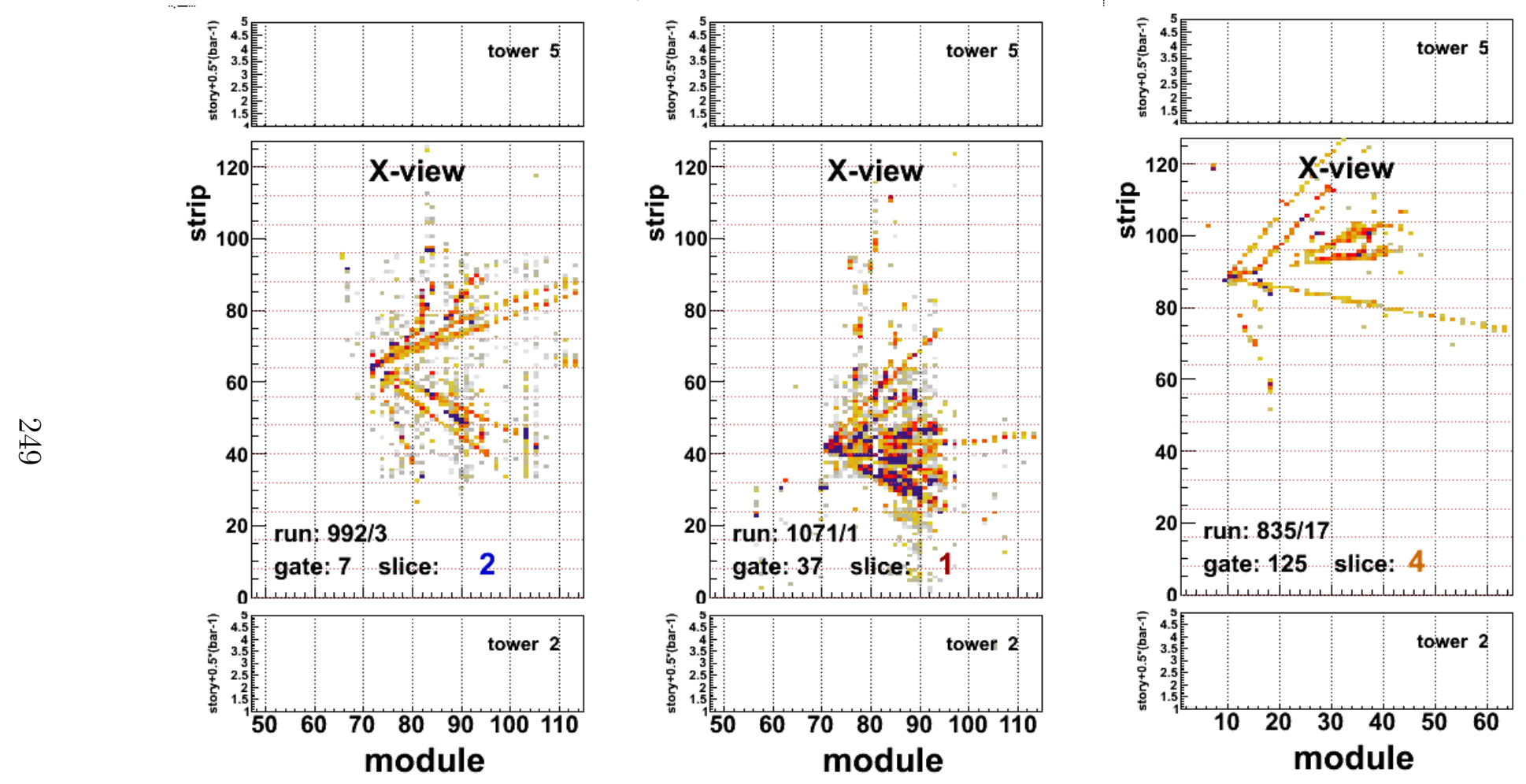

Figure 8.3: Neutrino event candidates in the MINER $\nu$ A detector. 
of the neutrino flux at the detector. MINER $\nu \mathrm{A}$ plans to use a combination techniques to constrain the flux, one of which will be repeating the analysis in this thesis to infer the neutrino flux by measuring the muon flux.

The MINER $\nu \mathrm{A}$ experiment will take data in the LE010/185 kA beam configuration and the ME100/200 kA beam configuration, which is very similar to the LE100/200 kA beam configuration. The muon distribution in the muon monitors and at the end of the decay volume is shown in Figure 4.16. The lower left plot shows the distribution of muons for the medium energy LE100/200 kA beam. In this configuration, and in the higher energy LE250/200 kA configuration, Muon Monitor 3 sees muons from the focusing peak. There is a 4 th alcove downstream of the 3rd alcove in the NuMI beam. The predicted threshold for muons to reach this alcove is $38 \mathrm{GeV}$. As can be seen in the figure, this corresponds to the muons that contributed to the unfocused high energy tail of the muon flux. There are plans to install a muon monitor within this fourth alcove. This muon monitor will provide an anchor for the unfocused high energy component of the muon flux. This will provide further sensitivity to the differential muon and neutrino flux spectrum in future analyses. 


\section{Appendix A}

\section{Pressure and Temperature Corrections for the Hadron and Muon Monitors}

\section{A.1 Introduction}

The secondary beam monitors (Hadron Monitor and 3 Muon Monitors) are Helium-filled ionization chamber arrays under continuous gas flow. Because of changes in ambient conditions such as barometric pressure or ambient temperature, the density of the He gas within the chambers changes, yielding several percent changes in the detectors' ionization signal per incident charged particle. Such changes must be factored out in order to compare chamber signals over long time periods.

To good approximation, one expects the chambers' response to vary linearly with gas density (increasing with pressure, decreasing with temperature). 
Further, the pressure and temperature should be fairly uniform throughout the individual arrays, given the low pressure-drop through the chambers. We foresee calibrating the chambers' response using pressure and temperature gauges installed at each ion chamber array, ${ }^{1}$ as shown in Figure A.1.

The chambers' response to pressure and temperature was studied in NuMI-B-1084 using a controlled bench setup with radionuclide sources at UTAustin. It is desirable to repeat such calibrations in situ, in case gas quality differences or pressure and temperature instrumentation differences exist. Further, the variation in response to pressure or temperature may be different for high energy beam particles than were the values obtained for low energy gamma's.

While the temperatures in the alcoves has been fairly constant, pressure variations of 20 Torr are expected to cause of order $3 \%$ change in chamber response. Thus, the corrections are of importance in order to maintain the monitors' $1 \%$ calibration specification.

\section{A.2 In-situ Corrections}

To the extent that a period of stable beam operation can be identified during periods in which pressure or temperature were observed to change, corrections may then be derived. Table A.1 shows 6 such periods of stable running in which ambient conditions were observed to vary significantly. As described in the table, the proton beam position, size, and intensity was relatively uniform

\footnotetext{
${ }^{1}$ In point of fact, the pressure gauges located directly at the detectors have failed due to radiation, and a redundant set of gauges located at the gas distribution rack (outside the radiation zone) are being used. These two sets of gauges were observed to track one another very well before the radiation damage of the gauges at the detectors.
} 


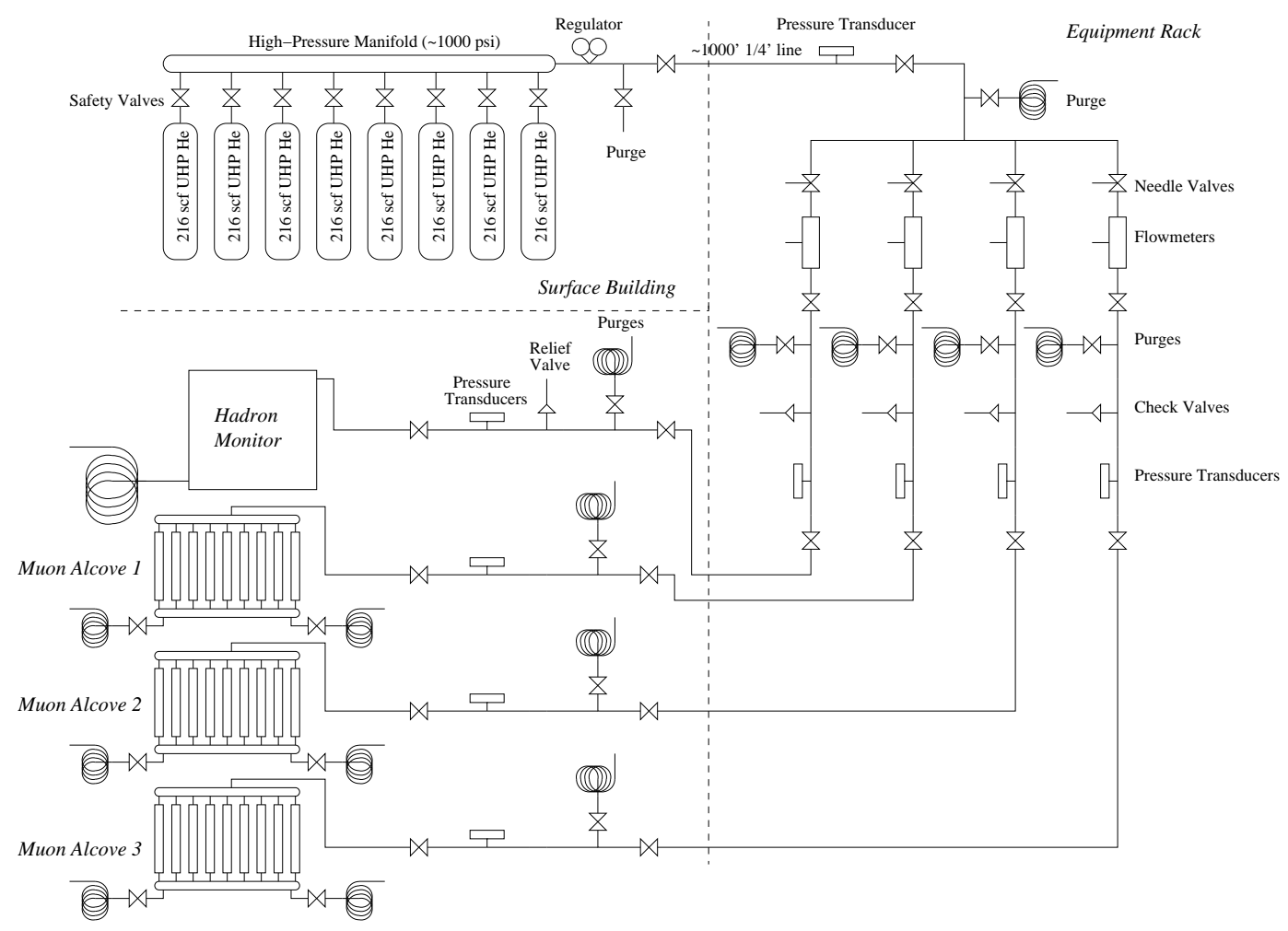

Figure A.1: Gas delivery system for the hadron and muon monitors. A highpressure line delivers He gas from a bottle pack at the surface to a distribution rack in the absorber bypass tunnel. The gas line is "Tee'd" into four parallel lines, each of whose flow is set by a manual needle valve. After the needle valves, the flow and pressure of each line are monitored at the gas distribution rack. A single gas line supplies each muon alcove and hadron monitor. The gas is purged to the room. Temperature-sensing resistive thermal devices (RTDs) are located at each detector, as is an in-series gas pressure gauge. Taken from Bob Zwaska's thesis. 


\begin{tabular}{|c|c|c|c|c|c|c|c|}
\hline & & Days Since & Helium & \multicolumn{4}{|c|}{ Cuts Applied } \\
\hline $\begin{array}{l}\text { Time } \\
\text { Period } \\
\end{array}$ & Dates & $\begin{array}{l}\text { Jan. } 1,2005 \\
\text { (UTC time) }\end{array}$ & $\begin{array}{c}\text { Flow Rate } \\
(\ell / \mathrm{hr} .) \\
\end{array}$ & $\begin{array}{c}-X_{T G T} \\
(\mathrm{~mm}) \\
\end{array}$ & $\begin{array}{l}Y_{T G T} \\
(\mathrm{~mm}) \\
\end{array}$ & $\begin{array}{c}A_{\text {beam }} \\
\left(\mathrm{mm}^{2}\right)\end{array}$ & $\begin{array}{l}\text { TORTGT } \\
\left(10^{12} \mathrm{ppp}\right) \\
\end{array}$ \\
\hline 1 & "Dec. 7-10, 2005 & "340.25-343.75 & $23(10)$ & "1.16-1.26 & none & $3.975-4.175$ & $27.0-27.8$ \\
\hline 2 & Jan. 8-13, 2006 & $372.25-377.75$ & $23(10)$ & $1.125-1.175$ & $1.0-1.2$ & $4.05-4.35$ & $21.6-22.2$ \\
\hline 3 & Feb. 16-18, 2006 & $411.25-413.25$ & $23(10)$ & $1.21-1.29$ & none & $3.51-3.71$ & $23.5-25.5$ \\
\hline 4 & Sep. 15-17, 2006 & $622.46-624.79$ & $23(30)$ & $0.8-1.4$ & $-0.2-+0.2$ & $3.5-3.6$ & 20-24 \\
\hline 5 & Sep. 21-22, 2006 & $628.88-629.50$ & $23(30)$ & $0.5-2.0$ & $-0.4-1.6$ & $3.75-3.85$ & 22.0 每2.5 \\
\hline 6 & Oct. $15-17,2006$ & $652.5-654.5$ & $23(30)$ & $1.1-1.2$ & $-0.05-+0.05$ & $3.06-3.46$ & $25-27$ \\
\hline 7 & Aug. 9 - Sep. $30,2005^{a}$ & 220.0-272 & $23(10)$ & $1.13-1.33$ & 0.98-1.08 & $3.9-4.1$ & $21-22$ \\
\hline 8 & Oct. 10 - Nov. 30,2005 & $282-283$ & $23(10)$ & 1.1-1.3 & $0.91-1.11$ & $4.21-4.41$ & $26.8-27.6$ \\
\hline
\end{tabular}

${ }^{a}$ Excluding Sept. 23-25 due to a gas bottle change-over.

Table A.1: Beam configurations and data sets accumulated for this analysis. The cuts on beam position, size, and intensity at the target are meant to restrict the variability in the beam seen for the various data periods. Time Periods 1-6 were used to study pressure variations, while $7 \& 8$ were used for study of temperature variations. 
during each of these periods (though not necessarily the same from period to period). The cuts shown in Table A.1 describe selections applied to these time periods to remove obvious beam effects. Even so, some beam-induced variations are seen.

\section{A.2.1 Pressure Corrections}

Figures A.2 through A.5 show the response of the Hadron and 3 Muon Monitors as a function of gas pressure during the 6 data periods of Table A.1. Clear correlations can be seen, though within each time period spill-to-spill variations and hour-to-hour variations result in systematic deviations from simple linear responses. For each data set, a linear fit was performed, with an "uncertainty" determined by maximum excursions which visually accommodate the data. These are summarized in Table A.2.

Further, the slope of chamber response as a function of pressure (to the extent it can be fitted to each data set) is not entirely consistent from data set to data set. Such might be a real effect, arising from different gas qualities during each period (the flow rate was changed, as indicated in Table A.1), or might be an artifact of the imprecise linear fits to the data.

\section{A.2.2 Temperature Corrections}

The temperature at the Hadron Monitor is largely dictated by the temperature of the absorber core, which increases as the beam power delivered to NuMI increases, and falls during low beam power periods or during beam off periods. Variations of several degrees Fahrenheit are observed. The temperatures in the muon alcoves are regulated by space heaters, and these are observed to 

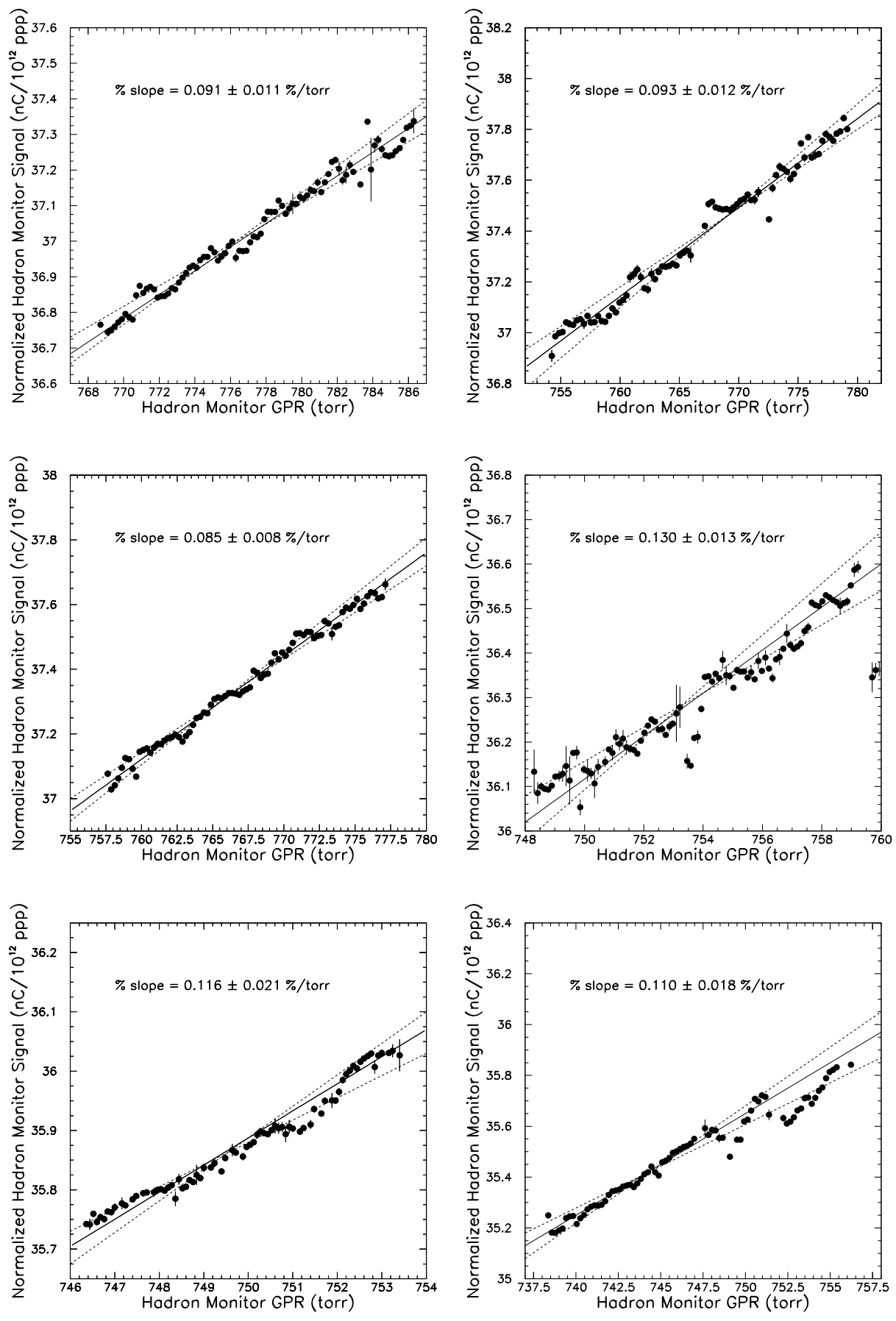

Figure A.2: Total Signal in the Hadron Monitor as a function of He gas pressure during the 6 data periods corresponding to Table A.1. 

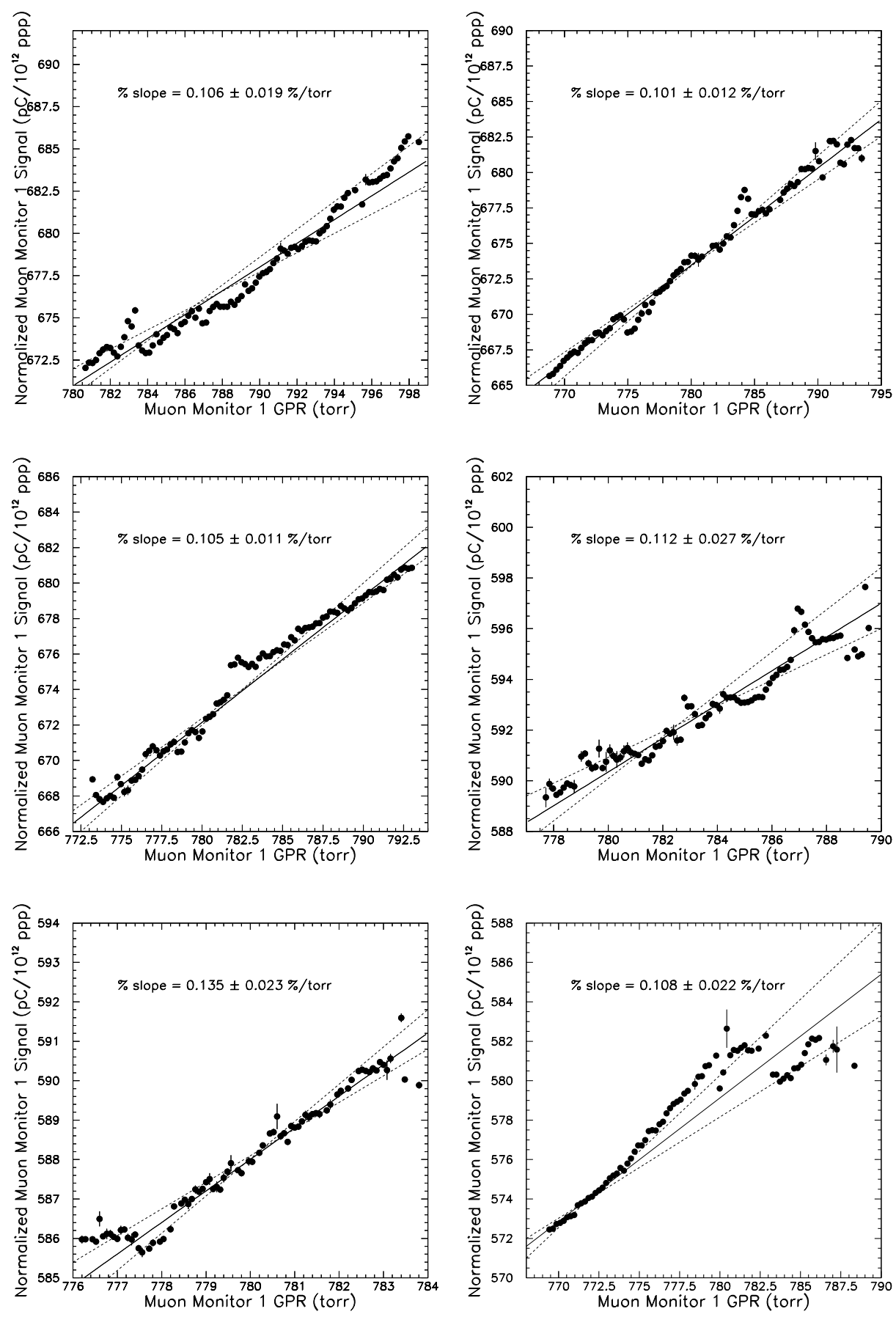

Figure A.3: Total Signal in Muon Monitor 1 as a function of He gas pressure during the 6 data periods corresponding to Table A.1. 

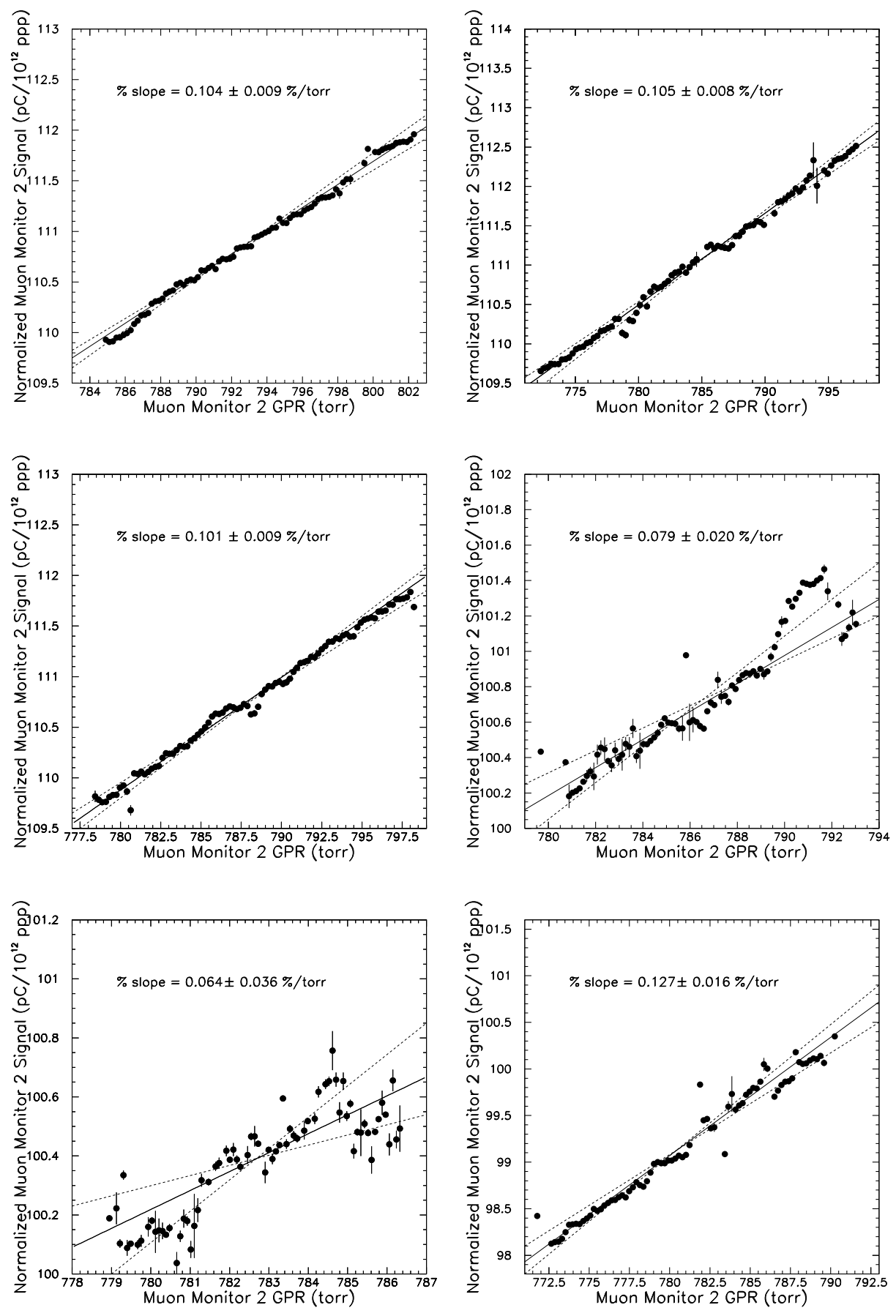

Figure A.4: Total Signal in Muon Monitor 2 as a function of He gas pressure during the 6 data periods corresponding to Table A.1. 

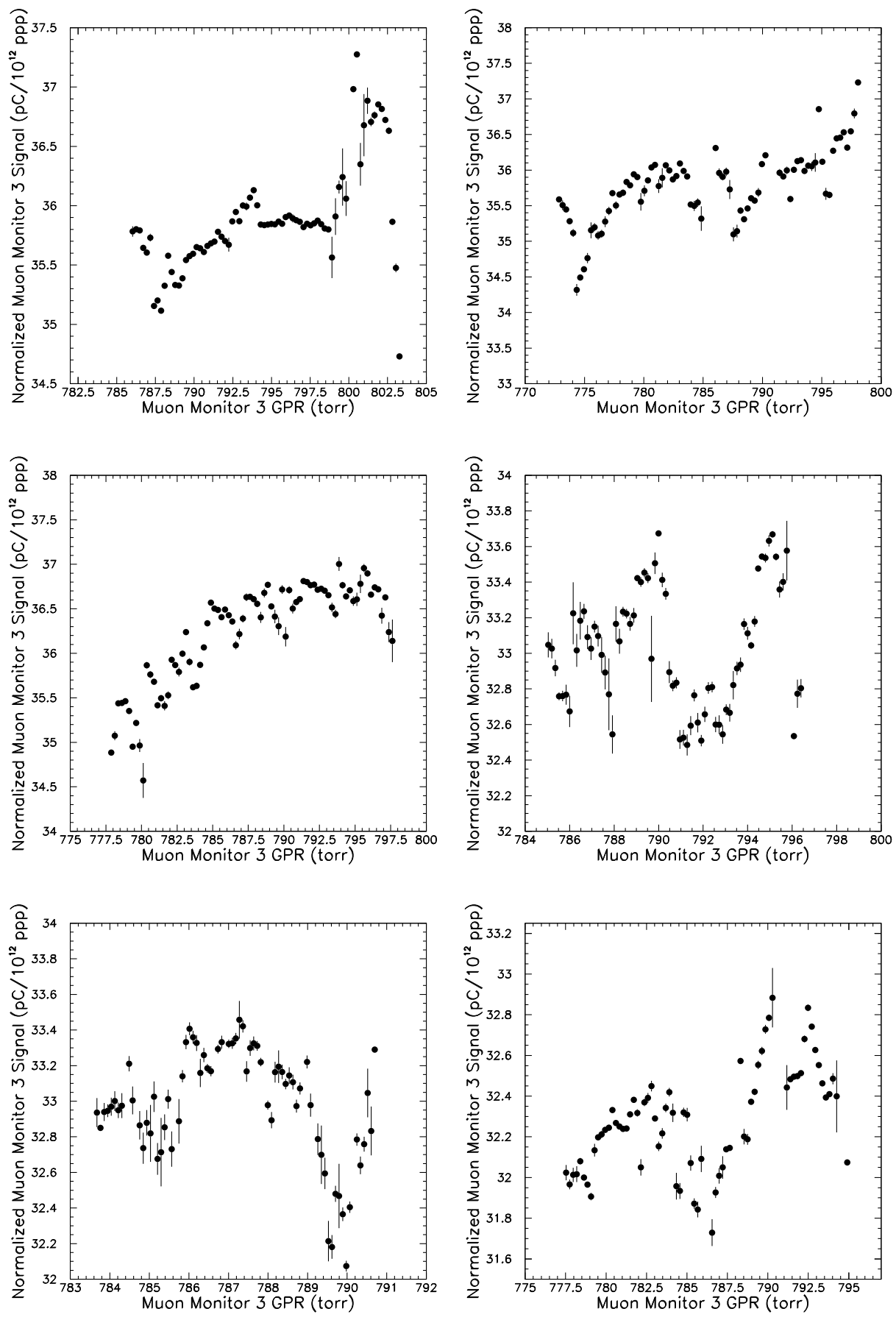

Figure A.5: Total Signal in Muon Monitor 3 as a function of He gas pressure during the 6 data periods corresponding to Table A.1. 


\begin{tabular}{c|c|c|c|c}
$\begin{array}{c}\text { Time } \\
\text { Period }\end{array}$ & $\begin{array}{c}\text { Hadron Monitor } \\
\text { \%/Torr) }\end{array}$ & $\begin{array}{c}\text { Muon Alcove 1 } \\
(\% / \text { Torr })\end{array}$ & $\begin{array}{c}\text { Muon Alcove 2 } \\
(\% / \text { Torr })\end{array}$ & $\begin{array}{c}\text { Muon Alcove 3 } \\
(\% / \text { Torr })\end{array}$ \\
\hline \hline 1 & $(0.091 \pm 0.011)$ & $(0.106 \pm 0.019)$ & $(0.104 \pm 0.009)$ & na \\
2 & $(0.093 \pm 0.012)$ & $(0.101 \pm 0.012)$ & $(0.105 \pm 0.008)$ & na \\
3 & $(0.085 \pm 0.008)$ & $(0.105 \pm 0.011)$ & $(0.101 \pm 0.009)$ & na \\
4 & $(0.130 \pm 0.013)$ & $(0.112 \pm 0.027)$ & $(0.079 \pm 0.020)$ & na \\
5 & $(0.116 \pm 0.021)$ & $(0.135 \pm 0.023)$ & $(0.064 \pm 0.036)$ & na \\
6 & $(0.110 \pm 0.018)$ & $(0.108 \pm 0.022)$ & $(0.127 \pm 0.016)$ & na \\
\hline \hline Average & $0.097 \pm 0.005$ & $0.107 \pm 0.007$ & $0.101 \pm 0.005$ & na \\
\hline
\end{tabular}

Table A.2: Observed variations in detector response with gas pressure for several data periods, and the average result. Reliable response variations were not measurable in Alcove 3. 
be largely constant within a degree Fahrenheit. Figures A.6 and A.7 show the response of the Hadron and 3 Muon Monitors as a function of temperature. The muon monitors show almost no correlation, largely because the vast majority of data is within a 1 degree span, with the few extraneous points at large temperature being exceptional. Given these observations, a correction factor of $(0.32 \pm 0.06) \% /{ }^{\circ} \mathrm{F}$ is obtained for the Hadron Monitor, and no correction will be applied for the muon monitors.

\section{A.3 Summary}

The pressure corrections are taken, for all 4 detector arrays, to be $(0.101 \pm$ 0.005)\%/Torr, and the temperature correction, applied only for the Hadron Monitor data, is taken to be $(-0.32 \pm 0.06) \% /{ }^{\circ} \mathrm{F}$. 

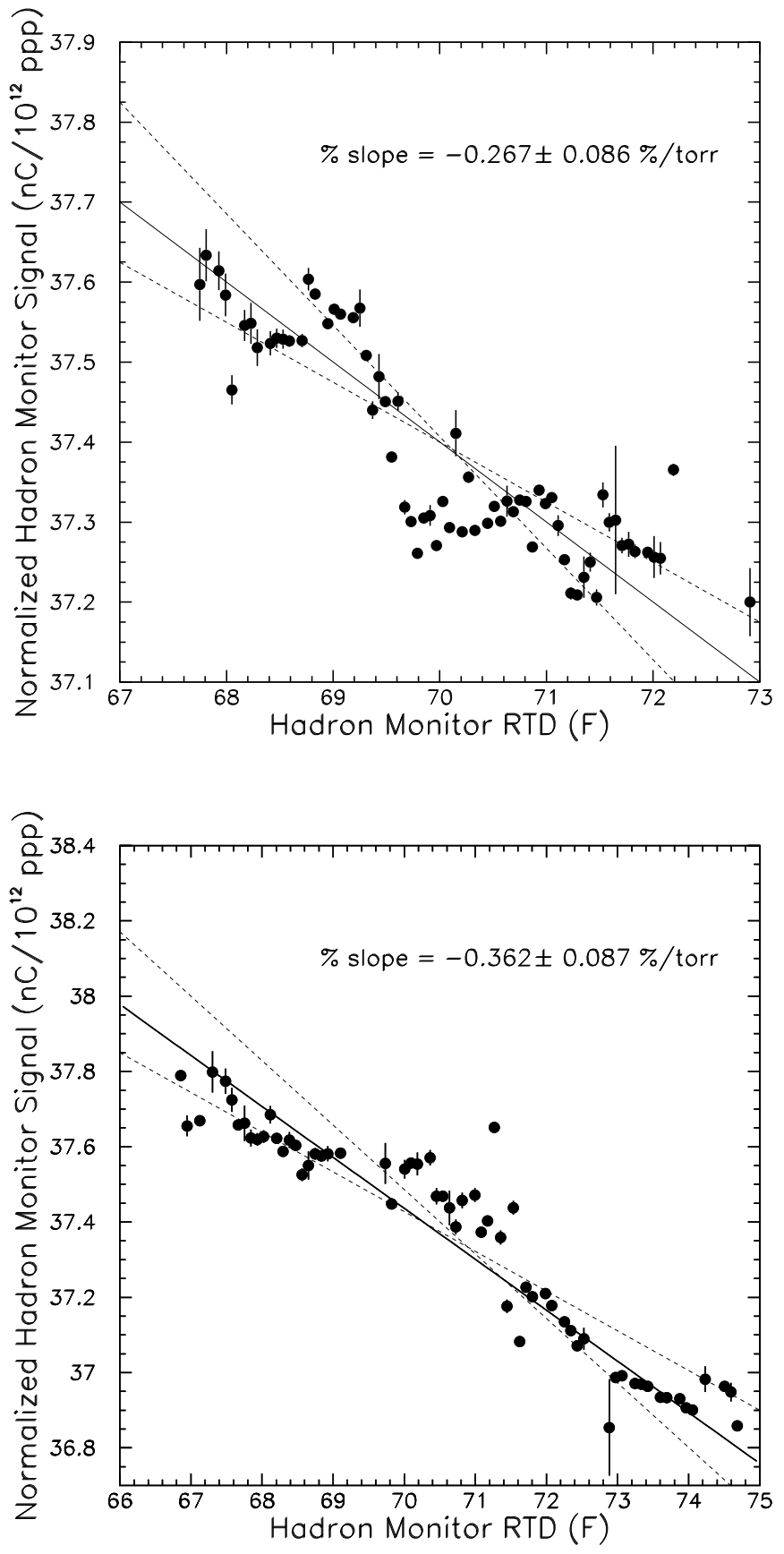

Figure A.6: Total Signal in the Hadron Monitor as a function of ambient temperature during the first two of the 6 data periods corresponding to Table A.1. 

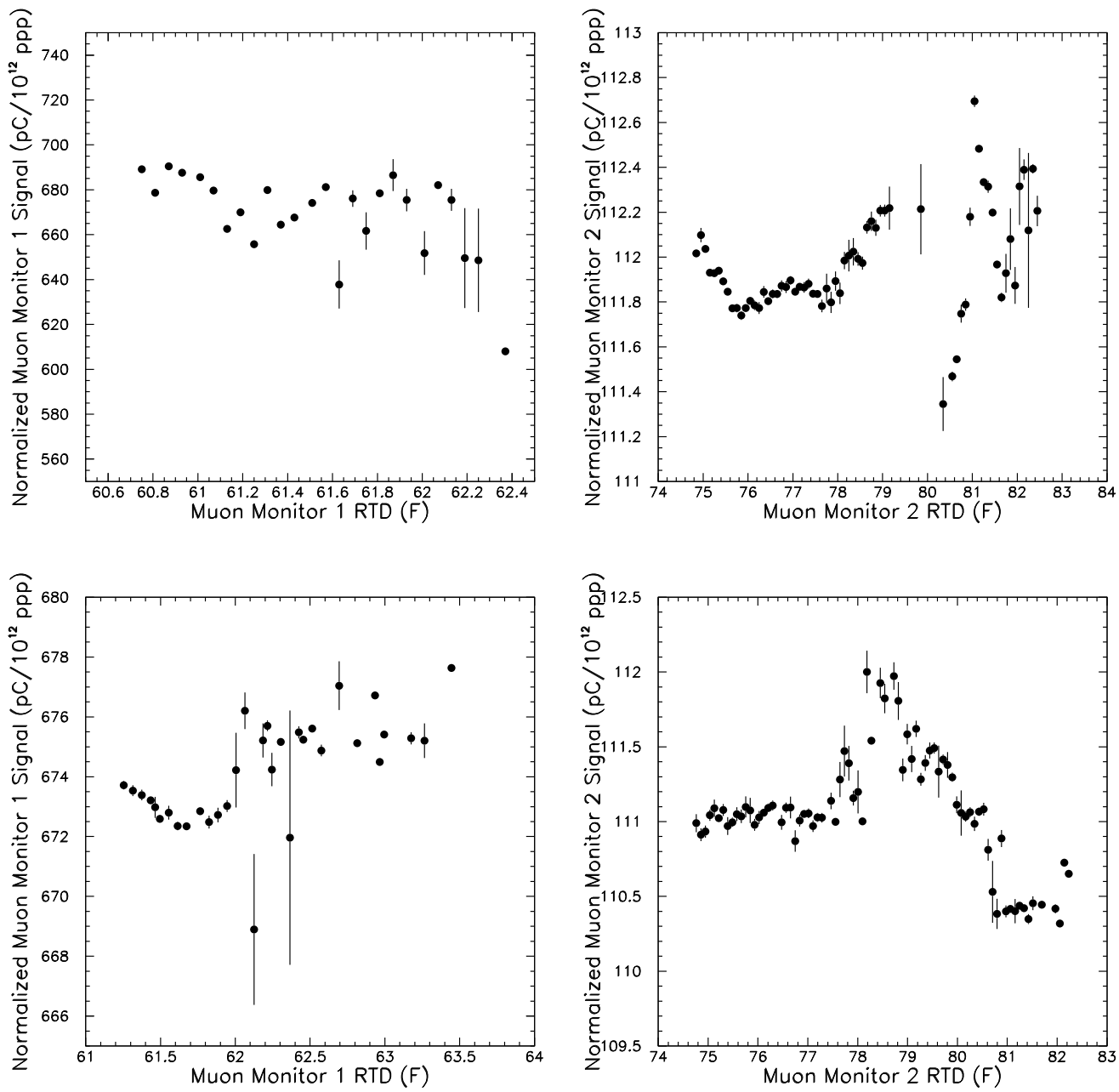

Figure A.7: Total Signal in Muon Monitors 1 (left column) and 2 (right column) as a function of ambient temperature during the first (top row) and second (bottom row) of the 6 data periods corresponding to Table A.1. 


\section{Appendix B}

\section{Alignment of the Proton Beam at the Target}

The hadron and muon monitors play an important role in aligning the target and horns with respect to the primary proton beam as well as monitoring the primary proton beam during normal operation.

It is important that the proton beam is transversely centered on the target for two reasons. First, the proton beam impinging on the edges of the target can cause damage to the target. Second, particles produced in primary proton-target interacts can undergo multiple re interacts upon escaping the target. Reinteractions tend to reduce the energy of particles escaping the target. The probability of reinteraction decreases as the pathlength to escape the target decreases. Thus particles produced nearest the edges of the target have a lower probability to reinteract. Particles produced nearest the edges of the target with have a higher energy than particles produced at the center of the target. Higher energy pions and kaons directly translates to a higher 
energy neutrino beam. It is important for neutrino experiments utilizing the neutrino beam to produce a consistent neutrino flux on a spill-by-spill basis over time.

There have been several times during the operation of NuMI when the target has been removed from the beamline temporarily and twice the target has been exchanged. Every time the target is removed from the beamline, it must be aligned upon reinsertion. The target is initially surveyed into place within the beamline. This procedure is very precise, positioning the target within several, $\sim 3 \mathrm{~mm}$, millimeters transversely of it's appropriate position. However, as the target is only $6.4 \mathrm{~mm}$ wide in the horizontal transverse dimension and the RMS proton beam transverse size is $1.2 \mathrm{~mm}$, this procedure alone is not precise enough.

The target is precisely aligned by scanning the proton beam in the horizontal and vertical directions across the target-baffle system. The signal in the hadron and muon monitors as the proton beam is scanned is used to determine the final position of the target. The target can be aligned to with fraction of millimeter, $\sim 0.5 \mathrm{~mm}$, using this procedure.

\section{B.1 The Hadron Monitor}

Figure B.1 shows the total signal from all chambers(pixels) of the hadron monitor as a function of the horizontal position of the proton beam as the proton beam is scanned horizontally across the face of the target-baffle system. The shaded regions show the position of the target and baffle as seen by the proton beam. The unshaded regions are the gaps in between the target and baffle where the proton beam, with the exception of the outer most protons in 

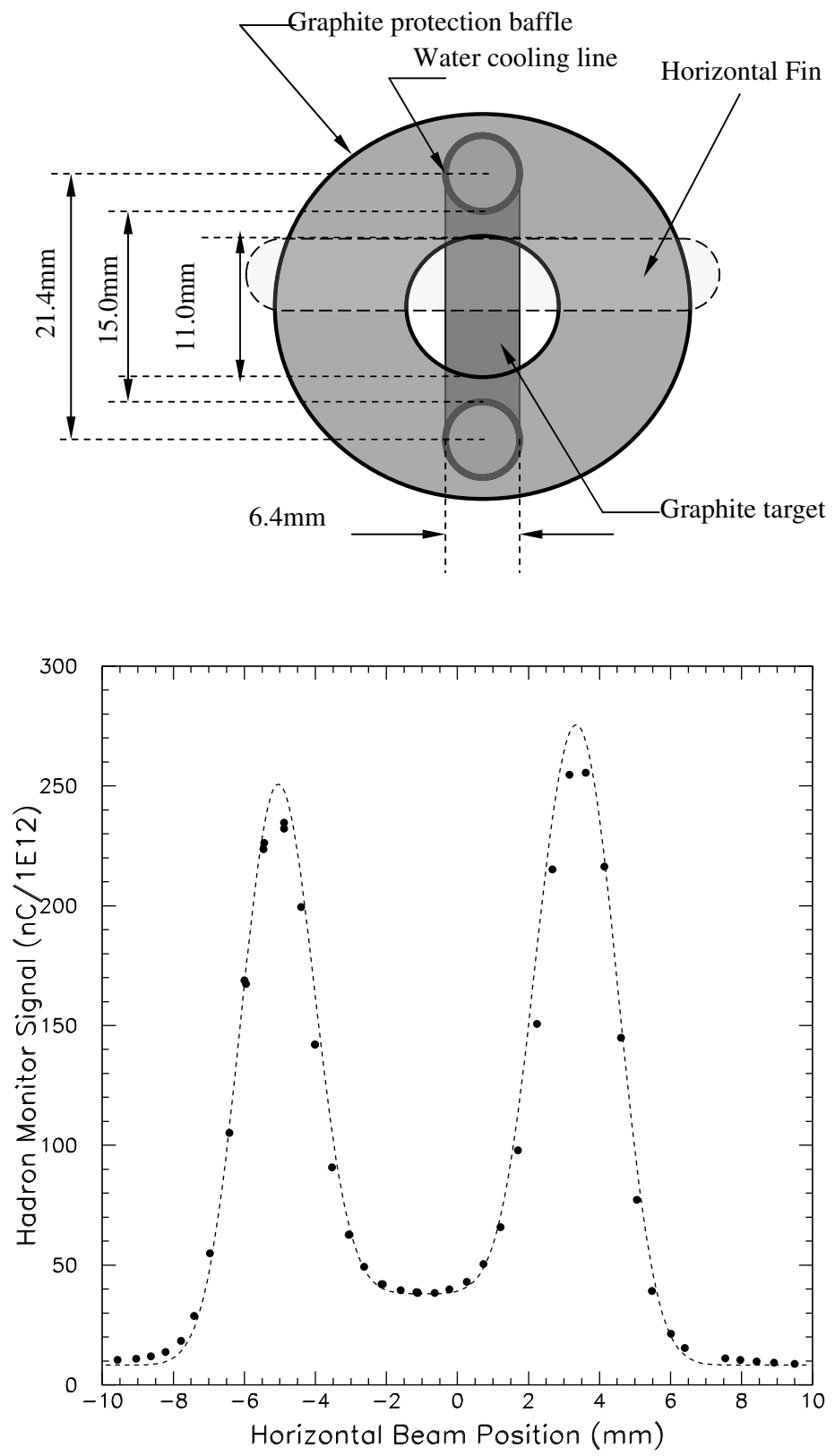

Figure B.1: The signal in the hadron monitor as the proton beam is scanned horizontally across the target-baffle system. The peaks in the signal occur when the proton beam passes through the gap(see top diagram) on either side of the target but before the baffle. The horizontal center of the target is determined by fitting to the central drop in charge and the center of the baffle is found by fitting to the outermost drops in signal. [31] 
the bunch, do not impact the target or baffle and is directly transported down to the absorber. Thus, it makes sense that the highest signal in the hadron monitor occurs when the proton beam is directed at the center of these gaps since the hadron monitor is sensitive to unreacted protons. The lowest signal in the hadron monitor when the beam is directed across the target occurs when the proton beam is exactly aligned with the center of the target. As the proton beam is directed near the edges of the target the hadron monitor signal increases for two reasons. The first is that, since the protons in the spill are contained within a Gaussian distribution with $\sigma=0.8 \mathrm{~mm}$ here, the protons in the tails of the distribution begin to pass through the target-baffle gap and miss the target all together as the proton beam is steered towards the target edges. The second reason is that protons impacting the target nearest the edge have a shorter pathlength over which to interact increasing the probability that protons will escape the target without interacting. As the proton beam moves from gaps onto the baffle the signal in the hadron monitor decreases because more of the proton beam impacts the baffle. When the proton beam is fully impacting the baffle the signal in the hadron monitor is lower than it was when the proton beam was at the center of the target because the baffle is $1.5 \mathrm{~m}$ in longitudinal length compared to the $1 \mathrm{~m}$ length of the target. Thus, less protons remain unreacted in passing through baffle than passing through the target. The fact that the hadron monitor signal is symmetric on either side of the target center indicates very good alignment of the target with respect to the baffle and it indicates that the target-baffle system is parallel to the beam center line. From the signal in the hadron monitor it is found that the horizontal center of the target is at $-0.95 \mathrm{~mm}$ and the center of the baffle is at $-0.75 \mathrm{~mm}$. 
Figure B.2 shows the total signal from all chambers(pixels) of the hadron monitor as a function of the vertical position of the proton beam as the proton beam is scanned vertically across the face of the target-baffle system. The explanations for the magnitude of the signal in the hadron monitor when the proton beam is positioned on the different regions of the target-baffle system is similar to that just described for the horizontal proton beam scan. However, with no gap between the target and baffle in the vertical direction the highest signal in the hadron monitor occurs when the proton beam is on the target.

As the vertical height of the target is $15 \mathrm{~mm}$ compared to the $11 \mathrm{~mm}$ hole of the baffle, it is possible for the target to be vertically off center but still not produce a gap between the target and the baffle. As mentioned in Section 2.1.1, there is a 48th target segment, called the horizontal fin, located $\sim 15.7 \mathrm{~cm}$ upstream of and rotated at $90^{\circ}$ to the main target segments. Thus the vertical height of the horizontal fin is $6.4 \mathrm{~mm}$, providing a gap between the baffle and this fin in the beam's-eye-view of the target-baffle system. The longitudinal length of this fin corresponds to $5 \%$ of an interaction length. This decreases the number of unreacted protons reaching the hadron monitor. However, a decrease in the signal from all of the pixels of the hadron monitor is not seen in Figure B.2, indicating that the decrease in unreacted protons is not measurable in this way. On the other hand, plotting the RMS beam size measured by the hadron monitor as a function of proton beam position, Figure B.3, shows that increased particle scattering resulting from the presence of the horizontal fin is measurable as indicated by the "bump" in the hadron monitor RMS near the vertical center of the target. From this it is determined that the vertical center of the target is at $\mathrm{y}=1.75 \mathrm{~mm}$. 

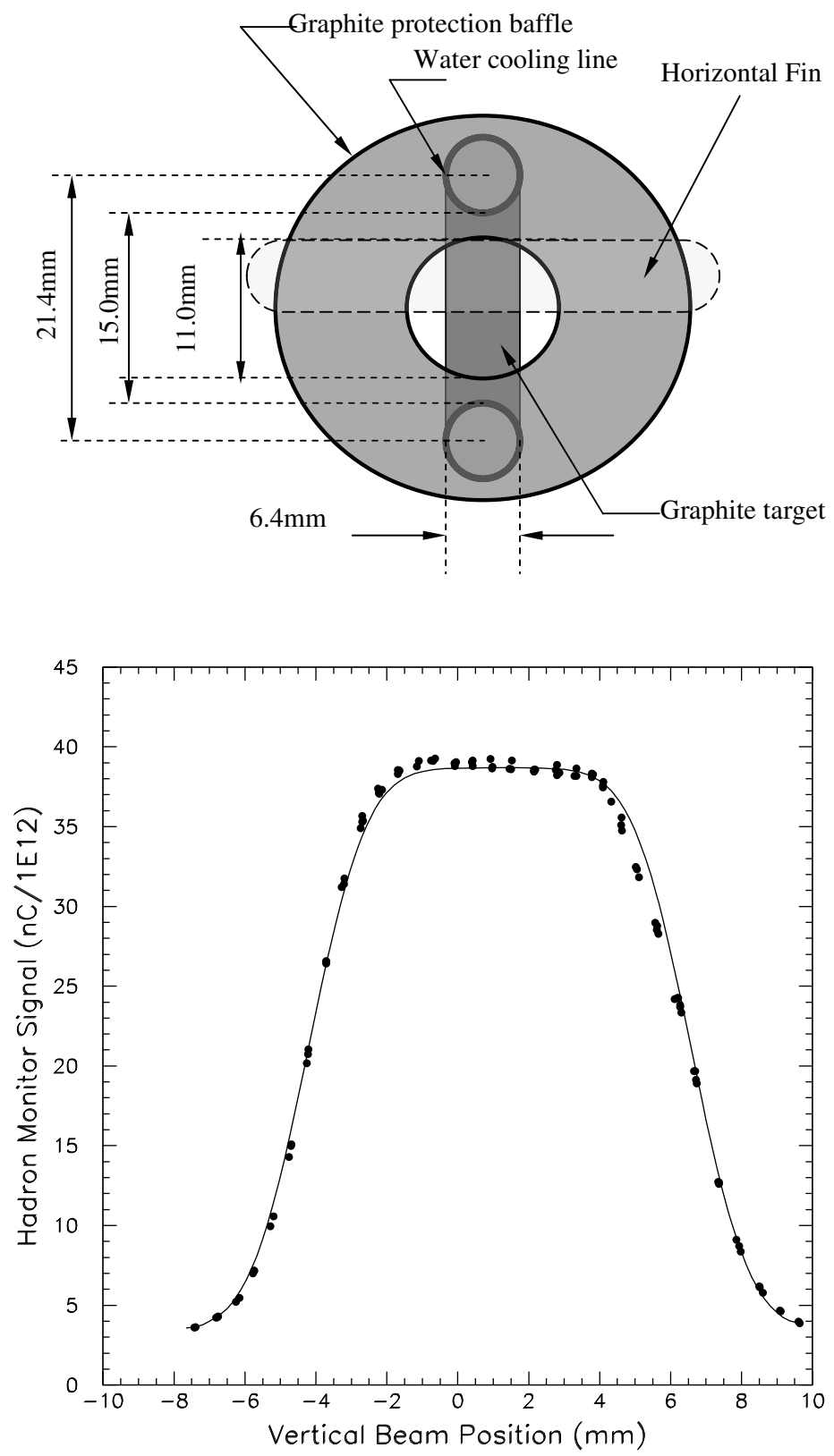

Figure B.2: The signal in the hadron monitor as the proton beam is scanned vertically across the target-baffle system at the horizontal center of the target. The maximum signal occurs when the beam is on the target and drops as the proton beam impinges upon the baffle. The vertical center of the baffle is found by fitting the data to 2 error functions. The baffle center is at $\mathrm{y}=1.2 \mathrm{~mm}$. [31] 

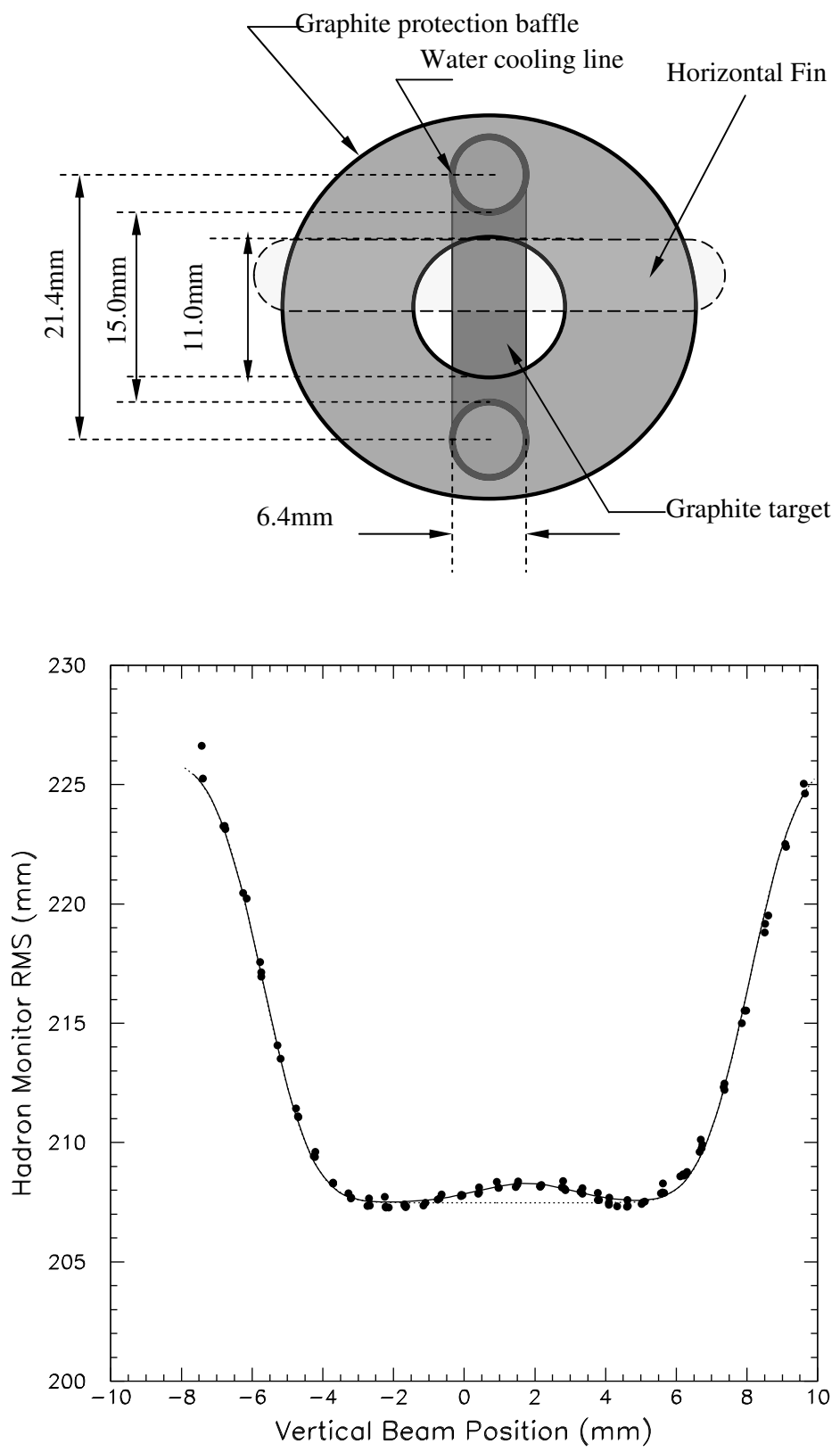

Figure B.3: The RMS size of the beam at the hadron monitor as the proton beam is scanned vertically across the target-baffle system at the horizontal center of the target. The location of the horizontal fin is deduced by examination of the "bump" in the RMS as the proton beam traverses the central target region. [31] 


\section{B.2 The Muon Monitors}

Figure B.4 shows the signal in muon monitors 1, 2 and 3 while the proton beam is scanned horizontally across the target-baffle system with and without horn focusing.

As a reminder, muon monitor 1 measures and integral of the muon flux for muons produced in the decay volume with momenta above $4 \mathrm{GeV}$. In the LE010 beam configuration the horns optimally focus $5 \mathrm{GeV} / \mathrm{c}$ pions and thus the peak of the muon(neutrino) flux occurs at a muon momentum(neutrino energy) of $5 \mathrm{GeV} / c(3 \mathrm{GeV})$. Thus the signal in muon monitor 1 is dominated by low momentum muons when the horns are pulsing. Thus in the LE010 beam configuration, Figure B.4, the signal in muon monitor 1 is greatest when the beam is centered on the target. The reason that the signal when the beam is impinging upon the baffle is lower than on the target is that although the baffle is $0.5 \mathrm{~m}$ longer than the target it is also $\sim 2 \mathrm{~m}$ further upstream of the horns than is the target. As discussed in Section 2.1.2, the further the source is away from the horns the higher the momentum of particle that is selected for full focusing. However, since the baffle is a half an interaction length longer than the target, the more reinteractions occur in the baffle moving the spectrum of particles escaping the baffle towards lower momenta. It is seen that when particles receive no focusing ("Horns Off") the signal in muon monitor 1 is largest when the proton beam passes through the gaps between the target and baffle and impinges on the hadron absorber producing interactions with in the absorber.

Muon monitor 2 measures and integral of the muon flux for muons produced in the decay volume with momenta above $11 \mathrm{GeV}$ so it sensitive 


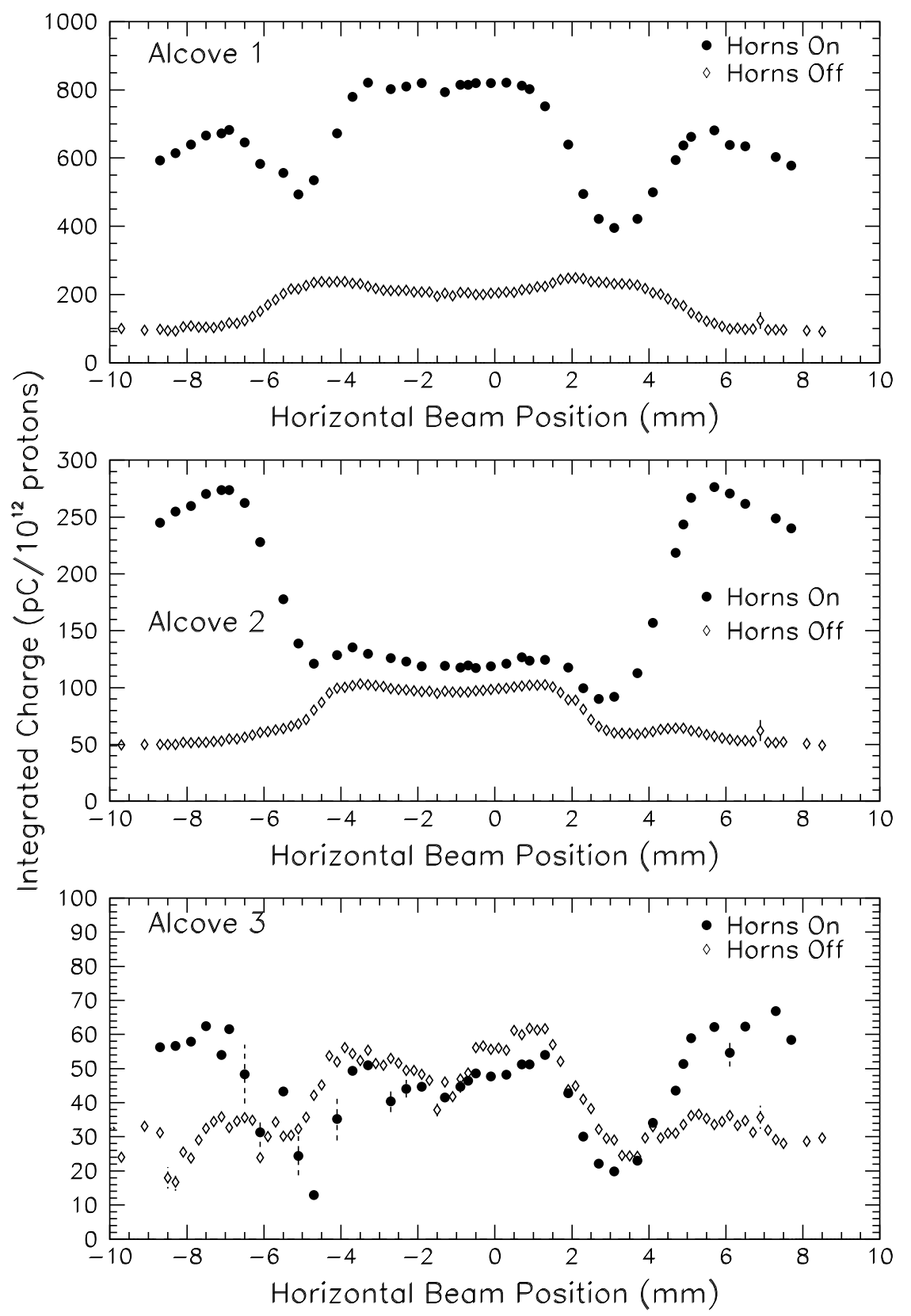

Figure B.4: The signal in Muon Monitors 1, 2, and 3 as the proton beam is scanned horizontally across the target/baffle system when in the LE010 target configuration and the horns are pulsing at $185 \mathrm{kA}$ and with the horns off( $0 \mathrm{kA})$. The target is centered at $\mathrm{x}=-0.95 \mathrm{~mm}$. The muon flux from the target and baffle varies for different focusing conditions and in the different monitors. [31] 
to higher energy muons. Without horn focusing the center of the target is most prominent, since, as just mentioned, the horns optimally focus higher momentum particles originating from the baffle but the large length of the baffle produces more low energy particles than even does the target. Muon Monitor 3, being sensitive to only the highest-energy muons, sees very little signal in LE010.

A very important aspect of these scans is that the flux of higher energy muons, as measured in muon monitors 2 and 3, drops noticeably when the proton beam impacts the center of the target, while as the proton beam is steered from the center of the target towards the edges the flux of higher energy muon increases before eventually decreasing as the beam passes over the gap. This is a result of reinteraction of pions in the target as mentioned previously. Particles produced in primary proton-target interactions nearest the edge of the target have less probability to reinteract before escaping out of the side of the target. Such can be a useful indication of position of the proton beam on the target and an important piece of data when estimating the constancy of the flux over time.

\section{B.3 Beam Position Corrections for the Muon Monitor Signals}

Under normal operating conditions the position of the proton beam at the target varies by less than $\sim 0.5 \mathrm{~mm}$. However, as just discussed, muon rates are affected by even slight offsets of the position of the proton beam on the target. Thus, when comparing muon rates, as is done for the horn current 
scans in Section 3.4, it is necessary to correct for slight offsets of the proton beam at the target.

Every time the target is removed and replaced in the beamline and every time the target is moved into a different longitudinal position, horizontal and vertical target scans are performed to check the alignment of the target-baffle system. So, there exist horizontal and vertical target scans performed in each beam configuration, LE000, LE010, LE100, LE150 and LE250. The signal in the muon monitors is plotted as a function of beam position as the proton beam is scanned across the target-baffle system. Based on the scans a correction is developed to account for the difference in muon rates measured with an offcenter proton beam compared to the muon rates when the proton beam is centered on the target.

Figures B.5 through B.7 show the results of six scans of the proton beam across the target and baffle in the horizontal direction. The center of the target is at $X=-1.2 \mathrm{~mm}$. The higher muon rates as the proton beam approaches the target edges confirms the lower attenuation of fast pions. The average position of the proton beam at the target during the horn scans varies from $\left\langle X_{T G T}\right\rangle=-0.84 \mathrm{~mm}$ to $-1.57 \mathrm{~mm}$.

Figures B.8 through B.10 show the results of six scans of the proton beam across the target and baffle in the vertical direction. The center of the target is at $X=1.1 \mathrm{~mm}$. The average position of the proton beam at the target during the horn scans varies from $\left\langle X_{T G T}\right\rangle=-0.84 \mathrm{~mm}$ to $-1.57 \mathrm{~mm}$.

It may be noted that the target scans performed in 2006 with $z_{\text {target }}=$ $150 \mathrm{~cm}$ and $250 \mathrm{~cm}$ show less definition, especially in Alcove 3. Following the Spring 2006 shutdown, the target was noted to be at a slight angle with respect to the proton beam axis. To obtain a better looking scan, a tilt was imposed 

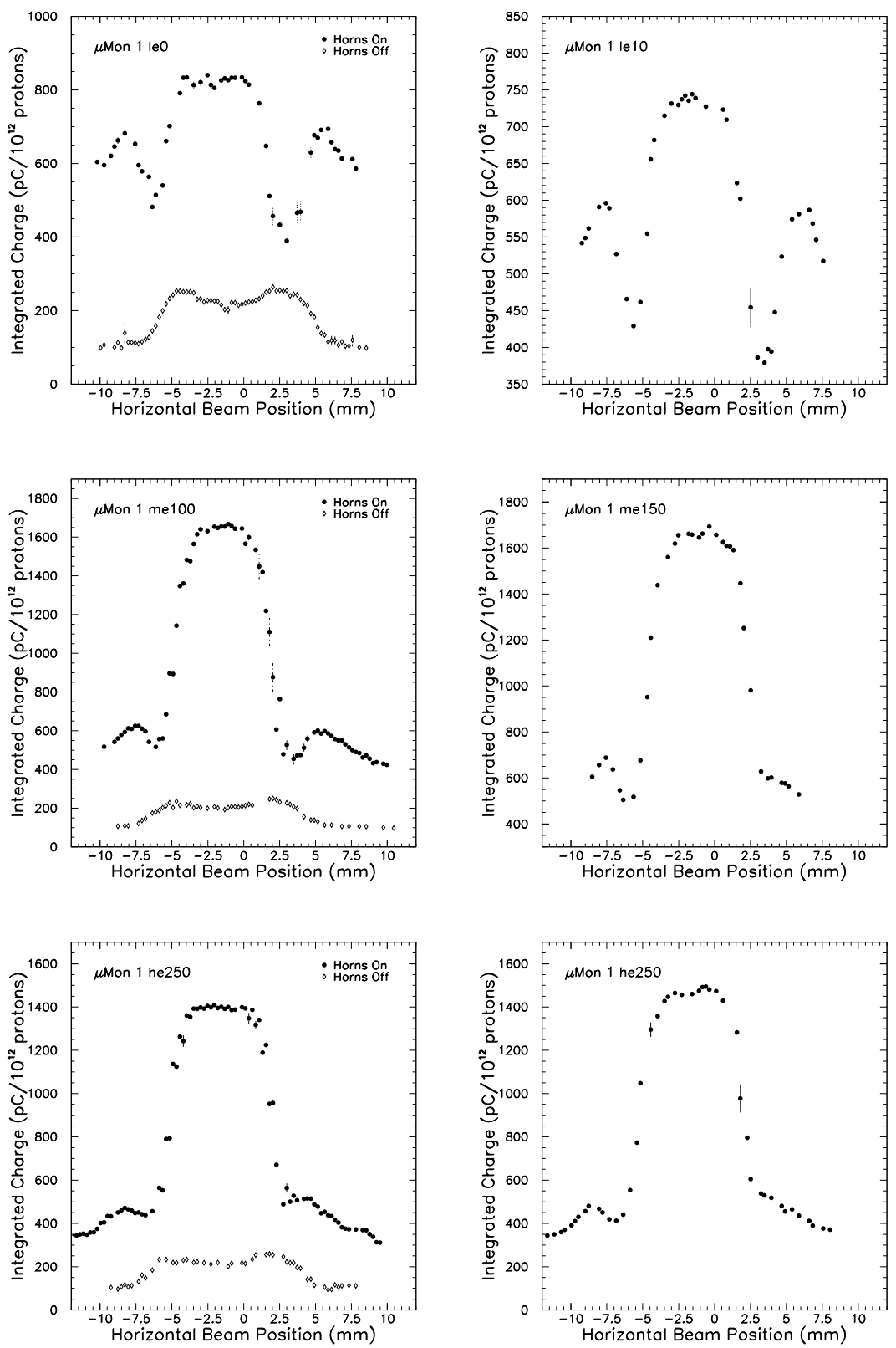

Figure B.5: Results of scans of the proton beam across the target and baffle in the horizontal direction. The muon flux in Muon Monitor 1 is measured with the target at the $z=0 \mathrm{~cm}, 10 \mathrm{~cm}, 100 \mathrm{~cm}, 150 \mathrm{~cm}$, and $250 \mathrm{~cm}$ (both Feb. ' 05 and June '06 data sets), both with and without the horns being pulsed. 

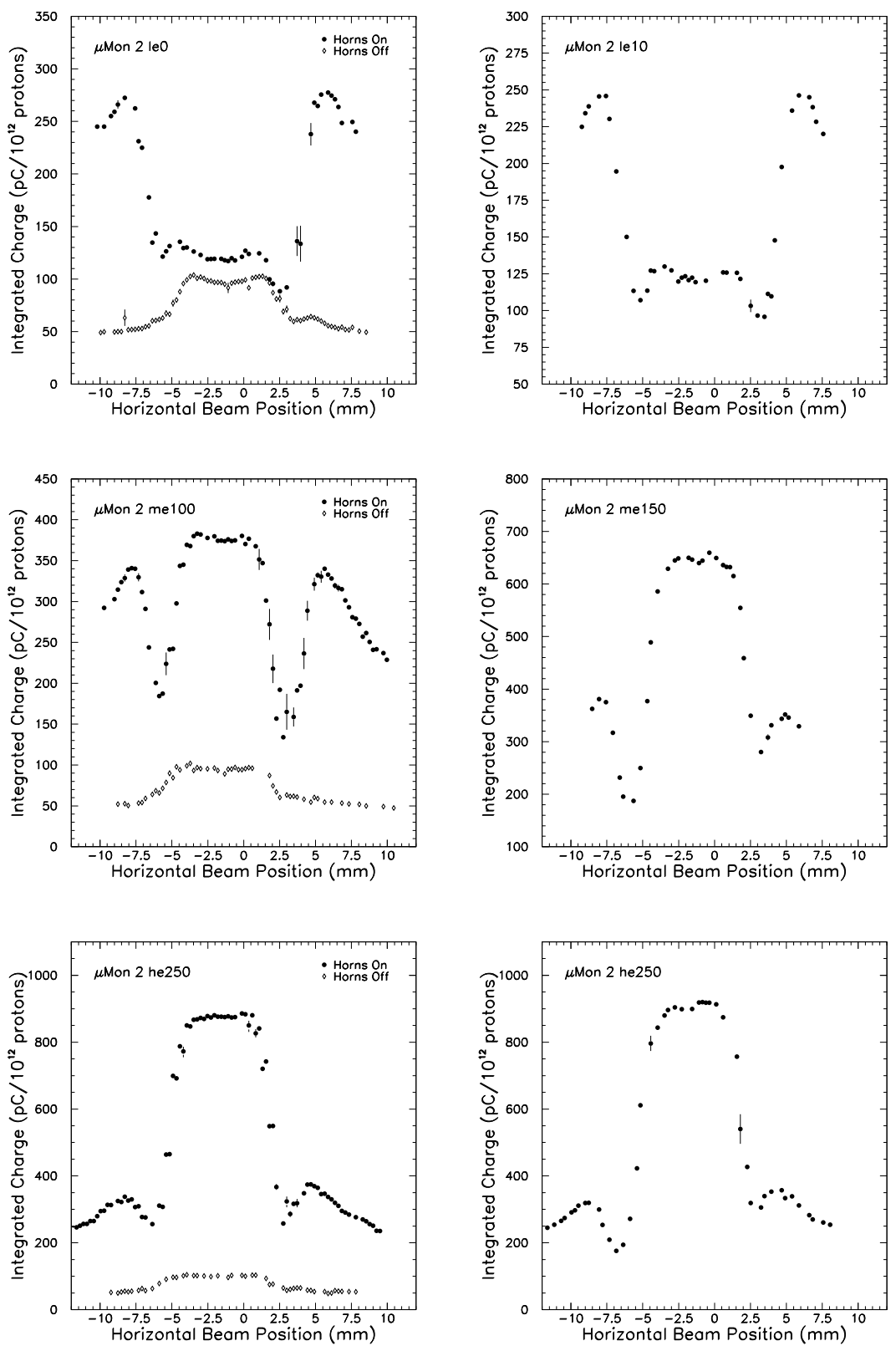

Figure B.6: Results of scans of the proton beam across the target and baffle in the horizontal direction. The muon flux in Muon Monitor 2 is measured with the target at the $z=0 \mathrm{~cm}, 10 \mathrm{~cm}, 100 \mathrm{~cm}, 150 \mathrm{~cm}$, and $250 \mathrm{~cm}$ (both Feb. ' 05 and June " 06 data sets), both with and without the horns being pulsed. 

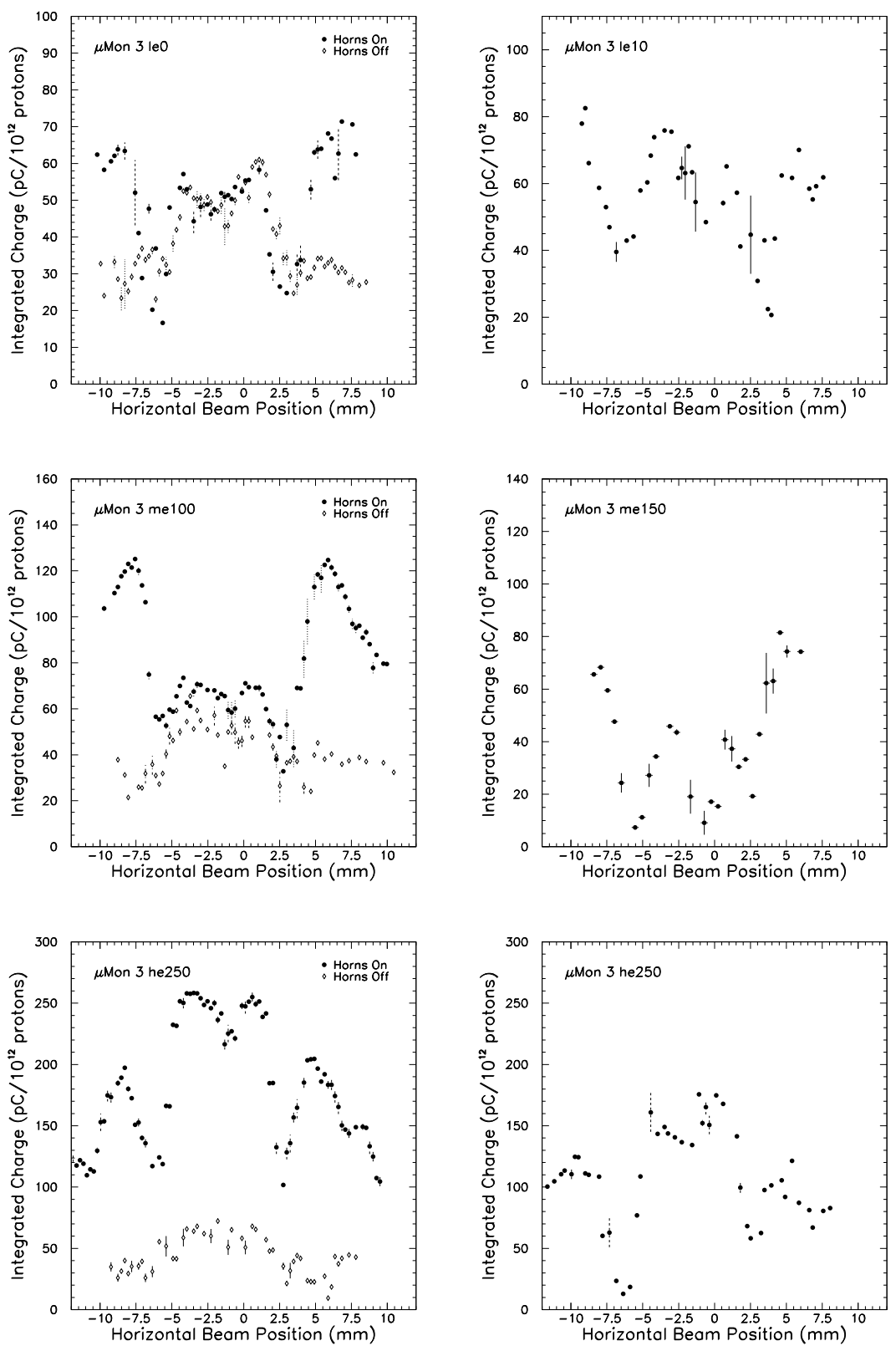

Figure B.7: Results of scans of the proton beam across the target and baffle in the horizontal direction. The muon flux in Muon Monitor 3 is measured with the target at the $z=0 \mathrm{~cm}, 10 \mathrm{~cm}, 100 \mathrm{~cm}, 150 \mathrm{~cm}$, and $250 \mathrm{~cm}$ (both Feb. '05 and June '06 data sets), both with and without the horns being pulsed. 

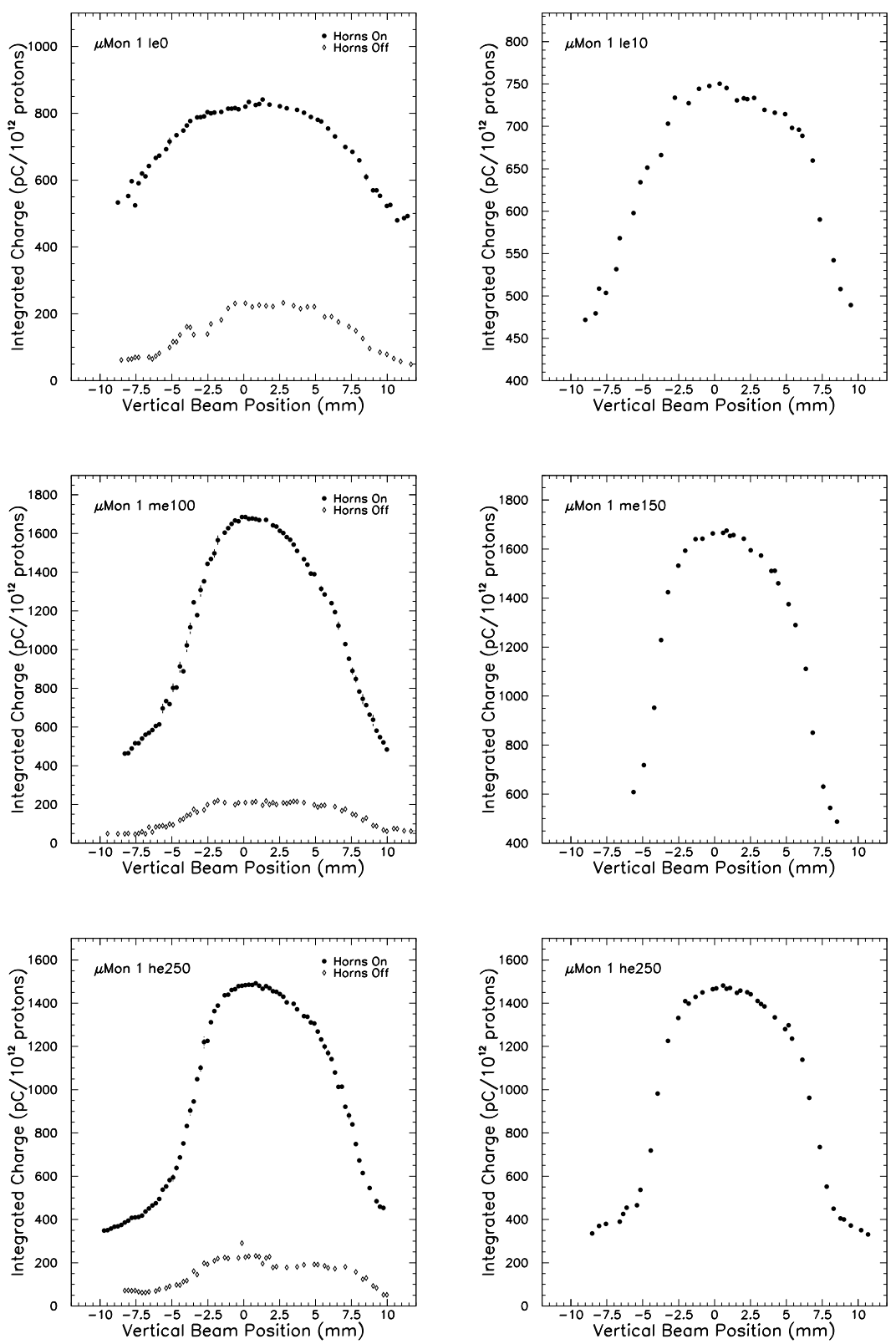

Figure B.8: Results of scans of the proton beam across the target and baffle in the vertical direction. The muon flux in Muon Monitor 1 is measured with the target at the $z=0 \mathrm{~cm}, 10 \mathrm{~cm}, 100 \mathrm{~cm}, 150 \mathrm{~cm}$, and $250 \mathrm{~cm}$ (both Feb. ' 05 and June ' 06 data sets), both with and without the horns being pulsed. 

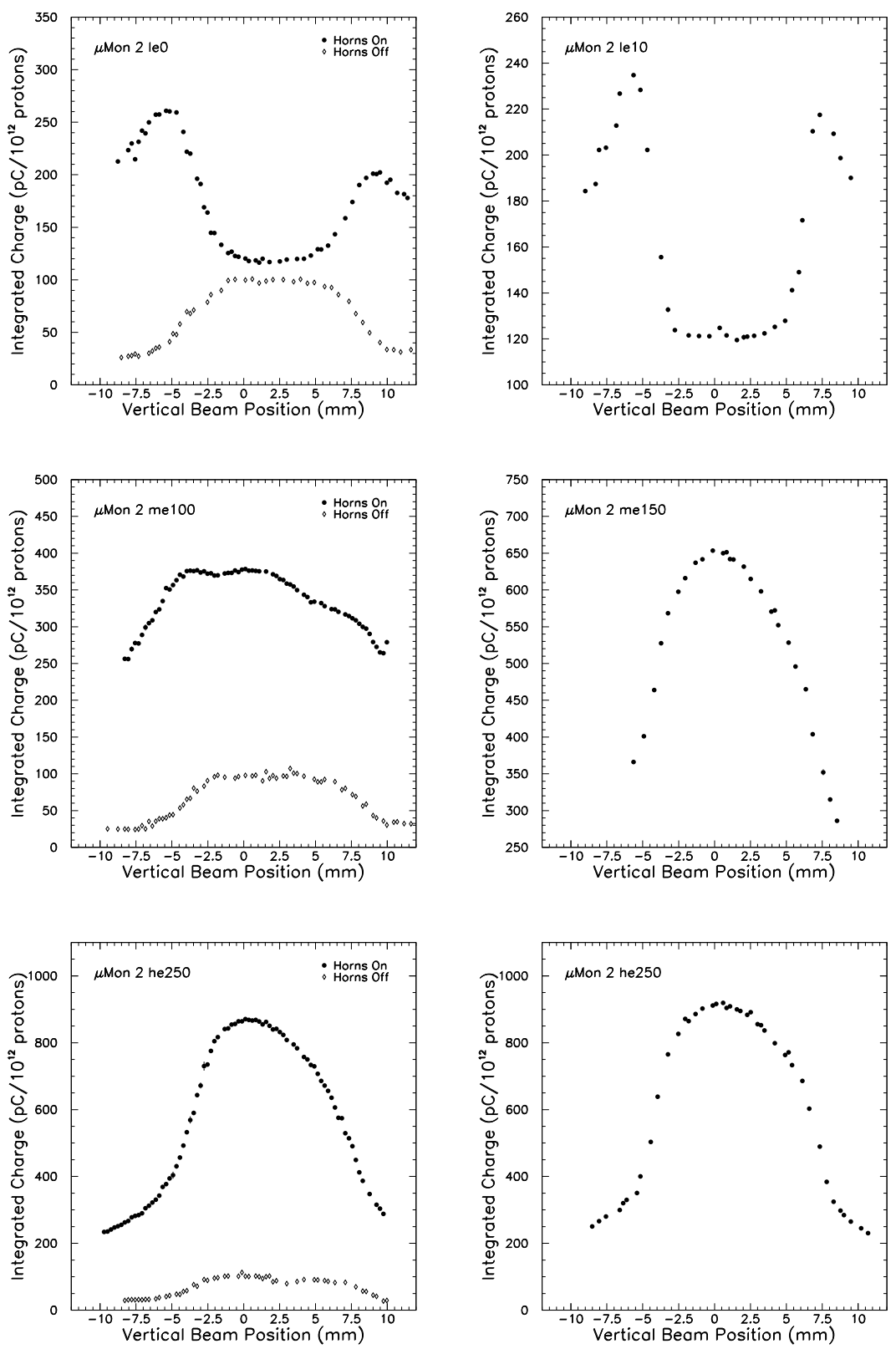

Figure B.9: Results of scans of the proton beam across the target and baffle in the vertical direction. The muon flux in Muon Monitor 2 is measured with the target at the $z=0 \mathrm{~cm}, 10 \mathrm{~cm}, 100 \mathrm{~cm}, 150 \mathrm{~cm}$, and $250 \mathrm{~cm}$ (both Feb. ' 05 and June ' 06 data sets), both with and without the horns being pulsed. 

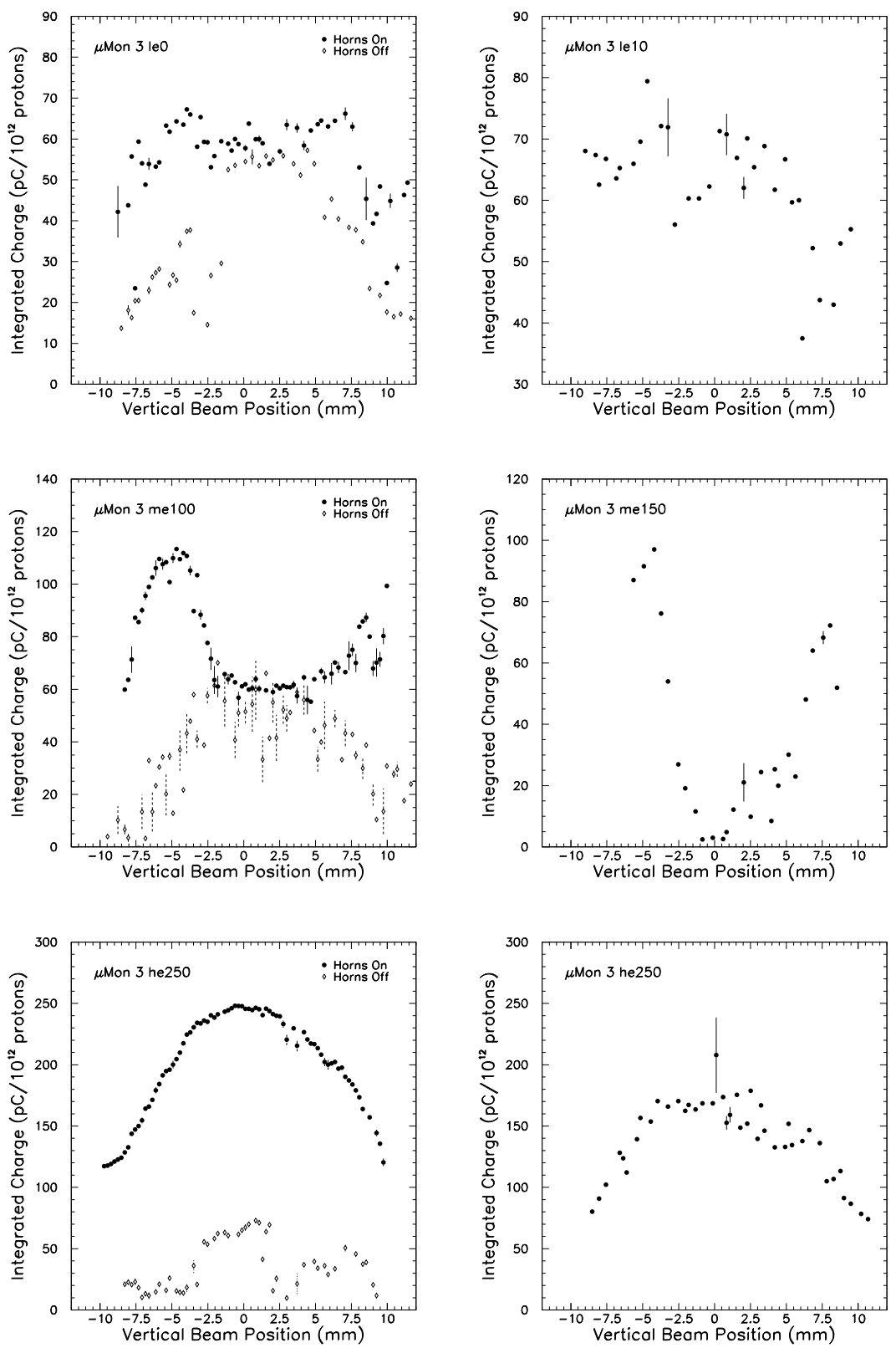

Figure B.10: Results of scans of the proton beam across the target and baffle in the vertical direction. The muon flux in Muon Monitor 3 is measured with the target at the $z=0 \mathrm{~cm}, 10 \mathrm{~cm}, 100 \mathrm{~cm}, 150 \mathrm{~cm}$, and $250 \mathrm{~cm}$ (both Feb. '05 and June '06 data sets), both with and without the horns being pulsed. 
in the proton beam horizontal direction. This tilt was not implemented at the time of the horn current scans in Figures 3.20 through 3.22, however. Therefore, the scans of the proton beam across the target in Figures B.5 through B.10 are shown with zero proton beam angle.

The data of Figures B.5 through B.10 are fit in the center region to 5 th-order polynomials to parameterize the response of the muon rates in each monitor as a function of proton beam position. In some cases, the fits do not describe the data particularly well or a fit to a flat function would equally well suffice. A correction which scales the muon rate at an off target center position to the rate at the center of the target is then calculated.

Table 3.5 lists the resulting corrections that are applied to the horn current scan data in each of the muon monitors, as well as the uncertainty on this correction, due to proton beam centering effects. 


\section{Appendix C}

\section{Flux Measurement Systematics}

Figures C.1 through C.6 show the individual components (Section 6.1) of the total error on the nominal fitted flux measurement after normalization (Section 6.2). 

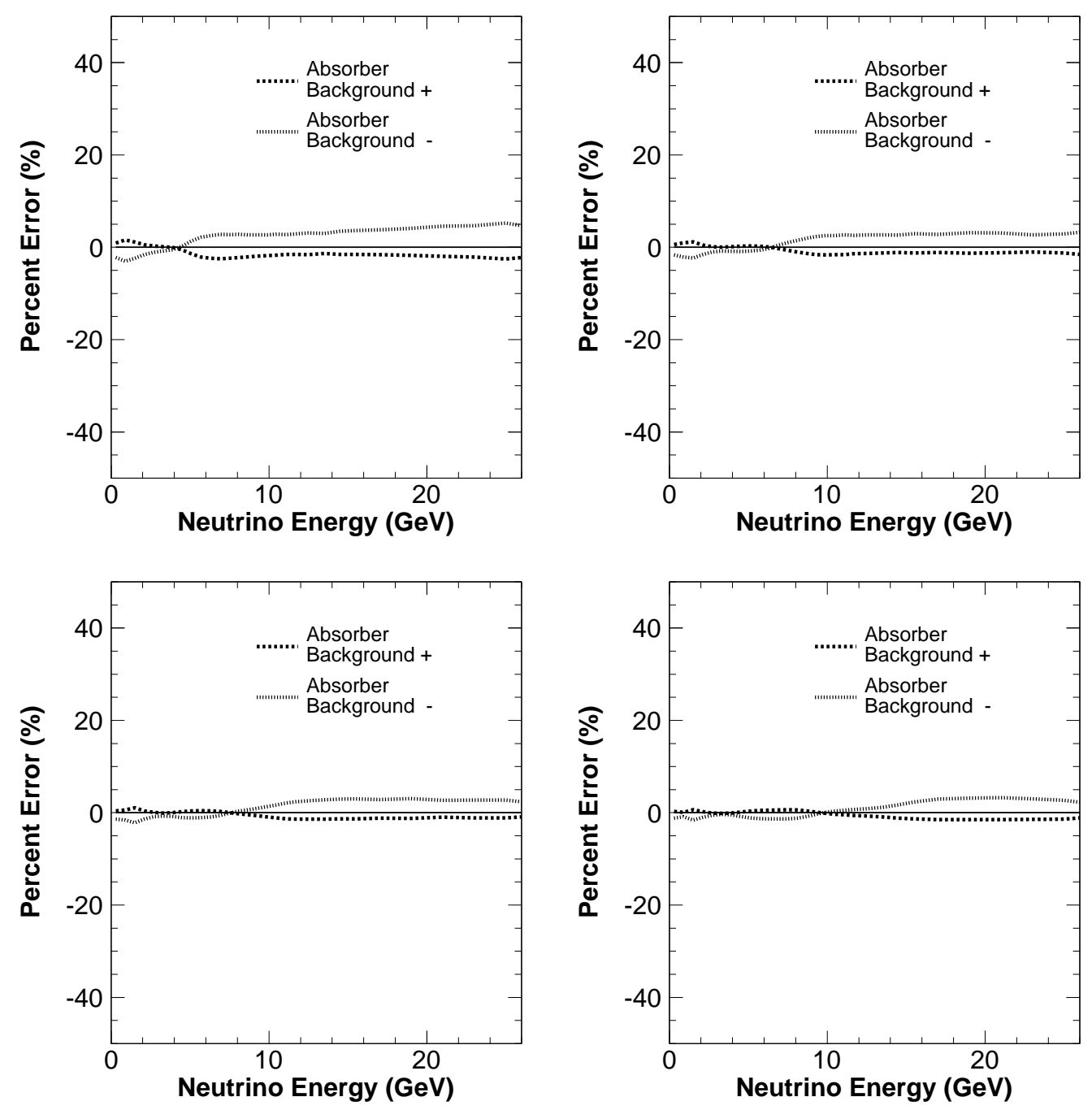

Figure C.1: The percent error on the neutrino flux measurement after normalization for the LE010/185kA(top left), LE100/200kA(top right), LE150/200kA(bottom left), and LE250/200kA(bottom right) beams from increasing $(+)$ or decreasing $(-)$ the absorber backgrounds in each muon monitor by $1 \sigma$. The + and - curves result from refitting for the flux after either increasing or decreasing the background. 

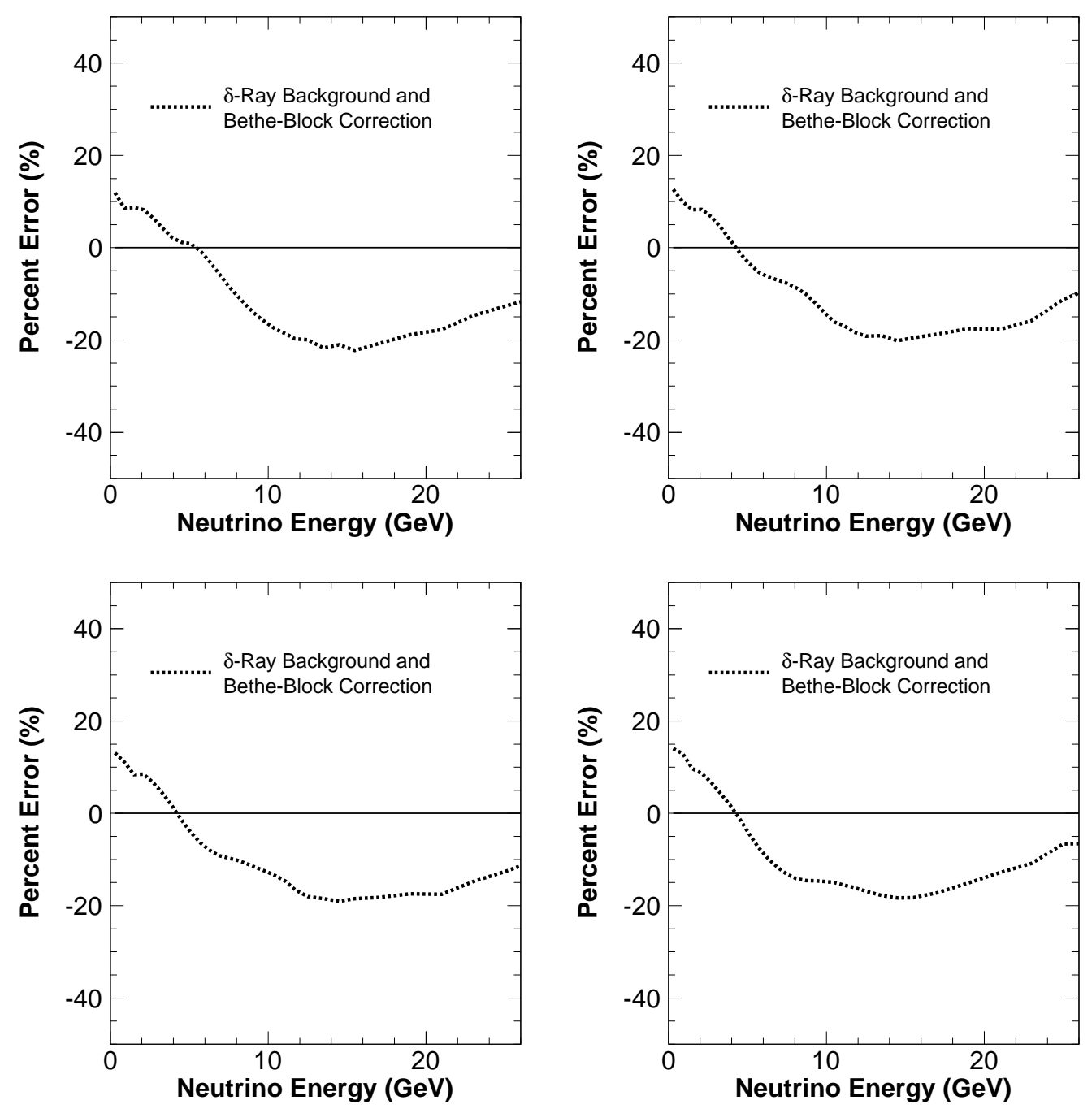

Figure C.2: The percent error on the neutrino flux measurement after normalization for the LE010/185kA(top left), LE100/200kA(top right), LE150/200kA(bottom left), and LE250/200kA(bottom right) beams from applying Bethe-Block to calculate the momentum dependent energy deposition of charged particles in the muon monitors and from including $\delta$-ray backgrounds in the muon monitors. The curve results from refitting for the cross section after applying these changes. 

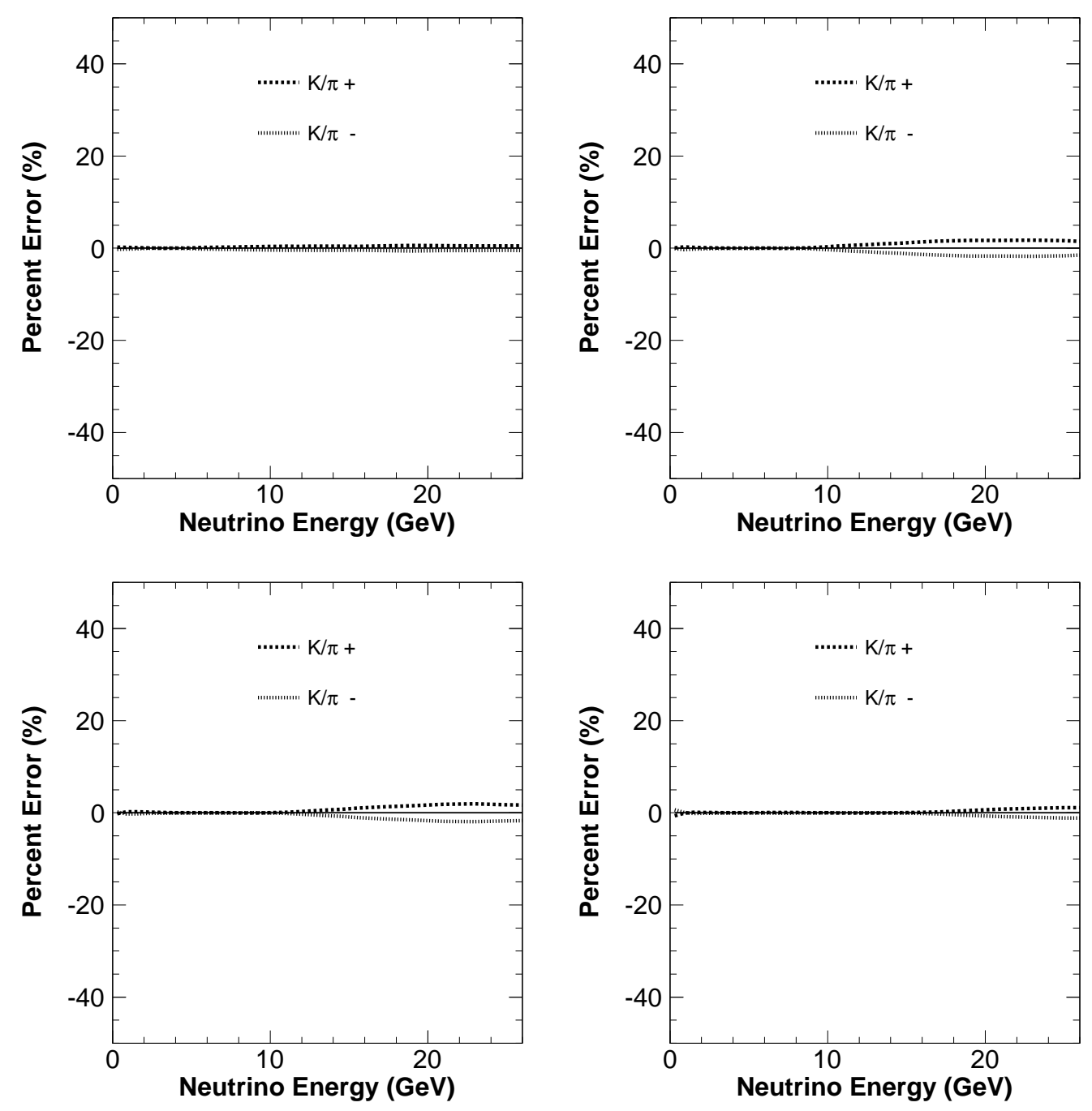

Figure C.3: The percent error on the neutrino flux measurement for after normalization the LE010/185kA(top left), LE100/200kA(top right), LE150/200kA(bottom left), and LE250/200kA(bottom right) beams from increasing $(+)$ or decreasing $(-)$ the Fluka05 $K^{+} / \pi^{+}$ratio by 10 percent. The + and - curves result from refitting for the flux after either increasing or decreasing the $K^{+} / \pi^{+}$ratio. 

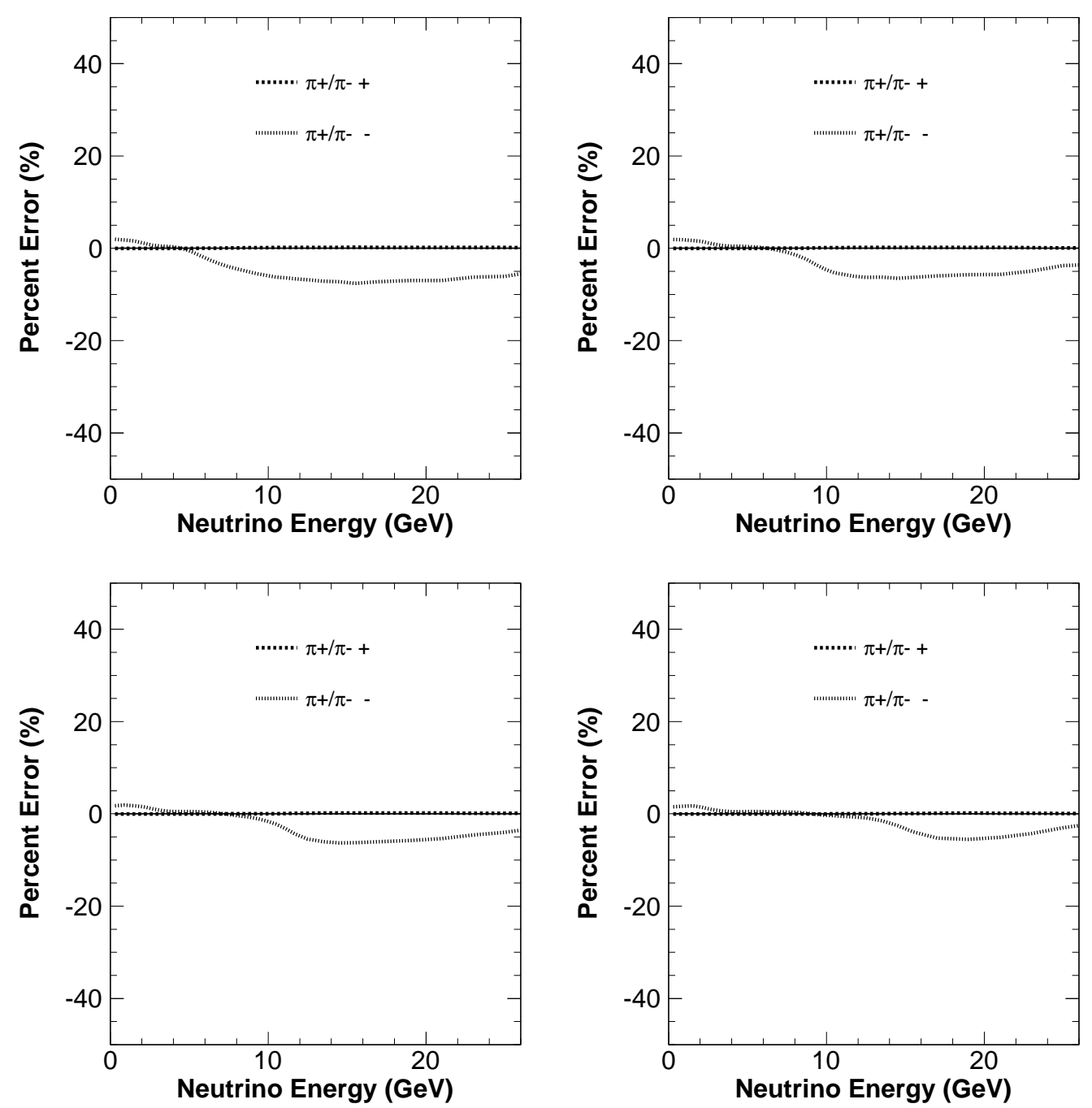

Figure C.4: The percent error on the neutrino flux measurement after normalization for the LE010/185kA(top left), LE100/200kA(top right), LE150/200kA(bottom left), and LE250/200kA(bottom right) beams from fixing the $\pi^{+} / \pi^{-}$ratio to the NA49 measurement fit with a fourth order polynomial $(+)$, as shown in Figure 4.9, or fixing the $\pi^{+} / \pi^{-}$ratio to the Fluka05 $\pi^{+} / \pi^{-}$ratio (-), also shown in Figure 4.9. The former results in an increase in the $\pi^{+} / \pi^{-}$ratio with respect to nominal(the NA49 measurement fit with a third order polynomial) and the latter results in a decrease in the $\pi^{+} / \pi^{-}$ ratio with respect to nominal. The + and - curves result from refitting for the neutrino flux after either increasing or decreasing the $\pi^{+} / \pi^{-}$ratio. 

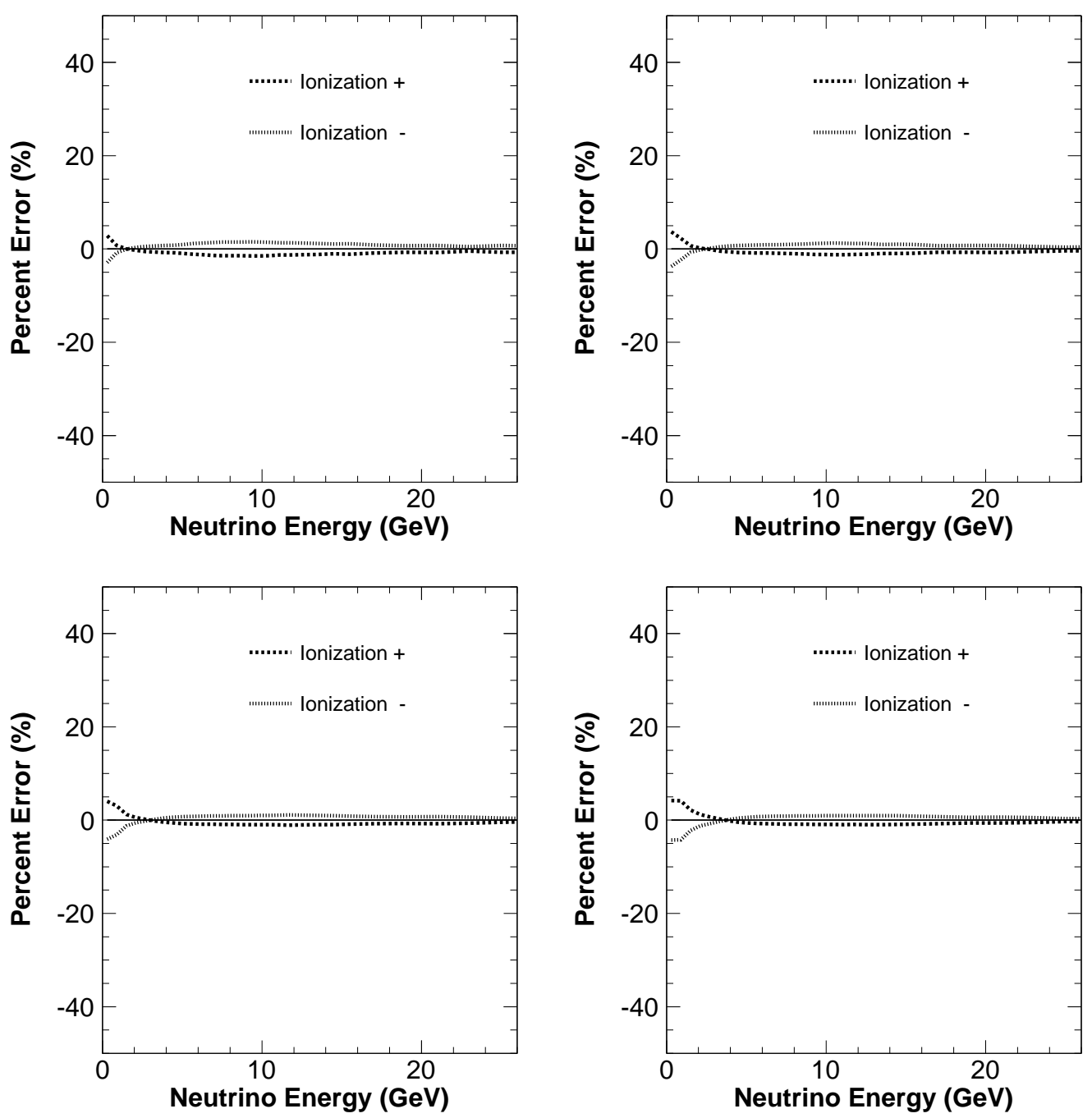

Figure C.5: The percent error on the neutrino flux measurement after normalization for the LE010/185kA(top left), LE100/200kA(top right), LE150/200kA(bottom left), and LE250/200kA(bottom right) beams from increasing $(+)$ or decreasing $(-)$ the muon monitor ionization scale factor by 10 percent. The + and - curves result from refitting for the flux after either increasing or decreasing the ionization scale factor. 

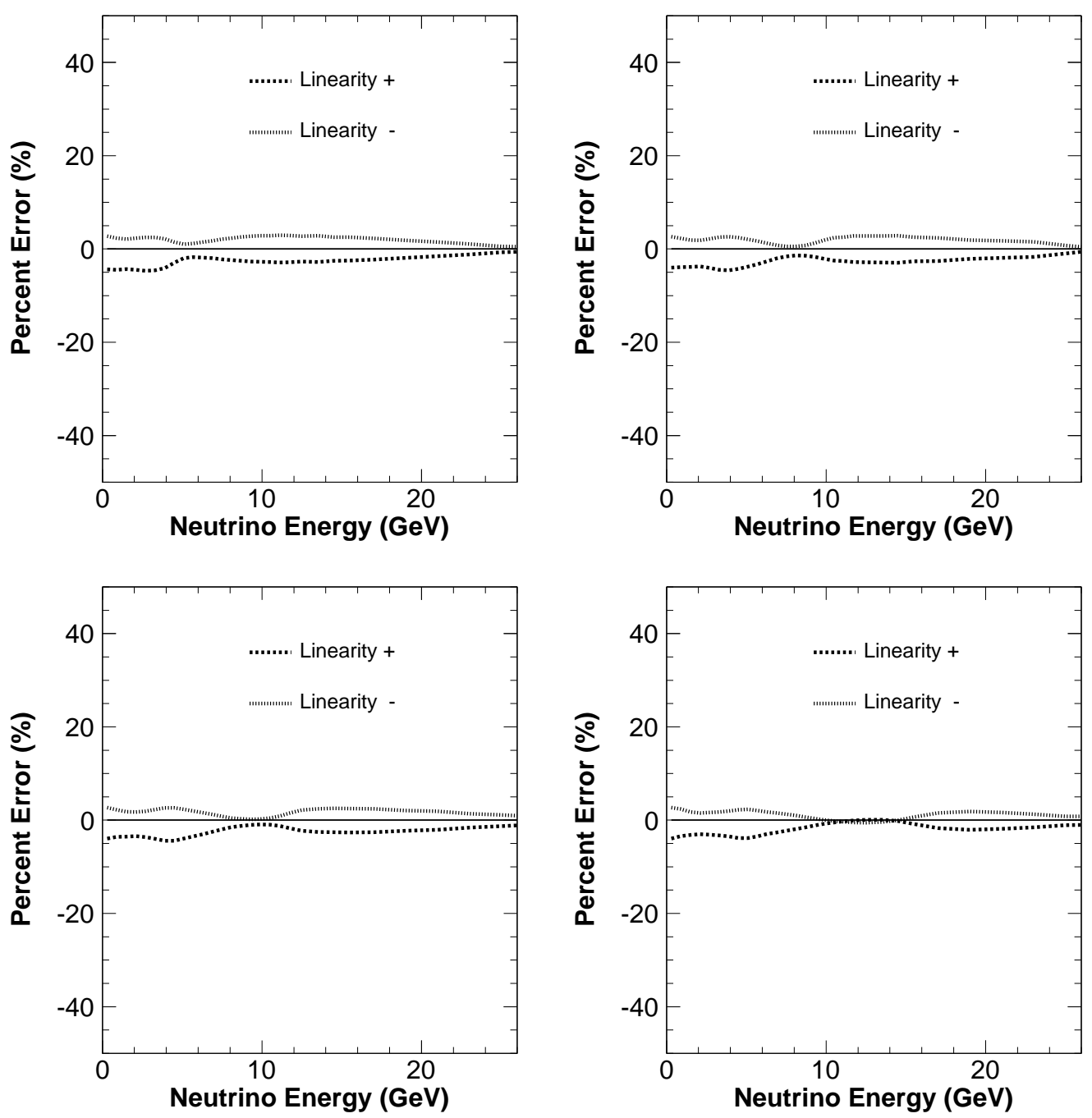

Figure C.6: The percent error on the neutrino flux measurement after normalization for the LE010/185kA(top left), LE100/200kA(top right), LE150/200kA(bottom left), and LE250/200kA(bottom right) beams from increasing $(+)$ or decreasing $(-)$ the muon monitor the linearity correction by $1 \sigma$. The + and - curves result from refitting for the flux after either increasing or decreasing the the linearity correction. 


\section{Appendix D}

\section{Cross Section Measurement Systematics}

This appendix elaborates on the discussion in Chapter 7 of the systematic uncertainties that contribute to the charged current $\nu_{\mu}$ cross section measurement. Figures D.1 through D.7 show systematics from detector and event selection sources, while Figures D.8 through D.13 show systematics stemming from the neutrino flux measurement. 


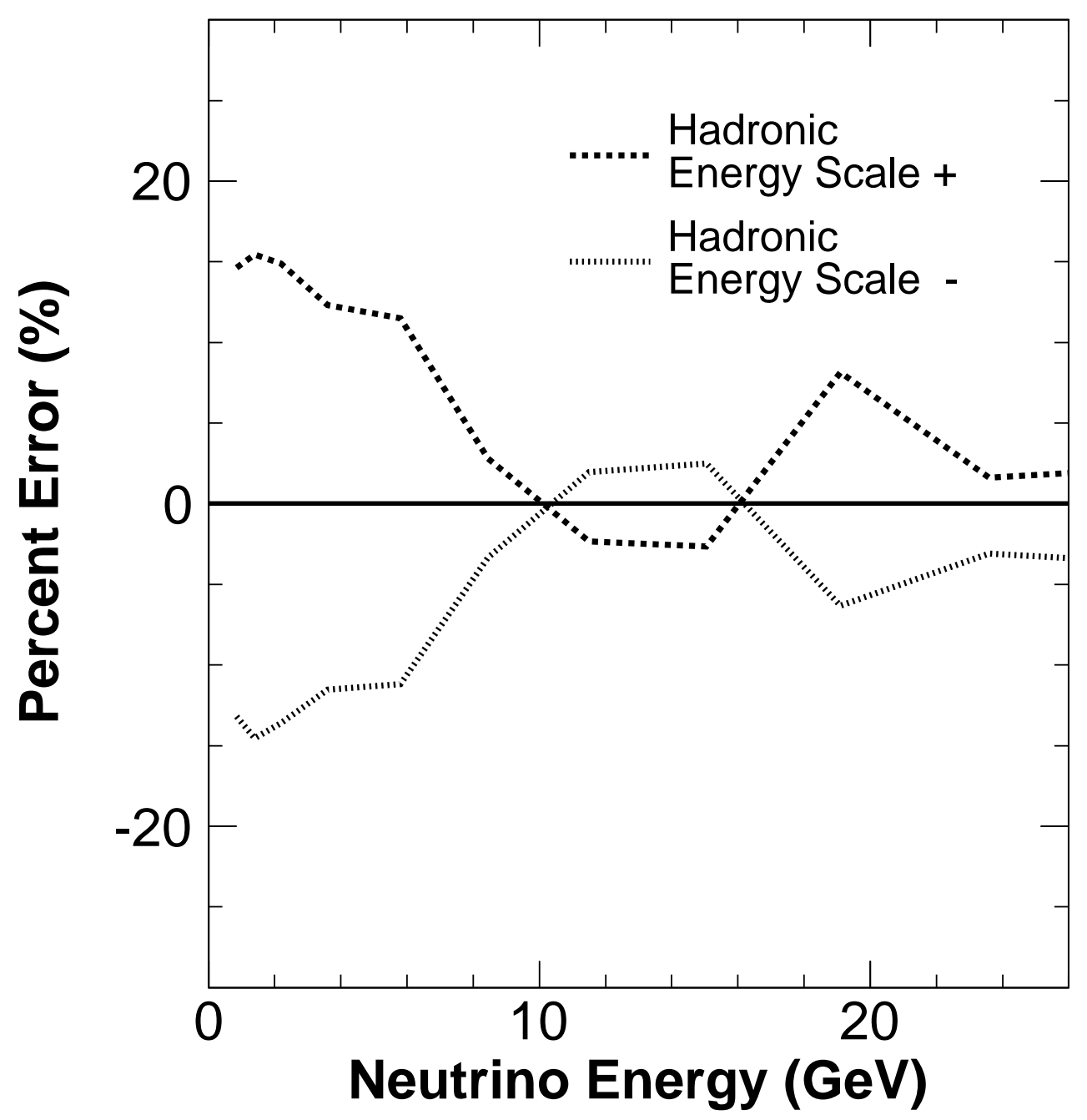

Figure D.1: The percent error on the cross section measurement from increasing $(+)$ or decreasing $(-)$ the hadronic energy scale by the amount shown in Figure 2.22. The + and - curves result from refitting for the cross section after either increasing or decreasing the hadronic energy scale. 


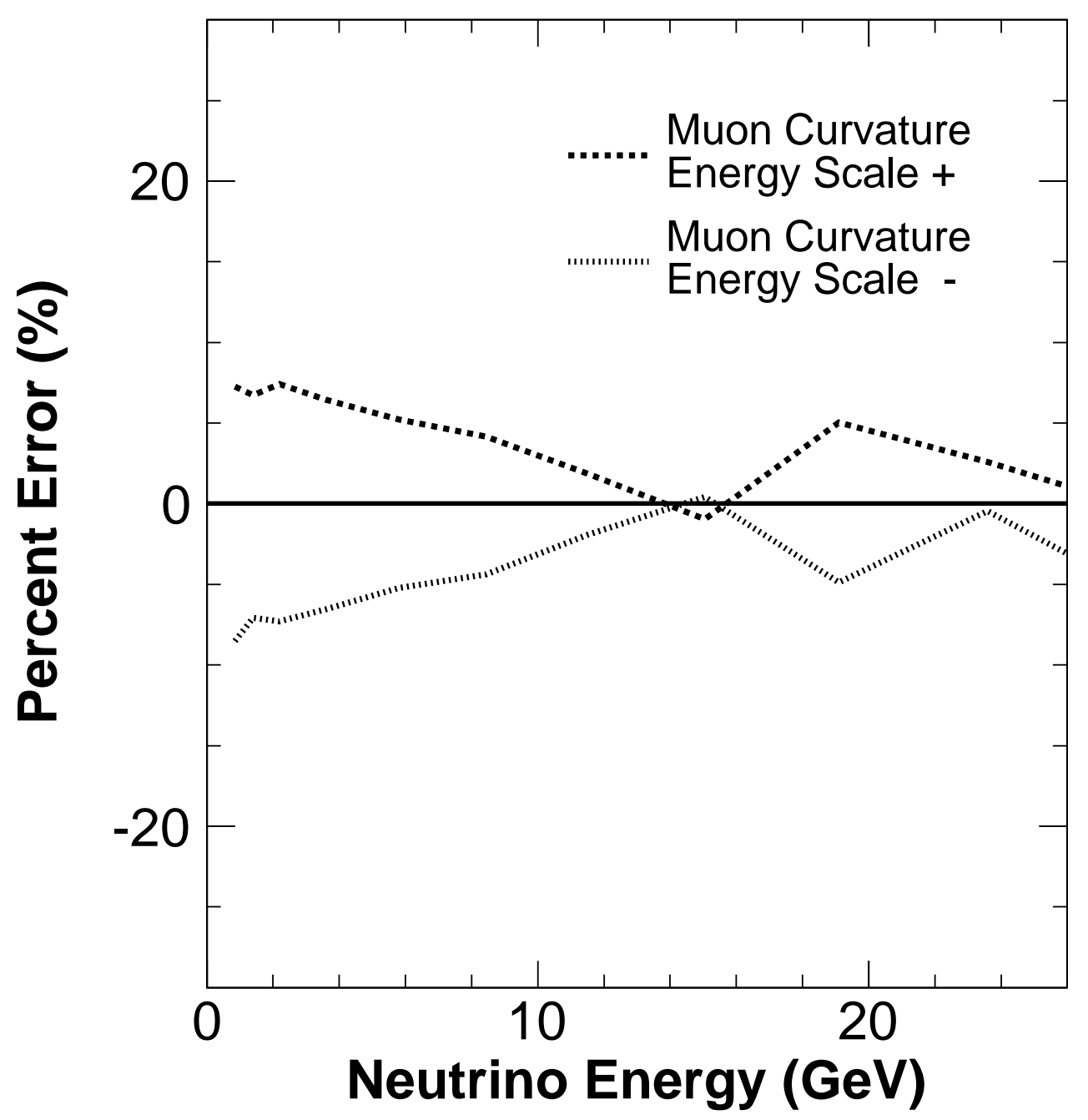

Figure D.2: The percent error on the cross section measurement from increasing $(+)$ or decreasing (-) the muon curvature energy scale by 4 percent. The + and - curves result from refitting for the cross section after either increasing or decreasing the muon curvature energy scale. 


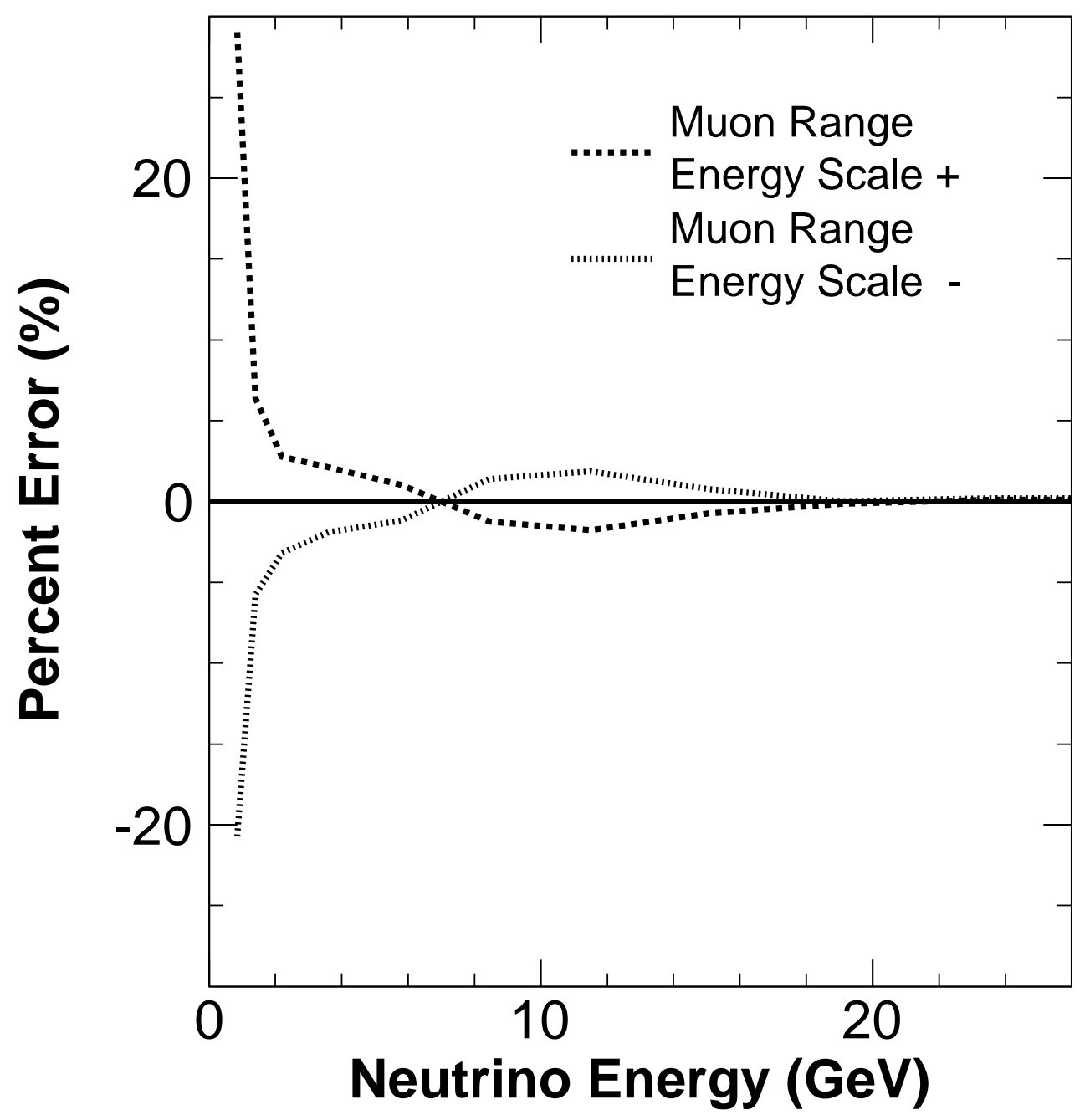

Figure D.3: The percent error on the cross section measurement from increasing $(+)$ or decreasing $(-)$ the muon range energy scale by 2 percent. The + and - curves result from refitting for the cross section after either increasing or decreasing the muon range energy scale. 


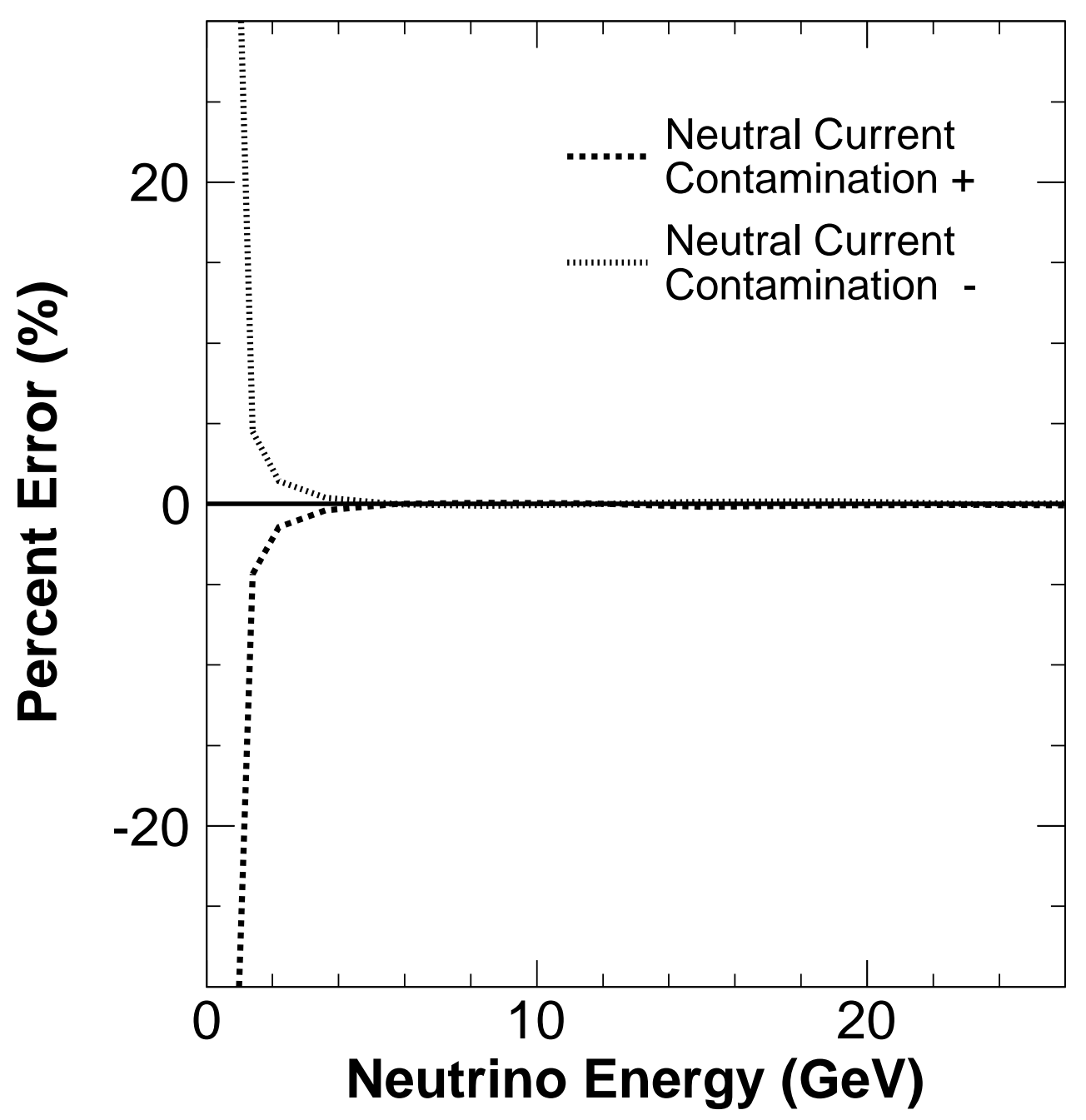

Figure D.4: The percent error on the cross section measurement from increasing $(+)$ or decreasing (-) the number of neutral current events in the charged current muon neutrino event sample by 50 percent. The + and - curves result from refitting for the cross section after either increasing or decreasing the number of neutral current events. 


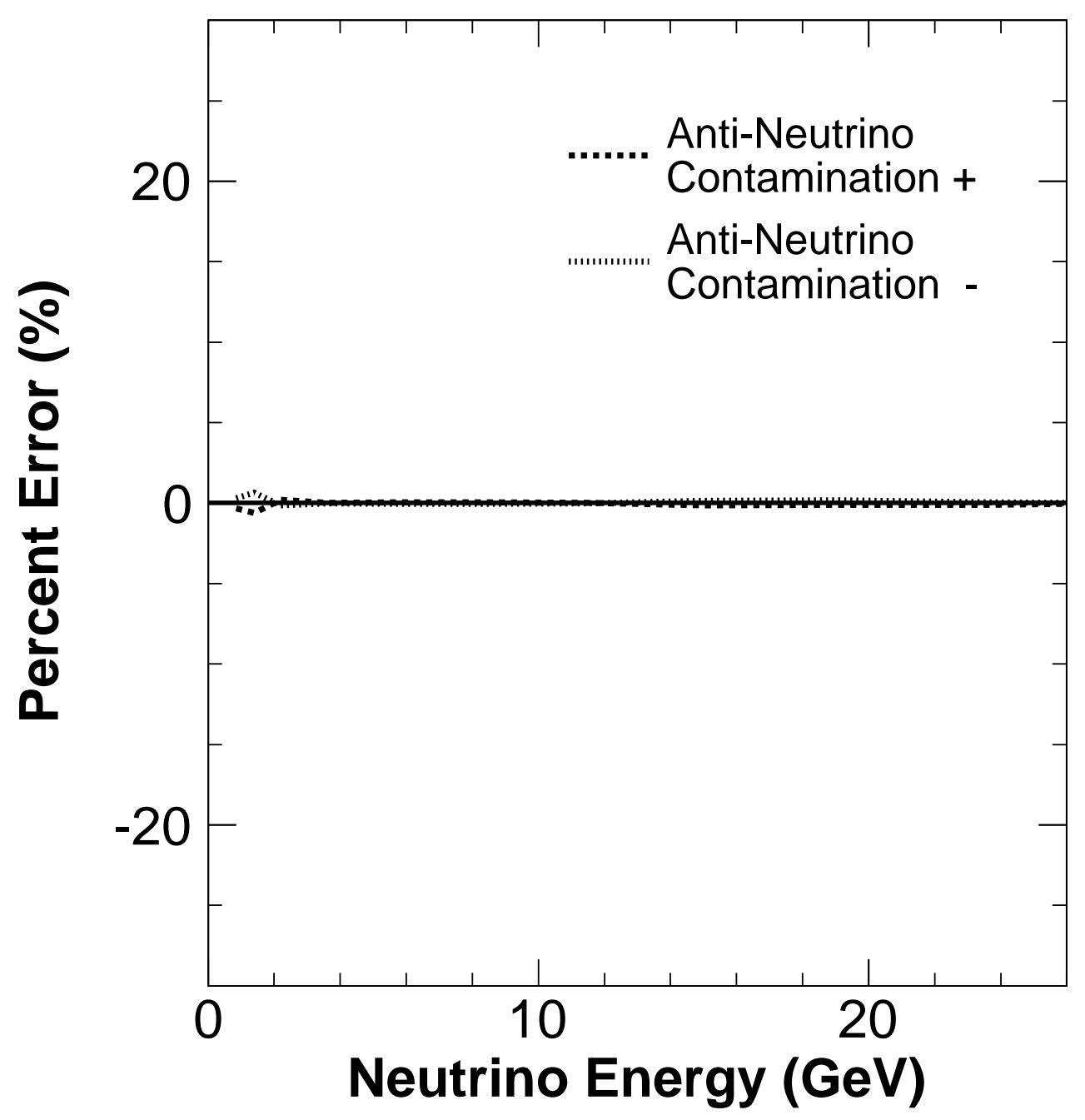

Figure D.5: The percent error on the cross section measurement from increasing (+) or decreasing (-) the number of charged current antineutrino events in the charged current muon neutrino event sample by 100 percent. The + and - curves result from refitting for the cross section after either increasing or decreasing the number of antineutrino events. 


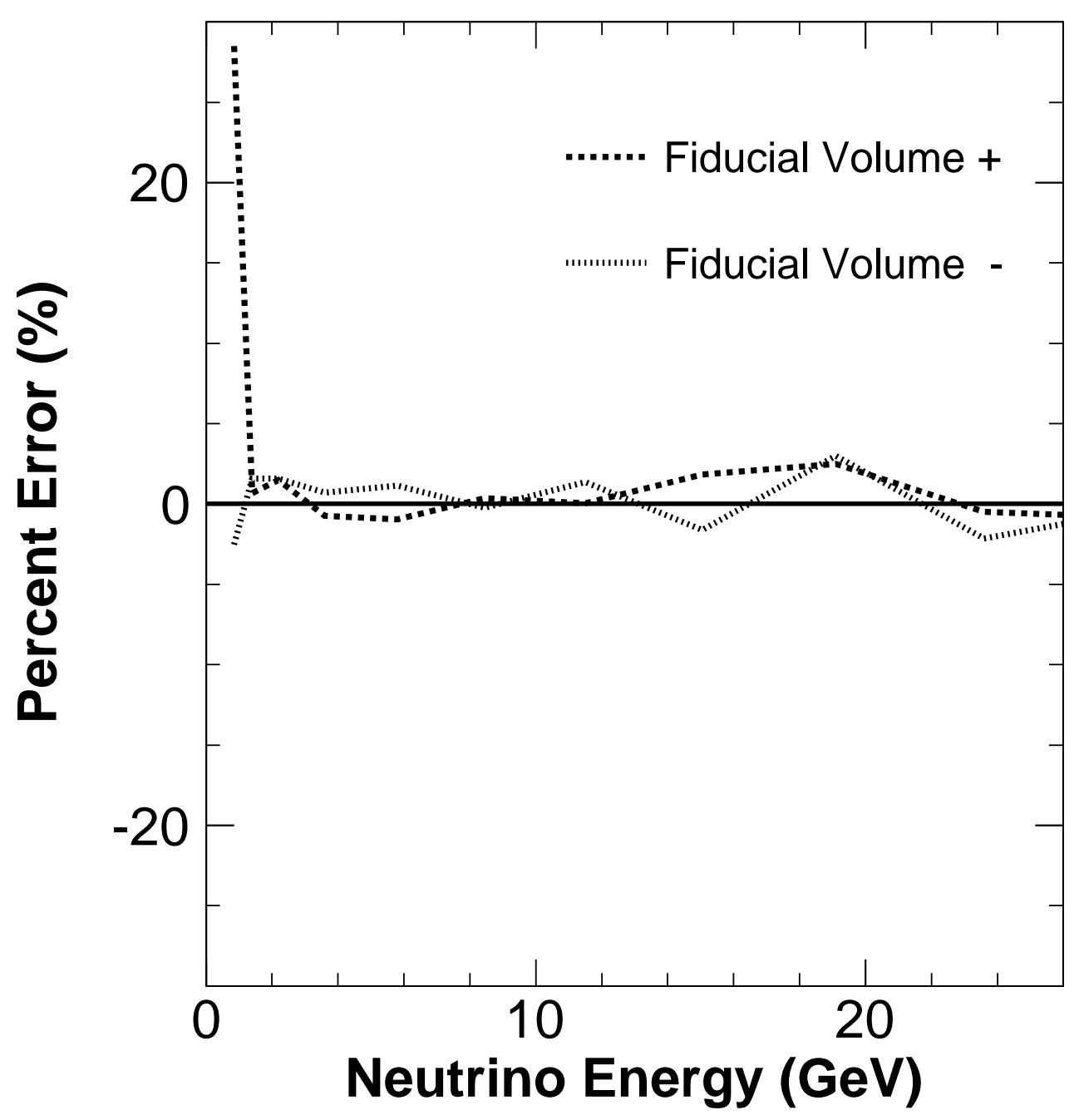

Figure D.6: The percent error on the cross section measurement from increasing $(+)$ or decreasing $(-)$ the fiducial volume by the vertex resolution in the transverse and longitudinal directions percent. Fiducial volume is defined to be a $3.267 \mathrm{~m}$ long and with a radius of $0.8 \mathrm{~m}$. The transverse resolution is $\sim 3$ strips which corresponds to $12 \mathrm{~cm}$ and the longitudinal resolution is 3 planes which corresponds to $18 \mathrm{~cm}$. The + and - curves result from refitting for the cross section after either increasing or decreasing the fiducial volume. 


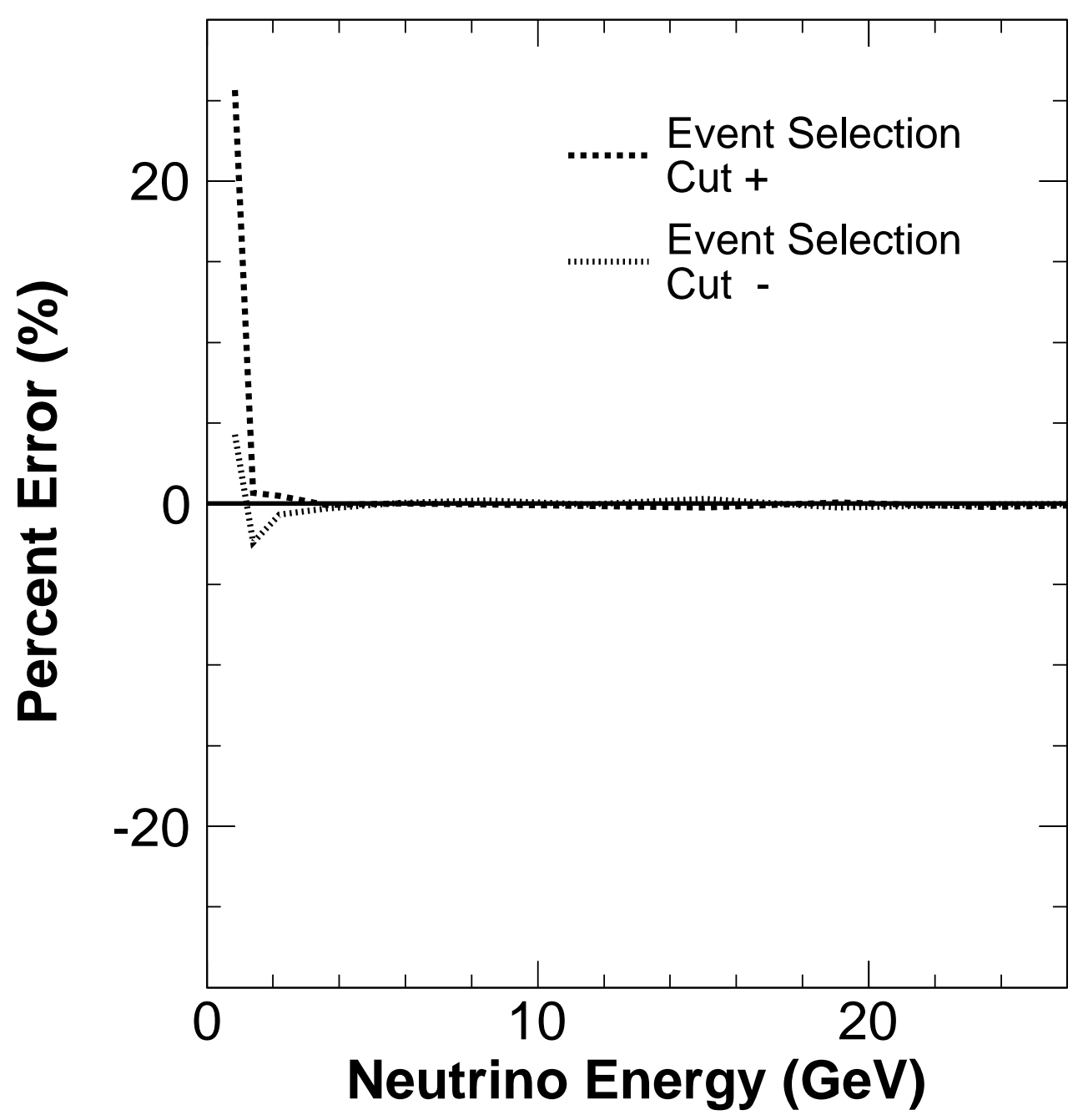

Figure D.7: The percent error on the cross section measurement from increasing $(+)$ or decreasing (-) the charged current event selection cut by 0.1 . The + and - curves result from refitting for the cross section after either increasing or decreasing the cut. 


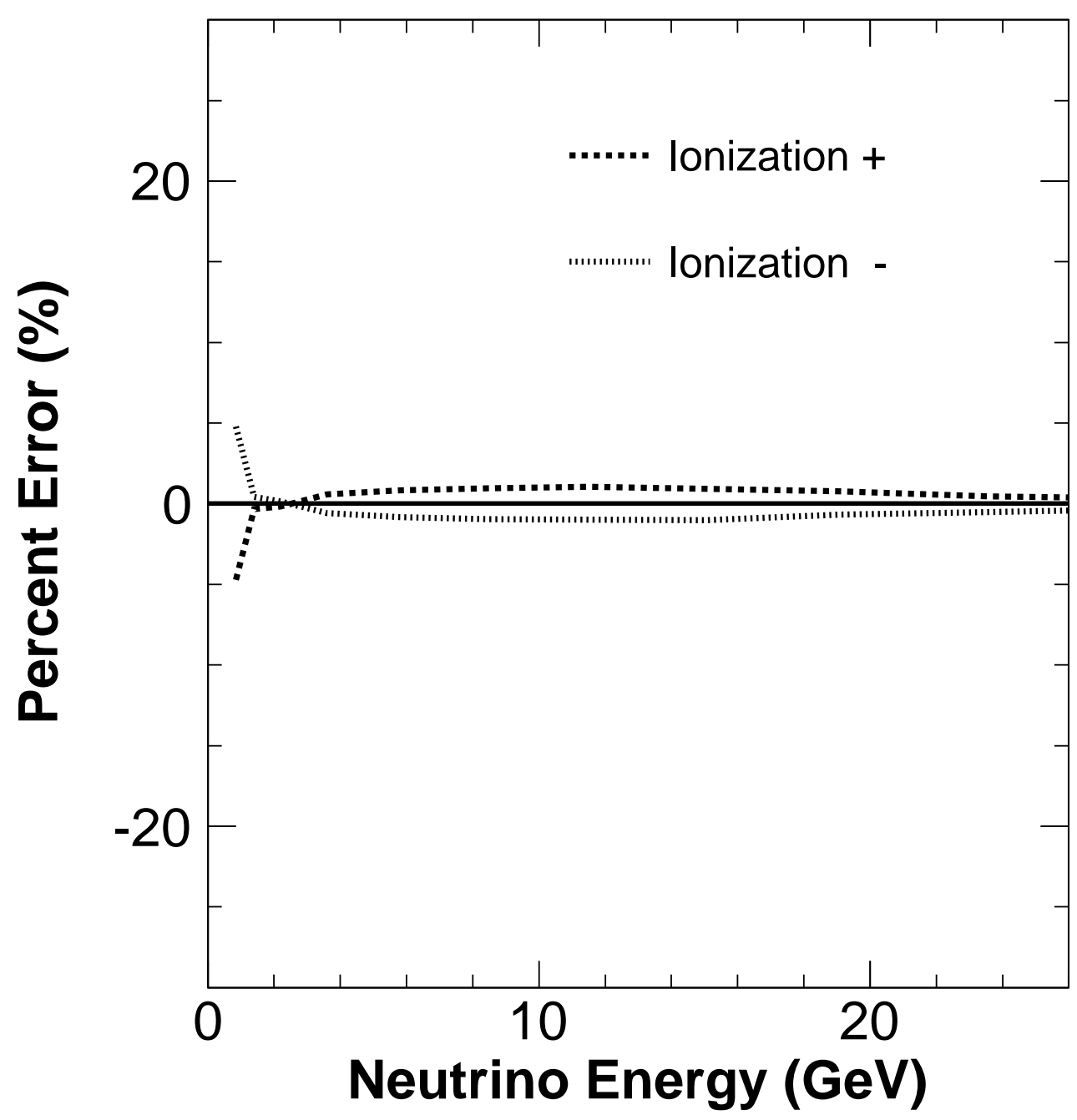

Figure D.8: The percent error on the cross section measurement from increasing $(+)$ or decreasing $(-)$ the muon monitor ionization scale factor by 10 percent. The + and - curves result from refitting for the cross section after either increasing or decreasing the ionization scale factor. 


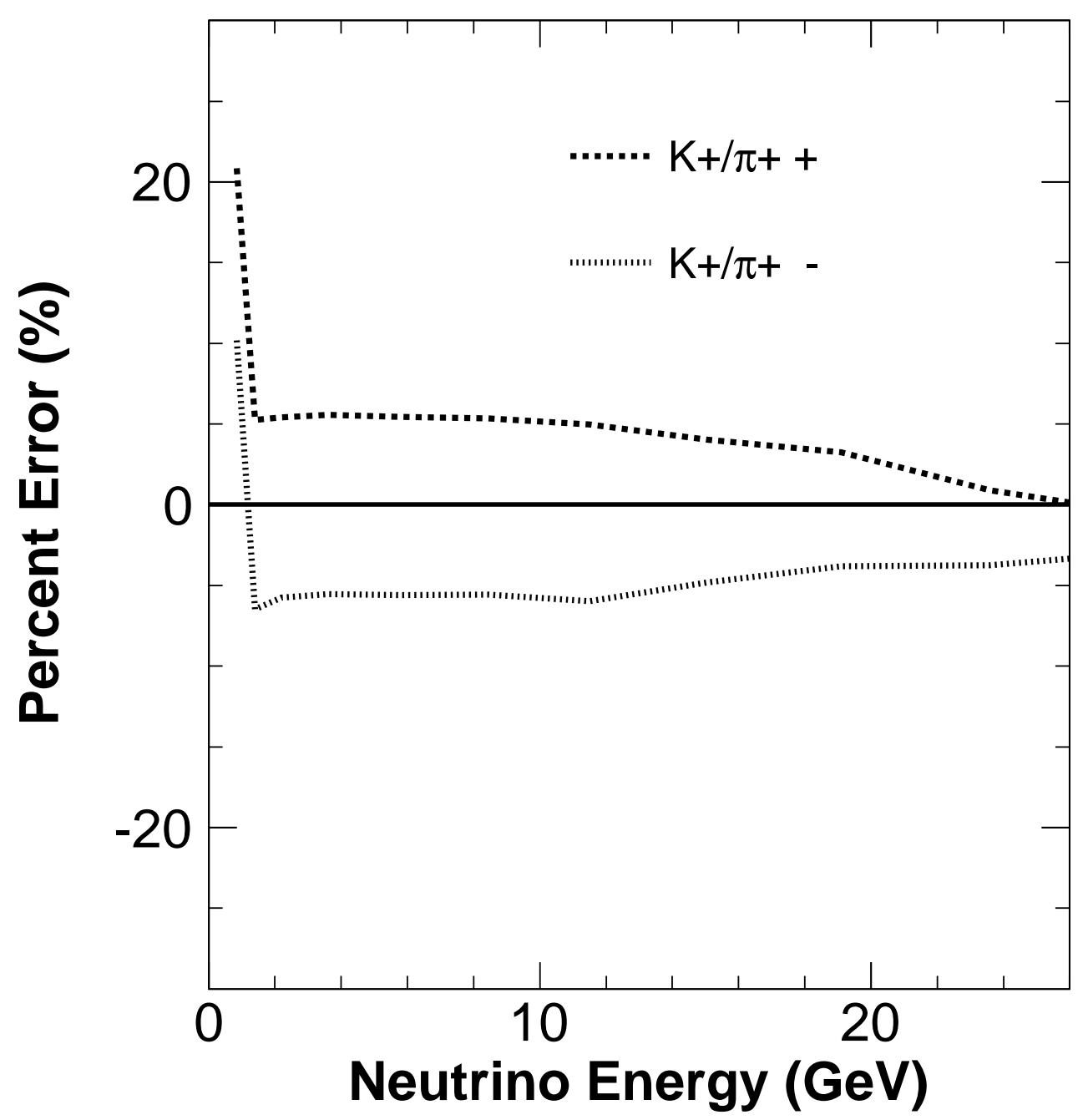

Figure D.9: The percent error on the cross section measurement from increasing $(+)$ or decreasing (-) the Fluka05 $K^{+} / \pi^{+}$ratio by 10 percent. The + and - curves result from refitting for the cross section after either increasing or decreasing the $K^{+} / \pi^{+}$ratio. 


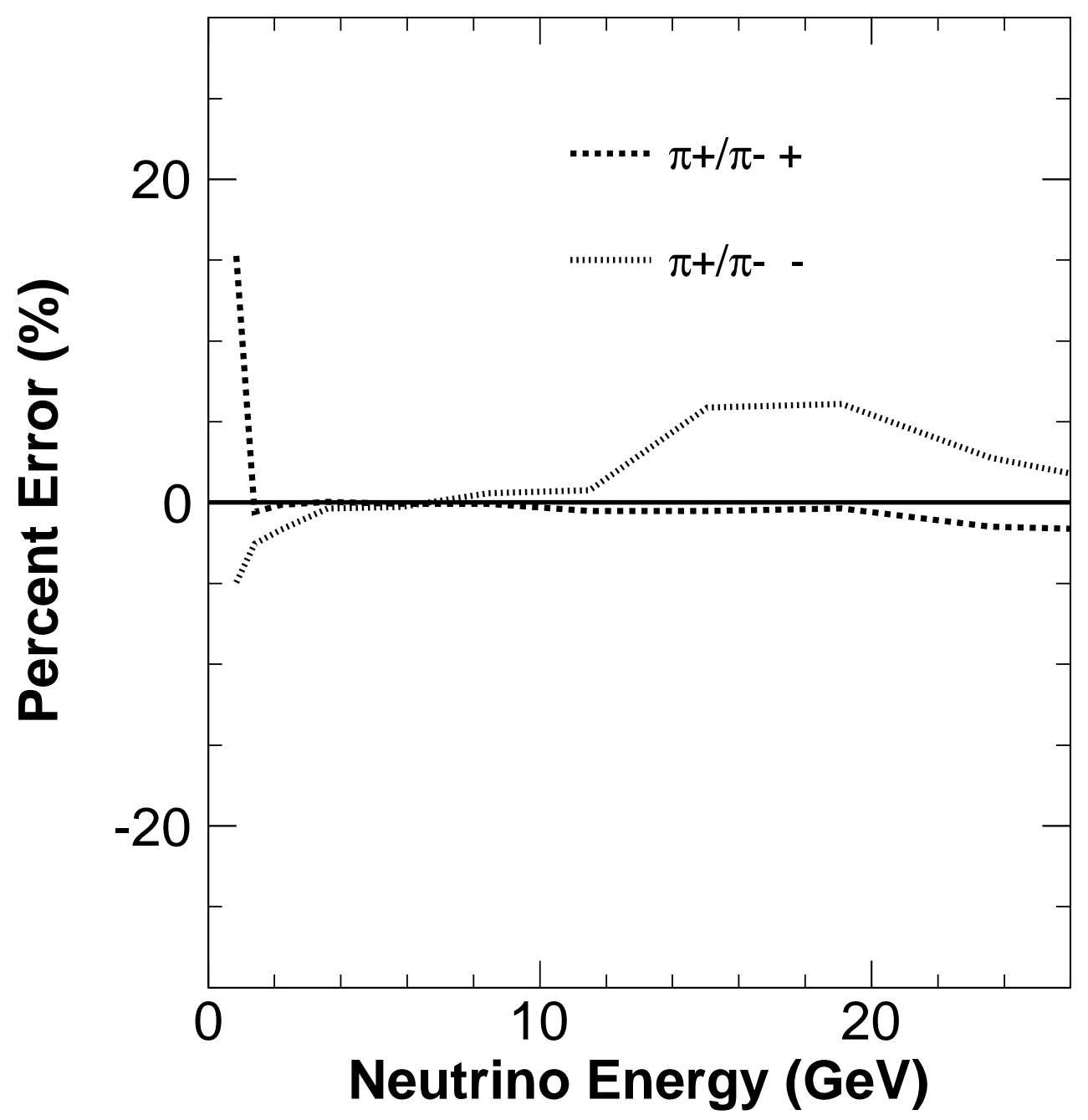

Figure D.10: The percent error on the cross section measurement from fixing the $\pi^{+} / \pi^{-}$ratio to the NA49 measurement fit with a fourth order polynomial $(+)$, as shown in Figure 4.9, or fixing the $\pi^{+} / \pi^{-}$ratio to the Fluka05 $\pi^{+} / \pi^{-}$ ratio (-), also shown in Figure 4.9. The former results in an increase in the $\pi^{+} / \pi^{-}$ratio with respect to nominal(the NA49 measurement fit with a third order polynomial) and the latter results in a decrease in the $\pi^{+} / \pi^{-}$ratio with respect to nominal. The + and - curves result from refitting for the cross section after either increasing or decreasing the $\pi^{+} / \pi^{-}$ratio. 


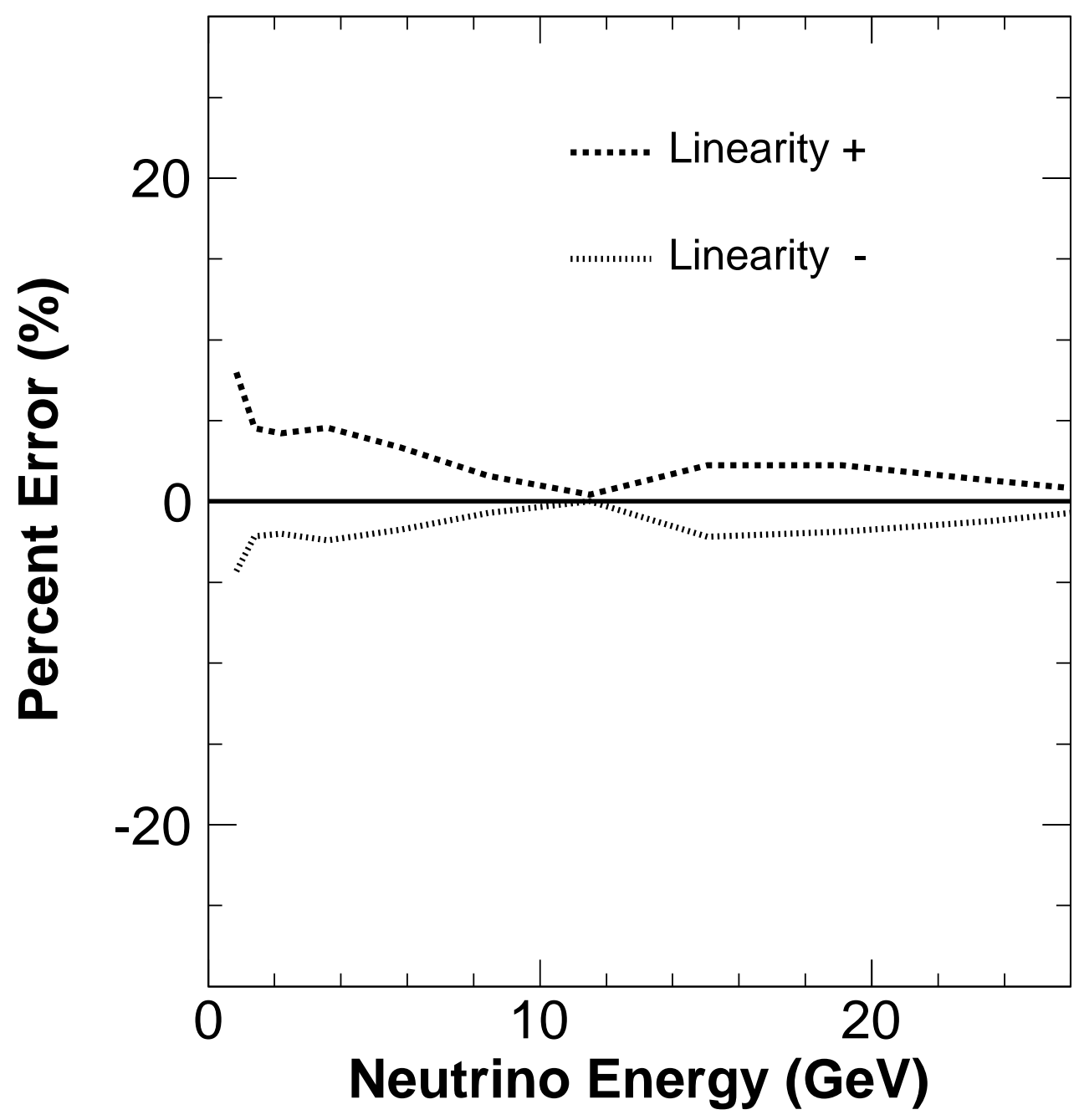

Figure D.11: The percent error on the cross section measurement from increasing $(+)$ or decreasing $(-)$ the muon monitor linearity correction by $1 \sigma$ as discussed in Section 3.1.4. The + and - curves result from refitting for the cross section after either increasing or decreasing the linearity correction. 


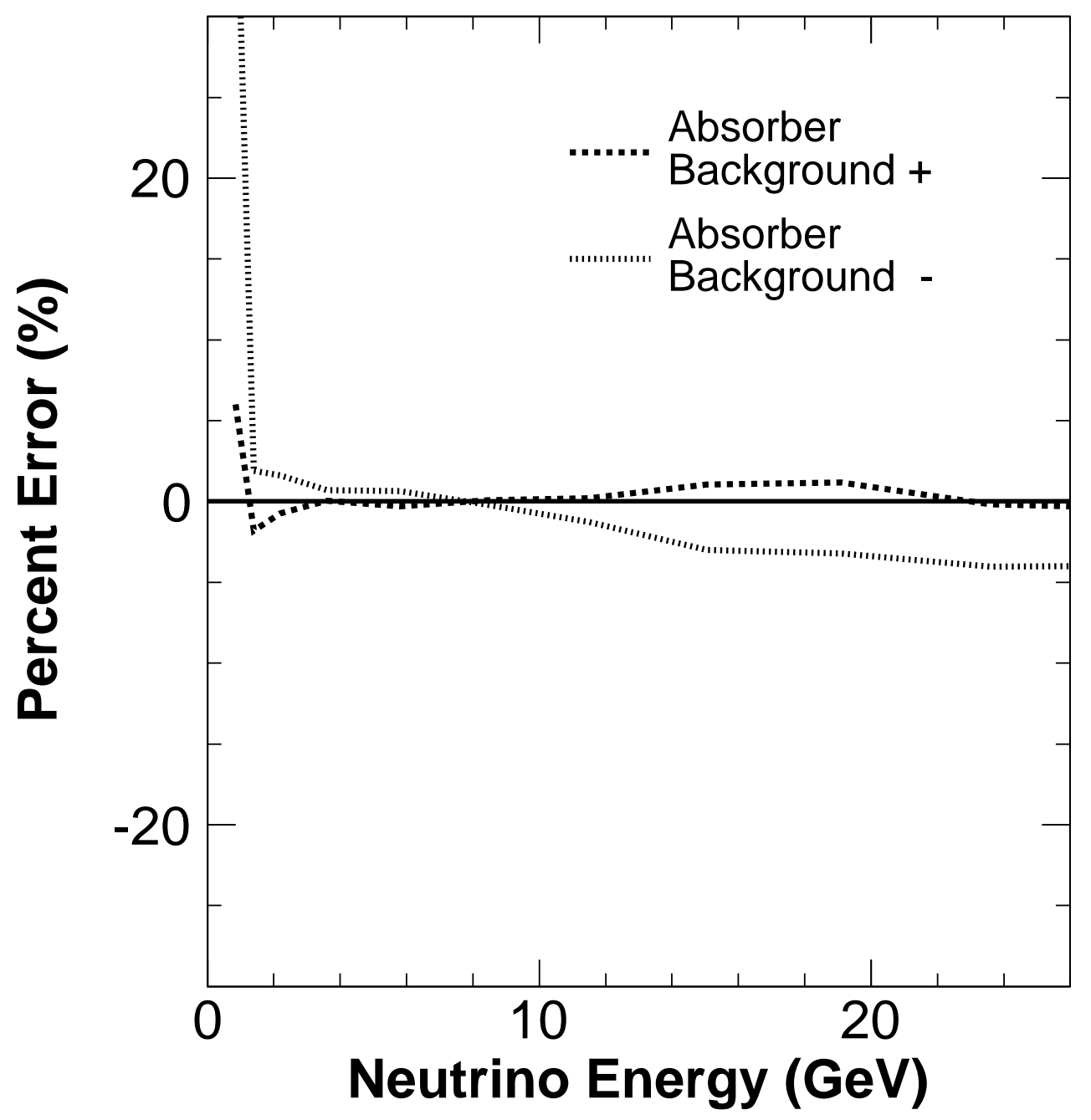

Figure D.12: The percent error on the cross section measurement from increasing $(+)$ or decreasing $(-)$ the absorber backgrounds in each muon monitor by $1 \sigma$ as discussed in Section 3.2. The + and - curves result from refitting for the cross section after either increasing or decreasing the background. 


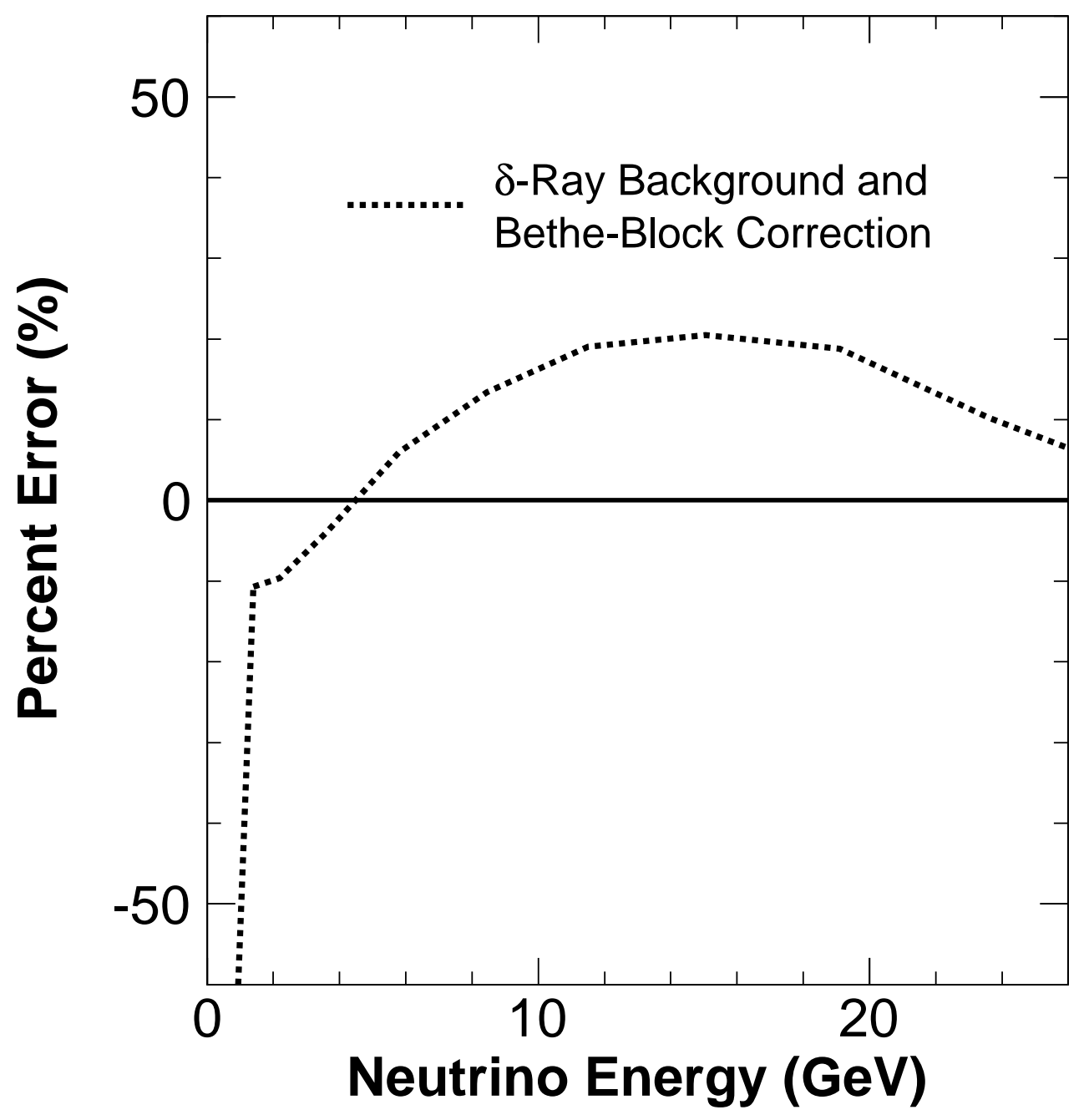

Figure D.13: The percent error on the cross section measurement from applying Bethe-Block to calculate the momentum dependent energy deposition of charged particles in the muon monitors and from including $\delta$-ray backgrounds in the muon monitors. The curve results from refitting for the cross section after applying these changes. 


\section{Bibliography}

[1] L. Brown, "The idea of the neutrino," Physics Today 81 (9) (1978) 23-28.

[2] H. Bethe and R. Peierls, "The "Neutrino"," Nature 133 (1943) 532.

[3] F. Reines and C. L. Cowan, "Free anti-neutrino absorption cross-section. I: Measurement of the free anti-neutrino absorption cross- section by protons," Phys. Rev. 113 (1959) 273-279.

[4] B. C. Barish et al., "Charged Current Neutrino and anti-neutrino Cross-Section Results from the CITFR Experiment," AIP Conf. Proc. 45 (1978) 305-329.

[5] D. H. Perkins, Introduction to High Energy Physics. Addison-Wesley Publishing Company, Inc., 1987.

[6] C. H. Llewellyn Smith, "Neutrino Reactions at Accelerator Energies," Phys. Rept. 3 (1972) 261-379.

[7] MiniBooNE Collaboration, A. A. Aguilar-Arevalo et al., "First Measurement of the Muon Neutrino Charged Current Quasielastic Double Differential Cross Section," arXiv:1002.2680 [hep-ex]. 
[8] K. S. Kuzmin, V. V. Lyubushkin, and V. A. Naumov, "Quasielastic axial-vector mass from experiments on neutrino-nucleus scattering," Eur. Phys. J. C54 (2008) 517-538, arXiv:0712.4384 [hep-ph].

[9] SKAT Collaboration, N. M. Agababyan et al., "The characteristics of neutrinonuclear reactions at $\mathrm{E}(\mathrm{nu})=1-\mathrm{GeV}-3-\mathrm{GeV}, "$ arXiv:hep-ex/0408128.

[10] MiniBooNE Collaboration, J. A. Nowak, "Measurement of the $\nu_{\mu}$ Charged Current $\pi^{+}$Production to Quasi-elastic Scattering Cross Section," AIP Conf. Proc. 1189 (2009) 351-354, arXiv:0909.2915 [hep-ex].

[11] H. Gallagher, "The NEUGEN neutrino event generator," Nucl. Phys. Proc. Suppl. 112 (2002) 188-194.

[12] R. Burns, "Determination of the neutrino flux." CERN Informal Conference on Experimental Neutrino Physics, CERN Yellow Report 65-32.

[13] D. Bloess et al., "Determination of the neutrino spectrum in the CERN 1967 neutrino experiment," Nucl. Instrum. Meth. 91 (1971) 605-612.

[14] H. W. Wachsmuth, "The neutrino spectrum for the CERN 1967 neutrino experiment." Proceedings of the CERN Neutrino Meeting, CERN Yellow Report 69-28.

[15] V. B. Anikeev et al., "Total cross-section measurements for muon-neutrino, anti- muon-neutrino interactions in $3-\mathrm{GeV}-30-\mathrm{GeV}$ 
energy range with IHEP-JINR neutrino detector," Z. Phys. C70 (1996) 39-46.

[16] A. P. Bugorsky et al., "Measurement System of Muon Fluxes for Neutrino Experiment at the IHEP Accelerator," Nucl. Instr. Meth. 146 (1977) 367 .

[17] P. S. Auchincloss, Measurement of the Total Cross Section for Neutrino - Nucleon Interactions. PhD thesis, 1987.

[18] C. L. Wang, "Pion, kaon, and anti-proton production between 10 and 70 bev," Phys. Rev. Lett. 25 (1970) 1068-1072.

[19] K2K Collaboration, M. H. Ahn et al., "Measurement of Neutrino Oscillation by the K2K Experiment," Phys. Rev. D74 (2006) 072003, arXiv:hep-ex/0606032.

[20] MiniBooNE Collaboration, A. A. Aguilar-Arevalo et al., "The Neutrino Flux prediction at MiniBooNE," Phys. Rev. D79 (2009) 072002, arXiv:0806.1449 [hep-ex].

[21] MINOS Collaboration, M. Kordosky and D. Petyt, "Study of Muon Neutrino Disappearance Using the Fermilab Main Injector Neutrino Beam," Phys. Rev. D77 (2008) 072002, arXiv:0711.0769 [hep-ex].

[22] v. Pavlović, Observation of Disappearance of Muon Neutrinos in the NuMI Beam. PhD thesis, The University of Texas, 2008. FERMILAB-THESIS-2008-59.

[23] S. E. Kopp, "Accelerator neutrino beams," Phys. Rept. 439 (2007) 101-159, arXiv:physics/0609129. 
[24] A. Abramov et al., "Calculations and Mapping od the Magnetic Field in the Prototype Horn 1." MINOS internal note 710, 2000.

[25] G. I. Budker. International Conference on Accelerators, Dubna, 1963.

[26] GEANT4 Collaboration, S. Agostinelli et al., "GEANT4: A simulation toolkit," Nucl. Instrum. Meth. A506 (2003) 250-303.

[27] J. Allison et al., "GEANT4 developments and applications," Nuclear Science, IEEE Transactions on $\mathbf{5 3}$ (Feb. 2006) no. 1, 270-278.

[28] K. Anderson et al., "The NuMI Technical Design Report." Fermi National Accelerator Laboratory, December, 2002.

[29] J. Koskinen, "Monte Carlo Description of the Geometry of the NuMI Beam for the Muon Monitors." MINOS internal note 2405, 2006.

[30] S. Kopp et al., "Secondary beam monitors for the numi facility at fnal," Nucl. Instrum. Meth. A568 (2006) 503, arXiv:physics/0607229.

[31] R. M. Zwaska, Accelerator Systems and Instrumentation for the NuMI Neutrino Beam. PhD thesis, The University of Texas, 2005. FERMILAB-THESIS-2005-73.

[32] D. Indurthy, S. Kopp, and Ž. Pavlović, "Systematic Uncertainties in the NuMI Beam Flux." MINOS internal note 1283, 2007.

[33] MIPP Collaboration, R. Webber et al., "Fermilab Recycler Ring BPM Upgrade Based on Digital Receiver Technology," AIP Conf. Proc. 732 (2004) 190-200. 
[34] S. Kopp et al., "Beam Test of a Segmented Foil SEM Grid," Nucl. Instrum. Meth. A554 (2005) 138, arXiv:physics/0507211.

[35] R. Zwaska et al., "Beam-Based Alignment of the NuMI Target Station Components at FNAL," Nucl. Instrum. Meth. A568 (2006) 548, arXiv:physics/0609106.

[36] MINOS Collaboration, D. G. Michael et al., "The Magnetized steel and scintillator calorimeters of the MINOS experiment," Nucl. Instrum. Meth. A596 (2008) 190-228, arXiv:0805.3170 [physics.ins-det].

[37] R. Ospanov, A measurement of muon neutrino disappearance with the MINOS detectors and NuMI beam. PhD thesis, The University of Texas, 2008. FERMILAB-THESIS-2008-04.

[38] T. H. Osiecki, A search for sterile neutrinos in MINOS. PhD thesis, The University of Texas. FERMILAB-THESIS-2007-77.

[39] S. Dytman, H. Gallagher, and M. Kordosky, "Systematic effects in MINOS: Nuclear effects and hadronic energy scale uncertainty," PoS NUFACT08 (2008) 041.

[40] M. A. Kordosky, Hadronic interactions in the MINOS detectors. PhD thesis, The University of Texas, 2004. FERMILAB-THESIS-2004-34.

[41] P. L. Vahle, Electromagnetic interactions in the MINOS detectors. PhD thesis, The University of Texas, 2004. FERMILAB-THESIS-2004-35.

[42] P. Adamson et al., "The MINOS calibration detector," Nucl. Instrum. Meth. A556 (2006) 119-133. 
[43] J. J. Hartnell, Measurement of the calorimetric energy scale in MINOS. $\mathrm{PhD}$ thesis, The University of Oxford, 2005.

FERMILAB-THESIS-2005-51.

[44] K. Lang and R. Ospanov, "A muon identification technique using the k-nearest neighbor algorithm." MINOS internal note 2737, 2008.

[45] R. M. Zwaska et al., "Beam tests of ionization chambers for the numi neutrino beam," IEEE Trans. Nucl. Sci. 50 (2003) 1129-1135, hep-ex/0212011.

[46] J. McDonald et al., "Ionization chambers for monitoring in high-intensity charged particle beams," Nucl. Instrum. Meth. A 496 (2003) 293-304.

[47] R. Keisler, "Neutron Induced Ionization in the NuMI Beam Monitors," 2005. Undergraduate Thesis.

[48] A. Fasso', A. Ferrari, J. Ranft, and P. Sala, "FLUKA: a multi-particle transport code." CERN 2005-10 (2005), INFN/TC_05/11, SLAC-R-773.

[49] R. Brun et al., "GEANT3 manual." CERN Program Library Long Writeup W5013, 1994. CERN Program Library Long Writeup W5013.

[50] H. W. Atherton et al., "Precise Measurements of Particle Production by 400-GeV/c Protons on Beryllium Targets,". CERN-80-07.

[51] D. S. Barton et al., "Experimental Study of the a-Dependence of Inclusive Hadron Fragmentation," Phys. Rev. D27 (1983) 2580. 
[52] NA56/SPY Collaboration, G. Ambrosini et al., "Measurement of charged particle production from $450-\mathrm{GeV} / \mathrm{c}$ protons on beryllium," Eur. Phys. J. C10 (1999) 605-627.

[53] NA49 Collaboration, C. Alt et al., "Inclusive production of charged pions in $\mathrm{p}+\mathrm{C}$ collisions at $158-\mathrm{GeV} / \mathrm{c}$ beam momentum," Eur. Phys. J. C49 (2007) 897-917, hep-ex/0606028.

[54] NA49 Collaboration, C. Alt et al., "Inclusive production of charged pions in $\mathrm{p}+\mathrm{C}$ collisions at $158-\mathrm{GeV} / \mathrm{c}$ beam momentum," Eur. Phys. J. C49 (2007) 897-917, hep-ex/0606028.

[55] MIPP Collaboration, J. Paley, "First Results from MIPP," AIP Conf. Proc. 981 (2008) 154-156.

[56] A. Fasso, A. Ferrari, J. Ranft, and P. Sala, "FLUKA: Present status and future developments,". Given at 4th International Conference on Calorimetry in High-energy Physics, La Biodola, Italy, 19-25 Sep 1993.

[57] N. V. Mokhov, The MARS Code System User's Guide. Fermilab-FN-628 (1995).

[58] M. Bonesini, A. Marchionni, F. Pietropaolo, and T. T. de Fatis, "On particle production for high energy neutrino beams," Eur. Phys. J. C20 (2001) 13-27, hep-ph/0101163.

[59] A. J. Malensek, "Empirical Formula for Thick Target Particle Production,". FERMILAB-FN-0341.

[60] R. P. Feynman, "Very High-Energy Collisions of Hadrons," Phys. Rev. Lett. 23 (Dec, 1969) 1415-1417. 
[61] E. Yen, "New scaling variable and early scaling in single-particle inclusive distributions for hadron-hadron collisions," Phys. Rev. D10 (Aug, 1974) 836-843.

[62] F. E. Taylor et al., "Analysis of radial scaling in single-particle inclusive reactions," Phys. Rev. D14 (Sep, 1976) 1217-1242.

[63] S. Kopp, Žarko Pavlović, and P. Vahle, "Fitting the Beam MC to the ND Data." MINOS internal note 1548, 2006.

[64] M. Kostin et al., "Proposal for continuously-variable neutrino beam energy for the NuMI facility,". FERMILAB-TM-2353-AD.

[65] I. Abt and B. Jongejans, "An Absolute Calibration of the Solid State Detectors in the Narrow Band Neutrino Beam at CERN," Nucl. Instr. Meth. A235 (1985) 85.

[66] E. Heijne, "Influence of muon induced secondary radiation on the muon flux measurement in the CERN neutrino beams." CERN-79-4, 1979.

[67] Particle Data Group Collaboration, W. M. Yao et al., "Review of particle physics," J. Phys. G33 (2006) 1-1232.

[68] CCFR/NuTeV Collaboration, U. K. Yang et al., "Extraction of $\mathrm{R}=$ $\operatorname{sigma}(\mathrm{L}) / \operatorname{sigma}(\mathrm{T})$ from CCFR nu/mu Fe and anti-nu/mu Fe differential cross sections," Phys. Rev. Lett. 87 (2001) 251802.

[69] CDHSW Collaboration, P. Berge et al., "A measurement of differential cross-sections and nucleon structure functions in charged-current neutrino interactions on iron," Z. Phys. C49 (1991) 187-223. 
[70] J. Boehm, "TrackLike Plane and VertexFinder Fix for Cedar." MINOS internal note 2661, 2007.

[71] NOMAD Collaboration, Q. Wu et al., "A Precise Measurement of the Muon Neutrino- NucleonInclusive Charged Current Cross-Section off an IsoscalarTarget in the Energy Range $2.5<\boldsymbol{E}_{\boldsymbol{\nu}}<\mathbf{4 0} \mathrm{GeV}$ by NOMAD," Phys. Lett. B660 (2008) 19-25, arXiv:0711.1183 [hep-ex].

[72] D. Bhattacharya, Neutrino and antineutrino inclusive charged-current cross section measurement with the MINOS near detector. PhD thesis. FERMILAB-THESIS-2009-11.

[73] S. Wojcicki, "Neutrino oscillation experiments using accelerators and reactors,". Given at 25th SLAC Summer Institute on Particle Physics: Physics of Leptons (SSI 97), Stanford, CA, 4-15 Aug 1997. 


\section{Vita}

Laura Jean Loiacono was born in Melrose Park, Illinois on August 27, 1981 to James and Donna Loiacono. After graduating from Victor J. Andrew High School in Tinley Park, Illinois in 1999, she attended Loyola University Chicago in Chicago, Illinois to study physics. During the summer she worked as an Undergraduate Research Assistant at the Advanced Photon Source at Argonne National Laboratory. In 2004, she graduated summa cum laude with a Bachelor of Science in physics and started as a graduate student in the Department of Physics at the University of Texas at Austin the same year. She began working on the MINOS experiment and NuMI beamline in 2006. In 2009, she earned a travel grant to attend the United States Particle Accelerator School held in Albuquerque, New Mexico.

Permanent Address: 3115 Helms Street,

Austin, TX 78705

This dissertation was typeset with $\operatorname{AT}_{\mathrm{EX}} 2_{\varepsilon}{ }^{1}$ by the author.

\footnotetext{
${ }^{1} \mathrm{LAT}_{\mathrm{E}} \mathrm{X} 2_{\varepsilon}$ is an extension of $\mathrm{LT}_{\mathrm{E}} \mathrm{X}$. $\mathrm{IAT}_{\mathrm{E}} \mathrm{X}$ is a collection of macros for $\mathrm{T}_{\mathrm{E}} \mathrm{X}$. $\mathrm{T}_{\mathrm{E}} \mathrm{X}$ is a trademark of the American Mathematical Society. The macros used in formatting this dissertation were written by Dinesh Das, Department of Computer Sciences, The University of Texas at Austin, and extended by Bert Kay, James A. Bednar, and Ayman El-Khashab.
} 